

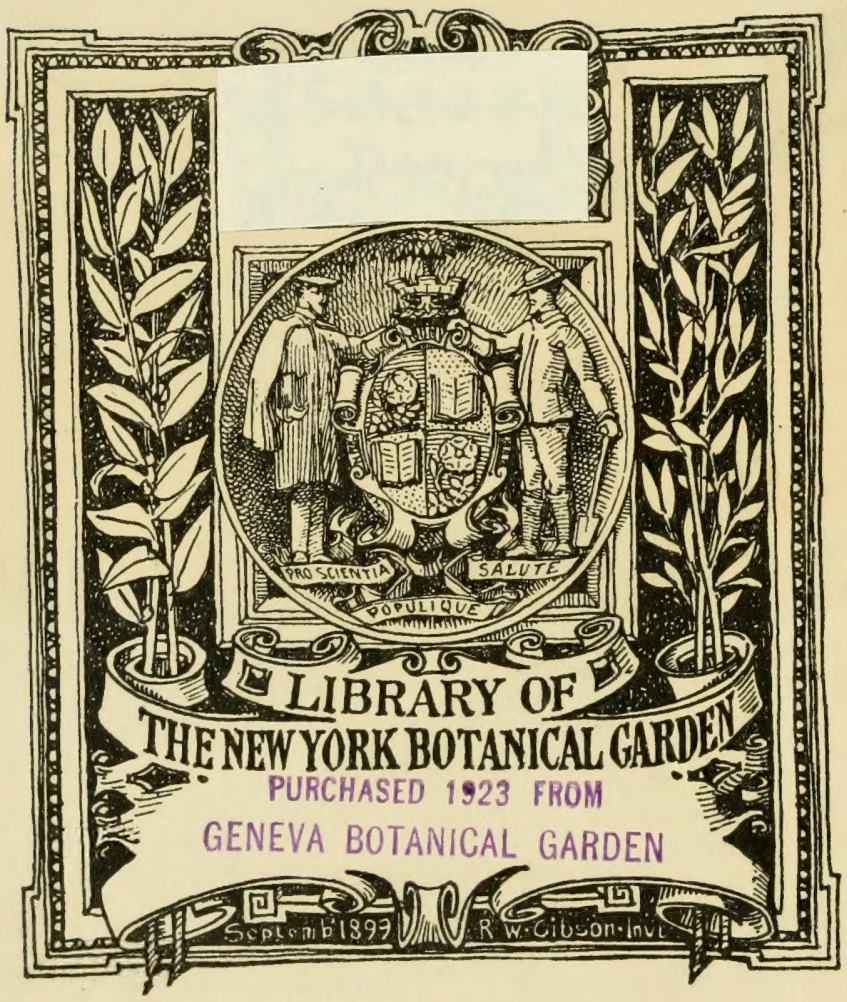




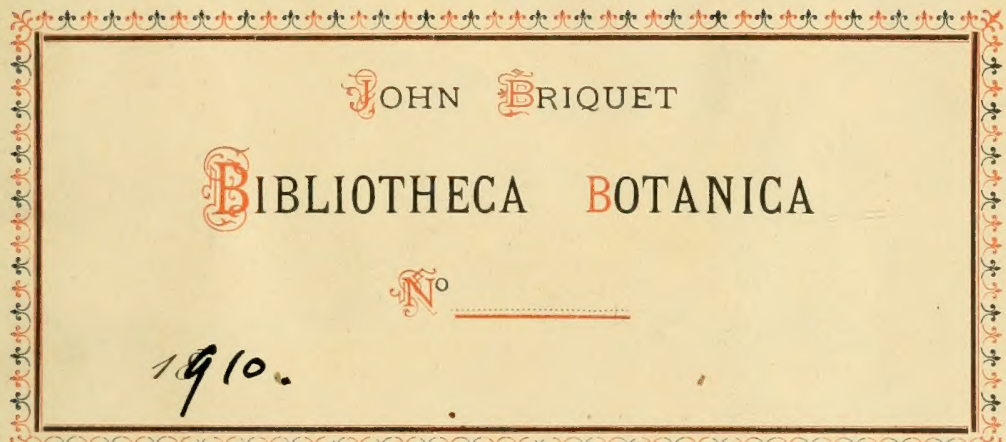

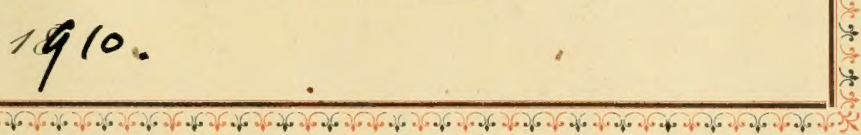

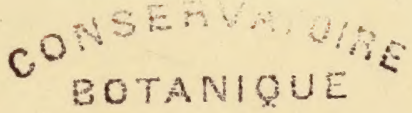

$-1-2 \times 3=$

VILL a beVlid

DUPLICATA DE LA BIBLIOTHÊQUE DU CONSHRVATOIRE BOTANIQUE DE GENEVF VENDU FN 1822 

CATAlOgue RAISONNÉ

DES ESPĖCES DU GENRE ROSIER. 



\section{CATALOGUE RAISONNÉ}

ou

ÉNUMÉRATION MÉTHODIQUE

DES ESPECES DU GENRE ROSIER

POUR

L'EUROPE, L'ASIE ET L'AFRIQUE

SPÉCIALEMENT

LES ROSIERS DE LA FRANCE ET DE L'ANGLETERRE

$$
\text { Pierre Par }
$$

M. Alfred DÉSÉGLISE

membre de plusieurs sociétés savantes franẹaises et étrangères

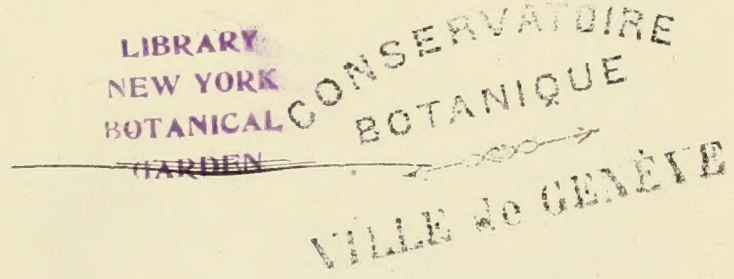

\section{GENEVE}

LIBRAIRIE DE CH. MENTZ, 2, PLACE DU MOLARD.

1877

DUPLICATA DE LA BIBLIOTHÊQUE DU CONSERVATOIRE BOTANIQUE DE GRNEVE VENDU EN 1922 
Extrait du tome XV (1876) du Bulletin de la Société royale de Botanique de Belgique. pp. 176-403,491_586 


\title{
A MONSIEUR
}

\section{Alexandre BOREAU,}

PROFESSEUR ET DIRECTEUR DU JARDIN BOTANIQUE D"ANGERS.

\author{
A MONSIEUR LE DOCTEUR
}

\section{Eugène RIPART,}

A BOURGES (CHER).

Témoignage d'une profonde reconnaissance, d'un sincère dévouement et hommage d'une estime sans bornes.

Alfred DÉSÉGLISE. 

Res maturales froprio privilegio munite persistunt. ut quedmadmodum errores in his commissi a nullo defendi, ila nec veritates obscrvationibus innixa, a tolo erudiorum orbe conculari possunt.

(LiNe, Sp. plant. (1762), praef.).

Les espèces paraissent être le but de la nature, comme elles sout l'objet de nos recherches, de nos methodes et de nos observations botaniques.

(VILLAns, Cat. de Strasbourg (1807), p. XXIX).

II sertil temps en effet que le juste respect pour l'autoriti de Linné, n’alłát pas jusqu'au fétichisme, et jusqu'à faire considérer même ses erreurs pour des artieles de foi.

(Plavenoy, Ann. se, nat., XVIII, p. 359).

Éfudier les plantes pour arriver à distinguer et à séparer tout ce que la nature elle-mème a séparé, en ohzervant en meme temps tous les points de contact (1)'ont mure rlles les diverses espèces, c'est ramener la science à son bul.

(Jondax, Asphodeles, Sess. extraord. de ha Soc. bot. de France à Grenoble, VH, p. 72S).

D'abord peu nombreuses et mal définies, les espèces du genre Rosier, mieux observées, ont pris, dans ces derniers temps, dans leur nombre un aecroissement considérable. Il m'a semblé qu'il ne serait pas sans intérèt aujourdhui de réunir et de coordonner de nouveau les faits épars dans différents écrits ou disséminés dans les grands herbiers, de former un tableau unique de toutes nos comnaissances sur les rosicrs, et d'y ajouter quelques réllexions que l'étude des auteurs el l'examen des types authentiques m'ont suggérées. 
C'est sans doute une grande témérité de vouloir, dans l'état actuel de la seince, dresser l'inventaire des nomhreuses espèces du genre Rosier, qui eroissent en Europe, en Asie et en Afrique. Leur nombre, qui va en augmentant chaque amnée, ne fera quaccroitre de plus en plus la confusion qui existe déjà dans leur nomenclature; mais les herbiers et les vastes bibliothèques de. MIM. De Candolle et Boissier, ont considérablement facilité mes recherehes; jy ai trousé une foule de doenments que j'eusse vainement cherehés ailleurs. Je viens exprimer de nouveau it MNI. De Candolle et Boissier, ma vive reconnaissance pour tous les renseignements qu'ils ont bien roulu mettre à ma disposition durant la rédaction du présent mémoire.

Selon que l'esprit de synthèse ou d'analyse prédomine dans la pensée des hommes qui s'oceupent de la fixation des espèces hotaniques, on voit le nombre de celles-ci diminuer on grandir. De ce point de départ apparaissent deux écoles, dont l'une est pour la multiplication des espèces, tandis que l'autre, poussée par la tendance contraire, tend sans cesse à en restreindre le nombre. Le travail le meilleur sera celui qui se trouvera le plus conforme ì la nature, et quand on voit les ètres tels qu'ils sont on est toujours dans le vrai.

Ne nous hâtons pas trop de généraliser, mais observons ineessamment et soyons convaincus qu'il n'est pas de fait particulier de si minime importance qu'il semble d'ahord, qui ne se rattache ì des lois naturelles. Je suis persuadé que le plus humble observateur peut, à l'aide d'une expérimentation très-simple, arriver it résoudre bien plus surrement une question queleonque d'espèce que le savant le plus distingué, qui, en portant son jugement sur la méme question, scrait disposé à ne tenir aucun compte de l'expérience. 
Le savant de nos jours ne s’éleve pas à des idées générales, en posant son pied sur le terrain mobile des hypotheses, mais en l'appuyant sur des fatts précis dont il déduit des eonséquences aussi rig̨oureuses que le permettent ses méthodes d'observation.

L'habitant des grandes villes condamné à étudier beaneoup plus en herbice que sur la nature vivante, se fait de l'espece une ide tout autre que l'observateur des champs. Il se forme de chaque espece un type idéal plus ou moins large, selon le nombre et l'état des spécimens qu'il a pu voir dans les herbiers.

Il faut dire aussi que bien des noms donnés comme nonveaux dans ces derniers temps, existaient depuis longtemps dans l'histoire de la bohanique, ignorés de la plupart des auteurs qui s'oceupent de faire des flores; l'érudition semble leur faire défant; eependant il y a un live qui peut les édilier sur les noms spéciliques publićs anciennement : c'est Steudel, Tomenclator botanicus. Ce sont de ces crreurs qui peurem itre passées à un jeune botaniste sans expérience, car sourent outre son defaut de jugement, les lives nécessaires lui manquent, et les types authentiques sont une lacune dans son herhicr ; mais, si ses erreurs doivent lui ère pardomées, en admettant que la science veuille bien consacrer l'usage des cirronstemes attemuntes, il ne doit pas en etre de meme pour celui on pour ceux qui ont un nom faisant autorici dans la science, car l'expérience est fille di temps et elle ne s'acepuiert qu'avec le nombre des années.

I ne flore est plus difficile a farre qu'une monographic ; puis nest-il pas du devoir du floriste comme dı monoEraple de mentionner tout ee qui a été écrit avant cux sur les questions quils traitent? Si on n'est pas à meme 
de distinguer l'espece d'un auteur, pourquoi la passer sous silence et croire qu'on se défait faeilement des espèces eritiques au moyen d'un oubli volontaire et qu'un trait de plume suffit pour rayer une espece de la nature? C'est une grave erreur : la vérité se fait toujours voir dans un temps plus ou moins éloigné; Ies faits existent quand mème et l'observation haséc sur l'hypothese est un non-sens.

In systime ctabli miguement sur des hypotheses of en dehors de faits spéciaur n'a pour hase que l'irlée d'm possible dont le contraire est possible an méme degré; et, dans de telles conditions, ce système est place sur l'extrème hord d'une pente glissante et rapide qui peut aboutir à l'absurde. La seience vit de certitudes ot non de possibilites. Alors on cherchera à prower par des théries inacceptables, puisqu'elles sont l'opposé des faits, que It $\boldsymbol{R}$. canina passe au $\boldsymbol{R}$. andegurensis, puis aux formes velues, de là au $\boldsymbol{R}$. tomentosa, puis au $\boldsymbol{R}$. rubiginosa, ef enfin finit par produre le $\boldsymbol{R}$. arensis et le $\boldsymbol{R}$. sempervirens. Wallroth, dans son II istoria Rosarum, a dejja fait une hesogne analogue ef personne n'a écouté Wallroth, sort réservé à fous ceux qui voudront condenser ce que la nature a séparé !

Je dirai aux partisans de cette école qu'ils feraient beancoup mieux de nadmetre que deux types dans les Rosiers : un samage qu'ils pourraient appeler $R$. sylecstris et l'autre cultivé, le $R$. saliea : ce qui a deja éte proposé il y a près de cinquante ans. Cette méthode de reduction a l'avantage de simplifier heaucoup les choses; mais je erois que ceux qui, dans la science, cherehent avant tout la verité, seront d'avis comme moi que les décisions de la fantaisie on de l'inexpérience ne méritent pas qu'ons'y arrête. 


\section{(11)}

Si l'école progressive a des lorts, je puis dire que l'école stationnaire en a de plus grands en negligeant de faire connaitre ce qui existe ef en traitant trop ligerement une foule de questions dont la solution de sa part est loin de présenter l'exactitude mène. C'est ec délain magristral qui fait dire anx jemes debutants phytolog̣istes: Que les types anciens ne sont d'aucune importance ì notre ciporpue, ces ricilles esperes elant plutot un amas confus de

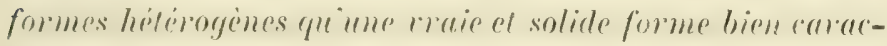
térisée, en sorte qu'on ne doit en tenir compte que d'une manière tout it fail sefondaire. Alors à quoi sert d'écrire tant de volumes si, par l'arrèt de la jeunesse, les vieux peres ne doivent plus itre comsultés? Pourquoi créer romme il l'envi des genres méconmaissables et des especes qui le sont plus encore? Gloriole, vanite, oceupations frivoles, suns ectat pour leur auteur et sans profit pour la science!

Vier et expliquer sont denx choses différentes, dont l'une ne remplace jamais l'autre : la negation est individuelle et laisse toujours le fait rebelle et incompatible; lexplication est collective ef soumet le fait au systeme général de la science positive. Tout en cherchant à rendre la botanique aussi facile que possible, cherchons avant tout a la rendre exacte et précise, persuadés que ac sont li les bases de la méthode qu'on doit suive pour délimiter les espèces.

Je dis que, quand une descriphion est compréhensible, ou que, d'autre part, ì l'aide d'un type authentique ou ì la vue d'une home grarure on recommais une plante, on doit adopter le nom imposé par son inventeur et non le rejeter par bon plaisir? Ces chicanes d'éeoles doivent ètre abandonnees. Le val savant romme le simple amateur ne 
demandent que la vérité et des faits présentés sans charlatanisme.

II ne suffit pas pour faire mo monographic de réunir des especes en plus ou moins grand nombre, supposant mème qu'on les distribue le plus méthodiquement possible; ce n'est point l'unique ohjet auquel doit s'attacher celui qui se livre à l'éfude spéciale d'un genre de plantes. Il doit etre historien, faire commatre toutes les particularités remarquables que peut offrir ce genre; foules les considerations doivent itre passérs en revue. Le hotaniste vraiment digne de ce nom n'a d'autre mobile que la recherche de la vérité. Il est en garde contre l'esprit de rouline. Il ne craint pas les mauraises especes, cest-idire celles qui n'existent pas dans la nature; il sait qu'elles servent à confirmer les bonnes, celles qui sont. Il n'est pas donné à l'homme de pouroir afteindre juscüà l'essence des choses, jusqu'aux espèces. Tous ses efforts en ce sens sont impuissants ou its ne produisent que des oeuvres frappées de stérilité.

Quant aux hybrides, ils peuvent ifre contestés, du moins la majeure partie des hybrides qui ont été crées lans ces derniers temps et sur lesquels leurs auteurs ne sont nullement d'aceord. Les hybridolitres sont à la botanique ce que les homéopathes sont à la médecine. Des plumes beaucoup plus puissantes que la mimue, se sont ocenpées de jeter l'anatheme contre nue nomenclature spécilique aussi barbare que contraire aux lois de la mature, celle de Schiede, qui fut le principal promoteur des hybrides spontanés; depuis lors des imitateurs fanatiques lui ont suceédé. M. Ch. Des Moulins, ce patient observateur, dans le Supplement final de son Calalogue de la Dordogne (1859), dit : "L'hybrilite spontaner est 
"possible, puisque nous pratiquous l'hybridation arti" licielle; mais celte hybridité spontanéc doit ètre rare, " sinon dans "In ertain nomlıre de genres déterminés, " du moins eu égard à l'ensemble du règne végétal. "Céte rareté proportionnelle est démontrée par la fixité " bien constatée d'un nombre immense d'espèces. " Or, si cette fixité n'était par la règle générale, "sujette à un petit nombre seulement d'exceptions, " la loi en un mot, - tout, depuis les temps historiques, "serait confusion dans le rigne végétal. Or encore, la " confusion n'est pas, ne doit pas, ne peut pas ètre la " loi dans les ourres de la suprème Sagesse. Tout est "réglé dans l'univers; tout doit ère réglé dans ehaeune " de ses parties. Confusion et loi sont deux idées qui "s'excluent d'une manière absolne; et l'hybridisme, " c'est la confusion, la rupture de la loi, partant l'excep" lion, l'anomalite. La méshode (ou le systeme), et la " nomenclature qui en est l'expression, doivent s'appli" quer exclusivement à ce qui est normal.

"L'hỵbridisme végétal n'est pas et ne saurait ètre la "Loi; done il doil êre nécessairement rare, et japplau" dis il la réserve prudente et sensée de Koch : Hybride "sunt eel sallem pro hybridis habentur (Syn., p. "389). " Si les caractères de ces formes sont constants, si " elles se reproduisent normalement et indéfiniment, ee " sont des espéces qu'on n’a pas jusqu’ici distinguées et " qu'il faut distinguer à l'avenir. Si non, ce sont des "accidents passagers, et il ne faut pas, en présence de " l'admirable harmonic de la création, - en présence de " la paix, ou, comme dit Saint-Augustin, en présence de "La trempuilité de l'ordre qui brille de toutes parts dans "les ouvres de Dien - il ne faut pas croire, dis-je, que 


\section{(14)}

" certains ètres non modifiés par arlifice s'écartent de la " rìgle, - assez fréquemment pour que ertle aberration " prenie une apparence de normalité - jusqu’à remplir " à l'égard d'autres ètres spécifiquement différents, la "double fonction de fécondateur et de fécondé, que la " mode du jour attribue alternativement, indifféremment " et si je l'osais dire, promiscuément, à une mème " espèce.

" Je mets fin à cette digression, fondée, je crois, sur " les principes les plus sains de la philosophie, de la " raison et de l'observation. Je sais qu'on peut se tromper " sur les faits. Mais dès qu'on ne se trompe pas sur les " principes, je me fais honneur de proclamer avee " M. Alexis Jordan que le principe est plus fort que le « fail, et que, si ces deux choses sont en contradiction, " il faut néeessairement que le fait ait été mal observé " ou mal interprété, ear le fait n'est dans l’ordre des " choses possibles, que parce qu’il est la réalisation d'un " principe; autrement il ne pourait avoir lieu. La philo" sophie la plus élémentaire enseigne que deux vérités ne " peuvent pas ètre opposées l'une à l'autre; or, qui dit " principe et qui dit fait, les proclame ígalement vérités." Fidèle à la loi que je me suis imposée de n'admettre aucune de ces espèces nominatives connues de leurs auterirs seulement, dont les descriptions sont toujours à venir, je les passe sous silence. Ces noms spéeifiques donnés à des plantes sans une description ou une planche sont nuls et non avenus. Les plantes dites inédites, publiées dans les exsiceata, sans une description sont dans les mèmes conditions.

Je ne chercherai pas ici à fortifier, par des raisonnements anteques, les opinions emises dans celle publication; 
je les donne avec convietion, toujours prèt à modifier ma manière de roir aussitot que des raisons plus solides m’apporteront de nourelles lumieres. C'est toujours aree la plus grande reconnaissance que je recerai toutes les observations que les botanistes vondront bien me communiquer; je leur soumets le résultat de mes recherches et de mes critiques; mon but a été de chercher la vérité et de rendre à chacun ee qui lui appartient; si ce mémoire obticnt leur approbation, elle sera pour moi la plus belle récompense!

J'ai cité dans le cours de ce mímoire les personnes qui m’ont communiqué des échantillons, souvent accompagnés de notes instructives; c'est pour moi un devoir, bien doux à remplir, de les citer ici et de leur témoigner mes bien vifs remerciments.

En première ligne, je dois placer M. Boreau, directeur du Jardin botanique d'Angers, pour la bienveillance qu'il a mise a me domer son avis chaque fois que je l'ai consulté, et en me procurant, au moyen de sa vaste correspondance, des échantillons de rosicrs, tant de la Franoe que des pays étrangers.

Des herborisations faites pendant de longues années dans le département du Cher, avec M. le docteur Ripart, sont pour moi des souvenirs agréahles ajoutés à une amitié sans bornes. Gustave Tourangin, qui fut mon premier guide dans la hotanique, ne peut plus recevoir mes remerciments, il est mort depuis trois atis, sa mémoire me sera toujours chère ! Le comte Jaubert, qui fut le promoteur de la botanique dans le centre de la France, dont la riche galerie botaniqur: m’a été si généreusement ouverte, ne pent plus recevoir meś remereiments, il a été enlevé à sa lamille, a ses nombreus amis of a la serence le "j decem- 
bre 1874; je garderai toujours un précieux souvenir du comte Jaubert; il faut espérer que parmi ses pelits-fils, il y en aura un qui se livrera à l'étule de la botanique et qui marchera sur les traces de l'auteur des Illustrationes plantarum orientalium.

A ees noms, j’ajouterai ceux de MM. I'abbé Puget, d'Annecy; l'abbé Boullu, de Lyon; Ozanon, de Lyon; Hanry, du Var; Pierrat et l'abbé Boulay, des Vosges; Lloyd, de Nantes; Lamolte, de Clermont; Grenier, de Besancon; Verlot, de Grenoble; Timbal-Lagrave, de Toulouse; Franchet, du Loir-et-Cher'; Mabile, d'Auxerre; Jullien, d'Orléans; Bornet, d'Antibes; l'abbé Chaboisseau, de Paris; Gencrier, de Nantes; Lamy, de Limoges; Paillot, de Besancon; Gariod, de Gap; Songeon, de Chambéry; Bordère, des Pyrenées.

En Angleterre, M. J.-G. Backer, de Londres, m'a procuré, outre des types authentiques de Woods, venant de lui-mème, tous les rosiers d'Angleterre, qui sont si peu répandus dans les herbiers du continent; je lui témoigne ma bien vive reconnaissance, et je le remercie sincèrement pour tous les renseignements qu'il m’a communiqués sur l'herbier de İinné et autres collections. - MII. AreherBriggs, de Plymouth; F.-II. Webb, de Liverpool.

En Belgique, M. Crépin, de Bruxelles. En Allemagne, M. Caspary, de Königsbergo feu Wirtgen, de Coblence. En Autriche, M. Kerner, d'Innsbruck. En Italie, M. Todaro. En Suisse, M. Rapin, de Genève; M. l'abbé Cotter, de Friboury; M. de la Soie, chanoine du St-Bernard à Bovernier; M. Christ, de Bâle.

Je prie toutes les persomnes que je viens de eiter, de recevoir l'expression de ma vive recomnaissance; puissentclles, en voyant l'usage que j’ai lait des secours qu'elles 
mont fournis, continuer à mhonorer de leur précieuse bienveillance!

Je déclare en outre que j’ai écrit ee que je pensais, sans aucune intention de nuire à qui que ce soit, mais seulement dans l'espoir que par ces diverses opinions on parviendra à trouver la vérité.

Genève, 10 mai 1873.

Rapport sur les Rosiers d'Europe de l'herbier de Linné, par M. J.-G. Baker (1).

L'herbier de Linné contient environ „̋0 échantillons de rosiers presyue tous en hon élat. Il y a 50 espéces d'Europe environ; la moitié sont étiquetées de la propre main de Limné : généralement il n'y a que le nom seul; quelquefois une note; parmi les autres, un grand nombre viennent de Jaequin 't sont accompagnés d'étiquettes chargées de nombreuses notes et collées sur la feuille; deux ou trois ont des étiquettes de quelque correspondant francais de Linné (du Roi?); il existe aussi sur quelques feuilles une note au erayon faite par Sir J.-E. Smith. Avec son herbier, on conserve l'exemplaire qui a appartenu i Limné de la seconde édition du Systema plantarum, qui contient beaucoup de notes manuserites d'une date plus réeente que le texte imprimé, des corrections de synonymes, etc.; je les ai toujours ajoutées quand elles m'ont paru avoir une certaine importance.

(1) M. Baker, de Londres, a bien voulu comparer les types de mon Essai monographique sur les Rosiers de France, avec ceux de l'herbier de Linné, collection conservée dans les galeries de la Société Linnéenne de Londres. Voici le résultat de ses recherches que je donne in extenso; ces notes recues en septembre 186\%, devaient paraitre de suite, quand des circonstances indépentantes de ma volonté m'rmpectremente le faire plus tot. 


\section{REMARQUE SUR LA COLONNE COPIÉE.}

Lin. herb. signifie : Étiquettes de la main de Linné dans l'herbier.

Lin. syst. " Notes manuserites de Linné dans l'exemplaire du Systema.

Jacq. herb. „ Etiquettes de la main de Jacquin collées sur les feuilles de l'herbier.

Smith, herb. " Notes de Smith dans l'herbier.

COPIÉ D'APRÉS L'IIERDIER OU LE LIVRE.

1. Eglanteria lutea, Sm. herb.

R. lutea Bauh. pin. 4̌̄̄, Ilall. 1l. succ. et R. eglanteria Tabern. Lin. Syst.

2. Bicolor. eglanieria var. Smith, herb.

5. Bicolor nobis. Germinibussubglobosis glabris, pedunculis inermibus, fol. cal. subaculeatis, petala intus rubicunda. Jacq. herb.

4. Rubiginosa. Differt a R. lutea foliis rugosis et sublus rubiginoso-glandulosis, qua in lutea sunt glaberrima glauca.

R. eglanteria anclorum nec Linnaei nec a Kramero, Jacquiniano, Crantzio observala.

$\boldsymbol{R}$. bicolor Jacq. forte est $\boldsymbol{R}$. lutea et varietas. Lin. herb.

5. Cinnamomea II.U. (hortus Upsaliensis). Lin. herb.

6. Foecundissima. Jacq. herb.

7. Cinnamomea avec une etiquette du correspondant francais.

8. Pimpinellifolia. Lin. herb.
ODSERYATIONS DE M, J, $=$ G, HAKER.

1. lutea ordinaire, feuilles légèrement velues en dessus; pétioles velus, mais trèspeu glanduleux; feuilles glanduleuses sur toute la surface inférieure; pédoncules tout à fait glabres.

2. lutea var. punicea Miller.

5. lutea var. punicea Miller.

4. Ceci montre que Linné distinguait clairement le $\boldsymbol{R}$. lutea du $\boldsymbol{R}$. rubiginosa. Cet échantillon, le seul de la collection étiqueté ruliginosa par lui, est évidemment ou votre rubiginosa ou le comosa. II a les styles fortement laineux; le fruit (non mûr) obovoïde, un peu aciculé à la base; les ficuilles un peu velues en dessus, très-glandulcuses en dessous, la foliole terminale ovale et même arrondie; les pétioles garnis de nombreux petits aiguillons inégaux; les bractées et les stipules supérieures nues sur le dos.

f̈, 6, 7. Cinnamomea ordinaire.

8. C'est notre spinosissima ordinaire en Angleterre, et votre plante n'est certainement pas le R. rubella Smith, pédoncule et fruit tout à fait nus; feuilles simplement dentées; pétioles sans glandes et à peine munis de quelques petits aiguillons; fruit (non mùr) subglobuleux; styles laineux. 
COPIÉ D'APRLS L'IIERTIER OU LE LIVRE.

9. Pimpinellifolia Iin. syst. (avec une barre transversale stir le non1). Sninosissima? Smith. herb. (avec une barre sur le point d'interrogation). L'échantillon vient de Jaequin étiqueté comme il suit : Ruse dustriace Crantz no 110 b) IIalleri a botanophyllo Ilalleriano fruticem accepi e montibus Generae. A R. Spinosissima, vix differt uisi corollis purpureis odorantibus, frutice minor, minus spinosa. Contra Hallerum foliola calycina apud nos simplicia nec pinnata; germen glabrum non hispidum ut xxx Crantzius.

10. Villosa. Ackero Sudermania. Usbeek. Lin. herb.

Caulis laevis, aculeis sub genicula 2 ad \& confertis. Petioli aculeati. Folia obtusa comentosa. Pedunculi hispidi. Germina globosa hispida. Petala rubra. Lin. Syst.

11. Rosa sylvestris virginiensis Parkinson. Cum R. Carolina L. non nilil convenit spinis stipularilms frueibus ovitis hispidis et licet Dill. diversam esse monet in hort. Eltham. p. 52j. Jaeq. herb. Smith a écrit :

R. pomifera melius. L. villosa horlorum el fl. Brilh. Smith. herl).

12. Sempervirens Lin. herb.

15. Gallica lac(y. herl), Caulis lacris vel aculeatus, petioli aculeati, folia supera nuda, subtus vix tomentosa subacuta. Pedunculi hispidi. Germina ovata basi hispida. Flores pleni rubri aut alli.

14. R. belgien Miller. Frutex humanac altitudinis. Caulex et rami fregueuter inermes, non nulli tamon aculais recurvis,
OBSEIVATIONS DE M. d, -G, BAKEIR

9. Je doute que vous rapportiez aussi celle espèce au $\boldsymbol{R}$. spinosissima. Il n'y a rien dans la collection étiqueté spinosissima par Linné. Cet échantillon a une lleur qui n'a pas plus d'un ponce de largreur, les feuilles simplement dentées, les pédoneules tout à fait lisses. C'est une plante tout à fait diflérente de celle que vous appelez $R$. A ustriace Crantz. Mais Jaequin aurait bien pu appliquer correctement un nom de Crantz.

10. C'est tout à fait le $R$. mollissima Firies. Des feuilles rugucuses molles grises sur les deux fac:s, très-légèrement glanduleuses en dessous; stipules molles comme les feuilles et tout a fait glanduleuses sur le dos; tube du calice globuleux, légèrement aciculé; sépales presque simples avec une longue pointe fenillée.

11. R. pomifera. Sans doute un exemplaire cultivé dans un jardin et venant de Jaequin. Notez que c'est le mollissima et non celui que Linné a nommé villosu. Consulter Fries Novit. fl. Suec.

12. Est-ce votre sempervirens ou votre scundens? Je n'en suis pas sûr. Feuilles larges, de deux pouces de longueur, largement ovales arrondies à la base. Stipules glanduleuses ciliées; colonne des styles très-velue; tous les sépales entiers et glanduleux sur toute leur surface extérieure.

15. R. Gallica.

1\%. Evidemment $R$ movinciretis. Fenilles longues de deux pouees, larges l'un demipouce, non cilices, poilues principalement 
COPIÉ D'APRÈS L'IIERBIER OU LE LIVRE.

OBSERVATIONS DE M. J.-G. BAKER.

obsiti. Flores corymbosi, omnium mihi rosarum maximi. Germen ovato-oblongum. Petala pallide incarnata extus albidena. Jacquin, herb.

15. "Rosula pulcherrima nana dicla. Rosa burdigabensis; rose de Dijon. In villosae varielas. Le correspondant francais (du Roi ?).

16. Rosu holosericen Miller. Fl. intense carmesinus odoris gratissima. Jun. Jul. Foliola magis ovata quam ut $R$. provinciali Mill. Nec non $\mathrm{xxx}$ duriara et nonnihil viscida. Flos etiam multo minor. Jacquin, herb.

17. Rosa moschata Mill. Frutex 4 pedalis ramosissimus. Caulis et rami aculeis copiosis recurvis muniti, foliola saepe septena tenerrima utrinque viridia subtus glandulosa odorem fortem $R$. eglanteria spargunt. Flos rubescens hemisphericus R. centifolia duplex minor fragrantissimus. Jacquin, herb.

18. Rosa.

Frutex humanae altitudinis. Aculei in caulibus rariores, folia utrinque villosa et tactu mollia inferne albida : flores copiosi corymbosi suaveolentes plani, minus pleni quam $\boldsymbol{R}$. centifolia cjusdemque coloris, germen turbinatum. Jacquin, herb.

19.

20. Gallica var. Lin. herb.

21.

22. Alpina Lin. herb. en dessous sur les nervures, quelques-unes des dents surchargées de dents accessoires; sépales prolongés en une longue pointe feuillée; tube du calice resserré au col; flcurs larges de trois pouces.

1๊. Série des rubiginosae. Échantillon en mauvais état; fleurs presque détruites par les insectes; folioles terminales petites, typiquement ovales, dentelures fines un peu surchargées de dents accessoires.

16. R. pumila. Feuilles plus petites que le $n^{0} 14$, doublement dentées, les dents secondaires glanduleuses, foliole terminale largement ovale.

17. Série des Gallicanae.

18. Série des Gallicanae.

\section{Série des Gallicanae.}

20. Je pense que c'est l'arvina. Styles réunis en colonne velue plus courte que les étamines, feuilles à nervures proéminentes en dessous, obovales et arrondies à la base, tube du calice glanduleux à la base, sépales laincux et légèrement glanduleux sur le dos.

21. Série des Grallicrinac.

22. Votre plante, mais à pédoncules hispides, ainsi que le tube du calice, et pétioles légèrement aciculés et garnis de soies. 
COPIÉ D'APRÈS L'HERBIER OU LE LIVRE.

25. Alpina Jacq. herb.

24. R. sylvestris pumila C. B, non Linnaei, An ejus R. Gallica? Lin. herb. Pumila Willd. Smith, herb.

2\%. Canina Lin. herb.

26. R. Cretica montana foliis subrotundis glutinosis et villosis. Jacq. herb.

R. glutinosa R. Graec. T. 482. Sm. herb.

27. R. dumetorum. E. B., 2579 ?

R. nubiginosa xxx Smith, herb.

28. R. Collina. Jacquin, herb.

29. Pendulina.

R. incrmis fructu longissimo. Liu. herb.

50. Alba. Lin. herb.

51. Monsp. (Montpellier). Lin. herb.

52. Monsp. id. Lin. herb.
OBSERYATIONS DE AI. J.-G. BAKER.

25. Deux échantillons avec une étiquette de la main de Jacquin. Un est $\boldsymbol{R}$. Alpina vera, l'autre est $\boldsymbol{R}$. Pyrenaica.

2:. Exactement $\boldsymbol{R}$. luteliana Lem. Dentelures simples; pétioles avec 5 ou 4 aiguillons recourbés, mais ni velus ni munis de soies; stipules légèrement ciliées-glanduleuses; pédoncules nus; sépales très-pinnatifides et fortement ciliés-glanduleux sur toute leur étendue.

26. R. glutinosa Sibth.

27. Le même que le $n^{\circ} 4$, mais sans aucune étiquette de Linné sur la fenille.

28. Votre plante.

Fcuilles glabres en dessus, velues en dessous principalement sur les nervures; foliole terminale presque ronde, toutes simplement dentées; pétioles velus et chargés de petites glandes ; tube du calice arrondi et pédoncules chargés de soies et d'aiguillons; styles très-velus.

29. C'est tout à fait votre plante. Linné et non Aiton est l'autorité originale pour le nom.

50. Exactement votre plante.

51. Alpina vera.

52. R. sepium Thuil., échantillon bien caractérisé. 


\section{HULTHEMIA.}

Dumort., Not. sur un nouv. genre de plante (1824); Boissier, f1. orient., II, p. 668 (1872); Lowea Lindley, hot. regiet. (1829), XV; Rhorlopsis Bunge in Ledeb., 11. Ah. $(1850)$, II, p. 22/; fonsae sp. Pallas; Jussicu; Willd.; Poir.; Pers.; Lindl.; Tratt.; DC.

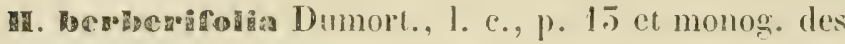
roses de la flore Bclge (1867), p. 59; Boissicr, l. c.; Rosa berberifolia Pallas, nov. act. Petrop., X, p. 579 ; Roessig, die rosen (1801), n":): ; Pers., syn. (1807), II, p. 47; Lindl., monog. rosar. (1820), p. 1; Thory, prod. du gen. rosier (1820), p. 5.); Trall., monog. ros. (IS25), II, p. 214; de Pronv., monog. dugemre ros. (1824), p. 25; $\boldsymbol{R}$. berberifolia var. Redouteana, Scringe, in DC., prod. (1825), II. p. 609; R. Persic Juss., gen. (1789), p. 452; IR. simplicifolia Salish., hort. Allert. (1796), p. 5̈.9; Poiret, enc. suppl. (1804), p. 276; Dum.-Cours., bot. cult. (1811), V, p. 467; $\boldsymbol{K}$. monophylle Desportes, roset. gal. (1827), p. 1; Lowea berberifolia Lindley, bot. reg. (1829), tab. 1261.

Icon. Olivier, voy., atl. tab. 45̄; Pallas, 1. c., fig. ら̋; Roessig, l. e.; Redouté, les roses (i82/), livrais. I, B.

Exsic. Aucher-Eloy, no 14.28; Kotschy, pl. Perse bor., $n^{\circ}$ 698; Karelin et Kiriloff, $n^{\circ} 230$.

IIAr. Mai, juin. - Perse. Amaden (Olivier), Assadabad (IIaussk.), Ies Monts Elvind (Olivier 1822, in herb. DC. - Aucher-Eloy), Téhéran (Kotschy). - Tartarie chinoise. Désert de la Songarie (Bunge in herb. DC. ; Turezanninoff in herb. Boissier).

Var. b. stenophylla Boiss., I. c., p. 669. - Iumilior, folia angustiora lineari-oblonga (Boiss.). - Perse. Téhéran (Kotschy, exs, no 20!).

Var. c. velutima Ser in DC., I. c.-Perse, herb. DC.

Ons. Introduit en France par Michaux et Olivier; en Angleterre, par Banks, 1790, d'après Desportes. 
ROSA.

Tourn., inst., I, p. 646, tab. 408; Lin., gen., nº 651; Juss., gen., p. 574; Lamarek, illust, tab. 440 ; Gaertn., fruct., I, p. 547, tab. 75 , f. 4 .

\section{Sect. I. - Symstylac.}

1). Sempervirentes.

R. Leschenaultiana Red.

Furope. Anglet. France. Asie. Afrique.

- thyrsiflora Leroy.

- multiflora Thunb.

- Luciae Franch. et Rochel.

- moschata Mliller

- Phoenicea Boissier

- Dupontii Déségl.

- Abyssinica R. Br.

- Brownii Tratt.

- ruscinonensis Déségl. et Gren

- longicuspis Bert.

- sempervirens Lin.

- scandens Miller

- prostrata JC.

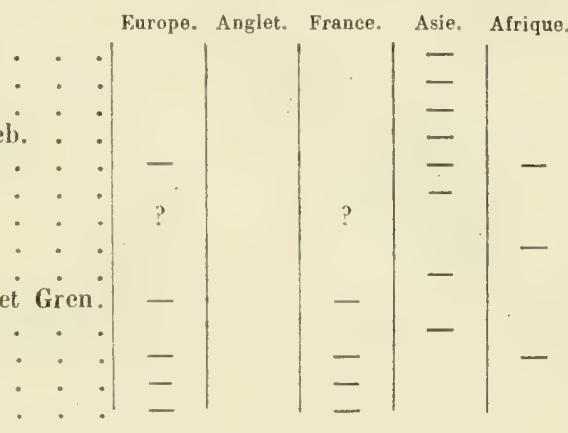

в). Arvenses.

R. bibracteata Bast

- conspicua Boreau.

- rusticana Déségl.

- Beggeriana Schr.

- arvensis IIuds.

- crronea Ripart

- ovata Lejeune.

- gallicoides Déségl.

c). Stylosae.

R. stylosa Desvaux

- Clotildea Timb.-Lagr.

- systyla Bast

— immitis Déségl.

- parvula Sauzé et Mail.

- virginea Ripart

- leucochroa Desvaux. 


\section{SECT. II. - Indicac.}

R. Indica Lin.

Europe. Anglet. France.

Asie. Afrique.

- Lawranceana Sweet.

-- semperflorens WVilld.

- longifolia Willd
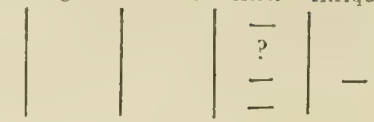

\section{Sect. III. - Raracatac.}

R. involucrata Roxb.

- bracteata Wendl.

- Lyellii Lindley

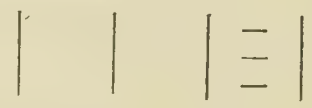

\section{Sect. IV. - Banksianac.}

R. Banksiae $\mathrm{Br}$.

- microcarpa Lindl.

- amoyensis IIance.

- Sinica Murr.

- hystrix Lindl.

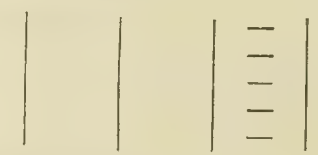

\section{Sect. V. - Gallicanac.}

R. hybrida Schleicher

- Polliniana Sprengel.

- arvina Krocker

- arenivaga Déségl.

- subinermis Chabert

- geminata Rau.

- Fourraci Déségl.

- Boraeana Béraud.

- Austriaca Crantz.

- incarnata Miller.

- virescens Déségl. .

- velutinaeflora Déségl. et Ozan.

- mirabilis Déségl.

- sylvatica Tausch.

- decipiens Boreau.

- opacifolia Chabert.

- galliea Lin.

- provincialis Ait.

— assimilis Déség!.

- pygmaca Bieberst.

- Czackiana Resser.

- Wolfgangiana Besser.

- pumila Lin. fil. 


\section{Sect. VI. - Centifoline.}

R. centifolia Lin.

Europe. Anglet. France.

Asie. Afrique.

- sancta Richard

- parvifolia IVilld.

- muscosa Ait.

- pomponia DC

- pulchella IVilld.

- turbinata Ait.

- Damascena Miller.
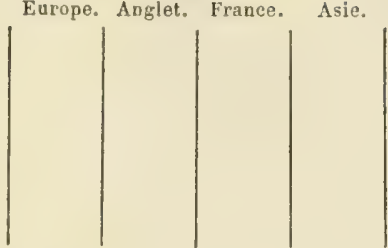

\section{Sect. VII. - Pimpinellifoliac.}

R. pimpinellifolia Lin.

- spinosissima Lin.

- Besseri Tratt

- Mathonneti Crépin

- consimilis Déségl.

- spreta Déségl.

- Ozanonii Déségl.

- mitissima Gmelin.

- Altaica Willd.

- oxyacantha Bieberst.

- Webbiana Wall

- albicans Godet.

- oxyodon Boissier.

- rubella Smith.

- reversa IV. et Kit.

- gentilis Sternb.

- myriacantha DC.

- Ripartii Déségl.

- dichroa Lerch.

Sect. VIII. - Sabiniae.

R. Sabini Woods.

- Doniana IV oods

- gracilis IV oods

- Wilsoni Borrer

- involuta Smith

- coronata Crépin

- Sabauda Rapin
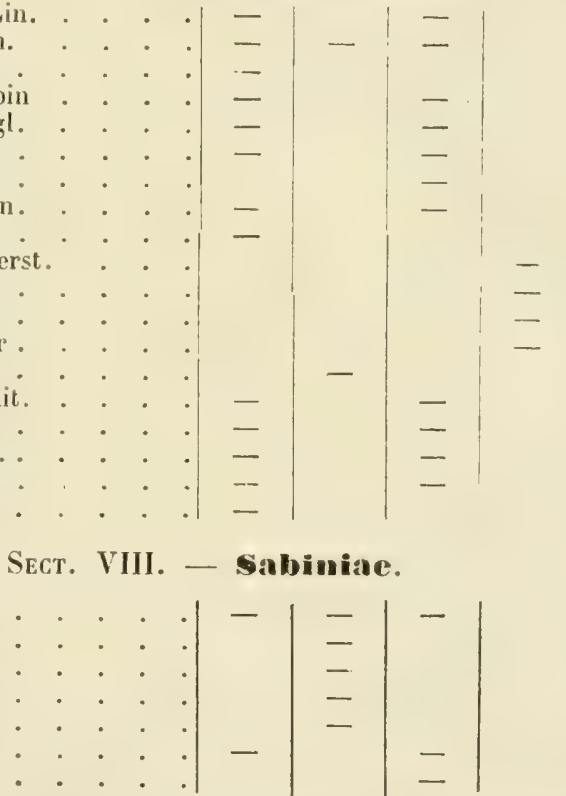

Segt. IX. - Cinnamomene.

R. cinnamomea Lin.

- IVilldenowii Spreng.

- Carelica Fries.

- Baltica Roth

- blanda Jacquin

- Fischeriana Besser

- laxa Retz

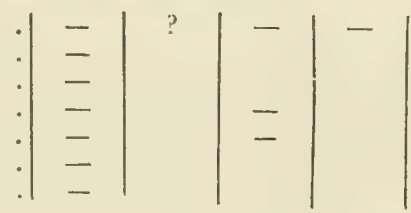


R. Dahurica Pallas

Eurspe. Anglet. France. Asie, Afrique.

- Silverhielmii Schr.

- Bungeana Boiss. et Buhse

- anserinacfolia Boissier

- Jacerans Boiss. et Buhse

- Lehmanniana Bunge.

- Cabulica Boissier.

- Elymaitica Boiss. et Hauss

- Orientalis Dupont.

- Kotsehyana Boiss.

- Kamtchatica Vent.

- rugosa Thunb.

- iwara Sieh.

- microphylla Roxb.

- macrophylla Lindl.

- sericea Lindl.

- dissimilis Déségl.

- Balearica Desfont.

Sect. X. - Alpinae.

R. alpina Lin.

- intercalaris Déségl.

- adjecta Déségl.

- Monspeliaca Gonan

- pendulina Ait.

- lagenaria Villars

- Pyrenaica Gouan .

- glandulosa Bellardi

Sect. XI. - VIontanac.

R. Franzonii Christ. .

- ferruginea Villars.

- montana Villars.

- Salaevensis Rapin.

- Perrieri Songeon.

- Caballicensis Puget

- Gorenkensis Besser

- oplisthes Boissier.

- falcata Puget

- Ilseana Crépin.

- glauea Villars.

- complicata Grenier

- intricata Grenier

- fugax Grenier.

- venosa Swartz.

- Crepiniana Déségl.

- subcristata Baker.

- alpestris Rapin

- Hibernica Smith

- Schultzii Ripart

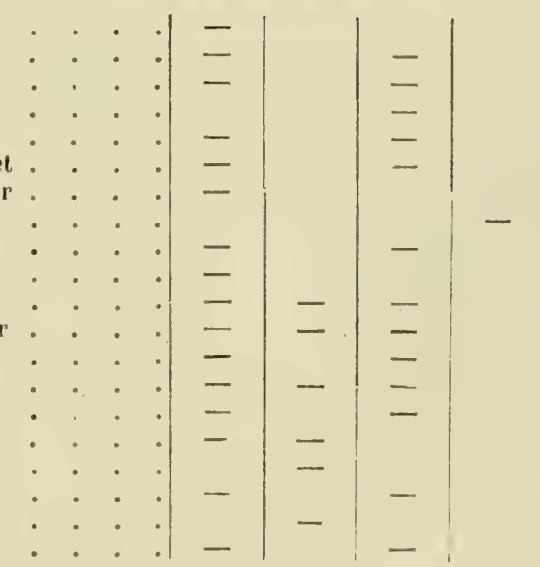


Sect. XII. - Caminne.

A). Nudae.

R. canina Lin.

Europe. Anglet. France. Asie. Afrique.

nitens Desvaux

- syntrichostyla Ripart.

- macroacantha Ripart.

- mucronulata Déséglise

- senticosa Achar

- fallens Déségl.

- aildita Déségl.

- callycina Bieberst. . . . . -

- armataStrven. . . . . -

- Transilvanica Selrr.

- Touranginiana Déségl. el Rip.. -

- ramosissima Rau

- Amansii Rip. et Déségl.

- globularis Franchet

- montivagar Déségl.

- spuria lósighi..

- sphaerica Grenier.

- exilis Crépin.

- aciphylla Rau.

B). Biserratar.

R. Carioti Chabert

- medioxima Déségl.

- Malmundariensis Lej..

- Nandonii Déségl.

- squarrosa Rau.

- rubellitlora Ripart

- rubescens Ripart.

- vinacea Baker.

- dumalis Bechst

- glaberrima Dumort.

- oblonga Déségl. et Rip.

- cladoleia Ripart.

- sylvularum Ripart

- insignis Rip. et Déségl.

- Chaboissaci Grenier.

- erinstyla kipart

- curticola Puget.

- stenocarpa Déségl.

- villosinscula hipart.

- armatissima Déségl. et Rip.

- stephanocarpa Déségl. et Rip.

- adseita Déségl. 
Europe Anglet. France. Asie. Afrique.

- macrocarpa Mérat.

- biserrata Mlérat

- sphaeroidea Ripart

. -

- brachypoda Déségl. et Rip.

- Armidac WVebb.

c). II ispidae.

R. Pouzini Tratt

- inconsiderata Déségl.

- Chavini Rapin.

- Wolfii de la Soie.

- Martini Grenier

- surculosa Woods

- abstenta Déségl.

- Andegavensis Bast.

- vinealis Ripart

- Verloti Crépin.

- Suberti Ripart.

- Rousselii Ripart

- interveniens Déségl.

- latebrosa Déségl.

- ambigua Lejeune.

- Kosinsciana Besser

- firma Puget

- aspernata Déségl.

- verticillacantha Jérat

- inconspicua Déségl.

- oenensis Kerner.

- Schotliana Seringe

- Acharii Bilb.

- Chaberti Déségl.

- IIaberiana Puget

- Waitziana Tratt.

- transmota Crépin.

- psilophylla Rau

- Aunieri Cariot.

- Laggeri Puget.

-- hamathodes Boissier

- Hampeana Griseb.

- Djimilensis Boissier

- Soongarica Bunge.

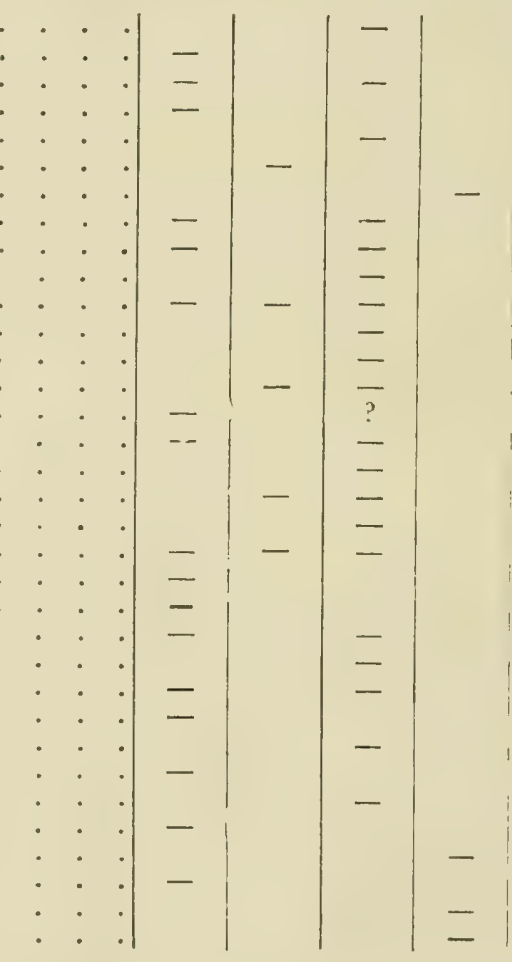

D). Pubescentes.

R. erythrantha Boreau .

- obtusifolia Desvaux

- brachiata Déségl .

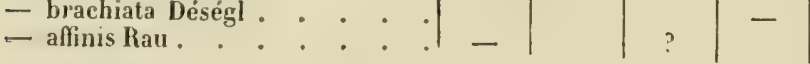




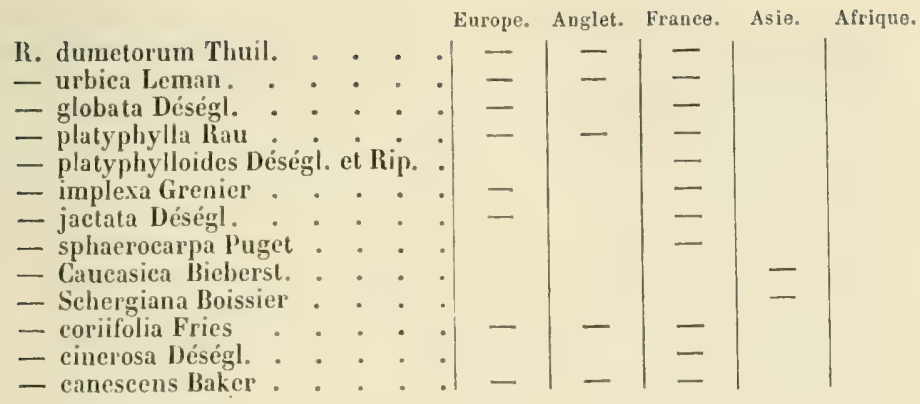

в). Collinae.

R. corymbifera Borkh.

- Numidiea firenier

- Deseglisei Boreau.

— imitala Déségl.

- Bellevallis Puget. -

- approximata Déségl.

- Friesii Scheutz.

- arguta Crépin.

- clivorum Scheutz.

- caesia Smith

- collina Jacquin

- cinerea Rapin.

- Boverneriana Lag. et de la Soic

- cerasifera Tímb.-Lagr.

- Friedlanderiana Besser

- saxatilis Steven

- Ratomsciana Besser.

- macrantha Desportes.

- Boreykiana Besser.

- alba Lin.

\section{Sect. XIII. - Eglanteriae.}

R. lutea Dalech.

- Maracandica Bunge.

- Phrygia Boissier.

-. hemisphaerica IIerm.

Sect. XIV. - Meubiginosac.

^). Tomentellae.

R. tomentella Leman

- similata Puget.

- Tiroliensis Kerner

$$
.|-|-\mid-1
$$




\section{$(50)$}

R. Borreri Woods

Europe. Anglet, France. Asie. Afrique.

- Bakeri Déségl.

- Valesiaca Lag. et Pug.

- Nebrodensis Gussone.

- viscida P'uget.

- viscosa Jan.

- Blondacana Ripart

B). Glandulosae.

R. insidiosa Ripart

- dryadea Ripart

- protea Ripart.

- consanguinea Grenier

- Godeti Grenier

- Colteti Puget

- marginata Wallroth

- trachyphylla kau.

- leucantha Bieberst.

- Wasserburgensis Kirschi.

- commutata Scheutz. .

- -

- Pugeli Boreau.

- nemorivaga Déségi. :

- decora Kermer.

- tlexuosa liaur.

- llexuosa liau.

- pseudo-flexuosa 0zanon

- speciosa Déségl.

- Jundzilliana Besser.

- nitidula Besser

- livescens Besser.

c). Pseudo-rubiginosae.

R. Ilungariea Kerner

- grandiflora Wallroth.

- sepium Thuil.

- vinodora Kerner.

- agrestis Savi .

- mentita Déségl.

- arvatica Puget.

- virgultorum Ripart

- Billetii l'uget

- Seraphini Viv.

- Cheriensis Déségl.

- Lugdunensis Déségl.

- Jordani Déségl.

- elliptica Tausch.

- Vuillantiana Boreau.

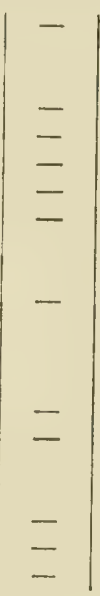




\section{(51)}

Eutope. Anglet. France. Asie. Afrique.

R. ladanifera Timb.-Lagr.

- Arabica Crépin

- Klukii Besser.

- subdola Déségl.

- Tuschetica Boissier

- Biturigensis Boreau.

D).Verae-rubiginosae.

R. apricorum Ripart.

- comosa Ripart.

- umbellata Leers.

- horrida Fischer

- permixta Déségl.

- septicola Déségl. .

- operta Puger

- echinocarpa Ripart.

- dimorphacantha Martinis.

- sylvicola Déségl. et Rip.

- Iberica Ster en.

- Aucheri Crépin

- asperrima Godet

- glutinosa Sibth

pustulosa Bertol.

- Heckeliana Tratt.

- Sicula Tratt.

- Orphanidis Boiss. et Reut.

- micrantha Smith.

- Horibunda Steven.

- diminuta Boreau.

- lactiflora Jéségl.

- Lemanii Boreau

- rotundifolia lichb.

\section{Sect. XV. - Tomentasac.}

A).Verae-tomentusae.

* Folioles toutes simplement dentées.

R. Didoensis Boissier.

- Armena Boissier

- Vanheurckiana Crépi

- Boissicri Crépin

- Balansaea Déségl.

- cineracens Dumort

- micans Déségl.

- Mareyana Boullu

- dumosa Puget.

- larinulenta Crépin 


\section{*** Feuilles doublement dentées.}

† Folioles à glandes éparses en dessous ou à nervures sceondaires relues.

R. foctida Bast

Europe. Anglet. France.

Asie. Afrig̨ue.

- abietina Grenier

-. Gisleri Puget.

- Sufferti Kirsehi.

- spinulifolia Dematra.

- vestiti Gudet

- terebenthinacea Besser

- Arduennensis Crépin.

- pulverulenta Bieberst.

- caryophyllacea Besser

- capnoides Kerner.

- cuspidata Bieberst

- cuspidatoides Crépin

- Genevensis Puget.

- scabriuscula Smith

- firinosa Rau

t十 Folioles églanduleuses en dessous.

R. Borkhausenii Tratt.

- collivaga Cottet

- velutina Clairville.

- tomentosa Smith.

- intromissa Crépin

- dimorpha Besser.

- Ledebourii Sprengel

- subglobosa Smith.

- Tunoniensis Déségl.

- confusa Puget.

- Annesiensis Déségl.

- Andrzeiowscii Besser.

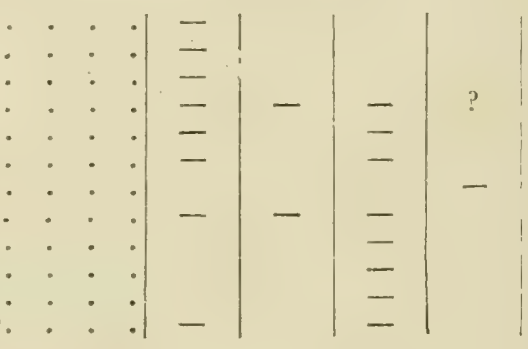

B). Pomiferae.

R. omissa Déségl

- Ruprechti Boissier. .

- Heldreichii Bois. et Reut.

- mollis Smith

- Scheutzii Christ.

- renusta Scheutz . . .

- Cremsensis Kerner

- Australis Kerner.

- resinosa Sternb.

- resinosoides Cripin

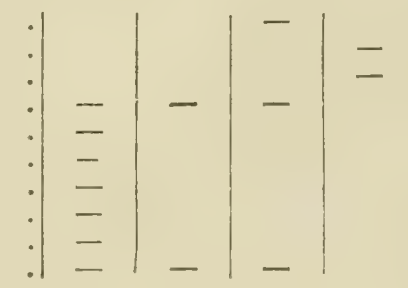




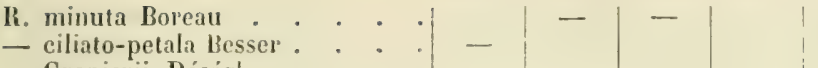

Europe. Anglet. France. Asie, Afrique.

- Grenierii Désénl.

- pomifera Herm.

- Friburgensis Lag. et P'ug.

- recondita Puget

- Murithii Puget

- Gaudini Puget.

- Gombensis Puget.

- proxima Cottet

\section{SECT. I. - Synstylac.}

Styles soudés ou rapprochés en colonne glabre ou hérissée (1).DC., cat.hort. monsp.(1815)), p. 157; Desvaux., journ. hot. (1815), II, p. 112; DC., in Seringe, mus. IIeliét. (1818), I, p. 2; Thory, prod. gen. ros. (1820), p. 155; Seringe, in DC., prod. (1825). II, p. 597; Desportes, ros. gall. (1827), p. 107; Duby, bot. gal. (1828), I, p. 17̋; Boreau, fl. cent. de la France (1840), II, p. 155; Déséglise, ess. monog., in Mém. soc. Acad. de M.-et-L. (1861), X, p. 49 extr., p. 9; Cariot, étud. des fleurs (1863), II, p. 167; Crépin, primit. monog. ros., fase. I (1869), p. 12; Cottet, énum. des ros. du Valais, in bull. Soc. Murith., fase. III (1874), p. 58; Systylae Lindley, monog. ros. (1820), p. 111; de Pronville, monog. du gen. ros. (182\%), p. 112; Godet, fl. jura (1855), p. 216; Baker, review of the british ros. (1864), p. 5ij et monog. of hrit. ros., in Linncan Society's Journ., MI, p. 258; Déséglise, class. of the spee. of the genus rosa in the naturalist (186:5),

(1) C'est La Chenal qui, le premier, a signalé la conformation particulière des styles dans le R. arvensis: Spec. med. immig. obs. bot. Basil, 17!:?). 
p. 509 ; Stylorhodon Dumort., not. sur un nouv. gen. de plantes (1824), p. 11, flor. belg. (1827), p. 94 et monog. des ros. de la fl. belge (1867), p. 64; Nitidae Rehb)., fl. cxcurs. (1850-52), II, p.625, part.; Nobiles Koch, syn. (184.̄), p. 25/, part.; Caninae sous-sect., Grenicr, fl. jura. (1864), p. 229; Stylosae Crép. 1. c., p. 15.

\section{A). Sempervirentes.}

1. $\left\{\begin{array}{c}\text { Pétioles glabres, églanduleux, aiguillonnés . . } 2 . \\ \text { Pétioles velus-glandulcux ou parsemés de poils } \\ \text { et de glandes . . . . } 4 .\end{array}\right.$

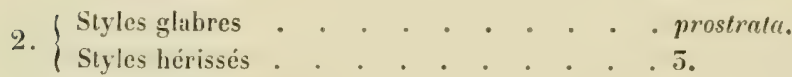

Folioles ovales acuminées, fleur blanche inodore,

5. fruit ovoïle rouge . . . . . . . sempervirens.

f Folioles oblongues ou ovales-obtuses, fleur blanche à odeur suave, fruit petit, sphérique . . scandens.

/ Folioles larges, ovales, glabres en dessus, pubescentes en dessous, styles velus, tube du calice glabre, fleur grande d'un blane rosé . . . Dupontii.

4. Folioles ovales-lancéolées, glabres en dessus, nervures secondaires parsemées de poils, tube du calice glanduleux, styles hérissés à la base, fleur blanche . . . . . . . . Ruscinonensis.

Obs. Les elefs dichotomiques ne sont établies que pour les rosiers indigènes de la France et de l'Angleterre.

1. R. Ceschenaultiana Relouté, les roses (1824), livr. 40, C; R. sempervirens var. Leschenaullianc Seringe, in DC., pr. II, p. 598 .

Icox. Redouté, l. c. ; Wight, pl. ind.-or., I, tah. 58.

Exs. Hohenacker, plant. ind.-or., no 1577.

Ha. Indes-Orientales. Montagne de Nil-Gherries. 
2. IR. thyrsifore Leroy! R. intermedia Carrière, revue horticole, NIII (1868), p. 270 (non Bose); Crépin, I. c., p. $125 ; R$. Wichurae K. Koch, wochens., $n^{\circ} 26$ ? (1869).

Icon. Revue horticole, XVII, fig. 29 et 50.

IIAs, - Le Japon! Non la Chine, comme le dit M. Carrière.

Ons. La description qui a été faite dans la Revue horticole, prouve bien de la négligence de la part de son auteur! Des mots ronflants pour étourdir le vulgaire, des détails impossibles, et les caractères principaux sont passés sous silenee! M. Crépin, l. c., a refait la description de ce rosicr.

ว. IR. multiflora Thumberg, fl. Japon. (1784), p. 214; Willd., sp., II, p. 1077 ; Pers., syn. (1807), II, p. כّ0; Lindl., monog. ros., p. 119 ; Tratt., monog. ros., I, p. 84; de Pronr., monog. du gen. ros., p. 119 ; Desportes, ros. gall., p. $110 ; R$. multiflora var. a. et b. Seringe in DC, prod., II, p. $598 ; \boldsymbol{R}$. Porida Poiret, dict. encycl., supp. IV, p. 723 ; R. Thunbergii Tratt., I. c., p. 86 .

IAs. - Le Japon (Thunberg); la Chine (Staunton).

Les échantillons de l'herbier DC. proviennent du jardin de Montpellier, 1816. - Introduit en Angleterre par Th. Evans, en 1804 et en France par Boursault en 1808, d'après Desportes.

4. R. Euciae Franch. et Rocheb., in bull. de la Soe. royale de botaniq. de Belgique (1871), X, p. 525; R. Wichuraiana Crépin, in herb. reg. Berol.

IIв. - Japon. Iokohama (Savatier), Ilakodate (Maximowicz in herb. Boissier!), Nagasaki 1862 (R. Oldham in herb. Boissier!).

:3. IR. mosehata Miller, diet. (1768), n ${ }^{\circ}$ 15; et éd. franc: (I781), II, 1). 526; Qner, fl. Es). VI (178k),

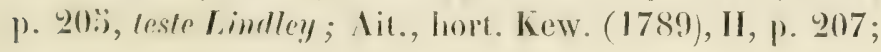


Willd., sp. (1797), II, p. 1074; et herl). $1^{\prime \prime} 984: 3 \mathrm{ex}$ Crépin; Desf., f1. Atl. (1798), I, p. 400; Gmelin, fl. Bad.-Als. (1806), II, p. 450; Pers., 1. c. p. 49; Dum.Cours., bot. cult. (1811), V, p. 482; Lindl., I. c., p. 121; Thory, prod. gen. ros. (1850), p. 158; Tratt., I. c., II, p. 9:3; de Pronv., 1. c., p. 121; Seringe, in DC., prod., II, p. 898 exel, val. h.; Desp., l. c., p. 111; Rehl)., fl. excurs. (1850), II, p. 62\%; R. opsostemma Ehrh., beitr. (1788), II, p. 72 ; R. arborea Pers., l. c., p. 50; Lindl., 1. c., p. 1/1; de Pronv., 1. e., p. 158; R. glandulifera Rosb. (test. Lindley); R. Brunonii var. arborea Seringe, 1. c., Desp., I. e., no 2478.

Icox. Jaequin, Schocnlor. (1798), III, tab. 280; et fragm., tah. 5/4, f. 5; Redouté, les roses (1824), liv. II, A; Roessig, tab. 27; d'après Pritzel, je cite: Miss Lawr., tab. 64; Guimpel et Schl., 51; Hayne, 11, 55.

Exs. Seringe, rosiers desséchẻs, nº 4! Kralik, pl. Tunetanac, $n^{\circ} 171$ ( sub. $R$. sempervirens L.).

IIab. Mai, juin. - Italie. Sicile : dans les bois montagneux de Madonie! (Todaro! J'ai recu ce rosier de M. Todaro, sous le nom de $R$. sempervirens var. luxurians). - Turquie d'Europe. Crète, île de Candie (Olivier 1822, in herb. DC. !). - Asie. Indes-Orient. (IIooker et Thomson!). - Afrique. Tunis, haies à Zaghouan (Kralik).

Ons. D'après Redouté, Olivier aurait vu le Rosa moschata alteindre 50 pieds de haut dans les jardins du roi de Perse à Ispahan. Indiqué en Espagne, d'après Quer; à l'île de Madère, selon Staunton. - Lindley, dans sa monographic, considere cette espece comme véritablement indigène dans le nord de l'Afrique, où elle s'étendrail de l'Egypte à Hogador, et de ce dernier point jusque dans l'ile de Madère. Cette plante se trouve aussi au Népaul où MM. Ilooker et Thomson l'ont observée. - Miquel (Ann. mus. bot. Lugduno-Batavi, III, p. 59), signale le R. moschata au Japon. - D'après M. Crépin (Primit. monog. ros. 1872), Meyen aurait rapporté le R. moschate (ll. pleno), de la Cordillière Saint-Fernand du 
Chili, et, dans l'herbicr du musée de Leyde, il y aurait un spéeimen à fleurs simples récolté dans une des iles Moluques : plante probablement introdute.

Aíton ignorait la patrie de ce rosicr; il était cultive en Angleterre par John Gerard, en 1596. Il était dans le jardin de Fugger, à Augsbourg, en $156 \%$.

6. R. Phoenicea Boissier, diagn., séric 1, fase. $\mathbf{X}$, p. 4 (1849) et 11. orient. (1872), II, p. 688.

Exs. Aucher-Eloy, $1^{0} 1455$ ! Kotsehy, $1^{\text {os }} 77$ ! 87 ! 1853 ! 532 ! Gaillardot, herb. de Syrie, $n^{\circ} 65$ !

IlA B. Juin. Les haies. - Turquie d'Asie. Mont Taurus (Kotsehy); - Syrie : Damas (Aucher-Eloy), Beslan (Kotschy), Aintab (Haussknecht), Tripoli, Beyrouth, Sidon (Boissier), haies du Nahr et Aoulé près de Saida (Gaillardot).

7. D. Dupontii Déségl., essai monog., in mém. soc. Acad. de Maine-et-Loire, X (1861), p. 58, extr., p. 18; R. nirea Dupont, non DC.; $R$. moschala var. rosea Seringe, in DC., prod., II, p. $598 ; R$. Damascena var. subalba Redouté, les roses (1824), livrais. 15, A; R. alba var. Damascene Poir., encyel., VI, p. 291, teste Redouté.

Icon. Botan. Reg. tab. 861 ! Redouté, 1. e. !

IIar. Juin. - Frence. Maine-el-Loire: Angers, fourneaux à chaux; transplanté au jardin botanique, d'une haie aujourd'hui détruite (Boreau, 18:32).

OBs. Thory cite comme patrie de eetle espèce les parties méridionales de l'Europe et dit qu'elle serait naturelle au sol de l'Espagne; mais on n'a aucune donnée certaine à cet égard.

§. IR. Abyssinica R. Br. in Salt., Mlyyss. app. 64; Lindl., 1. c., p. 116; Trall., 1. e., II, p. 99; Seringe, in DC., prod., II, p. 393 ; Ricland, loy. en Alyss. (1847), IV, p. 261; R. Schimperiana Ilochst et Steud. :

Icox. Lindley, I. c., tab. 15. 
Exs. Sehimper, Abyss. sectio prima, $n^{\circ} 189$.

Ilar. - A frique. Abyssinie : Vallée Chahagné prov. du Tigré (Richard), région inférieure des monts Scholoda (Schimper).

9. R. Brownii Tratl. I. c., II, p. 96; Sprengel, syst. (1825-27), II, p. 5.36; Swcet, hort. brit. (1859), p. 116; R. Brunonii Lind., I. c., 120; de Pronv., 1. e., p. 120; Seringe in DC., prod., II, p. 598.

Icon. Lindley, I. c., tab. 14!; Rot. mag. LXIX, tab. 4050 ; le texte dit: "fol. utrinque pilosis sublus glendulosis; "la figure 4050 représente une plante glabre; mème observation pour la planche de la flore des serres, IV, p. 566.

Haв. - Asie. Le Népaul (Wallich, 1819-21, in herb. DC.).

Var. b. nudiuseula Lindley, bot. reg., tab. 829 ; Ser. 1. c.; Desportes, ros. gal. $n^{\circ} 2476$.

Нав. - Asic. Le Népaul (Wallich, n689, in herb. Delessert).

10. RR. HRuseinoanensis Déségl. et Grenier, in Billotia (1864), p. 55; R. moschalu DC., fl. fr., IV, 1). 4.47 et Cat. hort. Monsp. (1815), p. 158 (non Miller); Duby, hot. gal., I, p. 175; Lois., 11. gal. (1828), I, p. 565; R. sempervirens var. pilosula Seringe, in DC., prod., II, p. 598; R. sempervirens var. I. moschate Gr. et Godr., 11. de Rr. (1848), I, p. $533 \%$

II Ar. Mai. - France. Pyrén.-Orient. : Prades (Coder, 1814; Thomas, 1820, in herb. 1)! !), Bonyult (Collin, in herh. Grenier), Perpignan (MonLagne); - Var. : Ilyères (Ilanry). - Italie. Sicile : Palerme (Todaro).

11. He. longicuspis Bert, mise. bot. XXI, in mem. Acad. Bonon. (1861), p. 201, tab. 15; Walpers, an. bot., VII, p. 878; R. sempervirens Hook. et Thom. (non Lin.).

ILs, - Asie. Ind.-Orient. : Khasia (herb. Boissier). Il diflère du II. sempervirens par ses pélioles el ses pédoncules légèrement relus, parsemés de quelques glandes fines, le tube du caliee velu à la base. 
12. He. sempenvipens 1. sp. 704; Miller, 1. e., 11"9; Willel., sp. II, p. 1072; Ait., I. c., II, p. 203; Gilibert, 1). d'Europe (1800), I, p. "385; DC. 11. fr. (180:3), IV, p. 446 ; Pers., l. c., p. 49; Desvaux, journ. bot., (1815), II, p. 115; DC., cat. hort. Monsp. (1815), p. 158; Lindl., l. e., p. 117, parl.; Thory, prod., p. 156; SaintAmans, fl. Agen. (1821), p. 205̃, exel. var. b.; de Prony., 1. e., p. 117, exel. var.; Lois., 1. e., p. 5̈9?, part.; Duby, 1. c., p. 173̈; Mutel, fl. fr. (185/), I, p. 587 ; Richt., col. Jot. Lin. (1840), p. 496, $n^{6} 5740$; Koch, syn. (1845), p. ¿23:3, exel. var.; Gonnet, fl. élément. de Fr. (1848), p. 482, exel. var.; Gr. et Godr., fl. de Fr. (1848), I, p. ̈3:3, exel. var. b.; Borean, 11. cent. de li France, éd. 2 (1849), n 631 , éd. 5 (18:37), $1^{\circ} 815$ et cat. de II.-et-L., p. 78; Delastre, 11. vien. (1842), l. $13 ̈ 7$; Arrond., 11. Toul. (18:4), p. 124; Déségl., I. c. p. 530 et extr., p. 1̋̈; de Mart.-Don., fl. Tarn (1864), p. 226 ; Boissier, Fl. orient. (1872), II, p. 688 ; R. moschatr liap. abr., p. 28 k et herb., texte Clos, révis. herb. Lapeyrouse; $\boldsymbol{k}$. atrovirens Viv., ll. ital., frag. 1, p. 4; R. alba Allioni, 11. Pedem. (1783), II, p. 159, non Lin.

Icox. Dillen, hort. Elhh., tab. 2946, 1. 518 ; Sibhorp, 11. Graeca, tah). 185; Bot. reg., tab. 46:3; Redoute, les lioses (1824), livatis. 15, C.; Clusius, hist. (1601); je cite d'après Lindley : Miss Lawr. tab. 4.

IIsB. Juillet. - France. Maine-et-Loire: Angers, Brise-Potière (Boreau), Saint-Barthclemy, Clsalomes (Boreau, catal.); - Vieme Entre Civray et Pressac (Boreau, Il. cent.); - Vendée: Luçon (P’ontarlier, in herb. Grenier); - Charente-inférieure : Fouras (Guillon), SaintLaurent-de-la-Prée (Lloyd); - Gard : Auduze (Grenier), Bagnols (Xatard, in herb. Grenier); - Iérault: Nontpellier ! - Aude: Narbonne (Delort), 
IIontague-Noire (Ozanon); - Iaute-Garonne : Toulouse (Arrondeau, flore); - Var: Fréjus (DC. 1807!), Hyères (Grenier). - Autriche. Dalmatic (Pelter). - Espagne. Saint-Filipe de Xantiva (Bourgeau), Pêna Castillo (Lang), Alyeziras (Boissier), Estepona (DC. 1850!). - Italie. Chivari (DC. 1808), Gènes (de Notaris, in herb. Grenier). - Turquie d'Europe Candie (Raulin, in herb. Boissier). - Grèce. Attique (de Heldreich), Athèn's (Boissier), Zante (Margot in herb. Boissier), Mont Taygète (Desprímux). - Afrique. Algérie : Alger (Bové), Bône (Mutel); - Maroc: 'Tanger' (Salzmann).

Var. b. microphylla DC., cat. monsp., p. 158 et fl. [r. (1815), V, p. 555 ; Seringe, 1. c.; Lois., 1. c.; Duby, 1. c.

IIAB. Mai, juin. - France. Hérault : bosquet Estor et bois de la Ramelte (DC. 1812 !), Béziers (Jullien). - Italie. Chivari (Turio, in herb. DC. 1808).

Obs. Le $R$. sempervirens L. aurait été introduit en Angleterre, vers $\mathbf{1 6 2 9}$, d'après Aiton, yui le dit originaire de la Germanie.

15. He. scandens Miller, dict., $n^{\circ} 8$, trad. franc., VI, p. 526; Boreau, fl. cent., èd. 5, II, p. 214, obs.; Déségl., l. c., p. 56 et extr., p. 16; R. sempervirens Tratt., 1. c., p. 97 (non Lin.); Guss., syn. sicul., I, p. 61 ; Rchb., fl. excurs., II, p. 623 ; Willd., herb. n० 9869, leste Crépin, primit. monog. ros., fasc. 2, p. 19 ; R. sempervirens $a$. scandens DC., f1. fr., V,

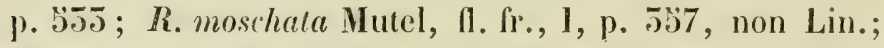
R. microphylla Dest'. fl. Atl.? I, p. 401, non Roxb.

IIs. Mai-juin. - France. Charente-inférienre : île de Ré (hipart); Gironde: Sainte-Foye (Chabert); - Dordogne : la Roquette (Puget); Lot-et-Garonne: Agen (Garroute); - Haute-Garonne : Toulouse (T'mbalLagrave); - Var : Le Lue (Hanry), Toulon (Iluet); - Alpes-maritimes: Antibes, Cannes (Bornet); - Aude: Narbonne à la Fenal (Martrin-Donos); - Vaucluse : Avignon (Grenier) ; - Pyrén.-Orient. : Bagnots ! - lle de Corse: Rogliano (Revellière in herb. Borean), Bonifacio (Kralik). Espagne. Pyrénées espagnoles: St-Lorinzo de la Muga (Bourgeau). Ilalic. Gènes (de Notaris in herb. Grenier), Palerme (Todaro), Ischia (Gussone in herb. DC. 1857), mont l'isan près d'Asciano (Savi), île de Sartaigne (Balbis, in herb. DC. 1826). - Turquie d'Europe. Constantinople (Boissier). 
Ons. I. Dans l'herbier DC., il y a un rosier étiqueté de sa main " $R$. verticillacantha Méral? n Romainville. C'est un $R$. scandens Miller, pris certainement dans un jardin.

OBs. II. L'espèce suivante nous étant inconnuc, nous rapportons in extenso la description donnéc par son auteur.

ak. Eroteri Tralt., 1. c., p. 98; R. scandens Brotero, Il. Iusit., II, p. 5 \&; R. sempervirens latifolia Thory, prod. p. 158 ; Seringe, 1. c.

Exs. Welwitsch, no 189 ?

Icox. Redouté, les roses (182㣙, livrais. $2 \ddot{b}, \mathrm{~A}$.

Frutex scandens 50-pedalis et ultra. Ramuli flaccidi, armati aculeis paucis, brevibus, rectis, validis, purpurasentibus, eeterum glabri. Folia alterna $2-5$ juga, foliolis $2-5$ pollicaribus, latis, obovatis, basi rotundatis, vel cordatis, apice acuminatis, simpliciter et argute serratis, utrinque glabris, lucidis, petiolis aculeis setisque glanduliferis sparsis munitis, stipulis linearibus, apice divaricatis, et glanduloso-serrulatis, acuminatis. Flores cymosi, longe peduneulati, magni; pedunculi hispido-glandulosi, subnutantes, centrali execpto, bibracteati. Urceoli plerumque glabri, elliptici; petala speciosa, rotundata, alha, quandoque extus roseo vittata. Fruetus nondum descripti (Trattinnick).

H.s. - - In Italia (Trattinnick).

Affinis $R$. sempervirenti, a qua tamen proecipue, urceolis glabris, segmentis 5 pinnatifidis, et petalis differt ('Tratt.).

14. Th. prostrata DC., cat. Monsp. (1815), p. 158 et 11. fr. (1815̋), V, p. 356; Lindl., l. c., p. 118; Arrondeau, 11. Toul., p. 12/4; Déségl., 1. c., p. \$6 et extr. p. 16; de Mar.-Don., florule du Tarn, p. 226; $\boldsymbol{R}$. semperrirens var. prostrata Desvaux, l. e., p. 115; de Pronv., I. c., p. 118; Saint-Amans, 11. Agen., p. 205; Mutel, I. c., p. 5.57; Gonnet, fl. élém. de la Fr. (1848), p.482; R. sempervivens var. lejostyla Koch, syn., p. $233 \%$; . sempervirens Lip., abr. p. 284, teste Clos, révis. de l'herh. Lapeyr., p. 260; $\boldsymbol{R}$. arensis var. prostrala Thory, prod., p. 15\%; Seringe in DC., prod., 1I, p. 597; Desportes, I. c., n²430; Duby, 1. (., p. 173; Lois., 1. e., p. 5\%9; R. arvensis Cíndolleance Trate, l. e., p. 104; R. humifusa Tratt., l. e., p. 102. 
Exs. Reichenbach, n"19:37; Willkomm, 11" 151 (sub nom. R. rubrifolia Vill.), no 196.

Haв. Mai-juin. - France, Lol-et-Garonne: Igen (Garroute, in herb. Ripart); - Gard : Le Vigan, Alson (Martin, in herb. Grenier); - Tarn : bois de Gaix près de Castres (de Larembergue), foret de Giroussens, loois d'Avignon près Parisot (de Martrin-Donos); - Aude : Axat (Loret, in herb. Grenier); - Haute-Garonne: bois de la Ramette près Toulouse (DC., 1807!), Toulouse (Baillet); - Bouches-du-Rhône : Aix à la Gaude (Eugène, in herb. Grenier); - Var : Le Lue (Ilanry). - Autriche. Illyrie : Trieste (Tommasini). - Esyngne. Billsto, Yrun, Fuenterrabia (Willkomm).

Var. obtusiuscula de Martr-Donos, I. c.

Tige couchée, à aiguillons épais, courts, coniques, inclinés, ceux des rameaux assez forts, dilatés; pétioles glabres, aiguillonnés; 5-7 folioles, ovales, la plupart un peu obtuses, fermes, glancescentes surtout en dessous, glabres et persistantes, celles des jeunes pousses rougeâtres ; pédoncules longs, solitaires ou en corymbe peu fourui, glabres ou munis de quelques rares soies glanduleuses; tube du calice oblong, glabre; sépales ovales, cuspidés, glabres ou rarement munis sur leurs bords de quelques glandes pédicellées; styles en colonne hérisée; fleur's blanches; fruit oblong (de Martrin-Donos).

II

Ons. J'ai vu, dans l'herbier DC, un spécimen cultivé en Angleterre du R. Ayreshirea hort. brit. (R. capreolata Neil? Edimb. phil. journ., no $\bar{J}$ 1. 102 ; R. arvensis var. Ayresteiren Seringe, in 1)C., prod., 11, p. 597) qui ne me semble pas difrérer du R. prostratu DC., si ce n'est qu'il a les folioles plus grandes. M. 'Timbal-Lagrave m'a envoyé un rosier de 'T'oulouse qui se rapporte exactement à l'échantillon de Llyel, qui est dans l'herbier $\mathbf{D C}$.

\section{B). Arvenses.}

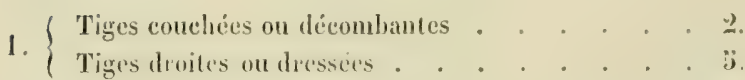

(Aiguillons uniformes, sans mélange de soies glandu-

2. lifères . . . . . . . . . . .

Aiguillons mélangés de soies glandulifères au sommet des rameaux . 

$5 .\left\{\begin{array}{c}\text { Pédoncules glabres, pétioles non glanduleux . . } \\ \text { Pédoneules plus ou moins glanduleux, pétioles por- } \\ \text { tant des glandes . . . . . . . . . . . }\end{array}\right.$

4. Iruit arrondi ou pyriforme

erronea.

I Fruit ovoïde-allongé.

̋. Folioles luisantes en dessus

mata.

Folioles non luisantes . . . . . . . . . bisracleatu. 6.

Arbrisseau élevé, corolle d'un beau blane, styles munis à la base de poils, fruit d'un rouge terne,

6. subarrondi .

conspicua.

Arbrisseau bas, touffu, corolle d'un blanc carıc, styles glabres, fruit rouge, ovoïde.

rusticance.

13̈. R. bibracteata Bastard, in DC., f1. fr. I, (1813), p. 557; Tratt. 1. c., p. 46; Boreau, l. c., éd. 2, 110 632 , éd. $5, n^{\circ} 814$ et Cat. de M.-et-Loire, p. 78; Arrondeau, 1. c., p. 12/; Déségl., l. e., p. 38 et extr., p. 18; de Martr.-Donos, fl. Tarn, p. 227; Cariot, 1. c., p. 167; Lloyd, fl. Ouest (1868), p. 180; Verlot, cat. pl. Dauph. (1872), 1. 112; R. arensis var. Bibracleata Seringe, in DC., prod., I, p. 397; Duby, bot. gal., I, p. 173; Lois.. 11. Gall., I, p. 539 ; Boreau, 11. cent., éd. 1, I, p. 185; Delastre, 11. vien., p. 13̈\%; Baker, monog. of brit. ros., in Linn. socicty's journ., NI, p. 242; R. arvensis var. multiflora Borcau, mém. de la soc. ind. d'Angers (1841), extr., p. $9 ; \boldsymbol{R}$. arvensis var. bracteata Gr. et Grodr., I. c., I, p. วัॐ̆; R. arensis var. umbellata Godet, f1. Jura (1855), p. 217; Reuter, Cat. Genève (1861), p 75; R. slylosa var. bracteala Saint-Amans, fl. Agen. (182I), p. 204; R. systyla Woods! brit. spec. of ros., in trans. soc. Limm. (1816), XII, p. 250 et herb. n 122, n $^{\circ}$ 126; Smith, Engl. flora, II, p. 59/; Lindley, I. c., p. 111; de Pronv., l. c., p. 115; Ilooker, brit. 11., éd. 2, p. 245 (non Bast.).

Icox. Engl. Jot., tab. $189 \ddot{.}$ 


\section{Exs. Billot, $n^{\circ} 1870$.}

IIs. Mai, juin. Ilaies. - France. Vendée : Fontenay-le-Comte (Letourneux) ; - Maine-et-Loire : Angers (Bastard, in herb. DC. 1815-17) Angers (Boreau); - Cher : Bourgneuf, près de lia forêt d'Allogrny ! - Isère: Crémieu (Verlot, catal.); - Tarn : Saint-Urcisse (Mart.-Donos); - Lozère : Mende (Prost, in herb. DC. 1815); - Ilaute-Garonne: Toulouse (Noulet, in herb. Grenier); - Loire-inférieure: Pornic (Dubouché in herb. Grenier). Angleterre. Sussex : (Woods ! échantillon récolté par lui--même et que je tiens de la générosité de II. J.-G. Baker); - Essex : Tipton. (E.-G. Varenne); - Devonshire: haies à Longloridge, Revelstoke, Plymouth (Areher-Briggs) ; - Cornwall : Burraton, Saint-Stephens (Archer Brigers).

16. R. conspicua Boreau, in mém. de la soc. Acad. de M.-ct-Loire, XII (1862), p. :3:3; R. corymbosa Bastard! inéd. in herb. DC. (non Ehrh., nec Bose).

ILs. - France. Maine-et-Loire: Angers, chemin de Saint-Barthélemy (1'opeau), route de Candé à Engrande, crètes de fossés tourbeux (Bastard, in herb. DC. 1811 !).

17. R. *usticana Déséglise, in Billotia (1865), p. 5/4 et extr., p. 2; R. arvensis var. oblusilte seringe, in DC., prod., II, p. 597 ?

Exs. Déséglise, herb. ros., n*1.

Hab. Juin. Haies, - France. Calvados : bois de Nanerbe près de Lisieux ! - Cher : Fontmoreau, Saint-Martin (Ripart), Marẹy, près de la Servanteric !

18. Ma. Reggearama Schrenk, enum. pl. nov., p. 75; Ledeb., fl. lioss., II, p. 82.

Glaberima; ramis decumbentibus, aculeis stipularibus rectis, stipulis omnibus conformilus oblongo-linearibus planis, auriculis denticulatis, petiolis aculeatis, foliolis "̈-7 (parvis decidais) oblongis simpliciter argute serratis, pedunculis uni-multifloris calycibustue subghobosis glabris 
bracteis latis einctis, laciniis calyeinis subintegerrimis, stylis in columnam staminibus breviorem cohacrentibus, ovalis, sessilibus.

Proxima R. arcensi, sed diversa aculeis raris stipularibus rectis, foliolis parvis, stylis in columnam brevem villosam cohacrentibus (Schrenk).

IIs. - In Siberiae Altaicac des. Songorn-Kirghisico ad. fl. Koksu.

Oss. N'avant vu aucun échantillon de ee rosier dans les herbiers de Genève, j’ai cru devoir rapporter la description donnée par l'auteur.

19. Mh. aryensis Hudson, 11. Angl. (1762), p. 192, éd. 2 (1798), p. 219; Willd., sp. II, p. 1066; Smith, fl. brit. (1804), II, p. 558 ; DC., fl. fr. (1803), IV, p. 458; Bastard, ess. fl. de Maine-et-Loire (1809), p. 187; Héral, fl. Par. (1812), p. 189; Desvaux, journ. bot. (1815), II, p. 115; Woods, I. c. (1816), p. 252; Seringe, mélanges (1818), I, p. 3; Lindley, I. e. (1840), p. 112, exel. var.; Saint-1mans, fl. Agen. (1821), p. 204; Tratt, I. c. (1824), II, p. 105; Smih, Engl. 11 (1824), II, p. 596; Ballis, f1. Lyon. (1827), p. 256; Dumort., fl. helg. (1827), p. 94, exl. var.; Chevalier, 11. génér. de Paris (1827), II, p. 688; Desportes, 1. c. (1828), $n^{n} 24 k$ k $n^{\circ}$ 24k3; Lorey et Duret, f1. Côte-d'Or (1851), I, p. 502; Host, 11. Aust. (1851), II, p. 26; Hooker, brit. fl. (1853), p. 24k; Borcau, fl. cent., èd. 1 (1840), nº 400, éd. 2 (1849), $1^{\circ}\left(6.5 .5\right.$, édiı. 5, (18:37), $n^{\circ} 813$ et Cat. de N.-et-Loire (18:39), p. 78; Koch, syn. (1845), p. 2:34; Godet, 11. juma (18\%5), p. 216; Arondeau, l. e. (18\%), p. 12/; Renter, Cat. Genève (1861), p. 75; Dumort., monog. ros, de la fl. belge(1867), p. 64, part.; Baker, Northumb. (1868), p. 164 et monog. of brit. ros., in Linn. Society's journ., XI, p. 24.1; Lloyd, fl. Ouest (1868), p. 180; Boissier, 11. 
orient. (1872), II, p. 688; Perard, Cat. Montlue. (1872), p. 8.j; R. repens Scopoli, 11. carn., ćd. 2 (1772), I, p. 533 ; Gmel., 11. Bad.-Als. (1806), II, p. 4.18; Rau, enum. ros. (1816), p. 40; Rehh., fl. exeurs. (1850), II, p. 62/4; Mulel, fl. fr. (185/), I, p. 5.6 ; Déséglise, l. c. (1861), X, p. 62 et extr., p. 22; Cariot, étud. des fleurs (186:3), II, p. 168; de Martrin-Donos, fl. du Tarn (186/), p. 227; K. Koch, dendrol., I, p. 261; R. serpens Wibel, Werth. (1799), p. 263; Kirsehleger, f1. d'Alsace (18:32), I, p. 242; R. sylvestris Herm., diss. (1762), $n^{\circ}$ 10; Poll., palat. (1776), II, p. 31; Roth. Catal. bot., fase. I (1797), p. 39; R. scandens Mönch, Verzeich. (1785), p. 118, non Miller; R. herporhodon Ehrh., beitr., II, (1788), p. 69; R. Halleri Krocker, f1. Siles. (1790), II, pars 1, p. 130; R. fusca Mönch, meth. (1794), p. 688; R. canina var. sylvestris Roth, f1. germ. (1789), II, p. つ60; R. sempervirens Roessig, die rosen (1800), p. 52 (non Limn.); R. glauca Dierb. heidelb. (1818), p. 140.

Icos. Custis, bot. mag. XLVI, tab. 20:14, mala; Jacquin, fragm., tab. 104; Baxter, brit. bot., V, tab. 523̈; Roessig, dic rosen, $n^{\circ} 52$; je cite d'après Pritzel les planches suivantes : Curtis, lond., IV, tab. 125; Schrank, f1. monac., IV, tab. 50:3 Guimpel, Holzgew. 95; d'après Lindley: Miss Lawrance, tab. 86.

Exs. Reichenbach, n 1752. Orphanides, flora gracea, $n^{\circ} 5108, n^{\circ} 5493$. Seringe, $n^{\circ} 1, n^{\circ} 4 \%$. Sendtner, $n^{\circ} 959$. Wirtgen, $n^{\circ} 82, n^{\circ} 180$. Billot, $n^{\circ} 5716$. Déséglise, $n^{\circ} 2$.

Hab. Juin. Ifaies, bois, landes. - Plante vulgaire en Franec, en Belgique, en Angleterre; rare en Éeosse; commune en Autriche, dans le Palatinat, la Silésie, la Carniole, l'Allemagne, la Suisse. Gussone, ne l'indíque pas en Sicile; je ne la vois pas non plus en Espagne ni en Portugal; cependant Seringe dans le modromus, dit: "In Europuc sylvis el sepibus. " Elle 
manque à l'Algérie; elle se retrouve dans la Turquie d'Europe, en Bosuic (Sendtner, exs. no 959), en Macédoine, Mont Korthiati (Orphanides, exs. $\left.n^{0} 5493\right)$; en Grèce, Mont Malevo (Orphanides, exs. no5108).

M. Boissier, dans sa flore d'Orient, dit pour l'aire géographique de celte espèce : Europa media et Australis ab Angliû, Belgio el Germaniu ad regionem Danubialem usque. n

Var. parvifonia de Martrin-Donos, I. c., p. 227.

Feuilles et fleurs très-petites; tiges presque dépourvues d'aiguillons.

IHar, - Tarn : forèt de Giroussens (de Martrin-Donos).

ODs. Voici les obserrations de M. Crépin, sur le R. arvensis de l'herbier de Willdenow, $n^{0} 9861$. " Ce numéro est représenté par trois feuilles simples. Fol. 1). Deux échantillons appartiennent au $R$. arvensis Iluds. Au milieu de la feuille, il y a un ramuscule qui appartient au groupe des Sepiaceae et qui paraît voisin du R. Jordani. Fol. 2, 5). Quatre rameaux florifères; $\boldsymbol{R}$. repens Hop. - No 9860. Ce numéro, $R$. arvensis L., est

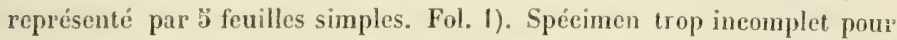
bien juger de la forme du $R$. arvensis à laquelle il appartient. Fol. 2). Un rameau florifére de la section des Caninae, trib. Biserralae ct voisin du R. oblonga Ripart. Fol. 5). Un rameau fructifére. Hème forme que celle du folio deux. Fol. 4). Un rameau fructifere. Forme de la section des Caninae, trib. Biserratae et qui peul être rapportée au $R$. viridicalu Puget. Fol. B). C'est une forme de la section des Cuninae, trib. Pubescentes et voisine du R. myriformis Déscigl. "(Crépin, primit. monog. ros., fasc. 2 (1872), p. 20 et p. 21).

20. R. erromea Ripart, in Crépin, primit. monog. ros. (1869), fasc. I, p. 12; R. arvensis Vill., Il. Dauph. (1789), III, p. ̈̈48; Krocker, I. c., p. 1/4 ; Gilibert, pl. d'Europe, I, p. ̈82; Thuillier, 1. Paris., p. 2̈0; Pers., syn., II, ). 1.7; Déséglise, 1. c., 1. 61 et extr., 1).21, non IIudson.

\section{Exs. Orphanides, flora Graeca, $\mathbf{1 1}^{\circ} 684$ !}

IIAB. Juin. Bois. - Angleterye. Youkshire: fourrés à Scawton (Baker). - France. Cher: Saint-Germain-des-bois, la Grange-Saint-Jean, bois de Fleuret (Ripart), bois de Saint-Florent! de Charron! et de Marmagne ! forêt du Rhin-du-bois ! - Haute-Savoie : Brenthonne (Puget). - Grèce. Mont Malevo (Orphanides, in herb. Boissier). 
OBS. J'ai recu de M. Kerner, d'Innsbruck, sous le nom de $\boldsymbol{R}$. baldensis Kerner, une forme assez curieuse du $\boldsymbol{R}$. arvensis, et sur laquelle il me serait assez difleile de me prononcer, n'ayant vu que des échantillons incomplcts. - $\boldsymbol{R}$. buldensis Kerner, mss. Caractères généraux du $R$. arvensis, dont il diffère par ses pétioles velues, glanduleux, aiguillonnés, ses folioles velues à poils apprimés en dessus, velues en dessous principalement sur les nerrures, la médiane portant en outre quelques petits acicules. Je ne connais pas la forme de la corolle et du fruit.

Har. - Autriche. Tyrol : au pied du mont Baldo (Kerner).

21. MR. ovata Lejeune, fl. de Spa (1811), II, p. 512; R. artensis var. orala Desvaux, journ. hot. (1815), II, p. 115, cxel. syn.; R. stylosa Mérat, fl. Par. (1812), p. 192 (non Desvatix); $\boldsymbol{R}$. controcersa Leman, inéd. in herl. Boreau; Rosier rompant Reynier, mém. soc. de Lausamne (1785)), I, p. 69; R seperina Sanzé et Maillard, fl. des Deux-Sèvres.

Icos. Reynier, l. c., tab. V. Redonte, les roses (182/4), livrais. 6, D.

Port du $\boldsymbol{R}$. arcensis, aiguillons dilatés en forme de disque ì la base, droits, crochus ou inclinés; pétioles pubescents, parsemés de glandes aiguillonnés en dessous; :)-7 folioles ovales, orales-elliptiques, les inférieures obtuses ou mème rétuses, glabres, vertes en dessus, glauques en dessous, à nervure médiane velue, villosité disparaissant en partie arec l'àge, simplement dentées, à dents ouvertes et terminées par un mueron; pédoneules portant des glandes fines stipitées plus on moins abondantes; tube du calice ovoide, glabre; fleur plus grande que dans le R. artensis; styles soudés en une longue colonne glabre; fruit ovoïde allongé.

Ifur. Juin. Inaies, bois. - Anglelerre. Devonshire: Plymouth (Bake:); - Yorkshire: Saint-John (Baker); Cheshire : (Webb) - Belgique. 


\section{(49)}

Prov. de Namur: Ilan-sur-Lesse (Crépin). - Frnnce. Deux-Sèvres: (Sauzet!); - Cher : bois de Varmagne ! la Touche près de Mehun! forèt de Fontmozeau, ruines du Château! Bourges, vignes du Château! la Servanterie près de Mehun! - Ilaute-Savoic: Arenthon (Puget).

22. IR. galsicoides Yoh.; R. stylosa var. gallicoides Baker, l. e., p. 240 .

Port et caractères généraux du $R$. arvensis, dont il diffère par ses tiges chargies au sommet de glandes fines, stipitées, violacées, mélangées avec des aiguillons, ces glandes se retrourent aussi sur les tiges stériles; ses folioles plus grandes, ovales-elliptiques, celles des jeunes pousses lancéolées ou moins cuspidées au sommet, les folioles adultes largement dentées, ì dents ouvertes, quelques-unes portent de petiles dents accessoires; les pédoncules sont solitaires ou réunis par 2-5̃, chargés de glandes fines violacées, ayant à leur base des bractées ovales, cuspidées, glabres en dessus, glanduleuses en dessous, plus courtes que les pédoncules; tube du calice perit, violacé-pruineux, obocoücle, portant ì sa base des glandes fines; divisions ealicinales appendiculées, bordées de glandes, saillantes sur le bouton, beaucoup plus courtes que la corolle; fleur grande; styles soudes en une longue colonne grèle, glabre, s'élevant au-dessus d'un disque conique saillant; fruit ovoïde allongé, rouge.

IIAB. Juillet. -- Angleterve. Warwickshire: Chesterton Wood near Myton (Bronwich). - Frunce. Loire-inféricure: Torton près de Nantes (de l'Isle Georget).

OBs. R. tuguriorum Willd., en. hort. Berol. (1809), p. 544 et herb., $\mathrm{n}^{\circ} \mathbf{9 8 5 1}$, le fol. 1 du $\mathrm{n}^{\circ} 9819$, les fol. 1 et 2 du no 9857 ; Lindl, 1. c., p. 159 ; Tratt., I. c., I, p. 76 ; Crépin, I. c., fasc. 5 (1872), p. 22 , cum descript. Sprengel et Steudel rapportent celte espice de Willdenow au R. arvensis. M. K. Koch, au R. repens. M. Crepin en fait une hybride ( $R$. arvensis $\times$ gallica ?). - Willdenow a décrit cette espèce à fleur 
pleine, sur une plante cultivéc au jardin de Berlin, dont la patrie est inconnue.

$$
\text { c). Stylosae. }
$$

Styles agglutinés en colonne plus ou moins saillante.

1 Pédoncules glabres ou presque glahres . . . . .

1. Pédoncules glanduleux . . . . . . . . 5.

2. $\left\{\begin{array}{l}\text { Pédoncules glabres } \\ \text { Pédoncules peu glanduleux. }\end{array}\right.$

Pétioles inermes, folioles petites elliptiques, tube du 5. $\left\{\begin{array}{l}\text { ealice ovoïde, fleur rose elair, fruit petit ovoïde parvula. } \\ \text { Pétioles aiguillonnés, folioles ovales-aiguës, tube du } \\ \text { ealice obovoïde, fleur d'un blanc pur, fruit petit, } \\ \text { sphérique, rouge . . . . . . . . virginea. }\end{array}\right.$

Pétioles velus, parsemés de glandes, aiguillonnés, feuilles grandes, ovales-elliptiques, glabres en des-

4. $\left\{\begin{array}{l}\text { sus, pubescentes en dessous, fleur rose . . . . . } \\ \text { Pétioles parsemés de poils blanchâtres, aiguillonnés, }\end{array}\right.$ Clotildea. feuilles ovales-aiguës, glabres, fleur blanche, onglet un peu jaunâtre, fruit petit, ovoïde, rouge . . . immitis.

5. $\left\{\begin{array}{l}\text { Folioles légèrement velues en dessus, corolle blanehe. stylosa. } \\ \text { Folioles glabres en dessus }\end{array}\right.$

6. $\left\{\begin{array}{l}\text { Corole rose, feuilles vertes, fruit d'un rouge sanguin. systyla. } \\ \text { Corolle blanche, onglet jaunâtre, feuilles devenant }\end{array}\right.$ d'un vert jaunâtre, fruit rouge-orangé . . . leucochroa.

25. R. stylosa Desvaux, journ. bot. (1809), II, p. 517 et (1815), II, p. 115; DC.. cat. Monsp., p. 158 et fl. fr. V, p. 556; Lejeune, f1. de Spa? (1811), II, p. 512 ; SaintAmans, 1. c., p. 204; Lois., 11. gall., I, p. 565 ; Desportes, ros. gal., p. 107; Mutel, fl. fr., I, p. 53\%; Boreau, bull. de la soc. ind. d'Angers (18/4), extr., p. 9, et fl. cent., éd. 2, no 656, éd. 5, no 818 et Cat. de M.-et-Loire, p. 78; Delastre, fl. de la Vienne, p. $15 \% 3$; Arrondeau, fl.toulous.? p. 124; Déségl., 1. c., p. 66, extr. p. 26 et in Billot, arch. de la fl. de Fr. et d'All., p. $5 \overline{5} 4$; de Martr.-Donos, l. c., 
p. 290; Cariol, élud. des fleurs (1863), II, p. 169; Lloyd, 11. de l'Oucst (1868), p. 179; Pérard, cat. de Montlue. (1872), p. 85); R. stylosa a. Destanxima Seringe, in DC., prodr., II, p. 390 excl. syn. Lindl. et Smith; Duby, 1. c., p. 17\%; Gr. et Godr., I. c., p. 583; R. serpenti-canina Kirschl., fl. d'Als., 1, p. 243.

Icov. Desvaux, l. c., tab. XIV.

Exs. Billot, $n^{\circ} 1485$; Déséglise, herb. ros., $n^{\circ} 40$.

IIab. Mai, juin. Haies, bois. - Prusse. Malmedy, d'après Lejune, flore de Spa. - France. Calvados: Saint-Pierre-des-Ifs (Boreau); - Maine-etLoire: Villebernier, Angers (Boreau); Vienne: Croutelle (Delastre); Deux-Sévres: indiqué dans ce départ. par MI. Sauzé et Maillard, dans leur catalogue; - Cher : bois de Gérissai, forêt d'Allogny (Ripart), forêt du Rhin-du-bois! bois de la Touche prés de Mehum! hois de Marmagne ! - Allier : Montluçon (Pérard, cat.); - Tarn-et-Garonne : Grizolles (Timbal-Lagrave); - Tarn : d'apres la florule de Nartrin-Donos, ce rosier serait A. C. ; 17 localités sont indiquées; - Jaute-Garonne: Toulouse (Arrondeau, flore); - Rhòne: Ecully (Chabert), Lyon à la Tête d'or (Jordan).

24. Clotaldea Timbal-Lagrave in Crépin, primit. monog. rosar., fasc. I (1869), p. 59 (non Timb. Lagr., bull. hist. nat. de Toulouse, IV, p. 172, 1871).

Fenilles grandes, ovales, ovales-elliptiques, arrondies aux deux extrémités, d’autres arrondies au sommet, cunéiformes à la base, glabres en dessus, pubescentes en dessous, complètement dépourtues de glandes, simplement dentées, à dents convergentes vers le sommet; pétioles velus, parsemés de glandes fines, faiblement aiguillonnés, quelquesuns sont mème inermes; stipules assez allongées, lancéolées, glabres, bordées de glandes, oreillettes aiguës, droites ou peu écartées; pédoncules 1-̄̄, parsemés de glandes fines peu ahondantes, munis it leur hase d'une bractée lancéolée, cuspilée an sommet, de couleur vincuse, giabre en 
dessus, parseméc de poils mous peu abondants en dessous, égalant les pédoncules, les pédoncules extéricurs sont munis en outre de deux petites bractées opposées plus courtes qu’eux; le pédoncule central est sans bractée; tube du calice ovoüde, glabre, d'une couleur pruineuse; divisions calicinales ovales, cuspidées all sommet, les extérieures glabres en dessous, munies d'appendices étroits filiformes, les extérieures entières, tomenteuses aux bords; styles agglutinés en colonne hérissée s'élevant au-dessus d'un disque un peu conique; fleur rose. - Description établie sur les échantillons recus de $\boldsymbol{M}$. Timbal-Lagrate en 1864 .

Han. - France. Haute-Garonne: bois de Bouconne près de la ferme du Bugué (Timbal-Lagrave).

Ons. Au mois de février 1864, j'ai reẹu de $\mathrm{M}$. Timbal-Lagrave un rosier venant du bois de Bouconne, près la métairie du bugué, sous le $n^{\circ} \mathbf{9 3}$, que j'ai laissé indéterminé en le rapportant au groupe du R. systyla. En 1869, M. Crépin, dans ses primitiae monog. ros., en a donné une diagnose sous le nom de R. Clotildea Timb.-Lagrave, et il place ce rosicr dans les Slylosue. En 1871, M. Timbal-Lagrave, dans le Bulletin d'histoire naturelle de Toulouse, IV, p. 172, donne de ce rosier la description suivante : Ie "R. Clotildec est peu répandu dans nos environs; il est cependant com" mun à Bouconne, du côté de Brax, près la métairie dite du Bugué. II " appartient à la section stylosae par ses styles un peu en colonne agglu" tinée. Les fleurs sont grandes et roses; les feuilles grandes, vertes en "dessus et glauques en dessous avec des glandes sur les pétioles et les " nervures. Le fruit est bleuâtre avant la maturité, puis rouge, glubulcux; "les sépales tombent quand le fruit devient rouge."

"J'incline à penser que cette plante est le R. suavis Arrondeau, mais " non le R. suavis Willd., comme le croit M. Noulet. n (Timbal-Lagrave).

Remarquons une chose, e'est que l'auteur de cette description ne fait aucune mention du port de l'arbrisseau, des aiguillons, de la dentition des folioles, ni si les styles sont glabres, velus ou hérissés. Les pédoncules sont-ils glabres on glanduleux? M. Timbal-Lagrave passe sous silence ees caractères, qui pourtant sont utiles à comnaitre. 


\section{( 55 )}

En 1872, j’ai prié M. Timbal-Lagrave de vouloir bien me procurer son type : le 4 janvier 1875, j’ai recu sous le nom de $\boldsymbol{R}$. Clotildea un mélange regrettable de la part d'un observatcur, tel que M. Timbal-Lagrave.

Voici ce que j'ai recu et que je conserve en herbier : l'échantillon en fleurs a les styles libres! très-courts, ylabres; les feuilles sont elliptiques, lancéolées, légèrement velues en dessus, pubesentes églanduleuses en dessous, doublement dentées, aiguillons grêles, droits. Cet échantillon appartient au groupe du $R$. comentose. L'échantillon en fi uit vert a les folioles grandes, ovales-elliptiques, glahres sur les deux faces, parsemées de glandes fines en dessous, la dentition des folioles est double et triple; les styles libres! courts, hérissés; les divisions calicinales redressées sur le fruit me paraissent persistantes. Cet échantillon appartient à ce que M. Timbal-Lagrave nomine $\boldsymbol{R}$. Tolosına et qui est une rubiginosac. Un troisième échantillon en fruit mûr, me semble être pris sur le même buisson que celui en fleurs; les feuilles et les aiguillons sont les mêmes.

Si l'échantillon en fleurs a les folioles dépourvues de glandes, je ne puis pas admettre que les glandes aient poussé comme des champignons sur les folioles du rameau en fruit vert, pour disparaitre ensuite sur celles du ramaux dont le fruit est à maturité. Le 7 février 1875, j’ai reç de nouveau de M. Timbal-Lagrave ce $R$. Clotildea, mais encore avec un mélange. Sur deux échantillons en fleurs, l'un a les folioles doublement dentées et appartient au groupe du $R$. tomentosn; l'autre est le vrai R. Clotilder ; ces deux échantillons ont les folioles églanduleuses en dessous et sont en contradiction avec le texte de l'auteur.

M. Timbal-Lagrave dit aussi qu'il penche à croire que cette plante serait le R. sunvis Arrondeau non Willd. M. Arrondeau, fl. Toulous. (IS52 4), p. 126, dit: a diguillons coniques, grèles, courbés; folioles glabres, d'un " vert foncé en dessus, pâles et glauques en dessous, orbiculaires, simple" ment dentées; styles courts; fleur's d'un rose foncé."

Ne comnaissant pas le type de $\boldsymbol{H}$. Arrondeau, je ne puis parler que d'après le texte; mais, alor's, je crois que la plante de M. Timbal-Lagrave est le contraire de celle de M. Arrondeau, car les échantillons recus cn 1864, 1875, 1875, ont les feuilles doublement dentées et nullement orbiculaires.

2:. R. systyla Bastard, essai 11. de M.-et-Loire, suppl. (1812), p. 51; Borean, fl. cent., éd. 2, n63̆4, éd. 5, n. 816 et mém. sor. indust. d'Angers (1844), extr., p. 9 
et catal. de M.-et-Loire, p. 78; Arrondeau, I. c., p. 12/, Déséglise, in Billot, annot. f1. de Fr. et d'Allem. (183̋3), p. 9, et mém. de la soc. Acad, de M.-et-L., X, p. 64 et extr., p. 24; de Martr.-Don., I. c., p. 228; Cariot, I. c., p. 168; Lloyd, l. c., p. 179; Verlot, eat. pl. du Dauph., p. 115; Godet, fl. Jura, p. 216 ; R. fastigiata Bastard! 1. c.; Lejeune, fl. de Spa (1811), II, p. 314; DC., It. fr. (1813), V, p. 553̈; Poir., encyelop. suppl., IV, p. 711; Tratt., I. c., II, p. 5; Déséglise, I. c., p. 65 ct extr., p. 5๊̄; de Martr.-Donos, I. c., p. 228; Cariol, I. c., p. 168; R. leucochroa var. angustuna Desv., journ. bot. (1815), II, p. 115; R. canina var. fastigiata Desvaux, I. c., p. 114; Seringe, in DC., prod., II, p. 615; Duby, I. c., p. 178; R. brevistyla var. DC., I1. fr., V, p. 557; R. rustica Leman! bull. philom. (1818), extr., p. 11, $n^{\circ} 28$; R. stylosa var. leucochroa Seringe, 1. c., p. \$99, part.; Duby, 1. c, p. 176 , part.; G. et Godr., I. c., p. కّ̋̋, part.; R. collina var. fastigiala Thory, prod., p. $70 ; \boldsymbol{R}$. serpenti-canina Kirschl., I. c.; R. stylosa Gaudin, fl. helv., III, p. 556 (non Desvaux); Rechb., fl. excurs., II, p. 624; Reuter, Cat. de Genève, p. 70; Dumort., monog. des ros. de la flore Belge, p. 64 part.; R. stylosa var. trivialis Gren., Il. Jura., p. 241 .

Icon. Redouté, les roses (1824), livrais. 11, B.

Exs. Billot, $n^{\circ} 1665$; Déséglise, $n^{\circ} 5, n^{\circ} 59$.

Ilaв. Juin. IIaies, bois, - Angleterre. Cornwall : haies à Burraton, Saint-Stephens, Saint-Mcllion (Brigrss); -- Glocestershire : Sydney (Purchass); - Devonshire: haies pres de Plymouth (Baker). - France. Loireinférieure: Thouré (Lloyd, flore); - Morbihan: Vannes (Lloyd, flore); Deux-Sèvres: Lassaudière (Sauzé); - Vendée: Napoléon-Vendée (Grenier); - Maine-et-Loire : Angers (Bastard, 1815 in herb. DC.), C. dans ce 
département (Boreau, catal.); - Loiret: le Briou, Ardon en Sologne (Jullien); - Indre: Chateauroux (Boreau); - Cher : C. C. dans ce département; - Haute-Vienne: Saint-Jean-Ligoure, coteau de la Roselle (Lamy); - Saòne-et-Loire: Autun (Carion), Chảlon-sur-Saône (Ozanon); Tarn: A. C. dans ce département (de Martrin-Donos, flore); - Lot-etGaronne: Pommaret pris d'Agen (Ozanon); - Haute-Garonne: Avignonet, Toulouse (Timbal-Lagrave); - Rhône: Lyon à Couzon, au pont d'Alay (Ozanon), Charbonnière, tour de Salvagny (Chabert), Tassin, Brouilly-Saint-Lager (Boullu); - Var: Ies Maures (IIanry); - HauteSaroie: Pringy, bois de Proméry et do Barioz, Thonon, Tessy (Puget). Suisse. Cant, de Genève: Carouge! - Cant. de Vaud: Nyon, Versoix, Coppet (Reuter, catal.); - Cant. de Bâle : Jura de Bâle (Christ).

Var. b. Ianceolata Lindley, monog, ros., p. 111; de Pronv. I. c., p. 115; R. brevislylu b. lanceolala Tratt., I. c., p. 47 ; R. slylosa var. ? lanceolata Seringe, in DC., prod., II, p. 599.

"Foliolis ovato-lanccolatis, fructo sphoeriro." -- Lindley.

Les feuilles sont plus grandes que dans le type, elliptiques-lancéolées, la terminale arrondic à la hase et plus ou moins acuminée au sommet, vertes, glabres en dessus, pubescentes sur les nervures, d'autres folioles n'ont de villosité que sur la côte médiane, simplement dentées; pétioles pubérulents ou glabres, parsenés de quelque rares petites glandes fines; pédoncules glabres ou munis de quelques soies glanduleuses peu abondantes, solıtaires ou réunis en corymbe de $5-13$ an sommet des rameaux; bractées ovales-acuminées, glabres, plus courtes que les pédoncules; tube du calice sphérique; styles en colonne, glabre, plus ou moins saillante, disque conique; fleur d'un rose clair; fruit rouge globuleux.

Нав. Mai-juin. Haies, bois. - Angleterre. Indiqué dans le midi de l'Irlande, par lindley. - France. Yonne : Auxerre (Mabile); - Vienne Ilontmorillon (Chaboisseau); - Cher : Berry, Boulon; - Rhône : CharJonnière (Chabert).

Ons. A l'exemple de De Candolle, flore française, j’avais séparé dans mon essai monographique des rosiers de la France le $\boldsymbol{R}$. fastiginta Bast., du $R$. systyla Bast., mais une étude plus approfondic de ces deux formes me fait adopter l'opinion de 11 . Boreau; le savant auteur de la flore du centre de la France dit en observation, p. 215: "Bastard a établi le R. systylu " sur des individus à rameaux uniflores et à longs styles, et le $R$. fastigiata " sur des pieds à fleurs en corymbe et à styles moins saillants, mais les "variations s'observent souvent dans le même buisson." 
Nous ne chercherons pas i éclaircir Ies observations plus ou moins judicicuses émises par M. Grenier, dans sa flore du Jura; pour lui, les $\boldsymbol{R}$. stylosa, $\boldsymbol{R}$. syslyla, $\boldsymbol{R}$. fastigiala et $\boldsymbol{R}$. leucochror, tout ne fait qu'un et, à l'en eroire, on trouverait sur le même pied toutes ces formes réunies: pour M. Grenier, a le $R$. stylosa Desv. édité en 1810, n'est que la forme pubescente atteignant son maximum de développement. "

M. Du Mortier, daus sa monographic des rosiers de la Belgique, sous le nom de $R$. stylosn, nous semble avoir en vue le $R$. systyla Bast., et le $\boldsymbol{R}$. leucochroa Desv. ? Il indique ee rosier dans le Luxembourg, et le dit répandu en Angleterre. C'est, il nous semble, le contraire: le $R$. systyla Bast. y est rare; Lindley et Woods ont pris pour le $\boldsymbol{R}$. systyla, le $\boldsymbol{R}$. bibracteata Bast., qui est loin d'y être commun.

26. IR. imsunitis Déséglise in mém. soc. Acad. de M.-et-Loire (1875), XXVIII, p. 17, extr., p. 1, deseript. de $q$. esp. nouv. du genre rosa.

Har. Juin. Bois. - France. Cher: bois de Marmagne! bois de Rouet près les vignes, commune de Mehun !

27. parovala Sauzé et Maillard, cat. du départ. des Deux-Sèvres (186/), p. 27; $R$, modesta Ripart, in Crépin, primit. monog. ros., fasc. I (1869), p. 59.

"Cette espèce inédite est commune dans le midi du "département; elle se distingue facilement du R. systyla " par ses fleurs beaucoup plus petites, ses pédoncules 》glabres, ses styles en colonne toujours saillante, par ses " tiges grèles et ses feuilles d'un vert tendre. " - Sauzé et Maillard, catal.

Arbrisseau à aiguillons rohustes, dilatés à la base, courbés ou presque droits, ceux des rameaux plus petits, écorce verdâtre ou violacée, tiges flexueuses; pétioles pubeseents, sillonnés en dessus, inermes, quelques pétioles très-faible-

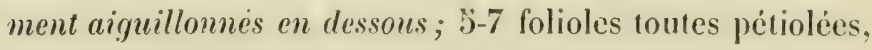
la terminale ovale, termince en pointe au sommet, les latérales elliptiques aiguës ou arrondies aux deux cxtrémites, 
petites, glabres, d'un vert clair en dessus, pubesentes sur les nervures, simplement dentées: stipules étroites. glabres, à oreillettes aignës, droites; pédoncules solitaires ou réunis en bouquet par trois. g'abres; tube du calice. ovoüde, oglabre; divisions ealicinales ovales, terminces en pointe, les extérieures appendiculées, les intérieures entières, saillantes sur le bouton, plus courtes que la corolle; fleur d'un rose clair; styles glahres, en colonne saillante; disque conique; fruit petit ovoïde.

Hab. Juin. Ilaies, bois. - France. Deux-Sèvres: (Sauzé !) ; - Cher : pacage de Bouy, commune de Berry, forêt de Fontmoreau, hois de Horthomier, Marçay près de Quincy ; - Ilaute-Garonne : Toulouse, à Laramette (Timbal-Lagrave).

28. Ris.ginea Ripart, in Déséglise, not. extr. de l'énum. des rosiers, the journ. of botany, juin 187/, extr. p. 1; R. leucochroa b. lactea floribus candidis Loisel.? notice, in Desvaux, journ. (1809), II, p. 257; Desportes, l. c., $n^{\circ} 2440$ (1).

Arbrisscau rohuste, touffu, aiguillons nombreux, dilatés à la base, recourbés au sommet, ceux des jennes rameaux moins forts; pétioles un peu velus au bord du sillon et à la naissance des folioles, quelques pétioles portent de petites glandes fines stipitées peu abondantes, aiguillonnés en dessous ; $\dddot{b}-7$ folioles ovales-aiguës ou ovales-arrondies, glabres, vertes en dessus, plus pàles en dessous, simplement dentées; stipules grlabres, bordées de glandes, oreillettes aiguës, divergentes; pédoncules 1-4, glabres, ayant à leur base des bractées ovales, cuspidées au sommet, glabres,

(1) Cette description a paru en juin 187k, dans le The Journal of Bolony, et je crois devoir la reproduire. 
égalant ou plus courtes que les pédoncules; tube du ealice obovoïde, glabre; divisions calicinales spatulées au sommet, les extérieures entières, saillantes sur le bouton, plus courtes que la cololle, réfléchies à l’anthèse, non persistantes; styles glabres en une colonne plus ou moins saillante, disque conique; fleur d'un blanc pur mème à l'onglet; fruit rouge, sphérique.

Ha b. Mai, juin. Haies, bois. - Angleterre. Lancaster (Wel:b). - France. Cher: Fussy (Ripart), hois de Rouet! Mehun! forêts de Fontmoreau! du Rhin-du-bois!, Berry!, Boursac!, Nierzon !, Aubusset! - Calvados : bois de Manerbe près de Lisicux!

29. Re. Ieraconea Desvaux, journ. bot. (1809), II, p. 516 , et (1815), II, p. 115̄; Loisel., nolice (1810), p. 81 excl. var. b.; DC. cat. Monsp., p. 1588; Saint-Amans, fl. Agen. (1821), p. 204; Desportes, 1. c., $n^{\circ} 2459$; Boreau, bull Soc. ind. d'Angers (18k k), extr. p.9 et fl. cent., éd. 2, n 6.3 , èd. $3, n^{\circ} 817$ ef catal. II.-et-Loire, p. 78; Déséglise, essai monog., I. c. X, p. 6 bै et extr., p. 25\%; de Vartr.-Donos, 1. c., p. 228; Cariot, I. c., p. 169; Lloyd, l. c., p. 179; R. brevistyla a. DC., fl. fr. (1815), V, p. 557; Thory, 1. c., p. 140; Tratt., 1. c., p. 47; R. stylosa var. lencochroa Seringe, in DC., prod., II (1823)), p. 399 ; Duby, 1. e., p. 176; Delastre, fl. de la Viemne (1842), p. 13̈7; Gomnet, fl. élément. de Ia Fr. (1848), p. 485; Gr. et Godr., 1. c., p. 55 ๖.

Icos. Desvaux, 1. c. $(1815)$, tab. XV; Redouté, les roses (1824), livrais. 6, A.

Haв. Mai, juin. IIaies, bois. - Angleterre. Devonshire : Egg, Buckland, Modbury, Newton (Briggs). - France. Vosges : Liezey! - Loire-inférieure: Thouaré (Lloyd); Vendée : Napoléon-Vendée (Pontarlier in herb. Grenier) ; - M.-et-L. : Anger's, Chalonnes (Boreau); - Deux-Sèvres : La-Mothe-Saint-lleray (Sauzé); - Vienne: Poiliers (Desvaux, in herb. 
Déséglise) ; - Cher : Saint-Florent! Saint-Martin-d'Auxigny! Berry! Mehem; - Tarn : A.C. dans ce département d'après de Martrin-Donos, flore; - Rho̊ne: Lyon à Charbonnière (Chabcrt), Villeurbane, Tassin (Ozanon); - Var: Hyères (Huet).

\section{SEct. II. - Hadỉcac.}

Crépin, primit. monog. rosar., fase. 2 (1872), in Bull. de la Soc. roy. de Botan. de Bolgique, XI, p. 158, extr. p. 22; Chinenses De., in Seringe, mus. helv. (1818), I, p. 2, pro part.; Smithiana Tratt., monog. ros. (1825), I, p. 88, exel. R. sinica.

Styles plus ou moins stuillants; stipules toutes étroites. Parson mode d'inflorescence, celte section a sa place marquée i coté de la section des Synstylac. - La section des Indicae est composée de formes dont la délimilation spécifique est encore très-obscure, ce qui est dir à ce qu'elles ont été toutes mimiticement déurites sur des plantes culticées et plus ou moins profondément modifiées par une longue cullure, nonseulement dans les jardins d'Europe, mais dans ceux de la Chine et du Japon. -- Crépin.

50. IR. Indica L., sp., 70̋̈; Pers, syn., II, p. 丂̆0; Tratt., l. e., p. 96; Richt., codex, p. 497, $1^{\circ} 574.0$

"Rami inermes; rarius armati una alterave spina tenuis" sima versus folia vel in petiolis. Folia pinnata : foliolis - quinis. subtus tomentosis, supra glabris, serratis : extimo " duplo majore. Pedunculi longi, nudi simplices. Calyx " incisus, laevis. Fruetus magnitudine sorbi aucupariae." (Lin.) Hab. in China.

OBs. - Lindley, dans sa monographie dont de Pronville a donné une traduction en langue française, dit: "II n'est peut-ètre plus temps de " demander ce que Limné a voulu désigner par Indica, puisque son carac" tère spécifique et sa description ne conviennent à aucune des espèces 
" indigènes de la Chine, du moins à celles qui composent cette tribu. Is? " figure de Petiver (tab. 5\%, f. 1lj sur laquelle il se fonde et en quoi il a "été suivi par Willdenow, Poiret et autres, appartient à une plante bien a différente qui a beaucoup de rapport avec le $R$. Banksiae et que j'ai " appelée microcarpa. J'ai tout fois examiné les échantillons de Linné et je " ne doute pas qu'ils n’appartiennent à cette espèce; mais ayant étudiẻ "pareillement le spécimen dont S. James Smith forme le type du " $\boldsymbol{R}$. sinica, je n'hésite pas à prononeer que cet échantillon est une " monstruosité de l'Indica de nos jardins. Les stipules sont étroites, poin"tues, finement dentées en leurs hords, les aiguillons droits, faibles, " inégaux, ce que l'on doit trouver dans l'Indica, et ne convient. pas au " port ou à l'état faible du Sinica. Ce nom m'ayant paru suranné, je l'ai "réservé pour la plante qui le porte dans l'horlus Kewonsis." (Lindley, monog., trad. de Prouville).

Voici ce que M. Crépin dit tles échantillons du $R$. Indica de l'herbier de Willdenow", n० 9875: " ce numéro est représenté par deux feuilles " simples. Fol. 1). Un rameau florifère. - Ne se rapporte nullement à la " diagnose de Linné, reproduite par Willdenow sur l'étiquette. Il doit " appartenir au R. Indica humilis, publié par Seringe dans ses roses " desséchées, no 39. - Fol. 2). Un rameau muni d'un ramuscule florifère. "-Appartient au R. Sinica Murray. n-Crépin.

Le $\boldsymbol{R}$. Indica de l'herbier DC, n'est pas celui décrit par Linné : une note écrite par De Candolle se troure dans son herbier, et donne la deseription suivante de ce qu'il prend pour $R$. Intica Lin.

a Sous-arbrisseau de כ̋-6 décimètres, écorce verte, aiguillons rougges, " recourbés, épars, stipules cilices de poils glanduleux, pétioles munis " d'aiguillons et de poils glanduleux, portant "ै folioles ailées, ovales a lancéolées, rouges en leurs borls, inégalement dentées en scie, glabres " et lisses; pédoncules garnis de poils glunduleux; ovaire glabre, ovale, " divisions du ealice dentelées, corolle d'un rose vif. Bengale. " (I C.).

I.e $R$. Indica Seringe et ce qui se trouve dans l'herbier DC., nous semblent rentrer dans te $R$. Bengalensis.

51. Th. Lawaraceana Sweet, hort. suburl, Lond. (18i8), p. 119, et hort. brit. (1859!), p. 216; Lindley, 1. c., p. 110; de Pronv., 1. c.: p. 110; Desportes, 1. c., p. 91; R. Laurentiae Andr. fase. 5;; Tratt., I. c. 1: p. 10\%; R. Lamentice subinemmis Tratt., L. ¿., p. 106; 
R. Indice var. Lawranceana Thory, prod., p. 151; Wallr., hist. ros. p. 101; R. Indica var. acuminala Scringe, in DE., prod., II, P. 601; $R$. Indica var. humilis Seringe, mélang., fase. I, p. \&4 et in DC., prod., II, p. $600 ; \boldsymbol{R}$. pusilla Mauritus cat. p. 1:3? teste Lindley; h. semperflorens var. minima Sims, hot. mag., tab. 1762.

Icos. Curtis, bot. mag., tab. 1762; Lindley, bot. reg., tab. כ̋58; Redouté, les roses (1824), livrais. כ̋, D., livrais. 59, A.

IIAB. - La Chine? - A élé introduit en Augleterre en 1810 ; à Paris par Noisette, mais j'ignore la date de son introduction en France.

Ons. Je passe sous silence le R. Noiselliana Thory (hybride obtenu du $R$. moschata fécondé par le $R$. Indica), et les rosiers à fleur double qui sont du domaine de l'horticulture, plutôt que des espèces botaniques, dont les origines sont des plus obseures, comme les noms sous lesquels ils figurent dans les catalogues

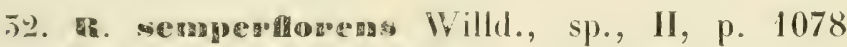
(1797), et enum. hort. (1809), p. 547; Jacq., hort. Schönbr., III, p. 17; Seringe, mélang., fase. 1, p. 11; Lindley, I. e., p. 108; R. dicersifolia Ventenat, jard. Culs, $n^{\circ} 5: 3$ (1800); R. Bengalensis var. Chinensis Pers. 1. e.; R. Sinica Tratl., I. c., p. 89 (non Hurray); R. atropurpurea Brotero, f1. Lusit. (180:3), II, p.488?; Seringe, in DC., prod., II, p. 601; $\because$. . Indica var. semperflorens Seringe, l. $\mathrm{e}$.

Icox. Curtis, bot. mag., VIII, tab. 284; Jacquin, hort., tab. 281, et obs., tab. \$3; Ventenat, tab. 55; Redouté, les hoses (1824), livrais. 15, B.; Roessig, die Rosen, tab. 19 ; d'après l'autorité de Lindley, je cite: Miss Lawrance, tal). 25; Smith, cxut., fasce 2, tal). 91 ; Prizel mentionne: Savi, fl. ilal., I, 58 et II, 2 ; Kerner, liort., 114 . 
Exs. Compagnie des Indes-Orient. (1829), $\mathrm{n}^{\circ}$ 686; Wallich in herb. DC, et herl). Delessert, échantillon cultivé au jardin de Calcuta ; Seringe, roses desséchées, $n^{\circ}$ "; Bourgeau, expédiı. du Mexique, no 2942.

IIı. - La Chine, passant pour être originaire du Bengale (Ventenat, l.c.). - Indes (Lambert, 1816, in herb. DC.); Bengale (Leschenault, 1821 in herb. DC.); Caleuta, 1829, in herb. DC. - Mexique. Orizaba (Bourgeau), Spont? - Brésil (Theremin, 1819, in herb. DC.), spont.? - Guyano hollandaise. Surinam (Wright, in herb. DC., 1829), spont.? - Afrique. Tanger, jardin du consulat du Danemark, où il forme d'énormes buissons à l'état sauvage, fleur simple (mai 1849, Reuter !).

OBs. Loiscleur-Deslonchamps, dans le dietionnaire des sciences naturelles, XLVI, p. 26f, dit : ce rosier, originaire de la Chine et des parties septentrionales du Bengale, a été introduit en Angleterre en 1771 et ec n'est guère que 20 ans après qu'il a été introduit en France. - Redoute dit qu'il a été introduit en Europe par les Anglais, qui l'ont rapporté de l'lnde, et il a fleuri pour la première fois en Angleterre vers l'année 1795. - D'après Desportes, son introduction en France date de l'année 1800.

5ร. R. Longifolia Willd., sp., II, p. 1079 et herb., no 9872; Pers., I. c., p. 30; Poirel, encyel. suppl., VI, p. 296; Tratt., I. c., I, p. 101 ; R. Indica var. Iongifolia Lindley, l. e., p. 1€6; Seringe, in DC., prod., II, p. 600; R. semperforens var. longifolin de Pronv., 1. c., p. 108 ; R. persicifolia Hortulan.

Icon. Redouté, les roses (1824), livrais. 18, A.

Har. - Les Indes

\section{Sect. III. - Baracteatae.}

Lindley, monog. ros. (1820), p. 7 ; de Pronville, monog. du genre rosicr, p. 28 ; Wendlandiana Trattinnick, monog. ros. (1815), II, p. 188 ; Chinenses §5. Seringe, in DC., prod, II, p. 602. 
54. Th. involucrata Roxb., in Lindley, l. c., p. 8; de Pronv., I. c., p. 29 ; Seringe, in DC., prod., II, p. 602 ; Desportes, I. e., p. 2; R. Lindleyana Tratt., l. e., p. 190 ; R. rlynophylla Thory, prod., p. 126 ; Seringe, 1. c., p. 606.

Icon. Bot. regist., tab. 759 ; Redouté, les roses (1824), livrais. 11, A.

Exs. Compagnie des Indes-Orient. (an. 1820), nº 696 , in herb. DC.

Ilan. - Les Indes et la Chine. - Les Indes (Lambert, 18!6, in herb. DC.) ; Assam (Colonel Jenkins, 182:, in herb. DC.).); Bengale orient. (Hooker et Thomson).

Introduit en Angleterre par Whitley, en 1820.

Oss. R. II ardii Paxton, mag. of botan., X, (IS45), p. 19\%. Iybride formé entre le $\boldsymbol{R}$. berberifolia et le $R$. involucrata. II n'a que les fleurs du premier, les feuilles ont aussi un aspect singulier, les pédoncules et les calices sont recouverts d'un duret épais et feutré. Cette plante était cultivée au jardin de Paris en 1868, d'où proviennent mes échantillons.

5:. ER. Buacteata Wendl., ob. $n^{\circ} 30$ et hort. Herren.; Pers., I. c., p. 50 ; Lindley, I. c., p. 10 ; de Pronv., I. c., p. 50 ; Tratt., I. c., II, p. 189 ; Seringe, in DC., prod., II, p. 602; Despories, I. c., p. 2 ; R. Hacarthea Dum.-Courset, bot. cult., éd. 2 (1811), V, p. 485.

Icon. Ventenat, jard. de Cels, lab. 28 ; Roessigr, die rosen, pl. 32; Redouté, les roses (1824), livr. 2, B. Je cite d'après Lindley: Wendland, hort. Iterren., 7, (a). 22 ; Miss Lawrance, tah. 28 sul, nom. R. lucida non Ehrh.

H.s. - La Chine.

Var. b. scabricanlis Lindl., I. c.; Seringe, I. c.; R. bracteata, Mönch, meth. sup., 290 ; Jacq., frag, p. 50. 
Icos. Jacquin, 1. c , tab. 5f, f. 2 ; Curtis, bot. mag., tab. 1377.

Ramis setigeris, aculeis minoribus, rectiusculis.

II $\mathrm{s}$, - La Chine.

OBs. Ce rosier, originaire de la Chine, a été introduit en Angleterre par Georgrs Staunton en 1793; introduit à Paris par Cels en 1795, d'après Desportes.

506. Te. Lyelli: Lindley, 1. c., p. 12; Tratt., 1. c., p. 191 ; Seringe in DC., prod., II, p. 601.

Icon. Lindley, I. c., tab. 1.

IIAB. - Le Népaul (Wallıch, 1829, n682, in herb. Delessert).

\section{Sect. IV. - Hanksianac.}

Lindley, monog. ros. (1820), p. 123̈; de Pronville, monog. du genre rosier, p. 123 ; Chinenses $\$ 2$ Seringe, in DC., prod., II, p. 601; Purshiana Tratt., monog., ros., II, p. 180 ; Aitoniana Tratt., I. c., p. 211.

57. Randian R. Br., in Ait., Kew., ed. alt., III, p. 258; Lindley, 1. c., p. 151; Thory, prod., p. 59 ; Tratt., l. c., p. 212; de Pronv., 1. c., p. 128; Seringe in DC., prod., II, p. 601 ; Desportes, roset. gall., p. 115 , $n^{\circ} 2495, n^{\circ} 2496$.

Icov. Curtis, bot. mag., tab. 1934, flore albo; Bot. reg., tab. 597, flore pleno; Roessig, die rosen, pl. 57 ; Redouté, les roses (1824), livrais. 50, A.

Hsis. - La Chine.

Ons. Introduit en Angleterre, en 1807, par IVilliam Ker; son introduction en Franec date de 1817, par Boursault. Le type est à fleur blanche; il y a une variété à fleur jaune introduite en $18 \%$ et plus répandue que le type dans les jardins.

58. 邪. micrecanpa Lindley, 1. c., p. 150; de Pronv., 
l. c., p. 128 ; Seringe, in DC., prod., II, p. 601 ; R. triphylla Roxb. in Lindl., I. c., p. 158 ?; Seringe, in DC., prod., II, p. 600 ?; R. cymosa Tratt., I. e., I, p. 87.

Icov. Lindley, 1. e., tal). 18; Petiver, gaz. 37, tab. 53, f. 11.

IIab. - La Chine.

Ors. Lindley, dans sa monographic, fait la remarque suivanle : "II ne "peut y avoir une preuve plus frappante de la connaissance imparfaite "que Linné avait des rosiers asiatiques, qu'en le voyant citer celui-ci bien " figuré par Petiver, comme étant l'Indica. Willdenow a reproduit cette " erreur, parce que probablement il regardait le $\boldsymbol{R}$. Indica Lin., comme " une plante qui lui était inconnue. " (Lindley, trad. de Pronville).

59. R. Amoyersis Inance in Seem., journ. of botan. (1868), VI, p. 297.

IIAB. - Chine. Autour de la ville d'Amoy (IIance).

40. 职. Sinica Murray, syst. veget.(1774), p. 594; Ait., Kew., éd. 2, III (1810), p. 261; Lindley, l. c., p. 126; de Pronv., I. e., p. 128; R. laevigata Michx, fl. Amer.hor. (1805), I, p. 295 ; Pursh., fl. Am.-sept. (1804), I, p. 543 ; Pers., syn. (1807), II, p. 49 ; Poir., encycl. suppl., VI, p. 29:3; Lindley, I. c., p. 123 ; Tratt., I. c., II, p. 184; de Pronv., l. e., p. 124; Seringe in DC., pr., II, p. 600; R. nicea DC., cat. Monsp. (1815), p. 157 (non Raf.); Seringe, 1. c., p. 599 ; Tratı., I. c., p. 185 ; Thory, prod., p. 57; R. ternata Poir., 1. c. (1810), p. 284; R. trifoliata Bose, dict. d'agricult., éd. 2 (1811), XIII, p. 280 ; R. Cherokensis Don, eat., éd. 8, p. 172.

Icon. Lindley, 1. c., lab. 16 ; Lindley, bot. regist., XXIII, tab. 1922; Curtis, bot. mag., tab). 2847; Redouté, les roses (1824). livrais., 9, B.

Exs. Compagnie des Indes orient. (182y), n०694. 
HАв. - La Chine (Expédition française en Chine, enllection du docteur Yvan, an. 1843-46, herb. Jaubert!); les échantillons de l'herbier DC. viennent du jardin hotanique de Montpellier ( 15 mai $180 \mathrm{~S}$ ), ils sont identiques a la plante distribuée par la compagnie des Indes et à celle que je possède de Louisiane.

Espèce remarquable par ses tolioles ternées, coriaces, glabres ; les divisions calicinales entières, les extérieures très-dilatées au sommet; le pédoncule dans sa partie supérieure et le tube du calice sont chargés de soies spiniformes assez longues; les styles sont velus; la fleur grande, blanche; les aiguillons petits, dilatés à la base, courbés ou crochus.

D'après Aiton, il a été introduit en Angleterre en 1799, par Philip Miller ; j'ignore la date de son introduction en France.

41. IR. hystrix Lindley, monog. ros. (1820), p. 129; Tratt., 1 c., II, p. 182; de Pronv., l. c., p. 127; Seringe in DC., prod., II, p. 559; R. cucumerina Tratt., I. c., p. 181 .

Icon. Lindley, 1. c., tab. 17.

Il diffère du R. Sinica, par les tiges qui sont plus aiguillonnées, les rameaux sont couverts de petites soies fines, mélangées de petits aiguillons inclinés ou droits; les pétioles sont armés de nombreux petits aiguillons grèles qui se retrouvent sur la nervure médiane des folioles; les divisions calicinales sont bordées au sommet de petites soies; les pédoncules et le tube du calice sont chargés de nombreuses soies spiniformes; les styles sont obscurément hérissés.

HAB. - Le Japon (Lindley); - la Chine (Fortune, herb. Désíglise).

Oes. R. recurva Roxb., in Lindl., l. c., p. 127; Tratt., 1. c., II, p. 227; Seringe, in DC., prod., II, p. 600. - Hab. le Népaul. - Espèce bien incertaine, dont il m'a été impossible de voir un type dans les herbiers.

\section{SЕст. V. - Gallicanac.}

DC., in Seringe, mus. Helv. (1818), p. 2 el p. 4; Besser, enum. Pod. et Volh. (1822), p. 60; Déséglise, 
essai monog., in mém. de la Soc. Acad. de M.-et-Loire, X (1861), p. 20 et extr., p. 10, et in the naturalist (1866), 10 20, P. 510; Cariot, étud. des lleurs (186\%), II, p. 169; Crépin, primil. monog. los., fasc. I (1869), p. 15; Cinnamomeae Seringe, in DC., prod., II, (182.'), p. 602, part.; Duby, bot. gall. (1828), I, p. 176, part.; Lorey et Duret, fl. de la Cóte-d'Or (1851), I, p. 50k, part.; Centifoliae Lindley, monog. ros. (1820), p. 68, part.; de Pronville, monog. du genre rosier (1824), p. 66, part.; Rehb., 11. excurs. (1850), II, p. 622, part.; Nobiles Koch, syn. (1845), p. $2 \% 3$, part.; Reuter, cat. de Genève (1861), p. 75, pait.; Diastylae, trib. Dimorphacanthae Godet, fl. du Jura (1855), p. 204.

Styles agglutinés en colonne velue, glabrc ou héris-

1. sée, mais non soudés . . . . . 2.

( Styles libres, glabres, hérissés ou laineux . . . 4.

Colonne stylaire égalant les étamines, corolle rose $2 .\left\{\begin{array}{c}\text { clair } \\ \text { Colonne stylaire plus courte que les étamines, } \\ \text { corolle rose . . . . . . . . . . . . . . }\end{array}\right.$

arvina.

5. $\left\{\begin{array}{l}\text { Styles relus, tube du calice grèle, obovoüde, glan- } \\ \text { duleux, fruit ovoïde, rouge orangé . . . . . }\end{array}\right.$ hybrida.

Styles glabres, tube du calice ovoïde, glabre, fruit oroïde, d'un rouge obscur

Polliniana.

4. Styles laineux . . . . . . . . . 17.

Styles glabres uu hérissés. . . . . . . .

๖. Styles glabres . . . . . . . . . 6.

Styles hérissés . . . . . . . . . . . ૪.

Arbrisseau, folioles ovales-elliptiques, doublement dentées, tube du calice obovoïde, glabre, fleur

6. grande, odorante, d'un beau rose, fruil ovoìde, rouge sale . . . . . . . . . areniungr.

Sous-arbrisspall. 
Pétioles pubescents glandu'cux, folioles ovales ou obtuses, dentées en scie, souvent surchargées de petites dents secondaires, la còte ayant quelques glandes, tube du calice ovoïde, glabre, fleur grande, d'un blanc rosé . . . . . . . .

Fourraei.

Pétioles glabres, glanduleux, aiguillonnés, folioles aiguës, vert sombre en dessus, velues sur les nervures, la còte glandulcuse, simplement dentées, tube du calice oblong, fleur grande, d'un blanc satiné, fruit ovoïde, rouge-brun .

opreifolia.

8. Fleur rouge foncé ou rcuge, à nuance veloutée . 9.

Fleur rose . . . . . . . . . 10.

Folioles oblongues-lancéolćes, d'un vert pâle en dessus, grisâtres en dessous, la côte légèrement velue et parsemée de glandes, doublement dentées, tube du calice ovoïle, glabre ou hispide à la base, fleur grande, rouge, à nuance velnutée, fruit ovoide.

virescens.

Folioles ovales, pubescentes en dessous, ne conservant à l'état adulte de la villosité que sur la côte, simplement dentées, tube du calice obovoïde, glanduleux, fleur grande, rouge foncé, à onglet court, jaunâtre, fruit pyriforme, rouge orangé .

velulinaeflora.

10. \{

Arbrisseau . . . . . . . . . . . . 15

Sous-arbrisseau.

15.

Folioles orbiculaires, tomenteuses en dessous, doublement dentées, fleur d'un beau rose, à onglet blanchâtre, brillant.

Folioles non orbiculaires.

- austriaca.

Folioles ovales-aiguës, blanchâtres, pubescentes en dessous, inégalement dentées, sépales bordés de glandes, tomenteux au sommet, fleur grande,

12. d'un beau rose . . . . . . . . . . sylvatica.

Folioles ovales-arrondies, blanchâtres, pubescentes en dessous, la côte glanduleuse, ì dents plus ou moins surchargées de dents accessoires, sépales glabres, fleur rose.

decipiens. 


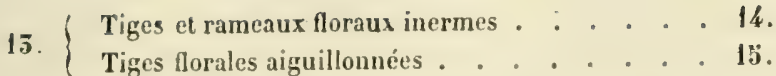

Pétioles aiguillonnés, folioles grandes, ovales-aiguës, cordiformes à la hase, nervures velues, la côte parsemée de glandes, simplement dentées, tube du calice ovoide, glabre, fleur grande, d'un beau rose.

\section{subinermis.}

Pétioles incrmes, folioles ovales-elliptiques, pâles, pubescentes en dessous, la côte glanduleuse, dentées en scie, à dents surdentées, tube du calice ovoìde, glanduleux, fleur d'un beau rose . . .

incarnala.

Folioles grandes, ovales, tube du calice globuleux,

10. fleur très-grande, d'un blanc lavẻ de rose, fruit arrondi, rouge sale . . . . . . . Boraeana.

Folioles médiocres, tube du calice ovoïde. . . . 16.

Folioles orbiculaires, pubescentes en dessous, pétales blanes, un peu rosés au sommet, fruit arrondi, rouge

16.

Folioles ovales, à nervures velues, villosité dispageminata. raissant arec l'âge, la côte velue et parsemée de glandes, simplement dentées, quelques-unes surchargées de dents accessoires, pétales grands, d'un beau rose, fruit rouge, obovor̈de.

nirabilis.

Folioles orbiculaires . . . . . . . 18 .

17. Folioles non orbiculaires. . . . . . 19.

Folioles doublement dentées, tube du calice ovoïde, arrondi, fleur rouge foncé, avec nuances veloutées, fruit globuleux, ro geâtre .

provincialis.

18. Folioles simplement dentées, en cour à la base, tube du calice oroide-oblong, fleur d'un beau rose nuancé de points blancs.

assimiles.

Fleur rouge . . . . . . . . . . 20.

19.

Fleur rose vif, onglet jaunâtre, folioles ovalesaiguës, nervure médiane velue, glanduleuse, tube du calice globulfux, fruit arrondi . . . . murulis. 
Fleur d'un rouge tris-foncé, folioles ovales-elliptiques, presque simplement dentées, fruit arrondi, rougeâtre . . . . . . . . . Gallica.

Fleur d'un rouge vif pâle en dehors et à l'onglet, folioles ovales, doublement dentées, fruit pyriforme, rouge orangé

pumila.

A). Styles rapprochés en colomne velue, hérissée on glubre.

42. R. Iydorida Schleicher, catal. $1815 \%$ Willd., herb., n 9838 , teste Crépin, I. c., fasc. 2 (1872), p. 4ว̈; Rchb., 1. c.. p. 625?; Mutel, fl. fr. (183/4), I, p. 535??; Borcau, fl. cent., éd. 2, n 660, éd. 3, n 829; Gr. et Godr., I. c., I, p. 5̌̉̌z; Arrondeau, fl. Toulous., p. 124; Reuter, 1. c., p. 75; Déséglise, I. c., p. 67 et extr., p. 27; Grenier, fl. Jura., p. 224; Cariot, 1. c , p. 170; R. Gallica var. hybrida Seringe, in DC., prod., II, p. 605 excl. syn. Rau; Godet, 1. c., P. 207; R. arvensis var, hylrida Lindley, I. c., j. 113 excl. syn. Rau; Lois., I1. gall., I, p. 361 ?; R. Axmanni Gmel., 11. Bad.-Als., IV, p. 567?; R. Gallicoserpens Kirschleger, 11. Als., I, P. 24k; R. agrestis Gmel, 1. c., II, p. 416, teste Seringe; R. Rhodani Chaber!! in Cariot, 1. c., p. 677, ex exempl. auth.

Exs. Schleicher, pl. Helv., cent. I, $1^{\circ} 54$; Seringe, roses desséchées, no 5/4; Schulız, exs. $n^{\circ}$ 1/46 et herb. norm., $n^{\circ}$ 47; Déséglise, herb. ros., $n^{\circ} 4$.

HAB. Mai, juin. Bois. - France. Cher : bois de la Brosse longeant Travaille Coquin (Blondeau, 1829, in herb. Déséglise), forêt de Fontmoreau (Ripart), forêt du Brouard près Levet! bois de Givray près de Bourges ! bois de Charron et de Marmagne! bois de Saint-Florent! - Puy-de-Dôme : bois de Lezoux (Lamotte); - Ilaute-Garonne: Bouconne a Toulouse (Timbal-Lagrave); - Rhòne: hois de Charhomière, Dardilly, Glésieux (Chabert), Brouilly-Saint-Lager, Tassin, Crapone (Boullu). - Suisse. Cant. de Genève : bois de la Bátie, Veyrier, Troënex, Compesières, lancy. - Bavière. Lac de Starnberg (Christ). 
45. IR. Polliaiana Sprengel, plant. min. cogn. pug. (1815), II, p. 66; Poiret, eneycl. supp., IV (1816), p. 715 ; Pollini, Viag. al lago de Garda, p. 129; Lindley, I. c. , p. 155?; Tratt., I. c., II, p. 101? R. Pollinaria de Pronville, I. e., p. 152 ? R. mmila b. Pollini, fl. Veron., II, p. 145 ; R. conica Chabert! in Cariot, I. c., p. 171; R. intermedia Chabert! in herb. Déséglise; R. areensisGallica Gremli! R. ambigens Gremli! R. canina-Gallica c. ambigens Gremli! R. sylicestris flore majore et rubente Seguier, fl. Veron., II, p. 311.

Icon. Pollini, I. c., tab. 1, f. 3.

Exs. Billot (suites), $n^{\circ} 5717$.

Sous-arbrisseau atteignant un mètre au plus, rameaux grèles, à écorce verte ou rougeàtre, les uns presque inermes, les autres armés d'aiguillons grèles en forme de disque à la base, droits ou légèrement inclinés, rougeàtres ou grisâtres, mélangés de soies pédicellées an sommet, ceux de la tige principale plus robustes; pétioles légèrement pubescents, glanduleux, aiguillonnés en dessous; 5-5 folioles ovales, arrondies à la base, aiguës au sommet ou ovales-elliptiques et d'autres tout à fait arrondies, vertes et glabres en dessus, glaucescentes en dessous, la còte glanduleuse et portant quelques poils, dentées en scie, à dents mucronées, quelques folioles ont les dents surchargées de petites glandes fines qui les font paraitre comme doublement dentées; stipules lancéolées, glabres, à oreillettes aiguës, droiles, à bords glanduleux ; pédoncules solitaires ou réunis par 2-14, assez longs, rougeâtres, portant des glandes pédicellées; des bractées qui se trouvent à la base du bouquet, l'une est dilatée ou terminée par trois folioles, l'autre est ovale, cuspidée, glabre; les pédoncules extérieurs ont 
deux petites bractées opposées, le pédoneule central en est dépourvu; tube du calice pruineux, ovoïde, glabre; divisions ealicinales terminées en pointe, grabres en dessous, les extérieures appendiculées, saillantes sur le houton, plus courtes que la corolle; styles glabres au-dessus d'un disque conique (comme dans l'échantillon de Sprengel) plus ou moins élevé; pétales roses, grands; fruit ovoïde, d'un rouge obscur.

OBs. La plante que je viens de décrire est identique à l'échantillon qui se trouve dans l'herhier DC., sous le nom de $R$. Polliniana, venant de Sprengel et donné sans localité en 1825, par de W elden. - M. Cariot, en décrivant le $\boldsymbol{R}$. conica, dit : "pétales aussi larges que longs, cordiformes" arrondis à l'onglet. n C'est une erreur, les pétales sont cunéiformes à la base, émarginés au sommet, d'après les beaux échantillons que j'ai de Chabert.

Han. Mai, juin. Bois. - France. Cher : bois de Charron près de Marmagne! - Rhône : bords des bois à Charbonnière, pont d'Alaï, Dardilly (Chabert). - Suisse. Cant. de Schaffhouse: Wirbelberg (Gremli), - Italie. Mont Baldo (Huguenin).

44. Fh. arviana Krocker, 11. Silesiaca (1790), II, p. 150 ; Rau, cnum. ros. (1816), p. 106; Tratt., I. c., I, p. 56, Relıb., I. c., p. 623 excl. syn.; Boreau, bull. de la soc. indust. d'Angers (1844), ext., p. 10, fl. cent., éd. 2, $\mathrm{n}^{\circ}$ 661, éd. 5, $\mathrm{n}^{\circ}$ 850, eatal. de N.-et-Loire, p. 79; Gonnet, fl. élém. de Franee, p. 482; Gr. et Godr., l. c., p. 53̈; Arrondeau, I. c., p. 125; Déséglise, l. c., p. 68 et extr., p. 28; Cariot, I. c., p. 170; R. Gallica var. arvina Seringe, in DC., prod., II, p. 604; R. serpenti-Gallica Kirschleg., I. c., p. 245; R. Gallico-stylosa Timb.-Lagr.! in herb. Ripart; $\boldsymbol{R}$. canino-Gallica Timb.-Lagr.! in herb. Déséglise.

HAB. Juin. Haies et bois. R. R. - France. Maine-et-Loire: Angers (Boreau); - Haute-Garonne: Toulouse à Boucomne (Timbal-Lagrave), 
Laramette à Tuulouse (Baillet); - Rhòne : Lyon à Charbonnière (Ozanon), vallon de Tassin (Chabert). - Slyrie. Grätz (Reichenbach, flora). Bavière. Retzbach près Wurtzbourg (Rau). - Silésie (Krocker).

B). Styles libres, hérissés ou glabres.

4). R. aneaniraga Déséglise in Jullien, catal. syst. de ฯฯ. pl. nouv. pour la flore orléanaise, in mém. de la soc. Acad. de M.-et-Loire, XII (1862), extr. p. 9.

Exs. Déséglise, herb. ros. $n^{\circ} 41$.

Haв. Juin. Haies. - Franne : Loiret : levée de la Loire à Orléans près le bois de l'Isle (Jullien), Saint-Denis-en-Val (Borean, in litt.).

46. R. subinen'mis Chabert, in Cariot, 1. c. p. 175.

Ilar. Juin. Haies, bois. - France. Rhône: Dardilly (Chabert), haies au Gau Francheville, Charbonnière? (Boullu).

47. M. gemanati Rau, énum. ros. (1816), p. 98 et p. 169; Tratt., I. c., II, p. 29; Rehb., I. c., p. 62/; Boreau, bull. de la soc. indust. d'Angers (18/4), extr., p. 10, fl. cent. éd. 2, no 638, éd. 5, nº 820; Gr. et Gorlr.,

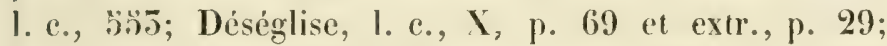
Cariot, I. c., p. 171; Fourreau, cat. pl. du cours du Rhòne (1869), p. 75; R. agrestis Kirschleger, I. c., I, p. 24/ an Gmel. ?; R. incomparabilis Chabert! in Cariot! I. c. p. 170 .

Icon. Redouté, les roses (182/), livrais. 25, e.

Exs. Billot (suites), $n^{\text {us }} 5578,5718$.

IIAB. Juin. Haies, bois. - France, Cher : Aubigny (Delastre in herb. Boissier) ; - Indre : Mézières (de Jouffroy, in herb. Grenier); - Rhône: J)ardilly, route de la tour de salvagny (Chabert), Charbonnièse, Craponne, Marcy, Saint-Lager, Méginant (Boullu). - Allemayne. Wurzhourg (Ratu); Francfort-sur-Mlein, forêt de Wilbeler (Rehb.). 


\section{(74)}

48. Rour. Fouci Déséglise, descript. de qq. esp. nour. de rosicrs, in mém. de la soc. Acad. de M.-et-Loire, XXVIII, (1875), p. 98, extr. p. 2; R. mixta Chabert! in Cariot, 1. c., p. 677 (non Trattinnick).

IIAs. Juin. Bois, haies - France. Rhòne : Charbonnière près de Lyon (Chabert), Sainte Consorce, Tassin à Méginant (Boullu).

49. R. Boracana Béraud, mém. de la soc. d'Agricult. d'Angers, V, p. 555 ; Borean, I. c., éd. 2, no 639 , éd. 5, n" 821 et catal. de M.-pt-Loire, p 78; Déséglise, essai monog. in mém. de la soc. acad. de M.-et-L., X, p. 70 et extr. p. 50; R. arvina Lloyd! f1. onest (1868), p. 181 (non Krocker).

II в. Juin. Haies. - Frunce. Loire-inférieure : Couerron (Boreau); Naine-et-Loire : Angers (Boreau)

50. R. Anstriaca Crantz, stirp. Austr. (1769), fasc. 2, p. 86 ; Poll., f1. Palat., II, P. 50 ; Tratt., I. c. I, p. 61 ; Boreau, fl. Cent., éd. 5, no 824; Déséglise, l. e., p. 71 et extr., p. 51 ; Cariot, 1. c., p. 171 ; Fourreau, I. c., p. 73 ; R. Gallica Roth, 11. Germ. (1789), II, p. 559 (non Lin.); Krocker, I. e., II, p. 143̈; R. Gallica var. hispida Seringe, in DC.. prod., II, p. 605; R. pumila Jaequin, fl. Austr., II, p. 59 (non Lin. fil.); Wallroth, ann. bot., p. 62; R. pumila var. hispida Rau, 1. c., p. 116; R. hispida Münchlaus. Hausv., V, p. 281 ; Schrank, baicr. 11., II, p. 4l, teste Trattinnick.

Icov. Jacquin, I. e., tab. 198.

Exs. Reichenbach, n²2ว̈0; Déséglise, herb. ros., $n^{\circ} 42$.

Ifır. Juin. IIaies, bois des terrains calcaires. - France. Cher : bois de Charron près de Marmagne! vignes de la Chapulle-saint-Ursin! bois de la Grange saint-Jean près de Levet! - Rhône : Lyon à Charbonnière (Chabert). - Autriche. Au pied de Freuenberges près de Langelois (Widerspach); - Illyrie: Trieste (Tommasini in herb. Boissier); - Croatie : Banat (Wierzbicki). - Italie. Mont Pastello près de Verone (Boissier). 
3̈1. R. incanonata Miller, diet., $n^{\circ}$ 19, trad. frane., VI, p. 527 ; Boreau, 1. c., éd 2, n 665, éd. 5, n $n^{\circ} 826$; Déséglise, I. c., p. 72, cxtr., p. 52 ; Fourreau, I. c.,p. 75; R. laevis Boullu, in litt.

Нав. Juin. Bois. - France. Cher : bois de Marmagne! bois de Contremoret près Bourges! bois de Giviay, commune de Trouy (Ripart); Loir-et-Cher : Chevemy, Fontaines en Sologne (Boreau, flore); - Khône : Tassin à Iléginant, Iarcy (Boullu), Charbonnière, Dardilly (Chabert); Ilaute-Garonne : Toulnuse à Colomiers (Timbal-Lagrave). - Suisse. Cant. de Genève : hois des Frères, près de Genève !

32. R. visescens Déséglise, I. c., p. 7j, et extr., p. 5j; Jullien, eat. syst. de qq. pl. nour. de la fl. Orléan, p. 8 ; R. Gallica Auet. pr. part.; R. Gallica var. inermis Seringe, in DC., prod., II, p. 604?

II ав. Juin. Ilaies, bois. - Frunce. Loiret : bois die Plissai, bords de la rivière des Montées près Orléans (Saint-Irilaire, 1803, in herb. du mus. d'Or'éans et 1×12, in herb. DC !) Saint-Jean-Ie-Blanc (Jullien).

5j. R. Teintharafora Déséglise et Ozanon, descript. de qq. esp. nouv. de rosiers, in mém. de la soc. Acarl. de M.et-Loire (1875), XXVIII, p. 100 et exir., p. 4; Fourreau, I. с, p. 75; R. Gallica var. velutinatlora Cariot, l. с., p. 175 .

HAB. Juin. Bois. - France. Rhöne : Lyon, bois de l'Éloile (Ozanon), Charbonnière (Chahert), colline de Brouilly, Saint-Lager (Boullu).

"̈4. R. mỉrabilis Déséglise, I. c., XXVIII, p. 101 et extr.; Fourreau, I. c., p. 75.

Har. Juin. Bois. - France. Cher : bois de Marmagne! - Rhône: vallon de Tassin, bois de Charbonnière (Chabert), Brouilly à Saint-Lagger, Iarcy-le-Loup (Boullu).

:3.) IR. Mvatica Tausch, in diar. bot. flor. dicto ann. ๖, II, p. 464; Tratl., I. c., I, p. 58; BI. et Fing., comp. 0. germ. (1825), I, p. 6.57; Seringe, in DC., prod., II, 
p. $62 \%$; Boreau, I. c., éd. 5, $\mathrm{n}^{\circ} 927$ et catal. de M.-ctLoire, p. 78; Déséglise, I. e., X, p. 74 et extr.. p. 25; Cariot, l. c., p. 172; R. pulchella Boreau, l. c., éd. 2, $\mathrm{n}^{\circ} 662$; Guépin, flore de Maine-et-Loire (18\%0), sup., p. 56 (non Willd.); R. triflora Chabert! in herb. Déséglise.

Exs. Déséglise, herb. ros., $n^{\circ} 45$.

Har. Juin. Bois, taillis. - Frone. Hine-et-Loire : Angers, Brissac (Boreau); - Cher: Trouy, bois des Dames (Ripart), bois de Marmagne! Saint-Florent! forét de Fontmoreau aux brûlis! - Nièvre : Iarzy (Boreau, flore); - Rhône : bois de l'Étoile (Ozanon), Charbonnière, pont d'Alä, Gau (Chabert) vigues de Brouilly-saint-Lager, Sainte-Consoree, Francheville au Gau, St-Genis-des-0llières (Boullu). - Alsace. Mutzig (Billot, in herb. Grenier) - Autriche. In umbrosis, cullinis in Bohemia (Trattinnick, I. c.).

\$6. RR. decipicans Boreau, 1. c., éd. 5 (1837), n 828; Déséglise, I. c., 1. $73 ̋$ et extr., p. 530 ; Cariot, I. c., p. 172; Fourreau, I c., p. 75; R. nemorum Ripart, in herb. Déséglise.

Ilab. Juin, juillet. Bois. - France, Cher : Montifaut, près de Bourges (Ripart), bois de Marmagne ! bois de la Grange-saint-Jean, près de Levet! forêt de Fontmorcau aux brûlis! - Rhône : Lycn au pont d'Alaï, Charbonnière (Chabert et Ozanon), Sainte-Consorce, Brouilly-saint-Lager (Boullu). - Suisse. Cant. de Genève : Le Vangeron près de Genève!

37. R. opacifolia Chabert! in Cariot, I. c., p. 677. Description communiquée par feu Chabert.

Racine non traçante, sous-arbrisseau bas de 5 à 6 décimètres, à rameaux dressés, fermes, armés d'aiguillons épars, grèles, un peu arqués, mèlés de soies glanduleuses. Pétioles glabres, un peu canaliculés, couverts de glandes rougeàtres, aiguillonnés en dessous. Stipules étroites, glabres, à oreillettes lancéolées, aiguës, divergentes, glan- 
duleuses aux bords. :3-7 folioles courtcment pétiolées, aignës, d'un vert sombre, glabres en dessus, opaques en dessous et poilues sur les nervures, à còte médiane glanduleuse, simplement dentées, à dents aiguës, mucronées ciliées. Pédoncules rougeàtres, hispides-glanduleux, munis de bractées lancéolées-aiguës, opposées, les pédoncules sont solitaires ou groupés par 2 ou J. Tube du calice glabre, oblong, hispide à la base. Sépales rougeàtres, les extérieurs pinnatifides, glabres, les intérieurs tomenteux en dedans sur les bords, les appendices sont étroits, glanduleux. Corolle très-grande, d'un blanc satiné. Styles glabres, un peu plus courts que les étamines, disque peu saillant. Fruit ovoïde, d'un rouge brun (Chabert).

Bien voisin du $R$. decipiens, dont il diffère par ses folioles plus petites, à dentelures plus aiguës, ses styles glabres, ses fleurs d'un blane satiné. A. D.

IIA, Juin. Ilaies. - France. Rhône: entre Charhonnière et Tassin, haies au Gau (Chabert)

\section{c). Styles libres, lameux.}

38. R. Gallica. L., sp.,70/4; Allioni, fl. Pedem. (178:3), II, p. 159, exel. syn. de Crantz; Gilibert, pl. d'Europe (1800), I, p. :84 ; Pers., syn., (1807), II, p.48; Willd., cnum plant. (1809), p. ¿40̈; Dum.-Cours., bot. cult., éd. 2 (1811), V, p. 474; Saint-Am., 11. Agen. (1821), p. 207 ; Tratt., I. c. (1825), I, p. 50 ; Balbis, 11. Lyon. (1827), I, p. 2:38; Dumort., fl. belgica (1827), p. 95; Chevalier, fl. génér. de Paris (1827), II, p. 696; Rchb), 11. excurs. (1850), II, p. 622; Richter, codex (1840), p. 497, $n^{\circ} 5742$; Gr. et Godr., I. e. (1848), I, p. 3.2 ; Boreau, I. c., éd. 5 (185\%7), no 822 et catal. de M.-etLoire (18;9), p. 78; Reuter, cat. de Genève (1861), 
p. 75; Déséglise, I. c. (1861), X, p. 76, et cxtr. p. 56 ; Cariol, 1. е. (1865), II, p. 175; Dumort., monog. des ros. de la fl. Belge (1867), p. 4.5; Loseos, pl. aragon. (1867), p. 150; Fourreau, I. c. (1869), p. 75; Verlot, pl. du Dauph. (1872), p. 115; Boissier, fl. Orient. (1872), II, p. 676; R. Gallica A. DC., fl. fr. (1805), IV, p. 444; Lindley, I. e. (1820), p. 68; R. Gallica var. officinalis Seringe, in DC., prod., II (1825), p. 605; Duby, I. c. (1828), p. 176; R. rubra Lam., fl. fr. (1778), III, p. 150; R. sylcatica Gatereau, fl. Montaub. (1789), p. 94; R. Belgica Brotero, fl. Lusit. (1801), I, p. 558 test. Lindley; R. blanda Brotero, I. c.? teste Lindley; R. semperflorens Desvaux, obs. (1818), p. 154 non Curtis nee Desf.; R. cordifolia IIost, fl. Austr. (1851), II, p. 25 teste Rchb.

Icon. Woodv., med., כ̄, 179; Nouveau Duhamel, VII, pl. 8; Sturm, flora, X. 54; Guimpel, holzgow., 89; Guimp. et Schl., 50 ; Hayne, II, 50; Wagner, 105 : ces planches sont citées d'aprés Pritzel. - Roessig, die rosen, $\mathbf{n}^{\circ} \mathbf{5 6}$; Lobel, ic., II, tab. 240; J. Bauh, hist., II, p. 54, f. 1.

Exs. Billot, $n^{0} 5.4$ bis.

Hıв. Juin. Bois. — La Belgique, la France, la Suisse, l'Autriche, l'Allemagne, l'Espagne. - Trattinuick dit : babitare seu sponte nasci videtur in IIspania et Gallia Australi. - M. Boissier, dans sa flore d'Orient, dit : Eurnpa media a Belgio, Germania ad Hispaniam, Italiam, Dalmatiam, Rossiam mediam et Australem. - Angleterre. Surrey près de Chartwood Wilson Saunders journ. of bot., 1871, IX, p. 275); spont.? -- France. Maine et-Loire : Saint-Gemmes-sur-Loire, Andard, coteau de Trèves (Boreau, catal.); - Sarthe : bois de Bouillon (Boreau, flore); - Vienne : Vezières, bois de Villiers (Boreau); - Loiret : Orléans, Saint-Denis-enVal (Boreau in litt.); - Loir-et-Cher: parc du Breuil (Franchet); Cher : bois de Marmagne! forèt du Rhin-du-bois à Jarry! - Puy-deDôme: bois de Lezoux (Lamotte); - Lozère : Chaldceorte! - Rhône : 
bois de l'Éioile prís de Lyon (Ozanon); Dardilly, Charbonnière (Clabert), pont d'Alai, Brouilly-saint-Lager (Broullu); - Basses-Alpes : Digne (Di. 1809!); - Alsuce. Mont Saint-(?)uentin près de Hetz (Fauché in herb. Boissier). - Suisse, Cant, de Genève: bois des Frères, Veyrier.

D’après Aiton, ce rosier était cultivé en Angleterre, en 1596, par John Gerard.

Obs. I. R. Gallica versicolor Tratt., monog. ros., I, p. 40 ; R. Gallica marmorea Seringe in DC., prod., II, p. 605. Icon. Roessig, die rosen, no 14; Redouté, les roses (1824), livrais. 11, C; Bot. mag., pl. 1794; Bot. regist., pl. 4 亿8.

Je ne connais la plante que cultivée, à fleur presque simple. Thory, prodrome du genre rosier, p. 92, dit : u celte variété croîl naturellement " près des frontières d'Espagne d'où elle m'a été rapportée par de Man" gourit. "

OBs. II. R. Gallica Willd, herb. no 9840; M. Crépin, 1. c, fase. 2, p. 57, dit : "Ce $n^{n}$ est représenté par cinq feuilles simples. Fol. 1). Paraît "être le R. Gallica tel qu'on l'entend ordinairement, mais je n'oserais me " prononeer. - Fol. 2 et כ). Deux ramuscirles florifères rappellant assez " le Rosa cultivé au jardin botanique d'Angers, que M. Boreau désigne a sous le nom de $\boldsymbol{R}$. provinialis. - Fol. 位. Je n'ose me prononcer sur " cette forme. - Fol. j). Parait devoir être rapporté au $R$. pumila."

Ons. III R. adenophylla Willd., enum. plant. (1809), p. 546 et herb. no 9837 ; Lindley, I. c., P. 9!; Tratt., I. c., I. p. 82.

M. Crépin, 1.c., p. 41, dit: "Cette espèce parait ètre une Gallicane " à folioles petites et prohablement produite dans les cultures. "Patrie inconnue.

39. M. provincialis Ait., KCW (1789), II, p. 204; Willd., énum. plant., 5ł引; Pers., syn., II, p. 48; Dum.Cours., l. c., V, p. 475; Boreau, l. c., éd. 5, no 825 et catal. de M.-et-Loire, p. 78 ; Déséglise, I. c., p. 77, et extr., p. 57; Cariot, 1. c., II, p. 175; R. Gallica Bastard. ess. fl. de II.-ct-L., p. 188.

Haв._L'Espagne et l'Italie, d'après diton et Willdenow.- La Bohème, I'Italie, l'Espagne, la France, selon Persoon. - Gussone, synopsis $\uparrow$. siculae, ne fait aucune mention de ce rosier. - Pohl, dans sa flore de 
Bohème, considère le $\boldsymbol{R}$. provincialis et le $R$. Gallica, conme variétés l'un de l'autre. - Borckhausen regarde le 1 . provincialis Miller comme étant la mêne espèce que le $R$. centifo'ia de Liuné.

France. Maine-et-Loire : naturalisé à Anger's, Avrillé, Beaucouzé, Faye (Boreau, catal.); - Loir-et-Cher : pare de Breuil, an spont.? (Franchel); - Cher : bois de Marmagne (Ripart), vignes de la Chapelle-st-Ursin :Khône: Charbonnière (Cariot, flore), Tassin (Boullu in Fourreau, cat.); Var: le Luc (IIanry). - Ilalie. Turin (herb. DC. 1:07), Paleme (Todaro!).

Ons. I. Loiseleur-Deslenchamps, dict. des sc. nat., dit que "le R. de a Provins a, dit-on, été rapporté de Syris a Provins par un comte de Brie, " au retour des croisades; mais rien n'est moins prouvé que ce fait; et il " parait au contraire que cette espèce a été connue de tnute antiquité et - que c'est probablement d'elle qu'Homère à vanté les vertus dans " l'llliade. " - D'après Aiton, ce rosier était cultivé en Angleterre en 1596 par John Gerard.

$O_{B S}$. II. R. provincialis Willd, herb. n $^{0}$ 9857, est réprésenté par dix feuilles simples. 11. Crépin, l. e., fait les remarques suivantes : Fol. 1 et 2 ;. "Ces deux spécimens appartiennent au $R$. tuguriorum Willd. Fol. 5). Je " ne puis me prononeer sur cette forme, dont le facies ne rappelle aucune" ment les gallicanae. C'est problement un hybride. Fol. 4). C'est une "Gallicane: Wallroth a écrit à côté - $R$. chamaerhodon v. gallicae var. " ô quae sequenta." Fol. 5). C'est la même forme ou à peu près que la " précédente et toutes les deux peuvent à la rigueur étre rapportées au " gallica tel qu'on l'entend ordinairement. Fol, 6). Pourrait bien apparte" nir au R. centifolia Lin. Fol. 7). Pourrait bien avoir quelques rapports s avec le $R$. damascena L. Fol. 8). Pourrait bien être une forme du “ $R$. pumila. Fol. 9). Me paraî être à peu près la mème forme que le a R. provincialis cultive au jardin botanique d'Angers; sculement les " folioles sont un peu pubescentes en dessous sur toute la surface. Fol. 10\%. "Appartient probablement au R. gallica."

60. Th. assimains Déséglise, descript. de qq. esp. nouv. de Ros., in mém. de la soc. Acad. de H.-et-Loire, (187j̃), XXVIII, p. 105, et extr., p. 7; R. cordifolia Chabert, in Cariot, 1. c., II, p. $673 ̋$ (non Host); Fourreau, I. c., p. 75. Pour la description voir Cariot, 1. c. 


\section{( 81$)$}

Har. Juin. Bois. - France. Rhône: Vallon de Ganches à Charbonnière (Chabert), Marcy-les-Roses près du bois de l'Étoile (Boullu).

61. 频. pysmaea M.-Bich., fl. Taur--Caue, I (1808), p. 597 et III (1819), p. 542; Tratt., I. c., I, p. 59; Seringe, in DC., prod., II, p. 60\%; R. Gallica var. pygmaea Boissier, 1. c., p. 676.

Icon. M.-Bieb., cent. pl. rar. Ross., I, tab. 2.

IIв. - In Tauria (M. B.).

Ons. M. Crépin, I. c., p. 47, a donné une deseription détaillée de l'échantillon qui existe dans l'herbier de Willdenow; sous le no 9835 et venant de Marschall von Bieberstein.

62. R. rupalis Déséglise, essai monog., in mém. de la soc. Acad. de M.-et-Loire (1861), X, p. 79, et extr., p. 59 ; Fourreau, 1. c., p. 75.

HaB. Juin, juillet. Haies, bois. - France. Cher: Moulon, près de Bourges (Blondeau 1850, in herb. Déséglise), pacage de la Servanterie près de Mehun; - Rhone : au Gau, au-dessus du pont d'Alay (Chabert), beis de l'Étoile (Ozanon), Tassin à Néginant (Boullu).

65. 䀧. Czackiana Besser, enum. Podol. et Volh., p. 61 et p. 66 ; Tratt., l. c., praef., p. VIII.

HAB, - Podolia ad Tyram (Besser, 1824, in herb. DC !).

Voici la description de l'échantillon de Besser conservé dans l'herbier DC. - Un rameau fortant 4 pétioles et deus fleurs. Le rameau a des grandes stipitées et de petits aiguillons fins. Les folioles sont grandes, oblongues-elliptique, ruguruses, coriaces, mesurant de 4 à 6 cent. de longueur, jusqu'à 5 cent. de largeur, glabres en dessus, pubescentes en dessous, nerveuses à nervures saillantes, la côte médiane parsemée de petites glandes, simplement dentées, à dents ciliées et surchargées de petites glandes. Pétioles pubescents-glanduleux, aiguillonnés en dessous. Stipules allongées, glabres, bordies de glandes, oreillettes divergentes. Pédoncules hispides-glanduleux. Tube du calice ovoüde, glanduleux et 
couvert de petites soies spiniformes, ees dernieres se retrouvent aussi an sommet des pédoncules. Divisions ealicinales, les intérieures cuspidées an sonmet, les extérieures spatulées, appendieulées, à appendices étroits, plus courtes que la corolle. Fleur grande. Styles hérissés.

Il y lieu de s'étonner de voir ce rosier passé sous silence par Seringe dans le prodromus.

64. R. Wolfgrangiama Bessel', 1. c., p. 67 ; Tratt., 1. c., II, praef. p, XVI.

A R. Czackiana, recedit iterum, cacterum illi proxima Rosa ab III. Colon. Ratomski in sylva prope Wiellor in distr. Rowniensi lecta, foliolis minus glandulosis, receptaculo graciliori, coarctura colli intra stamina conice elevata, petalis pallidis minoribus, stylis longissimis superne pubescentibus liberis, fructu setis evanescentibus subnudo pyriformi. (Besser.)

Besser place cette espèce de ses Gallicanac; mais est-ce bien une Gallicane? V'ayant pas vu un type de Besser daus l'herbier DC., ni dans celui de M. Boissier, jai eru devoir donner la courte description de Besser.

63. IR. pumila Lin. fil., sup. (1781), p. 262; M.-Bieb., fl. Tau.-Cauc., III, p. 5/42; Wahlenh., fl. Carp. (1814), p. 1500; Rau, cnum., p. 112, excl. var. b. ; Tratt., I. c., I, p. 43 ; Gmel., fl. Bad.-Als. (1826), IV, p. 564; Rchb., fl. excurs., II, p. 622 ; IIost, fl. Austr. (I8jI), II, P. 25 ; Borcau, I. c., éd. J̄, no 823 ; Guss., l. c., I, p. つ̋62; Déséglise, l. c., X, p. 78 et extr., p. 58 ; Cariot, l. c., II. p. 174; R. Gallica Auct., pr. part.; R. humilis Tausch? in diar. bot. flor., ann. 2, II, p. 463 ; Tratt., ?. c., p. 42.

Exs, Seringe, ros. desséch., $n^{\circ} 5 \overline{5} ;$ Billot., no $3 \breve{4} 4$ part. ; Déséglise, herb. ros., $\mathrm{n}^{0} 44 \mathrm{et} / 4 \mathrm{bis}$.

Ilar. Juin. Bois. - France. Loir-et-Cher: Cour Cheverny (Franchet); - Cher : bois de Narmagne, de Charron, petit bois des Vignes de la Cha- 


\section{( 85$)$}

pille-Saint-Ursin, forèt du Rhin-du-bois, bois de Givray près de Bourges; - Nièvre: Marzy (Boreau, flore); - Doubs : Mont Brégille à Besancon (Grenier); - Ilaute-Garonne: Toulouse (DC. 1807); - Rhône : Lyon, hois de l'Étnile (Ozanon), Charbonnière (Chahert), Tassin, Brouilly-SaintLager (Boullu), - Alsace. Mutzig ! - Suisse. Cant. de Genève : cnvirons de Genère. - Autriehe. Rehbergerthal près de Krems (Kerner); - Bohème : Leitmeritz (Kerner). - Allemagne. Franconie : les Monts Hoesselberg (Ilausser). - Italie. La Vénétie (Balbis, 1809, in herb. DC.). - Turquie d'Europe. Roumćlie: IIagion (Grisebach, in herb. Boissier); - Moldavie (Guebhard, 1818, in herb. DC.). - Russie l'Europe : la Podolie (Besser, 18æ4, in herb. DC.). - Grèce. Patras (Fauche, in herb. Boissier). - Turquie d'Asie. Anatolie, mont Alemdagh (Noë, in herb. Boissier).

OBs. Le R. pumilu de l'berbier de Willdenow, no 98399, est représenté par cinq feuilles simples. M. Crépin, 1. c., p. 58; fait les observations suivantes: "Fol. 1). Appartient au R. alpina L. - Fol. 2). Parait appar" tenir au R. pumila, nrais l'échantillon est trop incomplet pour pouvoir "garantir l'assimilation. - Fol. 5). Ressemble beaucoup au R. gallica “ Wibel. - Fol. 4). La destruction des styles dans ee spécimen m’empèche " de bien juger de cette forme, qui parait être bien voisine du R. virescens "Déségl. - Fol. 5). C'est la même forme que le $R$. pumila Wibel, fol. 5." - Introduit en Angleterre, d'après Aiton, en 1775 , par Kennedy et Lee.

\section{Sect. VI. - Centifoliae.}

DC., in Seringe, mus. helv. (1818), I, p. 5.

Doit-on réunir les espèces de ce groupe aux Gallicanae ou en faire une section séparéc? Voici la description que De Candolle donne pour les Centifoliae. "Styles libres; "fruit ovale; dicisions du calice pinnalifides; pédicelles " hérissés de poils glanduleux; folioles deux fois dentées en " scie; fleurs presque toujours doubles."

Cette division renferme sans contredit la partie la plus intéressante du genre pour les amateurs et les horticulteurs, mais d'un faible intérèt pour le botaniste, ear ces belles roses qui charment la vue sont des monstruosités pour lui, outre que la plupart sont sans patrie et sorties probablement des cultures. 
66. R. centifolia L., sp., 70k; Willd, en. plant., p. 345 ; Boissier, fl. Orient., II, p. 676.

Exs. Aucher-Eloy, no 4486 ! Kotschy, iter cilico, no 49.

IIAB...? - Cultivé en Angleterre en 1\$96, par John Gerard; j'ignore la date de son introduction en France.

Ons. Willd. herb. n $\mathbf{9 8 5 9}$; " ce numéro est représenté " par deux feuilles. - Fol. 1). Appartient-il au $\boldsymbol{k}$. cen" tifolia L.? je n'oserais me prononcer sur lui. -- Fol. 2). * Spécimen atteint de monstruosité dont il m'est impos" sible de déterminer le type spécifique " (Crépin, I. c., p. 44).

Kotschy, iter cilico, $n^{\circ} 49$, a distribué un rosier à fleur presque simple; est-ee le type du $\boldsymbol{R}$. centifolia L.? devant des matériaux incomplets, il devient très-difficile de se prononcer. Aucher-Eloy, $\mathrm{n}^{\circ} 4486$, a distribué un rosier venant d'Ispahan, à fleur presque simple, mais rien ne dit s'il est pris dans les cultures ou it l'état sauvage; M. Boissier, dans sa flore d'Orient, ne fait aucune mention de ce $n^{\circ}$ d'Aucher-Eloy. - Roessige, dans sa description économique et botanique des roses, a donné une dissertation tendant i prouver que le $\boldsymbol{R}$. canina $\mathbf{L}$. est le type véritable du Rosier à cent feuilles, lequel, selon lui, ne serait qu'une variété de ce premicr rosier, perfectionnée par la culture dans une longue suite de siècles (Thory, in Redouté, les roses, 182/4). - M. Bieberstein l'aurait trouvé spontané dans les forèts du Caucase oriental où, mème à l'état sauvage, on le rencontrerait à fleur double.

OBS. Les rosiers suivants font partie de cette section.

R. parvifulia Ehrh., beitr. VI, p. 97; Willd. herb, no 9870; Crépin, I. c., p, 42, - Hab. ? 
R. muscosu Ait.. Kew., 2, p. 207 ; DC., fl. fr., IV, p. 442; Willd., herb. no 9864 ; Icon. Ruessig, die rosen, no 6; Curtis, bot. mag., tab. 69; Redouté, les roses (1824), livrais. 2, D. ; livrais. 3, C. ; livrais. 7, B. Hab?

R. pomponia DC., 1. c., p. 445 ; R. Burgundiaca Desf, cat., p. 175. Icon. Curtis, bot. mag., tab. 407 ; Redouté, l. c., livrais. 3, D. - Hab. ¿

R. pulchella Willd., enum. plant., p. 3̋ว̆ et herb. $n^{\circ} 98326$; Crépin, l. c., p. 44. - Hab.?

R. turbinata Ait., 1. c. ; R. Francofurtana Gmel., fl. Bad.-Als., II, p. 408; R. campanulata Ehrh.

Exs. Wirtgen, p. crit., no $46 \overline{5}$. - Ilab.?

R. Damascena Miller, diet., no ้̋; Willd, herb. no 98ł1; Crépin, l. c., p. 45. - Hab. ?

R. sancla Richard, voy. en Abyss. (1847), IV, p. 292 ; Walpers, ann. bot., II, p. 466.

Hab.... Colitur circa Ecclesias in provincia Tigré.

Le port de ce rosier est à peu près celui du $R$. centifolia $\mathbf{L}$. ; mais il en differe par ses rameaux glabres, glauques, non glanduleux et armés seulement d'un petit nombre d'aiguillons recourbés, par ses fleurs beaucoup plus patites, à pédoncules glabres, glauques et non grlanduleux. Cette espèce est cultivéc dans les cours qui environnent les églises, dans une partic de l'Abyssinie (Richard).

\section{Sect. VII. - Pimpinellifoliae.}

DC., in Seringe, mus. Helv. (1818), I, p. 5 ; Lindley, monog. ros. (1821), p. 56, part. ; Besser, enum. Pod. et Volh. (1822), p. 60 ; Déséglise, ols. on the differ. meth. for the class. of the spec. of the genus rosa, in the Natu-

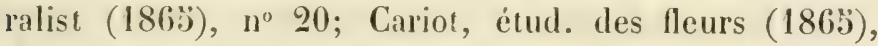
II, p. 174; Crépin, primit. monog. ros., fase. I (1869), 1. 14; ser. Woodsiana Tratt., monog. ros. (1825), II, p. 117, part.; Cinnamomene Seringe, in DC., prod., II, p. 176 , part.; Lorey et Duret, fl. de la Còte-d'Or (1831), p. 504, part.; Diastylae trib. leptacanlae Godet, 


\section{f. Jura (1855), p. 204, part.; Alpinae Reuter, Cat. de Genève (1861), p. 65, part.}

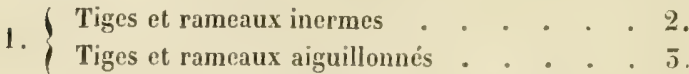

(Folioles petites, styles peu velus, fleur blanche. mitissima.

2. Folioles assez grandes, nervure médiane velue, tube du calice très-petit, styles laineux . . 0 zanonii.

Folioles doublement dentées, glanduleuses en

3. $\begin{aligned} & \text { dessous } . \\ & \text { Folioles simplement ou doublement dentées, }\end{aligned}$ églandulcuses . . . . . . . . . 5

Folioles à face inférieure portant des glandes nombreuses, pédoncules hispides-glanduleux,

4. Folioles à nervures secondaires peu glanduleuses ou églandulcuses, la nervure médiane glanduleuse, pédoncules peu glanduleux ou glabres

Ripartii.

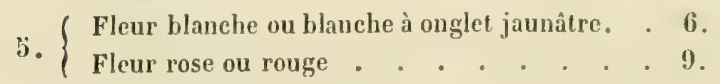

6. Stylesvelus . . . . . . . 7.

7. $\left\{\begin{array}{c}\text { Fruit gros, pyriforme, pétioles un peu pubéru- } \\ \text { lents, inermes } \\ \text { Fruit globuleux, pétioles glabres, aiguillonnés. spinosissima. }\end{array}\right.$

/ Styles glabres, folioles à côte médiane un peu
8. gelue dans le jeune âge, tube du calice petit,
tube du calice contracté au sommet . . spreta.
9. Folioles doublement dentées . . . . 10.
9. Folioles simplement ou irrégulièrement dentées. 11.
Folioles glabres, fleur d'un rouge foncé, fruit 10. $\left\{\begin{array}{l}\text { pendant, ovoïde, rouge. } \quad . \quad . \quad . \quad \text { rubella. } \\ \text { Folioles velues en dessous sur la côte de quel- } \\ \text { ques nervures secondaires, fleur d'un rose }\end{array}\right.$ clair . . ....... . reversa. 
Folioles simplement dentées, fruit globuleux, noirâtre à la maturité . . . . . . . pimpinellifolia.

11. Folioles simplement ou irrégulièrement dentées, fruit ovoïde ou arrondi, rouge à la maturite.

gentilis.

A). Folioles églanduleuses en dessous.

67. R. pimpinellifolia Lin., syst., $\mathbf{X}, \mathrm{n}^{\circ} 1, \mathrm{~A}$; syst., XII, $n^{\circ}$ 2; spec., ed. II, p. 70J; Willd., enum. plant., p. รૅ4ว̋ (non herb. no 9826); Krocker, fl. Siles., II, p. 145; Thuillier, fl. Par., p. 231 ; Pers., syn., II., p. 47; Gmelin, fl. Bad.-Als., II, p. 415; Raı, enum. ros., p. 62; Bl. et Fing., comp., I, p. 620; Richter, codex, p. 496, nº 5753; R. borealis Tratt., I. c., II, p. 141, part.; R. collina Schrank, bavar., $\mathrm{n}^{\circ}$ 774 (non Jacq.); R. chamaerhodon Villars? f1. Dauph., III, p. 353 ; R. a/finis Sternb., bot. Zig. (1826), beil. p. 80 (non Rau); R. pimpinellifolioalpina Kirschleger! fl. d'Als. (1852), I, p. 245 (non Rapin); $R$. pimpinellifolia var. rosea et var. affinis Koch, syn., p. 247; R. Scotica Miller, dict. no 5 , trad. franc., VI, p. $52 \% ; \boldsymbol{R}$. spinosissima var. flore roseo Hermann, diss. p. $6 ; \boldsymbol{R}$. campestris A. pimpinellifolia $\mathbf{W}$ allroth, hist. ros., p. 111; R. pumila spinosissima, flore rubro J. Bauhin, hist., II, p. 41; Tournefort, inst. (1729), p. 638.

Icos. Jacquin, fragm., tab. 107, f. 1; Redouté, les roses (1824), livrais. 24, C. (la plante figurée par Redouté est sortie d'un semis fait dans les pépinières de Descemet); livrais. 25, B.; livrais. 58, C. ; Loddiges, cab., p. 687; les planches suivantes sont citées d'après Pritzel: Nouveau Duhamel, VII, p. 16 ; Dietr., fl. bor., XII, 864; Regel, Gartenfl. (1862), 532 ; Guimpel, holzg., 86. 
Hab. - France. Seine-et-IIarne: Fontainebleau (Thuillicr, flore); Vosges : escarpements des hautes Vosges, le Ilohneck (Kirschleger, 18322! Boulay, 1860!); - Basses-Alpes : Gap et le Devoluy (Villars, flore). Silésie (Krocker, n.).

Oss. Linné, dans son Mantissa, p. 599, a fait la sup oression du R. pimęinellifolia en le réunissant au $R$. spinosissimo, mais sur quoi a-t-il basé celte réunion sans motif et si peu légitime? Gmelin et Rau, ayant cultivé pendant de longues années ees deux plantes, les considerent comme deux bonnes espèces. Dans notre Essai monographique sur les rosiers de la France, nous avons cherché à faire voir la confusion qu'il y a parmi les auteurs, pour le $\boldsymbol{R}$. pimpinellifolic el le $R$. spinosissima. Nous pensons que Tournefort connaissait les plai tes tout aussi bien que Linné! s'il n'cùt pas vu dans le R. pumila spinosissima flore rubro de J. Bauhin, une espèce tranchée, Tournefort u'cut pas manqué de rejeter ce synonyme en l'ajontant au $R$. campestris spinosissima flore albo odoralo de C. Baubin.

Je ne puis admettre qu'aucun botaniste du XVIIe siècle ait jamais confondu le $R$. campestris spinosissima flore alboodorato C. B., avec le $R$. pimpinellifolias Gerard; dans le thentrum botanicum de Parkinson, ils sont figurés comme tout à fait distincts.

Le $R$. spinosissima est seulement dans la première édition du species plantarum (1730); le R. pimpinellifolic est pablié pour la première fois dans le Syst. Nat. ed. X, p. 1026 (17i99) : mais, en réulité, Linné connaissait peu ces plantes; on peut en juger par les Amoenitales academicae éd. $2, \mathrm{~V}$, p. 220, où, en 17:\$8, il écrit lui-même ou fait écrire par son élève dans une énumération des arbrisseaux de la Suède " $R$. spinosissima per " totam Sueciam crescit, praecipue in acervis lapidum et ad agros, adeorjue " in sabuletis et montibus; altitudo ejus vix guna superat. " C'était sans doute le $R$. cinmamomea et c'est pourquoi Wahlenberg, dans sa flore de Suède, appelle $R$. spinosissima le $\boldsymbol{R}$. cinnamomea.

Smith, Engl. fora, dit que l'on doit rejeter ce nom (pimpinellifolia) obscur et employer celui de spinosissima pour ce que l'on donne ordinairement sous le nom de $R$. pimpinellifolia. Mais que deviennent les synonymes de Bauhin et de Tournefort? Avant de vouloir rejeter un nom, il faudrait voir si les bolanistes modernes ne font pas une confusion, préférant donner une hypothèse pour un fait acquis à la scirnce! Si nous consultons l'herbier de Linné, nous trouvons étiqueté un $R$. pimpinellifolice, qui est le $\boldsymbol{R}$. spinosissima ordinaire de l'Angleterre et de la Fiance.

L'herhier de Willdenow, no 9826, sous le nom de $\boldsymbol{R}$. pimpinellifolia, 
compremel quatre feuilles simples qui toutes appartiennent au R. sprimosissima, d'après l'étude particulière que M. Crépin vient de faire du genre Rosa de cetle collection.

Je ne suis pas éloigné de voir dans la plante de Sternberg, le $R$. pimpinellifolir, réudité ('n 1820 sous le nom de $k$. affinis Sternb. (non Rau, 1816).

6.8. E⿸. spingosissinas L., sp. (1764), 703 ; Herm., diss. (1762), \%. 6, part.; Crantz, stirp. Iustr. (1769), p. 84; Scopoli, fl. Cirn. (1772), I, p. 5 j゙ ; Leers, fl. herl).(177\%̈), p. 118; Inds., 11. Angl. (1778), p. 2 18, excl. syn. Gerard; Allioni, fl. Pedem. (178j̈), n“ 1794; Roth, tent. fl. Germ. (1788), I, p. 217, II, p. ว้ว̀ ; Villars, 1. c. (1789), III, p. öjö; Krocker, I. c. (1798), p. I/45;; Gilibert, pl. d'Europe (1800), I, p. 585); Smith, fl. Brit. (180/), II, p. כૅ) ; Gmelin, I. e. (180/), II, 1. 414; Sprengel. 11. Hal. (1806). p. 140̈; Pers., syn. (1807), II, p. 48; Mérat, 11. Par. (1812), p. 189; Rau, enume ros. (1816), p. ¿j8; Moots, monos. of roses, (1816), intrans. soc. limn., XII, p. 178 et herb), nos 7 à 13 ; Ilooker, brit. 11. (185.5), p. 22!) Déségrlise, ess. monog., in mém. de la soc. Acad. de M.-el-I., X (I861), p. 86 et extr., p. 46; Grenicr, 11. jura. (186/4), p. 226; R. spimosissime var. pusilla Woods, 1. c., P. 179; R. pimpinellifolia DC., fl. fొ. (1803̈), IV, p. 4.̄̄8; Mérat, l. с.; Bastard, essai f1. de V.-ct-Loire (1809), p. 187; Rehl)., 11. excurs. (1850), II, ). 612; Mulel, 11. fr. (185/4), 1,

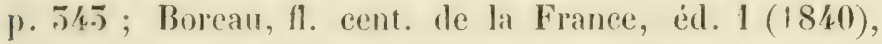

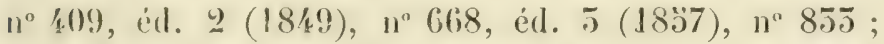
Godel, 11. jura (18.j.j), p. 2030 ; Reuter, cat. Geneve (186i), p. (i.); R. pimpinellifolia a. rulyaris Duby, bot. (1828), I, p. 177; R. pimpinellifolia a. collina Kirschleger 11. Ilsace (18.̈2), I, p. 24:ö; R. poteriifolia Besser, énum. Pod. et Volh. (1822), p. 62; Tratt., I. c., II, praef. p. xix; R. OEderiena Tratt., 1. c., P. 15̄.). 
Icos. Flora danica, tab. 598; Swenk, bot., tab. 539; Engl. bot., III, tal. 187, ćd. 5, tal). 461 (figure mauraise, qui représente le fruit rouge au lieu d'ètre noiràtre); Jacquin, frag., tab. 124; bot. regist., tab. 451; Roessig, die rosen, $n^{\circ} 9, n^{\circ} 25, n^{\circ} 59$; Redouté, les roses (1824), livrais. 7, C.; livrais. 40, A.

Exs. Karelin et Kiriloff (1840), $\mathrm{n}^{\circ} 249$; Schultz, $\mathrm{n}^{\circ}$ 1444; Billot, $\mathrm{n}^{\circ} 1182$, et his et ter; Fries, herb. norm., fasc. $X, n^{\circ} 52$; Déséglise, herl). ros., $n^{\circ} 6$.

Hab. Mai, juin. Bois, coteaux, broussailles. - Espèce commune dans toute l'Europe. M. Boissier, II. Orient., II, p. 675, dans la distribution géographique de cette espèce, dit: "Europa omnis, Sibiria, China borealis, India boreali-occidentalis. " Ial plante des Indes est différente de celle de l'Europe! - Gussone, synop. Sicul., n'indique pas le R. spinosissima en Sicile; Kirsehleger le dit rare dans le Grand-duché de Bade, nul dans le Kaisersthul en Brisgau; il manquerait en France, dans le département du Tarn, d'après la florule de Martrin-Monos; 11 . Arrondeau, dans sa flore Toulousaine, ne cite pas ce rosier.

Var. b. tomentella Boissier, 1. c.; "foliola minuta utrinque tomen" tella. - Hab. in monte Albo inter Djungutai et Kutuschi Daghestanix (Boissier)

Var. c. varibursensis Seringe, in DC., prod., II, p. 609; Déséglise, exs. herb. ros., no $ّ .-H a b . H a u t e s-A l p e s$, Villars-d'Arène (Ozanon).

69. R. Besseri Tratt., monog. ros., II, p. 128; Crépin, primit. monog. ros., fase. I, p. 40 ; R. microcarpa Besser, eat. crem. (1811), p. 20 et enum. Pod. et Volh., p. 18 et p. 62 (non Lindl., nee Thuub.); R. melanocarpa Link, enum. Berol., II, p. 377; Desportes, ros. gal., $\mathrm{n}^{\circ} 117 ; \boldsymbol{R}$. pimpinellifolia var. microcarpa Seringe, in DC., prod., II, p. 608.

Hab. - Russie d'Europe. La Volhynie (Besser, 1820, in herb. DC).

70. R. wathonneti Crépin, I. e., p. 42; R. Villarsiana Sieber; R. pellucina Arvet-Touvet! 


\section{(91)}

Exs. Karelin et Kiriloff (1840), $n^{\circ} 248$.

Hıв. - Siberie. Zmeofskoï (herls. DC., 1816); Mont Camuse (Fiseher de Gorenki, 1810, herb. DC.) ; Mont Tarbagatai ad torrentem Dschamybek et Terekty (Karelin et Kiriloff).

76. R. oxyacantha M.-Bich., fl. Taur.-Cauc., III, p. 5̇s; Tratt., 1. c., p. 138; Seringe, in DC., prod., II, p. 609; Ledebour, f1. Altaic., II, P. 228; Karel. et Kiriloff, enum, plant. Mlt., $n^{\circ} 525 ; \boldsymbol{R}$. pimpinellifolia var. oxyacantha Ledeb., t1. Ross., II, p. 74.

Exs. Karelin et Kiriloff (1840), n" 247.

H.s. - Désert de la Songarie (Karelin et Kiriloff, in herb. DC.).

OBs. L'échantillon conservé dans l'herbier DC. mesure 12 centim. de hatulcur; les aiguillons sont fins, droits, horizontaux, blanchàtres; pétioles velus, inermes; ;-7 folioles, d'un vert clair, glabres en dessus, glaucescentes en dessous, simplement dentées; divisions ealicinales entières, glabres ; tube du calice pelit, globuleux; les pédoncules portent quelques soies éparses ; styles laineux; fleur grande.

77. R. Webbiama Wallich, catal., n 682; Royle, ill. Himal., pl. 208; Walpers, repert. bot., II, p. 11; R. pimpinellifolia Ilooker et Thomson, exs. herh. des Ind.-Orient.

Haв. Juin. - Asie. Les monts Ilimalaya (Hooker et Thomson).

78. R. albicans Godet, in Boissicr, fl. Orient. (1872), II, p. 675 .

Exs. Bunge, $n^{\circ}$ 7; Haussknecht, $n^{\circ} 569$ A.

Haв. - Perse, Mont Parrow (Haussknecht, in herb. Boissier), Teheran (Bunge, in herb. Boissier).

79. Fr. oxyodon Boissier, fl. Orient., II, p. 674.

Hsв. - Le Caucase oriental (Ruprecht, in herb. Boissier).

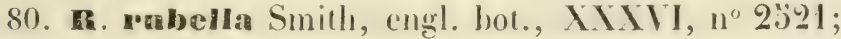
Woods, the brit. spec. ros. in trans. soe. Linn., XII, 
75. R. Ozanonii Déséglise, I c., p. 88, et extr., p. 48; Verlot, 1. c., obs.

Exs. Déséglise, herb. ros., $n^{\circ} 45 \%$.

HAB. Juin, juillet. Région des montagnes. - Isère: Saint-Eynard (Verlot); - Ilautes-Alpes: La Grave, le l'uy Vacher (Ozanon); - Savoie: mont Brizon!

Ons. R. pelrogenes Ozanon, in Crépin, primit. monog. ros. (1869), fusc. I, p. 40. - Je ne connais pas ce rosier; M. Crépin, dans sa clef analytique, dit: " tige et rameaux inermes, pédicelles et base du réceptacle "florifère plus ou moins hispides-glanduleux; folioles des rameaux flori" fères assez souvent doublement dentées."

74. R. midissima Gmelin, fl. Bad.-Als., IV, p. 558 ; Boreau, fl. cent. de la Fr., éd. 5, n ${ }^{\circ} 854 ; \boldsymbol{R}$. pimpinellifolia var. inermis DC., f1. fr., IV, p. 4.58; Seringe, in DC., prod., II, p. 609; R. pimpinellifolia var. mitissima Koch, syn., p. 21,7; R. pimpinellifolia b. montana Kirsch., fl. Als., I, p. 245; R. balloniana Herm., in Kirschı., I. c.

HAB. Juin. Broussailles des Montagnes. - France. Cantal; - Monts Dores; - Sources de la Loire, mont Gerbier (Borcau, flore); - Doubs: Besancon (Grenier); - Savoie : Nont Nivolet (Songeon); - Vosges : Champ du feu (DC., flore), ballon de Soulz, Hohneck, Hohenstaufen, ballon de Saint-Maurice (Kirschleger, flore). - Suisse. Cant. de Soleure : Ravallenfluk (Christ.).

7:3. R. Altaica Willd., enum. plant., p. 345; Besser, eat Crem. ann. 1816, p. 117; Tratt., I c., II, p. 120; Rehb., 11. excurs., II, p. 615; R. pimpinellifolia Pallas, fl. ross., II, p. 62; R. pimpinellifolia var. Altaica Seringe, in DC., prod., II, p. 608; var. grandiflora Ledebour, fl. ross., II, p. 75 ; R. spinosissima var. Pallasii Lindley, monog. ros., p. .̈1; R. grandiflora Lindley, I, e., p. 55; R. Sibirica Tratt., I. c., p. 250; R. hutescens var 1 . albis Link, enum. berol., II, p. 56. 
Exs. Sieber (1829), n 57, sub nom. R. Villarsiana Sieber; Maille, reliquiae, $n^{\circ} 1085$.

IIAb. Juin. Région des montagnes. - France. Vosges : Ballon de Saint-Mauriee (Pierrat); - Doulss : Crêt des Roches à pont de Roide (Paillot); - Isère: Saint-Christophe en Oisans (Boullu), en montant du Freney à l'Alpe du mont de Lans; mont Rachet (Verlot); - HautesAlpes : mont Gondran près de Briançon (Sieber, 1826), La Grave (Mathonnet); - Basses-Alpes : Saint-Paul de Vars (Arvet-Touvet); - IauteSavoie : Le Salive (Puget); - Savoie : mont Nivolet (Songeon). - Suisse. Jura de Soleure (Christ).

OBs. En 1829, Sieber a distribué en exsiccata un Rosn Villarsianu Sieber, j'ınnore si la description a paru; cette plante de Sieber me semble la mème que le $\boldsymbol{R}$. Mathonneti Crépin. M. Verlot, dans son excellent catalogue des plantes du Dauphiné, ne fait aueune mention de la plante de Sieber, ni de celle de Maille.

71. R. consimilis Déséglise, I. c., p. 90, et extr., p. 50; Verlot, cat. du Dauph., p. 115, obs.

Exs. Déséglise, herb. ros., $n^{\circ} 9$.

HAB. Juillet. - France. Lozère : (Prost, 1815, in herb. DC.); Hautes-Alpes : La Grave (Ozanon, Verlot), Villard-d'Arène (Verlot); Isère : Hontagne de la Salette (Verlot), Saint-Eynard (Ravaud, in herb. Grenier); - Haute-Savoie : Mont Veyrier près d'Annecy, Mandallaz (Puget); - Savoie : Mont Nivolet (Songeon). - Autriche. Illyrie : Slavnik près de Trieste ('Tommasini, in herb. Boissier).

79. ar. spreta Déséglise, l. c., p. 89, et extr., p. 49; Verlot, l. c.

Exs. Déséglise, herb. ros., $n^{\circ} 8$.

Haв. Juin, juillet. Région des montagnes. - France. PyrénéesOrient. : Eynes (Ripart); - Hautes-Alpes : La Grave(Ozanon), Villardd'Arène (Verlot), Rabou près de Gap (Grenier); - Isère : Montagne de Comboire près de Grenoble, Mont Rachet, Saint-Eynard (Verlot); Haute-Savoie : Montagne de Veyrier près d'Annecy (Pugel); - Savoie : dent de Nivolet près de Chambéry (Songeon). - Suisse. Valais : Mont Cornu (Orsini, in herb. Boissier). 
p. 177 et herbier $n^{\circ} 2$ a $n^{\circ} 6$; Lindley, 1. (.., p. 40; de Pronville, 1. c., p. 311; Tratt., I. c., II, p. 157; Smith, engl. flora, II, P. 374; Despories, ros. gall., p. 10; Rehb., fl. exeurs., II, p. 615; Hooker, hrit. flor. (1853), p. 228; Baker, Northumb. et Durh., p. 162; R. Candolleana elegans Thory, descript. nov. spee. gen. rosae (1819), p. 7; $R$. alpina var. rubella Seringe, in DC., prod., II, p. 612 .

Icon. Engl. bot., tab. 2321 et third edit., tab. 462; Thory, 1. e.

Hıв. - Angleterre. Coast of Durham (Winch, 1819, in herb. DC. ! Lyell, 1820, sans localité, in herb. DC.) ; je possède un échantillon cultivé venant de Woods !

81. R. areversa W. et Kit., hung., III, p. 295; Lindley, 1. c., p. 57; Tratt., l. c., p. 114; Desportes, l. c. 11 $^{\circ} 214$; Rchl., 1. c., p. 615; Koch, syn., p. 247; R. Klpina var. veversa Seringe, in DC., prod., II, p. 611; R. Wulfenii Trall.?, I. c., p. 200.

Icon. W. et Kit., 1. c., tab. 264.

Hab. Juin. Région des Montagnes. - France. Savoie : mont Nivolet, audessus de Monterminod (Songeon). - Autriche. Hongrie: les monts de Matra (W. et Kit.).

Ors. I. La plante que j'ai de la Savoie correspond à la planche 264 de la flore de Ilongrie. Les folioles sont ovales ou ovales-arrondies, glabres en dessus, velues en dessous sur la côte médiane et quelques-unes des nervures secondaires; les styles sont velus; les aiguillons sont grèles, dilatés à la base en forme de disque, longs, droits ou inclinés; les pédoncules solitaic es ou réunis par 2-5; la corolle est d'un rose clair ; je n'ai pas vu le fruit. Wald, et Kit. disent: feuilles a duplicatis serratis " la planche représente des folioles a dents simples, comme dans la plante de la Savoie; en présence d'échantillons incomplets et en l'absenee d'un type authentique, il est difficile d'affirmer. 
OBs. II. R. suavis Willd, en. berol. sup., p. 57; Link, en. hort. berol., II, p. 57; Desportes, I. c., no 79 ; Tratt., I.c., II, p. 154; Seringe, in DC., prod., II, p. 612 ; R. Alpina b. suavis Rchb., fl. excurs., II. p. 615.

Hab....? Cultivé au jardin botanique de Berlin en 1815, d'après Desportes; a été introduit en Angleterre en 1817, selon Sreet. II étail cultivé à Berlin depuis plus longtemps, puisque l'herbier de Kunth contient un échantilon récolté en 1806. Reichenbach dit que cette plante se trouve dans le sud de la Suisse : " in der südlichen Schweiz " - Willd., herb. n० 9848 ; M. Crépin dit : a M. K. Koch, dans sa dendrologie, rap" porte le $\boldsymbol{R}$. suavis Willd, au $\boldsymbol{R}$. reversa W. et K. En se basant sur "l'échantillon représentant le n 1898 , on est porté à admettre cette idena tification; cependant, dans cet échantillon, les folioles sont une fois plus a petites que dans le spécimen du $R$. recersa $n^{\circ} 9854$, à cóte seule velue - et glanduleuse. Dans l'herbier de Link, n’ 208, se trouve un ramusa cule en boutons du $R$. suavis, récolté dans le jardin botanique de "Berlin, identique avec le spécimen du no 9848 de l'herbier de IVilldenow. "Dans l'herbier de Kunth, no 162, la mème espèce récoltée en $1806^{\circ}$ dans a le même jardin botanique. "Crépin, l. c., fasc. 2 (1872), p. 51, p. 52.

82. Fi gentilis Sternb., bot. Ztg. (1826), beil., p. 79; Koch, syn., p. 247; R. rubella Godet! fl. jura, p. 205 (non Smith); Grenier, 11. jura, p. 227 ; R. pimpinellifolioalpina Rapin, in Reuter, cat. Genève (1861), p. 64 (non Kirschleger); $\boldsymbol{R}$. alpino-pimpinellifolia Reuter, 1. c.; Verlot, plant. du Dauph. (1872), p. 115.

HAB. Mai, juin. Région des montagnes. - France. Ain : Gex à la Faucille (Buullu); - Haute-Savoie : le Saleve au-lessus de Monnetier! Isère : mont Rachet, Villard-d'Arène. (Verlot). - Suisse. Cant. de Neuchâtel : sommet du Chaumont (Godet); - cant. de Soleure : Ravellen (Christ). - Autriche. Istrie : mont Maggiore.

в). Feuilles glanduleuses en dessous.

85. It. myriacantha DC., fl. fr. (1805) , IV, p. 459, V (181:3), p. 555; M.-Bicb., 11. Taur.-Cauc., III (1819), p. 12; Lindley, l. c., p. 530 ; Thory, prod., p. 44; Tratt., 
I. c., II, p. 88; Desportes, I. c., p. 12; Rcht., I. c., p. 615 ; R. myriarantha a. pumila Desvaux, journ. bot. (1815) II, p. 118; R. pimpinellifolia var. myriecenthe Seringe, in DC., prod., II, p. 608; Lois., 11. gal., I, p. 539 ; Duby, bot., I, p. 177; Koch, syn., p. 247; R. campestris e. myriacantha Wallr., hist. ros., p. $119 ;$ R. provincialis var. parvifolia M.-Bieb., I. e., p. 558 teste Wallroth; R. parcifolia Pallas, fl. ross., II, p. 62, test. Wallroth; R. spinosissima Gouan, fl. Monsp., p. 257; R. Granatensis Willkom. in Linnaea (18:42), XXV, p. 24! Walpers, Ann. bot., VII, p. 877.

ICon. Lindley, l. c., tab. 10; Redouté, les roses (182\%), livrais. 24, A, medioc.

\section{Exs. Willkomm (1845), no 1159 !}

IIAB. Juin. - France. Dauphiné (DC); - Ilérault : entre Montpellier et Mireval (DC., 8 mai 1807), Roquehaute (Ozanon); - Pyrénées-Orient. : Collioures! - Espagne. Sierra Alfacar (Willkomm, 1815, Reuter, 1849).

84. R. Riparaii Déséglise, 1. c., p. 87 et extr., p. 47; Verlot, eat. pl. du Dauph., p. $11 \bar{\jmath} ; R$. spinosissime Tratt., I. c., II, p. 118 (non Lin.); Rehb., I. c., p. 612; Mutel, il. fr., I, p. 543 ; R. pimpinellifolia var. c. Lloyd, 11. ouest (1868), p. 173 .

Exs. Billot, $n^{\circ}$ 5j78; Déséglise, herb. ros., $n^{\circ} 7$; Wirtgen, pl. crit., $n^{\circ} 75$ !, $n^{\circ} 127$ pro part.

IIAB. Mai, juin. Haies, bois, coteaux calcaires. - France. Loireinféricure : bourg de Batz! Le Pouliguen! - Cher : A. C. Saint-Florent, le Subdray, Northonnier, Trouy, Bourges, etc.; - Hautes-Alpes : mont Bayard (Verlot). - Prusse. Ockenheim près de Bingen (Wirtgen). Siberie (Herb. DC., sans date).

Ons. R. macrop odu Ripart! caractires du R. Ripartii, dont il diffère par son fruit gros, pomiforme, à pédoncules cbarnus au sommet.

II AB. - Cher : Viğnes de la Girange-St-Jean, Vignes de Givray (Ripart). 


\section{(97)}

8\%. R. dienzo: Lereh., in Ostr. bot. Zeitsch. (1872), XXII, 5, p. 145̆; Christ, die rosen, p. 72.

IlAB. - Juin. - Suisse. Cant. de Neuchâtel : château ruiné de Rochefort (Godet).

Feuilles vertes, pars mees de poils courts apprimés, ou glabres en dessus, velues en dessous principalement sur les nervures, portant en outre des glandes éparses, doublen"nt dentées; pétioles velus, parsemés de rares glandes fines, inermes.

\section{Sect. VIII. - Sabiniac.}

Crépin, primit. monog. ros. (1869), fase. I, p. 13̈; Pimpinellifoliae Lindley, I. c., p. 56, part.; série Biebersteiniana Tratt., I. e., I, p. 107, part.; Cimnamomeae Seringe, in DC., prod., II, p. 602, part.; Caninae Seringe, 1. c., p. 611 , part.

1. $\left\{\begin{array}{l}\text { Folioles doublement dentées } \\ \text { Folioles simplement dentées }\end{array}\right.$

2. Folioles églanduleuses en dessous. . . 5.

2. Folioles glandulcuses en dessous . . . 4 .

Folioles glabres sur les deux faces . . . . Sabunde.

5.

Folioles pubescentes sur le's deux faces, fieur

$\left\{\begin{array}{c}\text { blanche } \\ \text { Folioles parsemées de poils apprimés en des- }\end{array}\right.$ sus, velues en dessous, fleur rose . . Subini.

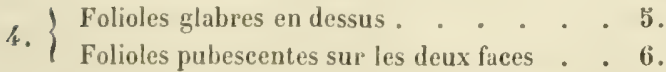

Folioles pubescentes et très-glanduleuses en dessous, tube du calice subglobuleux, glabre, pédoncule glabre, fleur d'un rose vif.

coronala submuda.

3. Folioles velues sur lesnervures en dessous, un peu glanduleuses à la base, tube du calice globuleux, hispide-glanduleux, pédoncules glandulcux, fleur rose à onglet blanc . . involue. 

(
Folioles portant de nombreuses glandes bru- nâtres en dessous, fleur d'un rose pâle, fruit subglobuleux ou ovoïds, rouge orangé. . coronata.

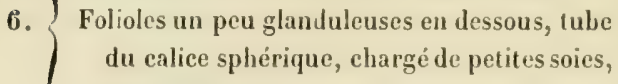 fleur d'un beau rose, blanche à la base, fruit globuleux
gracilis.

86. R. Sabian Woods, Irit. spec. of ros., in trans. lin. soc. (1816), XII, p. 188 et herb. $n^{\circ} 22$ a $n^{\circ} 24$; Lindley, l. c., p. 59, excl. var. J).; Smith, engl.f1. (1824), II, p. 580; de Pronv., monog. du genre ros., p. 63, exel. var. b.; Tratt., I. c., I, p. 150 ; Hooker, brit. fl. (1853), p. 252, exel. var. b. c.; Rehb., fl. excurs., II, p. 164, excl. var. b.; Babington, man., ed. 6, p. 124;

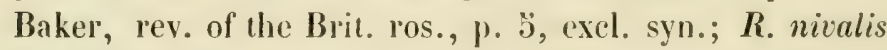
Don, hort. Cant., ed. 8, p. 170 ; R. incoluta var. Sabini Baker, monog. of Brit. ros. in Linn. Society's journ., XI, p. 205, part. exl. syn.

Icon. English bot., tab. 2954 , third ed., tab. 463.

Exs. Baker, herb. ros. brit., $n^{\circ} 1, n^{\circ} 2$.

Har. Juin, juillet. Haies, - Angleterre. Cheshire : près de Claughton (W ebb) ; - Warw ickshire : road side near Oakley Wood (Brunswich); Yorkshire : Cleveland (Baker). - France. Haute-Savoie : le mont Salève! - Suisse. Cant, de Soleure : Ravellen (Christ).

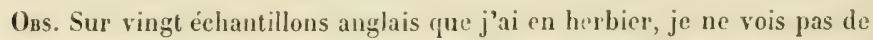
trace de glandes sur la face inférieure des folioles, même dans ceux distribués par M. Baker, herb. ros. brit., n० 1 et no 2 . Le rosier que j’ai récolté au Salève et celui que j’ai recu de M. Christ, venant du Jura soleurois, sont identiques à mes échantillons anglais.

87. H. Womian: Wroods, I. e., p. 183 et herb. $n^{\text {os }} 18$ a 20 ; Tratl., I. c., p. 15.5; Smilh, 1. c., p. 578 ; Desportes, ros. gal., $1^{\circ} 219 ; R$. Sabini var. Doniana 
Lindley, 1. 1., 1. ".9; Seringe, in DC., prod., II, p. 61:3; Rehb., I. c., p. 614; Hooker, I. c., p. 252 ; Babington, 1. c., p. 194; R. in olute var. Doniana Baker, monog. of brit. ros., in Limn. Society's journ., XI, p. 206.

Icov. English bot., tab. 2601.

Hıв. Juin. - Angleterre. Sussex (J. IVoods, in herb. Déséglise); Yorkshire: Thonton-le-Shief (Baker); - Cheshire (Webb).

Var. 1. gracheseens Baker, I. c., p. 206.

C'est une forme robuste d'Irlande récoltée à Antrim par le docteur' Moore. Feuilles à peu près de $\bar{z}-\overrightarrow{3} \frac{1}{2}$ pouces de long, ovales, maigrement poilues sur les deux faces, non glanduleuses; pédoncule aciculé et tube du calice elliptique, lisse. - Je n'ai pas vu cette forme.

Var. 2. Eobertsoni Baker, I. c.; R. Sabini var. b., engl. fl., II, p. 580; R. involuta Winch, geogr. distrib., \&l (non Smith).

Exs. Baker, herb. ros., $n^{0} 5$.

Intermédiaire entre $R$. Sabini et $R$. involuta. Les feuilles sont parsemées de poils apprimés en dessus dans le jeune âge, glabres quand elles sont adultes, velues en dessous sur les nervures, non glanduleuses; tube du ealice quelquefois mais non toujours lisse.

IL.в. - Angleterve. Yorkshire : les haics à Sowerby près Thirsk (Baker); - Northumberland : Newcastle (Winch, 1819, in herb. DC.).

S8. h. gacilis Woods, l. c., p. 186 et herb. no 21 ; Smith, I. c., p. 579 ; Trall., I. c., I, p. 151 ; R. Sabini var. gracilis lichb., I. c., p. 61/; Babington, I. c., p. 12/.

Icon. English bot., tab. 585.

IIAr. Juin. - Angleterre. Cumberland: Pooley-Bridge près d'Ambleside (Forster! recu de M. J-G. Baker); - Durham: Darlington (Hailstone, 1827); - Yorkshire, haies à Newsham Carr: collines à Ayton (Baker).

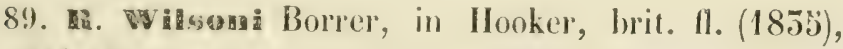
p. 2.51 ; Rehl)., fl. excurs., II, p. (ilk; Babington, I. c., p. 125 ; R. incolula var. I'ilsoni Baker, 1. e., p. 208. 
Icos. English bot., tah. 2725 et third edit., tah. 46\%.

Hab. Juin. - Angleterre. Carnarvonshire : bords du Ilenai près de Bangor (Wilson! Baker, Webb).

90. R. involuta Smith, 11. brit., III, (1804), p. 1598, et Engl. fl. (1824), II, p. 577 ; Woods, l. c., p. $185 \mathrm{et}$ herb. n $^{\circ} 17$; Lindley, l. c., p. 26 , excl. syn. Don; Tralt., 1. c., p. 152; Rehb., 11. cxeurs., II, p. 615; Hooker, brit. 11., p. $2 \overline{1}$; Babington, I. c., p. 125; Baker, rev. of the Brit. ros. (1864), p. 8; l'. incoluta var. Smithii Baker, monog. of Brit. ros., in Linn. Society's jourı. XI, p. 207, excl. syn. Rapin; R. pimpinellifolia var. imcoluta Seringe, in DC., prod., II, p. 609.

Icon. English bot., tab. $2068 \mathrm{et}$ tab. 2601.

Ilı. Région dus montagnes. - Angleterre. Les montagnes occidentales de l'Écosse. - Mon échantillon me vient de Woods, il a été récolté sur la plante cultive dans le jardin de Borrer. - Turner en 1816, Lyell en 1820 , ont envoyé ce rosier à DC.; les étiquettes sont sans indieation de localité.

Var. Ineviguta Baker, 1. c., p. 207.

Le pédoncule et le tube du calice glabres, ce dernier pelit, globuleux; les divisions calicinales tout à fait simples et non glanduleuses sur le dos.

Нав. - Anglelerre. Yorkshire: Broughton (Iaitstone, Baker). La même plante a été communiquée à M. Baker, par M. le docteur Moore, venant de Antrim et Derry; - Clıshire : près de IIoylake rivay station? (Webb).

Var. Moorel Baker, l. c.

HAв. Near the sea, Tamlaghbard, Derry. - Je n'ai pas vu cette variété a aiguillons plus vigoureux que dans les autres variétés, les grands $\ddot{b}$ ì 6 lignes de long, légèrenent courbés, larges ì la base de $\frac{3}{8}$ de pouce. Folioles presque glabres en dessus, faiblement velues et extrêmement glanduleuses en dessous. Pétioles à peine poilus, mais glanduleux et pourvus de nombreux acicules inégaux, les plus forts courbés en fiulx. Pédoncule et tube du calice hispides-glanduleux. Les sépales grands, de 8 a 9 lignes de longueur, légèrement pinnés. 
Var occidentalls Baker, l. c.: R. spinosissimn var. pilosa Lindley, I. c, p. 51 ; Hooker, l. c., p. 229 ; R. pimpinellifolia var. pilosa Seringe, in DC., prod., $1 \mathrm{I}, \mathrm{p} .609$.

Described by Lindley from an irish specimen still in the Hookeriam herbarium, the exact station not known (Baker).

II. Baker, in litt. 23 juin $186 \%$, me fait les observations suivantes sur cette curicuse forme : "Elle a lout à fait la feuille du $R$. vimpinellifolia " avee des dentelures doubles, la nervure médiane et le pétiole aciculés, " glanduleux et légèrement velus. Ie's sćpales, qui sont glanduleux sur le a dos, sont plus feuillés et dilatés à la pointe, deux d'entre cux sont " légèrement pinnatifides. "

91. IR. corponata Crépin, noles sur qq. pl. rar. ou crit. de la Belgique, in bull. de l'Acad. roy. de Belgique, XIV (1862), no 7 et extr. p. $2 \%$.

Exs. Wirtgen, no 270 et $270^{\text {bis }}$.

Hab. Mai-juin. Coteaux boisés. - Belgique. Prov, de Namur : Han-surLesse (Crépin). - France. Savoie: Praz-Flandet au-dessus de Chevron près d'Albertville? (Puget).

Var. subnuda Crépin, I. c., p. 26.

Har. Mai-juin. - Belgique. Prov. de Namur : Han-sur-Lesse, Wavreille (Crépin).

92. R. Sabauda Rapin, hull. soc. Hall, p. 175, et gruide cant. de laud., éd. 2, p. 191 ; Reuter, cat. genève (1861), p. 64; Grenier, f1. jura., p. 229.

Hab. Juin. Région des montagnes. - France. Haute-Savoie : le Mont Salève!

\section{Sect. IX. - Cinnamomeac.}

DC., in Seringe, mus. Helr. (1818), I, p. 2 ; Lindley, monng. ros. (1820), p. 15; Besser, enum. Pod. et Voll. (1822), p. 60; Seringe, in DC., prod. (1825), II, p. 602, part.; Duby, lot. Gall. (1828), I, p. 176, part.; Lorey 
et Duret, fl. Cotc-l'or (1851), I, p. 304 , part.; Déséglise, l. c., X (1861), p. 30 et extr., p. 10 et ols. on the differ. meth. prop. for the elass. of the spee. of the gen. rosa, in the naturalist (186:3), no 20, p. 511 ; Cottet, ínum. ros. du valais, in bull. soc. Murith., fase. $\delta$ (1874), p. 38 ; Cassiorhodon Du Horticr, IHulth. (1824), p. 11, florula Belgriea (1827), p. 95, monog. des ros. de la flore Belg. (1867), p. 45; Diastylae trib. dimorihacanthat Godet, fl. jura (1855), p. 204, part.
1. $\left\{\begin{array}{l}\text { Folioles pubescentes sur les deux faces } \\ \text { Folioles glabres sur lies deux faces. }\end{array}\right.$
cinnamomea.
2. $\left\{\begin{array}{l}\text { Rameaux aiguillonnés, folioles petites } \\ \text { Rameaux incrmes, folioles grundes . }\end{array}\right.$ 2.
Baltica.
blanda.

\section{Ph. cinnamsanac:a L., sp., 705; All , f1. Pedem.,} II, p. 158 ; Leers, 1. herb., p. 118 ; Moench, meth., p. 687; DC., fl. fr., IV , p. 4.59; Willd., enum. plant., p. $\$ 45$; Woods, 1. e., p. 17: et herb. $n^{\circ} 1$; Afzelius, ros. suec., fase. I, p. 7 ; Tratt, I. c., II, p. 171; Smith, engl. fl., II, p. 572 ; Lindley, I. c., p. 28; Du Mort., fl. belg., p. 95 et monog. des ros. de la flore de Belg., p. $4 \overline{5}$; Lois., fl. gall., I, p. 388 ; Duby, I. c., p. 176 ; Rechb., fl. excurs., II, p. 614, Host, fl. Austr., II, p. 26 ; Mutel, I. c., I, p. 347; Hooker, brit. fl., p. 227; Boreau, fl. cent., éd. $1, \mathrm{n}^{\circ} 410$, éd. $2, \mathrm{n}^{\circ} 666$, éd. $5, \mathrm{n}^{\circ} 851$; Kirsehleger, 11. Als., I, p. 246; Koch, syn. 2:8; Gonnet, ก. élem. de France, p. 477; Gr. et Godr., fl. de Fr., I, p. 536; Godel, 1. c., p. 207; Reuter, cat. genève (1861), p. 65; Déséglise, 1. c., p. 81, extr., p. 41; Grenier, fl. jura, p. 255; Cariol, 1. c., (186:3), II, p. 174; Baker, nat. hist. Irans. of Northumb. and Durh., II, p. 161; Boissier, I. e., II, p. 676 ; Balington, man., èd. 6, p. 125, R. cinnomomea a. majalis Rau, coum. ros., p. 355 ; Bl. et 
Fing., I. c., I, p. (622; Destaux, journ. bot. (1815), II, p. $120 ; R$. cimnamomea var. latifolia Seringe, in DC., prod., II, p. 606 ; R. cimnumomea var. collincola Seringe, I. c., p. 60:3 R. majalis Lerm., diss., p. 8; Retz., obs., fase. 5, p. 5.); Wahlenb., 11. Lap., $n^{\circ} 236$; Desf., 11. All., I, p. 400 ?; Lindley, l. e., p. 54; Tratt., I. e., II, p. 172; R. collimenla Ehrh., beitr., II, p. 70 ; R. simplex Scop., earn., I, P 5̈5; R. spinosissina Wahlemb., 11. Ups., p. 117, et fl. Suce., p. $516 ; \boldsymbol{R}$. mutica Müll., in 11. Danica, IV; Tratı., I. c., II, p. 169;R. Scopoliana Tratt., l. e., p. 229 ; R. turbinella Swartz in Spreng., syst., II, p. כ̋4 4 ; Seringe, I. c., p. 625; R. cinerea Sw.?

Icov. English hot, vol. 54, tah. 2588 (excl. Ie fruit); Redouté, les roses (1824), livrais. 10, D.; Flora Danica tab. 688; Svensk, bot., VIII, tab. 555 ?

Exs. Scheicher, $n^{\circ}$ 5ั5; Seringe, no 2; Schultz, n 648; Aucher-Eloy, $1^{\circ} 1 / 4.31$; Fries, herb. norm.. fase. 7, $n^{\circ} 45$ el $n^{\circ} 46$.

Haв. Mai, juin. - Suède. Laponie (Andersson, 1864, in herb. DC.). Norvège. Christiania (Buchinger). - Angleterre. Bois d'Aketon près de Pontefraet, Yorkshire, où il est probablement introduit (probably not a nalive) ; Birhhill, Galston, Ayrshire, d'apparence sauvage (apparently willd. - Hooker, brit. fl); - Bois près Alnwick (Baker, northumb. and Durh ) ; M. Baker, dans sa revue et sa monographie des rosiers anglais, ne fait aucune mention de ce rosier, ce qui porterait à croire qu'il n'existerait pas en Angleterre à l'état spontané : cependant Sweet, hort. Britannicus, regarde ce rosier comme spontané en Angleterre; un échantillon de Lyell, donné en 1820, existe dans I'herbier DC., mais sans localité. Aiton le dit natif du sud de l'Europe et cultivé en Angteterre en 15\%96, par John Gerard. - Belginue. En abondance près de Luxembourg, d'après M. Du Mortier. - France. Vosges (Mutel 11. fr.); - Neurthe: Nancy (Grenier) ; - Puy-de-Dòme . entre Sainte-Marguerite et le pont de Longue (Lecoq et Lam., catal.); - Creuse : Aubusson (Bridel, in herb. DC. !), 


\section{(104)}

montagnes de la Creuse où il est ecrtaimement spontané (Boreau, in litt., 50 mai 1871); - Jura: St-Loup (Puget). - Suisse. Cant. de Vaud: Lausanne (Schleicher); - cant. du Valais : Mont Catogne (de la Soie), Zermatt (Cottet), Loëches; - cant. de Fribourg : Furi (Lagger); cant. de Berne : rives de l'Aar à Berne (Seringe). - Autriche. Tyrol : entre Zirl et Onipontem (Kerner). - Allemagne. Lithuanic (Besser, 1824, in herb. DC.) ; - Bavière: Ingolstaat (Schonger). - Asie. Arménie (AucherEloy); Perse (Bélanger).

Seringe, dit: Ad ripas fluminum Europae et Americae? - Trattinnick, in Suecia, Dania, Anglia, nee non in Germania boreali. - M. Boissicr, Europa borealis et media in Alpinis, Sibiria omnis, Songaria.

D'après M. Crépin, le no 9819, R. cinnamomea de l'herbier de Willdenow, est représenté par huit feuilles simples. "Fol. 1). Appartient au * R. tuguriorum Willd. Fol. 2, 5, 4, ̋̈). Flore pleno. Fol. 6). Parait être a une forme du R. tomentella Lem. Fol. 7). Pourrait bien ètre une forme " du $R$. cinnumomea à folioles amples, allongées. Fol. 8), n’appartient pas a aux Cinnamomeae, pourrait bien ètre le $R$. coriifolia Fries. " (Crépin, 1. " c., fasc 2, p. 49).

OBs. I. R. foecundissima llünch. Ilausv., V, p. 279 ; Roth, f. Germ., II, p. כ̌ว7; R. cinnamomea Gmelin, f. Bad.-Als., II, p. 411 ; Lindley, 1. c., p. 28 ; Dematra, monog. ros. de Fribourg (1818), p. 4 ; $R$. cinnamomea var. foecundissima Koch, syn., p. 249; Renter, cat. Genève (1861), p. 63; $\boldsymbol{R}$. odore cinnamomi flore pleno C. Bauch., pin.; R. cinnamomea flore pleno Clusius, hist., I, p. 115 ; $R$. domestica Matth. 55, f. 1 ; Camerarius, epit., 98, f. 1.

Icos. Roessig, die rosen, tab. 5: Lindley, 1. e , tab. 3 ; Redouté, les roses (1824), livr. 57, B; flora Danica, tab. 1214 .

Exs. Seringe, no 29 ; Billot, no $5844^{\circ}$; Déséglise, herb ros., $n^{0} 58$.

IIAr. Mai, juin. Subspont. dans les haies. - France. Meurthe : ChâteauSalins (Maire); - Doubs: Besancon (Paillot); - Jura : Salins ; Landes : Mont-de-Marsan (Debeaux, in herb. Grenier) ; - Haule-Savoic : Pringy, Arenthon (Puget).

Ons. II. R. Ruvialis Müller, fl. Danica, tab. 868 ; Tratt., I. c., p. 174; Pers., syn., II, p. 47 ; R. cinnamomea var. b. Lindley, I. c., p. 28.

Je n'ai pas vu ec rosier, indiqué en Suisse par Hooker in Lindley et en Danemark par Vüller. La planche sos du fora Danica représente les 
divisions ealicinales plus courtes que lo bouton, l's feuilies ovales, a dentelures ouvertes assez plofondes.

Ons. III. R. aristata lapeyr., hist. pyr., p. 283; Lindley, I. c., p. 55; Tratt., I. c., II, p. 163; Seringe, in DC., prod., II, p. 606 ; Lois., 11. gall., I, p. 5:8; Desportes, roset gal., $n^{\circ}$ 48; Duby, I. c, II, p. 1009; Hutel, I. c., p. 555 ; Gonnet, 1. c., p. $479, \mathrm{n}^{\circ} 12$.

Haв. Mai, juin. - Lcs Pyrénées à Barrèges (Iapeyrouse).

"Cette espèce, admise par Lindley, Seringe, Dulyy, Loiseleur, Mutel u et Gonnet, omise par MII. Grenier et Godron, a sans doute disparu de "l'herbier; à sa place, se trouvent deux espèces: l'une le $R$. rubiginosa L., "l'autre le $R$. arvensis rar. bructeata Gr. et God." (Clos, révision de l'herbier de Lapeyrouse, p. 261).

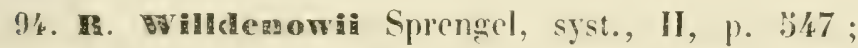
Seringe, in DC., prod., II, p. 621 ; Crépin, 1. c., fase. 2 (1872), in bull. soc. Jot. de Belg., XJ, p. I66, extr., p. 30 (non Trattimnick); R. microphylle Willd. Iserh. n 9828 (non Roxb.); R. arenaria M.-Bicb., in herb. Villd.

$$
\text { Haв. - Siberie. }
$$

9:. Th. Careliea Fries, sum. veget. scand. (I846), I, p. 171 ; Scheutz, in Crépin, 1. c., p. 243 et extr. p. 129.

Exs. Fries, herb. norm., fase. XIII, nº 99.

IAB. - Suède. Westerbotten (Dyhr, in herb. DC.; - Buchinger, in herb. Déséglise).

96. He. Ballica Roth, nov. pl. sper., p. 23/4; Tratt., 1. c., II, p. 57; Bhuff et Fing., comp., I, p. 625; Rchb., I. c., II, P. 615; Seringe, in DC., prod., II, p. 61:3; Boreau, précis des herb. faites en M.-et-L. (1862), extr. p. 19; R. lucida Koch, syn., p. 44k (non Ehrh.); R. spinosissima Pesneau, catal. de la Loire-inf. (1841), p. 181 ; R. pseudo-lucida Besser! in herb. DC.

Exs. Déséglise, herb. ros., $\mathrm{n}^{\circ} 46$. 
Hıs. Sahles maritimes. Juin. - France. Loire-inférieure : naturalisé dans les dunes de Pornichet (Ledantec, Prorost et Jullien); - Doubs : bois de Brégille a Besitmegon? (Paill,t). M. Paullot, daus sa lettre du l:j juıl. let $186 \%$, m'écrit: "II $\mathrm{m}$ 'a été impossible de remettre la main sur le " $R$. baltica, dont j'ai recueilli il y a deux ans un ćchantillon stérile dans " le bois de Brégille. n - Allemagne. IIambourg à Nienstäden (Lagger), IIambourg (Sonder in herb. Grenier)

97. PR. Blitad Ait., Ifort. Kew., éd. 1, II, p. 202 (1789); Jacquin, fragm. (1809), p. 70; Willd., sp., II, p. 1063 et herh., n" 9818; Tratt., L. e., II, p. 1'1; Rehb., fl. exeurs., II, p. 61/; R. fraxinifolia Gmel., fl. Bad.-Als. (1806), II, p. 412; Seringe, mélanges, I, p. 35 et mus. helv., I, p. 25; Lindley, 1. c., p. 26; Du Nortier, fl. helg., p. $9 \overrightarrow{3}$; Lej. et Courtois, comp., II., p. 140 ; Déséglise, in Billot, annot. Il. de Fr. et d'Allem., p. 293 ; R. Virginiana Miller, diet. $\mathrm{n}^{\circ} 1 \mathrm{v}$, trad. franc., VI, p. 526 ex Lindley; $R$. fraxine $\mathbf{W i l d}$.? enum. plant. sup., p. $77 ; \boldsymbol{R}$. cimnamomea .lérat, rex. 11. Paris (1845), p. 290 ; Coss. et Germ., 11. Paris (184:3), p. 181; R. cinnamomea var. globosa Desvaux, journ. bot. (1815), II, p. 120.

Icox. Jacquin, I. c., tab., 105; Bot. regist., tab. 438.

Exs. Seringe, $n^{\bullet} 28$; Wirtgen, pl. crit., fase. IX, $n^{\circ} 464$; Kickxia belgiea, $n^{\circ} 562$.

Hав. - Belgique. Prov. d'Anvers : Zammel (Van Haesendonck), Hersselt (Thielens); M. Du Mortier le signale pres de Huy; Lejeune et Courtois disent qu'il n'est pas rare vers Liége. - France. Loiret : Malesherbes (Cosson); - Seine-et-Oise: Dampierre! - Oise : Pierrefonds près de Compiègne (Ozanon); - Scine-t-Marne: Nemours (Mérat, revue fl. Par.); - Gironde: Bordeaux (Ozanon); - Côte-d'Or : Dijon (Jaenen); Lozère : Mende (Prost, 1815, in herb. DC.). - Suisse. Cant. de Genève : Bois des Frères près de Genève! - Allemagne. Coblence (Wirtgen).

98. R. Fischeriana Besser; Desportes, ros. gal., 
p. 114; R. saxalilis hort. genevensis! non Steven.

Arbrisseau de !- 2 metres, à tiges trés-fablement aiguillonnés, rameaux inermes, purpurins ou verdatres; petioles puberulents, inermes; $\bar{\partial}-\not j$ folioles d'un vert olancesent, chlahes en dessus, nervures relues, villosite disparaissant plus ou moins avec l'àge, mais la còte médiane reste toujours velue, elliptiques, les inféricures quelquefois ovales, doublenent dentées, à dents assez profondes, les secondaires terminées par une glande; stipules lancéolées, glibres, horlees de grlantes, à oreillettes aiguës; pédoncules solitaires ou réunis par i-j, courts, glabres, eachés par les bractées, le pédoncule solitaire porte à sa base une bractéc orale, cuspidée, plus longrue que le pédoncule, ceux réunis en houpuct portent a la hase deax bractées opposées, sourent une foliacée, le pédoneule central est dépourvu de bractées; tube dı calice petit, globuleux, glabre; divisions ealicinales entières, lancéolécs, spatulées au sommei, longuement saillantes sur le bouton, dépassant la corolle, réféchies à l'anlliese, ghlabres, les extérieures a hords glanduleux; styles libres, très-telus; flecir rouge; fruit ovoüde, rouge, couronné par les divisions calicinales rediessées, persistantes.

\section{Uaв. - Russie, ex Steudel.}

9!. घs. Bax Retz, in Hoffm., pliy!. B1., I, 59; Hornem., hafn., $n^{\circ} 11$, p. 472; Tratt., 1. c., II, p. 15; Scringe, in DC., prod., II, p. $600 ̈$.

Н.в. - siliérie.

100. Tr. Edusudea Pallas, fl. poss., II, p. 61 ; Lindley, 1. c., 1. 52 ; Trall., 1. c., II, 1. 170; Scringe, I. c., p. 606 ; Rchb., 11. excurs., II, p. 613 ?

HaB. - In Dahuriae et Mongoliae transalpinae apricis et betulis (Pallas), 


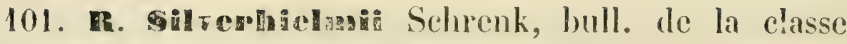
phys.-mathém. de l'Acad. imp. soe. de St-Pétersbourge, II, p. 193̈; Fischer, sup. cat. de St-Pétersh., 23 janv. 1844, p. 17; Walpers, repert., V, p. 649.

ILs. Juin. - In arundinetis ad II. Tsehu. - Des échantillons que j'ai en herbier, l'un vient du jardin botanique d'Angers (Boreau), l'autre a été récolté par moi dans celui de Genève; dans l'herbier de M. Boissier, les échantillons ont été pris dans un jardin.

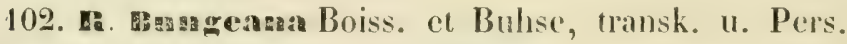
pflz. (1860), XII, p. 81; Walpers, an. bot., VII, p. 877 (1).

Icon. Boissicr et Buhse, I. c., Іab. VI.

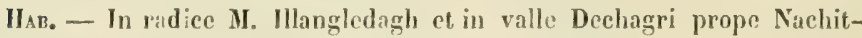
seliewan.

105. IR. angevinac folsia Boissicr, diagn., siric 1. , fase. 7, P. З1 et 11. Orienl., II, P. 677; R. Daëncnsis Boissier, diagn., sér. I, fase. 7, p. כ้l.

Exs. Aucher-Eloy, $n^{\circ} 14 \overline{7} 7$ ! $n^{\circ} 44833$ !; Kolschy, nos 42/4, $622,655,682$.

IlAB. - Perse (Aucher-Eloy); mont Kuh-Daëna et mont Elbrus (Kotsehy). - Les deux numéros d'Aucher-Eloy ne sont pas eités par M. Boissier dans sa flore d'Orient.

104. IR. Lacerans Boissier et Buhse, I. c., p. 85; Boissier, fl. Orient., II, p. 677.

II Ar. - Perse. Entre Nischapur et Nechhed, entre Herat et Tebbes (Bunge, in herb. Boissier).

(1) Dans Walpers, il y a une faute d'impression qui change le nom spécifique; c'est R. Bungeana qu'il faut dire et non R. Burgeanu comme l'écrit Walpers. 
103. Re. Lehanamaina: Bunge, pl. Lehm., p. 287; Walpers, ann. bot., IV, p. 656; Boissier, f1. Orient., II, p. 678 .

Har. - Asie, Samarcand (Lehmann, in herb. Boissier).

106. R. Cabulica Boissier, I. c., p. 678.

HAB. - In regni Cabulici Alpinis ad Erak et Sir i Chusma (Boissier).

Var. b. Latisplna Boissier, I. c.; R. latispina Boissier, diagn., sér. II, fase. 2, p. 49.

HAB. - In Alpinis regni Cabulici prope Kalou (Boissier).

107. Ely. Elymaraca Boissier et Haussk., in Boiss., fl. Orient., II, p. 673 .

Exs. Aucher-Eloy, no 14.59! Ce no n'est pas cité par II. Boissier; il est identique au R. Elymatica qui se trouve dans son herbier.

Uab. - La Perse (Aucher-Eloy), Teng Nalli (Boissier).

108. BR. Oriendalis Dupont, in DC., prod., II, p. 607; Boissier, fl. Orient., II, p. 680.

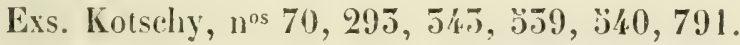

Has, Juin. - Région alpine inférieure du Taurus près de GulekMaden (Balansa), mont Elbrus près de Passgala; mont Gara (Kotschy).

Var. b. Oiympien. - Feuilles doublement dentées.

R. pygmaea var. Olympica Clement, sert. olymp., p. 40; R. glutinosa b. tomentella Boissier, l. c., p. 679. - Celte forme mo scmble plutôt appartenir au R. Orientalis, qu'au R. glutinosis Sibth.

HaB. - Olympe de Bithynie (Clement).

109. RR. Eúctgchyana Boissier, diagn., sér. I., fasc. 10, p. "̈, et II. Orient., II, p. 685.

Exs. Kotschy, no $6 \overline{3} 7$ ! 
HAB. Juin-juillet. - Perse. Mont Kuh-Daëna (Kotsehy).

Ons. Ce rosier, avec ses divisions calicinales entières, me semble plutót appartenir à la section Cinnamomeae qu'a celle des Tomentosae, où M. Boissier le place?

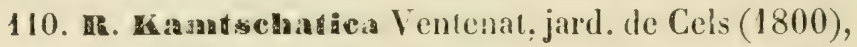
1ab. 67; Ait., Kew., ed. 2, III, p. 239; Pers., syn., II, p. 47; Lindley, 1. c., p. 6; Tratt., I. c., II, p. 125; Seringe, in DC., prod., II, p. 607; de Pronv., I. c., p. 27; Desportes, 1. e.; p. 2.

Icos. Ventenat, 1. c.; Bot. regist., tah. 419; Bot. mag., tab. 5149 .

HAB. Mai, - Kamtschatka.

111. M. ragosat Thunb, f1. Jap. (1784), p. 215; Pers., I. c., p. 48; Lindley, l. c., p. ॐ̈; Tratt., 1. c, II, p. 116 ; Seringe, in DC., prod., II, p. 607; Sieb. et Zuce., fl. Jap., p. 66; R. ferox Ait., Kew., ed. II, (1811), III, p. 262; Lindley, l. c., p. $\overrightarrow{3}$; de Pronv., l. c., p. 23; Desportes, 1. c., p. 1; R. Kimtchatica Thory, prod., p. 4ö; Cariot, étud. des flemrs (1863), III, P. 179; R. Kamtehatica var. ferox Seringe, I. c.; $\boldsymbol{R}$. echinata Dupont; R. Regeliana Lind. et André, illust. hort. (1872), p. 571 (non Reuter).

Icox. Miss Lawrance, Lal). $42 \mathrm{ex} \mathrm{Lindley;} \mathrm{Bot.} \mathrm{regist.,}$ tab. 420; Redouté, les roses (182/4), livrais. 5, A; Roessig, die rosen, tab. 60; Sieb. et Zuce., 1. c., tab. 28; Lindley, 1. e., tal. 19.

IIAB, - Le Japon. - Tous les échantilions que j’ai été à mềne de voir viennent des jardins et ceus que je possède en herbier ont la même origine. Sweet, hort. brit., dit que ce rosier a été introduit en Angleterre en 1796.

Var. onlabiuscula Crépin, l. c., fasc. 2, p. 5马; $R$. coruscans Waitz in Link, enum. hort. berol., II, p. 57; ; Tratt., I. c., II, p. 178; Vesportes, I. c., no 213 ; Seringe, in DC., prod., II, p. (610. 


\section{$(111)$}

IAв. ...? Il étail cultivé dans le jardin de Berlin en 1822, d'après Desportes; il a été introduit en Angleterre, sclon Sweet, en 1825.

112. ER. Iwara Siel), cat. rais. des pl. du Japon (18:36), p. 6; Regel, ind sem. hort. Petrop. (1861), p. 35 ; Koch, dendrol., I, p. 257.

IIAB. - Japon.

115. R. microphylla Roxb., in Lindley, 1. c., p. 9, p. 1/6; de Pronv., I. c., P. 10/4; Seringe, in DC., prod., II, p. 604; Desportes, I. c., p. 91, n 2029; IIardy, journ. des jard. (1828), p. 47; R. Roxburgii Tratt., I. c., II, p. $2 \overline{5} \overline{5}$.

Icox. Bot. regist., tab. 919; Bot. mag., tab. 5490.

HАв. - Chine (Wallich, 1819, in herb. DC.).

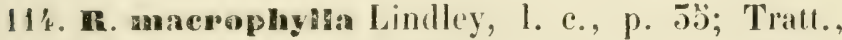
1. c., II, P. 166 ; Seringe, in DC., prod., II, P. 612; Don, hort. Cant. (1825), p. 198; Desportes, I. c., p. 8; R. velutina Royle.

Icox. Lindley, I. c., tab. 6; Wallich, pl. Asiat., pl. 117.

Exs. Wallich, pl. ind., $\mathrm{n}^{\circ} 690$.

Har. - Indes. Himalaya (Wallich, 1829, in herb. DC.), Kidrinath (Wallich, 1829, in herb. Delessert), Sikhim (Ilooker).

OBs. Je rapporte le $R$. velntinc Royle, qui était cultivé en 1868 au Musćum de Paris et dont j’ai des échantillons, au $R$. mrerophylla Lindl.

11\%. 趹. serece Lindley, I. c., p. $10 \%$; Seringe, in DC., frod., II, p. 615; de Pronv., I. e., p. 103̈; R. Wallichii Tratt., I. c., II, p. 195.

Icos. Lindley, 1. c., tab. 12; Bot. mag., tab. 5200.

Lxs. Wallich, pl. ind., nº 69 כ̆.

IIıB. - Indes. Kamaon (Wallich, 1826, in herb). DC.), Swinaghos (Webb, 1852, in herb. DC.), Sikkim (Hooker). 
116. R. dissimilis Déséglise, in the journ. of Botany, Juin 187/ et extr. p. 1; R. cinnamomea Karelin et Kiril., exs., $n^{\circ} 560$ (non Lin.), 1840.

ILAв. - Semipalantinsk (Kiriloff et Karelin, in herb. DC.).

117. R. maelian Bge, in Ledeb., fl. Mlt., II, p. 128; Bunge et Mey., sup. 11. Alt., $n^{\circ} 109$; Karelin et Kiriloff, enum. pl. Alt., $\mathrm{n}^{\circ}$ 556; Ledeb., fl. ross., II, p. 73 ; R. Alpina Pallas, fl. ross., II, p. 61.

Exs. Bunge, soc. imp. nat. cur. mosqu., $n^{\circ} \breve{59}$.

Haв. - Siberie. Semipalantinsk (Karelin et Kiriloff, 1840, in herb. DC.); Dalurie (Bunge, in herb. Boissier).

118. Hahearica Desfont., cat. Paris (180/), et exempl. authent. in herb. DC.; Pers., syn., II, p. 49 (1807); Dum.-Cours., bot. cul. (1811), V, p. 484; Déséglise, obs. sur les R. Balearica Desf. et R. vosag., Desp., in the journ. of Botany for Warch, 187/4, extr. p. 1; R. Carolina var. laevis, Seringe, in DC., prod., II, p. $60 \%$; R. Virginiana Tratt., l. c., II. p. 13̈.

Icon. Redouté, les Roses (1824), livrais. 7, A.

IIs. - Ile Nlajorque (Desfontaines! in herb. DC.).

Espèces douteuses ou peu connues.

12. ambiyodis C.-A. Meyer, flora, XXXII, 74ว̈; Walpers, ann. bot., II, p. 465.

HAB. - Kamtschatka.

ER. Gailclani-Ey aldemanoii Klotzsch, Prinz Walclemar Reise, p. 155; Walpers, 1. c., VII, p. 877.

H.s. - Llimalaya. 


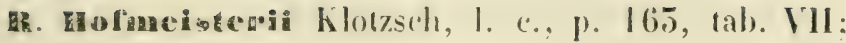
Walp., l. c., 878 .

$$
\text { IIмв. - Himalaya. }
$$

IR. Iñockeri Tratt., l. c., p. 251 ; Seringe, I. c., p. $623 ;$ R. pygmaea Krocker, 11. Siles., II, p. 134.

$$
\mathrm{H}_{\mathrm{AB}}, \text { - Silésie. }
$$

ER. meroicata Waitz, in Link, enum. hort. berol., II, p. 36 ; Tratt., 1. e., p. 175 ; Seringe, 1. c., p. 622.

HAB.....?

路. piatyacantha Schrenk, bull. Acad. St-Pétersb., p. 25\%; Ledebour, f1. ross., II, p. 75.

H.ı. -- Sibérie.

R. Gleberiana Schrenk, I. c., I, p. 80; Ledeb., I. c., p. 76; Walpers, repert. bot., V, p. 649 .

Нав. - Siliérie.

Re. 'Tazoica Steven, in M.-Bieb., fl. taur.-canc. (1808), I, p. 594; Lindley, 1. c., p. Ј̄।; Tratt., I. e., II, p. 9; Suringe, 1. c., p. 61 .

Haß. - In dumetis Tauriae.

\section{SEct. X. -- וpinac.}

Déséglise, 1. c., X, p. If et extr., p. 11, et obs. on the differ. meth. prop. for the classif. of the spec. of gen. ros., in the naturalist (186.3), $\mathbf{n}^{\prime \prime} 20,1 \% .511$; Crépin, primit. monog. ros., fase. I (1869), p. 14; Cottet, I. c., p. 58; Caniuae Seringe, 1. c., p. 611, part; Duby, 1. c., p. 177 part; Pimpinellifolice Lindley, I. c., p. 56, part.; Koch, 1, c., part; Diastylae trib. lepacanthae et trib. homaeacanthae, Godet, I. c. 
1. Rameaux munis de petits aiguillons . . . . 2

l Rameaux inermes. . . . . . . . .

2

Aiguillons fins, sétucés, styles velus, fruit ovoïde

Aiguillons plus robustes, styles laineux, fruit gros, ovoüde ou pyriforme

$\bar{\jmath}$

Styles hérissés. . . . . . . . . .

Styles velus . . . . . . . . ;

Pétioles inermes, feuilles vertes en dessus, fruit

oblong ou subglobuleux . . . . . Alpina.

4 Pétioles parsemés de petits aiguillons sétacés, feuil-

interealaris.

adjerta.

4.

;

les d'un vert sombre en dessus, fruit allongé en forme de fuscau.

lagenaria.

§. $\left\{\begin{array}{c}\text { Folioles portant quelques glandes éparses en } \\ \text { dessous . . . . . . . Pyrentica. }\end{array}\right.$

Folioles églanduleuses. . . . . . . . . 6 .

Folioles à dents profondes, irrégulières, pétales rouges, pâles en dehor's, onglet jaunâtre, fruit

6. $\left\{\begin{array}{c}\text { ovoïde ou subglobuleux. } \\ \text { Folioles a dents régulières, la côte parsemée de }\end{array}\right.$

Monspelinere. poils et de glandes, corolle d'un rose vif . . pendula.

119. R. Mpias L., spe., 70.̄; Jacep., 11. Austr., III, p. 4.̃); All., 11. Pedem., $1^{\circ}$ 1798; Roth, tent. 11. germ., II, pars prior, p. 339 ; Villars, 11. Dauph., III, p. 3329; Lam., 11. fr., III, p. 152; Krocker, fl. Siles., II, 1. 1.58; Gilibert, pl. d'Europe, I, p. 584; DC., II. fr., IV, p. 446 (cxel. var. b.); Gmelin, fl. Bad.-Ils., II, p. 429; Pers., syn., II, p. 49; Tratt., monog. ros., II, p. 498; Ballbis, f. Lyon., I, p. 260 ; Rchb., 11. excurs., II, 1). 615 ; Ilost, Il. Austr., II, p. 2/4; Richter, codex, p. 496, $11^{\circ} 5745$; Boreau, fl. cent., éd. 2, n 670 , éd. 5, n 586; Gonnet, 11. élem. de Fr., p. 477 ; Gr. et Godr., fl. de Fr., I, p. 乌̈3̆6, part.; Kirschleg., 11. Als., I, p. 246; Godet, fl. Jura, p. 206, part.; Déseglise, essai monog., p. 35; Fourreau, I. c., p. 75; Verlot, 1. c., p. 114; Boissier, 


\section{(11i)}

1. c., p. 675; R. Alpina va!? a culyaris Desvaux, journ. bot. (1815), II, p. 119 ; Scringe, in DC, prod., II, p. 611; Duby, I. c., I, p. 177 ; R. Alpina var. a. Bl. et Fing., l. c., p 6 \$1; Lois., 11. gall., I, p. 560 ; Mutel, fl. fr., I, p. 547; Godr., 11. Lorr., I, p. 218; Koch, syn., p. 248; R. rupestris Crantz, stirp. Austr., p. 8כ̆; R. inermis . Iiller, diet., n" 6, trad. franc., VI, p. 323 ; R. rinnamomea IIerm., dissert., p. 7 ; Rosa non spinosa Hallur, opuse, p. $218 ; R$. rubello fore simplici non spinosa J. Baul., hist., vol 2, p. 59 ; R. inermis, foliis septenis glabris, calycis segmentis indivisis Haller, helv., $n^{0} 1107$.

Icos. Jaequin, I. e., tab. 279 ; Bot. regist., tab. 424; Redoute, les roses (1824), livarais. 53 , c. (médioc.).

Exs. Seringe, $n^{\circ} 6, n^{\circ} 49, n^{\circ} 50$; Fries, herl. norm., fase. 9, n" 48; Diseglise, hul. ros., n० 10; Bourgeau, Alp. de la Savoie (1848), $n^{\circ} 79$.

HaB. Juin, juillet. - Montes Europac mediae et australis.

Var. setosa Seringe, in DC., prod., II, P. 612; R. Alpina hircina Desvaux, journ. bot. (1815), 11, p. 119? P'édonenles et tube du calice couverts de petites soies fines.

Ilas. Juillet. Région des montagnes. - France. Pyrénées-orientales (Coder, 1814, in horb. DC.); - Isère : Chamechaude, mont Rachet (Verlot); - Savoic : Col ùu Frêne au-dessous d'Aprenront (Songeon). Suisse. Berne (Seringe, 1817, in herb. LC.).

Var. globosa Desvaux, 1. e.; Seringe, 1. c. (excl. syn. canina ambigua Desv.); R. Alpina var. pedunculo valde hispido, ovurio glabro pendulo globoso DC., A. fr., V, 55:, $R$. oreinosa hilpart, in litt.! Pédoneules lisses ou glanduleux, tube du calice globuleux ou subglobuleux, ghahre.

ILAB. Juillet. Région des montagnes. - Frunce. Doubs : Norteau (herb. 
Grenier); - Vosges : ballon Saint-Maurice (1) (Pierrat); - Lozère : Mende (Prost, 1815, in herb. DC.); - Pyrénées orient.: Montlouis, pédoncules solitaires, glabres, tube du calice glabre (Ripart); - Alpes-maritimes : Forèt de Clans, pédoncules solitaires, glanduleux ainsi que le fruit (Bornet); - Isère: Chamechaude, pédoncules solitaires, jlanduleux, fruit glabre, divisions calicinales glabres (Verlot), Villard-de-Lans ì la Fauge, pédoncules glanduleux, solitaires ou réunis par deux, tube du calice et sépales glabres (Boullu); - Ilaute-Savoie: Ilabere-Lullin, pédoncules 1-2-4, glanduleux, sépales glabres, tube du calice glubre ou glanduleux; Allonzic r (Puget), Saint-Gervais sous le glacier de 'Trê-la-Tet, pédoncules très-lungs, peu glanduleux, fruit glabre, sépales parsemés de glandes sur le dos (Boullu), Foge, sépal's, fruit, pédoncules glanduleux (Puget), mont Salève! pédoncules et fruit glabres, sípales parsemés de glandes. - Suisse. Cant. de Vaud, : Creux-dessus près de Montbovon, pédoncules réunis por deux, glabres, fruil el sépales glabres. - Autriche. Schwargan, même forme que celle de la Suisse (Kerner). - Piémont : Val Pesio, péáoncules, fruit et sipales glabres (Bornet).

Var. pyriformin Seringe, in DC., prod., 1I, p. 612. Fruit court, pyriforme, glabre.

IIn. - France. Vosges : Le Ilohneck! - Suisse. Belpberg près de Berne (Seringe, 1815, in herb. vC.).

Var. helleboriun Seringe, 1, c.

Les trois échantillons de l'herbier DC. viennent du Jardin botanique de Moscou et ont été donné en 1821 par Goldhach. Forme très-curieuse qui, mieux étudiée, formera un type spécial; mais il est difficile de se prononcer sur des échantillons incomplets.

Ons. R. Alpina sorbinella Seringe, I, e, p. 611. L'échantillon qui existe dans l'herbier DC provient du Jartin botanique de Berne (1825), Seringe), et je ne vois pas de caractère pour le séparer du type. - R. Alpina hispidella Seringe, I. c., plante cultivée et qui me parait étrangère à cette section

(1) Pédoncules solitaires ou réunis par deux, hispides-glandulcux, fruit glanduleux ainsi que les divisions calicinales. - Il est probable qu'une elude plus approfondie de ces diffirentes formes fera separer ce que je crois devoir rémir pour le moment au type de Seringe. 
(herb. DC. sans loc.). - R. Alpina turbinata Seringe, l. c., p. 612; R. turbinatu Vill., fl. Dauph., III, p. 550 , ex Seringe; $R$. mixla Tratt., 1. c., I, p. 156 ex Seringe. Plante cultivée à lleur double (herb. DC.).

120. IR. integualang Déséglise, deseript. de qq. esp. nour. du genre rosa, in mém. soc. Acad. de M.-et-Loire, TXVIII (1875), p. 10k, extr.,p. 8; R. alpestris Déségl., ess. monog. obs., p. "3i) (non Rapin); R. editorum Ripart, in litt.!

Exs. Déséglise, herb. ros., n 59 .

HАв. Juillet. Région des montagnes. - France. Vosges: escarpements du Hohneck (Ozanon), ballon de Saint-Maurice, petit Rotabac (Picrat); - Pyrences orient. Montlouis (Ripart); Alpes-Maritimes : Colmiane près de Saint-Mlartin-de-Lantosque (Bornet); - Isère : Charminelle près de Voreppe, Saint-Christophe-en-()isans (Boullu), mont Saint-Eynard près de Grenoble (Verlot); - Hautes-Alpes : vallon de Puy-Vacher, cant. de la Grave (Ozanon); - IIaute-Savoie : les Voirons (Puget); - Savoic : mont Nivolet, au-dessus de Monterminod (Songeon).

121. Mr. adjecta Déséglise, I. c., XXVIII (1875), p. 103̈, extr., p. 9; R. intricala Déségl., ad amic. (non Grenier); Cottet, 1. c., p. 58; R. Alpina var. aculeata Seringe, in DC., prod., II, p. 611 excl. syn.; Reuter, eat. Genève (1861), p. 64.

Ilab. Juin-juillet. Région des moutagnes. - France. Vosges : ballon de Saint-Maurice (Pierrat): - Doubs: mont Suchet!; - Jura (Seringe, 1823, in herb. DC.); - Alpes-Haritimes: Vallon d'Auluebis, près de Saint-Martin-de-Lantosque (Bornet); - Pyrénées orient. : Montlouis (IRipart); - Haute-Loire : mont Mezene (Boullu); - Isère : SaintChristophe-en-Oisans, Villard-de-Lans (Boullu); - Savoie : mont Nivolet (Paris); - Haute-Savoie: Habère-Lullin, Brenthonne, mont de Coux-surCervens, Bourdignin (I'nget), bois de Notre-Dame-de-la-Gorge près de Saint-Gervais (Boullu), Chantemerle au-dessus de Samoëns! - Suisse. Cant. de Fribourg : pied de Lurqui près de la Cape au Moine! - Cant. du Valais : Bovernier, Zermatt (Cottet), broussailles près la cantine de Proz, chemin de Saint-Burnard! - Autriche. Tyrol : Val di Ledro (Kerner). 


\section{(118)}

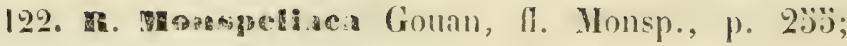
Déséglise, l. с., X, p. 96, cxlr., p. ॐ6; R. Alpinu var., DC., f. fr., V, p. $\$ 50$.

IIa B. Juin-juillet. Région des montagnes. - France. Les Cévennes : bois des Aubrets à l'Esperon (Roubicux, 1809, in herb. DC.); - Inautes-Alpes: La Grave (Ozanon); - Savoie : mont Cormet (Puget). - Suisse. Cant. de Fribourg: Grandvillars (Cottet); - cant. du Tessin: Valle W aggra (Christ). - Autriche. Tyrol : mont Baldo (Kerner).

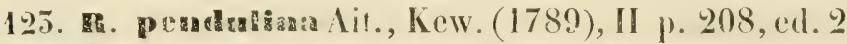
(1811), III, p. 26:3; Leers, 11. herh., p. 118; Pers., I. e., p. 49; Tratt., 1. с., II, p. 20'; Deseglise, 1. с., p. 97 ut extr., p. 57; $R$. Alpina var. penduline Desvaux, I. c., (1815), II, p. 119; Bl. ef Fing. I. c., I, p. 612; Lindley, 1. c., p. 57; R. Alpina var. Iatifolia Seringe, I. c., p. 612 ; R. Andrewsii Trall., 1. c., p. 2080 ; Seringe, 1. c., p. 625 .

Icos. Redour, les roses (1824), livrais. 7, D?; Miss Lawrance, tab. 91 ex Lindley.

Exs. Seringe, ros. desséchées, $n^{\circ} 26$ ?

Has. Juin, juillet. Région des montagnes. - Frence. Ilautes-Vosges (Pierrat); - Pyrénées-orient. (Coder, 1814, in herb. DC.); - Isère : SaintNizier près de Grenoble (Verlot); - Hautes-Alpes : La Grave, forêt des Fraux (Ozanon); - Basses-Alpes : Colmars (Loret, in herb. Grenier); Haute-Savoie: dents de Lanfond près d'.Inuecy, la Caille (P'uget); - Sivvoie : montagne de Saint-Cassin près de Chambéry (Songeon).

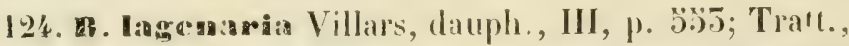
1. c., II, p. 202; Déséglise I. c., X, p. 97 et extr., p. 37; R. Alpina b. DC., fl. fi., IV, p. $446 ; R$. Alpina var. lagenaria Seringe, in DC., prod., II, P. 611; R. pendulina Rehb., fl. excurs., II, p. 615 .

IIsa. Juin, juillet. Région des montagnes. - France. Jura (Gaudin, 1814, in herb. DC.) ; - Lozrre: Nende (Prost, 1815, in herb. DC.) ; - Hautes- 
Alpes : Embrun (Villars, 1810, in herb. DC.); La Grave, forêt des Fraux (Ozanon); - Isère : Col de l'Are, près de Grenoble (Verlot); - HauteSavoie : Habère-Poche (Puget).

12\%. Dr. Pyosadea Gouan, ill. et obs. bot. (1775), p. 51 ; DC., 11. fr., IV, p. 446 ; Pers., I. c., p. 49; Tratt., I. с., II, p. 199; Rehb., I. е., p. 615̄; Host, fl. Austr., II, p. 24; Boreau, fl. cent. , éd. 5, n० 8.57?; Déséglise, 1. c.,

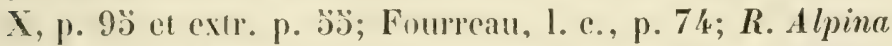
var. hispida Desv., 1. c., p. 119?; R. Alpina o DC., fl. fr., V, p. 556 ; R. Alpina var. Pyrenaica Seringe, in DC., prod., II, p. 611; Bl. et Fingr., 1. c., p. 641; Lois., 1. c., p. 560 ; Duby, 1. c , p. 177; Mutel, 1. c , p. 5/7; Gonnet, 1. c., P. 477; Godr.? fl. de Lorr., I, p. 218; R. hispida Krocker, fl. Siles. (1790), II, pars 1, p. 152 ex Tratt.; Bl. et Fing., l. c., p. 62 \%.

Icon. Gouan, I. e., tab. 19.

Exs. Huet du Pavillon, plant. Napolitanae, $n^{\circ} 511$; Bourgeau, Pyrén. Espagnoles, $n^{\circ} 15 \% 6$.

Obs. Depuis la publication de mon Essai monographique sur les rosiers, j’ai pu compléter mes premières notes données en 1861, ayant reẹu de diverses localités cette rare espece. Voici la description que jai établie sur les échantillons que j’ai pu me procurer.

Arbrisseau peu élevé (j’ai en herbier un échantillon complet avec racine qui mesure $\mathbf{5}(0)$ cent. de haut, les autres ont été pris sur des arbrisseaux de 1 mètre à 1 mètre .j0 (ent.), inerme, ou offraml sur les jeunes tiges quelques petits aiguillons grèles, droits, sélacés, blanchatres. rameaux grèles, à écorre rougeatre ou verdâtre; pétioles plus ou moins chargés de pelile's glandes fines, stipitées, inermes: ; $3-7-9$ folinles ordinairement petites, orales-ellip- 
liques, oucales-obuses ou arrondies, fermes, glabres, vertes en dessus, glangues en dessons, un peu nerveuses, la côte blanchatre portant des glandes fines, glandes qui se retrouvent aussi sur quelques nervures secondaires, doublement dentées, à dents grlanduleuses; slipules étroites ou larges, glabres en dessus, les unes plus au moins chargees en dessous de petiles glandes fines, les autres glabres, orcillettes obtuses ou un peu aiguers, dressées ou divergentes; pédoncules solitaires, glanduleux; tube du calice petit, grèle, glanduleux, ovoïde; divisions calicinales verdatres ou rougeatres, entières, lancéolées, spatulées au sommet, glanduleuses en dessus, alteignant presque la corolle; styles courts, velus; fleur rouge; fruit oroüde, rouge, hispide ou glahre, penché ou dressé, couronné par les divisions calieinales persistantes.

Haв. Juin, juillet. Région des montagnes. - France, Doubs : Pontarlier (Frenier); - Rhône: Mlont Pilat (Fourreau, catal.); - Ilautes-l'yrénées : Gèdre (Bordere), vallée d'Eynes (Schmilt, 1817, in hesb. DC.), Cirque de Gavarnie (Ozanon et Ripart), Cagire (Timbal-Lagrave). - Italie. Nont Corno (Huet du Pavillon, in herb. DC.). - Espagne. Pyrén. espagn. : Castanèze (Timbal-Lagrave), col de l'Ilópital de Vieila (Buurgeau). Turquie d'Europe. Bosnie (Sendtner).

Trattinnick indique aussi les Alpes de lit suisse; mais nous n'arons pas vu d'échantillons de ce rosicr venant de ce pays.

126. R. glazdulosa Bellardi, act. Taur. (1790), p. 230 ; DC., fl. fr., V, p. 5.59; Tratt.? I. c., p. 111; Rchl., I. c., p. 617 ; Crépin, I. c., fasc. 2 (1872), |. 29; Herb. Willd., $n^{\circ} 9867$.

Haв, - Ilalie.

\section{SEct. XI. - Montanae.}

Crépin, primit. monog. ros. (1869), fase. I, p. 15; Cottet, énum. des ros. du Valais, in hull. Soc. Murih. (1874), fasc. III, p. 58. 
1. Folioles simplement dentées . . . . .

1 Folioles doublement dentẻes. . . . . . 11.

Pédoncules lisses, sans glandes. . . . . 5

Aiguillons uniformes, ne dégénérant pas en soies

5. sétaceses.

diguillons mélangés au sommet des ramesux de soirs sétacées

s.

llibernice.

4. $\{$ Tube du calice globulcux . . . . . 5

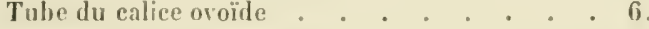

Aiguillons peu nombreux, folioles ovales-elliptiques, souvent rougeâtres, styles velus, fleur

亏. rose' fruit ghlohuleux . . . . . . .

Aiguillons nombreux, folioles orbiculaires, vertes, styles hérissés, fleur rose pâle, fruit gros, ovoïle

ferruginea.

Sclultzii.

(Styles hérissés, pétioles glabres, incrmes, fleur blanche ou rose clair, fruit gros, ellipsoïde .

Styles velus . . . . . . . . 7.

falcala.

7.

Folioles ovales ou ovales-obtuses, glauques, à nervures un peu rougeâtres, fleur d'un rose vif, fruit gros, rouge-orangé, subghlobuleux ou ovoïde

glaura.

Folioles ovales, frut ovoïde, rouge-sanguin, couronné par les divisions calicinales persistantes .

Crepiniena.

(Divisions calicinales entières, folioles glauques,

8. souvent rougôatres, fruit petit, globuleux ferruginea b. hispidula.

Divisions calicinales extéricures pinnatifides . . 9

9 Pétioles incrmes, folioles ovales, fleur d'un rose vif, fruil assez gros, globuleux . . . .

Folioles ovales-elliptiques, espacées sur le pétiole.

Tube du calice ovoïde-allongé, glabre, fleur'd'un rose vif pourpré, fruit très-gros, ovoïle-allongé, cou-

10. ronné par les divisions calicinales persistantes.

Tube du calice ovoüde, hispide à la base, fleur d'un rose foncé, fruit ovoïde, presque arrondi.

intricata.

10.

Sulacvensts.

Caballicensis.

11. Sédoncules glabres . . . . . . 12

l'édoncules glanduleux . . . . . . 14. 
12. $\left\{\begin{array}{r}\text { Pétioles pubérulents-glanduleux, folioles ovales- } \\ \text { aiguës, fruit globuleux, rouge, couronné par les }\end{array}\right.$

divisions calicinales persistantes. . . .

Pétioles glabres, glandulcux. . . . . . . 15

Folioles ovales, glauques, nerveuses, tube du calice ovoüde, fruit gros, presque arrondi, rouge, couronné par les divisions ealicinales persistantes. . . . . . .

Folioles ovales-aiguës, à côte portant quelques glandes, fruit ovoüde, divisions calieinales persistant jusqu’à la coloration du fruit

subcristuta.

N'MOSI

15.

Folioles portant quelques glandes éparses en

14. dessous, disparaissent en partic avec l'âge .

Folioles églanduleuses en dessous . . . . . 13

complicata.

alpestris.

13.

Folioles arrondies, pédoncules hérissés de longues

13. Soies glanduleuses, fleur d'un blane rosé, fruit gros, ovoïde, hérissé . . . . . . . .

Folioles ovales . . . . . . . . . . . . 16.

Rameaux floraux prestues inermes, folioles ovaleselliptiques, fruit très-gros, ovoüde-allongi, fleur

d'un pourpre vif, sépales glanduleux . . . Perrieri.

16. Rameaux floraux aiguillomnis, tube du calice ovoïde ou obovoïde, sépales glabres, bordés de quelques glandes, fruit globuleux

fugax.

montane.

127. ER. Wamanai Christ, die rosen der Schweiz (1875), p. 174 .

IIır. - Suisse. Cant. du Tessin : Mosno (Christ).

128. R. Fenongen Villars, prosp. (1779), p. 46; R. glauca Pourr., chlor. Narh. ace. Acarl. Toul. (1788), III, p. 326; Desfont.; cat. (1804), p. $17 \ddot{3}$ (non Villars); R. rubrifolia Villars, fl. Dauph. (1789), III, p. $\ddot{3} 49$; Bellardi, act. Tur. (1790), p. 22 ; Jacquin, fragm.. p. 70; DC..f1. fr., IV, p. 44:̈; Gmelin, fl. Bad.-Als., IV, p. 561 ; Pers., syn., II, p. 47; Lindley, monog. ros., 
p. 104; Tratt, monng. ros., II, p. 92; Bl. et Fing.; comp., I, p 626 ; Ballis, 11. Iyon., I, p. 2:99; Du Mortier, 13. Belgica, p. 94; Lois., 11. gall., I, p. 558 (excl. var. b.); Desportes, ros. gall.. p. 90; Rehb., fl. cxcurs., II, p. 621 ; Mutel, fl. fr., I, p. 552; Koch, syn., 249; Godr., fl. Lorr., I, p. 219; Gonnet, 11. élém. de France (1848), p. 480, excl. syn. Bell.; Gr. et Godr., fl. de Fr., I, p. 537 ; Borcau, 11. cent., éd. 2, (1849), no 672, éd. 5 (1537), no 8.8 ; Kirschleger, f1. 1s., 1, p. 246; Godet, f1. Jura, p. 208; Reuter, cat. Geneve (1861), p. 66; Déseghlise, I. e., X, p. 99, exir., p. "39; Grenier, 11. jur., p. 257; Cariot, étud. des fleurs (186.3), II, p. 175; J. Pardo, seric imperf. (1867), p. 150; Verlot, cat. pl. Dauph., p. 114; R. rubrifolia a lacvis Seringe, mus. Ifels. I, p. !) et in DC., prod., II, P. 609) Duby, hot., I, p. $177 ; R$. rubicunda IIaller fils, in Römer, arch. (1799), II, p. 6; R. glaue Desfont., cat. Paris. (1804), p. 173̈, non Villars; Dum.-Cours., bot. cult., éd. 2 (1811), V, p. 47̈̈; R. lurida Andr., ros., fase. 22 (ex Lindley); Tratt., 1. c., p. 91 ; R. cirnemomea oblonga Desv., journ. bot. (1815), II, p. 120.

Icon. Bellardi, I. c., tab. 9 et app. 11. Pedem., Lab. 4; Roessig, die rosen, tab. "3't Redouté, les roses (1824), livrais. I, D.

Exs. Bourgeau, Alp. de la Savoie (1848), n" 81, distribue sans nom spécifique; Botrgeau, pl. Pyrénées rspagnoles (1847), 1" 157; Seringe, ros desséch., n 8; Billot, exs., n 1185 .

Har. Juin, juillet. Région des montagnes. - Frence. Vosges: Le Ilohneck! - Doubs : mont Suchet, mont d'Or; - Cévennes : Espervu (Bouchet, 1807, in herb DC.); Lozère: Mende (Prost, 1815, in herb. DC.); - Puyde-Dime: les monts Dimes: - Cantal: Col Laurent entre Murat ef 
Aurillac (Bridel); - Pyrénées : Gavarnie (DC., 1807); - Ilautes-Alpes: La Grave (Ozanon); - Haute-Savoie: Malatrais au Brezon (Bourgeau), le Salève. - Suisse. Valais : Val Ferret, Fins-IIauls (Coltet), - Autriche. Tyrol: Val di Ledı (Kerner).

Yar. hispldula Seringe, nuus. helv., p. 8 et p. 12, et in DC., prod, II, p. 609; R. glunecscens Wulf., in Roemer, areh. (1515), II, p. 576 (non Desvaux); Tratt., 1. c., p. 95; R. cinnumomer var. gleuca Desv., journ. bot. (1815), II, p. 120; R. lividre Host, 1. Austr. (185!), II, p. 2̈; Willd., herb., n $9821 ; R$. Gutensteinensis catal. hort. univ. Vindob.; R. coerulescens Kerner ! in litt. Foliolis ovatis, floribus rubris, fructibus corymbosis lurvibus, pedunculis hispidulis (Seringe).

lcox. Seringe, mus. helv., tab. 1 ; Jacquin, fragm., tab. 106 ; Lindley, bot. reg., vol. 5, tab. $4 \overline{0} 0$.

Exs. Déséglise, herh. ros., no 11.

Ila B. Juin, juillet. Région des montagnes. - France. Puy-de-Dỏme: Le J'uy-de-Dòme (Lamotte); - גin : la Faucille (Michalet, in herb. Grenier); - Doubs : Morteau, ruisseau des Saules (Grenier); - Hautes. Alpes: au Grand-Pra-Bessé, aux Lauzières canton de la Grave (Ozanon); - Isire Saint-Eynard sur le chemin du Sappey et coteaux de Sassenage en allant à Saint-Nizicr (Verlot); - Haute-Savoie: Reyvroz, Annecy (Puget), Le Salève! - Autriche. Tyrol: Duliboden vallée de Gschnitz, Schwargan (Kerner). - Espagne. P'yrénées d'Aragon: vallée de Lessera ('Timbal-Lagrave).

Ons. "Lapeyrouse a dans son herbier, sous le nom de $R$ collina Murr., a deux plantes: l'une d'elles à sépales entiers, cadues, est le R. mbrifolia a Vill., et l'autre le R. sphaerica Gren."Clos, révis. herb. Lapeyr., p. 261.

129. R. moatana Chaix, in Villars, fl. Dauph., I, p. 546; III, p. 347; Gmelin?, II. Bad.-IIs., IV, 1. 56:3; Pers., I. c., p. 30; Tratı.?, I. c., p. ว0); Murith, hot. val., p. 91; Mutel?, I. c., p. 546; Gonnet, I. c., p. 477; Gr. et Godr., I c., p. 3\%8; Godet, I. c., p. 208; Reuter, 1. c., p. 63; Déséglise, l. c., p. 92, extr., p. 32; Grenier, f1. Jura, p. 250; Verlot, 1. c., p. 11 :); Fauconnel, herb. au Salève, p. 16/; R. Reynieri Haller fil., in Römer, 
arch., I, fasce. 2, p. 7; R. mbrifolia montana Gandin, 11. helv., III, p. 248 ; R. rubrifolia glandulosa Seringe, mus. Helv., I, p. 12 et in DC., prod., II, p. 610 (exel. syn. Bell.); R. glandulosa Déséglise, l. e., p. 91, extr., p. כै। (non Bell.); R. Salaevensis Verlot, I. c., p. I1つ้ (non Rapin); R. longepedumulata de la Soie in Christ, rosen der Schw. (1875), p. I80; Collel, 1. c., p. 59; R. Salaevensis forma longeperdunculata Christ, I. c., ex exempl. auth.!; R. sanguisorbifolia de la Soie!, I. e., p. 181 ex exempl. auth.!; Cottet, I. c. (non Don); R. Salaevensis forma sanguisorbella Christ, I. c.

Icon. Seringe, mus. IIelv., pl. 2, f. 5 et 4, mala.

Exs. Schleicher, "1" З̌̈; Billor, " 118k; Bourgean, Alp. de la Saroie, $n^{\circ} 77$.

IIsr. Juin, juillet. Région des montagnes. - France IJautes-Alpes. Charrance, Rahou (Blanc), bois Nondet (Waillard), La Grave aux Lauzières (Ozinon), Malacharre-sous-Aurouse pris de Gap (Gariod); - Basses-Alpes : Barcelonnette, au-dessus du col de Fours (Ozanon); - Isère : les Litages près de Saint-Christophe-en-Oisans (Boullu); - Alpes-Maritimes : Amen près de Pugget et vallon de Libaré (Bornet); - IIaute-Savoie : mont Salève à la grande gorge! - Savoic : Longefoy (Puget).-Suisse. Valais : Vercoring (Christ), Cretlaz-Gabioud (Puget), mont Clou à Bouvernier! graviers de la Drance près de Sembrancher! Finshauts! - Ilalie. Saint-Rémi, vallée d'Aoste!

Ons. I. Reichenbach indique ce rosier dans le Frioul ; il manquerait aux Vosges (M. Godron ne cite pas le $R$. montent dans sa flore), au Jura, à l'Auvergne, aux Pyrénées; Lapeyrouse l'indique dans celle dernière localité, mais M. Clos, dans la révision de l'herbier de Lapeyrouse, ne parle pas de ec rosier; il manque aussi à l'Espagne.

Ons. II. C'est la phrase rapportée par Hutel, dans sa flore française, qui mavail fait admettre pour la mêne espece diux noms diflérents: une étude plus approfondic du R. monkena m'a fait reconnaitie mon erreur. y. Verlot, dans son catalogue des plantes du Dauphiné, dit : "Quant à la 
" plante décrite par Mutel sous le nom de R. montune, elle est très-rliflé"rente de celle de Chaix; si l'on en juge d'après l'échantilhn de son "herbier, c'est un Rosier pompon (R. Remensis DC., 11. fr., no 5708) " d'origine cultirée (car les lleurs sont doubles), qu'il a décrit, en ajoutant "la phrase latine copiée sur l’étiquette du véritable $R$. montane donné " par Chaix à Villars et conservé dans son herbier. Cette maleneontreuse " description, formée d'éléments divers, doit être considérée 'comme " n'étant d'aucune valeur. Verlot, 1. c., p. 113."

Ons. III. Herbier de Willdenow, no 9842. R. montana; no \$8\$̄. R. Reynieri. " Dans ès numéros, les deux échantillons appartiennent a identiquement à la même forme! ( $\boldsymbol{R}$. montana) et eependant los deux " diagnoses de Willdenow sont différentes. "(Crépin, l. e., fisce. 2, p. 75).

Ors. IV. J'ai recu de MI. l'ablué I'oullu un rosier qui me semble différent du R. montanu Vill., mais n'ayant vu qu'un échantillon incomplet, je ne puis rien aflimer. $\boldsymbol{R}$. Rovaudi Boullu in litt.! Arlorisseau de 2 it 5 mètres, à rameaux grêles et inclinés, aiguillons faibles dilatés droits; pétioles inermes ou presque inermes, glahres; $5-7$ folioles ovales-aiguës, vertes en dessus, glaucescentes en dessous, doublement dentérs; pédoncule el tube du calice chargés de petites soies fines, blanchâtres, terminées par une grlande; sépales couverts de glandes en des ous; fruit non parvenu à la maturité, ovoïde, les sépales snnt redressés et paraissent persistants; styles peu velus.

H.в. - Isère : Corencon près de Villard-de-Lans (Boullu).

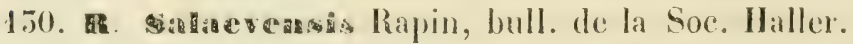
(1836), p. $178 \mathrm{rtg}$ guide du cant. de land (1862), 1). 191 ; Reuter, 1. е., p. 64; Foureau, 1. (․, ). 74.

Exs. Billot (suites), nº 5385.

Har. Juin, juillet. Région des montagnes, - France. Ilaute-Savoie : Mont Salève! - Savoic : Mont Nivolet (Paris, Songeon).

Ons. La plante de La Grase aux Lauzières (Ozanon), appartient au R. montana Vill. et non au R. Salaevensas. Quant aux indications de Le Devez, ltabou, Briançon, localités citées par M. Verlot, cat., p. 115 et 594, je n'ai pas vu d'échantillons et je doute jusqu'à plésent que le $\boldsymbol{R}$. Sulaevensis existe dans le Dauphiné, 
151. Persener Songeon, in Verlot, 1. ‘., p. 113 , sine deseript.

Deseription établie sur les notes et échantillons communiqués par M. Songeon.

Arhrisseau élevé de 2 mètres à 2 mèt. 30 cent., quelquefois fortement aiguillonné a la base ou inerme, aiguillons presque droits, subulís, hrusquement élargis à la base (Songeon, lettre du 9 décembre 1862); rameaux à écorec d'un pourpre vineux, les uns inermes, d'autres munis d'aiguillons droits, dilatés ì la hase en forme die disque; pétioles glabres, parsemés de glandes, aiguillonnés en dessous on inermes; folioles : $3-7$, glables, souvent lavées d'un rouge vincux en dessus, glauques en dessous, à nervure médiane portant à la base quelques glandes, ordinairement doublement dentées on simplement dentées et quelques dents surchargées de dents aceessoires; stipules larges, glabres, bordées de glandes, à oreillettes aiguës, divergentes; pédoncules solitaires ou réunis par 2-5, plus ou moins chargés de soies forles terminees par une glande, bractées ovales, euspidées chlabres, plus courtes que les pédoneules; tube du caliec violacé, ovöde, hispide-glanduleux à la base; divisions calicinales ovales, spatulées au sommet, glanduleuses en dessous, 2 entières à bord tomenteux, 5 pinnatifides ì appendices étroits, saillantes sur le bouton, n'anteignant pas la corolle; styles velus, disque peu saillant; fleur d'un pourpre clair; fruit rouge sunguin, murissant de bonne heure dans la région subalpine, gros, soucent pewhe par son propre poids, ellipsoide, eouronné par les divisions calicinales persistantes.

Exs. Billot (suites), $11^{\circ} 5584$. 
Itab. Juin, juillet. Région des montagnes. - France. Savoie: forêt d'Apremont près de Chambéry, mont Nivolet (Songeon), mont Margeriaz (Paris). - Italie. Saint-Rémi, vallée d'Aoste !

152. He. Caballieensis Puget in Déséglise, Billotia

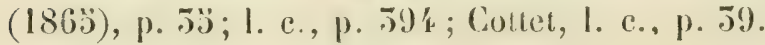

Exs. Déséglise, herb. ros., $\mathrm{n}^{\mathrm{s}} 16 \mathrm{et} 16 \mathrm{bis}$; Billot (suites), no 5.382.

IIar. Juin, juillet. Région des montagnes, - France. Ilaute-Savoic : Habère-Lullin, Habère-Poche, Reyvroz, les Voirons (Puget); - Savoie : Saint-Marcel près de Moutier's, mont Cenis (Puget); - Ilautes-Alpos: Chaudun près de Gap (Verlot, eatal.). - Suisse. Valais: Grüben près de Zermatt (Lagger).

155. R. Gonenkensis Besser; Desportes, 1. c., p. 10. Voici la deseription que jai élablie sur les deux échantillons conservés dans l'herbier DC.

Arbrisscau.......; ramuscules incrmes, à écorce verdàtre sur le spécimen en fleur, purpurine sur celui en fruit; pétioles inermes, tomenteux, portant quelques rares glandes fines; folioles $\ddot{-}-7$, grandes, elliptiques, grabres (d'un vert glaucescent sur le sec) en dessus, velues en dessous, i nervures très-saillantes, irrégulièrement dentées, ¿ dents grandes et quelques-unes accompagnées d'une dent accessoire, ciliées aux bords dans le ramuscule florifère et dépourvues de cils dans celui en fruit; stipules larges, glabres, oreillettes aiguës, dressées, un peu denticulées au sommet; pédoncules glabres ou faiblement hispides; bractées larges, glalıres, igalant les pédoneules; tube du calice glabre, globuleux; divisions calicinales longues, spatulés au sommel, hispides, glanduleuses en dessous, entières, ou des extérieures portant 1.2 pelits appendices courts, lilifurmes; sijles libres, velus; disque plan; fleur......; fruit a la maturiti rouge, globuleux, les 
divisions calicinales existent sur un fruit, l'autre trop avancé est écrasé.

HaB. - Circa Gorinki (Besser, 1824, in herb. DC.).

154. 足. oplistaes Boissier, fl. Orient, vol. II, p. 674.

II $\mathrm{AB}$. - Caucase oriental; Daghestan et le mont Borbalo (Ruprecht, in herb. Boissier).

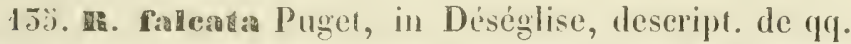
esp. nouv. du gen. rosa, mém. soc. Acad. de M.-et-Loire, XXVII (1875), p. 106, exll., p. 10; Fourreau, 1. с., p. 74; R. imponens lipart!; Cotter, l. c., p. 59, sine descript.

HAB. Juin. Région des montagnes. - France. Rhône : Chaponost (Boullu); - Itaute-Savoie : Habère-Lullin, haies près les chalets de la Clappaz (Puget); - Hautes-Alpes : Saint-Laurent-du-Cros près de Gap (Burle). - Suisse. Valais : Bovernier (de la Soie); - Cant. de Fribourg : Monthovon! Paribaz!

Ons. M. Ripart, dans sa lettre du 28 février 1875, me dit relativement à 'son R. imponens : "C'est un R. Reuleri à fruit ovale, allongé-elliptique, très " gros et à sépales persistant jusqu'à la maturité du fruit; vous m'avez " donné un exemplaire de H. l'abbé Puget, $\mathrm{n}^{\circ}$ 1079, que vous pouvez regar" der comme un type de cette espèce. " Ce numéro de mon herbier dont parle N. Riipart est justement le type du R. falcata Puget! que j'ai reeu de M. Puget et que j'ai distribué autrefois sous le nom de R. Reuteri forma?, d'où je conelus que $\boldsymbol{R}$. imponens Rip. est la même chose que le $\boldsymbol{R}$. falcala Puget. Le R. imponens Rip., ayant toujours été eité sans une description, e'est le nom de $\mathbf{M}$. Puget qui a la priorité. Dans ma description de $\mathbf{1 8 7 5}$, il faut exclure Déségl., herb. ros.. nº 60.

156. IR. Ilseana Crépin, primit. monog. ros. (1866), fasc. 1, p. 115 .

IIAn, - IIongrie. Ilradek dans la vallée du Waag.

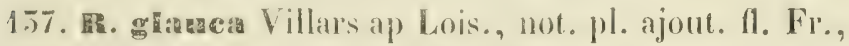
in Desv, journ. hot. (180!)), I1, p. .5.7 el ip. Lois., 1. c. 
extr. (1810), p. 80 (non Desf. cujus planta ad R. mubrifoliam spectat); Poiret, dict. encycl., sup. IV (1816), p. 716; Tratt., 1. c., II (1825), p. 225; R. Vosagiaca (Vosegiaca) Desportes, ros. gall.,(1828), p. 88; Déséglise, in the journ. of botany for March, 1874, extr. p. 5; R. canina var. glauca Desvaux, 1. c. (1815), II, p. 116; R. rubrifolia var. pimnatifida Seringe, mus. helv. (1818), fasc. 1, p. 11 et in DC., prod., II (1823), p. 610; Duby, 1. c., p. 177; R. rubrifolia var. Reuleri Godet!, f1. Jura (185̌5), p. 218; R. Reuleri Godet! in Reuter, cat. Genève (1861), p. 68; Déséglise, essai monog. in mém. soc. Acad. de M.-et-Loire (1861), X, p. 99, extr., p. 59; Grenier, fl. jur. (1864), p. 258 part.; Cariot, étud. des fleurs (1863̈), II, p. 173̈; Fourreau, cat. pl. du cours du Rhòne (1869), p. 74; Verlot, l. c. (1872), p. 115; R. monticola a Reuteri Rapin, guide cant. de Vaud (1862), p. 194; R. alpiphile ArvetTouvel!, ess. sur les pl. du Dauphiné (1871), p. 27 ex exempl. auth.!; R. montana germinibus glabris Schleicher, cat. $(1815)$, p. 46 .

Icon. Seringe, I. c., pl. 2, f. 2.

Exs. Déséglise, herb. ros , $n^{\circ} 60$; Billot (suites), $\mathrm{n}^{\circ} 5581$, et $5581^{\text {bis }}$; ce $\mathrm{n}^{\circ}$ bis est un très-mauvais échantillon distribué sous le nom de var. foliis biserralis; ce que nous avons reẹu a les feuilles simplement dentées.

II aв. Juin, juillet. Région des montagnes. - Angleterre. Lancashire : Sephton (Webb). - Belgique. Indiqué daus les montagnes boisées du Limbourg par Lejeune (revue de la flore de Spa); nous n'avons pas vu la plante belge. - France. Vosges : Champ du feu (Mougeot, 181/, in herb. DC. ! sub. nom. $R$. glaucae Vilar's), ballon de Saint-Naurice, Vagney (Pierrat), Gérardmer! - Puy-de-Dòme : Mont d'or, Saint-Nectaire (Lamy), 


\section{$(151)$}

Fontanat près de Clermont! - Jura : Salins, mont Poupet! - Doubs : Pontarlier à la Cluse ! - Isère : Chamechaude (Verlot), la Salette (Ozanon); - Lozère: Mende (Prost, 1815, in herb. DC.); - Pyrénées-0rientales (Coder, 181k, in herb. DC ) ; - Rhône : Francheville (Boullu) ; - BassesAlpes : Saint-Paul-de-Vars (Arvet-Touvet); - Irates-Alpes: La Grave (Ozanon), mont Bayard (Gariod); - Savoie : montagne des Chaires (Perrier), mont Joigny, mont Nivolet (Songeon); - Ilaute-Savoie : Reyvroz, Ilabìre-Lullin (l'uget), mont Salive (Rappin). - Swisse. Cant. de Fribourg: la Tine près de Monthovon! - Valais : Bovernier ! Salvan ! la Forclaz ! cant. de Berne: Adelboden au fond du Simenthat (Seringe, 1813, in herb. DC.) ; - indiqué par M. Christ, die rosen der Schweiz, dans les eantons de Vaud, Neuchâtel, Schaffhouse. - Autriche. Tyrol: Saint-Martin près de Hall, Mieders vallée de Stubai, Schwarzan (Keruer). - Prusse. IIarz (Wallroth, 1854, in herb. DC.), Schlosswald (Garke, in herb. de Königsberg), Kupyker Wald (Sanio, herb. de Königsberg). - Espagne. Pyrénées d'Aragon : vallée de Lessera (Timbal-Lagrave).

Obs. Je crois dervir donner ici la copie des observations que j’ai publiées en mars 1874 dans le Journal botanique de Londres sur le R. Vogesiaca Desportes.

1809. - Il est parlé pour la première fois du R. glauca Villars, par Loiseleur-Deslonchamps, en 1S09, dans sa notice sur les plantes i ajouler à la flore de France, éditée par Desvaux dans son journal botanique et dont le tirage à part a paru en 1810. " $R$. glaucu Villars, inédit. "R. germinibus ovatis pedunculisque glabris, calycinis laciniis pinna" tifidis, foliolis ovatis glaucis, aculeis sparsis. Ce rosier croît dans les " montagnes des Vosges; il m’a été communiqué par M. Mougeot. "

1815. - Desvaux, 1. c., série 2, II, p. 116, disposition méthodique des espèces de rosiers naturels au sol de la France, croit devoir réunir cette plante au R. canina et en fait sa variété " glauca ", R. glauca Vill. (non Desfont.); le R. glauca Desfont. est de 1808 et correspond au R. rubrifolia Villars.

1815. - De Candolle, flore francuise, V, p. 538, dit : a Sous le nom de " R. canina, je comprends tous les rusiers à fruit ovoïde, glabre ainsi que " le pédoncule; à folioles glabres, simplement dentées en scie ; à tiges et a pétioles munis d'aiguillons crochus, à styles libres, à fleurs variant du " rose vif au rose le plus pâle; quoique ce earactère exclue plusieurs des " variétés réunies à celte espèce par divers auteurs, il en reste encore un " nombre très-considérable, et parmi lesquelles il se trouvera probable- 
" ment quelques espèces dignes d'être admises : le $R$. glauca Vill., in Lois.,

" not., p. 80, remarquable par son feuillage glauque et le rose vif de ses " fleurs, parait être de ce nombre."

1820. - Lindley, monograph of roses, admet en synonyme avec un point de doute le $R$. glanca Vill, à son $\boldsymbol{R}$. canina.

1825. - Trattinnick, monographia rosacearum, place le R. glauca Vill., dans sa série des species minus cognitae.

1825. - Seringe, in DC., prod., admet au $R$. mubrifolia, une variété pinnatifida qui est le $R$. glauca Vill.!

Seringe, en décrivant le genre rosa pour le prodromus, ne fait aucune mention du $R$. glauca Vill., pas même comme synonyme! Il y a lieu de s'étonner d'un tel oubli de sa part, puisque la plante a été décrite en 1809, et que l'herbier DC. possédait le type depuis 1814 ! Le volume du prodromus date de 182:. Libre a Seringe de ne pas admettre le R. glauca Vill. comme espèce distincte, mais il était juste de le mentionner en synonyme, surtout dans un livre qui passe pour enregistrer tous les faits connus !

1828. - Duby, bot. gall., copie Seringe.

1828. - Desportes, rosetum gallicum, voyant que le $R$. glauca Villars, n'était pas celui décrit en 1808 par Desfontaines, lui donne le nom de R. Vosagiaca Desp. (1), R. glauca Vill., in Lois., not., p. 80.

1854. - Mutel, flore francaise, ne fait aucune mention du $R$. glauca Vill., dont il semble ignorer l'existence.

1845. - M. Godron, fore de Lorraine, dans la seconde édition, ne parle nullement de ce rosier; cependant I'herbier de Mougeot était à sa pertée. Il y a lieu de s'étonner d'un tel oubli, l'espece de Villars ayant pris naissance dans les Vosges !

1847. - Gonnet, flore élémentaire de la France, ne fait aucune mention de ce rosier.

1848. - MII. Grenier et Godron, fore de France, ne connaissaient sans doute pas ce rosier?

1852. - Kirschleger, flore d'Alsace, ne dit rien du R. glauca Vill.; il semble ignorer ce qui a été publié 40 ans avant lui !

1855. - Apparait, dans la flore du Jura de M. Godet, un R. mubifolia ß Reuteri, qui n'est pas autre chose que la variété pimatifida Seringe.

(I) Il y a certainement une faute de typographie, car e'est Vogesiaca qu'il faut écrire. 


\section{$(15 \overline{5})$}

1861. - Reuter, calologue de Genève; la variété établie par M. Godet, dans sa flore du Jura, p. 218, se change en espèce distincte dans le catalogue de Reuter et prend le nom de R. Reuteri Godet.

1861. - Nous même, dans notre essui sur les Rosiers, nous avons admis le $R$. Reuteri; mais nous gardions un doute sur cette espèce de nouvelle création, qui nous semblait devoir êrre décrite depuis longtemps, à cause de son abondance dans la région montagnarde où elle végète; il nous paraissait assez étrange yue ectte forme marquante n'eùt pas attiré l'attention des anciens monographes. En $185 \bar{y}$, herborisant dans Ies Vosges avee feu Billot, nous récoltimes ce rosier qui fut placé dans notre herbier sous le nom de $\boldsymbol{R}$. Vogesiaca Desp., détermination que notre savant maitre, MI. Boreau, avait bien voulu nous communiquer en 1834 .

1864. - 11. Grenier, qui dans sa flore de France n'admet que 25 espèces de rosiers, donne dans sa llore jurassique, pour le Jura seulement, l'énumération de 46 types dont six sont de sa création. Il admet sans rien corsulter du passé le $R$. Reuteri comme une nouveauté pour la flore francaise, mais, hélas ! qui avait été décrite 52 ans avant lui !

1871. - M. Arvet-Touvet, essai sur les pl. du Dauphiné, décrit comme espèce nouvelle, sous le nom de $\boldsymbol{R}$. alpiphila, le $\boldsymbol{R}$. Vogesiacc Desp., réédité en 1861 sous le nom de $R$. Reuteri.

R. glauca Desf. . . $1808=R$. rubrifolia Vill., 1789 .

"Vill. . $1809=R$. Vosagiaca Desp., 1828.

n Dierb . . $1818=R$. repens Scop., 1772.

" Schott. $1822=$ R.Schottiana Ser., 1825 (sub.R.canina var.).

R. Reuleri God. $\quad 1861=R$. Vosagiaca Desp., 1828.

R. alpiphila Ar.-T. $18 \pi /=$

id.

Le 5 avril 1874, M. Grenier me fit parvenir les notes suivantes sur lc R. glauca Vill.

"Lorsqu'en 1809, Villars donna à cette plante le nom spécifique de "glauca, ce nom avait déjà été oppliqué à d'autres espèces, mais à des a espèers antérieurement dénommées. Ce nom était done par le fait dispo" nible, et Villars a pu l'umployer légritimement pour désigner une espèce "nouvelle. Ainsi Pourret, en 1785, Desfontaines, en 1804, et après eux "Jaume-Sánt-Hlilaire ont imposé ce nom au $R$. ferruginea Villars (1779), " lequel ne diflére pas du R. mbrifolin Villars (1789). - Postérieurement a i 1809, Dierbach, dans son 11 heidelb., II,p.140 (1819), a substitué le nom " de $R$. glaner à celui de $R$. arvensis L.; Schot, d'aprés Besser, enum. Pod. " et Yohh.,p. (1'́ (1\$22), a donné ce nom à une forme du $R$. canina, ete. On 
a voit d'après cela que le nom eréé par Villars a récllement la priorité, et " qu'il doit ètre conservé, à la condition toutefois qu'on reprendra, pour "le $\boldsymbol{R}$. rubrifolia Vill., le nom plus ancien de $\boldsymbol{R}$ ferruginea Vill. (1779)."

"Veuillez remarquer que le $\boldsymbol{R}$. gluuea n'est pas de Desfontaines, et de " plus que le catalogue dans lequel cette dénomination a paru est de 1804 " et non de 1808. Le $R$. glauca, invoqué par Desfontaines pour remplacer le " $\boldsymbol{R}$. rubrifolia Vill., est de Pourret, et ce nom de Pourret est antéricur à * celui de $R$. rubrifolin Vill, jusque là Desfontaines avait raison, mais il " nous faut aller jusqu'au bout. Or voici la synonymic du R. mubrifolia "Villars."

" R. ferrugiana Villars, prosp. (1779), p. 46; R. glauca Pourret, chlor. "Narb., in act. Acad. Toul. (1788), III, p. 526 et herb, ; Desfont., catal. "(1804), p. 175 (non Villars); R. mbrifoliu Villars, f1. Dauph. (1789), III, "p. 549."

“ OBS. En 1779, dans son prospectus, Villars donne à cette plante le nom a de $\boldsymbol{R}$. ferruginen, faisant ainsi allusion à la teinte rembrunie qu'elle " prend souvent. Neuf ans plus tard, Pourret, qui n'avait sans doute pas a connu la publication de Villars, imposait à cette même plante le nom de " $R$. glauca (1788). L'année suivante (1789), Villars à qui la publicalion de " Pourret était probablement restée inconnue, trouvant que le nom de a $\boldsymbol{R}$. rubrifolia rappelait mieux la couleur de ce rosier, erut pouvoir " substituer ce dernier nom à celui de ferruginea qu'il avait proposé " d'ahord. Mais alors Villars n'était plus maitre de son espèce; il ne pouvait " pas plus en changer le nom que Pourret, qui, l'année auparavant, lui " avait à tort donné le non de $\boldsymbol{R}$. glauca, ear si Villars avait du renonecr " à son nom princeps, il aurait été condamné à adopter celui de Pourret " et très certainement telle n'était pas son intention."

" On comprend ainsi comment Desfontaines, en ne tenant pas compte du " premier nom créé par Villars, a été conduit à remplacer le $\boldsymbol{R}$, rubrifolia "Vill. (1789), par le R. glauca Pourr. (1788). Seulement Desfontaines, en " ne signalant pas Pourret comme auteur de ce dernier nom, a pu laisser ( involontairement croire qu'il était l'auteur du R. glauca. „ Grenier, lettre du 5 avril 1874.

158. đa. complicata Grenier, fi. jur. (1864), p. 259; Cottet, énum. des roses du Valais, in bull. de la Soe. Murith. (1874), fasc. 5, p. 59; R. Reuteri var. intermedia Grenier, 1. c.; R. inclinata Kerner, oest. bot. Zcitschrift, 


\section{( 153$)$}

11 11 (1869), extr. p. 2; Crépin, primit. monog. ros. lase. I (1869), p. 111; R. Reuteri var. b. Reuter, cat. de Genève (1861), p. 68; R. pennina de la Soie, in Cottet, l. c.

Exs. Déséglise, herb. ros., $n^{\circ} 64$.

IIıв. Juin, juillet. Région des montagnes. - Suède. Carlshaum (Scheutz). - Angleter. Yorkshire : près de Ried Swaledale; - Northumberland: haies près de Seaton Delaval (Baher). - France. Puy-de-Dòme: mont Dore (Lamy); - Doubs : route du fort de Joux à Pontarlier (Grenier); Haute-Loire: Ceyssac près le Puy (Boullu); - Iaute-Savoie : Reyrroz, IIabère-Poche, Habère-Lullin (Puget). -- Suisse. Cant. de Fribourg : la Cernaz près de Montbovon! - Valais : Mont Clou à Bovernier! Autriche. Tyrol: Mieder's, vallce de Stubai; Steinach, in valle fluvii Sill; mont Schlern près de Alzwang; Kranabitten près d'Onipontem; Ziel (Kerner). - Allemagne. Bade : Kaisersthal (Christ).

159. R. infricata Grenier, fl. jur. (1864), p. 259; R. transiens Kerner! oestr. bot. Zeitschreift (1870), no 1; R. Reuteri var. transiens Gren., l. e.; Christ, die rosen der Schw., p. 170; R. Rhoetica Kerner! in litt.

Caractères généraux du $\boldsymbol{R}$. glauca Vill., dont il diffère par ses pétioles peu ou pas glanduleux, ses pédoncules munis de quelques soies glanduleuses ainsi que le fruit; les folioles sont aussi simplement dentées.

H.в. Juin, juillet. Région des montagnes. - Suède. Halland : Getinge (Scheutz). - France. Cantal : Murat (Clisson); - Doubs : Pontarlier (Grenier); - Saône-et-Loire : Châlon-sur-Saône (Ozanon); - Ardèche : la Lourese (Boullu); - Isère : La Ferrière d'Allevard (Boullu); - IauteLoire : Ceyssac près le Puy-en-Velay (Boullu); - Haute-Savoie : HabèrePoche (Puget). - Autriche. Tyrol: Calvarienberg vallée de Gschnitz, Muliloden près de Trins, Saint-Leonhard vallée de IIernberg près de Brenner, Mieders Vallée de Stubai (Kerner).

140. I. Pugax Grenier, f1. jura. (186/4), p. 239; R. Deltusoici Lag. et Pug., in Cottet, l. c., sine descript.; 
R. discreta Ripart, in Crépin, 1. e., p. 16, sine descript.; Cottet, 1. e.; R. Sambrancheriana de la Soie, in Cotter, l. e., sine descript ; K. Reuterivar. allenophora Grenier, l. e.

Exs. Déséglise, hẹb. l'os., nº 13 et 1 s̆bis.

Il difère du R. glauca Vill. part ses pétioles glanduleus, ses folioles doublement dentées, ses pédonumles et fruits munis de quelques soies glanduleuses. - Il diflère du R. complicate par ses pédoncules et te fruit portant quelques soies. - Il differe du $R$. intricala par ses folioles doublement dentées.

IIAr. Juin, juillet. Région des montagnes. - Angleterre. Carnarvonshire près le pont de llenai (W (Wh)._- France. Douls: Pontarlier (Grenier): - Hautes-Pyrénées : Cirque de Gavarnie (Ripart); - Pyrénées-Orient. : Montlouis (Ripart; - Hautes-1/pes : Villard d'Arene (Ozanon); - IauteSavoie : Ilabère-Lullin, Habère-Poche (Puget). - Suisse. Vallais : Crettaz vallée d'Entremont, Bovermer (de la Soie), Mont Clou ! - Cant. de Fribourg: les Cases d'Allières près de Mlontbovon! - Autriche. Tyrol: Brenner, Roveredo; Boomgarten, vallée du Danube (Kerner).

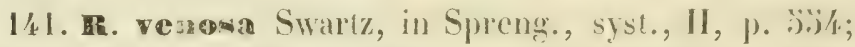
Besser, enum. Volh. et Pod., p. (i/; Seringe, in DC., prod., II, p. 6250 ; R. celsicola Irvet-Touvet, essai sur les pl. Dauph. (1871), p. 27.

"Germinibus ovatis pedunculisque glabris, laciniis caly" cibus elongatis subincisis margine tomentosis, aculeis "ramorum sparsis validis recurvis, petiolis inermibus " foliolisque diplicato-serratis venosis, subtus glaberrimis " glaucescentibus. Succia " (Sprengel).

La plante que nous avons en vue diffère de cette diagnose par les pétioles, dont les uns sont inermes (le plus grand nombre), d'autres sont faiblement aigruillonnés; les divisions calicinales sont aussi plus yue subincisis; le caractère des folioles convient parfaitement. 
Haz. Juin, juillet. Broussailles des montagnes. - France. Vosges : Saulxures, Comimont (Pierrat), Liezey, sur le chemin entre la forêt et le presbytère ubi eam legi $17 \mathrm{sept.} 18555$ ! - Rhône: Tassin? (Boullu); Isèse : forêt de Porte, derrière Chamechaude (Verlot), Villard-de-Lans (Boullu); - Hautes-A!pes : Lautaret (Apvet-Touvet); - Savoie : mont Joigny près de Chambéry (Sonģeon). - Suisse. Jura de Bâle (C'hrist). Prusse. Harz (Wallroth, 1854, in herb. DC.). - Italie. Sicile : Madonie (Todaro, recu sous le faux nom de $R$. montana).

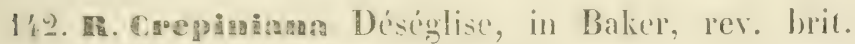
ros. ( $18(; 1)$, p. 28; Du Morr., monog. des ros. de la fl.

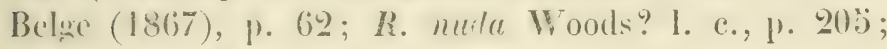
Tratt.? I. c., II, p. 25; $R$. forme etrange Crópin, not. 2, 1). $\because 7$.

$$
\text { Exs. Baker, } n^{\circ} 15 \text { ?, n० 21, no } 22 .
$$

Ilsв. Juin. Haies. - Angleterre, Yorkshire : Kilvington, Thirsk, Sowerby, Maunby, Earsden?(Baker). - Belgique. Province de Namur : Haw-sur-Lesse (Crépin).

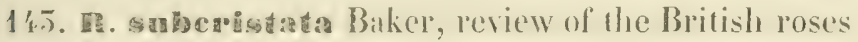
(18(i.), p. 29; R. Comentosa var \%. Yoods, l. c., p. 197 et lıerl)., "1" 1; R. cenina val. subcristala Baker, monog. of Brit. ros. (1870), in Linn. Society's jomrn., MI, p. 254, excl. syn. Gren. et Déségl.; $\boldsymbol{h}$. Caledoniae Borrer ex Baker.

Exs. Baker, herb. ros. brit., I $^{\circ} 25$.

Hab. Juin. Haies. - Angleterre. Yorkshire : Thirsk, Westerdale, (Baker); - Sussex : Dumbarton (Hailstone); - Cheshire : Kirby. Écosse. Argyleshire: Gleneroc (IIailstone); - Perthshire: Earn (Hailstone).

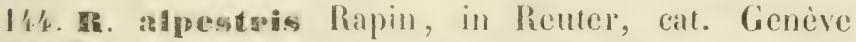
(1861), 1. 68; Grenier, 11. jur., p. 2.j.); R. momlicola h. relpestris Rapin, guide cant. Vaud (1869), p. 19k. 
H.ı. Juillet. Région des montagnes. - France. Ilaute-Savoie : te Salève. - Suisse. Valais : Barberine (Boullu); - eant, de Fribourg : Bonnondon (Rapin).

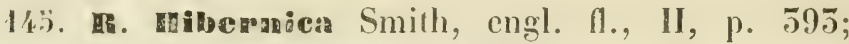
Woods, 1. c., p. 222 et herb. n० 107; Lindley, I c., p. 82; Trat.., I. c., I, p. 128; Seringe, in DC., prod., II, p. 617; de Pronv., 1. c., p. 85; Desportes, l. c., p. 79; Rehl., 1. c., II, P. 614; Iooker, lreit. I., p. 228; Baker, rev. of Brit. ros., p. 9 et monog. of Brit. ros., p. 209.

Icox. Engl. Bot., tab. 2196 et thind el., tab. 465.

IIar. Juin. Haies. - Angleterre. Cheshire: Great Meots (Webb). Irlande (Smitl)).

Var. alabra Baker, herb. ros. brit., $n^{0} 4$.

Yorkshire: près de Newton dans le Cleveland (Baker); - Cheshire : Great Meols (W ebb).

a Discovered in the counties of Derry and Down, particularly near a Belfast harbour, by M. Templcton, who, in consequence of its being " judged a new irish plant, received from a society at Dublin, " exemplo " raro el inaudito," a premium of 30 pounds sterling. This gentleman ". has many more claims to botanical distinction." Smith, engl. flora, vol. 2 , p. $5 ! 5$.

146. R. Selautzii Ripart, in Schultz, arch. de la flore de Fr. et d'Illem. (18:2), p. 234; Boreau, fl. cent., éd. 5, n 845 ; Déséglise, l. c., p. 105 et extr, p. 63 .

Exs. Schultz, herb. norm., nº 45; Déséglise, herb. ros., $n^{\circ} 47$.

Haв. Mai, juin. Haies, - France. Cher: les vallées à la Chapelle-saintUrlin (Tourangin), Bourges, haies des Vignes d'Auron (Ripart), Bourges près de Saint-Lazare! Vignes d'Asnières où j'ai r'écolté ce rosier en magnifique état de fructification le 50 juin 1858. - Suisse. Cant. de Fribourg: la Tine près de Montbovon! 


\section{Sect. XII. - Caminae.}

DC., in Seringe, mus. IIcly. (1818), I, p. 5; Lindley, monog. ros. (1821), p. 97, purt.; Besser, enum. Podol. et Volh. (1822), p. 60, excl. R. venosa Sw. R. terebenthinacere Besser; de Pronv., monos. genre ros. (1824), p. 97, part.; Seringe, in DC., prod. (182.;), II, 1) (311, parl.; Duby, hot. gall. (1828), I, p. 177, part.; R(hb., fl.

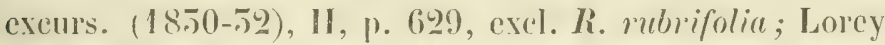
et Duret, 11. Cotc-l'Or (1851), I, p. 507, part.; Koch, syn. (1845), 1. 2̇0, part.; Déséglise, ess. monog., in mèm. de la Sne. Acad. de M.-et-Loire, I (1861), p. "30, extr., p. 12, 't in the Naturalist (1865), n०20, p.511; Grenier, ft. jur. (1864), p. 257, purt.; Router, eat. de Genève (1861), 1). 69, excl. R. stylosa; Cariot, étud. des fleurs (186\%), II, 1. 176; Fourreau, catal. pl. du cours du Rhòne (1869), p. 74; Crépin, primit. monog. ros. (1869), fase. I, 1. 17, excl. subd. G) tomentellae, II) scubralue; Diastylae trib. campylacanthae Godet, 11. Jura (18:5.5), a) pubescenti-tomentosae, c) mulae aut submudae, p. 204.

Clef dicholomiqne des subrlivisions de la section Caninat.
1. $\begin{aligned} & \text { Feuilles glabres sur les deux faces . . . . } 2 . \\ & \text { Fenilles plus ou moins pubesentes sur les deux } \\ & \text { faces ou seulement velues surles nervures } . \quad 4 .\end{aligned}$
Pétioles glabres ou à peu près, pédoncule et tube
2. du calice hispides-glanduleux, feuilles simple- ment ou doublement dentées . . . . . . Hispidac.
Pétioles glabres, pédoncule et tube du calice lisses. 5.
. Folioles doublement dentées. . . . . Biserralae.
4. I Pédoncule et tube du calice glabres. . . . Pubescentae.
- I Folioles simplement dentées . . . . Nudae.
I Pédoncule et tube du calice hispides-glanduleux . Collinae. 
1) Nudac Déséglise, different met. proposed for the class. of the spec. of the genus Rost, in the naturalist (1864), no 20, p. 511, part.; A) luleticnue et B) transitoriae Crépin, l. e.; Cottet, énum. des ros. du Valais (1874), in bull. de la Soc. Murith, fase. III, p. 59 et p. 40 .

Pétioles glabres ou à peu près, folioles simplement dentées, glabres, dépourvues de glandes et de villosité sur les nervures, pédoncule et fruit glabres.

1. $\left\{\begin{array}{l}\text { Styles glabres. } \\ \text { Styles hérissés ou velus } \\ \hline\end{array}\right.$

2. $\begin{gathered}\text { Petit arbrisseau à rameaux très-allongés . . } 5 . \\ \text { Arbrisscau à rameaux flexucux, folioles petites, } \\ \text { mucronées, fleur rose. . . . . . . mucronulata. }\end{gathered}$

( Aiguillons droits, folioles ovales, vertes, fleur

$5 .\{$ rose. . . . . . . . macroacantha.

Aiguillons inclinés ou courbés en faulx . . 4 .

Folioles d'un vert sombre, assez espacées sur

le pétiole, fleur blanche, assez grande . Plexibilis.

4. $\{$ Arbrisseau à rameaux très-florifères, folioles d'un vert jaunâtre, fleur d'un l,anc pur, onglet jaunâtre. . . . . . . . albo-lutescens obs.

3. Styles hérissés, fleur rose ou blanche . . 6

Styles assez longs, velus, fleur blanche, onglet jaunâtre . . . . . . . . syntrichostyla.

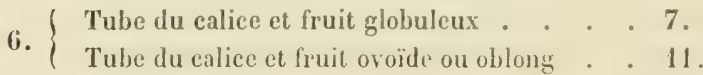

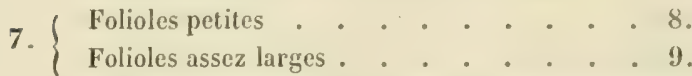

8. $\left\{\begin{array}{l}\text { Folioles ovales-arrondies, fleur petite, blanche Amansii. } \\ \text { Folioles-ovales cuspidées, fleur petite, d'un }\end{array}\right.$ blanc lavé de rose. . . . . . aciphylla.

Arbrisseau bas et touffu, folioles ovales un peu

9. acuminées, styles légèrement hérissés . . globuluris. Arbrisscau droit, non touffu. . . . 10. 


\section{(141)}

Rameaux purpurins, folioles ovales-oblongues 10. I médiane souvent rougeâtre, styles hérissés. montivaga.

Rameaux verts, folioles ovales-aiguës, d'un vert clair², styles hérissés en faisceau court.

11. $\left\{\begin{array}{l}\text { Folioles ovales, fruit ovoïde } \quad \cdot \cdot \cdot \cdot \cdot \\ \text { Folioles orbiculaires, fruit gros, oblong-allongé }\end{array}\right.$ sphaericr.

12.

12.

Folioles petites . . . . . . . . . 15.

Folioles assez larges . . . . . . . . 16

Rameaux courts, terminés par une touffe de folioles qui semblent sortir du mème point,

15. folioles ovales cuspidées, fleur rose carné .

Rameaux non terminés par une touffe de folioles.

Touranginiana.

15.

senticosa.

14.

Rameaux floraux courts, presque inermes,

14. tolioles ovales-arrondies, fleur rose pâle. - rrmosissima.

I Rameaux floraux plus ou moins allongés, aiguillonnés . . . . . . . . . 13.

Tiges florales et stériles chargées de nombreux aiguillons, folioles ovales, fruit petit, ovoïde horriduln obs.

1:. Tiges florales et stériles non ehargées de nombreux aiguillons, folioles rétrécies aux deux extrémités . . . . . . . . oxyphylla obs.

16. $\left\{\begin{array}{lll}\text { Pétioles parsemés de quelques poils } \ldots & 17 . \\ \text { Pétioles glabres . . . . . . . . . } 19 .\end{array}\right.$

17. $\left\{\begin{array}{l}\text { Rameaux purpurins, folioles ovales-aiguës, } \\ \text { fleur grande, d'un rose clair, styles faible- }\end{array}\right.$

18. $\{$ Folioles vertes . . . . fallens.

19 f Folioles très-luisantes . . . . nilens,

Folioles d'un vert mat . . . . . . . ceninu.

147. 卧. canina L., sp., 70\%; R. sepium Lam., fl. fr., (179:3), III, p. 129 (non Thuill.); R. Luletiuna Leman, bull. Philom. (1818), extr., p. 9, no 5 ; R. Surartzience Fries, nor.. .74? Seringe, in DC., prod ?, II, p. $61 \%$; 
R. fallax Puget, in Déségl., herl). ros., no 60, sine descript.; R. funitina Déséglise, ad amir.; Cottet, l. e., p. 59 , sine deseript.

Icon. Roessig, die rosen, tah. 21 et tah. 29; Redouté, les roses (1824), livrais. 28, A.; Flora Danica, IV, tab. 33\%; $X$, tab. 169:3; Swensk, bot., I, tab. 29, VIII, tab. \$34. Engl. bot., tab. 2611 et third edit., pl. 47 4.

Exs. Scringe, ros. dessieh., no 9; Fries, herb. norm., fase. VI, $\mathrm{n}^{\circ} 41$ ?, fase. VIII, $\mathrm{n}^{0} 43$ part.; Billot, $\mathrm{n}^{\circ} 22 \% 9$; Déséglise, herb. ros., $n^{\circ} 12$ et $n^{\circ} 60$; Baker, herb. ros. brit., $\mathrm{n}^{\circ} 12$; Wirtgen, plant. critiq., $\mathrm{n}^{0} 74, \mathrm{n}^{\circ} 466$; Aucher-Eloy, $n^{\circ} 14.51$ bis; Kotselyy, $n^{\circ}$ 2.77, $n^{\circ} 727$ ? Ce dernier $n^{\circ}$ présente un caractère singulier dans l'incurvation du sommet des rameaux florifères, probablement un état anormal?; de IIeldreich, no 2616 ? n $^{\circ} 2680$ ?; Orphanides, $n^{\circ} 2711$ ? tige et rameanx florifères inermes! probablement une espèce distincte du $R$. canina, mais il est difficile de se prononeer sur un échantillon ineomplet.

Hab. Mai, juin. Haies, broussailles, bois. - Espèce vulgaire en Europe. Je possède en herbier des échantillons de la suede de la Belgrique, d'Angleterre, de France, de la Suisse, de l'Autriche, de l'Allemagne, de l'Espagne, de la Turquie d'Europe. J'ai aussi ce rosier d'A frique. - Algérie : Tlemeen (Munby). - Traltinnick dit : in omni Europa et in Asia boreali.

Ors. I. D'après Loiseleur-Deslonchamps, le rosier doit son nom spécifique à la prétendue propriété que les anciens altribuaient à sa racine. Pline en parle comme d'un spécifique contre la rage; cette vertu miraculeuse fut, selon lui, révélée en songe à une mère dont le fils avait été mordu par un chien el l'emploi de ce remède guérit le malade.

OBs. II. Fries, herb. norm., fase. VIII, no $4 b^{\circ} ; R$. canina var. opaca Fries, nov. - Sous ce numéro, représenté par deux échantillons en fleurs, il y $a$ certain'ment une confusion et je doute que ces deux échantillons aient été pris sur le mème buisson? Celui du bas a les feuilles simplement dentées, ovales-cllipliques on ovales, les pétioles glabres, incrmes, les styles héris- 


\section{$(145)$}

sés : c'est tout simplement le $R$. cenina Lin. L'échantillon supérieur a les folioles, les unes ovales-aiguës, les autres arrondies, noublement dentées, les pétioles pubérulents, aiguillonnés, l's styles très-velus : $R$. dumalis Bechst?

Ces mélanges sont regrettables, surtuut quand ils viennent de la part d'un botaniste aussi éminent que II. E. Fries. J'ai vu la même confusion dans trois exemplaires de l'herbier' normal de Fries !

OBS. III. J'ai distribué un rosier sous le nom de $R$. fallax Puget, in Déségl., herb. ros., no 60, que je ne crois pas distinct du $R$. canina.Ilaute-Savoie: Pringy (Puget); - Savoie: mont Nivolet (Songeon); Cher : forêt de Nierzon!

148. ㅁ. gloucescens Desvaux, in Mérat, 11. Paris. (1812), p. 192 (non Wulf.); Boreau, catal. rais. du départ. de Maine-et-Loire (18:39), p. 79; R. conina val. glaucescens Desvaux, journ. Jot. (1815), II, J. 114; Seringe, in DC., prod., II, p. 615.

$\mathrm{H}_{\mathrm{AB}}$. Mai, juin. Bois, haies. - France. Maine-et-Loire : Angers (Bastard, 1815, in herb DC.); - Cher : Harmagne, la Chapelle-saint-Vrsin, bois de Soye, bois de Rouet près de Mehun; - Saône-et-Loire : Autun (Carion); - Isère : forèt de Portes, mont Rachet (Verlot); - Haute-Savoie : Habère-Poche (Puget); - Alsnce. Gros Wald près de Reichoffen! Autriche. Tyrol : Innsbruck (Kerner).

149. 16. вaiters Desvaux, in Nérat, fl. Par. (1812), p. 192; Boreau, 1. e.; $\boldsymbol{R}$. canina var. nitens Destaux, journ. bot. (1815), II, p. 114; Seringe, in DC., prot., p. 615 .

II AB. Mai, juin. Haies, - France. Vosges : Planois (Pierrat); - Cher : Givrai (Pipart), haies des vignes de la Chapelle-saint-Ursin, rignes de Couët près de Mehun, le Corpouay, commune de Berry; - Rhône : mont Genevre près de Lyon (IC), 1809; - Isere : le Sappey (Verlot); - IauteSavoic: l'ringy ( $\mathrm{P}^{\mathrm{B}}$ uget).

150. R. syathouchostgla Ripart., mss.

Arbrisseau peu élevé, ì rameaux flexucux, écorce verte, 


\section{$(144)$}

aiguillons des tiges forts, dilatés à la base, crochus, ceux des rameaux florifères plus petits, minces, allongés, dilatés it la base en forme de disque, courbés, les plus petits droits; pétioles glabres, très-légìrement reius en dessus à la base, parsemés de quelques glandes fines, sillomnés en dessus, aiguillonnés en dessous; $\dddot{3}-7$ folioles toutes pétiolées, la terminale arrondie ou un peu rétrécie à la base, glabres, d'un cert foncé en dessus plus pailes en dessous, ovalesaiguës, ovales-elliptiques, quelques-unes obtuses, simplement dentées, les folioles inférieures portent quelques rares dents aceessoires, on observe quelquefois sur la nervure médiane de la foliole terminale 1-5 petits acieules; stipules plus ou moins larges, glahres, bordées de glandes, oreillettes aiguës, divergentes; pédoneules lisses, solitaires ou en corymbe, portant a leur base des hractíes glabres, lancéolées ou ovales, cuspidées an sommel, égalant ou plus longues que les pédoneules; tube du calice ovoïde, glahre; divisions calieinales appendiculées au sommet, glabres, 2 entières ì bords tomenteux, 5 pinnatilides ì appendiees étroits, saillantes sur le bouton, égalant la corolle, réfléchies après l'anthèse, non persistantes sur le firuit; styles libres, tress-relus, simulant une colonne plus ou moins saitlante, disque conique; fleurblanche, it onglet jannitie ; fruit petil, ovoïde, rouge à la maturité.

Ce rosier n'appartient pas au groupe du $\boldsymbol{R}$. systyla, comme me l'écrit (25 févr. 1872) mon ami M. Ripart; les styles sont libres! et non soudés sur les échantillons reçus de $\mathbf{M}$. Ripart.

Han. Mai, juin. Bois, haies. - Anglelerre. Devonshire : Yeoem vale between Lee Nill Bridge et Yeo (Briggss); - Cormwall (Briggss). - Belgique. Prov. de Namur: Rochefort (Crépin). - France. Vosges : Rambervillers (Boulay); - Cher: vignes de Couët et bois de Rouet près de Mehun! - 
Isère : Saint-Christophe-en-Oisans (Boullu); - Rhoone : Ronzière (Chabert); - Lot-et-Garonne: Agen (Ripart); - Haute-Savoie: Ilabèrè-Poche, la Gille (Puget). - Autriche. Krems, Schwargan (Kerner). - Italie. Sicile : Madonie (Todaro).

\section{1, R. macroacantha Ripart, mss.}

Petit arlorisseau à rameaux très-allongés, grèles, étalés, dans le genre de ceux du R. repens, à écorce vineuse, aiguillons longss, dilatés à la base, droits; pétioles glabre's, portant quelques rares petites glandes, sillonnés en dessus, aiguillonnés en dessous; $\dddot{3}-7$ folioles toutes pétiolées, d'un vert clair, ovales ou ovales-clliptiques, glabres, simplement dentées; stipules allongées, gclabres, à oreillettes aiguës, droites ou divergentes, bordées de quelques petites glandes; pédoncules solitaires ou réunis par 2-5, glabres, longs, ayant deux petites bractées opposées, ovales, acuminées, glabres, bordées de glandes, égalant ou dépassant Ies pédoncules; tube du calice ovö̈le, glabre; divisions calicinales appendiculées au sommet, glabres, 2 entières à bords tomenteux, 5 pinnatifides à appendices étroits, saillantes sur le bouton, plus courtes que la corolle, réfléchies ¿ l'anthèse, non persistantes; styles glabres, disque presque plan; fleur rose, fruit ovoïde, rouge.

Très-remarquable par ses longs aiguillons droits, horizontaux, ni courbés, ni erochus; son port est différent aussi de celui du R. canina; il a besoin de se soutenir sur les autres arbrisscaux qui sont à sa portée; ses styles sont glabres.

Har. Mai, juin. Haies, bois.-France. Cher: Garenne de Turly près de Bourges (Ripaıt), boss de Givray près de Trony!

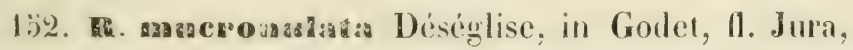
suppl. (1869), p. Tl; h. hololeia Ripart, in Cottel, 1. c. (187/), p. 58, sine descripu. ; R. firmula Godu, 1. c. ? 
Arbrisseau peu élevé, à rameaux flexucux, aiguillons dilatés à la base, droits et d'autres un peu arqués, assez robustes, ceux des rameaux florifères plus petits; pétioles glabres, parsemés de poils principalement it la hase et sillonnés en dessus, aiguillonnés ou inermes en dessous; כ̈-7 folioles toutes pétiolées, petites, ovales-elliptiques, aiguës, mucronées, vertes, un peu luisantes, glabres, simplement dentées, à dents aiguës, les supérieures conniventes; stipules glabres, bordées de glandes, oreillettes aiguës, les unes droites et d'autres divergentes; pédoneules ordinairement solitaires ou réunis deux ensemble, glahres, ayant à leur base deux bractées ovales-cuspidées, glabres, hordées de glandes, plus longues que les pédoncules; tube du calice ovoïde, glabre; divisions ealicinales appendiculées au sommet, glabres, les intéricures entières, à bords tomenteux, les extérieures pinnatifides, à appendices étroits, saillantes sur le houton, plus courles que la corolle, réfléchies à l'anthèse, caduques; styles glubres, s'élevant au-dessus d'un disque un pen conique; fleur rose; fruit ovoïde, rouge.

M. Godet attribue à mon R. mucromulata des feuilles " ordinairement doublement dentées; "'ce qui n'est pas, car mon type est à feuilles simplement dentées. M. Godet a décrit ce rosier, d'après l'avis de M. Grenier, sur un échantillon que j'avais communiqué sous ce nom provisoire à l'auteur de la flore de France.

IIAB, Juin. Bois. - Angleterre, Yorkshire: Thirsk (Baker). - France. Cher: haies de Turly près de Bourges (Ripart), bois de Gérissé près de Mehun! - Haute-Savole: Habère-Lullin à l'Arpettaz (Puget). - Alsace. Forêt de Vordersberg, côté des prairies de Niederhronn! - Suisse. Jura de Bâle (Christ);-Valais: Bovernier (Cottet).

Ues. Le R. oxyphylla Ripart, in litt, ne me semble différer du $R$. mucro- 
muluta que par ses folioles rétrécies aux deux extrémités et ses styles hérissés. - Cher : bois de Marmagne, Achères près de la forêt de Saint-Palais. - Alsuce. Berges de l'étang de Niederbronn!

155. ar. seaficosa Acharius, in act. Holm. (1815), p. 91; R. canina vulgaris b. Mutel, fl. fr., I, p. 552; R. canina b. senticosa Rehb., l. c., p. 620, excl. syn. Rau; Godet, 11. Jura, p. 21 "̈; Reuter, cat. de Genève (1861), P. 70; R. orthacantha Kerner! in Clurist, I. c., p. 168.

Icos. Acharius, 1. c., tab. 5; Swensk bot., VII, tab. 473 .

Port du R. caninit, à rameaux courts, terminés par une touffe de 4-6 feuilles, paraissan! sortir d'un point commun, aiguillons des liges robustes, dilatés à la base, droits ou courbés, ceux des rameaux plus petits, épars, souvent geminés au-dessous des pélioles; pétioles canaliculés, parsemés de poils à la base, glabres, du reste, aiguillonnés ou incrmes; "3-7 folioles toules pétiolées, petites, d'un vert gai, glal)res, fermes, orales-cuspidées ou ovales-clliptiques, simplement dentées, à dents fines, aiguës; slipules lancéolées, glabres, bordées de glandes fines, oreilletes aiguës, divergentes; pédoncules courts, ordin. solitaires, glabres, poriant à leur base une ou deux bractées ovales-cuspidées, glabres, plus longues qu'eux; tube du ealice petit, ovö̈de, glabre; divisions calicinales appendiculées au sommet, glabres, 2 entières à hords tomenteux, 5 pinnatilides à appendices étroits, linéaires, saillantes sur le bouton, plus courles que la corolle, réfléchies à l'anthèse, caduques ì la roloration du fruit; syles courts, hérissés; disque plan; lleur d'un blanc carné; firuit ocoüde presque arrondi, ronge.

Hab. Mai, juin. Haies, bois - Frrnce. Cher : petit bois des Vignes de la Chapello-saint-Ursin ! - Hhône : Lyon au grand camp (Ozanon); Ilaute-Savoie : Arenthon (Juget), le nont Saleve, dans le chemin de la 


\section{(1/48)}

Croisette, d'après Reuter. - Surise. Cant. de Neufchâtel : d'après M. Godet, il serait assez commun aux environs de Neuchàtel. - Autriche. Tyrol: Mieders, vallée de Stubai (Kerner).

\section{3้k. R. Aexibilis Nов.}

Arbrisseau de 1 mètre à 1 mètre 30 cent. de hauteur, à rameaux flexueux retombants et cherchant un appui sur les arbrisseaux voisins; aiguillons des tiges nombrcux, robustes, dilatés à la base, inclinés ou courbés en faulx au sommet, égaux, ceux des rameaux florifères rares, espacés, plus petits et de mème forme; écorce verdâtre, celle des jeunes pousses d'une couleur pruineuse; pétioles glabres, sillonnés, parsemés de poils blanchàtres dans le sillon, aiguillonnés en dessous; 7 folioles d'un vert sombre en lessus, non luisantes, toutes pétiolulées, assez espacées sur le pétiole, orales, orales-elliptiques ou arrondies an sommet, rétrécies à la base, les inférieures plus petites, quelquefois obtuses, glabres, simplement dentées; stipules plus ou moins larges, glabres, bordées de glandes, oreillettes aiguës, droites ou divergentes; pédoncules glabres, en corymbe de 4-8-12-20 ou rinnis en cyme bifide ou trifide, le corymbe porte à sa base des bractées ovales-cuspidées, glabres, a bords glanduleux, les cymes trifides ont à leur base deux bractées opposées, les pédoncules exiérieurs portent deux petites bractées, le pédoncule intérieur en est dépourvu; les cymes bifides ont les mèmes bractées, mais un seul pédoncule porte deux bractées, l'autre en est privé; tube du calice ovoüde, glabre; divisions calicinales ovales, glabres, spatulées au sommet, deux entières, trois pinnatifides à appendices larges, ciliés, saillantes sur le bouton, plus courtes que la corolle, rélléchies ì l'anthèse, non persistantes; styles com.ts, glabres; disque conirque; fieur assez grande, blanche; étamines blanchatres, i anflieres 
jaunes; fruit rouge, ovoïde, ceux des corymbes ou des cymes trifides, le fruit du centre est obovoïde.

HAB. Mai, juin. Haies, bois. - Angleterre. Yorkshire : les haies à Sowerby? (Baker). - France. Cher : Clairières du bois de Rouet, commune de Mehun près des vignes.

133. R. fallews Déséglise, in Fourreau, l. c. (1869), p. $7 \ddot{3}$, sine descript.; Cottet, I. c., p. 40, sine descript.

Exs. Déséglise, herb. ros., $n^{\circ} 15$.

Arbrisseau élevé, à rameaux flexueux, aiguillons des tiges robustes, dilatés et comprimés à la base, inclinés an sommet ou presque droits, ceux des rameaux florifères peu abondants, espacés et plus petits; pétioles sillonnés et parsemés de poils en dessus, portant sur le bord du sillon quelques rare's petites glandes, aiguillonnés en dessous; "3-7 folioles toutes pétiolulées, vertes, glabres, ovales arrondies à la base, terminées en pointe courte, ovales-elliptiques, arrondies et parfois rétuses, simplement dentées, à dents plus ou moins régulières; stipules glabres, bordées de çlandes, oreillettes aiguës, peu divergentes, les supérieures dilatées; pédoncules solitaires ou réunis en bouquet, glabres, ayant à leur hase des bractées ovales-cuspidées, glabres, bordées de grlandes, égalant ou dépassant les pédoncules; tube du calice ovö̈le, glabre; divisions calicinales ovales, spatulées all sommet, glabres, 2 entières, 5 pinnatifides à appendices étroits, saillantes sur le bouton, plus courtes que la corolle, réfléchies à l'anthèse, caduques avant la maturité du fruit; styles courts, hérissés; disque peu saillant; fleur grande, d'un rose pảle, à pétales émarginés au sommet; fruit ovoïde, rouge.

IlAs. Mai, juin. IIaies, bois. - France. Cher : bois d'Yèvre près de Vierzon, bois de Marmagne; - Rhône: Craponne (Boullu); - HauteSavoie: Thonon, Arenthon, Habèrc-Lullin (Puget.) 
Ors. $R$. albo-lutescens Ripart, in litt., nous parait bien voisin de l'espèce précédente. M. Ripart, dans sa lettre du 25 févr. 1875, dit : a Les fleurs a sont d'un blanc pur avec l'onglet jaune, les filets des étamines sont d'un a jaune prononcé, les styles libres et glabres, tout le feuillage est d'un " vert un peu jaunatre ; c'est un arbrisseau élevé, à long rameaux très"florifères," - II $\mathrm{Ab}$. Mai, juin. - Cher : les Aix-d'Angillon (Ripart); - Rhône : Lyon (Ozanon); - Haute-Savoie : Thonon? (Puget).

1\%6. IR. addita Déséglise, notes extl. de l'énum. des rosiers, in the journal of Botany, for June 1874, et extr., p. 2 ; $\boldsymbol{R}$. coriacea Crépin (non Opiz); $\boldsymbol{R}$. canima var. b. coriacea Boissier, fl. orient:, II, p. 68 วั.

Exs. Kotsehy, no 265, n०6.6.

Haв. Juin. Région des montagnes. - Perse. mont Elbrus à Passgala (Kotschy), entre Nischapur et Mechhed (Bunge, in herb. Boissier).

Ons. Il y a une faute de typographie dans le journal anglais : $R$. coricen, c'est $\boldsymbol{R}$. coriacea qu'il faut lire.

157. R. calycina M.-Bieb., f1. Taur.-Cauc., III, p. 549; Tratt., monog. ros., II, p. 8.

Has. Juin. - Russie d'Europe. Odessa.

Ce rosier manque aux herbiers de MM. De Candolle et Boissier. Seringe ne fail aucune mention du $\boldsymbol{R}$. calycina, pas même dans ses species non satis notae. - Rehb, $\mathrm{fl}$. exeurs, admet le $R$. calycina en synonyme au R. coriifolia, à tort selon nous, puisque cedernier a les feuilles pubescentes et que le $\boldsymbol{R}$. calycina les a utrinque glaberrimis.

158. G. armata Steven; Besser, enum. Pod. et Volh., p. 62 ; Tratt., l. c., p. 224 .

Ce rosier ne se trouve pas dans les herbiers de MII. De Candolle et Boissier, quoique dans le premier surtout les types de Besser soient si richement représentés.

159. R. Trassilvatenica Schur, enum. pl. trans., p. 202 ; Walpers, ann. hot., VII, p. 879. 


\section{( 151$)$}

HAB. - Hangestein près de Kronstadt.

Celte espèce n'est pas représentée dans les herbiers de MM. De Candolle et Boissier.

160. Th. Tounanginiana Déségl. el Ripart, I. c., X, p. 162 et extr., p. 62 ; Cariot., I. c., II, p. 176 ; Fourreau, l. e., p. 74 .

IIab. Juin. Haies. - France. Cher : hairs du chemin de Bourges à Givrai (Ripart), haies des vignes du château à Bourges, la Servanterie près de Mehun; - Rhône : Lyon (Chabert); - Haute-Savoie : Arenthon (Puget). - Italie. Sicile: Palerme (Todaro).

161. R. Manosisgima Rau, enum. ros., p. 74, sub. R. canina var.; Déséğlise, l. c., p. 105 et extr., p. 65; Cariot, 1. c., II, p. 177 ; Fourreau, 1. c., p. $74 ; R$. canina var. ramosissima Bl. et Fing., comp., I, p. 627.

Hıв. Juin. Haies, bois. - France. Cher: haies des vignes des Macheriots près de Bourges, pacage de Bouy commune de Berry, Mehun, forêt du Rhin-du-Bois, Trouy, Vierzon, au bois d'Yèvre; - Marıe : Reims (de Belley); - Saône-et-Loire : Saint-Forgent près d'Autun (Carion); Rhòne : Lyon, au-dessus de Couzon (Boreau); Doubs : Montmahoux (Paillot).

162. R. Amansii Déséglise et Ripart, in the journal of Botany for June 187/, extr., p. 5; R. Aginensis Ripart (non Desp.).

IIs. Juin. Ilaies. - France. Lot-et-Garonne : haies autour du château d'Arasse près d'Agen (Garroute, Ripart).

OBs. J'ai reçu d'Angleterre, du comté Nord d'York, de M. Baker, un rosier qui se rapproche beaucoup du $R$. Amansii par ses fruits et ses styles, mais l'échantillon etant dépourvu de feuilles, je ne puis pas me prononcer définitivement.

165. R. globularis Franchet, in Boreau, fl. cent. de la Fr., éd. 5, n 839 ; Déséglise, ess. monog., p. 104, extr., p. 6'; Cariot, I. c., II. p. 177 ; Grenier, fl. jura? p. 242. 
IA в. Juin. Haies. -- France. Loir-et-Cher : Carrières de Bcaumont (Franchet); - Rhône : Francheville (Chabert) ; - Doubs : Besançon ! Alsace. Forêt de Vordersberg près de Niederbronn!

16\%. Th. masazivaga Déséglise, descript. de qu. esp. nouv. du genre rosa (1875), in mém. Soc. Acad. de M.-et-L., XXVIII, p. 107 et extr., p. 11 ; Fourreau, I. c., p. 74; Verlot, I. c., p. 114, obs.; Cottet, I. c., p. $40 ; R$. monticola Déséglise, olim.

Exs. Déség̨lise, herb. ros., $1^{0} 61$; Billot (suites), $\mathrm{n}^{\circ} 5580$.

НАВ. Juin, juillet. Broussailles des montagnes. - France. Ilautes-Alpes : Gap (Burle); - Isère : mont Rachet (Verlot); - Savoie : mont Nivolet (Songeon); - Ilaute-Savoie : mont Sion, IIabere-Lullin, IJabèe-Poche, Saint-Germain-sur-Talloires, Pringy (Puget).... Snisse. Valais : Fins-Ilauts (de la Soie), vallée de Biun (Cottet), mont Clon! - Cant. de Fribourg : Paribaz, contrefort de la Cape au Moine! - Autriche. Tyrol: Mieders, vallée de Stubai.(Kerner).

163. R. speria Puget, in Déséglise, 1. e., XXVIII, p. 109, extr., p. 15 ; Fourreau, 1. c., p. 74 ; Coltet, 1. c., p. 40 .

Exs. Déséglise, herb. ros., $n^{\circ} 49$; Billot (suites), $n^{\circ} 5379$.

HAv. Juin, juillet. Broussailles des montagnes. - France. Vosges : Labresse (Pierrat); - Isère: forêt de Porte (Verlot); - Haute-Savoie : Pringy, Epagny, Saint Martin, Annecy-le-Vieux, montagne de l'offiège (Puget). - Suisse. Cant. de Fribourg : Lurgui base de la Cape au Moine! - Autriche. Tyrol : Madona del monte ad Roveredo (Kerner); - Autriche infér. : Krems (Kerner).

166. R. sphaerica Grenier, in Billot, archiv. de la fl. de Fr. et l'Allem., p. 555; Boreau, 1. c., éd. 5, $n^{n} 841$; Déséglise, ess. monog., in mém. Soc. Acarl. de M. etLoire, X, p. 104, et extr., p. (4; Reuter, I. c., p. 70 ; 


\section{$(135)$}

Cariot., 1. c., II, p. 177; Fourreau, 1. c., p. 74 ; Verlot, 1. e., p. $114 ; R$. canina var. globosa Desv., journ. bot. (1815), II, P. 114; R. camina var. sphaerica Godet, 1 . Jura, suppl., p. 75 .

\section{Exs. Billot, $n^{\circ} 1479$.}

Ias. Juin. IIaies, bois. - Angleterre. Cheshire: Hoylake (W' ebb); Cornwall ; haies près de Kingsmill (Briggs). - Belgique. Prov. de Nanur Rochefort, Han-sur-Lesse, Wavreille (Crépin). - France. Vosges : Saulxures (Pierrat) ; - Loiret : Orléans (Jullien) ; - Cher : C., Trouy, Bourges, Mehun, Allogny, Vierzon, ete. ; - Puy-de-Dôme : Clermont ; Doubs : Pontarlier (Grenier); - Jura: Saint-Loup (Puget); - Rhône : Lyon à Charbonnière (Chabert), Villenrbanne (Ozanon); -..- Isèrc : torêt de Porte (Verlot); - Hautes-Alpes : Charrance près de Gap (Burle); Iaute-Savoie : IJabère-Lullin, Argonnex (Puget), mont Salève à Monnetier! - Suisse. Cant. de Vaud: Chescières (Rapin); - Valais : Sion, Sembrancher (Cottet). - Autriche. Autr-infér. : Krems (Kerner); Tyrol : Trins, vallée de Gschnitz (Kerner).

167. R. exis Crépin, in bull. de la Soc. roy. de Botan. de Belgique (1868). VII, 1 $^{\circ}$, extr., p. I.

Hab. - I'russe rhénane. Vallée de la Nahe.

168. 埴. anciphylla Rau, enum. ros., p. 69; Tratt., 1. c., II, P. 22; Bl. et Fing., comp., I, P. 624; Rehl)., fl. excurs., II, P. 619; Bor., fl. cent., éd. 5, n 84/; Déséglise, 1. c., p. 106, et extr., p.66; Cariot, I. c., 1. 177 ; R. canina var. aciphylla Lindl., monog. ros., p. 99; Seringe, in DC., prod., II, P. 614; R. sphaerica var. aciphylla Grenier, 11. jur., p. 242.

Icon. Rau, I. e., pl. 1; Redouté, les roses (1824), livrais. 59, B.

IIAB. Mai, juin. Lieux sces et pierreux. - France. Cher : forêt de Fontmorean! petit hois anx Loups dans le's vignes de la Chappelle-saint-L'rsiu! bois de Roum près de Mehun! la Chapelle-saint-Ursin et Brécy (Ripart), 
Bourges (Tourangin); - Doulss : Brégille à Bt'sancon (Paillot); - Rhóne : indiqué à Beaunont par M. Cariol, mais nous n'avons pas i u la plante de cette localité. - Suisse. Cant. de Fribourg: Montbovon! - Bavière. Wurzbourg (Rau, 1817, in herb. DC.). - Belgique. Prov. de Namur: Jemelle (Crépin).

Oss. $\boldsymbol{R}$. horvidula Déségl., in herb.; $R$. ferox Chabert (non Ait.). J'ai reçu de feu Chabert uu rosier sous le nom de $R$. ferox Chabert ! qui est très-remarquable, mais sur lequel je ne puis pas me prononcer, ne le comnaissant que par deux échantillons en fruit, - Il a le port du R. aciphylla, dont il differe par les tiges chargées d'aiguillons inégaux, dilatés à la base, inclincs ou droits, ceux des rameaux floriferes beaucoup moins nombreux, espacés, petits, droits ; le fruit est petit, rouge, ovoïde ; les folioles un peu plus grandes que dans le $\boldsymbol{R}$. acipylla; les folioles sont irrégulièrement dentées.

IIAB. - Rhône : Francheville, derrière le château (Chobert); - Cher : haies de Saint-Elny-de-Gy, près du village.

\section{в). Biserratae.}

Crépin, primit. monog. ros., fase. I (1869), p. 17 ; Cottet, ros. du Valais, in bull. Soe. Murith. (1874), nº $\overline{5}$, p. 40 ; Nudae Déséglise, olss. on the differ. meth. prop. for the class. of the spee. gen. rosa, in the Naturalist (1863), n.20, p. 511 , part.

Pétioles glabres ou à peu près, folioles doublement dentées, glabres, dépourvues de glandes et de villosité sur les nervures; pédoncule et ube du calice lisses.

1. Styles glabres ou presque glabres. . . . 2.

1 Styles velus ou hérissés . . . . . 10

2. $\left\{\begin{array}{l}\text { Pétioles velus ou légèrement velus . . . } 5 . \\ \text { Pétioles glabres, parsemés de glandes ou églan- } \\ \text { duleux }\end{array}\right.$

3. $\left\{\begin{array}{c}\text { Folioles orbiculaires, d'un vert glauque, fleur } \\ \text { assez grande, d'un beau rose, fruit ovoïde medioximn. } \\ \text { Folioles orales, vertes, fleur d'un rose clair, fruit }\end{array}\right.$ arrondi. . . . . . . villosiuscults. 
Rameaux floraux inermes ou portant de rares

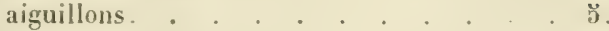

Rameaux floraux aiguillonmés. . . . . . 7

Tiges faibles, retombantes, rameaux courts, inermes ou munis de rares petits aiguillons, folioles ovales-aiguës, tube du calice petit, ellipsoïde, fleur rose, fruit grêle, ellipsoïde, rouge-orangé.

Rameaux florifères inermes . . . . . .

Folioles d'un vert sombre en dessus, fleur rose clair, styles très-obscurément hérissés, fruit ovoilde.

cladolcia.

Folioles d'un vert clair, fleur blanche, fruit ovoüle presrgue arrondi.

glaberrima.

7 Rameaux floraux courts, étalés, folioles assez. petites, ovales-aiguës, fruit petit, ovoïde . . curticole Rameaux floraux plus ou moins allongés. . . 8 .

Tube du calice ovoïde, styles entièrement glabres 9 .

8. Tube du calice ovoïde-allongé, fleur rose, styles obscurément hérissés . . . . . . oblongn.

9. Folioles ovales-arrondies, fleur blanche . . Carioti.
- Fulioles ovales, à dents aiguës, fleur rose.
Chaboisaei.

10. Styles velus . . . . . . . . 11 .

Styles hérissés . . . . . . . . 12

Styles très-relus, simulant une colonne courte, folioles orales, les inférieures subobtuses, fleur rose clair, fruit globuleux . . . . . erioslyla.

11.

Styles velus, petit arbrisscau, folioles ovales, d'un vert glauceseent, pédoncule très-court, fruit gros, obovoïde, couronné par les sépales persistants

steplennocarpa.

19. Fruit ovoïde ou ovoïde allongé. . . . . 15.

Fruit globuleux. . . . . . . 20.

15. Folioles petites . . . . . . . 14

Folioles assez larges . . . . . . 16.

Tige couverte de nombreux aiguillons inégaux,

horizontaux, fleur grande, d'un rose pâle, fruit

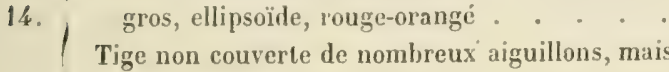
armalissima. aiguillonnée . . . . . . 15 


\section{$(156)$}

diguillons rapprochés sur les rameaux florifères,

15. $\left\{\begin{array}{l}\text { flur rose, fruit ovoïde : . . . . . } \\ \text { Aiguillons épars, fleur blanche, fruit ovoïde- }\end{array}\right.$

squervosa. allongé.

adscilu.

16. I Pédoncule court. . . . . . . 17

16. Pédoncule plus ou moins allongé . . . 18.

Pédoncule long de $\mathbf{5}-\mathbf{4}$ millim., fleur rose, fruit

17. $\left\{\begin{array}{l}\text { obovoïde, affectant une forme pyriforme. } \\ \text { Pédoncule court, ayant plus de } 4 \text { millim., fleur }\end{array}\right.$ rose clair, fruit obovoïde-allongé . . . . insignis.

18. Fleur rose ou blanche . . . . . . . dumalis

18. Fleur d'un rose vif . . . . . . . 19.

19. $\left\{\begin{array}{c}\text { Folioles latérales presque sessiles, ovales-ellip- } \\ \text { tiques, a'un vert non luisant en dessus. } \\ \text { Folioles latérales pétiolées, ovales-aiguës, d'un }\end{array}\right.$ vert luisant en dessus . . . . . . . rubescens.

Jeunes pousses d'un rouge vineux ou les stipules,

20. les pétioles, les bractées, lís nervures lavés d'un rouge vineux . . . . . . . 21.

Jeunes pousses peu ou point d'un rouge vineux. 느.

Jeunes pousses fortement lavées d'an rouge

vineus, fruit gros, presque arrondi. . . Malmundarionsis.

21 Pélioles, stipules, bractées, nervures des folioles lavés d'un rouge vineux, fruit subglobuleux, folioles-elliptiques . . . . . vinucer.

22. I Petit sous-arbrisseau, fruit petit, globuleux . . sylvularmm.

Arbrisseau élevé, fruit gros . . . . . . 25.

25. Feuilles luisantes en dessus . . . . sphaeroidea.

Feuilles non luisantes eu dessus . Folioles ovales-aiguës, fleur rose pâle, fruit très-
(

24. gros, de la grosseur d'une petite noix. . macrocurpa.

Folioles ovales, d'un vert sombre, fleur rose. . biserrala.

169. BR. Camodo Chabert, in Cariot, étud. des fleurs (186:3), II, P. 677; Fourreau, I. c, p. 74 (sinc descript.); Crépin, primit. monog. ros. (1869), fase. I, p. 44.

Description établie sur les notes et échantillons recus de feu Cliabert. 
Racine non traçante; arbrisscau de 2 à 5 mètres, touflu, rohuste, aiguillons très-nombreux sur les tiges qui partent de la souche, crochus, ceux des rameaux moins nombreux, courbés en faux, rougeàtres; pétioles glabres, canalieulés, parsemés de glandes, munis de quelques poils à l’insertion des folioles, aiguillonnés en dessous, à acicules se prolongeant jusque sur la ncrure médiane, une partie des nervures médianes ont ce caractere, mais pas toules; stipules lancéolées, Iongues, glabres, bordées de glandes, ì oreillettes aiguës, divergentes; toutes pétiolées, glalmres, ovales-arrondies ou ohtuses, vertes en dessus, plus pàles en dessous, doublement dentées, i dents ourertes, les principales mucronées, les secondaires glanduleuses; pédoncules lisses, solitaires ou groupés par $\bar{j}-4$, munis de hractés acumines, dont une souvent termince par un appendice foliaire, glabres, plus longues que les pédoncules extéricurs, égalant celui du centre; tube du calice glabre, ovö̈de, arrondi à la base, contracté au sommet; divisions calicinales spatulées an sommet, glabres en dessous, 2 entières à bords tomenteux, 5 pinnatifides, saillantes sur le bouton, plas courtes que la corolle, réfléchies à l'anthèse, eaduques; styles courts; disque conique; fleur de grandeur moyenne, blanche, à pétales obeordés; fruit ovoüde, arrondi à la base, un peu atténué au sommet, rouge en septembre, pulpeux en octobre.

HAn. Juin. Haies. - Frunce. Cher : haies des vallées à la Chapelle-saintUrsin près de Bourges (Tourangin); - Rhòne : Lyon au Gau au-dessus du pont d'Alay (Chabert); - Aude : montagne Noire. Ie Mas-Cabardis (Ozanon).

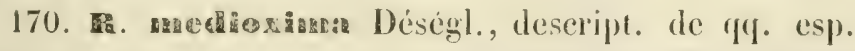
nour. du genre Rosa, in mém. soc. Acad. de M.et-Loire, XXVIII (1875), p. 110 et extr., p. 16; Cirépin, I. c., p. 17; Verlot, eat. pl. du Dauph., p. 59/. 
Ilar. Juin. IIaies, buissons, - Franee. Cher : haies de Roulon, commune de Berry !- Isère : forêt de Porte desrière Chamechaude (Verlot); Haute-Savoic : la Malveria sur Annecy-le-Vicux, Thonon (Puget). Alsace. Niederbronn! - Autriche. Tyrol : Lienz (Kerner).

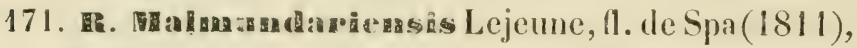
I, p. 251; Desportes, ros. gall., $1^{\circ}$ 1986; Boreau, I. c., éd. 2, II, p. 178, éd. 5, " 10 $^{\circ}$; Déséglise, ess. monog., in mém. soc. Acad. de M.-et-Loire, X, p. 107 et extr., p. 67; de Martr.-Don., 11. du Tarn, p. 229; Cariot, I. c., p. 177; Foureau, I. c., p. 74 ; Pérard, cat. de Montluç., P. $85 ; \boldsymbol{R}$. canina var. Malmumeriensis Chevalier, $\mathbf{f}$. génér. de Paris, II, P. 694; R. canina var. ambigua Seringe, in DC., prod., II, p. 614.

Icon. Redouté, les roses (182/), livr. 26, B. mala.

Exs. Déséglise, herb). ros., $n^{\circ}$ 48; Billot (suites), $n^{\circ} \mathbf{5 7 2 0}$ sul. R. affinis Rau. Il peut se faire que ce soit le $\boldsymbol{R}$. a/finis de M. Grenier, mais non celui de Rau. Rau dit : foliolis "supra pubescentibus, subtus glancesecntibus glabris." Les échantillons distribués ont les feuilles littéralement glabres sur les deux faces. Orphanides, fl. graec., $n^{\circ} 5550 ? n^{\circ} 2755$ ?

IIAB. Juin. Ilaies, bois. - Angleterre. Yorkshire : wood by the canal side Wood Fewton (Hiailstonc); - Devonshire : Leigham Egg Buchland (Briggs). - Prusse. Malmedy (Lejeunc). - France. Indre : Mers, bois du Magner (Boreau); - Cher : A. C., Contremoret, Bourges, bois de Marmagne, Melun, etc.; - Puy-de-Dòme : entre les Gazeriers et Sussat, les Vergnes près de Riom, Saint-Pardoux (Lamotte), Clermont! - Mllier : Montluçon (Pérard, catal); - Doubs : nnont Brégille (Grenier); - Jura : Salins (herb. Grenier); - Saône-et-Loire : Châlon-sur-Saône, Chagny, Tessey (Ozanon); - Ain : Ambronay; - Gard : le Vigan (Diomède, in Lerl, Grenier); - Aude : Montagne-Noire, le Mascabardès (Ozamon); Tarn : Saint-Urcisse (Martrin-Donos); - llhı̀ne: Talssin, Ecully; - Isère : 


\section{( 159$)$}

vallon J.-J. Rousseau à Pariset (Verlot); - Var : le Lue (Ilanry);-- IlauteSavoie : Thonon, Pringy, Reyvroz (Puget).

OBS. Les folioles de ce rosier sont doublement dentées, contrairement ¿ l'assertion de M. Du Mortier (monog. des roses de la fl. belge); Lejeune, 1. c., ne parle pas de ce caractère dans sa flore de Spa, mais dans la revue de la flore de Spa, il les dit doublement dentées (duplicato-serratis). Nul doute ne peut s'élever à cet égard.

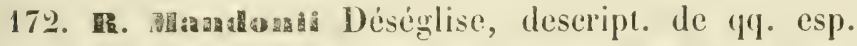
nour du genre rosa, in mém. soc. Lead. de M.-et-Loire, XXVIII (1875), p. 111 et extr., p. 1引̆; R. canina Mandon, pl. Maderenses (1860-1866), exs. no 98 (non Linn.).

HAB. - Madère, in dumetosis, jardin da serra, 1000 à 1500 mèt. d'alt. (Mandon)

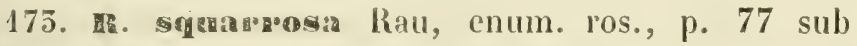
R. canina var.; Bor., 11. cent., éd. 5, n 8/5̄; Déséglise, ess. monog., extr., 1). 68; de Mart.-Donos, I. e., p. 250 ; Cariot, 1. c., II, p. 178; Fourreau, I. c., p. 74; Verlot, I. e., p. 114, obs.; R. canina var. squarrosa Seringe, in DC., prod., II, p. 614; B1. et Fing., l. c., p. 627; R. canina b. H.-Bieb., 11. 'Taur.-Cauc., I, p. 400, ex Rau.

\section{Exs. Billot (suites), $n^{0} 5719$.}

IIıв. Juin. Bois. - France. Vosges: forêt de Rambervillers (Boulay); - Cher : forêt du Rhin-du-bois, bois de Rouet près de Mehun ; - Saôneet-Loire : Clıâlons-sur-Sâne (Ozanon); - Ain : avant le pont de la Cadette près de Lyon (Chabert); — Tarn: la Souzière (de Martrin-Donos) ; Basses-Alpes : Barcelonnette (Ozanon); - Rhòne : Francheville (Boullu), pont d'Alay (Ozanon); - Haute-Savoie : Pringy, Habère-Lullin, lit Margeriaz (P'uget). - Suisse. Cant. de Fribourg : la Cernaz près de Montbovon (Coltet). - Autriche. Tyrol : Aichholz près de Kapaun (Keraer).

Oss. J'ai reçu de feu Chabert un rosier sous le nom de $R$. serrulate Chab. qui est bien roisin du R. squarrosa; il en a les caractères géné- 
raux et n'en diffire que par ses slyles glabres et sa fleur blanche. Voici la description que je tiens de Chabert: " $\boldsymbol{R}$. serrulata Chabert. Arbrissean élevé, i rameaux droits, touffus, aiguillons rohustes, nombreux sur les tiges, courbés atu sommet, ceux des rameaux moins nombreux, plus petits, presque droits; pétioles parsemés de quelques poils à I'insertion des folioles, portant des glandes fines, aiguillonnés; $\check{b}-7$ folioles les unes obtuses d'autres ovales, glabres, vertes en dessus, plus pâles en dessous, nervure médiane portant quelques glandes surtout i la base, doublement d’ntées, à dents

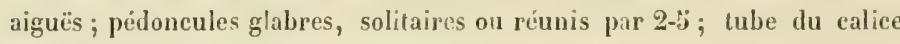
ovol̈de, glabre; divisions calicinales glahres on dessous, 2 entièes, 5 pinuatifides, plus courtes que la corolle; styles glabres, disque conique; fleur blanche; fruit rouge, ovoïde. "

IIAB. - Rhône: haies près de la ferme de la Glande sur le mont Verdin, au Gau (Chabert).

17\%. 泥. apbentifora Ripart, in Déséglise, I. c, p. 109 et cxtr. p. 69; Fourreau, 1. c., p. 74 .

Haв. Juin. Haies. - France. Cher: Saint-Éloy-de-Gy (Ripart), Marçay près de la Servanterie? - Puy-dc-Dôme: Saint-Pardoux (Lamolte); - Rhône : Lyon à Dardilly (Ozanon).

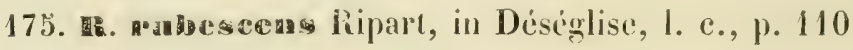
et extr., p. 70; Cotiet, I. c., P. 40.

Isı. Juin. IIaies. - France. - Cher : la Chapelle-Saint-Urbin (Ripart), le Corponay, près de Saint-Eloy-rle-Gy. - Suisse. valais : vallée de Binn (Cottet).

176. 哟. Eanacea Baker, review of the British roses (186/), p. 32 .

Exs. Baker, herb. ros. brit., $n^{\circ} 28$.

II ав. Mai, juin. IIaies. - Angleterre. Yorkshire : Sowerby (Baker).

177. Re. Alusaris Bechstcin, forstb., p. 241; Tratt, 1. c., II, p. 24; Boreau, l. c., éd. 5, I' 847; Déséglise, 1. c., p. 111 et extr., p. 71 ; de Martr.-Don., I. c., p. 250; Grenier, 11. jura., p. 244, exel. var. b.; Cariot, 1. e., p. 178; Baker, I. c., p. 2\%; Pérard, I. c., p. 85; Fourreau, 1. c., p. 74; Verlot, I. c., p. 144; R. canina Leman, bull. 


\section{(161)}

phil. (1818), extr., p. 9, et plurim anct.; R. canina var. glandulose Rau, 1. e., p. 7ั̈; BI. et Fing., 1. c., p. 627; R. canina var. slipularis Chevalier, 1. c., p. 695; R. canina var. sarmentosa Godet, 1. c., p. 213 ; Reuter, 1. c., p. 70; $R$. canina var. dumalis Baker, monog. of brit. ros. in Linn. society`s journ., XI, p. 227; R. stipularis Mérat, 11. Par. (1812), p. 192; Seringe, in DC., prod., 1I, p. 625;

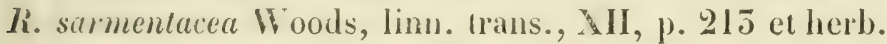
n० 79 à 84; Smill, engl. 11., II, p. 590; Tratl., I. c., p. 59 ; R. glancophylla Winch, geogr. distrib. (1829), p. 45; R. sepium var. stipularis Desvaux, journ. bot. (1815), II, p. $116 ; \boldsymbol{R}$. fissispina Wierzbicki; R. rumulosa Godron, fl. Lorr., éd. 2, I, p. 251.

Icon. Engl. bot., tab. 2595 .

Lxs. Seringe, ros. desséch., n 9; Reichenbach, n 1731 (sub. R. tortuosa non Wierzb.), no 1956 (sub. R. Rissispina Wierzb.); Billot, $1^{\circ}$ 2260, $1^{\circ}$ 2062; Wirtgen, pl. crit., $n^{0 s} 73,76,233,463,437,381$; Déséglise, herb. ros., $n^{0 s} 14$ el 14 bis; Baker, herb. ros. brit., $n^{1 / 5} 15$ et 14; Bourgeau, pl. d'Espayne (1865), n²454.

$\mathrm{II}_{\mathrm{B}}$. Hai, juin. Buissons de la plaine et des montagnes. - Très-répandu en Europe.

Ons. 1. Le no 173̈1, publié par licichenbach, est-il bien le R. tortuosa Wierzb.? Je ne conuais pas la deseription de ce rosier, mais je possède un type de Wirrzbichi qui est completement difiérent de la plante publice par Reichenbach. Ce $R$. Lorluosa Wierzb., est tout simplement le $R$. Andegavensin Bist.! Cehui de Rerichenhach, que j’â été à mème de voir dans l'herb. de $\mathbf{M}$. Boissier, est le $\boldsymbol{R}$. dumalis.

OBs. II. R. erythrellu Ripart cst un $\boldsymbol{R}$. dumulis à fleur tiès-grande, d'un "rose vif rappelant la couleur des gallicanes n (Ripart, in litt. 25 fév. 1875). Je ne connais ce rosier que par deux échantillows en tleur; je n’ai pas vu le fruit; je ne vois pas trop cu quoi il peut être diflér:nt du R. dumalis; 
une étude plus approfondie de ce rosier fera probablement reconnaitre d'autres ca:actères que celui tiré de la fleur.

OBs. III. R. rhynchocarpa Ripart, mss. - M. Ripart, dans sa lettre du 6 déeemb. 1875, me dit: Ce rosier a les feuilles irrégulièrement dentées, " non glanduleuses, glabres; styles glabres, à peine munis de quelques " poils; c'est son fruit surtout qui le caractérise, il est obovoïde, mais la " partie supérieure est amincie, de telle sorte qu'elle est plus étroite que "le disque auquel elle sert de support."

НАв. - France. Cher : bois de la Grange-saint-Jean (Ripart). Belgique. Prov, de Namur : Han-sur-Lesse (Crẻpin).

178. R. glaberrima Du Mort., fl. belgica (1827), p. 94 et monog. des ros. de la fl. helge $(1867)$, p. 63.

Inar. Juillet. - Belgique. Tournay (Du Hortier), - France. Cher : forêt du Rhin-du-bois? - Haute-Savoie: broussailles du petit Salève près le château de Monnctier! - Autriche. Tyrol: Ponale ad lacum Benacum (Kerner). - Itulie. Les haies à Sta-Margherita a Montici près de Florence (Levicr).

179. R. oblonga Déségl. et Ripart.

Arbrisseau de 1 mètre à 1 mèt. 30 cent. de hauteur, à rameaux flexueux, munis d'aiguillons dilatés à la base, arqués, plus faibles et presque droits sur les jeunes tiges; pétioles glabres, parsemés de quelques rares glandes, aignuillonnés en dessous; acicules se prolongeant sur la nervure médiane de quelques folioles; ovales-elliptiques, d'un rept clair luisant en dessus, glabres, fermes, doublement dentées, à dents secondaires glanduleuses; stipules glabres, étroites, bordées de glandes, oreillettes ordin. droites; pédoncules solitaires ou réunis par trois, glabres; tube du calice obovoïde ou ovoïdeallongé, glabre; divisions calicinales spatulées au sommet, glabres, 2 entières, Jo pinnatifides à appendices étroits, saillantes sur le bouton, plus courtes que la corolle, réflèthics it l'anthèse, caduques; styles obseuré- 


\section{$(165)$}

ment hérissés, presque glabres; disque un peu conique; fleur assez grande, rose; fruit rouge, ellipsoïde.

IIaв. Mai, juin. Bois, haies.-France. Cher : bois de Marmagne (Ripart), la Servanterie et Vlarçay près de Itchun, Fontiley près de Bourgo's, forêt de Fontmoreau, forêt d'Allogny, Allouis; - Haute-Savoie : Epagny (Puget), - Suisse. Cant. de Fribourg: Alontbovon.

180. aR. clandoleara Ripart, in Crépin, l. c., fase. I, p. 44 (sine descript.); Verlot, I. c., p. 594 ; Coltet, I. c., p. 40 (sine descript.).

Port du $\boldsymbol{R}$. dumalis, remarquable par ses rameanx florifères inermes et ses liges sarmenteuses n'ayant pas ou trèspeu d'aiguillons; pétioles glabres, sillomés en dessus, parsemés de quelques poils et de rares glandes fines, inermes, quelques pétioles très-faiblement acieulés; 5-7 folioles, la terminale ovale ou ohovale ou elliptique, rétrécie aux deux extrémités, les latérales otales, ovales-elliptiques, les inférienres quelques-mes obluses, d'un vert sombre en dessus, glabres, doublement dentées; stipules glabres, à oreillettes aiguës, droites, à hords ghlanduleux ef un peu scrulées all sommet; pédoncules solitaires ou réunis par 2-ö, glabres, ayant i leur base une ou deux bractées assez grandes souvent terminées par un appendice trifolié, glalires, plus longues que les pédoncules; tube du ealice ovoüle, glabre ; divisions calicinales spatulées au sommet, 2 entières à hords tomenteur en dessous, 5 pinnatifides ì appendices assez longs, saillantes sur le bouton, réiléchies à l'andhese, caduques; siyles glabres ou très-obscurément herisses; disque conique; fleur d'un rose elair; fruit rouge, ovoïde.

II.B. Mai, juin. Haies, bois. - Belgique. Prov. de Namur : Rochefort (Crépin). - Frence. Cher: Bourges, haies des vignes d'Auron (Ripart), P'erre-Lati, press de Bougras! communal du Nueste pres de Brécy! Bouy 


\section{(164)}

commune de Berry ! for'c̀ du Rhin-du-bois ! la Servanterie et les vignes de Couët près de Mehun ! - Autriche. Autr -infér. : Sehwargan (Kerner); - Tyrol : entre Ziel et Fragenstein (Kerner).

\section{R. sylvularum Ripart, Mss.}

Petit sous-arbrisseau à rameaux vineux ou verdatres, grèles, avec des aiguillons droits, dilatés ì la base en forme de disque ou un peu inclinés, ceux des tiges plus robustes, comprimés à la base; pétioles glabres, parsemés de glandes fines peu abondantes, aiguillonnés en dessous, quelques pétioles sont aussi inermes; 507 folioles petites, orales, ovales-obtuses, glabres, d'un vert sombre en dessus, plus pàles en dessous, doublement dentées; stipules glabres, à oreillettes droites; pédoncules solitaires ou groupés par 2-5̄, glabres, courts, ayant à leur base des bractées ovales cuspidées, glabres, plus longues qu'eux; tube du ealice ovoïde, glabre; divisions calicinales glabres en dessous, 2 entières, 5 pimmatifides à appendices étroits, saillantes sur le bouton, plus couries que la corolle, réfléchies à l'anthèse, caduques; styles faiblement hérissés; disque conique; fleur rose; fruit petit, rouge, globuleux.

IIn. Juin. Haies, buissons. - Belgique. Prov. de Namur : Rochefort, au Mont Rival (Crépin). - France. Cher : Saint-Germain-des-bois (Ripart); - Saône-et-Loire : Châlons-sur-San̂ne au péage (Ozanon); - Isère : vallon J.-J. Rousseau près de Grenoble (Verlot); - Haute-Savoie : Pringy, Thonon (Puget). - Suisse. Cant. de Fribourg : Montbovon! - Cant. de Bâle: Jura de Bâle (Christ). - Autriche. Autriche-inférieure: Krems (Kerner).

182. AR. Ragignis Déségl. et Riparı, in Déségl., descript. de qq. esp. nouv. du genre Rosa, in mém. soc. Acad. de M.-ct-Loire, XXVIII (1875), p. 112 et extr., p. 16; $\boldsymbol{R}$. canina var. insignis Grenier, f1. jur., p. 245.

Hab. Juin. Haies. - Belgique. Prov. de Nanur : Rochefort (Crépin). - 


\section{(163)}

France. Cher : Bourgneuf (Ripart), la Servanterie! Bourges près le moulin Bàtard! - Loir-et-Cher : la Buissonnière commune de Maray! - Allier : les Gazeriers (Lamotte); - Rhône : Francheville à Chaponost (Chabert); Isère : mont Rachet (Verlot); - Saroie : Méry (Puget); - Ilaute-Savoie : Annecy-le-Vieux (Puget).

185. Th. Chaboissaci Grenier, 11. jura., p. 241, obs.; Fourreau, l. c., p. 74 .

Exs. Déséglise, herb. ros., $n^{\circ} 62$ et 62 bis.

Haв. Juin. Haies, buissons. - Angleterre. Yorkshire : haies près de Thirsk (Baker). - France. Vosges : Rambervillers (Boulay), Rémiremont! - Meurthe: Nancy (Godron, in herb. Grenicr); - Seine-inférieure : Yvetot (Lebel); - Vienne : le Poirat, commune de Pindray (Chaboisseau, in herb. Grenicr); - Cher : Quiney ! Graire! Berry! Trouy ! entre la forêt de Saint-Palais et Achères! -- Allier : Vichy (Blanc, in herb. Grenier); - Jura : Salins ! - Haute-Garonne : Toulouse (TimbalLagrave); - Gard : le Vigan (Grenier); — Rhône : Lyon à la Tête d'or, Saint-Genis-des-Ollières (Boullu); - Haute-Savoie: Pringy (Puget). Suisse. Cant. de Bâle : Bâle (Christ). - Vénétie. Garda ad lacum Benacum (Kerner).

\section{R. eriostyla Ripart et Déséglise.}

Arbrisseau peu élevé, aiguillons plus ou moins nombreux, inclinés ou droits, dilatés à la base; pétioles presque inermes, parsemés de poils el de glandes; ; $;-7$ folioles ovales, ovales-elliptiques, les inférieures assez généralement sub-obtuses, glabres, doublement dentées, à dents secondaires ordin. terminées par une glande; stipules rrlabres, oreillettes dressées ou peu divergentes, bordées de glandes; pédoncules glabres, solitaires ou réunis par 2-4 en bouquet, portant à leur base des bracties ovaleseuspidées, glabres, plus longues qu'eux; tube du calice ovoïle, glabre; divisions ealicinales 2 entières à bords tomenteux, 5 pimnatifides à appendiees linéaires bordés de 
glandes, saillantes sur le bouton, égalant la corolle, réfléchiesà l'anthèse, non persistantes; styles libres, très-relus, simulant une colonne courte; disque un peu conique; fleur d'un rose clair; fruil globuleux, dans ecux rémis en bouquet, le fruit central est souvent obovoüde.

Ha B. Juin. Ilaies, hois - Angleterre. Carnarvonshire (Webb). France. Haute-Savoie : Brenthonue, Habère-Lullin, Saint-Martin (Pugct); - Ilaute-Garonne: Boussens (Timbal-Lagrave). - Autriche. Autricheinfér. : Scharwgan (Kerner).

185. R. curdicola Puget, in Déségl., descripr. de qq. esp. nouv. du genre Rosa, in mém. soe. Acad. de M.-ctLoire, XXVIII (1875), p. 114 et extr., p. 18.

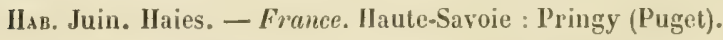

486. IR. stenocanpa Déséglise, l. c., p. 115 et cxtr., p. 17.

IIab. Juin. IJaies. - France, Ilaute-Savoie : Annecy-le-Vieux, buissons aux Barattes (Puget). - Suisse. Cant. de Fribourg : Cernaz près de Montbovon!

187. IR. villosiuscula Ripart, in Crépin, primit. monog. ros. (1869), fase. I, p. 4.8, sine descript.; Cottet, 1. e., p. 40, sine descript.

Arbrisseau peu élevé, à liges munies d'aiguillons asse\% nombreux, dilatés, comprimés is la base, crochus ou inclinés au sommet, souvent géminés sous les pétioles; pétioles velus, à villosité courte et peu lournie, parsemés de glandes, aiguillonnés ou inermes; "艹-7 folioles, la terminale souvent terminée en pointe courte, ovales-arrondies, ou ovales-elliptigues, glabres, fermes, nerveuses, la nervure médiane porte aussi quelques petits acieules, vertes en dessus, glauques en dessous, doublement dentées; stipules étroites, glabres, oreillettes aiguës, droites ou disergentes; 


\section{( 167$)$}

pédoncules solitaires ou réunis par 2-4, glabres, ayant ì leur base des bractées ovales-cuspidées, glabres, plus longues qu'eux; tube du calice glabre, ovö̈de; divisions calieinales 2 entières à bords tomenteux, 5 pimnatifides à appendices portant aux bords quelques glandes, réfléchies it l'anthèse, saillantes sur le bouton, plus courtes que la corolle, eaduques; styles glabres; disque conique; fleur rose clair; fruit arrondi dans les fruits en bouquet, le central affecte une forme obovoïde.

IIAr. Juin. Ilaics. - Belgique. Prov de Namur: IIan-sur-Lesse (Crépin). - France. Cher : Saint-Germain-des-bois, vignes de Givrai près de Bourges (Ripart). - Suisse. Cant. de Fribourg : les cases d'Allıères; Valais : vallée de Pinn (Cottet). - Autriche. Tyrol : Leopoldsruh (Kerner).

188. R. armatissiuna Déségl. et Ripart, I. c., XXVIII (1875), p. 114 et extr.p. 18.

IIAr. Juin. Haies. - France. Cher : carrières de la Chapelle-saint-Ursin.

189. 奋. stephanocarpa Déséglise et Ripart, l. c., p. 115 et extr., p. 19.

HAr. Buissons et haies des coteaux calcaires. - France. Cher : coteaux de l'Yèvre, rive gauche à Therrieux près de Savigny-en-Septaine.

190. Ar. adscita Déséglise, descript. de qฯ. esp. nouv. du genre Rosa, in Billotia (1866), p. 54; Verlot, l. c., p. 594 .

Exs. Déséglise, herlo. ros,, no $ّ 0$.

Hab. Mai, juin. Ilaies, buissons. - France. Cher : earrières de la Chapelle-saint-Ursin. - M. Verlot indique ce rosier à Gap.

191. Fr. megalocarpa Déségl., descript. de qq. esp. nouv. du genre Rosa, in mém. soe. Acad. de M.-et-Loire, XXVIII (1875), p. 117 et extr., p. 21; R. macrocarpa Boissier, f1. Orient., II, p. 684. (non Méral, nee Rochel). 
Has. - Perse. Vallée de Djimil (Lazistan), alt 2,000 mèt. (Balanso, in herb. Boissier).

192. R. macasazoa Mérat, 0. Par. (1812), p. 190; Seringe, in DC., prod., II, p. 625̄; R. crnina var. macrocarpa Cheval., 11. génér. des env. de Paris, II, p. 695.

Arbrisseau élevé, à lameaux flexucux, à écorce vincuse ou verdâtre, aiguillons des tiges robustes, dilatés à la base, courbés au sommel, ceux des rameaux moins forts, épars, presque droits ou inclinćs; pétioles glabres, portant quelques rares petites glandes fines, sillonnés en dessus, aiguillonnés en dessous; $\ddot{3}-7$ folioles toutes pétiolées, la terminale arrondie ou un peu rérécie i la base, formes, glabres, ovales-aiguës on ocales-arrondies, ì nerr ures secondaires plus ou moins saillantes, la médiane porte aussi quelques petits acieules, doublement dentées; stipules larges, glabres, it oreillettes aiguës, diveregentes; pédoncules courts, glabres, solitaires ou réunis par 2-5, portant à leur base des bractées ovales-acuminées, glabres, plus longues que le pédoncule; tulse du calice ovoüle, glabre; divisions caliciuales appendiculées au sommer, glabres en dessous, 2 entières à bords tomenteux, $\bar{j}$ pinnatifides à appendices bordés de quelques glandes, non persistantes; styles courts, hérissés; disque presque plan; fleur d'un rose pàle; fruit très-gros, rouge, slabre, globuleux, de la grosseur d'une petile noix!

IIAв. Juin. Haies. - France. Environs de Paris (Mérat, fl.); - Cher: haies des vignes de Mlontilaut près de Bourges! haies de Maregay près de Quiney! - Suisse. Cant. de Schafhouse: Siblingen (Gremli).

Oвs. J'ai vainement elierché le type de Mérat dans l'herbier Delessert et dans ceux de MM. De Candolle et Boissier; la plante que j'ai cer rue scmble se rapporter à la description incomplète donnée par Mérat, qui ne dit pas si les folioles sont simplement ou doublement dentées, et ne fait 
aucune mention des styles ni des divisions calicinales. Voici la description donnée en 1812 par Mérat.

* R. macrocarpa, $\mathrm{N}$. tige de 5 à 6 pieds, à aiguillons peu courbés ; " folioles ovales, pointues, dentées, non glanduleuses; péliole presque " aiguillonné, un peu glandulcux; pédoncule et fruit glabres, ce dernier " globuleux, du volume d'une petite noix ; 2-5 fleurs ensemble, de coulcur " rose pâle." Mérat.

Lindley rapporte à tort le $R$ : mucrocarpa Nićrat en synonyme au R. sepium Thuill., car Mérat, dit "folioles non glanduleuses."

195. R. bisersoata Mérat, fl. Par. (18/2), p. 190; Leman, bull. philom. (1818), extr. p. 12; Thory, prod. du genre ros., p. 101; Tratt., I. c., II, p. 55, Boreau, 1. cent., éd. 5, $n^{\circ} 848$; Reuter, I. c., p. 70 ; Déséglise, ros. monog., in mém. soe. Acad. de M.-et-Loire, X, p. 112 et extr., p. 72; de Mart.-Donos, 1. c., p. 250; Cariot, I. c., p. 178; Fourreau, I. c., p. 7\&; R. sepium var. nitens Desvaux, journ. bot. (18:5), II, p. 117 ; R. canina rar. Meratiana Seringe, in DC., prod., II, p. 61'作; R. canina var. biserrata Cheval., 1. c., p. 695; IIutel, 凡1. fr., I, p. 53̈2; Gonnet, fl. élém. de Fr., p. 480.

Icox. Redouté, les roses (182/), livais. 27, C. mala.

IIAB. Juin. Haies, buissons. - France. Loiret : Saint-Gabriel près d'Orléans (Jullien), la Chapelle, Cereottes (Boreau, in litt.); - Cher : route de Bourges à Soye! Berry! Villalin près de Quincy! vignes de Couët près de Mebun! - Haute-Savoie : Ilabère-Lullin (Puget). Autriche. Autr.-infér. : Krems (Kerner).

194. R. sphaesoidea Ripart, mss.

Arbrisseau ayant le port du $R$. dumalis; aiguillons robustes, dilatés, comprimés à la base, crochus ou inclinés an sommet, souvent nuls sur les tigges florifères; pétioles wabres, sillonnes en dessus, parsemés de glandes fines, aignillomnés en dessous; ; $\ddot{b}-7$-folioles ovales-aiguës ou 
elliptiques, glabres, d'un vert luissant en dessus, glaucescentes en dessous, la nervure médiane d'un grand nombre de folioles porte quelques petits acicules, doublement dentées; stipules assez larges, glabres, bordées de glandes, oreillettes aiguës, droites ou divergentes; pédoncules glabres, réunis ordin. en corymbe, cachés par de larges bractées ovales-acuminées, glabres, bordées de glindes; tube du calice ovoïde, glabre; divisions calicinales appendiculées au sommet, glabres en dessous, 2 entières à bords tomenteux, 5 pinnatifides à appendices bordés de glandes, saillantes sur le bouton, plus courtes que la corolle, non persistantes; styles hérissés; disque presque plan; fleur l'un rose clair' fruit assez gros, arrondi, rouge.

$\mathrm{H}_{\mathrm{AB}}$, Mai, juin. Haies, bois. - Belgique. Prov. de Nanur : Rochefort (Crépin). - France. Cher : La Chapelle-saint-Ursin (Ripart), Marçay près de Mehun, Berry, Savigny-en-Septaine, vignes d'Auron près de Bourges, bois de Roeset, route de Bourges à Saint-Amand; - Iaute-Savoie: Thonon (Puget). - Autriche. Autr.-infér. : Alaunthal près de Krems (Kerner).

\section{R. brachypoda Déséglise et Ripart.}

Arbrisseau élevé; aiguillons dilatés, comprimés à la base, inclinés ou droits, ceux des rameaux florifères plus petits, rameaux à écorce vineuse, les jeunes pousses souvent lavées d'un rouge vineux; pétioles glabres, sillonnés en dessus, parsemés de rares glandes, inermes ou quelquesuns faiblement aiguillonnés; $\dddot{b}-7$ folioles ovales, ovaleselliptiques ou obtuses, glabres, d'un vert clair en dessus, plus pâles en dessous, à nervure médiane ayant principalement à la base quelgues petits acieules, doublement dentées; stipules glabres, bordées de glandes, oreillettes aiguës, droites: pédoncules très-courts (5-4 millim.), glabres, réunis en bouquet, ayant à leur base des bractées 
ovales, appendiculées au sommet, glabres, bordées de glandes, plus longues que les pédoneules; tube du calice ovö̈de-allongé, glabre; divisions calicinales glabres, spatulées an sommet, 2 entières, 5 pinnatifides à appendices courts, filiformes, plus courles que la corolle, réfléchies à l'anthese, puis se relevant et caduques avant la coloration du fruit; styles hérissés; disque un peu conique; flem rose on d'un rose pále; fruit gros, rouge, obovoïde affectant une forme pyriforme.

Ilas. Mai, juin. Bois, haies. - France. Cher : bois de Soye (Ripart), vignes de la Chapelle-saint-Ursin, vignes de Couët près de Mehun, forêt d'Allogny.

196. Mr. midas Webb; Bourgeau, exs., plantac canaricuses, $\mathrm{n}^{\circ} 532$.

Has. - Ile Palma, cumbre de Garafia (Bourgeau). — Afrique. Madère (Masson, in herb. Boissier).

\section{c). Hispidae.}

Déséglise, ohs. on the differ. meth. propos. for the classif. of the spee. of the gen. rosa, in the Yaturalist, $11^{\circ} 20$, p. 512 (1865), part.; Crépin, primit. monog. ros., fase. I (1869), p. 18; Cottet, I. c., p. 40.

Pétioles glalores ou à peu près, plus ou moins parsemés de grandes, feuilles glabres, à nervures secondaires dépourvues de glandes, simplement ou doublement dentées, pédoncule et tube du ealice plus ou moins hispides-grlanduleux.
1. $\left\{\begin{array}{l}\text { Aiguill.ns dégénérant au sommet des rameaux } \\ \text { floriferes en soies sétacées . . . . . } 2 . \\ \text { Aiguillons ne dégénérant pas en soies. } 4\end{array}\right.$ 
2. $\left\{\begin{array}{l}\text { Folioles doublcment dentées } . . \\ \text { Folioles irrégulièrement dentées, styles glabres }\end{array}\right.$ óu très-obscurément hérissés . . . . .

Folioles suborbiculaires, divisions calicinales glabres sur le dos, fleur grande, d'un beau rose.

5. Folioles ovales, d'un vert sombre, divisions calicinales parsemées de glandes, fleur moyenne, rose

interveniens.

Chaberti:

lalebrosa.

4. Folioles simplement ou irrégulièrement dentées §ै.

Folioles doublement dentées . . . . . . 12

Folioles ovales-arrondies, irrégulièrement dentées, la majeure partie à dents simples, d’autres

3. \{ folioles doublement dentées, styles velus, fleur rose . • . . . . • . . .

Folioles toutes doublement dentées . . . . 6 .

Styles glabres, fleur blanche, folioles ovales, tuhe du calice ovoïde, fruit subglobuleux . . edita, ohs.

Styles velus ou hérissés . . . . . . 7 .

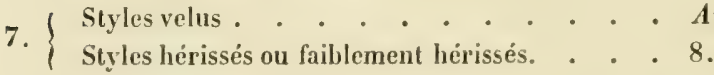

Styles hérissés . • • • • • • • • . 9.

Styles faiblement hérissés, presque glabres, folioles médiocres, ovales-arrondies, tube du calice glanduleux, divisions calicinales glandulcuses, fleur rose clair, fruit obovoïde . . Rorisselii. fruit petit, presque arrondi. . . . . surculosa.

Tube du calice ovoïde . . . . . . . 10.

(Folioles larges, ovales-arrondies, fleur grande, d'un beau rose . . . . . . . . transmola.

Folioles médiocres, ovales . . . . . 11.

Pédoncules parsemés de soies glanduleuses, tube du calice glabre, fleur grande, d'un beau rose, fruit gros, ovoïde-allongé, affectant une forme

11. ellipsoïde

vinealis.

Pédonculesglandulcux, tube du calice glanduleux, fleur rose clair, fruit ovoide . . . . .

Andegavensis. 
12. Folioles petites, styles glabres, fleur petite, rose. Pousine.

12. Folioles plus ou moins grandes . . . . 15.

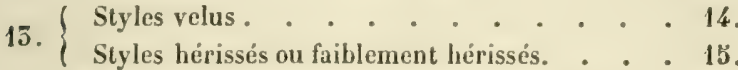

Styles hérissés ou faiblement hérissés. • • 15.

Folioles ovales, tube du calice globuleux, fruit globuleux . . . . . . . . . Martini.

14. Folioles assez larges, suborbiculaires, tube du calice ovoïde, fleur grande, rose, fruit gros, ellipsoîde

Acharii.

1\%. S Folioles ovales-arrondies, tubẻ du calice ovoïde, glabre, fleur grande, d'un beau rose, fruit ovoïde Folioles ovales, ovales-aiguës ou elliptiques . .

psilophylla.

16.

Péduncule et tube du calice chargés de petites

16. soics glanduleuses, folioles ovales . . . .

Pédoncule et tube du calice non chargés de soies

aspernata.

17.

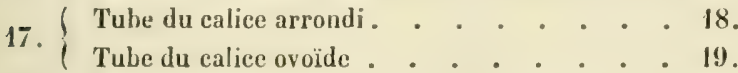

Folioles ovales aiguës, styles faiblement hérissés,

18. tleur rose, fruit subglobuleux . . . firma.

Folioles ovales-elliptiques, styles très-hérissés, fleur grande, d'un beau rose, fruit obovoïde . Haberiana.

19. $\left\{\begin{array}{c}\text { Folioles ovales-aiguës . . . . . . . . } \\ \text { Folioles ovales, fleur grande, d'un rose carné, } \\ \text { fruit gros, ovoïde. . . . . . . . . }\end{array}\right.$

Chavini.

Tube du calice ovoïde-allongé, glabre, fleur rose clair, aiguillons épars. . . . . . . Sulserti.

20. Tubedu calice ov oïde, glabre ou glanduleux, fleur rose, aiguillons en spirale ou formant presque un verticille.

inconspicua.

197. R. Ponzài Tratt., I. c., II, p. 112; Desportes, I. c., n 1955; Déséglise, essai monog., in mém. soc. Acad. de M.-et-Loire (1861), X. p. 115, et extr., p. 75; Cariol, II, p. 676; Verlot, I. c., p. 115; R. micrantha DC., fl. fr., V, p. 559 (non Smith); Poiret, diet. encycl., IV, p. 714; R. graveolens Gren. et Godr., l. c., p. 561, pr.part. (non Grenier, fl. jura.); R. Diomedis Grenier! in Billot, exs. 


\section{Exs. Billot (suites), 11 ${ }^{\text {os }} 5721$ ! 5712 bis! 5850 !}

Hab. Juin. - France. Ilérault : Saint-Loup pres de Hontpellier (DC. !) ; - Bouches-du-Rhòne: Marseille (Roux, in herb. Grenier); - Gard : Anduze (Miergue), Vigan (Tuezkiewicz); - Var: bois des Maures aux Escarcets, colline des Melen au Camnet (Janry); - Aude : montagne Noire, le Mas-Cabardès (Ozanon); - Isère : château de Verna près de Crémieu (Boullu), vallon J.-J. Rousseau à Pariset, la bastille de Grenoble, Comboires (Verlot); - Rhône : Couzon (Boullu).

Ors. I. M. Grenier, dans le Billotia, p. 121, attribue au R. Pouzini des styles hispidules. M. Grenier ne fait done pas attention aux exemplaires qu'il possède? Le no 5830 de Billot ( $R$. Diomedis Gren.), qui a dû lui passer sous les yeux, a les styles trìs-glabres ! comme les numéros de la mème collection, 5721 et 5721 bis. Je ne vois aucune différence cntre ces numéros pour les séparer.

Seringe, dans le Prodromus, ne s'est nullement préoceupé du type de l'herbier DC., ni du texte de la flore francaise; DC. dit : "les feuilles sont " très-glabres, ovales, petites. " Seringe : "foliolis minimis rubiginoso" glandulosis glabris; " erreur aceptée sans contròle pas MII. Grenier et Godron, dans leur flore de France.

Quant ax $n^{\circ} 5831$, publié par les eontinuateurs de Billot sous le nom de R. Pouzin var. subintrans, R. subintrans Gren,, e'est un rosier de la section Rubiginosae et non une Caninae; nous reparlerons de ce $n^{0}$ en son lieu et place. Cette forme avait été signalée en 1852 par M.H. Boissier et Reuter.

Ons. II. J'ai recu de France, d'Espagne et d'Algérie, un rosier bien voisin du R. Pouzini Tratt., dont il diffère par ses styles obscurément hérissés, ses fouilles plus larges, ses fruits plus gros. Serait-ce le R. Pouzini a. nuda Grenier, in Billotia, p. 121, non Tratt. ? M. Grenier dit : folioles lancéolées : "mes échantillons ont, dans la plante d'Espagne, les folioles ovales, les inféricures orbiculaires; dans l'échantillon de France, elles sont grandes, ovales, arrondies à la base ou elliptiques; dans ceux d'Algérie, les folioles sont ovales, les inférieures elliptiques ou subobtuses.

Je connais seulement cette forme par des échantillons trop incomplets pour pouroir porter un jugement quelconque. - France. Aude: le MasCabardes (Ozanon). - Espagne. Rochers au-dessus de la ville de Venasque en Aragon (T'imbal-Lagrave). - Algrovie. Oasis de Mnechounès près de 


\section{(175)}

Biskra (Balansa) : plante distribuée par M. Balansa en 18\%;, sous le nom de $\boldsymbol{R}$. canina L. var. collina, sans numéro d'ordre.

OBs. III. Une autre forme que je possède a tous les caraclères du R. Pouzini, mais en diffère par ses styles tris-hérissés presque velus, le pédoncule peu ou pas glanduleux. - France. Iterault: Roquehaute près de Béziers (Ozanon); - Aude : le Mas-Cabardès (Ozanon); - Isère : entre le Sappey et la forêt de Porte (Verlot), le Sappey près de Grenoble (Boullu).

198. R. inconsiderata Déséglise, deseript. de qq. esp. nouv. de Ros., in mém. Soc. Acad. de M.-et-Loire (1875), XXVIII, p. 117 et extr., p. 21; R. Hispanica Boiss. et Reuter, pugil., p. 44 (non Niller); R. rubiginosa Cosson, in Bourgeau, pl. exs. (non Lin.).

Exs. Bourgeau, pl. d'Espagne (1849), no 19:; année 1831 , $n^{\circ} 1161$, ce dernier $n^{\circ}$ pro part., représenté par deux échantillons en fruits; l'un est R. rubiginosa Auct., l'autre R. Hispanica Boiss. et Reut. ! plante qui ne ressemble pas beaucoup à une rubigineuse. - En 185̆/4, M. Bourgeau, toujours sous le visa de M. Cosson, a distribué encore le $\boldsymbol{R}$. IIspanica sous le nom fautif de R. rubiginosa L.; l'étiquette ne porte pas un numéro d'ordre, mais seulement l'année 1834 .

II AB. Juillet. Région des montagnes. Espagne. Sierra d'Antequerra (Reuter), Sierra de las Nieves (Bourgeau, $\left.n^{\circ} 195\right)$, Sierra Nevada (Bourgeau, $n^{\circ} 1161$, pr. part.), Aleala de IIenares prís de Madrid (Bourgeau, no 1134), Escurial (Reuter).

Ons. R. Mispanica var. Nevadensis Boiss, et Reut., 1. c., ayant les folioles glanduleuses en dessous, est étranger à eette section; ses styles sont trèsvelus d'après l'échantillon authentique que je possède.

R. II ispanicu var. Escurialensis Boiss. et Reut., 1. c.; d'après les échantillons que j’ai été à même d'examiner dans l'herbier de M. Boissier et qui sont les mèmes que celui que je possède, je ne vois aucune différence avec le type pour on faire une sous-rariété d'après le style du jour. 
199. R. Chavim Rapin, in Reuter, cat. de Genève (1861), p. 69; Rapin, guide Vaud., éd. 2, p. 19:3; Gregnier, f1. jur., p. 256 ; Godet, fl. Jura, sup. ( 1869$)$, p. 75; Fourreau, 1. c., p. 74 ?; Verlot, 1. c , p. 115?; Cottet, 1. c., p. 4 .

Hab. Juin, juillet. Broussailles de la région subalpine. - France. IlauteSivoie : le mont Salève à la base de la grande gorge (Rapin), la Croisette. - Suisse. Valais: mont Clou au-dessous de Bovernier! - Cant. de Fribourg : la Cernaz près de Montbovon (Cottet).

200. Th. Wolfi De la Soie, mss., in Cottet, 1. c., sine descript.

Arbrisseau de 1 mètre à 1 mètre 50 cent. de hauteur; aiguillons des tiges longs, droits, dilatés à la base, souvent géminés sous les pétioles, rameaux droits; les jeunes pousses ont les feuilles souvent d'une teinte vineuse; pétioles çlabres, parsemés de glandes, aiguillonnés en dessous; $\breve{-7}$ folioles espacées sur le pétiole, la terminale aiguë aux deux extrémités ou rétrécie à la base, arrondic an sommet, terminée en pointe courte, les laiérales obocales, cunéiformes à la base ou ovales, d'autre's principalement dans les inférieures ovales-elliptiques, d'un vert sombre, glabres sur les deux faces, la còte porte quelques petits acieules, doublement dentées, à dents principales aigues, ouvertes, terninées par un petit mucron; stipules gglabres, bordées de glandes, oreillettes aiguës, droites ou peu divergentes; pédoncules solitaires ou réunis en bouquet peu fourni, chargés de petits acieules fins, terminés par une glande noiràtre; bractées ovales-cuspidées, glabres, bordées de glandes, un peu serrulées au sommet, égalant ou plus courtes que les pédoncules; ube du calice ovoïde, concert comme les pédoncules de petits acicules; divisions 
calicinales longues, spatulées au sommet, glanduleuses en dessous, 2 entières, $\bar{\jmath}$ pinnatifides à appendices étroits, filiformes, non persistantes sur le fruit; styles courts, obscurément hérissés; disque plan; lleur rose lavée de blane; fruit ovoïle.

Шав. Juin. Broussailles de la région subalpine. - Suisse. Valais: Borernier (De la Soie).

201. 모. Wartini Grenier, 11. jur. (186/4), P. 242; Verlot, 1. c., p. 59\%.

Hıв. - France. Lozère : Bagnols-les-Bains (Grenier); - Gard : Auriat, près d'Aumessas (Martin, in herb. Grenirr); - Isère : la Fauge au Villardde-Lans (Boullu).

202. Ar. surenlosa Woods, trans. of the Linn. societ. (1816), XII, p. 228 et herb. n० 117 et $n^{\circ} 121$; Tratt., 1. c., II, p. 40; R. canina var. surculosa Smith, engl. fl., II, p. 594; IIooker, lorit. fl., p. 359 excl. syn. Swartz; Babington, man., ed. 6, p. 123?; Baker, monog. of brit. ros., in journ. soc. linn., XI, p. 226.

Je conmais ce rosier par un échantillon de Borrer que je tiens de la libéralité de M. J.-G. Baker; voici la description qu'en donne Woods.

"Frutex octo-pedalis, laxus, habitu Rosam caninam vel potius $\boldsymbol{R}$. Bora reri inter el $\boldsymbol{R}$. curvensem referens.

- Rami diffusi, atro-purpurei vel intense fusci, juniores glaucescentes, " nune copiose aculeati nune fere inermes; aculei fortissimi, uncinati, " nune bino-stipulares, nune solitarii, sparsi. Petioli supra tantum sparse " pilosi, aliouquin g'abri, acule is fortibus uncinalis muniti. Stipulae spathu" latae vel lineares, nunc serratx, nunc basi glanduloso-ciliatæ, nunc nisi " apicem ver'sus integerrinae, glabrae, interdum margine pilosae, ex flori" bus propriores latiores et demum folid deficientibus in bracteas ellipti" cas, acuminatas, immutatae. Foliola 7, par superius ef foliolum impar " ceteris majora, acie suprapue nerro tantum pilis raris instructa, celliptica 
* vel subrolunda, acuminata, impar basi cordatum vel ovatum, serrata,

a subtus glabra, obscura, junior'a purpurascentia. Pedunculi 1-24, hic " illic setis sparsis, tenerrimis, pilisve muniti. Receptaculum ovatum, - fuscum, glabrum, diseo convexo. Calycis foliola triangulari-elliptica, " acuta fere usque ad basin divisa, pinnis lanceolatis vel lineari-lanceola" tis, nervosis, integerrimis. Flores rubeseentes. Styli subporrecti, villosi; - stigmata in globulum congesta. Fructus late ellipticus, ruber. n

About Albourne, IIenfield, West Grimstead, and elsewhere in Sussex. Borrer (Woods). L'échantillon que je possède vient de IVest Grimstead (Borrer).

Oss. J'ai récolté dans le département du Cher un rosier qui se rapproche beaucoup du $R$. surculosa Woods; il en diffère par ses styles glabres et sa fleur blanche.

16. edita Déséglise, herb. - Arbrisseau élevé; rameaux flexueux, les uns inermes, d'autres aiguillonnés, à aiguillons dilatés à la base, inclinés au sommet, écorce verdâtre ou vineuse; pédoncules réunis par 4-3-7-10 en corymbe, glabres, quelques-uns portent de rares glandes (comme dans l'éclıantillon de Borrer); styles glabres; disque plan (dans l'échantillon anglais, les styles sont entourés de poils i la base et glabres au sommet dans le spécimen en fruit, obscurément hérissés dans l'échantillon en fleur); divisions calicinales plus courtes que la corolle (exactement conformées comme dans le type anglais); étamines d'un beau jaune; fleur blanche (Woods dit: flores rubescentes); tube du calice ovoide, glabre; fruit petit, subglobuleux, altenué au sommet (de mème forme que dans le spéeimen de Borrer! mais Woods dit: late ellipticus); feuilles glabres, simplement dentées.

II Bruère, dans le sentier qui passe sous le viaduc du chemin de fer.

203. R. abstenta Nob.; $R$. canina var. collina Boissier, fl. Orient., II, p. 685 (non Jacq., et exel. syn.).

Exs. Kotschy, $n^{\circ} 5 / 47$ !

Description établie sur un magnifique échantillon de la collection Kotschy, que je possède.

Arlorisseau....; d'après l'échantillon, les rameaux paraissent tortueux, peu aiguillomnés, à aiguillons petits, dilatés 
eomprimés à la base, crochus ou inclinés au sommet; pétioles glabres. parsemés de rares glandes, munis de quelques poils blanes surtout à l'insertion des folioles et qui me simblent cadues à l'ìge adulte, aiguillonnés; 丂̆-7 folioles toutes pétiolées, ovales, ovales-elliptiques, d'autres obovales, glabres sur les deux faces, épaisses, fermes (me paraissant d'un vert glateeseent), nervures secondaires apparentes, quelques folioles ont la nervure médiane munie de quelques petits acicules, d'autres l'ont parsemée de poils et enfin il y a des folioles à nervure médiane parfaitement lisses, simplement dentées, à dents aiģuës; stipules glahres, bordées de glandes, à oreillettes aiguës, droites; pédoncules solitaires ou billores, parsemés de glandes, ayant à leur base deux bractées opposées, assez larges, ovales-cuspidées, lavées d'une couleur vineuse, glabres, bordées de glandes, plus longrues que les pédoncules; tube du calice...... ; divisions calicinales longuement cuspidées au sommet, 2 entières glabres en dessous, j pinnatifides glanduleuses en dessous à appendices étroits, filiformes; styles glabres; disque conique; fleur...... fruit pas cncore arrivé à la maturité, hispide, globuleux.

Har. Juin. - Perse australe. Mont Kuh Delu, prop. Schiras (Kotschy).

OBs. M. Boissier cite aussi un $n^{\circ} 257$ de Kotsehy (1) à l'appui de son R. canina var. collina; le numéro est étranger aux Caninae hispidue.

204. Fr. Andegavensis Bastard, essai fl. de M.-etLoire (1809), p. 189 et suppl. (1812), p. 29; Loisel., notice(1810), p.81; DC., f1. fi., V (181:3), p. ว̈59; Lenan,

(1) Il y a certainement une erreur de typographic dans sa flore d'Orient? ce $n^{\prime}$ est pas $n^{\circ} 257$, mais bien 257 qu'il faut lire. 


\section{( 180$)$}

bull. philom. (1818), extr. p. 9; Thory, 1. c., p. 107; Tratt., I. c., II, p. 56; Rehb., fl. excurs., $\mathrm{n}^{\circ} 4005$; Boreau, l. e., éd. $2, \mathrm{n}^{\circ} 676$, éd. $5, \mathrm{n}^{\circ} 836$ et catal. de M.-et-Loire, p. 79; Arrondeau. fl. Toulous., p. 126; Reuter, eat. Genève (1861), p. 70 ?; Déséglise, in Billot, arch. de la fl. de Fr. et d'Allem., p. 534 et in mén. soc. Acad. de M.-et-L., X, p. 11 है et extr., p. 7 3̈; Baker, rev. of the Brit. ros., p. 51; de Hart.-Don., I. c., p. 251; Cariot, l. c., II, p. 178; Fourreau, 1. c., p. 74; Verlot, I. c., p. $115 ;$ R. canina var. grandidentata Desvaux, jour. bot. (1815), II, p. 115; Saint-Am., fl. Agen., p. 203̈; R. canina var. glandulifera Woods, 1. c., p. 223 et herb., $n^{\circ} 112$ et $n^{\circ} 114$; $R$. canina var. hispida Desvaux, I. c.; Seringe, in DC., prod., II, p. 614; Duby, bot. gall., I, p. 178; Delastre, fl. de la Vienne, p. 159; Boreau, 1. c., éd. 1, II, p. 158; Kirschleger, fl. Als., I, p. 248; R. canina var. Andegavensis Desportes, 1. c., p. 88; Baker, monog. of Brit. ros., in Linn. Soeiety's journ., Xl, p. 251; Dumort., I. c., p. 60; R. canina var. hirlelle Gren. et Godr.? I. c., p. 538; R. canina var. glandulosa Grenier, fl. jur., p. 24.5; Godet, fl. Jura, sup., p. 75; R. sepium var. intermedia Desvaux? I. c., p. 117; R. dumetorum var. hispida Cheval., I. c., II, p. 694; R. sempervirens Bastard, I. e., p. 188 non L.; Rau, enum. ros., p. 120; R. Raui Tratt., l. c., p. 53; Bl. et Fing., 1. c., p. 651 ; R. tortuosa Wierzbicki, ex exempl. auth.!; R. dolosa Godet, l. c., suppl., p. 72 (non Wendl.) vu exempl. auth. herb. Rapin!

Icon. Redouté, les roses (1824), livrais. 16, B.

Exs, Wirtgen, pl. crit, $n^{\circ} 545 \%$; Déséglise, herb. ros., $101 \%$. 
Has. Mai, juin. Ilaies, bois. - Belgique. Prov. de Namur : Rochefort (Crépin). - France. Vosges : Labresse, Presle(Pierrat); - Maine-et-Loire: Angers (Boreau), Anjou (Bastard, 1815, in herb. DC.); - Loire-inférieure: Saint-Aignan près de Nantes (Lloyd); - Loiret : Orléans (Saint-Hilaire, 1812, in herb. DC.), Saint-Denis-en-Val (Jullien); - Cher : C. Bourges, Berry, Mehun, Vierzon, elc. ; - Allier : les Gazeriers (Lamotte); - Còted'Or: Meursault (Ozanon), Maxilly ! - Saône-et-Loire : Chålons-sur-Saône (Ozanon); - Aude: le Mas-Cabardès (Ozanon); - Haute-Garonne : Toulouse (Timbal-Lagrave); - Rhône: Lyon (Boreau); - Isère : le Sappey et la forèt de Porte, Paritet (Verlot); - Haute-Savoie : HabèrePoche (Puget). - Prusse. Coblence (Wirtgen). - Autriche. Csiklova in Banat (W ierabicki). - Suisse. Cant. de Neuchàtel : abbaye de Bevaix (Godet, in herb. Rapin); Valais : Bovernier (de la Soie); - cant. de Fribourg : Montbovon (Cottet).

OBs. Le R. Andegavensis présente les formes suivantes. Nous ne leur consacrons pas de numéros spéciaux, mais sans vouloir pour cela diminuer en rien leur importance.

20k/1. R. agraria Ripart! R. agrestina Ripart olim.

Exs. Déséglise, herb. ros., n ${ }^{\circ}$ 18? Billot (suites), $n^{\text {os }} 5722,1476$.

Port du R. Andegarensis, aiguillons robustes, dilatés, comprimés à la base, inclinés ou droits, ceux des rameaux florifères plus grèles; pétioles glabres, glanduleux, parsemés de poils dans le sillon, aiguillonnés; $\breve{-7}$ folioles ovales-aiguës, ovales-elliptiques, quelques-unes subobtuses, glabres, vertes en dessus, glaucescentes en dessous, simplement dentées; stipules assez grandes, glabres, bordées de glandes, oreillettes aiguës, droites ou divergentes; pédoncules solitaires ou en bouquet, parsemés de quelques soies glanduleuses, ayant à leur base de larges bractées ovales-euspidées une souvent rifoliée, grlabres, plus longues que les pédoncules; tube du calice ovoïde ou obovoïde, glabre ou hispide à la base; divisions calicinales glabres en dessous, 2 entieres à bords tomenteux, 5 pimnatifides, 
saillantes sur le bouton, plus courtes que la corolle, rélléchies à l’anthèse, caduques; styles glatres ou très-obscurément hérissés; disque un peu saillant; fleur d'un rose pâle; fruit ovoïde, rouge.

Has. Mai, juin. Haies, bois. - Belgique. Prov. de Namur : Ave (Crépin). -France. Vosges: Cornimont (Pierrat); - Loir-et-Cher : Menetou-surCher, Salbris: - Cher : la Roulanne commune de Nisdun, Achères, Bourges, la Servauterie, Mehun, Marmagne, Berry ; - Puy-de-Dome : Clermont ; - Doubs : Besançon (Paillot); - Rhône : mont Verdun (Chabert), Craponne (Boullu); - Hautes-Alpes : Gap (Burle); - Haute-Savoie: Thonon (Puget).

20//2. Fo. Lemaitrei Ripart, in Verlot, cat. pl. Dauph., p. 394 .

Voisin du R. Andegarensis Bast., dont il diffère par ses styles glabres, ses folioles plus petites, douhlement dentées. (Ripart, in litt.).

IAs. Juin, juillet. Iaies, - Angleterre. Cornwall : Saint-Johs et Irelay (Briggs). - France, Cher : Fussy (Ripart); Hautes-Alpes : indiqué par N. Verlot à Freissinouse près de Gap. - Suisse. Jura de Bâle (Christ).

20\%/5. Fh. comdensata Puget, in Billotia, p. 118.

Exs. Billot (suites), $n^{\circ} 5846$.

Voisin du $R$. Andegacensis Bast., dont il differe par son port touffu, ses folioles plus courtes et plus obtuses, ses stipules plus grandes, ses pédoncules moins hispides, ses styles velus, son fruit subarrondi.

НАв. Juin, juillet. Broussailles de la région montagneuse. - HauteSavoie: Habère-Poche, Ilabère-Lullin (Puget).

\section{4/4. R. purpnrascens Ripart, mss.}

Voisin du R. Andegarensis Bast., dont il diffère, d'après les notes de M. Puget, par ses rameaux et tiges rougeàtres, aiguillons droits ou peu inclinés, ses stipules et bractées rougeàtres, ses pédoncules lisses ou légèrement glandu- 


\section{( 183 )}

leux, fleur grande, d'un beau rose, feuilles simplement dentées, styles velus.

Haв. - Habère-Lullin (Ripart), environs de Mouticrs, Bellevaux, montagne de Veyrier (Puget).

\section{4/5. R. obtusa Ripart, mss.}

Voisin du R. Andegravensis Bast., dont il diffère par ses folioles doublement dentées, tuhe du calice petit, ovoïde, glabre ou hispide à la base, styles glabres, fruit petit, arrondi.

Hab. Bois, haies. - Cher: Garenne de Turly près de Bourges (Ripart), le Colombier près de Vierzon.

203. R. rinealis Ripart, in Déségl., deseript. de qฯ. esp. nouv., Billotia, p. 56 et extr., p. 4 .

Exs. Déséglise, herb. ros., n ${ }^{\circ} 19$.

$H_{\mathrm{AB}}$. Mai juin. Haies. - France. Cher : la Grange-Saint-Jean, Givrai, Quatre-Vents, Turly (Ripart); - Loir-et-Cher : route de Menetou-surCher à Maray! - Hautes-Alpes : village de Manse près de Gap (Burle). Suisse, Cant. de Fribourg : la Tine près de Montbovon (Cottet).

206. R. verloti Crépin, primit. monog. ros., fase. I, p. 55, sine descript.; Verlot, l. c., p. 594 .

Ce rosier nous étant connu par un échantillon en fruit seulement et manquant de notes, nous ne pouvons pas étahlir une description. M. Verlot, dans son catalogue, aurait dì au moins donner les caractères différentiels.

II৯в. - Isère : le Sappey (Verlot, Boullu).

207. ER. Suberti Ripart, mss.; Verlot, I. c., p. 594, sine descript.

Deseription étahlie sur les notes et échantillons reçus de M. Ripart.

Port du $R$. Andegarensis Bast.; aiguillons des rameaux 
dilatés comprimés à la bas e, crochus ou incline's au sommet; pétioles glalu'es, parsemés de glandes, les uns inermes, d'autres aiguillonnés en dessous; : b-7 folioles ovales-aiguës ou obtuses, glabres, vertes en dessus, glaueescentes en dessous, la nervure médiane de plusieurs folioles porte quelques petits acieules, doublement dentées; stipules étroites, glabres, borlées de glandes, oreillettes aiguës, droites ou divergentes, il y a quelques stipules portant sur le dos des glandes; pérloneules solitaires ou réunis par 2-5, faiblement hispides, munis à leur base de bractées ovales-cuspidées, glabres, bordées le glandes, plus longues ou égalant les pédoncules; tube du calice ovoüdeallongé, un peu contracté au sommet, glalsre; divisions calieinales parsemées de glandes peu abondantes en dessous, 2 entières à borls tomenteux, $\bar{j}$ pimmatifides à appendices bordés de glandes, saillantes sur le bouton, plus courtes que la corolle, non persistantes; styles hérissés; disque presque plan; fleur d'un rose clair; fruit oroüde ou obovoïde, rouge.

IlAB. Mai, juin. Bois, haies. - Angleterre. Devonshire: Woodlands (Briggs). - Belgique. Prov. de Namur: Rochefort (Crépin). - France. Cher : Gérissai, les Quatre-Vents (Ripart), forèt du Rbin-du-bois; Douls : Pontarlier (Grenier); - Pyrén -Orient. : fond de Combes (Ripart); - Loire: Pelussin à la Nadeleine (Boullu); — Rhône : Crapoune (Boullu). - Suisse. Jura de Bâle (Christ).

208. R. IRonsseläi Ripart, mss.; R. dubic Bastard inéd. (1815), in herb. DC. (non Wib.).

Port du R. Andegavensis Bast.; pétioles parsemés de quelques rares glandes fines, faiblement aiguillomnés en dessous ou inermes; 5 folioles acales-arrondies ou briècement aiguës, subobtuses, quelques folioles sont aussi orbirulaives, de moyenne grandeur; plus petites que celles du 
R. Andegarensis, glabres, d'un vert clair en dessus, plus páles en dessous, simplement dentées, a dents aiguës; stipules étroites, glabres, bordées de fines glandes, oreillettes aiguës, droites ou divergentes, les supérieures plus larges; pédoncules ordin. solitaires, portant de fines glandes; bractées ovales-cuspidées, bordées de glandes, ordin. plus longues que les pédoncules; tube du calice ovoïde, glanduleux; divisions calicinales spatulées au sommet, parsemées le glandes en dessous, 2 entières à bords romenteux, e pinnatifides à appendices lancéolés, saillantes sur le bouton, plus courtes que la corolle, non persistantes; styles glabres ou très-obscurément hérissés, quelques cils sont à la hase; disque un peu conique; fleur rose; fruit rouge, obovoïde ou ovoïde, presque arrondi.

IIse. Mai, juin. Ilaies, bois. - France. Anjou (Bastard! 1813, in herb. DC.); - Cher : Gareme de Turly près de Bourges (Ripart) : - Gard : bords des routes à Aumessas (Grenier); - Isère : le Sappey (Verlot).

209. Rr. interveniens Nob.; $R$. occulta Crépin?, 1. c., p. 52.

Port du R. Andegavensis Bast.; écorce des rameaux vineuse ou verdatre, aiguillons des rameaux longs, horizontaux, droits, dilatés à la base en forme de disque, espacés, dégénérant en aiguillons sétacés sur les rameaux florifères; pétioles glabres, parsemés de fines glandes, aiguillonnés en dessous, folioles médiocres, oxales-arrondies, orales, brièvement aiguës ou subobtuses, glabres, d'un vert clair en dessus, plus pâles en dessous, irrégulièrement dentées, il y a des folioles à dentelure simple, d'autres ì dents surchargées de dents aceessoires; stipules étroites, glabres, borlées de glandes, oreillettes aiguës, divergentes; pédoncules ordin. solitaires, hispides-glanduleux, portant des liractées ovales, cuspidées-glabres, plus courtes que 
les pédoncules; tube du calice ovoïde, glabre; divisions calicinales spatulées au sommet, glabres en dessous, 2 entières à bords tomenteux, 5 pinnatifides, saillantes sur le bouton, plus courtes que la corolle, réfléchies à l'anthèse, caduques; styles glabres ou très-obscurément hérissés; disque presque plan; fleur rose; fruit rouge, ovoïde, atténué au sommet, arrondi à la base.

HAB. Mai, juin. Bois. - France. Cher : bois de Givrai, commune de Trouy près de Bourges!

210. R. Iatehrosa Déséglise, notes extr. de l'énum. des rosiers, in the journ. of botany for June $1874 \mathrm{et}$ extr., p. $4 ; \boldsymbol{R}$. occulta Crépin ?, l. c.

HAn. Juin. Haies, bois. - Angleterre. Devonshire : Boxhill, Pennycross, Jamerton, entre Saltash passage et Ernesettle, IIareston, Brixton, haies à Ifemerdon, Plympton, Sainte Mary (Briggs). - France. Cher : bois de Marmagne!

211. R. ambigna Lejeune, revue fl. de Spa, p. 98 ; Seringe, in DC., prod., II, p. 625; R. canina var. Lejeunii Du Mort., fl. belgica, p. 94; R. canina var. Andegavensis Du Mort., monog. des ros. de la fi. Belge, p. 60.

"Germinibus oviformibus glabris, glandulis pedunculis a raris, pedunculis raro glandulosis, foliolis utrinque " glaberrimis duplicato-serratis acuminatis, bracteis pétio" lisque glanduloso-ciliatis. " (Lejeune).

Hab. - Belgique. Verviers et Ensival (Lejeune, fl.), Rochefort? (Crépin). - France. Savoie : Chambéry ? (Paris).

Ons. Ce rosier est complètement passé sous silence dans la flore générale de Belgique (1853), par M. Mathieu; il en est de même dans le manuel de la fors de Belgique (1860), et primitiae monographiac rosurum (1869) de M. Crépin.

En 1861, j'ai reçu de M. Crépin un échantillon étiqueté par lui $\boldsymbol{R}$. Andegavensis Bast.; R. ambigua Lejeune! trop incomplet pour me faire une idée de cette espèce. Cet échantillon de M. Crépin est une sommité florale en fruit, sans aiguillons. Je remarqque que les folioles sont 
simplement dentées (Lejeune dit : duplicato-serratis), les styles sont glabres, les pétioles lisses, églanduleux et inermes.

212. R. Gosingciana Besser, enum. Pod. et Volh. (1822), p. 60, ex exempl. auth. !; Tratt., 1. c., II, p. 48; Boreau, I. c., éd. 5, $n^{\circ} 857$ et catal. de M.-et-Loire, p. 79; Déséglise, ess. monog., extr., p. 76; Fourreau, 1. c., p. 74; R. canina var. intermedia Desvaux, obs. (1818), p. $157 ; R$. canina var. rolundifolia Seringe, in DC., prod., II, p. 615; R. stipulacantha Bastard! inéd. (181 วँ), in herb. DC.

\section{Exs. Unio itiner., 1859.}

IIab. Juin. IIaies, hois. - Russic d'Europe. Podolie (Besser, 1820, 1824, in herb. DC.), Volhynie (Iohenacker). - France. Maine-et-Loire : Anjou (Bastard, 1815, in herb. DC.), Angers, Chalonn's, Beaulieu (Boreau, catal.); - Loiret : Saint-Denis-en-Yal près d'Orléans (Jullien); - Cher : SaintFlorent, Lazenay près de Bourges, Quincy; - Puy-de-Dome : SaintPardoux (Iamotte) ; - Doubs : mont Rosmmont à Besançon ! - Saône-etLoire : Autun (Carion); - Isère : le Sappey (Verlot); - Rhỏne : Lyon au bois de l'Etoile (Ozanon); - Haute-Savoie : Bellevaux (Puget).

Ons. En 1861, dans notre essai des rosiers de la France, nous avons admis ce rosier d'après la grande autorité de $\mathbf{1 1}$. Boreau, puis en comparant nos échantillons à un spécimen que nous possédons en herbier de l'Cnio iliner., ann. 1859, récolté en Vollyynie par 1I. Hohenacker. Depuis nous avons pu étudier et voir un type authentique de Besser dans l'herbicr de M. Alph De Candolle. Ce que nous avons publié sous le nom de R. Kosinsciana est identique à la plante de Besser.

Bastard, dont le coup d'oeil étail si juste quand il s'agissait de distinguer une espèce, avait distribué en $181 \%$, sous le nom de $R$. stipulacantha Bast., un rosier qui est le $\boldsymbol{R}$. Kosinscirnn ; mais, Bastard n'ayant jamais donné une description de son espèce, Besser a la priorité.

215. RR. frma Puget, in Billotia, p. 118; Crépin, 1. c., 1). ว̈2.

Exs. Billot (suites), $n^{\circ} 5847$. 


\section{( 188$)$}

НАв. Juin, - France. Haule-Savoie : Bellevaux, Argonnex, Reyvroz (Puget); - Jura : Salins.

214. T. aspermata Déséglise, notes extr. de l'énum. des ros., in the journ. of botany for June 187/4 et extr., p. כ่ R. saxatilis Boreau! 11. cent., éd. 2, n 678, éd. 5, $n^{\circ} 859$, non Steven; $\boldsymbol{R}$. glandulosa Bor., 1. c., éd. 1, $\mathbf{n}^{\circ} 408$, excl. syn.; R. verticillacantha Baker, monog. of British ros., obs., p. $252 ; R$. aspratilis Crépin?

HAB. Juin. Haies. - Angleterre. Devonshire: Warleigh Wood, Woodlands (Briggs); - indiqué par M. Baker dans Somersetshire, près de Bridge-W Wter et Weston-super-Mare. - France. Nièvre : coteaux de la Charité (Boreau); - Isère : le Sappey (Verlot).

21\%. R. verticillacantha Mérat, f1. Par. (1812), p. 190; Leman, bull. philom. (1818), extr., p. 9; Thory, 1. c., p. 107; Seringe, in DC., prod., II, p. 622.

- Tige de 5-5" pieds, ayant des aiguillons petits, courbes, 4-b゙ ensemble, " presque semi-verticillés; folioles ovales, à dents non glanduleuses, gla* bres; pétioles glabres, très-légèrement glanduleux; pédoncule et fruit * hérissês de poils glanduleux ; folioles du calice presque simples, très" glanduleuses; fleurs solitaires, d'un rose pâle. Se trouve dans les buis- sois au Calvaire. Si cette plante n'avait pas le fruit globuleux, ce serait " le R. Pyrenaica Gou. " (Mérat).

J'ai vainement cherché dans les herbiers de MII. Delesseri, De Candolle et Boissier, le type de Mérat. Dans sa flore des environs de Paris, Mérat ne dit pas si les folioles sont simplement ou doublement dentées; il dit seulement des "folio!es ovales à dents non glanduleuses n; Leman dit : feuilles bidentées.

216. R. inconspicua Nob.; R. verticillacantha plurim. auct., non Mérat.

Arbrisseau à aiguillous nombreux, dilatés à la base, lecourbés au sommet ou presque droits, en spirale et forment presque un verticille autour de la tige; pélioles parsemés de poils à l'insertion des folioles, glanduleux, 


\section{( 189$)$}

aiguillonnés; 3̈-7 folioles ovales-aiguës, ovales-elliptiques, quelques-unes subobtuses, de médiocre grandeur, glabres, vertes en dessus, glauques ou glauceseentes en dessous, la nervure médiane porte quelques petites glandes, doublement dentées, à dents secondaires glanduleuses; stipules glabres, à bords glanduleux, oreillettes aiguës, droites ou peu divergentes; pédoncules solitaires ou réunis en bouquet peu fourni, hispides-glanduleux, bractées opposées, ovales-cuspidées, une souvent trifoliée, glabres, bordées de glandes, plus longues ou égalant les pédoncules; tube du calice ovoïde, glabre ou hispide; divisions calicinales glabres sur le dos, 2 entières à bords tomenteux, $\bar{\jmath}$ pinnatifides à appendices bordés de glandes, saillantes sur le bouton, plus courtes que la corolle, non persistantes; styles hérissés; disque plus on moins élevé; fleur assez grande, rose; fruit rouge, ovoïde.

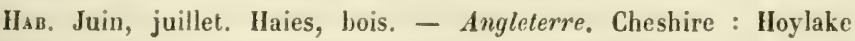
(Wehb). - France. Indre : Sainte-Lizaigne; - Loir-et-Cher : Bcaumont (Franchet); - Creuse : Chamborand (de Cessac); - Cher : Mehun, Berry, vignes de la Chapelle-saint-Ursin; - Saône-et-Loire : Chagny, Thésé (Ozanon). - Autriche. Autriche-supér. : Vorderssorder (Kerner).

Ors. Une forme que j’ai récoltée en fleur seulement en $185 \%$, dans le départ. du Cher, diffère du $R$. inconspicua par ses pétioles à partie interstipulaire glanduleuse sur le dos, ses styles glabres. - Pacage de Langenot, commune de Berry. - M. Lamotte m'a envoyé la même forme de l'Allier : haies à la Roubière près les Gazeriers (Lamotte).

217. R. OEnensis Kerner, Oesterr. bot. Zeitschrift, $n^{\circ} 11,1869$ et extr., p. 4 .

HAB. - Autriche. Autr.-infér. : Schargan (Kerner); - Tyrol : Mühlau, Kranabitten, Sennenhofen, Lemenhof (Kerner).

218. R. Schottiana Seringe, in DC., prod., II, p. 615 (sub R. canina var. Schottiana); R. glauca Schott. ap. Besser, enum. Pod. et Volh., p. 6/4 non Villars; 
Crépin, 1. c., p. 32; R. virgata Gremli! ex exempl. auth.!

Voici la description minutieuse étahlie sur l'échantillon de Besser, conservé dans l'herbier DC. - Besser a ajouté en synonyme sur son étiquette $\boldsymbol{R}$. inermis M.-Bieb.

Arbrisseau....; aiguillons....; rameaux florifères grè̀les, inermes; pétioles glabres, parsemés de glandes fines, aiguillonnés ou inermes en dessous; §ั-7 folioles ovales-acuminées, ovales-elliptiques, glabres, vertes en dessus, glauques en dessous, la nervure médiane lisse ou portant quelques fines glandes, les nervures secondaires un peu saillantes, doublement dentées, à dents secondaires glanduleuses; stipules étroites, allongées, glabres, bordées de glandes, oreillettes aiguës, divergentes, quelques stipules inférieures ont la partie interstipulaire parsemée de glandes peu abondantes; pédoncules solitaires ou réunis par 2-4, légèrement glanduleux, les uns affectent une forme penchée, les autres sont droits, bractée lancéolée, la pointe prenant une forme foliacée, glabre, bordée de glandes, plus longue que les pédoncules; tube du calice ovoïde, contracté au sommet, glabre ou légèrement glanduleux à la base; divisions calicinales longues, lancéolées, spatulées au sommet, glanduleuses sur le dos, 2 entières à bords tomenteux, 5 pinnatifides, longuement saillantes sur le bouton, dépassant la corolle, réfléchics à l'anthèse; styles courts, velus; disque peu apparent ; fleur..... (difficile de reconnaitre la couleur sur un ancien échantillon).

IIı. - Russie d'Europe. Podolie (Besser, 1820, in herb. DC.). - Suisse. Cant. de Schaffhouse : Unterhallau, Wilchingen et Schaffhouse (Gremli). - Aulriche. Autriche-infér. : mont Kegel près de Stein, vallée du Danube (Kerner).

219. R. Acharii Bilb., in Rechb., fl. excurs., II, 


\section{(191)}

p. 619; Boreau, fl. cent., éd. 5, n 846; Déséglise, 1. c., p. 118 et extr., p. 78 ; Cariot, 1. c., p. 179; R. Timeroyi Chabert, in Cariot, 1. c., p. 180 ; ex exempl. auth. !

Icos. Svensk botanik, IX, pl. 577.

IIas. - Puy-de-Dôme : Fontanat près de Clermont ! - Hhône : Lyon au-dessus du pont d'Alai (Chabert).

OBs. Il nous a été impossible de voir un type de ce rosier dans les herbiers à notre disposition Nous avons vu dans la bibliothèque de M. Boissier l'ouvrage suédois Svensk botanil, où la plante est décrite (en langue suédoise) et figurée; la planche représente assez bien la plante que nous avons en vue, seulement les pétioles sont figurés glabres et non ligèrement velus, les bractées sont plus courtes que les pédoncules, les sépales sont figurés avec des appendices plus petits que ceux de notre plante; la description étant en langue suédoisr, il nous a été impossible de nous en servir; nous avons eu recours à Reichenbach.

II. Cariot déerit sous le nom de $\boldsymbol{R}$. Timeroyi Chabert une plante différente de celle distribuce par Chabert. L'auteur de l'étude des feurs attrilue aux folioles des nerrures secondaires glanduleuses, ce qui n'existe pas sur les échantillons types que nous tenons de feu Chabert. Nous ignorons ce que peut-ètre ce $R$. Timeroyi décrit avec des folioles glanduleuses, car nous admettons que H. Cariot, tout en copiant une description doit voir si les caractères assignés sont rigoureusement exacts. La description originale que nous avons de feu Chabert lui-même; pour son $\boldsymbol{R}$. Timeroyi, dit : u la nerrure médiane seule parsemée de glandes. n

220. IR. Chaberti Déséglise, in Cariot, l. c., p. 180. Description établie sur les notes et échantillons recus de feu Chabert.

Arbrisseau de 1-2 mètres, touffu, à écorce rougeâtre ou verdatre, aiguillons épars, dilatés, crochus ou droits, dégénérant sur les rameaux florifères, en aignillons fins, sétacis, les uns terminés par une glande, les autres sans glande; pétioles glabres, glanduleux, aiguillonnés ; 5, rar. 7 folioles, suborlhiculaires, subobtuses ou ovales-arrondies, glabres, vertes en dessus, glauques en dessous, doublement 


\section{(192)}

dentées, à dents aiguës, les secondaires glanduleuses, nervures secondaires un peu apparentes, la còte médiane porte quelques glandes; stipules larges, glabres, bordées de glandes, oreillettes acuminées, droites ou peu divergentes; pédoncules solitaires ou réunis par 5-4, hispidesglanduleux, bractées ovales-acuminées, glabres, bordées de glandes, ordin. plus longues que les pédoncules; tube du calice glabre, ovoüde, contracté au sommet; divisions calicinales glabres sur le dos, spatulées au sommet, 2 entières, 3 pinnatifides à appendices bordés de glandes, saillantes sur le bouton, atteignant presque la corolle, caduques; styles courts, hérissés; disque presque plan ; fleur grande, d'un beau rose; fruit rouge, ovoïde.

II AB. Juin. - France. Rhône : vallon de Dardilly, Charbonnière (Chabert) Ste-Consorce près de Lyon (Boullu).

221. R. Haberiana Puget, in Déségl., descript. de qฯ. esp. nouv. de ros., in Billotia, p. 57 et extr., p. 5 .

Exs. Déséglise, herb. ros., no 20 et 20 bis; Billot (suites), no 5585 .

Has. Juin, juillet. Région des montagnes. - France. Haute-Savoie: Hahère-Lullin, Habère-Poche (Puget). - Suisse. Cant. de Fribourg : haies ì Montbovon où M. Cottet me l'a fait récolter.

222. Fh. Waitziana Tratt., 1. c., I, p. 57 ; Seringe, in DC., prod., II, p. 625; BI. et Fing., I. c., p. 65/4; Rehb., I. c., p. 621 .

HAs. - Allemagne. Altenbourg en Saxe. Je n'ai pas vu ce rosier.

245. R. transusota Crépin, 1. c., p. 18; R. psilophylla Boreau, fl. cent., éd. 2, n 679, éd. 5, no 860 et catal. de M.-et-Loire, p. 79 (non Rau). 
H/B. Mai, juin. IIaies. - France. M.et-Loire : Angers, Saint-Barthelemy (Boreau); - Haute-Saroie: Bellevaux (Puget),

224. R. psingpaym Rau, enum. ros., p. 101; Tratt., 1. c , I, p. 27!; BI. et Fing., 1. c., p. 628?; Rehb., 1. c., p. 619; Gr. et Godr., I. c., p. 358 ?; Grenier, 1l. jura., p. 225?; Boullu, in Billotia, p. 125?; R. gallico-canina Reuter, in Godet, Il. Jura, p. 218 ; Reuter, eat. de Genève (1861), p. 75, non Kirschleg.; Rapin, l. c., p. 196.

Exs. Billot, $n^{\circ} 5577$.

Hıв. Mai, juin. Haies, bois. - Suisse. Cant. de Genève : Compesières (Chavin), Onex (Rapin), Lancy.

Ons. M. Rapin m'a donné une forme récoltée par lui à Onex près de Genève, dont les liges florifères sont plus ou moins chargées d'aiguillons fins sétacés. - Sous le nom de $R$. psilophylla Rau, les auteurs font une confusion; nous-mème, dans notre essai monog., nous avons mal interprété le texte de Rau, et ce que nous avons donné comme $\boldsymbol{R}$. siplophylla est étranger ì la section Caninae. A quelle forme ce nom de $R$. psilophylla Rau s'applique-t-il exactement? Trattimnich dit : foliolis supra pubescentibus; ce qui ne concorde pas avec le caractère assigné par Rau : foliolis glaberrimis. MII. Grenier et Godron décrivent le R. psilophylla avec des feuilles glabres ou pubescentes et dommant pour localités Lyon et Angers : les ćchantillons que nous avons été à même de recevoir de ces localités ont des feuilles glabres.

225. A 1. c., p. 74; Boullu, in Billotia, p. 122.

Exs. Billot (suites), $n^{\circ} 5725$.

HAB. Juin. Haies. - France. Rhône : lıaies à Francheville (Boullu), au Gau au-dessus du pont d'Alai (Chabert).

226. R. Lagrener Puget, Mss.

Description établic sur les notes et échantillons reous de M. Puget.

Arbrisseau assez élevé, à jeunes rameaux lavés d'un pourpre vincux ou verdalres, aiguillons épars, faibles, 
droits ou peu inclinés, dilatés ì la base' pétioles canalienlés, un peu pubescents dans le sillon, parsemés de glandes, aiguillonnés en dessous; $\ddot{3}-7$ folioles ovales-aiguës ou ovales-elliptiques, quelques-unes obtuses, glabres, d'un vert sombre en dessus, glauques en dessous, la nervure médiane porte de rares petites glandes, doublement dentées, à dents secondaires glanduleuses; stipules glabres, ì oreillettes aiguës, droites ou divergentes, les stipules inférieures sont quelques fois parsemées de glandes; pédoncules hispides-gianduleux, solitaires ou en corymbe peu fourni, munis de bractées ovales-cuspidées, glabres, plus longues qu'cux; tulbe du calice ovoïde, hispide-glanduleux; sépales glanduleux sur le dos, terminés en spatule, 2 entiers, 5 pinnatifides, rélléchis à l'anthèse, puis redressés, persistants jusqu à la coloration du fruit; styles courts, hérissés; fleur d'un beau rose; fruit ovö̈de ou obovoïde, d'un beau rouge.

Ilan Juin, juillet. Rérion des montagnes. - Suisse. Cant. de Fribourg : Ouhanna (Lagger), les Cases d'Allieres (Puget), la Tine prìs de Montbovon où M. Cottet me l'a fait récolter.

227. R. haematodes Boissier, fl. Orient.; II, p. 684. IfAB. - Le Caucase oriental (Ruprecht, in herb. Boissier).

228. Fr. Irampeana Grisebach; Crépin, 1. c., p. 108.

IIAв. - In rupibus Rosstrappe IIercyniae graniticis et in agri Gottingensis saxis calcareis prope IIeiligenstadt.

229. R. Minailensis Boissier, l. c., p. 675.

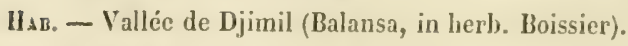

250. R. Soongondea Binge, in Ledeh., fi. Alt., II, p. 227 ; Walpers, repert., 1I, p. 11. 
$H_{A B}$, - La Songarie.

Je ne connais pas ce rosier, dont pas un échantillon n'existe dans les herisiers de Genève; peut-être même ce rosier est-il étranger à cette section?

\section{D) Pubescentes.}

Crépin, primit. monog. ros., fase. I (1869), p. 19 ; Cottet, énum. ros. du Valais, in bull. Soc. Murith. (1874), $n^{\circ} 5$, p. 41; Villosae Déséglise, obs. on the differ. meth. propos, for the classif. of the species of the gen. Rosa, in the Naturalist (1865), no 20, p. 512 , part.

Pétioles velus ou tomenteux, folioles simplement dentées, rarement doublement dentées, plus ou moins velues en dessus ou en dessous, rarement glabres avee la nervure médiane seule velue, pédoneule lisse, styles velus, hérissés ou glabres.

1. Folioles simplement dentées . . . . , 2.

Folioles doublement ou irrégulièrement dentées 12.

2. $\left\{\begin{array}{l}\text { Styles velus. } \\ \text { Styles hérissés ou obscurément hérissés. }\end{array}\right.$

(Fleur d'un rose vif, folioles petites, velues en

5. dessous . . . . . . . . erythrantha.

Fleur rose ou rose clair . . . . . 4.

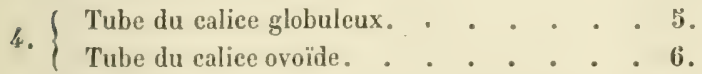

Folioles assez larges, orbiculaires, glabres en dessus, nervures velues, fleur rose clair, fruit

$3 .\left\{\begin{array}{l}\text { oroide } \\ \text { Folioles médiocres, ovales-arrondies, pubesen- }\end{array}\right.$ platyphylla. les en dessus, velues grrisâtres en dessous, fleur rose, fruit arrondi . . . . . . coriifolin.

Folioles petites, à pubescence cendrée sur les

6. $\left\{\begin{array}{l}\text { deux faces, fleur rose, fruit arrondi . cinerosa, } \\ \text { Folioles non pubescentes sur les deux faces. . } 7 .\end{array}\right.$ 


\section{( 196$)$}

Folioles ovales-aiguës, parsemées de poils en dessus, velues sur les nervures, fleur rose clair, fruit ovoïde

urbicu.

Folioles ovales-aiguës ou obtuses, glabres en dessus, nervure médiane seule velue, fleur rose clair, fruit gros, arrondi, rouge salc.

globala.

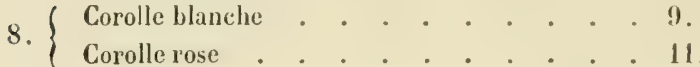

9. $\int$ Folioles pubescentes sur les deux faces, presque

- toutes obtuses, tube du calice ovoïde ou

Folioles non pubescentes sur les deux faces. - 10.

10.

Pétioles et nervure médiane seuls velus, fruit ovoïde . . . . . . . .

Pétioles velus, folioles glabres en dessus, velues en dessous sur les nervures, fruitgros, arrondi

implexa.

Folioles ovales-arrondies, parsemées de poils en dessus, pubescentes en dessous . . .

sphaerocuipa.

11.

Folioles ovales-aiguës, glabres en dessus, parse-

dumetorum. mées en dessous sur les nervures de poils qui disparaissent avec l'âğe, la côte scule reste velue, fruit ovoüde

platyplyylloides.

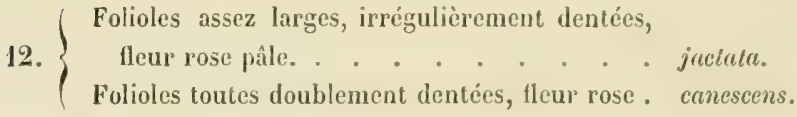

251. R. erythrantha Boreau, fl. cent. de lat Fr., ćd. 5, $n^{0} 850$ et catal. M.-ct-Loile, p. 79; Déséglise, essai monog., in mém. soc. Acad. de M.-et-Loire, X, p. 121 et extr., p. 81 .

HAB. Juin. Haies, - France. Naine-ct-Loire: Anger's, Saumur (Boreau); - Loiret : haies du faubourg Saint-Jean près d'Orléans (Jullien).

252. Th. obtasifulia Desvaux, joutu. loot. ( 1809$)$, II, p. 517; Loisel., notice (1810), P. 82; Tratt., I. c., I,

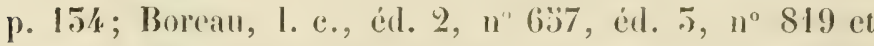
catal. de M.-ct-Ioire, 1). 78; Gr. cl Godr., 1. c., 1. 33̈7; 
Déséglise, I. c., p. 121, extr., 81; de Mart.-Don., fl. Tarn, p. 25!; Cariot, I. c., p. 181; Fourreau, 1. c., p. 75; Verlot, 1. c., p. II6; R. canina var. oblusifolia Desvaux, 1. e. (1815), II, p. II"̈ et obs. (1818), p. 1307; Seringe, in DC., prod., II, p. 615; Delastre, fl. Vienne, p. 1:59; Lloyd, 11. Ouest (1868), P. 176; R. canina var. leucantha Guépin, 11. M.-ct-Loire (1858), ए. 5.57; R. leucantha Bastard, I. e., suppl. (1812), p. 52 (non Loisel., nee

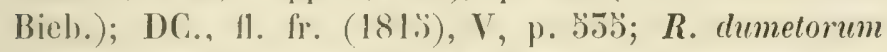
Leman!, hull. philon. (1818), extr., p. 9, ex exempl. R. dumetorum var. obtusifolia Cheval., I. c. (1827), II, p. 692; R. collina var. lencantha Thory, 1. c., p. 72; Desportes, I. c., p. 89; R. collina var. obtusifolia Du Mort., monog. ros. de la 1l. belge (1867), p. 58.

\section{Icon. Redouté les Roses (1824), livrais. 11, B.}

Exs. Schultz, herl, norm., $n^{\circ} 475$; Billot, $n^{\circ} 1664$; Déséglise, herb. ros., $\mathrm{n}^{\circ} 21$.

IIs. Nai, juin. Haies, bois. - Angleterre, Cornwall : près de Craflhole (Briggs); - Devonshire : près d'Elburton (Briggs). - France. Maine-etLoire : Angers (Desraux, 1809, in herb. DC.), bords de la Loire à Angers (Bastard, 1813, in herb. DC..); - Vienne : Pindray près de Montmorillon (Chaboisseau); - Seine: haies i Longchamp (Leman, 1810, in herb. DC.); Loiret : Maison rouge près d'Orléans (Jullien), la Ferté-St-Aubin (Boreau); - Clıer : C., Berry, Mehun, Allogny, ete. ; - Corrèze : Tulle ; - Doubs : Besançon (Grenier); Saône-et-Loire : Autun (Carion); - Lot-et-Garonne : Agen (de Pommaret); - Ilérault : Montpellier (DC., 1807); - HauteGaronne : Toulouse (Timbal-Lagrave); - Rhône: Lyon à Dardilly (Ozanon), Charbomiere (Boullu); - Hautes-Alpes : Gap (Burle); - Var : Aix (Grenier) : - Haute-Savoie : Habère-Lullin, Thonon (Puget). Alsace. forèt de Wasenbourg près de Nicderbronn. - Suisse. Cant. de Schaffhouse, où il serait C. (Christ). - Autriche. Autr.-infér. : Baumgarten pris de Mautern vallée du Danuhe (Kerner) ; - Tyrol : Madona del monte ad Raveredo (Kerner). - Allemagne. Bavière : Iarberg (Christ). 
Ors. 1. R. leucanthre Loisel., notice (1810), p. 82; Mérat, 1. c., p. 195(1812), non Bieb.; R. stylosa corymbosu Desvaux, l. c. (1815), V, p. 115; R. canina var. leucantha Delastre, 1. c., p. 159.

Je n'ai pas vu le type de Loiseleur-Deslonchamps, mais en présence de sa description, ce rosier ne me semble qu'une forme du $R$. oblusifolia à pédoncules nombreux raprochés en curymbe, a dis isions calicinales ayant les appendices larges et la pointe dilatée.

IIАв. Juin. Haies, bois. - France. Eure-et-Loire: Dreux (Loisel., I. c.); - Cher : Berry ! bois de Faix près de Vierzon ! - Yonne : Auxerre (Mabile). - Angleterre. Cornwall : haies à Penters Cross (Briggs).

OBs. II. J'ai vu dans l'herbier DC. un rosier étiquetté par Desvaux : R. puberula Desv. (l'étiquette ne porte pas de localité el seulement la date 1809 ); ce rosier est le $\boldsymbol{R}$. obtusifolia. - Le $\boldsymbol{R}$. dumetorum Leman (non Thuill.), d'après un échantillon authentique qui se trouve dans l'herbier DC., est le $R$. oblusifolia Desv. !

Ons. III. Un rosier distribué par de IIeldreich sans numéro, suus le nom de a $\boldsymbol{R}$. canina L. var. In montibus tophaceis supra Bouldour. Pisidiae. mai 1845 n me semble bien voisin du $R$. obtusifolia, dont il a les caractères: les styles sont moins hérissés et les folioles moins pubescentes dans la plante d'Orient. Je ne parle que d'apres l'echantillon que je possède en fleur seulement; peut-ètre distinct de $\boldsymbol{R}$. olsusifolia, mais il est difficile de se prononcer sur un échantillon incomplet; unr clıose certaine, c'est que ce n'est pas le $R$. canina $\mathbf{I}_{\text {. }}$.

Obs. IV. En 1864, j’ai recu de M. Timbal-Lagrave quatre numéros d'un rosier étiqueté par lui-même $R$. curvula T'imb.-Lag. ! J'ignore si l'auteur a donné une description de ce rosier, ne la trouvant pas dans les ourrages qui sont à ma disposition. The confusion très-grande rigne dans ces 4 numéros. N• 65. R. curvula Timb.-Lag. ; Espagne. Pyrén. d'Aragon : vallée de Venasque. Ne me parait pas autre chose que le R. leucomlha Loisel.; le coractère des divisions calicinales convient très bien. No 64 . $R$. curvula Timb.-Lag. ; Espagne. Pyrén. d'Aragon : vallée de Lessera. Est le R. coriifolia Fries. Nos 91, 92. R. curvulı Timb.-Lag.; Espagne. Pyrén. d'Aragon : vallée de Lessera. Ces deux numéros sont le R. glauer Villars!

255. ER. brachiata Désiglise, descript. de qq. esp. nouv. de ros., in mém. soc. Acad. de M.-el-Loire, IXVIII 
(1875), p. 105, extr., p. 7; R. Orienlalis hort. generensis! (non Dupont).

Hab. - La Perse (Bélanger).

25\%. I2. afisais Rat, cnum. ros. (1816), p. 79 ; Tratt., I. c., II, p. $26 ;$ Bl. et Fing., I. c., p. 628, excl. syn. Wools; Rchb., I. c., p. (i20; Grenicr, 1. jura., p. 245"?

$\mathrm{H}_{\mathrm{AB}}$, - Inveni hanc rosam propre Retzbach in sepibus (Rau). - France. Cher: bois d'Yèvre près de Vicrzon? ubi eam legi 50 mai 1854.

Ons. Mes recherches ont été infructueuses pour trouver un type authentique de Rau dans les herbiers qui sont à ma disposition.

La plante pullice par les continuatears de Billot, no $5720, R$. affinis Ran, n'est pas la plante de Rau !, mais bien le R. Malmundariensis Lejeune, du moins pour ce que j’ai recu. Rau dit: " petiolis indique « villosis subinermibus;.... foliolis supra pubescentibus subtus glau" cescentibus glabris. " Dans le $n^{\circ} 57 \pm 0$, les pétioles sont glabres, les folioles glabres sur les deux faces.

25:). IR. dametogean Thuillier, fl. Paris (1799), p. 230) (non Chevalier); Vérat, I. c. (1812), p. 189; Woods, 1. c., (1816), XII, p. 217 et herb. n 95 à 97; Rau, l. с., p 85\%?; DC., fl., fr., V (1813), p. 5ّ̋4, excl. syn. Gmel.; Tratt., I. c., II, p. $2 \%$ \%; Bl. et Fing., I. c., 1. (5.)1; R(hl)., 11. excurs., $n^{\circ} 5997$ ?; Boreau, bull. Soc. ind. d'Angers (18/4), extr., p. 11 et 11. cent., éd. 2, $\mathrm{n}^{\circ}$ 674, éd. 5, $\mathrm{n}^{\circ}$ 8329, cat. de M.-et-Loire, p. 79; Gussone, syn. Sicul., I, p. 366 ; Gr. et Godr., l. c., p. 3:38?; Godet, 11. Jura, p. 215?; Déséglise, 1. c., p. 125 ctextr., p. 85; de Vart.-Donos, 1. c., p. 252; Grenier, fl. juras., 1. 247, part.; Cariot, 1. c., II, p. 181; Fourreau, I. c., p. 7亏; Baker, rev. brit. ros., p. 27; Verlot, I. c., p. 116 ; R. canina var. dumelorum Desvaux, I. c. (1815), II, 1. I1: et ols. (1818), p. 1\%7; Seringe, in DC., prod., 
II, p. 614 (excl. syn. Rau, Besser, Smith); Dul)y, l. c. 1, p. 178; Delastre, fl. Vienne, p. 139; Baker, monog. of Brit. ros., p. 229; R. canina 2 race pubescens Borean, I. c., éd. 1, II, p. 1.j8; R. canina collina var. Reuter, I. c., p. $70 ; R$. coliena DC., fl. fr., IV., p. $44 \mathrm{I}$ (non Jaeq.); Seringe, mélang. (1818), I, p. 40 exel. syn. Borkh.; R. collina var. dumetorum Thory, I. c., p. 7 1; Du Mort., fl. Belg., p. 94; R. pilosa var. vulyaris Desportes, I. c., p. 88; R. Borkhansii Gaud., fl. helv., ex Seringe!; R. solstitialis Besser, primit. fl. gal., I, p. .224?; Trall., I. c., p. 10 ?; R. submitis Grenier, archiv. de la fl. de Fr. et d'Allem., p. 552 ?

Icon. Redouté, les roses (1824), livrais. 5.), D; Engl. bot. 2579 ? et 2610 ?

Exs. Schleicher, n” 48; Seringe, ros. desséch., n Fries, herb. norm., fase. $7, n^{\circ} 45$; Sendiner, $n^{\circ} 941$; Baker, herb. ros. brit., $n^{\circ} 19 ;$ Billot, $n^{\text {os }} 147.3$ et $1473^{1 / i}$; Wirtgen, pl. crit., $n^{\circ} 77$.

Had. Mai, juin. Haies, bois. - Plante commune en Europe.- Suède. Upsal (Fries). - Angleteme. Yorkshire: Thirsk, Sowerby (Baker); - Middlesex : environs de Londres (Woods); - Devonshire: Egar, Buckland (Briggs); - Cornwall : Millbrook, Jrevol (Briggs). - Belgique. Prov. de Namur : Rochefort (Crépin). - France. Naine-et-Loire: bords de la Loire (Bastard, 1815, in herb. DC.), Angers (Boreau); - Scine : environs de Paris (Thuillier); Loiret: Ardon en Sologne (Iullien); - Loir-et-Cher: Maray !; - Cher : C , Mehun, Bourges, Allogny, etc.; - Puy-de-Dôme : Nurol (Lamy), Clermont!; - Allier : les Gazeriers (Lamolte); - Doubs : Besançon (Grenier); - Saône-et-Loire: Autun (Carion);-Aude : montagne Noire, le Mas-Cabardès (Ozanon); - Rhòne: pont d'Alai (Chabert), Couzon (Ozanon), Tassin (Boullu); — Isère : mont Rachet (Verlot); — AlpesMaritimes: Saint-Martin-de-Lentosque (Bornet); - Haute-Savoie : Bellevaux, Arenthon (Puget), - Suisse. Valais: Bovernier (de la soie); cant. de Bâle : Jura de Rảle (Christ). - Autriche. Autriche-inféricure : 
Pfeffermühle, Klingelbach pres de Bergern (Kerner); - Tyrol : Val di Ledro (Kerner). - Vénélic. Verone (Kerner). - Turquie d'Europe. Bosnie (Sendtner, 1847, in herb. Boissier).

Ons. Le R. solstitiulis Besser est considéré par M. Grenier, dans sa flore jurassique, comme étant la même plante que le $R$. coriifolia Fries : cette assimilation me parait douteuse. Le type de Besser, que j’ai vu dans l'herbier DC., est plus voisin du $R$. dumelorum Thuill. que du $R$. coriifolia Fries. J'ai admis à la plante de Thuillier le synonyme de Besser avec doute, quoique considéré comme étant identique par plusicurs auteurs. Je suis plutòt porté à séparer l'espèce de Besser, d'après le type authentique que j'ai vu, mais pas assez complet pour me prononeer définitivement, ne connaissant pas la forme du fruit. - Je crois devoir douner une description établie sur l'unique échantillon conservé dans l'herbier DC.

L'étiquette de Besser est ainsi concuc: R. collina var. \% milti. Rosa solstitialis primil. fl. gall. 1820. L'étiquelte ne cite pas la localité.

Échantillon haut de 13 cent. ; rameau armé de 12 à $1 / 4$ petits aiguillons géminés, dilatés à la base, droits ou courbés; le rameau porte 8 pétioles pubescents, églanduleux, faiblement aiguillonnés en-dessous; ovales, la terminale un peu acuminée, elliptiques ou obtuses, arrondies a la base, quelques folioles de la base du rameau sont eunéiformes à la base, arrondies au sommet, de moyenne grandeur, pubescentes sur les deux faces, les nervures secondaires velues, blanchâtres, un peu saillantes, irrégulièrement dentées en scie, à dents surchargées de dents aceessoires dents principales aiguies, ciliées, quelques folioles ont des dents profondes, 2 pétioles situés à la base du rameau portent 9 folioles qui ont les nervures seulement velues, l'intervalle est glabre; stipules allongées, glabres en dessus, légèrement velues en dessous, à oreillettes aiguës, dressées, les stipules sont à bords ciliés et parsemés de quelques glandes fines; pédoncules courts, solitaires ou réunis par trnis, glabres; hractées ovales cuspidées, glabres, plus longues que les pédoncules; tube du calice ovoïde, glabre; divisions ealicinales courtes, appendiculées au sommet, à pointe velue, à bords tomenteux, glabres en dessous, 2 entières, $\overline{5}$ pimatifides ì appendices eiliés; styles courts, velus ; disque un peu conique; pétales courts, échancrés au sommet; fruit...... Trattinnick dit a fructus ovalus corcineus " glabris. In en. pl. volh. et porl., p. 65, cel. Besser suam R. solstitialem " pro varietate $0 ., \boldsymbol{R}$. collinae declarat. Sed nimis ambigui sunt limites inter " species et varietates rosarum! Nobis videtur $R$. solstitialis in eodem "sensu species esse, in quo R. fastigiula, uncinclla, calycina, saxulilis, 


\section{(202)}

"dumalis, psiophilla ef plures aliae protalibus deelarapi consueverunt." Trattinnick. I. c

256. Mr. beba Leman, bull. philom. (1818), cxtr.. p. 9; Boreau, 1. c., éd. 5 , no 833 et catal. Ir.-ct-Loire, p. 79; Déséglise, I. c., p. 124 et exir., p. 84; de Martr.Don., I. c., p. 252; Grenier, fl. juras., p. 246, excl. var. b.; Cariot, I. c., p. 181; Fourreau, 1. c., p. 75; Baker, rev. of the Brit. ros., p. 26; Pérard, cat. de Montlue., p. 82; Verlot, 1. e., p. 116; R. collina var. urbica Du Mort., monog. des ros. de la il. belge, p. 58; R. collina var. $\beta$. et $\%$ Woods, 1. e., p. 219 et herb. 96, 98-105 ex Baker; R. canina var. wrbica Baker, monog. of British ros., p. 228; R. Forsleri Smils, Engl. 1. (1824), II, p. 592 ex Baker ; $\boldsymbol{R}$. ranealis Puget, in Déséglise exs.; Fourreau, 1. c., p. 73̈; R. semiglabre Ripart; R. villosiuscula Boullu (non Ripart), in Billotia, p. 120; Verlot, l. c., p. 594; Fourreau, I. e.; R. trichoneura Ripart, in Crépin, l. с., p. 59; Verlot, I. c.; Fourreau, I. с.; k. obscura Puget.

Icon. Engl. bot., XXXVII, tab. 2611.

Exs. Wirtgen, pl. crit., $n^{\text {os }}$ 4.68, 1699; Kralik, plantes de Corse, $1^{\circ} 576$; Déséglise, herl. ros., $1^{\circ}$ 22, 110 $^{\circ} 66$; Baker, herb. ros. Jrit., $1^{\circ} 16$; Billot (suites), $1^{\text {os }} 572 / 4,5849$; de IIeldreich a distribué ce rosier sous le nom de « $\boldsymbol{R}$. canina L. var., Creta in sylcaticis montium. Mai 1846, " sans numéro d'ordre.

Har. Mai, juin. Haies, bois. - Suède. Blekinge (Scheutz). - Angleterre. Yorkshire: Thirsk, Sowerby (Baker); - Devonshire : Iaira (Briggs). France, Maine-et-Loire: Angers (Boreau); - Loiret : Orléans (Jullien); - Cher : C., Berry, Graire, Mehun, Marmagne, ete. ; - Puy-de-Dôme : Clermont ; - Allier : entre les Gazeriers et Susset (Lamotte); - Nièrre : 
Germigny (Jaubert); - Ain : Ilauteville (Franchet); - Jura : Salins, mont Belin ; - Saône-et-Loire : Autun (Carion), Châlons-sur-Saône (Ozanon); - Gard: Anduze (Miergue), le Vigan (Tuezkiewicz); - Isère : Pariset, le Sappey, mont Rachet, Grenoble (Verlot); - Haute-Savoie : Thonon, Pringy, Reyrroz (Puget); - Savoic : Chambéry (Paris), base de Margeriaz (Songeon), Moutiers (Puget); - Corse : Quensa (Kralik). - Suisse. Jura de Bâle (Christ). - Autriche. Autriche-inféricure : Schwargan (Kerner). - Prusse. Coblence (Wirtgen). - Espagne. Pyrén. d'Aragon: vallée de Lessera (Timbal-Lagrave). - Turquie d'Europe. Ile de Crête (de Helureieh).

Ons. Ce rosier présente les formes suivantes, que nous ne conmaissons qu'imparfaitement, nous ne leur consacrons pas de numéros spéciaux, sans vouloir pour cela diminuer en rien leur valeur spécifique.

256/1. 此. nuncalis Puget, in Déséglise, herb. ros., n" 66 ; Cottet, 1. c., p. 41 .

Caractères généraux du $R$. wbica Lem., dont il diffère par ses pétioles inermes, ses feuilles glabres en dessus, ses bractées glałres, ses styles hérissés, son fruit obovoüde. Voici la description de M. Puget.

Arbrisseau élevé, à tiges verditres, longues; aiguillons forts, dilatés à la base, crochus sur les vieilles tiges, les tiges florifères souvent inermes; pétioles velus, inermes; 13-7 folioles ovales, d'un vert paile, glabres en dessus, velues en dessous sur les nervures, simplement dentées; stipules à bords ciliés-glanduleux, les supérieures dilatées, à oreillettes droites; pédoneules solitaires ou en corymbe, glabres; bractécs glabres, ovales-acuminées, plus longnes que les pédoncules; tube du calice ovoüdeallongé, glabre; divisions calicinales glabres en dessous, 2 entieres, 5 pimnatifides; styles courts, hérissés, disque presine plan; fleur blanche ou d'un rose très-pale; fruit oboroüde, rouge sanguin à la maturité.

IIхв. Mai, juin. Haies. - Hautc-Savoie: Thonon les haies sur Crêtes et les 
bois autour du marais de Cessé (Puget); -- Rhône : Lyon a Charbonnières, indiqué par Fourreau, mais je ne connais pas la plante. - Suisse. Valais : Bovernier, Mlayen-de-Sion (Cottet).

256/2. Ex. semairlabara Ripart, in Crepin, primit. monog. ros., fase. I, p. 19 ef p. 89 sine deseript.; Verlot, I. c., p. 594; Cottet, I. c., p. 4.1.

Caractères généraux du $R$. urbica Lem., dont il se distingue par lespétioles et la nervure seuls velus (Ripart, in litt., 25 février $187 \bar{\jmath}$ ).

IIAn. Juin. - Angleterre. Devonshire : Efford Holbeton, près de Yealm Bridge (Briggs); - Yorkshire : près de Thomton-le-Shief (Baker). Belgique. Prov. de Namur : Rochefort (Crépin). - Frence. Cher : Garenne de 'Turly près de Bourges (Ripart), petit hois des vignes de la Chapellesaint-Ursin, Mchun, Savigny-en-Septaine, Bourges; - Rhône : Oullins (Boullu), Charbonnière (Chabert), Tassin (Ozanon); -- Mpes-Maritimes : Saint-Martin-de-Dentosque (Bornet) - Suisse. Valais : Bagnes (Cottet); - cant. de Bâle : Jura de Bâle (Christ).

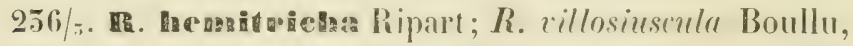
in Billotia, p. 120 (uen Ripart); Fourreau, 1. c., p. 7 :3.

Caractères généraux dı R. urbice Lem., dont il differe par ses pétioles velus et ghlandnlenx, les folioles double. ment dentées (Ripart, in litt., 25 févr. 1875).

IIAB. - Angleterre. Yorkshire : Iaies à Marrick Moor (Baňer); - Devonshire: haies entre Wiverton et Blackpool, Brixton (Briggs). - Écosse. Lanarkshire : Bonington (Ilailstone). - France. Creuse : Grand-bourg (de Cessac); - Isère : Charvieux (Boullu). - Suisse. Jura de Bâle (Christ). - Autriche. Autriche-infér. : Raumgarten, vallée du Danube (Kerner).

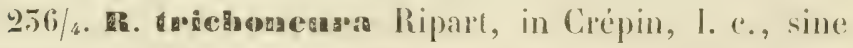
deseript.; Verlot, 1. c., p. 593̈; Cottet, 1. c., p. 41.

Caractères généraux du R. urbia Lem., dont il diffère. par les nervures médianes el latriales relues, ses fleurs roses, ses styles faiblement hírisser, les fenilles sont aussi simplement dentées (Ripart, in lit., 25 férr. 1875). 


\section{( 205$)$}

Has. - Cher : Turly près de Bourges (Ripart); - Pyrénées-Orient. : Montlouis (Kipart).

256/5. RR. obsenara Puret, in Fourreau, cat. des pl. du cours du Rhòne (1869), p. 75 (sine deseript.).

Description ébablie sur les notes et échantillons recus de M. Puget.

Arbrisseau de 1-2 mietres, à rameaux flexueux, verdatres; aiguillons inçgaux, rob!nstes, dilatés, crochus; pétioles pubeseents, porlant quelyues rares glandes fines, aig̨uillonnés en dessous; glabres en dessus, velues en dessous sur les nervures, simplement dentées; stipules glabres en dessus, pubescente's en dessous, à bords ciliés granduleux; pédoncules glabres, solitaires ou réunis par 2-4, portant a leur base des bractées ovales, puheseentes en dessons, égalant ou dépassant les pédoncules; tulse du calice obovoürle, glabre; divisions calicinales glabres sur le dos, 2 entières, 5 pinnatificles, plus courtes ugue la corolle, styles légèrement hérissés, disque ennique; fleurs grandes blanches ou d'un rose très-clair; fruit allongé, ovoöde ou obovoüde allongé.

HaB. Belyique. Prov. de Namur : Rochefort (Crépin). - France. HauteSavoie : Pringy, Argonnex (Puget). - Suisse. Schaflhousse (Gremli). ltalie. Sicile : Palerme (Todaro).

257. He. globata Déséglise, in Crépin, 1. c., lase. I, p. 58 ; sine rlescript.; Fourreau, I. c., p. 75.

Arbrisseau de 1 à 2 mitres de haut, rameaux rerts ou l'ongeitres, anmés d'aiguillons dilatés à la base, crochus ou inclines; pretioles pubeseents, inermes ou faiblement aipuillonnés; ; "j-Tl lolioles ovales-aigguës ou obtuses, glabres en desist whes en dessous sur la nervure médiane, simplenem dentes; stipulesglahres, oreilletesaiguës, droites;

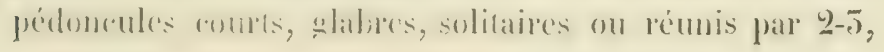


ayant i leur base des bractées ovales-cuspidées, glabres cn dessus, pubescentes en dessous, plus longues que les pédoncules; tube du calice oroüde, glabre; divisions calicinales glabres sur le dos, 2 entières, 3 pimnatilides à appendices ciliés, plus courtes que la corolle, rélléchies à l'anthèse, puis caduques; styles velus, disque peu saillant; fleur d'un rose clair; fruit gros, arrondi, d'un rouge sale.

Hsв, Mai, juin. Broussailles, haies. - France. Savoie: Méry près de Chambéry (Puget); - Ilaute-Saroie : montagne de Veyrier, Mloutiers (Puget); Suisse. Valais : Col de Salvan (Cottet); - cant. de Fribourg : Montbovon.

258. 路. platyphyll Rau, 1. c., p. 82; Tratt., I. c., II, P. 28; BI. et Fing., I. c., p. 628; Rchb., I. c., $n^{\circ}$ 5994; Boreau, 1. c., éd. 5̄, no 85 k el cat. de M.-etLoire, p. 79; Déséglise, 1. c., p. 125 et extr., p. 85; Grenier, fl. juras., p. 215; Cariot, I. c., II, p. 181; Baker, rev. of the British ros., p. 26; Verlot, 1. c., p. 116; Fourreau, 1. c., p. 75; Coltet, 1. c., p. 41; R. arvensis Roth, tent. 11. Germ., II, p. 53\%, exel. syn.; R. opaca Grenier, in Billot, arch. de la 4 . de Fr. et d'Allem., p. 552; R. Chapusii Grodet, 11. Jura, sup., p. 75.

Exs. Billot, no 1478, no 2261; Baker, herb. ros. brit., $n^{\circ} 18$ ?

IlAB. Juin. Ilaies, buissons. - Angleterre. Yorkshire: Thirsk (Baker); - Devonshire: Warleigh (Briggs). - France. Cher : la Servanterie près de Mehun, route de Bourges à Soye, Boursac près d'Allogny; - Yonne : Auxerre (Mabile); - Doubs : Pontarlier (Grenier); - Rhòne: Charbonnière (Boullu); - Ilautes-Alpes : mont Bayard sur Gap (Burle); - IlauteSavoie: Thonon, Habère-Lullin (Puget); - Savoie : Moutier's (I'uget). Suisse. Valais : au-dessus des maisons de la fontaine Feuillée au Fully (Cottet); - cant. de Frihourg. : Monthovon. - Ilatic. Sieile : Palerme (Todaro). 


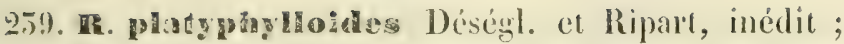
Fourreau, 1. c. p. 7̋̈, sine deseript.; Cottet, 1. c., p. 41 . Arbrisseau aiguillons des vieilles tiges robustes, dilatés ì la base, crochus, ceux de's rameaux florifères petits, dilatés a la base en forme de disque ou dilatés-comprimés a la base, droits ou inclinés au sommet; pétioles velus, portant quelques rares glandes, inermes ou faiblement aiguillonnés; "̈-7 folioles orales-aiguës, ocales-elliptiques, subobluses ou orbirulaires, rertes et glabres en dessus, glaucesecutes en dessous, parsemees de poils sur les nervures qui disparaissent ace l'aige, la coile seule reste velue, simplement denteses, ¿̀ dents terminées par un mueron; pédoncules glabres, solitaires ou réun par par $\mathbf{1}-5$; bractées ovales-cuspidés, glalures, ciliées, égalant ou dépassant les pédoncules: tube du ralice ovö̈le, glabre; divisions ealicinales, 2 enticres, $\overline{~ p i n n a t i f i d e s, ~ g l a b r e s ~ s u r ~ l e ~ d o s, ~ p l u s ~}$ courtes que la corolle, non persistantes; styles obscurément hérissés; disque presque plan ; fleur d'un rose clair; fruit ovoïde.

IIAB. Mai, juin. Ilaies. - France. Cher : Turly près de Bourges (Ripart);-Puy-de-Dome: Clermont ; - Saòne-et-Loire : Châlons-surSaône (Ozanon); - Rhône : Lyon à Tassin (Ozanoin); - Maute-Saroie : Pringy, Saint-Martin près d'Annecy, Brenthonne (Puget).

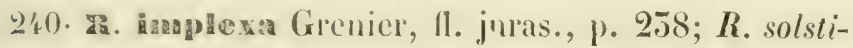
tialis var. demudata Gren., I. c.

Har. - France. Doubs: Pontarlier : (Grenier) ; - Isère : Saint-Christophe-en-Oisans. - Suisse. Jura de Bâle (Christ). - Autriche. Tyrol: Mieders, vallée de Stubai (Kerner).

2ł. IR. jactata Déséglise, notes extr. de l'énum. des ros., in the journal of hotany, for Jume $1875 \mathrm{et}$ extr., p. $\bar{x}$; R. uncinella Auct., an Besser? 
Exs. Lnio itincl., annéc 185̄8? annéc 1.859; Déséglise, herb. ros., $n^{\circ} 67$; Billot (suites), n 5587 .

HAв. Juin, juillet. Haies, bois. - Russie d'Europe. Volhynie (IIohenacker, 1859), Tyrae (Besser!) - France. Ilaute-Savoie : Ilabère-Lullin, Habère-Poche, Saint Germain-sur-Talloires (Puget) ; - Saveie : Puy-Gros près de Chambéry (Paris); - Isère : forèt de Porte, derrière Chanechaude (Verlot).

OBs. Le R. uncinella Bess., publié par l'Unio iliner., an. 1858 et venant du Caucase, diffère de celui publié par la même société en 185̄y : par ses divisions calicinales glanduleuses sur le dos, à appendiees étroits, ses styles velus, les folioles sont à dents plus fines, mais aussi irrégulièrement dentées; ee n'est certainement pas le $\boldsymbol{R}$. uncinclla Besser.

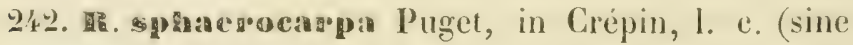
descript.).

Deseription établie sur les notes et échantillons recus de M. Puget.

Exsı, Déséglise, herł). rosar., $1^{\circ}$ (88; Billot, n० 5387 bis.

Arbrisseau assez éleré, touffu, rameaux courts, bruns, portant des aiguillons robustes, dilatés à la base, arqués, rameaux florifères presque inermes; pétioles relus, inermes on faiblement aiguillonnés; "j-7 folioles oxales-obluses ou suborbiculaires, glabres, d'un vert sombre en dessus, plus pailes et velues sur les nervures en dessous, simplement dentées; stipules glabres, larges, à bords ciliés-glanduleux, oreillettes aiguës, droites ou dirergentes; pédoneules lisses, courts, solitaires ou en boupuet peu fourni, portant à leur base de larges bractées glabres, soment denticulces au sommet; tube du calice subglobuleux, lisse; divisions calicinales glabres sur le dos, உ2 entières, 5 pimnatifides, égalant la corolle, non persistantes; siyles courts, obscurément hérisses, disque peu saillant; fleur hlanche; fruit gros, arrondi d'un rouge sale à la maturité. 
HAB. Juin, juillet. Ifaies de la région montagnarde. - Haute-Savoie : Ilabère-Lullin (Puget).

2'5. Casacanica Pallas, f1. Ross. (1788), I, pars 2, p. 62; Marsch.-Bieb., fl. Taur.-Cauc. (1808), I, p. 400, III (1819), p. 5̋̈1; Ait., Kew., ed. 2, III, p. 298; Tratt., mon. ros., I, p. 116, exel. syn. Lindley; Seringe, in DC., pr., II, p. 615̆; Desp., ros. gall., p. 86, exel. syn. Lindley; Rehb., 11. exeurs., p. 621?

IIAB. - Ibérie.

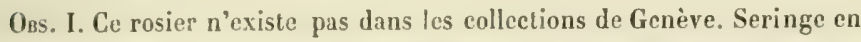
décrivant cette espèce pour le prodromus, a mis simplement la diagnose de Bieherstein en ajoulant on variété le $R$. Cancasica de Lindley. Reste à savoir ce que peut être l'espèce de Lindley.

Le monographe anglais avait certainement en vue un type différent de celui de Pallas! La planche de Lindley représente une plante à feuilles simplement dentées (le texte de Lindley dit le contraire: the serratures are always double), les pédoncules sont hispides. Seringe, 1. c., fait de cette plante le $R$. Caucasica $\beta$. Lindleyana, en ajoutant en synonyme le R. leucantha M.-B., fl. Taur.-Cauc., sup., 5̋I ? Nonne R. caninae var.? An species propria?

Ons. II. J'ai vu dans l'herbier DC. un rosier distribué par Besser en 1820, étiqueté $R$. Cancusica cult. in hort. Crem., de graines du Caucase. Échanti!lon peu conforme à la description de Bieberstein.

Voici les notes prises sur le type de Besser : Échantillon haut de 50 cent., aiguillons de la branche dilatés, droits ou inclinés. Pétioles tomenteux, aiguillonnés en dessous. Folioles 5-7, d'un vert clair, glabres en dessus, glauques en dessous, la nervure médiane velue, un certain nombre de nervures secondaires sont velues, mais pas toutes, ovales, ovales-obtuses ou ohovales, simplement dentées, ì dents fines, aiguës. Stipules glabres, it bords ciliés-glanduleux. Pédoncules longs ( 2 cent.), glabres, bractées ovales-cuspidées, glabres, ciliées, plus courtes qu'eux. Tube du calice ovoïde, glabre. Divisions calicinales terminées en une longue pointe Ienticulé et ciliée, glabres surie dos, 2 cntières, 5 pinnatifides, rélléchies à l'anthise. Styles ohseurément hérissés; disque presque plan. Il est difficile de se prononcer pour la couleur de la fleur sur un ancien échantillon. Je n’ai pas vu le fruit. 
244. Af. Schergkana Boissier, 11. Orient., II, p. 696. Exs. Kotschy, $n^{\circ} 150$.

HAs. - In monte Schergi supra Zebdani in Antilibano (Kotehy, in herb. Boissier).

243. n. cornfolia Fries, novit., ed. 1 (181/4),p. 55 et ed. 2 (1828), p. 1/47; Sprengel, syst. veget., II, p. 3\%34; Seringe, in DC., prod., II, p. 625 ; Rehb., I. e., p. 625; Godet, I. c., p. 215; Reuter, 1. c., p. 69; Déséglise, essai monog., p. 86 ; Cariot, I. e., p. 182; Baker, rev. of British roses, p. 50; Du Vortier, l. e., p. 61; Verlot, l. c., p. 116; Fourreau, 1. c., p. $73 ;$ R. crassifolia Wallm., in Liljebl., fl. Suec., p. 180 ex Sprengel; R. sepium Swartz, ex Sprengel; R. bractescens Woods, 1. c., p. 216 , herh., $n^{\circ} 90, n^{\circ} 91$; Smith., engl. 11., II, p. 591; Hooker, brit. f1. (1855), p. 242; R. frutetorum Besser, cat. hort. Crem., sup. 5, p. 20 et enum. Pod. et Volh., p. 18; Tratt., I. c., II, praef., p. 8; Boreau, 1. c., éd., 2, $n^{\circ} 67.3$, éd. 5, $n^{\circ} 853 ; \boldsymbol{R}$. terebinthinacea Grenier, in Billot, areh. de la 11. de Fr. et d'Allem., p. 555, non Besser; R. solstitialis Grenier, fl. juras, 1). 257 (non Besser); R. cunina var. coriifolia Baker, monog. of British roses, p. 253.

Icon. Engl. bot., third edit., pl. 472.

Exs. Fries, herls. norm., fasc. VI, $1^{\circ}$ 4. 5 ; Scheicher, $\mathrm{n}^{\circ}$ 48; Unio itiner., an. 1859?; Billot, $1^{\circ}$ 1480; Baker, herb. ros. brit., $n^{\circ}$ 2/4; Déséglise, herl. ros., $n^{\circ} 2 \bar{\jmath}$.

Ilar. Juin. Broussailles de la région montagneuse, - Suède. Smaland: Fenrsjö (Fries); - IIalland : Varberg (Scheutz); - Bleckingie : Ellcholm (Scheutz); - Kronoberg : WV exiö (Scheutz), - Russie d'Ẻerope. Russie : Kioviam (Hohenacker); - Volhynic : Podnaïow (Besser); - Galicic (Besser, 1820, in herb. DC). - ingleterre. Yorkshire: Tirsk (Baker); Cheshire: Kisby (Webb); - Devonshire: près de Synlam, Plymouth 
(Briggs). - Écosse. Pertshire : Dunkeld (Ilailstone). - Frunce. Puy-deDòme : les Vergnes près de Riom (Lamotte); - Cantal : Murat (Clisson); -- Doubs : Pontarlier (Grenier); - Pyrénées-Orient. : Eynes (Ripart) ; Haute-Garonne : Bagnères-de-Luchon (Timbal-Lagrave); - Rhône : Tassin, Craponne (Boullu), Grange-Bruyere (Chabert) ; - Isère : Villarsde-Lans (Boullu), le Sappey (Verlot); - Hautes-Alpes: la Grave (Ozanon), col de Bayard près de Gap, Rabou (Burle); Lozère : Pélérinage de SaintPrivat près de Mendes (Prost, in herb. DC.); - Haute-Savoic: Reyrroz (Puget), le Salère; - Saroie : Chambéry, base de Margeriaz (Sungeon). Suisse. Valais : Sembrancher, Bovernier, Salvan, etc.; - cant. de Bâle : Jura de Bâle (Christ). - Aulriche. Autriche-infér. : Krems (Kerner); Tyrol : Múhlau, Krambiehel, val di Non, entre Rocheta et Cles (Kerner). - Espagne. Pyrenées d'Aragon: vallée de l'Essera (Timbal). - Italie. St-Oyen, vallée d'Aoste.

246. IR. cinerosa Nob.; R. cinerascens Cariot, I. c., II, p. 182 (non Du Mort.).

HAB. Juin. Haies. - France. Loire: Chalmazelle (Cariot).

247. H. camescens Baker, rev. of the British roses, p. 28 (non Besser); R. Lomentosa var. incana Woods, l. c., p. 205?; R. caesia var. incana IIooker, 1. c., p. 212?; R. canina var. incana Baker, monog. of British roses.

Exs. Baker, herb. ros. brit., $\mathrm{n}^{\circ} 20$.

IIAB. Juin. IIaies. - Angleterre. Yorkshire : Thirsk, entre Keld et Muker (Baker); - Devonshire : Blackpool (Briggs). - France. Ilautc-Garonne : Toulouse (Ripart). - Autriche. Tyrol : Mieders, vallée de Stubai (Kerner).

Ons. I. Voici une forme voisine du $R$. canescens Baker, qui peut-être en est distincte, mais n'ayant vu que des échantillons incomplets, je ne puis rien affirmer :

R. amblyquyln Ripart, in litteris! a les feuilles ovales-arrondies, obluses: c'est un R. obrusifolin dont les pétioles sont glanduleux et les feuilles doublement dentées, les styles glabres, fleur blanche ou à peine carnée; la forme des feuilles le distingue du R. canescens (Ripart, litt, 25 fér. 1873). 
II AB, - France. Cher: Montpensier (Ripart); - Gard: Anduze (Miergue). - Suisse. Valais : Sembrancher. - Autriche. Tyrol : Madona del monte ad Roveredo, Mont Baldo (Kerner).

OBs. II. at. Guepini Desvaux, fl. d'Anjou, p. $52 \%$; R. collina var. constricta Guépin, fl. Maine-et-Loire.

Il a l'aspect du $R$. collina Jacq., dont il diffère par ses pétioles peu velus, ses folioles beaucoup moins velues, a villosité n'existant que sur les nervures, ses folioles doublement dentées, ses pédoncules glabres, sa fleur. - Il est aussi voisin du $R$. Friedlanderiana Besser, dont il diffère par ses pétioles très-légèrement velus, ses aiguillons ne dégénérant pas en soies sur les rameaux, ses folioles plus grandes, ovales-elliptiques, ovales-arrondies, ses pédoncules et le tube du calice glabres.

HAB. Juin. IIaies. - Belgique. Prov. de Namur: Rochefort? (Crépin). France, Naine-et-Loire: Angers, Sainte-Gemme (Boreau).

\section{E) Collinae.}

Crépin, primit. monog. ros., fase. I (1869), p. 42; Cottet, énum. ros. du Valais, in hull. soc. Murith., fasc. 5 (1874), p. 42; Villosae Déséglise, differ. meth. proposed for the Class. of the spec. of the gen. ros., in the Naturalist (1865), n²0, p. 5̄12, parı.; Caninae L, Déséglise, essai monogr., p. 15; Tomentellae Crépin, I. c., p. 20, part.

Pétioles velus ou tomenteux, folioles plus ou moins pubescentes, simplement ou doublenent dentées, pédoncule hispide-glanduleux.

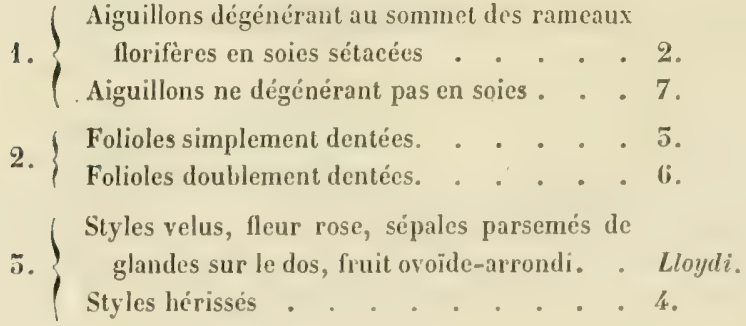




\section{$(215)$}

Folioles médiocres, ovales-arrondies, glabres en dessus, nervure médiane velue, tube du calice hispide à la base, styles obscurément hérissés, presque glabres, fleur rose . fallaciosa.

Folioles grandes. . . . . . . . 5.

Folioles orales-aiguës, glabres en dessus, ner-

i. Folioles ovales-elliptiques, presque glabres en macranthe. dessus, pubescentes grisâtres en dessous, flcur rose, à onglet blanchâtre . . . .

Styles hérissés, folioles assez grandes, ovales ou ovales-elliptiques, glabres en dessus,

6. $\left\{\begin{array}{l}\text { velues en dessous, fleur grande, rose : } \\ \text { Styles velus, folioles suborbiculaires, pubes- }\end{array}\right.$ approximata. centes en dessous, nervure médiane portant quelques glandes, fleur rose . . . . Folioles simplement dentées . . . . . 8.

7. Folioles irrégulièrement dentées ou doublement dentées. . . . . . . 17.

8. 1 Stylesvelus . . . . . . . . . 9.

๑. Styles hérissés . . . . . . . . 12.

9. Tube du calice globuleux . . . . . 10.

Tube du calice non globuleux . . . . 11.

Folioles ovales-arrondies, pubescentes en dessus, velues-grisâtres en dessous, fruit trèsgros, globulcux, couronné par les sépales

10. persistant jusqu'à la coloration du fruit, neur rose. . . . . . . . • .

Folioles elliptiques, pubeseentes en dessus, velues-tomenteuses en dessous, fleur rose carmin, tube du calice hispide.. . . .

Folioles ovales-arrondies, glabres ou presque Boverneriana. glabres en dessus, pubescentes en dessous, pédoneules glanduleux, fleur rose, fruil gros, wrö̈le . . . . . . . . . .

collina.

11. Folioles ovales-aiguës, glabres en dessus, nercerasifera. vures velues en dessous, pédoncules parsemés de quelques soies, sépales persistant jusqu'à la maturité du fruit, fleur rose, fruit obovoïde . . . . . . . Bellavallis. 
12. Fleur blanche . . . . . 15.

Fleur rose. . . . . . . . . 13.

13. $\left\{\begin{array}{c}\text { Fruit pyriforme, folioles ovales-elliptiques,par- } \\ \text { semées de poils en dessus, nervures velues }\end{array}\right.$

en dessous . . . . . . . . imilata.

Fruit non pyriforme. . . . . . 14.

Folioles ovales-elliptiques, glabres, vert sombre en dessus, nervure médiane velue, fruit

14. $\left\{\begin{array}{c}\text { nvolde on subarrondi. } \cdot \cdot \cdot \cdot \cdot \cdot \cdot \cdot \\ \text { Folioles ovales-obtuses, parsemées de poils en }\end{array}\right.$ dessus, fruit obovoïde. . . . . . . trichoider.

IVédoncules réunis en corymbe, folioles ovales,

I5. aiguës aux deux extrémités - . . . corymbifera.

Folioles orales-aiguës, velues principalement

en dessous, pétioles inermes, fruit petit,

16. $\{$ ovoïde ou arrondi . . . . . . .

Folioles ovales-elliptiques, glabres en dessus, nervures velues . . . . . . incerta.

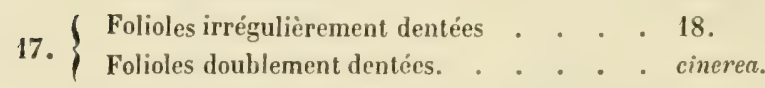

Pétioles tomenteux, parsemés de glandes, faiblement aiguillonnés, folioles ovales-aiguës, tube du calice ovoïde, hispide à la base, pédoncule parsemé de quelques soies, fleur

rose, styles velus
Arbrisseau peu élevé, très-touffu, rameaux

caesia.

18. presque inermes, folioles ovales-aiguës, tube du calice petit, sphérique, fleur rose pàle . arcana.

248. R. conymbifera Borkh., holzart., p. 519 ; Gmel., fl. Bad.-Als., II. p. 424, exel. syn.; Tratı., I. c., II, p. 21; B1. et Fing., I. e., I, p. 625; Borcau, 1. c., éd. 2, II, p. 178, éd. 5, n 849; Déséglise, I. c., p. 127, extr., p. 87; Cariot, I. c., II, p. 182; Fourreau, I. c., p. 75 ; syluestris Tabern., p. 149:3, $1^{\circ} 10$; Rchb., $\mathrm{fl}$. 
cxeurs., II, p. $620 ;$ R. sepium Rau, I. c., p, 80, non Thuill.

\section{Exs. Wirtgen, $n^{\circ} 251$.}

IIsa. Mai, juin. IIaies, huissons. - Belgique. Prov, de Namur : Rochefort (Crépin). - France. Cher: La Servanterie près de Mehun ! - Rhône: route de la Tour de Salvagny (Chabert); - Haute-Savoie: Thonon (Puget); - Var: Le Luc (Hanry). - Prusse-Rhénane: Eifel (Wirtgen).

Ons. J'ai recu d'Angleterre de M. Baker un rosier voisin du R. corymbiferi Borkh., dont il diffère par les divisions calicinales parsemées de quelques glandes sur le dos, ses styles plus hérissés, les folioles simplement dentées, quelques dents profondes surchargées de dents accessoires. M. Baker a mis en synonyme sur son étiquette: $\boldsymbol{R}$. tortuosa Wierzb., in Rchb. fl. germ. exs. $n^{\circ} 1731$ ! Il est possible que ce soit le $R$. tortuosa de Relıb., mais d'aprís un type authentique de Wierzbicki que je possède en herbicr, le numéro de Reichenbach y est étranger ! la plante de Wierzbicki est tout simplement pour nous, d'après le type de l'auteur, le R. Andegawensis Bast.! Je propose pour le rosier d'Angleterre le nom de $R$. incertu Déségl. - Arlurisseau à aiguillons des rameaux dilatés à la base, crochus, tiges arquées, à branches flexibles ascendantes; pétioles velus, aiguillonnés, les inférieurs du ramuscule inermes; 5 -7 folioles ovaleselliptiques, glibres en dessus, velues en dessous sur les nervures, à villosité dispurcissanl avec l'ige, la cute senle resle velue, simplement dentées, quelques dents profondes surchargres de dents accessoires; stipules étroites, glabres, it oreilleltes diverarentes; pédoncules solitaires ou réunis par 2-5̃-4, faiblement aciculis, bractées glalres, terminées en une pointe souvent denticuléc, plus longurs que les pédoncules; tube du calice ovoïde, glabre; divisions calicinales spatulées au sommet, parsemées de fines glandes sur le dos, 2 entieres, 5 pinnatifides, caduques avant que le fruit change de couleur; styles hérissés; fruit ovoïde, rouge, médiocre.

IIAB. Juin, juillet. Haies. - Angleterre. Yorkshire: Sowerby, Thirsk, Studley (Baker).

24!). Ia. Namidiea Grenier, in Déséglise, notes extr. de l'enmm. des rosiers, ap. the journ. botan., for June 1874 , exir., p. ๖.

II.ı. - Alyérie. Haies autour de Constantine (Coste, in herb. Grenier). 
230. IR. Besceglimei Boreau, 11. cent. de la Fr., éd. 5, n" 83̈1; Désíglise, essai monog., in mém. soc. Acad. de M.-et-Loire, X, p. 128 et extr., p. 88; Cariot, I. c., p. 183; Verlot, 1. c., p. $116 ;$ R. urbica var. glandulosa Grenier?, fl. juras., p. 247; R. collina var. Deseglisei Du Mort., I. c., p. 58 .

Exs. Willkomm, it. hisp., $n^{\circ} 77$ ? Billot (suites), $n^{\circ} 3589 b i s ?$ Il me semble exister de İa confusion parmi les échantillons distribués? Les fleurs et les fruits ont été pris sur des buissons différents; il suffit de voir pour s'en convaincre! les styles de l'échantillon en fleurs sont presque laineux, les folioles sont grandes : l'échantillon en fruits a les styles obseurément hérissés, presque glabres, les folioles petites.

Ha b. Juin. Haies, bois. - Belgique. I'rov. de Namur : Rochefort (Crépin). - France. Cher : C., liaies de la Bertherie près d'Allogny, forêt du Rhindu-Bois, haies des vignes de Plante-fou ì Mehun, forêt de Fontmoreau, bois de Morthomier, bois de Marmagne, Cerbois, Vierzon, bois de Soye, Saulzais-le-Potier, la Rouhanne ; - Saône-et-Loire : Châlons-sur-Saône (Ozanon), Saint-Forgent près d'Autun (Carion); - Rhône: Lyon bois de l'Étoile, Charbonnière (Ozanon), Dardilly (Chabert); 一 Jura : sinint-Loup (Puget); - Haute-Savoie: Thonon (Puget). - Alsace. Haies à Niederbronn! - Suisse. Jura de Bâte (Christ).

OBs. I. J'ai reçu sous le nom de R. Deseglisei, un rosier qui me parait tout différent de notre type, j'ai vu trop peu d'échantillons pour me prononcer; j’ai nommé ce rosier en herbier $R$. arcance : en voiei la description que j’ai pu établir sur les notes et échantillons reçus de M. Puget.

250/, т. ихсаиa Déségl, herb.

Arbrisseau peu élevé, très-touffu, à rumeaux courts, presque inermes ou faiblement aiguillonnés; pétioles tomenteux, inermes; ; $;-7$ folioles ovalesaiguës ou elliptiques, parsemées de poils courts en dessus, velues en dessous, șimplement dentées, quelques folioles ont des dents surchargées de petiles dents accessoirrs, les folioles sont dentées à partir des trois quarts du limbe; stipules étroites, à bords ciliés, oreillettes aiguës, Iroites; pédon - 
cules solitaires ou reunis par 2-7, lisses, parsemés de quelques petits acieule's fins terminés par une glande ; bractées ovales-cuspidées, glabres en dessus, velues en dessous, plus longues que les pédoncules; tube du calice petit, sphérique, glabre; divisions calicinales spatulées au sommet, pursemées de glandes sur lo dos, 2 entières, 5 pinnatifides; styles formant une tête très-velue; disque plan; fleur pelite, d'un rose très-pále ; fruit petit, arrondi.

Voisin du R. Deseglisei Bor., dont il differe par son port touffu, ses rameaux courts, moins aiguillonnés, ses folioles irrégulièrement dentées, le tube du calice petit, sphérique, ses divisions calicinales, ses styles trèsvelus.

$\mathrm{H}_{\mathrm{AB}}$. Mai, juin. Broussailles de la région des montagnes. - France. Hautes Alpes : Gap? (Burle); - Haute-Savoie : Moutiers à Ia Chaudanne (Puget). - Suisse. Valais : chapelle Lorette au Bourg-Saint-Pierre.

OBS. II. Le R. hispidule Rip. (non Bast.) ne m'étant comnu que par un seul échantillon en fruit, je ne puis me prononeer définitirement; voici la description que j’ai pu établir sur les notes communiquées par I. Puget.

\section{0/2. It. hispidula Ripart.}

Arbrisseau peu élevé, à rameaux touffus, aiguillons petits, rougeâtres sur les tiges florifères, robustes, dilatés à la base, erochus ou inclinés sur les vieilles tigres; pétioles velus, aiguillonnés en dessous; ; $;-7$ folioles pétiolées, ovales-elliptiques ou obtuses, glabres, d'un vert sombre $e n$ dessus, pâles e'n dissous, a nervure médiane velue, simplement dentérs; stipules glabres ou très-faiblement velues en dessous, à bords ciliés et un peu glanduleux, oreillettes droites; pédoncules courts, lisses ou parsemés de rares petits acicules, solitaires ou en bouquet, portant à leur base de larges bractées ovales-cuspidées les dépassant; tube du calice ovoüde, lisse, divisions calicinales spatulées au sommet, 2 cutières, 5 pinnatifides, églandulruses, réfléchies à l'anthèse, caduques; styles hérissés; fleur blanche; fruit ovoïde on subarrondi.

HaB. Bois. - Ilaute-Savoie: Habère-Lullin, bois du Milieu (Puget).

Oss. III. Le R. trichoidea Ripart m'est peu connu; voici la nole que je tiens de M. Puget, avec un échantillon à l'appui.

$230 / 3$ ז. tricholdea Ripart.

Arbrisseau élevé, aiguillons petits, crochus, nuls ou rares et plus petits sur les rameaux florifères; pétioles velus, inermes ou rarement un peu 
2iguillonnós; ;-7 folioles courtement pétiolées, largement ovales-obtuses, légèrement parsemées de poils apprimés en dessus, pubesecntes en dessous, simplement dentées; stipules glabres en dessus, à orcillettes un peu pubescentes en-dessous, eiliées-granduleuses anx bords, droites ou peu divergentes; pédoncules solitaires ou en corymbe peu fourni, portant de rares glandes fines, souvent quelques poils, 'munis à leur base de bractées à pointe un peu velue, plus longues qu'eux; tube du calice obovoüde, lisse; divisions calicinales appendiculées, velues, égalant la corolle, refléchies à l'anthèse, caduques; styles saillants, hérissés; fleur blanche; fruit olovoüde (Puget).

IIse. - Haute-Savoie : colline du Paradis-sur-Salins près de Moutiers (Puget).

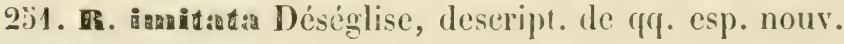
de ros., in mém. soc. Acad. de M.-el-Loire (1875), XXVIII, p. 120 et extr., p. 24; R. pyriformis Désiglise, in Billot, exs., non Swartz; Fourreau, l. c., p. 75 ; Cottet, 1. c.. p. 42 .

\section{Exs. Billot (suites), nº 2588 .}

Haв. Mai, juin. Ilaies, bois. - Belgique. Prov. de Namur: Han-surLesse (Crépin). - France. Cher: ronte de Bourges à Achères, pic Montaigu, Achères près de la Loge; - Doubs: Besancon (Grenier); Rhône: Lyon au pont d'Alay (†zanon), Francheville (Boullu); - HateSavoie: Habère-Poche (Puget). - Suisse. Cant. de Fribourg: la Tine près de Montbovon ; - Valais : Valorsine, Fins-Hauts (Cottet).

2:2. BR. Bellavalliss Puget, in Déséglise, I. c., XXVIII, p. 121 et extr., p. 25; Cottet, l. c., p. 42.

Exs. Déséglise, herb. ros., $\mathrm{n}^{\circ} 69 ;$ Billot (suites), $n^{\circ} 5590$.

Haв. Juin, juillet. Buissons de la région des montagnes, - HautesPyrénées: Gèdre (Ripart); - Isère : Saint-Christophe-en-Oisans (Boullu). - Savnic : forêt d'Apremont près de Chambéry (Songeon); - HauteSavoie: Bellevaux, haies des Mouilles de Terramont, Jambaz (Puget). - 
Suisse. Valais: Bovernier (De la Soic). - Autriche. Tyrol: entre Matre et Steinach (Kerner).

255. a․ approxinata Déséglise, descript. de qฯ. esp. nouv. de ros., in Billotia, p. 57, extr., p. 5.

Exs. Déséglise, herb. ros., $n^{\circ} 31$.

IIAB. - France. Puy-de-Dôme : broussailles rocailleuses sur le versant N. de Mirabelle-aux-Vergnes près de Riom (Lamotte).

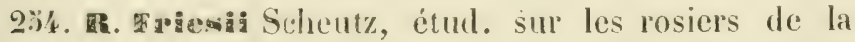
Scandinavie (1872), p. 59; Crépin, 1. c., fasc. 2, p. 128; R. collina Fries, sum. veget., p. $4 \overline{5}$ et p. 175 (non Jacquin).

Exs. Fries, heri). norm., fase. VI, n 42 .

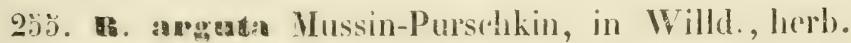
no 9866; Crépin, I. c., fasc. 2, p. 78 (1872), cum descript.

$\mathrm{H}_{\mathrm{AB}}$, - Le Caucase.

236. Th. clivenum Scheutz, l. c., p. 28; Crépin, 1. c., p. 126 .

Haв. - Suède. Lyckeby (Scheutz).

2:7. 2R. caesia Smith, Engl. Lot., tal). 2367; Woods, I. (., P. 212 et herl). no 78; Tratt., I. c., II, P. 14; Smith, engl. fl., II, 1). 389 ; IIost, 11. Austr., II, p. 21 ?

Icon. Engl. bot, thil'd edit., tab. $475 \mathrm{bis}$.

IHAB. Juin, juillet. - Angleterre. Derbyshire : Rerpton (Baker); Staffordshire : entre Alstonfield et Ilam (P'urchas).

2:8. Ar. collina Jaequin, 19. Austr. (1774), II, p. J̈8; Pers., syn. (1807), II, p. 30; Dum.-Cours, bot. cult. (1811), V, P. 482; Rau, I. е. (1816), p. 16.̄; Krocker, fl. Siles., IV (182.), p. I19; Tratl., I. c., II (1825), p. 2; 
B1. et Fing., 1. c. (1825), p. (6.52; Gmel., 1. c., IV (182(3), p. 562; Du Mort., 11. Belg. (1827), p. 9/4 excl. var.; Desportes, I. c. (1828), $\mathrm{n}^{\circ} 2029$; Rehl., I. c. (1850), p. 620; IIost, I. c. (18.1), II, p. 222; Borcau, hull. soc. ind. d'Angers (184/4), cxtr., p. 11, 11. cent., éd. 2) (1849), 1 ${ }^{\circ} 681$, éd. 3 (183̌7), n862, part.; Gonnet, I. c. (1848), p. 479 excl. var.; Arrondean, 1. e. (18:;/), p. 126; Déséglise, 1. c., X (1861), p. 129, extr. p. 89; de Vart.-Don., 1. c. (186/), p. 252; Cariol, I. c. (186ij), II, P. 185; Fourreau, I. e. (1869), p. $7 \dddot{3} ;$ R. campestris Fries, 1 . Halland. (1818), p. 86 ex Desportes; R. collinc var. a. Thory, 1. c. (1820), p. 69; Du Mort., monog. ros.(I867), p. $37 ; R$. dumetorum var. collina Cheval., 11. Paris (1827), II, p. 692; R. dumetorume var. glandulosu Grenier, I. e. (1864), p. 247; R. canina j̄e race hispida J. Boreau, J. с. (1840), II, p. 158; R. canina var. colline Gr. et Godr., 1. e. (1848), I, p. 358 ; Baker, monog. of Brit. ros. (1870); $\boldsymbol{R}$. rigidula Puget et Lagg.

\section{Icos. Jacquin, I. c., tab. 197 opt.!}

IIAB. Juin. Haies. - France. Cher : bois de la Forêt à Saint-Florent, bois de Charron, commune de Marmagne; - Yonne: Auxerre (Mabile); - Rhône : pont d'Alay (Chabert), Craponne au Gau (Boullu); - IlautesAlpes : Laye, sur le mont Bayard (Burle); - Savoie: Salins près de Moutiers (P'uget). - Anglefrere. Deronshire: Plymouth (Briggs). - Suisse. Cant. de Genève: Carouge près le pont de fer; - cant. de Schaffhouse : Jura de Schafhouse (Christ); - cant. d'Uri : Oberintschi (Puget). Autriche. Autr.-inférieure: Oberbeigern près de Mautern (Kerner); Tyrol : Ponale ad lacum Benacum (Kerner). - Prusse. Wurzbourg (Rau, 1817 ! in herb. DC.).

OBs. I. Le R. collina que j'ai décrit en 1861, dans mon essai monographique des rosiers de la France, est identique ati type qui existe dans l'herbier de Linné, d'après l'examen que M. J.-G. Baker a bien voulu faire. La majeure partie des auteurs confondent ce beau rosier arec le 
$\boldsymbol{R}$. dumetorum Thuill. et le $\boldsymbol{R}$. oblusifolia Dest. J'ai vu dans l'herbier de II. Boissier un rosier stiqueté par II. Godet, comme un type du R. collina Jacq.! le $\boldsymbol{R}$. obtusifolia Desv. - J'ai reçu aussi le $\boldsymbol{R}$. collina d'Autriche, venant de la localité classique, que M. Kerner m'a cnvoyé; il est identique à ma plante. - L'English botany (ISOS), XXVII, tab. 1898, sous le nom de $R$. collina Jacq., représente le $R$. systyla Bast.-J'ai pu consulter, dans la riche bibliothèque de $\mathbf{M}$. Nph. De Candolle, le Flora Austriaca de Jacquin, et la belle planche qu'il donme de son type représente bien la plante que j’ai 'u rue. Je ne vois pas, comme le dit M. Crépin, primit. monog. ros., fasc. 1 , p. $6 k$, que le type de Jacquin paraisse obscur. Les auteurs qui décrivent ce rosier ont sans doute pu consulter le Flora Austriaca?

OBs. II. J'ai reçu de MI. Ozanon et Songeon, un rosier qui differe du $\boldsymbol{R}$. collina Jacq. par ses pétioles dépourvus de glandes, inermes en dessous, la nervure médiane non glanduleuse, les pédoncules très-peu lispiles : aspect du R. clumetorum Thuill., dont il diliere par ses divisions calicinales parsemées de glandes, ses pédoncules hispides.

HAв. - Rhòne: Lyon au pont d'Alay (Ozanon); - Savoie: Saint-Jean de la Porte (Songeon), bois Champion près de Moutiers (Puget).

Ous. III. J'ai reçu de M. Puget un rosier qui se rapproche beaucoup du $\boldsymbol{R}$. collina Jàeq., dont il diffère par ses pétioles églanduleux et inermes, ses folioles moins velues en dessous, ses styles allongés, très-velus comme laineux, les pédoneules et le fruit lisses, parsemés de fins et courts petits acicules peu nombreux.

IIab. - Savoie: Salins près de Moutiers (Puget).

Ons. IV. Une troisième forme qui diffère du type de Jacquin par les pétioles églanduleux, les styles glibres ou très-obscurément hérissés, les folioles plus petites.

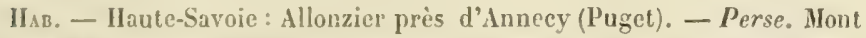

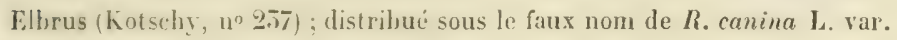
dumelorum.

Ons. V. M. Crépin, I. c., fas. 2, p. 80 , dit que le $R$. collina qui se trouve

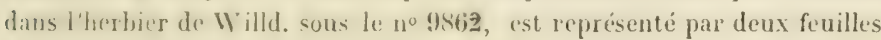
simples: la prenière si ce n'est pas une forme du $\boldsymbol{R}$. coriifolia $\mathrm{Fr}_{\mathbf{r}}$., est une forme se rapprochant du $\boldsymbol{R}$. dumetorum Thuill.; la seconde feuille serait une forme du $R$. arvensis. II. Crépin ajoute: "D’après les maté- 
" riaux de son herbier, il est bien difficile de savoir ee que Willdenow " entendait par son $\boldsymbol{R}$. collina."

2i38/1. ER. Rollacósa Nob.; R. collina Boreau, catal. de Maine-et-Loire (1859), p. 79.

Ce rosier est une forme intermédiaire entre le $\boldsymbol{R}$. collina Jacq. et le $\boldsymbol{R}$. Friedlanderiana Besser. Il diffère du R. collina par ses aiguillons dégénérant en soies sétacées églanduleuses au sommet des rameaux, ses pétioles lisses, glanduleux, non pubescents, ses folioles irrégulièrement dentées, velues seulement sur la nervure médiane, ses stipules glabres, ses styles glabres ou obscurément hérissés. - Par ses aiguillons, il se rapproche du $\boldsymbol{R}$. Friedlanderiana Besser, dont il diffère par ses pétioles lisses, glanduleux, non pubescents, les folioles non régulièrement doublement dentées, à nervure médiane: seule velue, ses stipules glabres, ses bractées, le tube du calice et ses styles.

HAB. Mai, juin. - Maine-et-Loire: Angers (Boreau); - Rhône : SainteConsorce près de Lyon? (Boullu).

238/2. Th. Ioy Nob. R. collina Lloyd, non Jacq.

Ce rosier est intermédiaire entre $R$. collina Jact., et R. Friedlanriana Bess. Il differe du premier par ses aiguillons dégénérant en suies sétacées; du second par ses folioles simplement dentées. Voici la deseription que j’ai pu établir sur un magnifique échantillon reçu de M. Lloyd, spécimen pris sur un pied cultivé rapporté de SaintSébastien.

Arbrisseau.......; aiguillons des rameaux dégénérant en aiguillons fins, sétacés, églanduleux; pétioles tomenteuxglanduleux, aiguillonnés; $\ddot{j}-7$ folioles ovales-arrondies, d'un vert sombre et glabre en dessus, à nervure médiane seule velue en dessous, simplement dentées, à dents ouvertes et profondes; pédoncules 1-4-9, hispides-glanduleux, 


\section{( 245$)$}

bractées ovales-acuminées, glabres en dessus, faiblement velues en dessous, plus courtes que les pédoncules; divisions calicinales longues, spatulées au sommet, glabres et parsemíes de grlandes sur le dos, 2 entières, 5 pinnatifides, saillantes sur le bouton; styles velus; fleur rose.

IIAв, - Loire-inférieure: Saint-Sébastien (Lloyd).

239. 조. canerega Rapin, in Grenier, 11. juras., p. 258 (non Sw.); R. solstilialis var. glandulosa Grenier, l. c.;

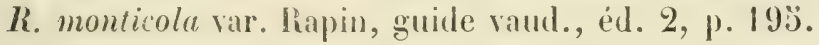

IIas. Juin. Broussailles de la région des montagnes. - France. HauteSavoie: le mont Salève (Rapin).

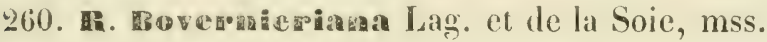

Arirrisseau peu élevé, à aiguillons épar's, dilatés à la base, crochus ou inclinés, souvent geminés, rameaux flexueux, à écorece verdatre ou vineuse; pétioles tomenteux, incrmes ou aiguillonnés; $\ddot{~ ¿-7 ~ f o l i o l e s ~ o v a l e s-a r r o n d i e s ~ o u ~ e l l i p t i q u e s, ~}$ fermes, pubescentes en dessus, velues-grisatres en dessous, à nervures un peu apparentes, simplement dentées, a dents ouvertes, plusieurs surchargées de petites dents accessoires, ciliées; stipules larges, légèrement velues au sommet, oreilletes arrondies, divergentes, à bords ciliés; pédoncules groupés par $\mathbf{2}_{-5}$ ou solitaires, hispides-glandu* leux, bractées larges, souvent terminées par un appendice foliacé, plus longues que les pédoncules; tube du caliee globuleux, lisse ou hispide, glanduleux à la base; divisions calicinales spatulées au sommet, parsemées de glandes sur le dos, 2 entieres à bords tomenteux, 5 pinnatifides à appendices ciliés, saillantes sur le bouton, plus courtes que la corolle, rélléchies ì l'anthèse puis relevées, non conniventes, persistantes jusqu’à la maturité du fruit; styles courts, velus; fleur rose; fruit très-gros, globuleux, d'un beau rouge. 
Ilar. Juin, juillet. Région des mulagnes. - Surde. IIolmeslarl, Helsingborg (Scheutz). - France. Hautes-Pyrénécs : Gèdre (Bordère); - Isère : Saint-Christophe-en-Oisans (Boullu). - Suisse. Valais : col de la Forcla route de Martigny, Bovernier.

261. Th. cenpasiea Timbal-Lagrave, not. sur une excurs. bot. à Bagnères de Luchon, in bull. soc. bot. de France (1869), XI, extr. p. 22.

HAB. Juillet. Région des montagnes. - Espagne. Pyrén.-d'Aragon : entre Sarlé et Venasque ('Timbal-Lagrave).

262. HR. Fored1:anderoana Besser, cat. hort. Crem. (1816), et enum Pod. et Voll., P. 6.); Tratt., 1. c., II, pracf., p. 9; Boreau, 1. c., éd. 2, II, p. 180, éd. 5, $n^{\circ} 86 \bar{j}$ et cat. de M.-el-Loire, p. 79; Déséglise, I. c., p. $1 \overline{0} 0$ et extr., p. 90; Cariot, I. c., II, ए. I8.̄; Fourreau, I. c., p. 73 ; $\boldsymbol{R}$. canima var. pilosiuscula Seringe, in DC., prod., II, p. 615 part.

Un magnifinue exemplaire type de Besser conservé dans l'herbier DC. est identique à la plante décrite par M. Boreau et par moi. Toici les notes minuticuses que jai prises sur l'échantillon de Besser; l'étiquette porte pour localité a ad Tyram. 1820. »

Lin rameau portant 4 ramuscules en fleurs. Aiguillons dégénérant en soies fines, églanduleuses (caractère omis par M. Boreau et par moi : nos plantes ont effectivement ce caractère). Feuilles suborbiculaires, vertes, glabres en dessus, pàles, velues cu dessous principalement sur les nervures, les nervures secondaires apparentes blanchàtres, la cóte médiane de quelques folioles porte 1-2 petits acicules ou quelques rares glandes, doublement dentées, a lents principales ciliées, les secondaires glanduleuses. Stipules à oreillettes élargies, aigü̈s au sommet et portant des glandes éparses, ciliées-gilandu- 


\section{( 225 )}

Ieuses aux bords. Pédoncules hispides-glanduleux, bractées plus longues qu'eux. Tube du calice subglobuleux, contracté au sommet, lisse ou hispide à la base. Divisions calicinales glabres sur le dos, hordées de quelques rares glandes, 2 entières à bords tomenteux, 5 pinnatifides, réfléchies à l'anthèse, non persistantes. Styles courts, hérissés; disque presque plan. Fruit ovoïde-arrondi.

Har. Juin. Haies, bois. - France. Maine-et-Loire : Angers (Boreau) ; Cher : forêl de Fontmoreau aux Brulis!; - Hhône: Lyon à Dardilly (Chabert), Tassin aux Torrets, Chaponost (Boullu).

OBS. Voici une note prise en 1865 sur le port et la forme des aiguillons du R. Friedlanderiana du Départ. du Cher.

Arbrisseau haut de 1 metre 50 cent, à rameaux decombants ou flexucux; aiguillons des tiges assez nombreux, robustes, dilatés à la lase, presque droits ou un peu crochus au sommet, blanchâtres, cenx des rameaux petits, crochus ou droits, rougeâtres, dégénérant en aiguillons fins, sétacés, non glanduleux ; le fruit est d'un rouge orangé, ovoïde, contracté au sommet.

Seringc, dans l'herbier DC., a réuni sous le nom de $\boldsymbol{R}$. canina var. pilosiuscula, les $R$. Ratomsciana Besser, R. Friedlanderiana Besser; $\boldsymbol{R}$. collina Rau, $\boldsymbol{R}$. nilidult Besser.

J'ai reçu de Saulxures (Vosges), de M. Pierrat, un rosier voisin du R. Friedlanderiana Besser, dont il differe par ses rameaux inermes et ses styles glabres; probablenent un type distinct? Mes échantillons sont trop incomplets pour pouvoir porter un jugement.

265. R. saxatilis Steven, in Bicb., fl. Taur.-Cauc., III (1819), p. 548; Tratt., monog. ros., II, p. 11; Ser., in DC., pr., II, p. 614; Crépin, primit. mon. ros., fase. 1, p. 19 et p. 65.

Il m'a été impossible de voir un type authentique, ce rosier ne se trouvant pas dans les herhiers de MAI. DC. et Boissier. Voici la deseription de Bieberstein.

"Germinilus ovatis peduneulisque undique hispidis, " aculeis caulinis sparsis validis compressis recurvis, "petiolis villoso-glandulosis aculeotis, foliolis (majusculis) 
" ovatis inacqualiter arguc scrratis : serraturis sublu"plicatis, utringue glabriusculis subus pallidioribus; " floribus subcorymbosis."

"Affinis proxime praecedentibus (nitidula et Juna dzilliana). Foliola in universum nomihil latiora : serra" turis haul ita subiliter serulatis, sacpe simplicibus. "Irispiditas germinum atque pedunculorum insignis per "totam germinum superficiem extenditur. Flores saepis" sime plures aggregati mediocres. Styli hirsuti. Fructus " $\boldsymbol{R}$. collinue, eui practer glabritiem foliolorum, hace " etiam valde est affinis. " (Bieberstein).

HАв, - In Tauria, sat frequens (Trattinnick).

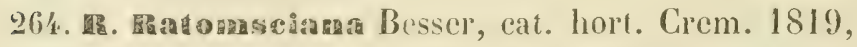
et enum. Pod. et Folh., p. 6:3; R. humilis Besser, cat. hort. Crem., sup. IV, p. 19 (non Tausch); Tratt., I. c., praef. p. 10.

Exs. Steven, pl. Rossiae rar. (sans numéro).

II AB. - Russie d'Europe. Podolic (Besser ! Steven !). Le type de Besser existe dans l'herbier DC. et son étiquette est ainsi conçue: a $R$. Ratom"sciana Mihi. R. humilis Mihi in eat. hort. Crem. conf. not. Nomen " quoniam, frutex jam $\bar{J}$ pedalis nec ex ordine pumilarum. Specimen " mutavi cultam e spontanea 1820 .

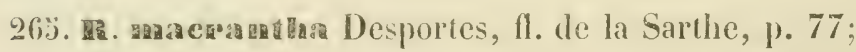
Boreau, l. c., éd. 2, nº 580, éd. 5, no 861 et catal. de H.-et-Loire, 1. 79; Gomnet, I. c., p. 180; Gr. et Godr.,

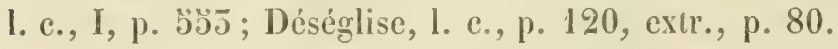

IIs. Mai, juin. - France. Sarthe : la Flèche (Boreau); - Maine-ct-Loire: Angers (Boreau); - Loiret : Chanteau (Boreau); - Rhune: Charbonnière (Boullu),

OBS. Les aiguillons dégénèrent au sommet des rameaux en aiguillons fins, sétacés, églanduleux.

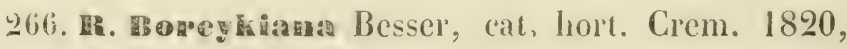


et cenum. Pod. et Volh., p. 63̈; Tratt., 1. c., II, p. 22כ̈; Rehb., l. e., p. 622.

Description établic sur un pied cultive au jardin botanique de la ville de Genève (Suisse).

Arbrisscau ayant le port (lu $R$. albu; aiguillons des tiges griles, unilormes, courlés ou inclinés, dégénérant cn soies granduleuses au sommet des rameaux. Périoles tomenteux, glanduleux, aiguillonnés ou inermes. "̈-7 folioles grandes, larges, ovales-ellipirjues, la terminale arrondie a la base, aiguë au sommet, ghlabres ou parsemées de poils courts apprimés en dessus, puhescentes-grisitres en dessous, à neprures secondaires saillantes, blanchatres, la nervure médiane parsemée de quelyues grandes, simplement dentées, à dents oureries, mucronces, cilices. Stipules grandes, glabres en dessus, ghabres on relues en dessons, mreilletes aignës, diverones, bords cilićs-glanduleux. Pédoneules soliaires ou réunis par 2-5-3-7 en corymbe, hispides-ylanduleus. Bractées orales-cuspidées, sourent foliacées au sommet, égalant les pédoncules, glabres, à bords glanduleux, les corymbes ont a la base 2 bractées ovales, opposées, les pédoncules exlérieurs ont vers leur liers supéricur 2 petites bractées plus comrtes qu’eux, le pédoneule central est dépourvu de bractées. Tube du calice ovoïle, glabre, ayant it la base quelques glandes stipitces. Divisions calicinales longuement appendiculées, a pointe sourent denticulée, hispides-glanduleuses sur le dos, saillantes sur le houton, plus courtes que la corolle, 2 entières, $\bar{x}$ pinnatitides, réfléchies à l’anthèse, caduques. Styles herissés ; disque presque plan. Fleur grande, d'un heau rose i ongrle blanchàtre, pétales obcordés. Fruit rouge, ovoüde, précoce, presque en maturité dans les jardins vers le $15 ้$ août. 


\section{( 228 )}

II AD. Juin. - Russie d'Europe. Podolia australi (Besser, 1820, in herb. DC.). - Frence. Jaute-Garonne: hois de Fonsorbes près de Toulouse? (Timbal-Lagruve). - La plante de Toulouse a les styles glabres, les feuilles moins grandes, les aiguillons plus robustes et les soies glanduleuses rares sur les rameaux florifères.

267. ㄱ. Imar L., sp., 705; IIerm, diss., p. 14; Miller, - diei., n 16; Lour., fl. Coch., I, p. 596; Roh, cent. fl. Germ., II, pars prior; p. ̋61; Moench, meth., p. 689; Lam., fl. fr., III, p. 150?; Krocker, l. c., II, p. 148; Gilinert, pl. d'Etrope, I, p. 582; DC., 11. fr., IV, p. 448; Gmel., I. c., II, p. 427; Pers., I. c., II, p. 49; Willd., enum. pl., ’. 548; Dum.-Cours., I. c., V, p. 484; Rau, l. c., p. 9^; Leman, 1. c., extr., p. 11; Seringe, mél. bot., I, 1. 5:3 M.-Bich., I. с., III, p. 532; Lindley, l. с., p. 81; Trait., I. e., II, p. 4; Seringe, in DC., prod., II, p. 621; Bl. ei Fing., I. c., I, p. 65s; Balbis, f1. Lyon., I, p. 265; Du Nori., fl. Belgica, p. 94, et monog. des ros., p. 46; Chevalier, I. c., II, p. 697; Loisel., I. c., I, p. 562; Desportes, l. c., p. 74; Duby, I. c., I, p. 179; Rchb., I. c., II, p. 625; Lorey et Dur., I. c., p. 5̄l1; Mutel, I. c., I,

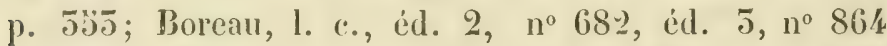
et catal., I.-et-Loire, p. 79; Gonnet, l. c., p. 480; Kirschleger, I. c., I, p. 249; Déséglise, I. c., X, p. 151 et extr., p. 91; Grenier, 1. c., p. 226; Boissier, l. c., II, p. 684; R. usitatissima Gatereau, f1. Montaub., p. 9/4; R. procera Salisb., hort. Allert., 539 ex Desportes; R. canina $^{\text {e }}$ race hispida c. Boreau, 1. c., éd. 1, II, p. 158; Delastre, fl. Vienne, p. 109 .

Icon. Flora Danica, VII, tab. 1215 ; Roessig, die rosen, tab. 15, tab. 25, f. 1, tab. 54; Redouté, les roses (1824), livrais. 6, B.; Blackwell, lıerb., tab. 75; d'après Thory, je 
cite : Besler, hort. Eyst. vern., ord. 6, tab. 5; Miss Lawr., tab. 25; Nouv. Duhamel, VII, n 16, f. 1.

Exs. Seringe, roses desséch., $\mathrm{n}^{\mathrm{os}} 50$ et $\mathbf{3 1}$.

İa. Juin. - Russie d'Europe. Occurit in Iberia, d'après IV ilhelms in herb. Gorenkensi (Bieb., l. c.). - Asie. Colchide près de Kutais (Ruprecht, v. s. s. ! in herb. Boissier). - Afrique. Algérie : lieux montagneux d'Ouleed-Ourid près de Tlemeen (Munby!), flore semi pleno.

Ons. Je n'ai vu d'échantillons que do deux localités où ce rosier soit spontané : celui récolté par Ruprecht, et l'échantillon que je possède en herbier venant de Munby. Les localités françaises données par les auteurs sont très-incertaines au point de vue de l'indigénat.

Linné dit : in Europa, indication vague. - Loureiro, in China et Cochinchina; je crois que Loureiro a bien en vue le $R$. alba L., mais je ne le certific pas, n'ayant rien vu de ces régions qui puisse se rapporter à ce rosier. - Gmelin, ad horlorum sepes, in hortis, locisque funerariis, et arbustis. - DC., fl. fr. : cet arbrisseau crồt dans les haies, sur les collines, on le cultive dans tons les jardins. - Gilibert, pl. d'Europe : dans les haies aux Broteux. M. Cariot, dans sa flore, n'admet pas ec rosies comme étant spontané; il dit: cultivé dans les jardins, se trouve quelquefois subspontané près des habitations. - Fourreau, dans son catalogue des plantes du cours du Rhône, n'admet pas ce rosier comme appartenant à sa flore. Persoon, synopsis: colit. in hortis. - IVilldenow, enum. pl. : in Europa anstrali; ce rosier est représenté dans son herbier sous le $n^{0} 9876$, par une feuille simple portant un ramuseule florifère du $\boldsymbol{R}$. alba. M. Crépin ne dit pas si cet échantillon provient d'un jardin ou s'il est spontané. - Dumont-Courset : l'Europe, l'Autriche. Host, fl. austriaca, ne fail aucune mention de ce rosier. - Desvaux, journ. bot., dit: le $R$. alba $n^{9} a$ jamais été trouvé sauvage. - Lindley donne pour patrie à ce rosier : Piémont, Cochinchine, Fionie, France, Hesse et Saxe, localités copiées dans les flores sans preuve à l'appui; la localité du Piémont est donnée sur' l'autorité d'Allioni; le $R$. alba de la Flore du Piémont est le $R$. sempervirens Lin. - Trattinnick : a Lusitanice usque ad Caucasum. - Seringe, in DC., prod. : in Germania? - Balbis : cultivé dans les jardins. I) Mortier, fl. belgica: bois montagneux de Juslenville, gì et lì dans les haies. La localité de Juslenville est prise d'après Lejeune, mais le $R$. alba y est-il spontané? Puis est-ce bien Ie $R$. alba? - Chevallier, flore de 


\section{( 250$)$}

Paris : on le cultive dans les parterres. - Desportes : la France, l'Allemugne, le Piémont, le Caucase, localités copiées dans Lindley. -- Duby, bot. gall. : in hortis frequentissmme culta. - Lorey et Duret, dans la flore de la Côte-d'Or, disent : celte espèce est indigène el cultivée dans tores les jardins. - Gussone, syn. sieul. : R. alba, R. centifolia et $R$. Damascena Siculue indigenae ex Ucria, sed certe cullae, nec spontunene ullibi proveniunt. - M. Boreau : les haies, et indique les départements de Saône-et-Loire, Loiret (la plante que nous avons de M. Jullien est bien le $R$. alba, mais certainement non spontanẻ (quoique à fleur simple), Maine-et-Loire; II. Boreau, dans son catalogue de ce dernier département dit : peut-être n'est-elle pas naturelle au département. - Carion, catalogue de Saône-etLoire, dit : nous ne mentionnons pas le R. alba quoiqu'il soit indiqué d'après notre témoignage dans la Flore du Centre, la haie où il existait ayant été arrachée et le rosier détruit. - Gonnet, fl. de Fr. : les haies, buissons, mais rare, cultivé. - MM. Grenier et Godron excluent avec raison ce rosier de la Flore de France. - Kirschleger : naturalisé dans les haies, les clotures ct planté dans tous les jardins des paysans. - Grenier, fl. jurassique: cù et là dans les haies surtout dans le voisinage des habitations, cette plantc n'est pas indigène. - Aiton, Kew., dit ce rosier natif d'Europe ct son introduction en Angleterre r'emonterait à l'année 1597.

\section{Sect. XIII. - Eglanteriae.}

DC., divisions des roses, in Seringe, mus. Helv. (1818), II, ]. 5, excl. R. berberifolia; Déséglise, I. c.,p. 82, extr., p. 42, et in the naturalist, $n^{\circ} 20$, p. 512; Cimnamomene Seringe, in DC, prod., II, p. 602, part.; Duby, 1. c., I, p. 176, part.; Pimpinellifolice Koch, syn., 246, part.; Luteae Crépin, 1. c., fase. 2 (1872), ¡. 97; séric III $D u$ Pontiana Tratt., l. c., I, p. 75, part.; séric VII Roessigiuna Tratt.: 1. c., II, p. 30 .

268. Th. Iutea Daléchamp, hist. plant. (1587), I, p. 126 ; Miller, diet., n 11, éd. franc. (178:i), VI, p. 526; Moench, meth. (179/4), p. 688; Lam., 11. fr. (1793), III, P. 152; Gmel., 1. c. (1806), II, p. 405̄; Biroli, fl. Agon. (1808), 
1, p. 170; Willd., enum. pl. (1809), p. 345; Dum.Cours., l. c. (1811), V, p. 4(67; Rau, I. c. (1816), p. 157; Balh. et Noc., fl. Ticin. (1816), I, p. 408; Lindley, 1. c. (1829), p. 84; Krocker, 1. e. (1820), pars II, p. 110; de Pronville, I. c. (1824), p. 88; Chevalier, I. c. (1827), II, p. 688; Rehb., l. c. (1850), II, p. 612; Koch, syn. (184. ) , p. 24.6; Lee. et Lam., catal. (1847), p. 137; Borcau, 1. c., él. 2 (1849), no 667, éd. 5 (1837), no 852; Kirschleger, I. c.(1852), I, p. 242; Déséglise, 1. c. (1861), 1, p. 82, extr., p. 42 ; Du Mort., monog. des ros. (1867), p. 32; Verlot, l.c. (1872), p. 116; Boissicr, 1. е. (1872), II, p. 671; R. foetida IIerm., diss. (1769), p. 18; Allioni, fl. Pcdem. (1783), no 1792; R. Eglanteria L., sp. (17(6/), 705, pr. part.; Roth, l. c. (1789), II, pars prior, p. 3.j.5; Krocker, 1. e. (1790), II, pars prior, p. 159; DC., fl. fr. (1803), IV, p. 457; Giliberl, 1. с. (1806), I, p. :85̄; Pers., syn. (1807), II, p. 47; Mérat, 11. Paris (1812), p. 189; Seringe, mél. (1818), I, p. 19; Thory, prod. (1820), p. 99; Saint-Amans, fl. Igen. (I82I), p. 206; Tratl., I. e. (1825), II, p. 51; BI. et Fing., 1. c. (182:3), I, p. 621; Du Mort., 11. Belgica (1827), p. 95; Loisel., 1. c. (1828), I, p. 560; Desportes, I. c. (1828), 1. 80; Lorey et Dur., I. c. (1851), p. 506; Irutel, I. c.(1854), I, p. 54/; Delastre, 1. c.(1842), p. 160; Gonmet, I. c. (1848), P. $476 ; R$. Eglanteria A lutea Seringe, in DC., prod. (I823), II, p. 607 ; Duby, I. e. (1828), I, p. $176 ; R$. eerea Rocssig, die rosen (1500), $\mathrm{n}^{\circ} 2 ; \boldsymbol{R}$. chlorophylla var. unicolor Ehrh., beitr. (1787), II, P. 69; R. vulpina Wallroth, hist. (1828), P. 201; R. punicen Viller, n 15, l. c.; Roessig, l. e., no 3 ; R. bicolor dace., hort. lint. (1770), vol. 1, p. 1; R. chlorophlylla var. bicolor Ehrh., I. c., p. $70 ; R$. Eglanteria 
var. bicolor DC., 1. c., p. 457; Seringe, in DC., prod., II, p. 607 .

Icon. Lobel, II, pl. 209 ; Curtis, bot. mag., tab. 565, tab. 1077; Roessig, tab. 2, tab. 今’; Jacquin, 1. c., tab. I ; Redouté, les roses (182/4), livrais. 8, A et B, livrais. 22, B.

Exs. Aucher-Eloy, no 1452; Iaussknecht, no 567a; de Noé (18ว2), no 697; Kotschy, $n^{\circ} 297$; Seringe, $n^{\text {os }} 11$ et 12.

Hab. Mai, juin. - Europe. Spont.? Turquie d'Europe. Moldavie (Guebhard, 1828, in herb. DC. spont. ?). - Asie. Anaiolic, mont S:pylo (Aucher-Eloy); - Arménie : Baibut (Ituet da Parillon, 1955, in herb. Boissier); - Syrie boréale : Aintab ế Marasch (IIaussknecht, 136\%, in herb. Boissier), Diarbekir (de Noé, 1852, in herb. Boissier); - Perse : Sihna et Kermanchah (IIutssknecht, in herb. Loissier), Schiras (Kotschy); - Caucase : Baku (Seidlitz, 1367, in herb. Boissier); - Indes-Orient. : Kischtwarr, à une alt. de 7:00 pieds (Hooker eí Thomson, in herb. DC.).

OBS. Indiqué en France dans les flores, mais certainement pas spontané et étranger à la flore française; il se trouve ẹà et là dans des haies, mais échappé des jardins. Voici le relevé des diverses localités citées dans les flores de France et d'Allemagne, qui sont loin de prouver que ce rosicr y soit spontané. D'après ce que j'ai vu, je crois que la patrie de ce rosier serait l'Asie. Son introduction en Europe remonte probablement à unc époque très-ancienne? Il aurait été introduit en Angleterre, d'après Desportes, par John Gerard en 1596.

1719. - Garidel, dans son histoire des plantes de la Provence, dit : “ j’ai " trouvé cette espèce dans quelques haies du quartier des Fea nouillères, et au-dessous du quartier dit le Camp de Mante, "proche la métairie du sieur Burle."

1755. - Vaillant : environs de Paris.

1762. - Hermann : Afrique, Égypte.

1764. - Linné : IIelvetia, Anglia.

1789. - Allioni : Piémont, collines autour d'Alliano.

1789. - Villars dit ce rosier cultivé dans les jardins, qu'il vient peutêtre naturellement en Provence, mais qu'il ne l'a pas rencontré ; il. eite les localites du Poët et de Sisteron d'après de Leuze. 


\section{$(255)$}

1790. - Krocker : Nuper in horto pomaria in Klein Dels; Vidi; e foro * emtam accepi ante aliquot annos : in hortis indigenatum " nacta est. "

1805. - De Candolle : a les haies de Soissons (Poiret), il est cultivé dans les jardins; " puis il cite les localités de Villars, Allioni, Vaillant. J'ai vu dans l'herbier DC. ce rosier récolté par Requien en 1810 à Avignon, sauvage dans les haies.

1806. - Gmelin : in Aegypto. Colitur passim in hortis et arbustis anglicis.

1806. - Gilibert : a Je ne peux assurer si ce rosier est spontané en a Lithuanic : on l'apporte comme déraciné dans la forêt de a Bobrowzyzna; mais ce qui est certain, je l'ai cent fois trouvé " dans les jardins abandonnés des paysans. - Observé dans a les haies de Saint-Lager en Beaujolais."

1807. - Persoon : in hortis.

1808. - Biroli : in summis rupibus montium Novariensium Orfano, cimae Mullerce et in valle de Vegezzo.

1809. - Willdenow : in Germania, Anglia. - Il est à remarquer que pas une flore anglaise n'admet ce rosier comme indigène. Woods ne parle nullement du $R$. lutea, dans son synopsis of the British species of rosa. Lindley, dans sa monographie, cite les localités d'après les flores d'Allioni, W ibel, Rau; puis à la fin de ses observations il dit : a the only spontaneous specimens I a have seen were gathered near Avignon by M. Requien, and " are in the possession of M. Hooker."

1811. - Clairville l'indique en Suisse à la Sarraz (Vaud).

1811. - Dumont-Courset : Allemagne, Italie.

1812. - Mérat: se trouve dans les haies au-dessus du village d'Andresysur-Seine.

1816. - Balbis et Nocea : in sepibus collium traspadanorum Papiensium circa vicum Casteggio, loco dicto la Freccia rossa.

1818. - Seringe : la patrie de cette rose est entièrement inconnue; elle fait l'ornement des bosquets par ses belles fleurs simples et jaunes.

1820. - Thory : ce rosier croit spontanément en France, en Angleterre, en Allemagne, en Italie et en Espagne.

1821. - Saint-Amans : les haies R. R. R. aux environs de Talives près d'Agen. 


\section{$(254)$}

1825. - Trattinnick rapporte les localités citées par Thory.

1825. - De Candolle : patric inconnue.

1825. - Blufl et Fingerhut : in sepibus, sed rarior.

1827. - Chevalier : l'espèce eroit dans les haies, rarc.

1827. - Dumortier : in dumelis et sepibus.

1828. - Loiscleur patria incerta, in hortis colitur et interdum in sepibus dumetisque reperitur; circa Lutetiam, Andresis.

1828. - Desportes : l'Allemagne, l’Italie, la France.

1828. - Dulsy : in sepibus prope Avenionem (Requien) et in Galloprovincia ex Villars.

1850. - Reichenbach copie les lucalités données par Allioni, Biroli, Balbis et Nocea.

1851. - Lorey et Duret : cette espèce est généralement cultivée, il en existe une haie au-dessous de Sombernon sur la route de Paris.

1854. - Mutel copie les localités indiquées par De Candolle, fl. fr.

1842. - Delastre : cultivé. Quelques haies aux environs de Londun, R. R.

1845. - Koch : patria secundum DC. ignota ; in ditione fl. nost. passim in sepibus quasi sponte provenit.

1843. - Cosson et Germain : naturalisé dans les haies, et aux environs des villages.

1847. - Lecoq et Lamotte : R. R. spontané. Puy-de-Dôme : haies do la butte de Montpensier et du pré Monsieur près Aigueperse, haies piès Cournon. - Haute-Loire : au-dessus du vieux pont d'Estrouilhas près le Puy.

1848. - Gonnet : Sisteron et le Poët (d'après Villars), Avignon، Malesherbes, Dreux, ees deux dernières localités sans doute copiées dans la flore de MM. Cosson el Germain.

1852. - Kirschleger : naturalisé dans les haies et les clòtures, dans les jardins des paysans.

1837. - Boreau : naturalisé dans quelques haies. - Indre : Chateanroux, faubourg Saint-Christophe. - Loir-et-Cher : Herbilly près de Mer.

1859. - M. Boreau, dans son catalogue du département de Maine-etLoire : Ons. plusieurs autres rosiers ont été observés dans les haies, mais ne peuvent être considérés comme spontanés. $\boldsymbol{R}$. Iutea, haie à l'entrée de la route de Nantes.

1872. - Verlot : il est bien douteux que cette espèce ait été rencontréc réellement sauvage, mais elle est très-souvent cultivée dans les jardins. 
269. R. Vhapacandica Bunge, pl. Lehm., p. 287 , Boissicr, 1. c., II, p. 671.

IIAB. - Asie. In valle superiori fluvii Sarafschan supra Samarkand Turkestania (Bunge, in herb. Boissier).

270. AR. Elhrygia Boissier, amn. sc. nat., 4e série, II, p. 249 ; Walpers, ann. bot., IV, p. 653.

$\mathrm{IL}_{\mathrm{AB}}$ - Asie-mincure. Phrygie occidentale (Tchihatchef, exs. $\mathrm{n}^{0} 178$, in herb. Boissier).

Obs. Deux fautes de typographie existent dans le texte des annales des sciences naturelles : $\boldsymbol{R}$. Phygia pour $\boldsymbol{R}$. Plirygia; puis à l'hab. a in Physia occ. " pour "Plirygia occ. ".

II. Boissier dit que ce rosier a les peur's jaunes, et dans sa flore orient., II, p. 686, il regarde cette plante comme étant le R. muiginosa Lin. Si ce rosier a réellenent la fleur jaune, comme l'a écrit M. Boissier, il ne peut pas être le $\boldsymbol{R}$. rubiginosa $\mathbf{L}$. Nous voulons bien réunir autant que possible, mais encore faut-il que les réunions proposées soient justifiées et ne se trouvent pas en contradiction avec les textes!

271. Th. Hernispluacerea Ilerm., diss. (1762), p. 18, $n^{0}$ xiv ; Koch, dendrol., I, p. 226; Déséglise, not. extr. de l'énum. des rosiers, in the journ. of botany for June 1874, extr., p. 6; R. glaucophylla Ehrh., beitr. (1788), II, p. 69 ; R. sulphuren diton, Kew. (1789), II, p. 201; Villu., spec. (1797), II, p. 1063̈; DC., I. c. (1805), IV, p. 458; Gmel., I. е., II, p. 404; Pers., I. c., II, p. 47; Dum.-Cours., 1. с., V, p. 478; Lindley, 1. c., p. 46; Thory, I. c., p. 125; Savi, trattato degl. allir. del. Tose. (1822), II, p. 171; Krocker, fl. Siles., sup. (1822), IV, par's 2, p. 111 ; de Pronville, l. c., p. 74; Seringe, in DC., prod., II, p. 608; Desportes, 1. c., p. 11 ; Rehb., I. e., II, p. $61 \%$; Cariot, I. c., II, p. 182; Boissier, fl. Orient., II, 1. 672; R. Luteu Brotero, 11. Lusit. (1801), I, p. $557 \mathrm{ex}$ Lindley; R. Repini Boissier et Bal.! in Boiss., diagn. (18:9), sér. 2, fasc. 6, p. 72; Boissier! 11. Oriental., II, 


\section{( 256$)$}

p. 672 ; R. flava pleno flore Clusius, hist., I, p. 114 et curae poster. (1611), p. 15.

Icon. Clusius, curac post., p. 15 (le dessinateur a sans doute oubliẻ de figurer les stipules ?); Roessig, die rosen, tab. 43; Botan. reg., I, p. 46; Redouté, les roses (1824), livrais. I, C.; Boissier et Buhse, l, c., tab. VI, f. 1; d'après l'autorité de Lindley, je cite les gravures suivantes n'ayant pas les ouvrages ì ma disposition : Parkinson, parad., tab. 41/4, f. 6; Miss Lawr., ros., tab. 77.

Exs. Seringe, ros. desséch., $n^{\circ} 15 ;$ Balansa, pl. d'Orient (1857), no 1171 ; Tchihatchef, exs. (183̈8), $\mathrm{n}^{\circ}$ 212, in herb. Boissier!; Buhse (1847), $\mathrm{n}^{\circ} 541$, in herb. Boissier !

IIs. Mai. - Phrygie : Ouchak (Balansa); - Galatic: mont Elmadagh (Boissier); - Cappadoce : Césarée (Balansa); - Arménie : Erzinghan (Tchihatchef, in herb. Boissier); - 'Perse horéale : mont Elbrus (Buhse, in herb Boissier).

Ons. J'ai expliqué, dans le journal botanique de Londres, les motifs qui me font considérer le $R$. Rapini Boiss. comme étant le type à fleur simple du R. hemisphaerica Herm.

\section{SECT. XIV. - Rubiginosae.}

DC. in Seringe, mus. Helv. (1818), I, p. 2 et p. 4; Lindley, l. c., p. 84, part.; de Fronville, 1. c. p. 86, part.; Rehb., l. c., II, p. 617, exel. R. psilophylla; Déséglise, l. c., p. 55, extr. p. 15; Reuter, 1. c., p. 71 ; Crépin, I. c., fasc. 1, p. 25; Cottet, 1. c., p. 42; Caninae Seringe, in DC, prod., II, p. 611 pari.; Duby, I. c., I, p. 177, part; Lorey et Duret, I. c., I, p. 507, part.; Caninae G. tomentellae, Crépin, 1. c., p. 20; Cottet, I. c., p. 42 ; Caninae H. scabratae, Crépin, I. c., p. 20 ; Cottet, 1. c., p. 42; Glandulosae Crépin, I. c., p. 21 ; Diastylae trib. campylacanthae B resinoso-glandulosae Godet, I. c., p. 204. 


\section{a) Tomentellae.}

Folioles plus ou moins pubescentes ou à nervures secondaires seules velues ou glabres, à glandes peu abondantes non odorantes; pédoncules glabres ou hispides glanduleux.

1. Styles glabres. . . . . . . . . 2.

Styles velus ou hérissés . . . . . . 5.

CFolioles velues en dessous. . . . . . 3.

2. Folioles glabres ou velues sur les nervures secondaires . . . . . . . . 4.

5. (Tube du calice ellipsoïde, fleur rose clair, fruit petit, ellipsoïde, rouge. . . . . . . similata.

Tube du calice ovoïde, fleur rose, fruit ovoïde . Borreri.

( Rameaux floraux inermes, folioles grandes, velues sur les nervures secondaires, fleur d'un beau rose • • . . . • • . . .

Rameaux floraux aiguillonnés, folioles glabres, tube du calice grêle, ovoïdc . . . . . . subintrans.

Styles velus, tube du calice grêle, ovoïde, neur

๖. d'un blanc carné, folioles petites, glabres,

Styles hérissés ou obscurément hérissés . . . 6.

6. Folioles velues en dessous . . . . . . 7.

Folioles glabres en dessous . . . . . 8.

7. Folioles ovales-elliptiques, d'un vert foncé, divitomentella. sions ealicinales parsemées de glandes sur le dos, persistantes jusqu'à la coloration du fruit, fleur rose, fruit ovoïde, rouge . . . . .

Bakeri.

Folioles médiocres, ovales-arrondies, styles obscurément hérissés, fleur petite, d'un beau rose, pédoncules hispides-glanduleux, fruit ovoïde, rouge violacé . . . . . . . . viscida.

Folioles assez grandes, ovales cuspidées, d'un vert obscur, styles hérissés, pédoneules parsemés de quelques glandes, fleur grande, rose pâle, fruit assez gros, ovoïde-arrondi.

Blondaeana. 
272. R. Comentella Leman, bull. philom. (1818), extr. p. 10; Boreau, 1. с., éd. 2 (1849), 110 685, éd. 5, $n^{\circ} 863$ et catal. de M.-ct-Loire (1839)), P. 79; Reuter, at. Geneve (1861), p. 71; Déséglise, in Billot, arch. de la fl. de Fr. et d'Allem., p. 5.̃̌ et essai monog. in mém. Soc. Acad. de M.-et-Loire, $X$, p. 152 extr., p. 92; Manceau, bull. Soe. d'Agricult. se. et arts de la Sarthe (1862), extr., p. 8; Grenier, 11. juras., p. 247; Baker, Engl. hot. third edit. (1864), III, p. 217 et review of the Brit. Roses in the naturalist (186/4), p. 102 extr., p. 55; Cariot, I. e., II, p. 184; Du Mort., monog. ros. luelg. p. 30 exel. var.; Lloyd, fl. Ouest (1868), p. 177; Fourreau, 1. c., p. 73 ; Pérard, I. c., p. 82; Verlot, 1. c., p. 116; Cottet, I. c., p. 42; R. inodora IIooker, 11. lond., n. s. 1. 117, ex Baker; R. rubiginosa var. C. Rapin, I. c., p. 101; R. tomentosa var. dumetorum Gaud., 11. helv., III, p. 552 , ex Reuter; R. canina var. tomentella Baker, monog. of Brit. ros. in Linn. Society's journ., XI, p. 251.

Exs. Billot, n 1477; Baker, herh. ros. brit., no 29; Bourgeau, Alp. de La Savoie (18/8) 1" 80, sub. R. canina var. fastigiata (R. fastigiala Bast., teste Cosson!); Déséglise, herb. ros., $\mathrm{n}^{\circ} 70$.

Ilsв. Mai, juin. Ilaies, bois. - Angleterre. Yorkshire: Thirsk, Think, Sowerby (Baker); - Devonshire : hroussailles près de Biekleigh, Lynham, Pennyerots (Brigrgs). - Belgique. Prov. de Namur : Rochefort (Crépin). Frence. Heurthe : Nancy (Mathicu); - Maine-et-Loire: Angers (Boreau); - Aisne : forêt de Villers-Cotterêts (Questier);- Loir-et-Cher : Salbris;Indre: Sainte-Lizaigne; - Cher : C. Bourges, Allogny, Allouis, Epineuille-Fleuriel, ete.; - Puy-de-Dòme : les Vergnes près de Riom (Lamotte); Allicer : Areon près d'lihreuil (Lamolte); - Doulss : Mont Brégille près de Besancon; - Saône-et-Loire: Châlons-sur-Saône à Thésé (Ozanon); Lot-et-Garonne 'Agen (de Pommaret); - Rhône: Lyon au pont d'Alay (Ozanon), Dardilly, Roncière (Chabert), Tassin, Chapoly, Couzon, Beaumont (Boullu), indiqué comme AC. dans ee département par M. Cariot; 
- Isère: Noreppe à Chalais (Verlot) ; - Var : le Luc (IIanry) ; - Alpesmaritimes: Saint-Martin-de-Lantosque (Bornet); - Savoie : rochers de la Cave au Brezon (Bourgeau); - Haute-Savoic: Pringy, Conflans (P'uget), le Iont Salève; - Iautes-Alpes: Gap (Burle). - Alsace. Jagerthal, Reichoffen. - Suisse. Valais : Sembrancher, Sion (Cottet); - indiqué par M. Christ, dans les cantons de Schafhouse, Uri et du Tessin. Autriche. Tyrol: entre Ilall et Saint-Martin, Fragenstein, Vorarlberg (Kerner).

OBs. I. R. villosulu Paillot, rev, !itt. Franche-Comté (1867), p. 562 et in Billotia (1869), p. 119 ; exs. Billot (suites), n 5848. - Ce numéro est représenté par des exemplaires bien mauvais, des sommités florales dépourvues de vieux bois et d'aiguillons, des brins qui ne sont pas admissibles dans une collection comme celle de Billot.

I. Paillot attribue à son $R$. villosula, "des aiguillons géminés sous les a feuilles, dilntés ì la base, arqués au sommet, ceux des rameaux presque "droits subulés, " caractère qu'il est impossible de voir sur les brins distribués. "Les folioles lniérales sessiles fortement velues en dessous, " elles sont toutes pétiolées et les nervures seulement velues dans l'échantillon en fleur et presque glabres dans celui en fruit. a Sépales courls, presque " entiers, velus sur les deux faces; oles sépales, dans les échantillons distribués, sont pinnatifides, à appendices larges, glabres sur le dos. - Ces observations me portent à croire que le $\boldsymbol{R}$. villosula distribué sous le no 5848 n'est pas celui décrit par. M. Paillot, dans le Billotia, p. 119 et p. 120.

Ons. II. R. concinna Lagger et Puget inéd.; R. tomentella forma concinna Christ, die Rosen Schw., p 128?

Arbrisseau assez élevé, à rameaux verts, munis d'aiguillons crochus, rares sur les tiges florifères, plus robustes sur les vieilles tiges. Pétioles pubescents-glanduleux, aiguillonnés en dessous; 5 -7 folioles ovales-aiguës ou ovialrs-obtuses, glalmes, plus ou moins parsemées en dessous de glandes sur les nervures; nervures secondaires pubescentes et glanduleuses, doublement dentées, à dents sccordaires glanduleuses; slipules étroites, glabres, bordées de glandes, à oreillattes aiguës peu divergentes; pédoncules solitaires ou réunis par $2-5$, pen et irrigulièrement hispides-glanduleux; tube du calice ovoïde, lisse ou un peu hispide ; divisions calicinales appendiculées, à appendices courls bordés de glandes, parsemées de glandes en dehors, réfféchies à l'anthèse, caduques; styles courts, glabres on presque glabres; fleur d'un rose clair; fruit de grosseur médinere, 
ovoïde ou presigue arrondi, contracté au sommet, lisse ou un peu glanduleux à la base (Puget).

Hab. Juin. - Suisse. Cant. d'Uri : Altorf (Puget).

275. IR. sismailata Puget, in Déséglise, descrijt. yq. esp. nouv. de ros. in Billotia (186/4), 1).58, extr., \%. (6; Cottet, 1. c., p. $4 \overline{5}$.

Exs. Déséglise, herb. ros., n²4.

$\mathrm{H}_{\mathrm{AB}}$. Juin. Broussailles, - France. Yonne: Auxerre (Mabile); - Lozère: Bagnols-les-Bains (Martin, in herb. Grenier); - Ilaute-Loire : Fise (Lamotte, in herb. Grenier); - Isère: Villard-de-Lans (Boullu); Saroie : Saint-Nicolas-la-Chapelle (P’uget), chemin de l'Arpetlaz au-dessus des Ilerys (Perrier, in herb.Grenier);-Haute-Savoie : Arenthon, bords de l'Arve, Pringy (Puget), Conflans (Perrier). - Suisse. Valais: Bovernier (Cottet).

274. Be. Tymoliensis Kerner, OEsterr. hot. Zeitschrift (1869), $\mathrm{n}^{\circ} 11$, extr. p. 7.

Hab. - Autriche. Tyrol: Steinach entre Trins et Gschnitz, Schönberg (Kerner).

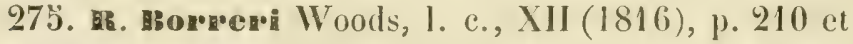
herl). no 71; Tratt., 1. c., II, p. 15; Smith, Engl. fl. (1824), II, p. 588; Baker, in Engl. bot., third edit. (1864), III, p. 21 k et rev. of the Brit. ros., p. 20; R. rubiginosa var. inodora Lindl., I. c., p. 88; R. inodora Rehb., l. c., p. 618 (non Fries); R. canina var. Borreri Baker.

Icon. Engl. bot., tab. 2579 et third edit. pl. 471 mala. HAB. - Angleterre. Kenl: Knockhoet (Baker); - Yorkshire : près de Wetherby Grange (IIailstone). - Belgique. Prov. de Namur: Rochefort (Crépin).

276. R. Bakeni Déséglise, in the journ. of botany, $n^{\circ} 21$, sept. 1864, p. 267; Baker, rev. of the Brit. ros. 


\section{(2/1)}

(1864), p. .j4; R. Tomentella Baker, North Yorkshire, p. 22) non Leman; R. canine var. Bakeri, Baker, mon. of Brit. ros., p. 257.

Icos. Engl. bot, third ed., tab. 47j.

Exs. Baker, herb. ros. brit., nº 50 .

HaB. Juin. Broussailles. - Angleterre. Northumberland: fourrés à Nolywell Dene (Baker); - Yorkshire : haies à Sowerby, Thirsk (Baker).

277. Ma. Valesolaca Lag. et Pug. mss.; Cottet, I. c., p. 栏, sine deseript.; R. mirrantha forma Valesiace Christ, 1. c., p. 112 .

Arbrisseau de 1 mètre à $1^{\mathrm{m}} \partial 0$ de haut, à rameaux flexueux; liges florifüres toujours inermes, les inféricures portent de rares aiguillons crochus; pétioles canaliculés en dessus, peu aignillonnés en dessous, parsemés de glandes et de poils yui disparaissent en partie avec l'ige; 5-7 fol. grandex, largement ocales ou obtuses, glabres, d'un ierl sombre en dessus, glaupues et parsemées en dessous de rares glandes sur les nervures secondares; ces demieres sont aussi légerement velaes, la nervure médiane velue ef pars'mée de glandes, doublement dentées, a dents secondaires terminées par une glande; stipules grandes, glabres, bordées de glandes, les inférieures un peu glanduleuses en dessous, à oreillettes droites; pédon. cules rémis par trois, formant aussi des corymbes de 6-9, rarement solitaires, hispides glanduleux, munis a leur base de 2 bractées opposées, lancéolées, glabres, bordées de glandes et dépassant les pédoncules; tube du calice ovö̈le, contracté au sommet, glabre; divisions calieinales ovales-lancécées, ¿2 entières, $\bar{\jmath}$ pinnatifides, parsenées de mandes fines en dessous, saillantes sur le houton, plus 
courtes que la corolle, réfléchice i l'anthese, caduques; styles un peu agglutinés à la base, glabres, disque saillant; fleur d'un bean rose; fruit petit, ovoüde, rétréci et comme étranglé au sommet.

IIs. Juin. Bronssailles. - Suisse. Valais: Vollège, vallée d'Entremont (de la Soie), rocailles de la route de Bovernicr it Sembraneher! Sion.

278. Hebrodengin Gussone, syn. Sicul. (1942), I, p. 565; R. mbiginose var. parvifolia Seringe, in DC., prod., II, p. 616 excl. syn.; R. Ilispanica var. Neculensis Boiss. et Reut., pugil. (1852), p. 44 .

Je n'ai pas vu le type de Gussone et je emnais seulement ce rosier par deux échantilons venant de la localité de Madonie (Sicile), recus de M. Todaro. L'échantillon que je possèle d'Espagne et ecux de France sont identiques a mes spécimens de la sieile; les aiguillons, les folioles, les divisions calieinales, les styles, roncordent avee la plante reçue de M. Todaro. Les pédoncules, dans les ichantillons d'Espagne et de France, sont un pen parsemés de glandes fines peu abondantes; dans mes échantillons siciliens, je vois des pédoncules glahres et des pédoncules parsemés de petites glandes.

$\mathrm{H}_{A B}$. - France. Ain : Thoiry, base du Reculet (Seringe, in herb. DC., 1825!); - Haute-Savoie : Argentière (Boullu). - Espagne. Sierra Nevada (Reuter). - Ilalie. Sicile : Madonic (Todaro).

Var. B. - Diffère du type par ses aiguillons plus robustes, inclinés ou crochus, les divisions calicinales glabres, styles glabres. R. subinurans Grenier in Billotia; exc. Billot (suites) nº 5851. - HАB. Ilaute-Garonne: Toulouse (Timbal); - Gard : le Vigan (Tuezkiewiez) ; - Ilautes-dipes: Gap (Grenier).

279. vis. viscià Puger, in Crépin, primit. monog̣. ros., fase. I, p. 20 , sine descript. 
Arbrisseau à rameaux violacés on verditres; aiguillons épars assez nombreux, robustes, longs, dilatés à la base, recourbés, ceux des rameaux florifères plus petits; pétioles glanduleux, parsemés de poils blanchatres en dessus,

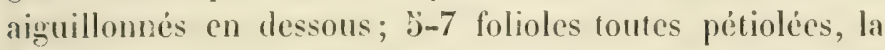
terminale arrondie à la base, termince en pointe courte au sommet, ovales-arrondies, glabres, d'un vert sombre en dessus, glauceseentes et glanduleuses en dessous sur Ies nervures, doublement dentées, à dents secondaires glanduleuses; stipules assez larges, glabres, à bords glanduleux; oreillentes aiguës, divergentes; pédoncules solitaires ou en bouquet peu fourni, courts, hispides-glanduleux, cachés par de largres bractées ovales-acuminces, glabres, bordées de slandes; tube dı calice ovoüde, hispide-glanduleux; divisions ealicinales ovales, appendiculées au sommet, glanduleuses en dessous, à glandes peu abondantes, 2 entieres ì bords tomenteux, $\bar{j}$ pimnatifides à appendices courts, étroits, bordes de cylandes, saillantes sur le bouton, égalant presque la corolle, étalées à l'anthèse puis réfléchies, non persistantes; styles courts, glabres ou très-obscurement hirissés; disque saillant; fleur pelite, d'un bean rose; fruit ovoüde, hispide, d'un rouge violacé à la maturité.

11Ав. Mlai, juin. Bois, haies. - France. Jura : Lons-le-Saulnier, colline de l'Hermitage (Puget); - Ilaute-Savoie: Thonon (Puget); - Alpesmaritimes : de Roquebillière à Bertemont (Bornet).

580. R. viscosa Jan, eat., p. 8; Gussone, I. c., I, p. 565 .

Ilab. Juin, juillet. - Ilalie. Sicile: Madonie (Gussone). - Je ne connais pas ce rosier, qui ne se trouve pas dans l's herbiers de Genève.

281. In. Blondacana Ripart, in Déségliser, cssai 
monog., in mém. Soc. Acad. dr M.-ct-Loire, X (1861), p. $15 \overline{5}$, extr., p. 95; Baker, rev. of the British ros., p. 54 ; Cottet, 1. c., p. 42; R. trachyphylla Boreau, 1. c., èd. 5, n'688 non Rau; R. trachyphylla var. Blondaeana Du Mort., І. c., p. 59; R. trachyphylla var. nuda Grenier, $\mathbf{1}$. juras., p. 214.

Exs. Déséglise, herb. ros., $n^{\circ} 52$.

IIAB. Juin. IJaies, bois. - Angleterre, Cheshire: West Kirby (Webb). - Belgique. Prov. de Namur: Rochefort (Crépin). - France. Loir-etCher : Cour Cheverny (Franchet); - Loiret: Maison rouge, route de la Chapelle, St-Denis-en-Val (Julien); - Cher : C. Bourges (Ripart), Marmagne, Mehun, Allouis, Saint-Florent, Vierzon, etc.; - Yonne : Auxerre (Mabile); -- Doubs: Besançon (Grenier). - Suisse. Valais : Mont Ravoire près de Bovernier.

OBS. Ce rosier présenterait les formes suivantes que nous ne connaissons qu'imparfaitement.

1. Q8. vinetorum Ripart, mss. - Caractères généraux du R. Blondacana, dont il diffère par ses pétioles plus aiguillonnés, ses stipules dépourvues de glandes en dessous, ses styles obscurément hérissés, sa fleur d'un blane carné (Ripart).

HAB. Cher : Vignes de Turly près de Bourgers (Ripart), vignes d'Asnières près de Bourges.

2. It: controversa Ripart, mss.

Petit sous-arbrisseau à port beaucoup plus grèle que les $\boldsymbol{R}$. Blondaeana et $\boldsymbol{R}$. semi-glendulosa; ses aiguillons plus évidés, ses tiges plus minces et ses folioles plus petites. Il diflere surtout des deux par ses styles glibres; les pédoncules et le tube du calice sont presque aussi grlanduleux que ceux du R. Andegavensis (Ripart).

HAB. IIaies des vignes. Juin. - Cher: Bourges (Ripart); - Aude : le Mas Cabardès (Ozanon).

5. It. practerita Ripart, mss.

Styles velus, fruit arrondi, pédoncules lisses ou portant quelqques rares soies glanduleuses avortées (Ripart). 
Haß. Cher : bois de Givai près de Bourges (Ripart). - Belgique. Prov. de Namur : Rochefort (Crépin).

4. R. seml-siandutosa Ripart, niss.

Pédoncules glabres; styles glabres on olseuriment hérissés; dirisions calicinales églanduleuses en dessous (Ripart).

IIı. Cher : Bourges, Saint-Martin (Ripart), Allouis, Mehun; - Allier : Chassagne près les Gazeriers (Lamotte).

\section{B) Glandulosae.}

Aiguillons droits, inclinés ou un peu crochus, dégénérant quelquefois en aiguillous sétacés et grlanduleux, fenilles ordinairement grandes à glandes peu ou pas odorantes, pédoncules hispides-grlanduleux, corolle ordinairement grande.

1. Rameaux floraux incrmes . . . . . . 2 .

1 Rameaux floraux aiguillonnés . . . . 5.

Pétioles glabres, glanduleux, folioles ovaleselliptiques, tube du calice ovoïde, hispide à la base, sépales glanduleux sur le dos, fleur d'un rose vif.

Godeti.

2. Pétioles tomenteux, glanduleux, folioles orales, triplement dentées, tube du calice ovoïde-allongé, hispide-glanduleux, sépales couverts de glandes sur le dos, fleur grande, d'un rose pourpre.

Wasserburgnsis.

Aiguillons dégénérant au sommet des ra-

meaux en soies sétacées . . . . . . 4

Aiguillons ne dégénérant pas en soies sétacées. 9.

(Folioles dépourvues de villosité sur les deux

4. $\left\{\begin{array}{c}\text { faces } \\ \text { Folioles pubescentes en dessous } \\ .\end{array}\right.$

Divisions calicinales glabres, tube du calice

5. Solabre. Disions calicmales glanduleuses sur le dos, insi iosit. tube du calice hispide-glanduleux 


\section{( 246$)$}

Folioles orales-obtuses, irrégulièrement den. tées, styles obscurément hérissés, fleur

(6. rose, fruil ovoüde

dryadea.

Folioles grandes, ovales-elliptiques, doublement et triplement dentées, styles hérissés, fleur purpurine, fruit obovoïde-allongé.

protea.

7.

Arbrisseau, folioles grandes, ovales-elliptiques, styles velus, fleur grande, d'un beau

rose, fruit ovoïde. . . . . . . .

specioste.

Sous-arbrisseau ne formant pas buisson . . 8 .

8. Pétioles velus parsemés de quelques glandes,

nemorivagre.

pseudo-plexuosa suave, fruit gros, obovoïde ou arrondi, d'un

9. $\left\{\begin{array}{c}\text { Sous-arbrisseau ne formant pas buisson, } \\ \text { folioles ovales-aiguës, fleur rose, fruit glo- }\end{array}\right.$

buleux . . . . . . . Pugeti.

Arbrisseau plus ou moins élevé. . . . 10.

Fleur d'un rose foncé, à odeur suave, folioles plus ou moins chargées de glandes visqueu-

10. ses odorantes, fruit obovoïde . . .

Fleur d'un beau rose, folioles à glandes non

odorantes . . . . . . . 11.

subolida.

Folioles larges de trois centimètres au moins. Jundzilliana.

11. Folioles dépassant rarement deux centimètres. . . . . . . . . Alexuosa.

282. Insidiosa Ripart, mss.; R. psilophilla Déséglise exs. non Rau; $R$. depressa Gremli?

Exs. Déséglise, herb. ros., $n^{\circ} 65$.

Arbrisseau à aiguillons robustes dilatés comprimés à la basc, inclinés, ceux des rameaux et des tiges florifères 


\section{$(247)$}

inégaux, épars, les plus robustes dilatés comprimés à la base, inclinés, les plus petits dilatés en forme de disque droits dégénérant en soies sétacées églanduleuses; pétioles hérissés de poils courts à la hase, parsemés de glandes fines stipitées, incrmes ou faiblement aiguillonnés; folioles §-7, ovales-arrondies ou obtuses, coriaces, glabres, vertes en dessus, glancescentes en dessous, la còte médianc glanduleuse, les nervures secondaires parsemées de quelques glandes, doublement dentées; stipules glabres en dessus, parsernées de glandes en dessous, oreillettes aiguës divergentes, bords glanduleux; pédoncules solitaires ou réunis par 2-4 en bouquet peu fourni, hispides-glanduleux; tlibe du calice perit, ovoüde, contracté au sommet, glabre; divisions calicinales spatulées au sommet, glabres sur le dos. 2 entières, $\vec{\jmath}$ pimnatifides, à appendices bordés de glandes, saillantes sur le houton, rélléchies à l'anthèse, non persistantes; styles hérissés, disque conique; fleur rose; fruit ovoïde, rouge.

Haв. Mai, juin. IIaies, - France. Cher : Trouy, Grange-Saint-Jean près de Bourges (Ripart). - Suisse. Lant. de Schaffhouse? (Gremli). Autriche. T'yrol : 'Trins, vallée de Gschnitz (Kerner).

\section{R. dryatea Ripart, mss.}

Arbrisseau à aignillons robustes, dilatés à la base, arqués, dégénérant au sommet des rameaux en aiguillons sélacés; pétioles parsemés de poils courts, plus ou moins chargés de grlandes fines stipitées, inermes ou faiblement aiguillonnés; $3-7$ folioles orales-aiguës obluses ou orbirulaires, coriaces, fermes, glabres, vertes en dessus, glaucescentes en dessous, la còte médiane porte des glandes et quelques petits acicules, les nervures secondaires ont quelques glandes, irrégulierement denties ì dents aiguës profondes, les unes simplement dentées et d'autres dou- 
blement dentées; stipules glabres sur les deux faces, oreillettes droites on divergentes; pédoneules en boupuet peu fourni, hispides-glanduleux, courts, bractece ovales acuminées, glabres, plus longures que les pédoncules; tube du calice ovoüde, hispide-glanduleux, à glandes fines; divisions calicinales spatulées au sommet, glanduleuses sur le dos, 2 entières à bords tomenteux, 3 pinnatifides à appendices étroits bordés de glandes, réfléchies à l'anthèse, eaduques; styles obscurément hérissés, disque presque plan; fleur grande, rose; fruit ovoüde, celui du centre obovoïde, d'un beau rouge.

IIs, Mai, juin, Bois. - France. Cher: bois des Dames commune de Trouy (Ripart), haies des vignes de Trouy près le bois de Givray.

\section{R. protea Ripart, mss.}

Albrisseau élevé, rameaux à écorce purpurine ou verditre, flexueux retombants, aignillons dilatés à la base, inclinés, dégénérant aı sommet en soies sétacées ģlanduleuses; pétioles parsemés de poils dans le sillon, glanduleux, aiguillonnés en dessous; ; $)-7$ folioles assez grandes; ovales-clliptiques, vertes en dessus, glaucescentes en dessons, glabres, la còte médiane galandulcuse et quelques nervures secondaires parsemécs de glandes, doublement et triplement dentées, ì dents secondaires glanduleuses; stipules longues, glabres, bordées de glandes, oreillettes divergentes; pédoncules très-courts, solitaires ou en bouquet peu fourni, hispides-glanduleux; tube du ealice ovoïde, hispide-glanduleux; divisions calicinales longues, spatulées au sommet, glanduleuses sur le dos, 2 entières, 5 pimnatifides à appendices bordés de glandes, saillantes sur le bouton, plus courtes que la corolle, réfléchies it l'anthèse, caduques; styles hérissés, disque conique; 
fleur grande, d'un beau potrpre; fruit rouge, obovoüdeallongé, ce qui lui donne une forme ellipsoïde.

Hab. Mai, Juin. ILaies. - France. Cher: haies des vignes de Trouy près de la Grange-Saint-Jean (Ripart).

28:. R. consanguinea Grenier, fl. juras. (1864), p. 220\%; R. gallico-umbellata Rapin, in Reuter, cat. Genève (1861), p. 72.

IIsB. Juin. - Suisse. Cant. de Genève: Veyrier près de Genève.

Ors. Les feuilles ne sont pas subpubescentes en dessous, comme le dit M. Grenier; elles sont glabres, couvertes de glandes fines, roussâtres, la côte médianc porte quelques poils : clles ne sont pas ovales-aiguës, comme le dil Reuter, mais bien ovales-arrondies ou ovales ellipliques; les divisions calicinales sont très-glanduleuses sur le dos.

286. B. Fodeti Grenier, in Godet, fl. Jura, suppl. p. 75 .

Exs. Billot, no 2061 et 2061 ter.

Has. Juin. - France. Neurthe : Nancy, earrières de Balin (Mathieu). Alsuce. Ruines du Château d'Andlau (Mathieu), forêt de Gros Wald près de Reichoffen! forêt de Vordersberg près de Niederbronn! - Suisse. Cant. de Neuchâtel: Mont de Chaumont (Grenier); - cant. de Bâle : Jura de Bâle (Christ).

\section{R. Cotteti Puget! mss.}

Description étahlie sur les notes et échantillons reçus tle M. Pugget; j’ai vu aussi ce rosier cultivé dans le jardin de M. Cottet.

Arbrisseau de 1-2 mètres, ì rameaux munis d'aiguillons Iongs, grciles, droits, dilatés à la base en forme de disque, hanchâtres ou de couleur faure; pétioles velus, parsemés de glandes fines, aiguillomnés en dessous; 3-7 folioles, oraleselliptiques, vertes, glahores ou parsemées de poils courts apprimés en dessus, glaurgues con dessous, nerveuses, 
la cote médiane glandulenser, les norvures secondaires portent aussi quelques glandes qui disparaissent avere l'ìge, doublement deutées; stipules glubres, opoillelles aigues divergentes; pédoncules solitaires ou réunis par 2-4, hispides-glanduleux; bractées ovales-acmminées, ylahres en dessus, pubescentes en dessous, à hords cilićs-glanduleux, égalant ou dépassant les pédoncules; tube du ealice ovoüde, hispide-glanduleux; divisions calicinales, gylanduleuses sur le dos, spatulées au sommet, 2 entières, 5 pinnatifides portent $1-4$ appendices courts, saillantes sur le bouton, dépassant la corolle, réfléchies à l'anthèse, puis redressées, comniventes, persistentes; styles hérissés; fleur rose; fruit arrondi on ovoüde, tun peu atténué au sommet.

IIAB, Juin. Broussailles, - Suisse. Cant. de Fribourg : aux cases d'Allières (Cottet); indiqué dans te canton de Vaud, vallée de l'Ilongrin (Favrat).

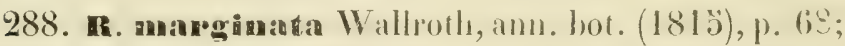
Lindley, 1. e., p. 58; Trall., 1. c., 11, \%. 14k; Seringe. in DC., prod., II, p. 60\%; 131. et Fing., 1. c., 1). 626; Rehb., I. c., II, p. 625̄; Reuter? I. c., p. 66; R. villosa var. G. Wallr., hist. ros. (1828), P. $2: 5 \overline{5} ; R$. Iomenlose b. marginata Rapin? gouide, 1). 192; h. spinutifolia b. denudala Grenier, 11. juras., p. 250 \%; R. trachyphylla Grenier? 1. e., p. 245 non Rau.

HAB. Juin. Broussailles, - Allemagne. Bennstadt. L'étiquctte de Wallroth in herb. DC., 1854 ! ne fait aucune mention de la localité.

Ons. Qu'est-ce que le R. marginata W allroth? plante que je crois peu connue des botanisles. Lindley, Trattinnich, Blulf, Fingeruth, Scringe, Reichenbach, se contentent de rapporter la description originale de Wallroth, sans faire aucun commentaire; le type étail probablement à celte époque comme aujourd'hui à peu près inconnu. 


\section{( 251$)$}

En 1828, Wallroth, dans son II istorin rostrum assimile ce rosier an R. villose L., exemple suiri avee empressement par Koch, dans son Synopsis. Ce rapprochement est assez singulier. Le $\boldsymbol{R}$. marginata de France, de Suisse et d'Angleterre, est-il bien celui décrit dans l'Annus botanicus? J'en doute, car ec type de Wallroth n'est pas une Rubiginosae, comme le disent les auteurs qui admettent ce rosier comme espèce distincte.

M. Grenier m'a envoyé plusieurs échantillons de son $R$. trachyphylla, qui n'est certainement pas celui de Rau, mais bien le $R$. marginata Auct. non Wallroth. - La plante d'Angleterre que je possède en herbier se rapporterait mieux à la description de Wallroth, mais les folioles ont quelques glandes en dessous. - J'ai consulté dans l'herbier DC. le type du R. marginala Wallroth, ex ipso! qui se trouve dans cetle collection depuis 1854; voici la description minutieuse que j’ai pu établir.

Échantillon haut de 18 ecutimetres, portant deux ranuscules avee fruits et 4 pétioles avec des folioles.

Aiguillons du rameau petits, dilatés à la base en forme de disque,droits, blanchâtres ou vineux, dégénérant au sonmet en aiguillons sétacés églanduleux. Pétioles lisses, parscmés de glandes fines, aiguillonnés en dessous. Folioles ॐ, ovales, non acuminées, glabres, la nervure médiane porte à la base de rares glandes, doublenent dentées, hrs principales terminées par un mucron, les secondaires par une glande. Slipules étroites, glabres sur les deux faces, bordées de glandes fines, oreillettes aiguës, droites. Tube du calice....... Pédoncules solitaires ou biflores, hispides-glanduleux, ayant à leur base une bractée glabre, ovale, terminée par un appendice en forme de feuille, plus longue que le pédoncule. Divisions calicinales lancéolies spatulées au sommet, parsemées sur le dos de quelques glandes fines peu abondantes, 2 entières, 5 pinnatifides, à appendices eourts, étroits, hordés de glandes, étalées et un peu redressées sur le fruit, persistant probablement jusqu’à la maturité du fruit? Styles courts, trèsvelus, disque plan. Flcur..... Fruil petit, globuleux.

Ce $\boldsymbol{R}$. marginata Wallr., n'est pas une Rubiginosae, mais bien une Caninae hispidae!

J'ai en herbier le R. marginata Auct. non Wallroth, des localités suivantes: Angleterre. Yorkshire: haies à Kilvington (Baker). - Écosse. l'erthshire: fourrés à Glen Shee (Baker). - France. Haute-Savoie: le Mont Salève ! - Doubs: Pontarlier, chemin de Charpillot (Grenier). Suisse, Cant. de Fribourg : Comballaz près de Montbovon (Cottet). 
289. R. trachyptoylla Rau, chum. ros., P. 19/4; Lindley, 1. c., p. 142; Thory, I. c., p. 99; Tratt., I. c., II, p. 54; Bl. et Fing., l. c., p. 626; Rchb., I. c., II, p. 619; Sprengel, syst., I, p. 3.). p. 22; Arrondeau, 1. c., p. 126\%; Déséglise, l. c., p. 15うे, extr., p. 93; de Martr.-Don., I. c., p. 252; Cariot, 1. c., II, p. 184? Du Mort., monog. ros. Belg., p. 59?

Exs. Wirtgen, pl. crit., $n^{\circ} 25, n^{\circ} 25$ bis, $n^{\circ} 254$ ?; Billot, n" 2061 bis? échantillon tres-mauvais, sur lequel il est difficile de se prononeer.

IIsb. Juin, juillet. Broussailles. - France. Je doute que ce rosier appartienne à la flore française. Il est indiqué dans les départements: Ilaute-Garonne: Toulouse à Larramet, Boucomne (Arrondeau, flore); - Tarn : Moulin d'Avignon, Grillac (de Martrin-Donos, flore) ; - Rhône : en allant d'Écully à Charbonnière (Cariot, flore). - Suisse. Cant. de Schallouse : Jura de Sehallouse (Christ). - Allemagne. Bavière: Wurtzbourg (Rau) ; - Prusse rhénane : Coblence (Wirtgen).

Var. b. campestris Du Nortier, 1. c., p. 59; R. campestris 1)u Mort. 11. Belgica (1827), p. 95 non Swartz, nee Wallr.

Je ne connais pas ce rosier considéré comme une espèce distincte en 1827 et comme une variété du $R$. trachyphylla en 1867.

Ons. Seringe, d'après le type de Rau, avait raison de placer ce rosier dans les Caninue haspidae; mais Rau en décrivant son espèce avait en vue une autre plante, qui certainement appartient au groupe des Rubiginosac, à cause des glandes qui sont indiquées sur les nervures. Seringe devait avant tout se reporter au texte et non à un échantillon très-incomplet qui a pu ètre distribué par erreur sous le nom de $R$. trachyphylla.

Description établie sur l'échantillon de Rau, conservé dans l'herbier DC. L'étiquette porte: IVurtzhourg 1817. Rau a certainement fait une confusion, ear il a distribué sous te nom de $R$. trachyphylle, une plante que je considère, d'après l'examen que j'en ai fait, comme appartenant au groupe du $\boldsymbol{R}$. Andegavensis.

Échantillon mesurant 10 centimètres de hauteur, portant trois pétioles avec folioles et un bouton non épanouị. Remuscule ayant 7 petits aiguillons fins, les plus longs ont 4 millim., les plus petits deux milli- 


\section{$(25 \overline{5})$}

mètres de longueur, droits ou courbés, dilatés à la base en forme de disque, de couleur fauve (sur le sec). Pétioles glanduleux, aiguillonnés en dessous et parsemés surtout ì la hase de petits poils courts, blanes. Folioles 5, toutes pétiolulées, le pétiole inférieur porte des folioles elliptiques, la terminale aiguë aux deux extrémités, mesurant en longueur 2: millim., les latérales un peu arrondies aux deux bouts, mesurant $130-20$ millim. de longueur; les folioles des deux pétioles supérieurs sont ovales-arrondies, presque obtuses, ayant 20 millim. de longueur et 17 millim. de largeur, glabres sur les deux faces, nerveuses, à nervures secondaires saillantes, églanduleuses; la côte est parsemée de quelques petites glandes fines, doublement dentées, les dents prineipales aiguës, convergentes au sommet, les secondaires terminées par une glande. Pédoncule solitaire, court, parsemé de quelques glandes fines avortées comme celles du $R$. arvensis. Tube du calice petit, obovoide presque subglobuleux, glabre. Divisions calicinales courtes, glabres sur le dos, les extéricures pinnatifides à appendiees courts, étroits.

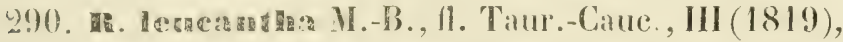
p. 5.j2 (non Loisel); Link, cmmm. Lor1. Berol., II, p. 599; Desportes, I. c., p. 91; R. Biebersleiniana Tratt., I. c., II, p. כّ.

\section{IIsm. - Le Caucase.}

(Mas. Je possède en herbier une plante de l'Unio itiner. an. 1859: in fruticetis mrope IIclenendorf Georg. Cancase, "récoltéc par Hohenacker. - L'échantillon en fleurs a les pédoncules latéraux glabres, le pédoncule central glabre et parsemé de quelques rares glandes, le tube du caliee et les divisions ealicinales glabres; dans les échantillons en boutons non épanouis, les pédoncules et le tube du calice sont un peu velus, les divisions calicinales relues et parsemées de glandes.

Cette forme récoltéc par Itohenacker constitue-t-elle le R. lencantha M.-B.? dans le flora Taur.-Carne, IIl, p. \%氵32, l'autcur dit : germinibus.... basi pedunculisque hispidis. J'ai vu dans l'herbier DC, un R. leucantha étiqueté par Besser et provenant de son jardin bolanique, plante qu'il cultivait comme venant dn Canease. Voici la description établie sur ce type de Besser, conservé dans l'herbier DC. et envoyé en 1824.

Aiguillons du rameau dilatés ì la biase, inclinés ou un peu courbés au sommet, mesurant -b̆ millim. de longuenr. Pétioles un peu velus dans le 
sillon surtout à la base, le reste lisse, parsemés de gullundes fines stipitées, aiguillonnés en dessous. Folioles 3̈-7, grandes, toutes pétiolulées, ovalesarrondies, les plus grandes mesurant $2 \frac{1}{2}-\overline{5}$ cent. de largeur sur 4 cent. de longueur, glabres sur les deux faces, vertes en dessus, glauques en dessous; nervures plus ou moins saillantes, les secondaires parsemées de fines glandes, la còte médiane porte des glandes et quelques petits acicules, doublement dentées, les dents prineipales terminées par un mucron, les secondaires par une glande. Stipules longues, glabres sur les deux faces, glanduleuses en dessous principalement au sommet, oreillettes aiguës, un peu denticulées, droites. Pédoncules réunis en bouquet par 5-4, hispides-glanduleux. Tube du culiee ovölde, hispide-ghanduleux. Divisions calicinales spatulées au sommet, glanduleuses en dessous, 2 entières à bords tomenteux, 5 pinnatifides à appendices courts presque filiformes, bordés de quelques glandes, saillantes sur le bouton, rélléchies à l’anthèse. Styles très-obscurément hérissés presque glabres, disque conique. Fleur grande. Fruit.......

291. M. wasseabuargensis Kirschl.! 11. Als. (1832), I, p. 247.

Je connais ce rosier par trois beaux spécimens recus en 18\%6, de Kirschleger mème. Ce curieux rosier n'aurait-il qu’une localité unique? Mes échantillons sont seulement en fleurs, je n’ai pas vu le fruit. Je donne in extenso la description de Kirsehleger.

Arbuste touffu, haut de 2 mètres, à écoree cannelle; vieux tronce et rameaux floriferes inermes; turions à aiguillons soustipulaires geminés, étroits, subulés, droits; écorce du reste complètement lisse et glabre; stiputes conniventes plus ou moins appliquées l'une contre l'autre, à oreilletles dressies; folioles $30-7$, un peu roides, ovales, à dents triplement dentées; pétioles tomenteux, glanduleux et aignillonnes en dessous; face inférieure des folioles d'un revl très-pile, glabre, al lexeeption de la nervure médiane

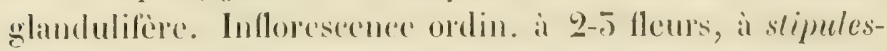

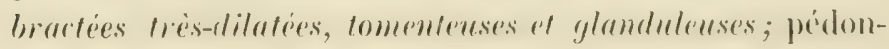




\section{( 255 )}

cules, ureéoles ovoürles-allongés et segments ealicinaux (les deux (xtépienres pinnatifides) chargés de glandes trèsdensex, longues de $2-\overline{5}$ millim. Corolle grande, rose pourpre. Styles trìs-velus; carpelles ì stipes très-courts. Vosges. Vallée de Münster, près du château ruiné de Wasserhourg derrice Soulzhach (Kirschleger).

Oss. La description de Kirschleger est incomplète; ses échantillons vont me permettre de signaler plusicurs omissions. Les pétioles sont tomenteux-glanduleux, faiblement aiguillonnés en dessous ou inermes. Les folioles sont ovales-ellipliques, les dernières folioles du pétiole plus petites ordin. obtuses, d'un vert sombre en dessus, glaucescentes en dessous, la côte médiane glanduleuse; les nervures secondaires sont parsemées de quelques glandes. Les stipulıs glabres en dessus, tomenteuses et parsemées de quelques rares glandes en dessous. Les pédoncules longs, chargés de soies glanduleuses; bractées ovales, cuspidées, tomenteuses sur les deux faces, plus contes yuc les pirbuncules, bordées de glandes. Divisions calicinales ovales, terminées en longue pointe spatulée, couvertes en dessous de glandes et de petits acicules. Le tube du calice ovoide, chargé de soies glanduleuses.

292. He. comanamata Seheutz, I. c., p. 20; Crépin, 1. c., fasc. 2, p. 128 .

HAB. - Suède. Asarum (Christ).

295. ar. subolida Déséglise, descrip. de qq. esp. nouv. de ros., in mém. Soc. Acad. de M.-et-Loire, XIVIII (1875), p. 12/, extr., p. 28; R. terebinthinacea Déséglise, ess. monog., p. 119 (non Besser).

Exs. Déséglise, herb. ros., $n^{\circ} 50$.

Ifar. Juin. Bois. - France. Saône-et-Loire: Brouailles près de Louhans (Moniez), Châlons-sur-Saône (Ozanon); - Rhône: Lyon au pont d'Allay (Ozamon), Tassin à l’Aigu (Boullu).

29)4. Pager. Borean, in Déséglise, 1. e. (1861), X, p. 156 , extr., p. 96; Citriot, 1. c., II, p. 183; Fourreau, 
1. c., p. $7 \ddot{3}$; R. foetidn Reuter! at. Gencre (1861), p. 72 non Bastard; R. hispidecerpe Chabert! in Cariot, 1. c. (1863), p. $677 \mathrm{ex}$ exempl. authent.

Exs. Déséglise, herb. ros., 11" 27; Billot (suites), $\mathrm{n}^{\circ} 5591$.

Har. Juin. Les bois. - Frence. Saône-ct-Loire : Châlons-sur-Saône, bois de (iivry (Ozanon); - Ain : la Cadette près de la Pape (Aunier, Chabert); - Rhône : route d'Écully à Charbonnière (Chabert), Chaponost (Boullu); - Haute-Savoie : Annecy (Boreau), Pringy, bois de Tessy sur Épangy, bois dẹ̦'Barioz (P'uget); - Haute-Garonne : bois de Laramette près de Toulouse, Nailloux, hois de la Tesnque (Timbal-Lagrarce), Moulin Grammont (Baillet), Toulouse (Ripart).

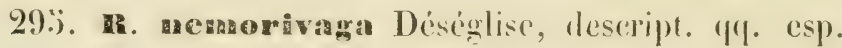
nouv. de ros., in Billotia (1864), p. 40, extr., p. 8; R. selulifera Timb.-Lagr., in herb. Déséglise.

Exs. Déséglise, herb. ros., no 26.

Ilab. Mai, juin. Bois. - France. Cher : forêt de Fontmoreau! - Rhône: hois de l'Étoile à Charbonnière, Tassin (Boullu); - Ifaute-Garonne : Laramette (Timbal-Lagrave). - Autriche. Bohème: Leitmeritz près de Pokratitz (Kerner).

OBs. R. Thomasii Puget.

Je connais ce rosier par deux échantillons trop incomplets pour me prononeer; je dome en entier la description de M. Puget.

Arbrisseau peu élevé, à rameaux bruns ou rougeâtres, munis de rares petits aiguillons dilatés à la base en forme de disque, arqués, courts; pétioles velus aiguillonnés en dessous, portant quelques glandes fines: 5-7 folioles petites, ovales-arrondies ou obtuses, vertes et glabres en dessus, glauques pubescentes en dessous, parsemérs de rares glandes fines sur les nervures secondaires et la côte médiane, doublement dentées; stipules étroites, glabres, à bords glanduleux-ciliés, oreillettes courtes, droites ou peu divergentes; pédoncules solitaires ou en corymbe peu fourni, hispides-glanduleux; tube du calier oroïde, lisse ou hispide-glanduleux à la base; sépales 2 entiers, 5 pimatifides, plus courts que la corolle, non persistants; styles courls, hérissés; lleur d'un rose elair ; fruit petit, arrondi, d'un beau rouge à la maturité. 


\section{$(25 \%)$}

II Ar. Région des montagnes. - Suisse. Cant. de Fribourg: La Gotalaz (Puget).

296. dR. decorea Kerner, in litt.; $\boldsymbol{R}$. amoena Kerner, olim, non auce.

Description établie sur de nombreux et magnifiques échantillons recus de M. Kerner.

Arbrisseau....., rameaux floraux à écorce vincuse ou verditre, inermes, les jeunes pousses ont des aiguillons dilatés à la base, inclinés ou droits, de couleur fauve; il se trouve aussi des jeunes pousses inermes; pétioles parsemés de glandes tres-fines, inermes ou faiblement aiguillonnes; : j-7 folioles fermes, coriaces, glabres, d'un vert clair en dessus, glauques 'n 'essous, nervures blanchatres, les secondaires parsemées de quelpues rares grlandes, la còte médiane glandulcuse, ovales-lancéolées, un peu acuminces, doublement et triplement dentées, les dents principales grandes, onvertes, terminćes par un petil mucron, les secondaires grlanduleuses; stipules glabres, parsemées de glandes en dessous, a bords glanduleux, oreilletes aiguës droites; pédoncules solitaires ou par 2-5̄, glabres; tube du calice ovoïde-allongé, glabre ; divisions calicinales cuspidées au sommet, glabres sur le dos, 2 entières, 5 pinnafides à appendices prespue filiformes, bordés de que lques glandes, plus comres que la corolle, eaduques; styles trìsvelus, disque plan; fleur grande, rose; fruit arroudi.

HaB. - Autriche. Tyrol: Alaunthal près la ville de Krems (Kerner).

297. R. Mexuosa Rau, enum. ros. (1816), p. 127, non Ratf.; Tratt., I. c., II, p. 74; BI. et Fing., I. c., p. 65/;

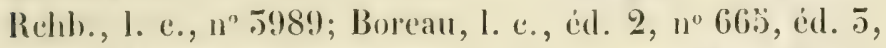
$n^{\circ} 867$; Déséglise, essai monog., p. 97; de Martr.-Don., 1. c., p. 25.5; Cariot, 1. c., p. 18/; Fourreau, 1. c., p. 75̈; 
R. rubiginosu var. Aexuosu Limlley? I. c., 1. 88; Desportes, 1. c., no $195 \%$; R. foetida Boreau, l. c., éd. 1, 404, excl. syn.; R. colline var. flexuse Du Mort., I. c., p. $\check{8} 8$.

Hab. Juin. Haies, bois. - France. Vosges : Château d'Andlau en descendant vers le Rüppelholz (Boulay); - Cher: Plou, Poisicux (Blondean, 185̃, qui la connaissait aussi à Marmagne et la nommait R. montena Vill.), forêt du Rhin-du-bois! bois de Rouct et de la Touche, commune de Mehun! bois de Marmagne! bois des Dames, commune de Trouy! bois du Corpouay près de Saint-Éloy-de-Gy! Usages de Cerbois! bois de Galambert! la Servanterie ! - Rhône : Lyon bois de l'Etoile, Tassin (Ozanon), Charbonnière, Francheville (Chabert); - Ain : Lyon à la Pape (Ozanon). - Alsace. Bois montagneux du Jaegerthal près de Niederbonn! - Autriche. Autriche infér. : Krems (Kerner); - Bohème: Babitz près de Leitmeritz (Kerner).

298. Th. pseudo-flexuosin Ozanon, in Déséğlise, descript. de qq. esp. nouv. de ros. (1864), p. 42, extr., p. 10; Fourreau, 1. c., p. 75 .

Exs. Déséglise, herb. ros., $n^{\circ}$ 28; Billot (suites), $\mathrm{n}^{\circ} \mathbf{5} 852$.

IlAB. Juin. Bois. - France. Rhône : Lyon à Charbonnière (Ozanon), Tassin à Méginant, Chapoly (Boullu).

299. R. speciosa Déséglise, 1. e., p. 59, extr., p. 7 (1864); Fourreau, 1. c., p. 75.

Exs. Déséglise, herb. ros., n² 25; Unio itiner., an. 1859; Billot (suites), n 5592 .

Hab. Juin. Bois. - France. Cher: bois de Charron et de Narmagne! - Rhône: Lyon à Charbonnière (Ozanon), Tassin, Francheville, SaintGenis-des-Oltières (Bullu). - Russie-d'Europe. Volhynie(Ilohenacker).

500. R. Mundzilliama Besser! enum. Pol. et Volh. (1822), p. 46 et p. 67, ex exempl. aulh.!; Rehb., 1. с., 
11"4015; Borean, I. c., éd. 5, nº 868; Déséglise, in Billot, annot. fl. de Fr. et d'Allem., p. 126 et ess. monog., p. 98; de Martr.-Don., p. 252; Cariot, 1. c., p. 183; Fourreau, I. c., p. 7丂̈; R. Jundzilli Besser, cat. hort. Crem., an. 1816, p. 117; M.-B , Il. Taur--Cauc., III, (I8I9), p. 5/7, excl. syn. DC.; Trat., I. c., II, p. 77; R. glandulosa Besser, cat. hort. Crem., an. 1811, supp. 5, p. 20, non Bellardi; R. reticulate Kerner, OEsterr. bot. zeitschrift. (1869), $\mathrm{n}^{\circ} 11$, extr., p. 8.

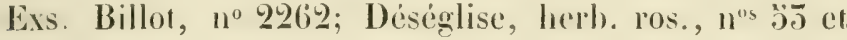
วั5 his.

Har. Juin. Haies, loois. - Russie d'Europe. Volhynie (Besser, in herb. Déséglise). - Franee. Vosges : Rambervillers (Boulay); - Loir-et-Cher : Gières (Franchet); - Cher : Saint-Martin-d'Auxigny (Ripart), Montifaut près de Valio! Roulon et bois de Gérissai commune de Berry! forêt du Rhin-du-bois! La Servanterie! Vaubert! Saint-Florent! - Rhône : pont d'Alay près de Lyon, Charbonnière (Chabert), Saint-Lager (Boullu); Indiqué dans le départ. du Tarn, par M. de Martrin-\$onos, dans sa florule, - Suisse. Cant. de Schaffhouse (Christ). Mon étiquette ne porte que cette indication vague. - Autriche. Autriche-infér. : Oberbergern près de Mautern, Rehbergerthal près de Krems (Kerner).

Var. B. Besser, enum. Pod, et Volh., p. 67. Foliis minoribus, basi minus rotundatis et glandulosis, petiolis pubescentibus, aculeis paucissimis. E Kuna Pod. Aust. (Besser).

Cette variété est representée dans l'herbier DC. par un échantillon venant de Besser même; l'étiquette est ainsi : " $\boldsymbol{k}$. Jundrillii var. b. E Podol. 1824, Besser!"

Échantillon haut de 25 centim., portant quatre ramuscules; le rameau est inerme ou presque inerme; $\overline{5}$ à 4 petits aiguillons longs de 2 millim, dilatés à la base en forme de disque, droits, le's ramuscules portent des arguillons sétacés entanduleus, épars, peu ahondants. Pétioles pubeseents, ayant quelques rares glandes stipitées, inermes ou faiblement aiguillonnés. Folioles 5-7, médiocres, espacées sur le pétiole, la terminale ovale-arrondie, termnée en pointe eutrte, sur les pétioles inférieurs la terminale est obovale, arrondie au sommot, cunéiforme à la base, les folioles latérales 
ovales-elliptiques ou obovales, fermes, vertes, glabres en dessus, glaucescentes, pubescentes en dessous, à nervures blauchâtres, saillantes, églanduleuses, simplement dentées, à dents ciliées. Stipules glabres en dessus, parsemées de poils en dessous, oreillettes aiguës, peu divergentes, presque droites. Pédoncules solitaires, Jongs, chargés de glandes fines stipitées. Tube du calice ovoïde, hispide à la base. Divisions calicinales longues, terminées en une longue pointe spatulée, glanduleuses sur le dos, 2 entières, 5 pinnatifides à appendices bordés de glandes. Styles courts, hérissés, disque presque plan. Fleur grande. Fruit...

Cette variété de Besser est certainement étrangère au $R$. Jundzilliuna et même à la section Rubiginosae!

Var. C. Besser, 1. c.; eadem aculeis fortibus, inferne compressis parum arcuatis. Charkowia (Besser).

Var. D. Besser, 1. c.; foliolis oblongis sesquipollicaribus et ultra, sublus eglandulosis, serraturis divergentibus, aculeis similibus, fructibus globosis. E distr. Rown (Besser).

Var. E. Foliolis var. B, minoribus, subtus minus glaucis, aculeis var. C, ramulis floriferis, superne nudis, receptaculo magis subgloboso, floribus carneis. E Silesia (Besser).

Var. F. Foliolis var. B, sed subtus magis glandulosa, aculeis var. C, ramis floriferis eaeteris magis setoso-aculeatis. E Krasninki Pod. Austr. (Besser).

Var. G. Foliolis praegressi omnibus minoribus, subtus valde glandulosis, petiolis et costis pubeseentibus, aculeis var. $\mathrm{B}$, pedunculis et ealycibus parce setosis. E Krasnenki 'Besser).

Hae nisi intermediis jungeretur cum $R$. Jundzilliana ab affinitatem cum $\boldsymbol{R}$. provinciali inter gullicanas militante, potius ad rubigineas trans. ferri deberet (Besser).

Ons. 1. Ces cinq dernières variétés ne sont pas représentées dans l'herbier DC. ; j'ai rapporté in extenso les diagnoses de Besser qui sont dans l'énum. Pod. et Volh.

OBS. II. En 1811, Besser, cat. hort. Cremen., Sup. ̄, p. 20, décrit un R. glandulosa (non Bellardi 1786-1801). - R. (glandulosa) calycis tuho elliptico, laciniis, pedunculis, petiolis costisque foliolorum glandulosohispidis; foliolis elliptico-ovatis biserratis subus glaucescentibus, aculeis subreflexis sparsis. Besser in Bieberst, I, c.

En 1816, Besser, cat. hort. Cremen., p. 117, donne le nom de R. Jundsilli à son R. glamduloser. 


\section{( 261$)$}

R. germinibus ovatis basi pedunculisque hispidis, aculeis caulinis raris hasi dilatatis eompressis recurviuseulis, petiolis glanduloso-villosis aculeatis, foliolis (majuseulis) ovatis argute glanduloso-biserratis, subtus glaucesecntibus subvillosis : venis glandulosis, floribus subcorymbosis. Besser in Bicberst., 1. c.

En 1S22, Besser, enum. Pod. et Volh., p. 46, conserve le nom de R. Jundzilli; puis dans le même livre, p. 67 , il lui assigne le nom de R. Jundzilliann, ajoutant à ce type six variétés, dont une de ces variétés est représentée par un échantillon dans l'herb. DC.

J'ai done dû prendre pour base du $R$. Jundzilliana le type mème de Besser que je possède en herbier et non la plante distribuée par Hohenacker quoique renant de la Volhynie.

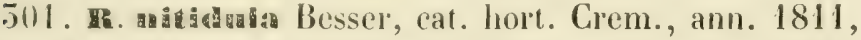
sup. 4, 1. 万o el ann. I8I6, \%. 118, enum. Pod. et Volh., 1). 20 et p. 67 ; Biel., 11. Taur.-Cauc. (1819), III, 1. 5/47; 'Tralt., mon. los., II, p. 76; R. canina pilosiuscula Ser., in DC., prod., II, P. 615 part.; $R$. arguta Stev. ex Bieb.

Foici la description minutieuse que j’ai pu établir sur un yype de Besser conservé dans l'herbier DC. L'étiquette porte: "h. nitidula Mihi, “at. Crem. specimen eulum e specim. spontaneo. Besser, 1820. »

Un tris-grand echantillon couvant presque une feuille d'hephice. Aiguillons unls sur le rameau, sur 8 ramuscules qui sont sur. le rameau, cinq portent 1-5 petits aiguillons fins non sétacés granduleux. Pétioles pubérulenis, g̈landuleux, aignillonnés en dessous. Folioles 5̈-7, médiocres, ovales, quelpues-mues un peu pointues au sommet, vertes, glabres ('n dessus, glaucescentes et pars'mées en dessous de poils qui doivent disparaitre avec l'àge, un grand nombre de folioles sont pourvues de poils et d'autres en sont dépourvars, glanduleuses sur

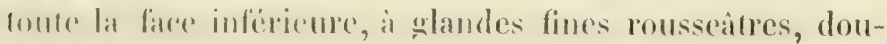


blement dentées. Stipules glabres en dessus, parsemées de grandes en dessous au sommet, la partic intra-stipulaire glanduleuse. Pédoncules solitaires, hispides-glanduleux, braciées ovales, plus longues que les pédoncules. Tube du calice petit, globuleux, lisse ou hispide à la base. Divisions calicinales longues, glanduleuses sur le dos, spatulées au sommet, à pointe un peu denticulée, 2 entières, 5 pinnatifides ìappendices bordés de glandes, réfléchies à l'anthèse. Styles courts hérissés, disque presque plan. Fleur petite.

II a. Cum affinibus in Tauriae nemorilus atque dumetis passim occuirt (Bieberstein).

Obs. Dans l'herbier DC., se trouve un autre spécimen de Besser ainsi nommé : “ $R$. nitidula var. b. mihi. E Podol. Austr. ad Hypanim. Besser, 1824. "Voici la description que j'ai pu établir ì l’aide de l'échantillon de Besser.

Rameau portant des aiguillons dilatés à la base, courbés au sommet, dégénérant en soies glanduleuses. Pétioles velus-glanduleux, inermes et faiblement aiguillonnés. Folioles 5̈7, ovales-elliptiques, ovales-arrondies ou obovales, parscmées de poils courts en dessus, la majeure partic des folioles ont des glandes à la face supérieure, plus ou moins couvertes de glandes rousseâtres en dessous et de quelques poils, la nervure médiane plus ou moins velue, doublement dentées. Stipules glabres en dessus, glanduleuses en dessous; oreillettes aiguës, droites, bordées de glandes. Pédoncules parsemés de glandes, bractée petite, ovale, acuminée, glabre, bordéc de glandes égalant ou dépassant les pédoncules. Tube du crelice ovoïde, lisse, hispide à la base. Divisions calicinales glanduleuses sur le dos, terminées en pointe plus ou moins denticulée à denticules glanduleux, 2 entières, 5 pinnatifides à appendices bordés de glandes réfléchies à l'anthèse. Slyles hérissés, disque plan. Fleur grande.

J'ai souligné les earactères différentiels. Je n'ai vu les fruits ni du type ni de la variété; les échantillons sont seulement en fleurs dans l'herbier DC. Cettevariété ne se trouve mentionnée ni par Besser, ni par Bieberstein, ni par Trattinnick.

502. IR. Iivescens Besser, cat. hort. Crem., an. 1811, 
sup. 4, p. 19, an. 1816, p. 118 et enum. Pod. et Volh., p. 20, p. 67 ; Tratt., I. c., I, p. 60.

HAB. - La Pologne australe (Trattinnick). - Il existe dans l'herb. DC., un type de Besser, mais l'étiquette ne porte pas la localité et seulement l'année 1820.

Var. B. major Besser, in herb. DC.! - L'étiquette de Besser est ainsi : « R. livescens b. major Mihi, ad Ilypaniam, 1824, Besser. " Je ne vois cette forme mentionnée ni par Besser ui par Trattinnick; voici la description que j'ai pu établir sur l'échantillon de Besser.

Rameau et ramuscules inermes. Pétioles lisses, glanduleux, aiguillonnés. Folioles 5-7, grandes, ovales-elliptiques, vertes, glabres en dessus, glanques en dessous, nervure médiane et nervures secondaires parsemées de glandes, doublement dentées. Stipules allongées, glabres en dessus, parsemées de glandes en dessous, oreillettes aiguës, divergentes. Pédoncules hispides-glanduleux, bractées fígalant les pédoncules. Tube du ealice ovoïde, glanduleux à la base. Divisions calicinales spatulées au sommet, parsemées de fines gylandes sur le dos, 2 entières, 5 pinnatifides à appendices bordés de glandes. Styles très-velus, disque plan. Fleur détruite par les insectes. Fruit...

\section{c.) Pseudo-rubiginosae.}

Déseglise olss. on the difl. meth. proposed for the classif. of the spec. ros., in the Vaturalist, $n^{\circ} 20$ (1863), p. .5.5; Rubiginosae a) sepiacene Crépin, monog. ros., fase. I (1869), 1). 25; Cottet, énum. ros. du Valais, in bull. soc. Murith. (1874), fase. 5, p. 42.

1.

( \iguillons uniformes, ne dégénérant pas en soies

1 sétacés gylanduleuses. . . . . . . . 2.

| Aiguillons dégénérant en soies sétacées glanduleuses sur les rameaux florifères. . . . . 15.

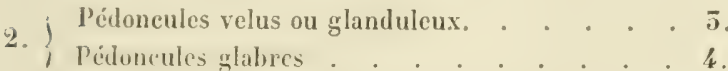

Pedonentes glabres . • . • • . . 4

P'édoncules velus, pétioles aiguillonnés, folioles ovales ou obovales, styles velus, fleur rose, fruit

-) aruondi

Billelii.

| Pédoncules glanduleux, pétioles inermes, folioles très-atténuées à la base, styles hérissés, fleur petite, rose, fruit rouge orangé, ovoïde.

Iadamifere. 


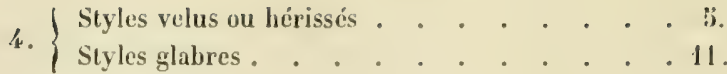

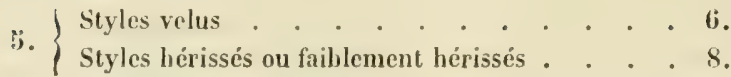

Folioles petites, tube du calice petit, globuleux,

6. fleur petite, rose, fruit petit, sphérique, couronné par les sépales persistants . . . . Lugdunensis.

Folioles non petites . . . . . . . . 7.

( Pétioles lisses, couverts de glandes, tube du calice

7. subglobuleux, fleur rose, fruit gros, arrondi .

Jordani. ovoïde, fleur blanche, fruit globuleux

Vaillantiana.

Arbrisseau à rameaux couverts lie petits aiguillons, folioles très-petites, ovales-arrondies, tube du calice petit, globuleux, styles glabres ou

8. obscurément hérissés, fleur petite, rose, fruit petit, subglobuleux. . . . . . . .

Arbrisseau à rameaux non couverts de petits aiguillons . . . . . . . . . 9.

Seraphini.

Pétioles pubérulents, glanduleux, tube du calice obovoïde, styles hérissés, fleur blanche carnée,

9. fruit gros, ellipsoïde, couronné par les sépales persistants . . . . . . . . . . Pétioles lisses glanduleux . . . . . . 10.

Tube du ealice ovoïde-allongé, styles presque glabres, fleur blanche ou rosée, fruit ovoïde-

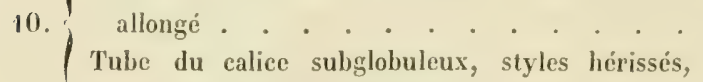
Cheriensis. sepium. fleur blanche, fruit globuleux . . . . virgullorum.

Petit arbrisseau, rameaux floraux aiguillonnés, iolioles petites, ovalcs-aiguës, tube du calice

11. ovoïde, fleur blanche, fruit ovoïde . . . . .

Arbrisseau à rameaux floraux inermes ou presque inermes. . . . . . . . . 12

agrestis. 


\section{( $26 \ddot{3})$}

calice ellipsoüde, fleur petite, hanche, fruit

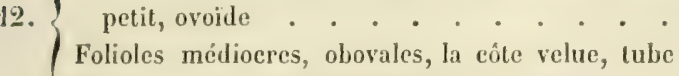

mentila.

du calice ovoide, fleur blanche, fruit ovoïde. .

arvatice.

Folioles ovales-clliptiques, pédoncules hispides glanduleux, divisions calicinales glanduleuses sur le dos, tube du calice oroîde, lleur rosée,

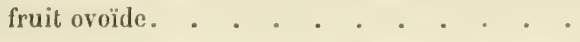

subdota.

15.

Folioles arrondies ou obtuses, pédoneules lisses, divisions calicinales glanduleuses sur le dos, persistantes sur le fruit, tube du ealice petit, globuleux, fleur blanche, fruit globuleux - Biturigensis.

505. R. Hungarica Kerner, Uester. bot. Zeitschr. (1869), p. 254; Crépin, 1. c., p. 69.

Hab. - Autriche. Hongrie : au pied du Piliserberges (Kerner).

504. MR. grandiflora Wallroth, ann. bot.(1815), p. 66 ; Tratt., l. c., II, p. 51 ; Bl. et Fing., l. c., p. 650 ; Desportes, 1. e., p. 82 ; R. rubiginosa var. grandiflora Seringe, in DC., prod., II, p. 616.

\section{ILs. - In Saxonia, ad marginem nemorum (Wallroth).}

OBS. Je ne connais pas ce rosier, qui peut-être, ne doit pas faire partic de cette subdivision.

503. R. sepimm Thuillier, fl. Paris (1799), p. 252; Bastard, l. c. (1809), p. 189; Mẻrat, I. e. (1812), p. 192 ; DC., I. c. (1815), V, p. 358 , excl. syn.; Leman, 1. c. (1818), extr., p. 10; Tratt., l. c. (1825), II, p. 52; B1. et Fing., I. c. (1825), p. 629; Desportes, I. c. (1828), $n^{\circ} 1979$; IIost, l. c. (1851), II, p. 21 ?; IIooker, British flora (185:i), p. 258 ?; Boreau, l. c. éd. 2 (1849), $n^{\circ} 683 \%$, éd. $5(1837)$, no 870 et eatal. II.-et-Loire (1859), p. 80 ; Godet, I. c. (1853), p. 21/4; Arrondean, 
1. c. $(18.34)$, 1. 126 ; Reuter, 1. c. (18(i1), 1. 72; Déséglise, I. c., p. 14.5, extr., p. 105 (1861); de Martr.Don., 1. c. (186/), p. 25, Grenier, 11. juras. (186/), p. 230 , excl. var. b.; Cariot, I. e. (1865), p. 188; Du

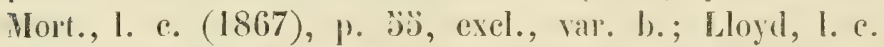
(1868), p. 177; Foureau, l. c. (1869), p. 73; Perard, 1. c. (1869), p. 82; Verlot, I. c. (1872), p. 117; Gottet, I. c. $(1874)$, p. $42 ; R$. canina var. sepium DC., I. c.

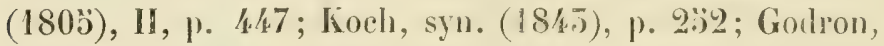
1. Lorr. (1845), I, p. 221 ; Coss. et Germ., fl. Par. (1840), p. 179; R. sepium var. rosece Desvaux, journ. bot. (1815), II, p. 116; Thory, I. c. (1820), p. 115; R. rubiginosa var. sepium Saint-1mans, I. c. (1821), p. 206; Seringe, in DC., prod., II (182.3), p. 617, excl. syn. Hall., Rau; Du Mort., 11. Belgica (1827), p. 95; Chevalier, I. c. (1827), 1 , p. 691 ; Duly, bot. (1828), I, p. 178; Gr. et God., I. c. (1848), I, p. Ӟ00 ; Gomnet, I. e. (1848), p.4.79; hirschleger, I. c. (18:52), I, p. 249; R. inodora Mries! novit. (1814), I, p. 9; Seringe, I. c., p. (616; Babington? man., éd. 6, p. 12k; R. stylosa var. glandulosa Seringe, 1. c., p. 399; R. glutinosa Schulz, Starg., sup., 27, non Smith; R. pseudo-sppium Callay!; Cottet, 1. c., p. 42.

Icon. Redoute, les roses (1824), livmis. 22, c. médiocre; Engl. bot., third. edit., tal,. k70? la figure en fruit a les aiguillons horizontaux, la figure en bouton a le pédoncule hispide-glanduleux, la figure aree feuilles et fleur ouverte pourrait à la rigueur ètre prise pour le $\boldsymbol{R}$. sepium. Cette planche est la reproduction de la planche 265 de l'English botany, ed. I.

Exs. Reichenbach, $n^{\circ}$ 1898; Fries, herb. norm. fase. 
X, no $ّ 1$ ! Billot, nos 1870, 1871 bis, ter et quater ; Seringe, $n^{\circ} 47$ !; Déséglise, herb. 1’os., $n^{\circ} 50$.

IIAB. Juin, juillet. Haies, bois. - Ce rosier est généralement répandı en Europe. Nanquerait-il en Espagne, en Portugal, en Asie? Je n'ai pas vu un seul échantillon de $\boldsymbol{R}$. sepium venant de ces pays.

Var. B. pulseseens Rapin, in Reuter, cat. de Genève, p. 75. - Fenilles it folioles pubémulentes en dessous ainsi que le pétiole.

IIs. - France. Allier : haies à Arẹon près d'Ebreuil (Lamotte); Saroie : près de IJontmélian (Songeon); base du Salève. - Suisse. Jura de Bùie (Christ). - Ilalie. Florence (Levier).

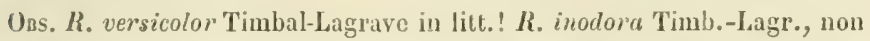
Iries. - Je donne in extenso la note de II. Timbal-Lagrave, que j’ai reçuc avec la plante; j'ai vu trop peu d'exemplaires pour me prononcer; mais je crois que e'est une espèce particullère et bien différente du R. sepium Thuil. Fleurs grandes, $\overline{5}$ ou 4 au sommet des rameaux, rose-pourpre vif mais pàlissant jusqu'au rose tendre selon que l'anthèse est plus ou moins an ancée ; pédonculcs tins, glabres; ovaire elliptique, glabre; sépales fortement pinnatilides et mème bipimnatifides, glanduleux, ovales, cordés au sommet, très-velus en dedans; étamines à filets longs, glabres; styles courts, très-velus; bractées ovales, cuspidées, glanduleuses; feuilles à pétioles glanduleux, obovales, doublement dentées, glanduleuses en dessous, glabres en dessus, dents très-profondes; rameaux courts, diffus, inermes; arbrisseau de 1 mètre, un peu diffus, peu épincux ('TimbalLagrave, leltre du 4 févr. 1873).

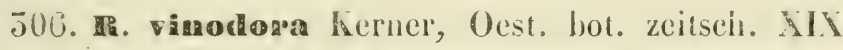
(1869), 1. 529, сxtr., p. ̈̈; Crépin, 1. c., p. 11:3.

Ilas. - A utriche. Tyrol : Innsbruck, entre Zirl et Fragenstein (Kerner).

507. 1R. agmestis Savi, 1. Pis. (1798), I, P. 473 et Trattato degli alberi della Toscana (1811), I, p. 190 (non Gmel.); Pollini, 11. Veron. (1822), II, P. 144; Desportes, 1. c., no 1984; Rehb., 1. c., p. 619; Gussone, syn. Sicul., I, p. Ӟ6̈̈; Boreau, l. c., éd. J (1837), 
11" 871 et catal. I.-et-Loire, p. 80 ; Déséglise, in Billot, annot. 11. de Fr. et d'Allem., p. 127 et ess. monog., in mém. Soc. Acad. de M.-et-Loire, X, p. 14/, cxtr.. p.10/; Kirschleger, I. e., III, p. 566\%; de Martr.-Don., l. с., p. 25/4; Cariot, 1. c., p. 186; R. sepium rar. parviflora Bastari, 1. c., sup., p.51; Pérard, I. c., p. 82; R. sepium var. alba Desvaux, journ. bot. (1815), II, p. 116; R. sepium var. arrestis Carion, cat. S.-ct-Loire (18:39), p. 42; Grenier, 1. c., p. 2:30; Du Mort., l. c., p. ̋̈6; R. sepium var. myrlifolia Thory, l. c., p. 114; R. myrtifolia IIaller fils in Schleicher, cat. plant. IIelv. exsiccatarum, al, anno 179/4 et seqq., sine deseript.; Desportes, I. c., no 1981; Boreau, 1. c., éd. 2, II, p. 181; R. albiflora Opitz, in Tratt., l. c., I, praef., p. 58? Bl. et Fing., 1. c., p. 645; R. rubiginosa flore albo Pollini, viag., pp. 18,$89 ; \boldsymbol{R}$. sylvestris foliis odoratis Seguier, fl. Veron., II, p. 512 .

Icov. Pollini, fl. Veron., tab. 2, f. 4; Redouté, les roses (1824), livrais. 57, D.

Exs. Seringe, no 10!; Billot, $n^{\circ}$ 2265̄; Déséglise, herb. l'os. $n^{\circ} 5 \overline{5}$.

IIAB. Juin. Lieux pierreux. - France. Maine-et-Loire : Angers (Bastard, 1810, in herb. DC.); - Cher, G.; - Allier : Montluȩon (Pérard, Catal.); - Saône-et-Loire : Monthélon près d'Autun (Carion), Châlonssur-Saòne (Ozanon); - Lozère : Mende (Prost, 181\%, in herb. DC.); Gard : Anduze (Miergue); - Iaute-Garonne : Toulouse (Timbal-Lagrave); - Rhóne : Chaponost (Boullu); - Isère : la Bastille de Grenoble (Verlot); - Var: le Luc (Hanry); - Alpes-maritimes : Antibes (Bornet); - Savoie : Chambéry (Songeon). - Suisse. Valais : Bovernier! - Italie. Florence (Levier), Palerme (Todaro).

508. mentita Désiglise, descript. qฯ. esp. nour. ros., in Billotia (1864), p. 行, extr., p. 11. 
Exs. Déséglise, herb. ros., $n^{0} 51$.

II.s. Alai, juin. Broussailles. - France. Haute-Savoie: Thonon it la pornte de Ripaille (Puget).

509. H. arvatica Puget, in Baker, review of the British roses (186/), p.5̄ et in Engl. hot., third edit., II (1864), p. 517; Cariot, I. c., II, p. 186; Fourreau, 1. c., p. $7 \check{3}$; Cottet, 1. e., p. 4s̄; R. canina var. areatica Baker, monog. British roses, p. 229; R. trachyphylla var. arvatica 1) Mort., I. c., p. 59?

Exs. Baker, herb. ros. brit., $n^{\text {os }} 25$ ?, 26?, 27.

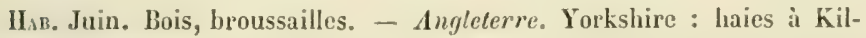
vinglon (Baker). - France. Savoie : Méry près d'Aix, Salins près de Moutitrs (Puget); - IIante-Savoie : montagne d'Annecy-le-Vieux près de la Ilalveria, la Margeriaz, Thonon route d'Orsier.

510. IR. virgultorum Ripart, in Déséglise, I. e., (186\%), p. 44, extr., p. 12; Fourreau, 1. c., p. 73; Virlot, 1. c., p. 117; Cottet, 1. c., p. 45; R. neglecta Ripart, olim (non Leman); R. sepium var. sphaerocarpa Cariot, l. c., p. 186 ?

Exs. Déséglise, herb. ros., $\mathrm{n}^{\circ} 52$.

Uan. Mai, juin. Haies, bois. - France. Cher : Bourges (Ripart), la Chapelle-Saint-Lrsin, Mareay près de Quincy, Valio, Saint-Eloy-de-Gy, Mehim; - Allier : les Gazeriers (Lamotte); - Saône-et-Loire : Châlonssur-Saône (Ozanon); - Rhône : Mont Cindre (Chabert), Charbonnière (Ozanon), Vernaison (Boullu); - Isère : la Bastille de Grenoble (Verlot); - Ilautes-Alpes : Gap (Burle); - Ilaute-Savoie : Thonon, pointe de Ripaille (Puget). - Suisse. Valais : Bovernier. - Autriche. Tyrol : Laserz (Kerner).

511. R. Brilletii Puget, in Crépin, primit. monog. ros., fasc. 1 (1869), p. 116.

Exs. Billot (Suites), $11^{\circ} 5.394$; Kotseliy, Perse bor'., $n^{\circ} 276$ ? 
IIab. Juin. Broussailles. - France. Haute-Garonne: Toulouse ('T'imbalLagrave); - Savoie : Salins près de Mouticrs (Puget).

512. R. Seraptrani Viviani, al. fl. Ilal. fragm. (1808), J) 67 et 01. cors. spec. nov. (182/), p. 8; Geringe, in DC., prod., II, p. 623̈, Despories, I. c., n 1989; Gusonne, 1. с., p. 5̌64; R. rubiginosa var. C. b. Mutel, fl. fr., I, 1. 530 ; R. graveolens var. Corsica Gren. et Godr., I. c., I. p. כ̌61; de Marsilly, catal. pl. Corse, p. 56 .

Exs. Aucher-Eloy, $n^{\circ} 14 \overline{\mathrm{o}} \mathrm{k}$; Kralik, jul. corses, 1849, sans numéro.

Hab. Juin. - France. Hle de Corse : mont Coscione (de Forestier), Bastia (Kralik). - Ilalie. Toseane : Pise (Savi); - Sicile : Madonie ('T'odaro). - Grèce (Aucher-Éloy).

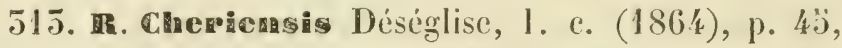
extl., p. 15̄; Verlot, I. c., p. 117; Cotlet, I. c., p. 42; R. Boullui Gandoger, soc. Dauph. (1874).

Exs. Déséglise, herb. ros., $n^{\circ} \breve{4}$.

IIar. Mai, juin. Broussailles, haies. - France. Cher : Carrières de la Chapelle-Saint-Ursin! - Rhône : Saint-Genis-les-0Hieres, Montmelas, mont Thoux près de Lyon (Boullu); - Haute-Savoie : Saint-Gervais (Boullu); - Hautes-Alpes : mont Bayard sur Gap, Charrance (Gariod); - BassesAlpes : route de Gap à Barcelonnette (Ozanon). - Suisse. Cant. de FriJourg: Montbovon route d'Ayre (Cottel); - Valais: Bovernier (de la Soie). - Autriche. Tyrol : Leopoldstadt (Kerner).

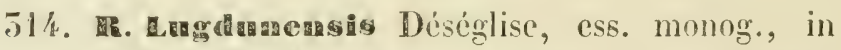
mém. Soc. Acal. de M.-et-Loire, X (1861), p. 14, extr., 1. 101; Cariot, 1. c., p. 186; Fomreau, 1. (2., 1. 73 ; h. graceolens var. eriophora Grenier, 11. juras. (1864), p. 249 (non Gu. et Godr. Il. de Fr.); R. graceolens V'erlos! i. e., p. 117 (non Gr. et Godr.); R. graveolens Gr. et Godr., I. c., I, p. $乡 60$, part.

Exs. Billot (suites), no 5855, excl. syn. Rau. 


\section{$(279)$}

II.ı. Mai, juin. IIaics, broussailles. - France. Rhône : route de Villeurbanne au Molard (Chabert), Tascin ì l'Aiga (Boullu), mont Cindre (Ozanon); - Ain : Echeys (Chabert); - Isère : Pierre-Frête (Ciłaberi), la Salette, Villard-de-Lans, mont Anoysin près de Crémieu, Saint-Christophe-en-Oisans (Boullu), Pariset en allant à Saint-Nizier (Vealot); Hautes-Alpes : entre Brusinel el les Barrayues (Gariod); - Ilaute-Savoic : Arenthon (Pugel); - Savoie : Salins près de Moutiers (Puget), Chambéry (Paris), Bellecombette (Songeoni; - Ardèche : Lonvese (Boullu). Suisse. Valais : mont havoire près de lovernier, Sembrancher, Sion (Cottet).

Var. 13. maceacanada. Aiguillons moins nombreux sur les rameaux; arbrisseau plus éleve; feuilles plus grandes; fruit gros, sphérigue; fleur petite, d'un rose vif' d'après Chabert. - France. lihône : (ntre Champagny et Dardilly (Chabert), mont d'Or au-dessus de Couzon (Boullu); Isère : Saint-Nizier près de Grenoble (Verlot). - Suisse. Valais : Bovernier.

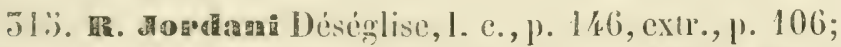
R. rubiginose Mahlenbere, fl. Carp., p. 130 ex Rau; R. rubiginosa var. glabra liau, enum. ros., p. 157; Bl. et Fing., 1. c., I, p. (58; Boreau, 1. c., éd. 5, II, 1. 737; R. rebiginosa var. d. Bochst. Forstb., $1042 \mathrm{ex}$ Rau; R.graveolens var. muda Grenier, 11. jur., p. 249?

Inas. Juin. Broussailles, haies. - Belgique. Prov. de Namur : Rochefort? (Crépin). - France. Puy-de-Dòme : Clermont, escarpements de la route de Clermont a Bordaaux; - Yonne : Auxerre (Mabile); - Isere : Villard-de-Lans (Boullu); - Loire : Lupé (Boullu). - Autriche. Tyrol : Anthol\%, Miedors, vallée de Stubai (Kerner).

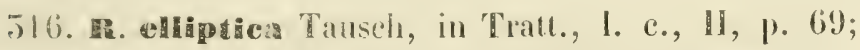
Seringe, in DC., plod., II, p. 623; Bl. et Fing., l. e., p. (i.)6; R. rubiginose Grimpel, deatsch. Holzart., I, p. 121, tab. 91 ex Trallinnick.

IIav. Juin, juillet. - Autriche. La Boheme. Je n'ai pas vu ce rosia placé par Seringe dans le Prodromus au rang des espèces douteuses.

\section{7. 뜨. Vaillantiana Boreau.}


Arbrisseau de 1-2 mètres, à tiges munies d'aiguillons robustes dilatés à la base, arqués ou presque droits, quelques fois nuls sur les rameaux florifères; pétioles pubérulents, glanduleux, aiguillonnés en dessous ou inermes; 3-7 folioles ohovales, d'un vert clair, parsemées de petits poils apprimés en dessus, poils disparaissant à l'état adulte, pubescentes-rglanduleuses en dessous, à grlandes fauves, il y a aussi quelques folioles inférieures qui portent à la face supérieure des glandes, doublement dentées à dents glanduleuses; stipules glabres en dessus, glanduleuses en dessous, oreillettes divergentes; pédoncules solitaires ou en bouquet, lisses, courts; bractées de la base du bouquet ovales-acuminées, glabres en dessus, parsemées de glandes en dessous, plus longues que les pédoncules; tube du calice ovoüle ou subglobuleux, lisse, divisions ealicinales longues, terminées en pointe souvent denticulée, lisses en dessous, tomenteuses en dedans, 2 entières à bords tomenteux, 5 pinnatifides à appendices linéaires bordés de glandes, réfléchies à l'anthèse, puis redressées, couronnant le fruit avant la maturité, non persistantes; styles courts, velus; fleur blanche; fruit gros, globuleux.

IIab. Mai, juin. Broussailles. - France. Savoie : bois Champion près de Moutiers, Salins (Puget), collines de Chanaz et de Bellecombette près Chambéry (Songeon); - Ilautes-Alpes : Charrance, Gap (Burle).

518. Hadanifena Timb.-Lagr', précis des herboris. ann. 1870, in bull. soc. hist. nat. de Toulouse, IV (1871), p. 175 .

IIab. Bois. - France. Ilaute-Garonne : bois de Balma à Toulouse (Timbal-Lagrave).

OBs. R. heteropliylla Timb.-Lagrave, in litt.! (non Woods). "Fleurs "blanches légèrement roses, de petite taille, ordin. \& ì au sommet des 


\section{$(275)$}

"rameaux principaux, solitaires sur les rameaux latéraux; pédoncules " courts, glanduleux; ovaire sphérique, glabre, d'un vert rougeâtre; " sépales lancéolés-ovales, longuement cuspidés, pimatifides, glanduleux, "dents peu nombreuses; pétales obovales, cordés au sommet, de petite " taille, jamais étalés; étamines à filets longs, blanc-jaunâtre à la base; "styles hérissés; fruit globuleux, rouge foncé bientôt rougge-noir; sépales "quoique desséchés persistant longtemps."

" Arbrisseau très-touffu, d'un mètre de hauteur, offrant de gros " rameaux qui donnent des tiges grêles, flexucuses, inermes, ces rameaux "portent des feuilles tantôt ovales, tantòt elliptiques souvent sur le même "rameau, plus ou moins atténuées à la base, dentées, dents glanduleuses " ćcartées, d'un vert sombre en dessus, hérissées-glanduleuses en dessons, " de 5 i 7 folioles, les inférieures plus petites, la supérieure longuement "pétiolée. " (Timbal-Lagrave, lettre, \& févr. 1875). - Ilaute-Garonne : Toulouse (Timbal-Lagrave).

519. IR. Anabica Crépin, 1. c., fase. 1, p. 125; R. mbiginosa var. Arabica Boissier, fl. orient., II, p. 687.

Exs. Unio itiner., an. 185̄̌, $n^{\circ}$ 446; Bové, $n^{\circ} 180$; Schimper, $\mathrm{n}^{\circ} 725$.

Hab. Mai, juin. - Arabie. Mont Horeb ou de Sainte-Catherine (Sehimper, Bové).

520. R. Klukii Besser, cat. hort. Crem., an. 1816, p. 118 et enum. Pod. et Vollı., pp. 46, 67; M.-Bieb., fl. Taur--Cauc., III (1819), \%. 546; Tratt., I. c., II, p. 70; BI. et Fing., I. c., p. 629; R. balsamica Besser, cat. Crem., an. 1811, sup. IV, p. 18.

IIAB. - La Crimée.

521. Rr. subdola Nob.; R. Klukii Boreau, l. c., èd. 2, $n^{\circ}$ 684, éd. 5, no 869 (non Besser); Déséglise, I. c., p. 140, extr., p. 100; Grenier, 11. juras., p. 248?

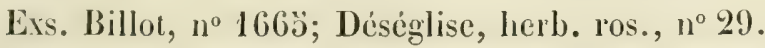

Arbrisseau droit, élevé, à aiguillons assez nombreux, robustes, dilatés à la base, droits inclinés ou arqués, déyéné- 
raml en aiguillons sétaces, les mus termines par une glande, les aulres ceglanduleux; pélioles lisses ou parsemés de poils courts, dhatres de glandes, aiguillomés en dessous; fololes toutes pétiolées, la terminale arromlie an sommet, rétrécic it la hase ou aiguë aux deux extrémités, les latérales ovales-elliptiques, ovales-aignës, quelques folioles infericures subohuses, coriaces, rudes an toncher, glabres, d'un vert luisant en dessus, il y a aussi quelques folioles porfant it la fare supericure des glantes iparses, nervures swillantes of mu peu parsemées de poils courts, couvertes de glandes en dessous, douhlement dentées à dents ghanduleuses; stipules étroites, les unes ghibres sur les deax fares, d'antres glabres en dessus phas ou moins parseméces de glandes en dessous, cilices-mhaluleuses, oreillettes aiguës, divergentes; pédoncules hispides-glanduleux, munis de bractées orales, acminees, glabres en dessus, gुlanduleuses en rlessous, égalant ou dépassant les pédoitcules; tube du calice ovoïde, contracté au sommet, glabre ou hisprie à la base; divisions calicinales en pointe bordée de glandes, glanduleuses sur le dos, denx entières, trois pinnatifides à aprendices étroits hordés de glandes, saillantes sur le bonton, phas courtes que la corolle, réllechies a lanthise, puis redressées, couromant le fruit arant la maturité, non persistantes; styles hérissés, disque plan; fleur grande, rosée; fruit ovoüde, arrondi à la base, un peu attenué au sommet.

Ilı. Juin. IIaies. - France. Loirel : bois de l'Isle près d'Orléans (fullien); - Cher: la Chapolle-Saint-Ursin, la Servanterie près de Hlehun, route de Soye à Bourges; - Yonne: Auxerre (Ribile); - Lot-et-Giaronue : Agen, Saint-imans, 1808, in herb. DC).

Ons. M. Boreau a pris comme moi pour hase du $R$. Klukii, la description donnce par Sprengel, syst., Jl, p. 505. Le type du R. Klukii Besser manque dans l'herbier SC. Ayant demandé des jenseignements sur co 
resier à M. Crépin qui avait à eette époque entre les mains les types de Bieberstein, conservés dans les herbiers du Muséum de Saint-Pétersbourg, H. Crépin a bien voulu m'envoyer un dessin d'un des échantillons authentiques de l'herbicr de Bieberstein accompagné de notes comparatives établics sur la plante de Crimce et sur celle de France. Toici les notes de 31 . Crépin.

* Dans l'herbier de Bicherstein, se trouvent, dans la chemise du R. Kilukii, trois échantillons de ee type sur lesquels je rais rous donner des détails.

10 "Rosu balsamica Besser. Ex Tauria (Iocalité en Russe) sine dubio. "

" C'est un rameau florifère assez grand. Les axes ne présentent aucunc

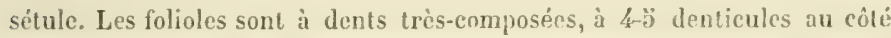
inférieur et 2 ou 5 au côté supéricur; clles sont presque gllabres arec de rares poils à la face supérieure, à côte un peu velue à la face inférieure avec quelques rares poils ẹa et là et des glandes ćparses moins apparentes que dans votre $R$. Ḱlukii de la Serranterie. Les folioles paraissent aroir été moins blanchâtres ou moins pâles en d’ssous que dans votre plante; cn outre elles ne sont pas atténuées comme daus la plante francaisc. Les pétioles sont beaucoup moins glandulcux dans le $R$. Kluliii et arec quelques rares poils. Les pédicelles sont beaucoup plus courts; ils sont lisses ainsi que le réceplacle. Sépales à bords moins glanduleux, moins pinnatifides et moins grands. n

"Somme toute, la plante de Crimée ne peut être identifice avec la vôtre.

$2^{\circ}$ "R. Ucrlsnmica Mihi, sup. IIII. " Je vous ai calyué et dessiné l'échantillon entier. Folioles plus arrondies, mais même dentelure que dans le $n^{\circ}$ 1. Pétioles plus velus. Pédicelles courts, lisses ainsi que le réceptacle. Sépales non glanduleux sur le dos, glanduleux aux bords. Parait être spécifiquement identique avee le $n^{\circ} 1$. Les styles sont abondamment velus.n

50 "Balsamiea Besser. - Rosa rubiginosn "ffinis. Ex Tauria a. 1810. An ad R. argulam Stev."

"A part le mot bulsamica Besser qui parait avoir été écrit par Besser, le peste de l'étiquette est de M.-Bieb."

"C"est la même forme que le $n^{\circ} 1$. Je pense quavece ecs renseignements vous pourrez former les éléments de la distinction du $R$. Filukii de la Criméc d'avec le vôtre. La deseription de M.-Bieb. correspond bien aux échantillons deson herbier. "(Crépin in litteris, 51 jany. 1870).

Ots. II. Jans ma description du R. Klukii, in Billot, ann. Il. de Fr. et 
d'Allem. (1853), p. 10 et essai monogr., 11. 100, j’ai complelement oublié de faire la mention des aiguillons dégénerant en soies glanduleuses. les pédoncules sont toujour's hispides-grlanduleux; e'est en suivant Sprengel que j'ai attribué des pédoncules glahres ou hispides, ce qui fait roir que Sprengel ne commaissail pas très-bien le type de Besser.

II. Grenier, dans sa flore du Jura, p. 248, décrit un $\boldsymbol{R}$. Klukii, dont la description a été ealquée sur la mienne et il cite avec ecrtitude le $n^{\circ} 166 \%$ publié par Billot; puis il donne la localité de Genève. Il a pris ma description sans se préoccuper du type qu'il a entre les mains. M. Grenier m'écrivait le 25 janvier 1874 : a Ce que j’ai publié sous le nom de R. Klukii, dans ma flore du Jura, n'est pas la plante de Besser; mais ce que j'ai décrit sous le nom de R. graveolens Grenier, 11. jur., p. 248, est très-exactement la plante de Besser. Votre R. Jordani ne liffère du R. Kluliii que par ses pétioles glabres et glanduleux non tomenteux. Le $R$. Klulii de ma flore du Jura est l'hybride nomme $R$. Gallico-umbellata Rapin. in Reut. cat., p. 72. " (Grenier).

Qu'est ce que le $R$. graveolens Gren., plante décrite en 1840 dans la fl. de Fr. I, p. J60? Une réunion bảtarde d'une Canine avec des Rubigineu. ses, la var. a. est le $R$. Lugdunensis! la var. b. le $R$. Pouzini, la var. c. le $R$. Seraphini. M. Grenier, dans sa flore du Jura, me semble ne pas comnaitre le type décrit par lui dans sa llore de France, puisque le type primitif devient sa var. b.! M. Grenier, llore du Jura, dit : " $R$. graveolens Gren., fl. de Fr., var. a. nuda. R. Jordani Déségl. Je possède qualques exemplaires de cette variété dont les pédoncules réunis en corymbe sont les uns glabres et les autres hispides-glanduleux. " Avant d'avancer une hypothèse pour un fait acquis, M. Grenier est-il certain de connaitre le R. rubiginosa glabra Rau; le doute peut être permis!

M. Grenier a pris probablement la solution du $R$. Klukii dans la flore de M. Godet. Ce dernier auteur dit dans son suppl., p. 78 : "R. graveolens Gren. R. Klukii Besser! (non Bor, ni Rap.). - Obs. Le R. Klukii Besser n'est point celui de Boreau, ni de Rapin et se rapporte au R. graveolens. Besser dit posilivement de son $\boldsymbol{R}$. Klukï " pédoneules et tube du calice glabres " (conf. M.-B. fl. Taur.-Cauc. III, p. 516), et tel est en effet le R. Klukii que je tiens de Besser lui-même! et qui est identique avec le R. graveolens Gren. " (Godet).

Admettant que ce soit vrai, nous demanderons à M. Godet s'il ignore les lois de la nomenclature botanique. Pourquoi prendre le nom le plus nouveau à la place du plus ancien? La nomenclature botanique a ses lois 
que chaque phrtographe doit suive sans intervertir l'ordre de la synonymic selon son caprice!

D'apres ee que je connais du R. Klukii authentique, M. Godet me semble ne pas avoir regardé bien attentivement son type rue je ne conteste nullement. - I. Cariot, éludes des fleurs (1865), II, p. 187, décrit un R. graveolens qui certainement n'est pas ce que les auteurs de la flore de France ant eu en vue. Chabert m'a envoyé plusieurs échantillons de ce qu'il nommait $\boldsymbol{R}$. graveolens, $\mathrm{t}$ provenant de la localité " entre Champagne el Dardilly. " J'ai reconnu le $R$. Lugdunensis et non autre chose.

522. Me. Tuschetica Boissier, f1. orient., II, p. 675.

Ilsr. - In regione subalpina et alpina Tuschetiae et Daghestaniae prope Beshita Diklo (Boissier).

525̄. R. Biturigensis Boreau, I. c., éd. 2 (18/9), II, p. 650 , éd. 5, n $65 \ddot{3}$; Déséglise, l. c., p. 143̈, extr., p. 103 .

Exs. Schulız, no 1440; herb. norm., n 44; Déséglise, herb. ros., $n^{\circ} 54$.

IIar. Mai, juin. Haics. - Frence. Cher : C. autour de Bourges! la Chapelle-Saint-Ursin!

\section{D.) Verae-rubiginosae.}

Rubiginosae b. micranthae, c. suavifoliae Crépin, I. c., p. 24.4, p. 24ว̈; Cottet, 1. c., p. 4.5; Rubiginosae trib. 5. Déséglise obs. differ. meth. proposed for the class. of the spec. of the gen. ros. (186ij), in the Naturalist, $\mathrm{n}^{\circ} 20$, p. 515 , part.

Feuilles courertes en dessous de glandes odorantes; aiguillons des tiges robustes, crochus ou courbés en faul, droits, dégénérant souvent en aiguillons fins, grèles, sétacés, glanduleux ou églanduleux; pédoncules hispidesglanduleux; divisions ealicinales caduques ou persistantes. 
(Aiguillons dégénérant au sommet des rameaux en

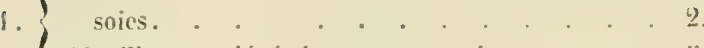

Aiguillons ne dégénérant pas en soies . . . . "..

2 Styles hérissés. . . . . . . . 5

Styles velus ou glabres . . . . . . . . 4.

/ Feuilles ovales-arrondies, pédoncules hispidesglandulenx, fleur rose, fruit arrondi, rouge sanguin . . . . . . . . .

apricorum.

7. Feuilles ovales, pédoncules hérissés d'aiguillons en forme de soies, fleur petite, rose, fruit ovoüle, rouge-orangé, couronné par les divisions calicintiles

comosa.

Arbrisseau élevé, styles velus, pédoncules ordin. réunis en bouquet, fleur d'un rose vif, fruit

4.

ovoïde arrondi . . . . . . . . .

umbellatu.

Petit arbrisscau, aiguillons longs, inclinés ou droits, styles glabres, lleur petite, rose, fruit ovoïde, rouge . . . . . . . . . sylvicola.

i). Styles glabres .

6.

1 Styles hérissús.

12.

( Rameaux floraux grèles, allongés, inemes, fleur (j)

Rameaux floraux aiguillonnés

operta.

$\cdot 7$

f Fleur blanche, folioles petites, ovales-arrondies,

7. fruit petit, ovoüde, rouge-orangé. . . lactiflora.

I Fleur rose. . . . . . . . . 8.

Petit arbrisseau, folioles tres-petites, tube du calice petit, ovö̈le, fleur très-petite, rose, fruit

$\therefore$ petit, ovuïde-arrondi.

Arbrisseau plus ou moins élevé . . . . . . . 9.

9. Tube du calice ovoïde. . . . . . . 10

9. Tube du calice oblong ou subglobuleux. . . . II

10. Folioles ovales, fleur rose. . . . . . mermixtu.

dimimulu.

o. . Folioles elliptiques, fleur rose clair . . . . micranllee. 
Tube du calice subgloluleux, folioles ovales, licur rose, fruit arrondi, rouge sanguin . . . . septicolre.

Tube du calice oblong, feuilles petites, oraleselliptiques, fruit petil, ovol̈le, rouge.

\section{Lemanii.}

f Styles hérisés, folioles ovales, parsemées de tquelques glandes en dessus, tube du calice hérissé de soies, fleur rose, onglet blanc, fruit gros, ovoilde-arrondi, arbrisseau it aiguillons nombreux, les plus petits droits . . . . . echinocarpa.

Styles velus, folioles petites, arrondies, églanduleuses en dessus, tube du calice très-petit, subglobuleux, glabre, fleur rose foncé, fruit globuleux, petit arbrisseau à aiguilions grêles, longa, piespue droits. . . . . . . rolundifolia.

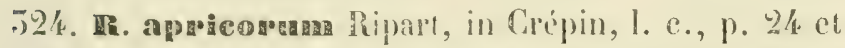
p. 72, sine descript.; R. rubiginosa Auct. pro part.; R. rubiginosa L., mant., 564, pro part., non herl).

Exs. Billot (strites), no 30393 ; Wirtgen, pl. crit., n०81\%; Fries, herb. norm., lase. 5, no 41 ?

Abrisseau élevé, touffu, rameux, chargé d'aiguillons nombreux, rolustes, dilatés comprimés a la base, crochus ou courbés en faulx, roussilres ou blanchitres, dégénérant sourent au sommet des lameaux en aiguillons fins sítacis; peitioles pubescents ğlanduleux, aiguillonnés en dessons; $\ddot{-7}$ folioles médiocres, orales-arrondies, ovalesclliptiques, parsemécs de poils apprimés en dessus, changées en dessous de glandes fauves odorantes, nervures velues principalrment la còte, domblement dentées à dents

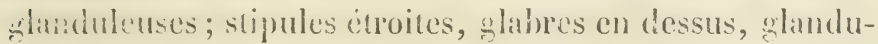
leuses ca dessons, bordées de galandes, oreilletes aiguës diverentes; pédonemles solitaires ou en eorymbe peu fommi, hispides-glanduleux, munis de bractés ovales aruminecs, mlabres sur les deux faces ou glabres en dessus, 
parsemées de quelques rares glandes en dessous, bords glanduleux, plus longues que les pédoncules; lube du calice petit, ovoüde, contracté au sommet, glabre ou hispide à la base; divisions calicinales glanduleuses sur le dos, spatulées au sommet, deux entières, trois pinnatifides à appendices courts bordés de glandes, saillantes sur le bouton, réfléchies à l'anthèse, puis redressées, eaduques; styles très-hérissés, disque presque plan; fleur rose; fruit arrondi, d'un rouge sanguin à la maturité.

Linné a eu en vue sous le nom de $\boldsymbol{R}$. rutiginosa plutôt un groupe de formes diverses qu'une forme spéciale; il en est pour le $\boldsymbol{R}$. mubiginosa comme pour le $\boldsymbol{R}$. villosa $\mathrm{L}_{\text {. : }}$ ces noms doivent être délaissés.

IIAr. IIaies, bois. Juin, juillet. - Espèce vulgaire dans l'Europe centrale, d'après mes échantillons d'herbier. J'ai ce rosier d'Angleterre, de douze départements de la France, de l'Autriche, de l'Allemagne et des Pyrénées d'Aragon.

Obs. R. Canariensis Nob.

M. Bourgeau, plantae canarienses, no 343 , a distribué sous le nom de R. rubiginosa var. umbellata Lindl. un type curieux, mais qui n'est ni le $\boldsymbol{R}$. rubiginosa, ni le $\boldsymbol{R}$. umbellala. Je doute qu'il appartienne à la section Rubiginosae à cause de ses longs styles qui me paraissent soudés en colonne? - J'ignore si ce rosicr a recu un nom. Voici la description que j'ai pu établir avec les échantillons de mon herbier.

Un de mes échantillons mesure 50 centim. de hauteur et porte un tout petit aiguillon dilaté ì la base, presque droit an sommet; l'autre échantillon mesure 10 centim. de hauteur, est inerme, l'écorce est vineuse; pétioles pubérulents, parsemés de quelques glandes fines, inermes ou aiguillonnés; les folioles, au nombre de 3.7, sont obtuses, vertes, glabres ou parsemées de poils courts apprimés et de quelques glandes en dessus, glaucescentes glanduleuses en dessous, à nervures légèrement velues, doublement dentées, les dents principales terminées par un mueron calleux les autres par une glande; pédoneules hispides-glanduleux, en cyme bifide ou trifide; un de mes échantillons a 10 fleurs réunies par $2,5,2,5$; tube du calice ovoïde, hispide à la base; divisions calicinales terminées en pointe dilatée denticulée, glabres et parsemées de quelques 


\section{(281)}

glandes sur le dos, 2 entières, 5 pinnatifides à appendices bordés de glandes, réfléchies à l'anthèse; styles simulant une colonne (comme dans le $R$. hybrida) longue de: $\&$ millim., laineuse, disque plan; fleur...... il est difficile de se prononcer pour la couleur sur un échantillon ancien; fruit......

Haв. Juillet. - Ténériffe, vallée de Bajamar (Bourgeau 1846).

523̈. R. comosa Ripart, in Schultz, arch. de la fl. de Fr. et d'Allem. (1852), p. $25 \%$; Déséglise, ess. mon., in mém. Soc. Acad. de M.-et-Loire, X (1861), p. 1ั̋̃, cxtr., p. 115; Cariot, 1. c., II, p. 188; Fourreau, 1. c., p. 76; R. rubiginosa L., herb.! non mant.; $R$. rubiginosa var. comosa Du Mort., l. c., p. 5้4.

Icov. Flora danica, tab. 870 ; Engl. bot., XIV, pl. 991 et third ed., tab. 468 (très-mauvaise figure qui représente un $R$. rubiginose avec des divisions calicinales entières!); Roessig, die rosen, tab. 25, f. 5; Jacquin, fl. Austr., I, tab. 50?; Svensk botanik, VII, tab. 465.

Exs. Seringe, $n^{\text {os }} 7$ ? 40 ?; Schultz, herb. norm., $n^{\circ} 46$ ! Reliquiae Mailleanae, $n^{\circ} 605$; Déséglise, herb. ros., $n^{\circ} 55$; Billot (suites), $\mathrm{n}^{\circ} 5596,5597$; Wirtgen, pl. crit., $n^{\circ} 582$ ?

Iıв. Juin, juillet. Haies, broussailles. - Écosse. Argyleshire : Tarbet (IIailstone). - Angleterre. Devonshire : près de Lee Mill Bridge (Briggs). - Belyique. Prov. de Namur : Rochefort (Crépin). - France. Vosges : forèt de Rambervillers! - Loiret : bois de l'Isle près d'Orléans (Jullien); - Cher : Bourges (Ripart), carrières de la Chapelle-Saint-Ursin, Montifaut près de Bourges, Les Landes commune de Berry, Allouis, la Servanterie; - Puy-de-Dóme : vignes de Coudes (Lamotte); - Doubs : mont Brégille près de Besançon (Paillot); - Côte-d'Or : Larrey (Boullu); - Ardèche : Anmonay (Boullu); - Hautes-Pyrénées : Gèdre (Ozanon); - Rhône : Lyon à Villcurbanne (Boreau), route de Brinda à Chàteau-Vicux (Chabert), Chaponost, Saint-Laurent-de-Vaulx (Boullu); - Isère : Villard-de-Lans (Boullu); - Hautes-Alpes : mont Bayard sur Gap (Gariod), Charrance (Burle); - Haute-Savoie : Fessy près de Thonon, Pringy (Puget); - 
Savoie : entre Clanaz et les Charmettes (Songeon), Brides près de Moutiers (Puget); - Suisse. Valais : Lourtier, la Ravoire, Chemin (Cottet, de la Soic), Col de la Forclaz, route de Martigny! - cant. de Bâle : Jura de Bâle (Christ). - Autriche. Autriche-infér. : Alaunthal près de Krems (Kerner); - Tyrol : Mühlau, Lienz, Pöllant (Kerner). - Prusse. Lyek (Caspary). - Espagne. Pyrénées d'Aragon: Sarlé en Aragon (TimbalLagrave).

526. H. umbellata Leers, fl. herb. (1775), p. 117 et p. 286; Gmel., I. c., II, p. 425; DC., fl. fr., V (1815), p. 5๊52; Rau, I. c., p. 140; Tratt., I. c., II, p. 5ૅ; Bl. et Fing., 1. c., p. 655; Desportes, 1. c., no 1956; Rchb., 1. c., $\mathrm{n}^{\circ} 5990$; Boreau, l. c., éd. 2, II, p. 181, éd. 5, no 874; Reuter, I. c., p. 72; Déséglise, I. c., p. 15̋1, extr., p. 111; de Martr.-Don., 1. c., p. 255; Cariot, 1. c., p. 188; Fourreau, 1. c., p. 76; R. rubiginosa var. umbellata Lindley, I. c., p. 87; Seringe, in DC., prod., II, p. 616; Du Mort., fl. belgica, p. 95 et monog. ros. de la fl. belge, p. 5ૅ5; Grenier, f1. juras., p. 252; R. rubiginosa var. triflora Willd., enum., p. 5้46; Tratt., I. c., p. 65 ; Bl. et Fing., 1. c., p. 659; Rau, 1. c., p. 154; Desportes, I. c., $\mathbf{n}^{\circ}$ 1924; Seringe, mus. helv., I, p. 29; R. เемиiglandulosa Mérat, fl. Par. (1812), p. 189?; R. sempervirens Roth, fl. germ., I, p. 218 et II, p. 556 , excl. syn.

Exs. Wirtgen, pl. crit., $n^{\circ} 79, n^{\circ} 470$.

Hab. Juin, juillet. Haies, - France. Naine-et-Loire : serait C. dans ce département d'après le catalogue de Boreau; - Seine : le Calvaire près de Paris (Lallemand, 1815, in herb. DC.); - Loiret: Orléans (Jullien); - Cher: Allouis, Mehun, Lazenay près de Bourges; - Puy-de-Dôme : Clermont; - Haute-Garonne : Toulouse (DC. 1807); - Tarn : serait C. dans ce département d'après la florule de M. de Matrin-Donos; - Rhône : Charbonnière (Chabert), Lyon (Boreau); - Hautes-Alpes : Saint-Laurentdu-Cros, Coréo près de Gap (Burle); - IIaute-Savoic : au pied du Salève, Argentière. - Suisse. Valais : Bovernier (de la Soie). - Allemagne. Obermendig, Coblence (Wirtgen). 


\section{( 285$)$}

527. R. horrida Fischer, cat. hort. Gorenk. (1812), p. 66 (non Spreng.); Besser, cat. hort. Crem. (1816), p. 117; R. provincialis M.-Bieb., fl. Taur.-Caue., I (1808), p. 596 (non Ait.); R. ferox M.-Bieb., cent. pl. rar. ross. (1810), I, tab. 57 et fl. Taur.-Cauc., III, (1819), p.559 (non Thunb.); Tratt., l. c., p. 87; Boissier, fi. orient., II, p. 687; $\boldsymbol{R}$. rigida Willd., herb., $\mathbf{n}^{\circ} 982 \overline{5}$; R. rubiginosa var. minor Ledeb., fl. ross., p. 80.

\section{HAB. - Le Caucase.}

528. permixta Déséglise, I. c.,p. 147, ext., p.107; de Martr.-Don., 1. c., p. 25/, Fourreau, 1. c., p. 76; Cottet, 1. c., p. 45; R. rubiginosa Ait., Kew., 2, p. 206; Krocker, II. Siles., II, p. 159; Smith, fl. Brit., II, p. 540; Spreng., fl. halens., p. 146; Pers., syn., II, p. 49; Wallr., ann. bot., p. 64; Roth, fl. germ., II, p. 538 ; Mérat, I. c., p. 190; Gussone, I. c., I, p. 5̋6̈; R. rubiginosa a. vulgaris Rau, I. c., p. $150 ; \boldsymbol{R}$. eglanteria Woods, l. c., p. 206 et herb., nos 61-66; R. suarifolia Lightef. p. $262 ; \boldsymbol{R}$. micrantha var. permixta Grenier, fl. juras., p. 251; Du Nort., I. e., p. 53 ; R. rubiginosa var. permixta Baker, monog. of British roses in journ. Linn. Societ., XI, p. 220; R. Salvanensis de la Soie, in Cottet, I. c., p. 45̄; R. Lusseri Lag. et Pug., in bull. Soe. Murith., fasc. 2 (1875), p. 42, Cottet, I. c., p. 45. - Le R. permixta est le $\boldsymbol{R}$. rubiginosa des auteurs qui déerivent ce rosier avec le fruit ovale, tandis que Linné dit : germinibus globosis.

Exs. Déséylise, herb., ros., no 72; Bourgeau, pl. d'Espagne, $\mathrm{n}^{\circ} 2455$ ?

II $\mathrm{AB}$. Juin, juillet. Haies, bois. - Angleterre. Surrey (Baker). - Belgique. Prov. de Namur Rochefort (Crépin). - France. Vosges : Ro- 


\section{(284)}

chesson (Pierrat); - Maine-et-Loire : Champigny-le-Sec (Boreau); Haute-Vienne : route du Dorat à Lathus (Lamy); - Loiret : Orléans (Jullien); - Cher : C. dans ce département ! - Allier : les Gazcriers, Gannat (Lamotte); - Côte-d'Or : Meursault (Ozanon): - Saône-et-Loire : Chàlons-sur-Saône (Ozanon); - Aude : Mas-Cabardès (Ozanon); — Tarnet-Garonne : Grizolles (Timbal-Lagrave); -- Haute-Garonne : Toulouse (Timbal-Lagrave); - Pyrénées : Saint-Sauveur (Lamy); - Rhòne : Lyon à Charbonnière (Ozanon), Lyon-Balmes-Viennoises (Boreau), Francheville (Boullu); - Isère : Grenoble (Verlot), Balbins (Boullu); - IIautesAlpes: Gap (Burle); - Haute-Savoie : Pringy, Thonon (Puget), Chanoine (Ripart), le mont Salève. - Suisse. Valais : Bovernier, Salvan. Autriche. Tyrol: Lengenficld, Tharn (Kerner). - Prusse. K œnigsberg (Caspary). - Espagne. Avila (Bourgeau). - Italic. Vénétie: S. Vigilio prope Gardu ad lacum Benacum (Kerner); - Sicile: Palerme (Todaro); - Toscane : Pise (Bertoloni, 1810, in herb. DC.).

Ons R. rubiginosa Bourgeau, $p l$. d'Espagne, 18å1, no 1161, forme qui se rapproche du $R$. permixta, mais en diffère par ses rameaux floraux incrmes, ses folioles glanduleuses sur les deux faces, ses styles hérissés. - Sierra Nevada à Guejar de la Sierra (Bourgeau).

529. R. septicola Déséglise, I. c., p. 149, extr. p. 109; Fourreau, I. c., p. 76 ; Cottet, 1. e., p. 45; R. micrantha var. septicola Grenier, 1. c., p. 252; Du Mort., I. c., p. 5̌5; R. rubiginosa auct. pro part.

Exs. Déséglise, herb. ros., $\mathrm{n}^{\circ} 5 \%$; Billot (suites), $\mathrm{n}^{\text {os }} 5596$ bis, 5720̈; Wirtgen, pl. crit., n ${ }^{\circ}$ 80; Schultz, herb. norm., $\mathrm{n}^{\circ} 45$.

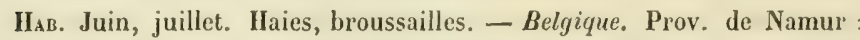
Ivoir (Crépin); - prov. de Luxembourg : Grune (Crépin). - France. Vosges : Planois (Pierrat); Loiret: Maison-Fort près d'Orléans (Jullien); Cher : CC. dans ce département! - Creuse : Grand-Bourg (de Cessac); Saôuc-et-Loire : Autun, Broye (Carion); Châlons-sur-Saône à Dracy-le-Fort (Ozanon); - Ilaute-Garonne : St-Martory (Timbal-Lagrave); - Rhône : Tassin (Boullu), Dardilly (Boreau); - Isère : la Salette près de Corps (Ozanon), Pariset à Grenoble (Verlot); - Haute-Savoie : Thonon (Puget). - Prusse. Coblence (Wirtgen). 
Ons. I. Malgré les beaux spécimens de $R$. sphaerophora que j’ai de M. Ripart, je ne puis pas saisir les différences spécifiques, je ne vois que du $R$. septicola dans ces échantillons. Voici une note que M. Ripart m'a envoyée. a $R$. spllaerophora Ripart. Foliis in pagina superiore leviter pubesentibus; praesertim secundum nervos glandulosis. Fructibus omnin" globosis; floribus minimis roseis; calycis tubo subgloboso. Stylis glabris. " (Ripart in litteris 25 fevr. 1875). - Hab. - Cher : Carrieres de la Chapelle-Saint-Ursin (Ripart).

OBs. II. R. resinosa Lejeune, revue (1824), p. 96, no 728 (non Stcrnb.); R. rubiginose var. resinosa Wallroth, ann. bot., p. 65; Du Mort., fl. Belgica, p. 95 et monog. ros. belg., p. כ̋ .

Hıв. - Prusse. Malmedy. - Belgique. Wegnez (Lejeune). Je n'ai pas vu ce rosier, que M. Du Mortier place dans sa monographie des roses de la flore Belge à côté du R. echinocarpa Rip.

550. R. operta Puget, in Crépin, primit. monog. ros., fase, 1, p. 66, sine descript.

Arbrisseau assez élevé, rameaux floraux sarmenteux, inermes ou munis au sommet de quelques petits aiguillons aciculaires, aiguillons des vieilles tiges forts, dilatés à la base; pétioles pubérulents, glanduleux, aiguillonnés ; §̌-7 folioles ovales, obovales ou arrondies, glabres en dessus, couvertes en dessous de glandes, la nervure médiane velue, doublement dentées; stipules glabres sur les deux faces ou glabres en dessus, pubérulentes, parsemées de glandes en dessous, oreillettes aiguës, divergentes; pédoncules hispides-glanduleux, portant à leur base de petites bractées plus courtes qu'eux; tube du calice ovoüde-allongé, lisse ou hispide; divisions calicinales 2 entières, $\tilde{3}$ pinnatifides à appendices bordés de glandes, non persistantes; styles courts, glabres, disque presque plan; fleur rose; fruit ovoïde.

II.в. Juin, juillet. Haies, bois. - Franoe. Cher : Rhin-du-Bois, Roulon, commune de Berry, Boursac près d'Allogny, bois de Rouet, commune de 
Mehun; - Khône : entre Francheville et Chaponost (Puget); - Savoie . Thonon (Puget). - Alsace. Forêt de Vordersberg près de Niederbronn!

531. R. cchinocarpa Ripart, in Déséglise, 1. c., p. 150, extr., p. 110; Fourreau, 1. c., p. 76; Cottet, I. c., p. $4 \overline{5} ; \boldsymbol{R}$. rubiginosa var. echinocarpa Grenier, fl. juras., p. 249; Du Mort., monogr. Ros. belg., p. 54; R. rubiginosa var. parviflora Saint-Amans, fl. Agen. (1821), p. 206.

\section{Exs. Wirtgen, pl. crit., $n^{\circ} 471$ ?}

IAв. Juin, juillet. Haies, broussailles. - Angleterre. Surrey : Box Hill (Woods), 1850, in herb. Déséglise). - Écosse. Pertshire : IIill of Kinwone (IIailstone), - Belgique. Prov, de Namur : Rochefort (Crépin). - France. Cher : haies des Vignes de Couët près de Mehun (Ripart); Indre : Vignes de Sainte-Lizaigne! - Loir-et-Cher : haies des Vignes de la Buissonnière, commune de Maray! - Lot-et-Garonne : Agen (SaintAmans, 1807, in herb. DC.);- Lozère: Mende (Prost, 1815, in herb. DC.); - Hautes-Alpes : col de Bayard, versant de Laye (Burle); - Savoie : Salins (Puget), colline de Bellecombette près de Chambéry (Songeon). Alsace. Forêt de Gros-W Wald près de Reichoffen! haies des vignes de Niederbronn où il est C.!, haies du chemin de Niederbronn au Soegerthal du côté des près vis-à-vis la maison forestière. - Suisse. Valais : broussailles de chemin, au-dessus de Bovernier, à une alt. de 1200 mèt.; cant. de Bâle : environs de Bâle (Christ). - Autriche. Autr.-infér. : mont Sehaberg près de Mautern, vallée du Danube (Kerner); - Tyrol : Lengenfeld (Kerner). - Prusse. Lyck (Sanio), Kiauten, Kapkeim (Caspary).

OBs. Le $R$. rubiginosa Willd, herb., n 9865 , est représenté par six feuilles simples; M. Crépin, I. c., fase. 2, p. 87, considère les folios 1 et 2 comme appartenant au $\boldsymbol{R}$. echinocarpa Ripart.

552. R. dimorphacantha Martinis, bull. soc. roy. de bot. de Belgique, VII (1868), p. 248-20̈0; Crépin, I. c., fasc. I, p. 24 et p. 72.

Haв. - La Belgique. - Je n'ai pas vu ce rosier.

555. R. sylvicola Déségl. et Ripart, in Déségl., 
descript. qq. esp: nouv. Ros., in mém. soc. acad. de M.-ct-Loire, XXVIII (1873), p. 122, extr., p. 26 ; R. rubiginosa var. sylvicola Baker, l. c., p. 220.

IАв. Mai, juin. Bois, buissons. - Angleterre. Yorkshire : bord de la route à Gunnerside Swaledale (Baker). - France. Cher : petit bois aux Loups dans les vignes de la Chapelle-Saint-Ursin! - Hautes-Alpes: villa Robert près de Gap (Burle).

53ว4. An. Iberica Stev., in M.-Bieb., fl. Taur.-Cauc., III (1819), p. 545; Tratt., I. c., II, p. 67; Seringe, in DC., prod., II, p. 617; R. Isaurae Tratt., l. c., p. 72; R. rubiginosa var. Iberica Boissier, 11. Orient., II, p.687, excl. syn. Besser.

Exs. Unio itiner., an. 1858.

Haв. - La Perse.Té héran (Olivier, 1822, in herb. DC.), Chuschangurberg (Buhse, in herb. Boissier).

535. B. Aucheri Crépin, I. c., fase. I, p. 123 ; Boissier, I. c., p. 687.

Exs. Kotschy, pl. Perse boréale, 1846, no 276 !

Hab. Juin. - Perse. Mont Elbrus près de Passgala (Kotschy).

5356. asperrima Godet, in Boissier, fl. Orient., II, p. 678.

Exs. Haussknecht, $n^{\circ} 511$ !

HAB. - Perse. Mont Kuh Nur (Haussknecht, in herb. Boissier).

557. R. glutimosa Sibthorp et Smith, prod. (1806), I, p. 548; Lindley, I. c., p. 95\%; Tratt., I. c., II, p. 84; de Pronville, 1. c., p. 9\%; Rehb., l. c., II, p. 614; Gussone, l. c., I, p. 679, excl. syn. Bieb.; R. rubiginosa var. Crelica Thory, prod., p. 110; Seringe, in DC., prod., II, p. (616; R. Cretica Tratt., p. 85; Desportes, 1. c., no 1976; 
R. Libanolica Boissier, diagn., sćrie I, fase. 10 (1849), p. 4 .

Icos. Cupani, Panph., ed. 1, tab. 61, ex Smith et Sibthorp, fl. Graec., tab. 482; Redouté, les Roses, livrais. 10, a ?

Exs. Aucher-Éloy, nos 1429 ! 1450!; Kotschy, $n^{\circ s} 186$, $515,567,926$; Blanchet, $n^{\circ 5} 51630,5166,5167$; Balansa, $n^{\circ} 582$; de Heldreich, $n^{\text {os }} 566$ bis, 754, 926, 1045, 1049, 2671, 2678, 2681?; Orphanides, nos 412, 425; Büchler, $\mathrm{n}^{\circ} 208$.

J'ai vu les localités citées tant dans l'herbier de M. Boissier que dans le mien; je eite les deux numéros d'Aucher, qui ne figurent pas dans la collection de M. Boissier.

Haв. - Juin. Région des montagnes. - Italie. Sicile : Madonie (Todaro). - Grèce. Taygette et mont Olenos (Aucher-Éloy, de IIeldreich), mont Parnasse (Orphanides, de Heldreich). - Macédoine. Mont Athos (AucherÉloy! Orphanides). - Anutolie. Mont Gheidagh (de Ileldreich). - Syrie. Mont Liban (La Billardière, Boissier, Blanchet, Kotschy). - Cappadoce. Césarée (Balansa). - Arménie. Erzerum (IIuet du Pavillon).

558. ㅅ. pustnlosa Bertol., fl. ital., V (1842); Gussone, I. c., I, p. 569 (non M.-Bieb.); Tenore, fl. nap., IV, p. 287; R. glutinosa var. b. Rehb., I. c., p. 614.

II AB. - Italie. Sicile : Busambra (Todaro).

559. MR. Meckeliama Tratt., II, p. 8\%; Seringe, in DC., prod., II, p. 624; Gussone, I. c., p. 562; Boissier, 1. c., II, p. 680 .

Exs. Huct du Pavillon, pl. napolit., no 515 ?; Orphanides, fl. Graec., $1^{\text {os }} 422$ ?, 2562 ?; de Heldreich, $n^{\text {ns }} 566$ ? 2679 ?.

Il $\mathrm{AB}$ - Juin, juillet. - Italie. Sicile : Madonie (Todaro), Calabre 
(Huct du Pavillon). - Grice. Mont Parnasse (Orphanides), Taygette, Olenos (de IIeldreich).

540. Sicula Tratt., I. c., II, p. 86 ; Seringe, in DC., prod., II, p. 624.

$\mathrm{IIAB}_{\mathrm{A}}$ - Italic. Sicile (Trattinnick.).

541. R. Orphanidis Boissier et Reut., in Boissier, diagn., sér. 2., fase. 2, p. §0; Boissier, fl. Orient., II, p. 680 .

Exs. de Heldreich., n 24/2.

II AB. Juin, juillet. - Mont Olympe (de Ileldreich, in herb. Boissier).

542. R. micantha Smith., engl. bot. (1812), XXXV, tab. 2490; Woods, I. c., p. 209 et herb. nos $^{\circ 7}$ à 70 ; Tratt., I. c., II, p. 75; Smith, engl. flora, II, p. 587; Rehb., I. c., p. 617; Hooker, brit. flor. (1853), p. 256; Reuter, 1.c., p. 71; Baker, review of the British roses, p. $18 \mathrm{et}$ in Boswell Syme, engl. bot., third edit. (1864), III, p. 211; Lloyd, f1. Ouest (1868), p. 178; R. nemoralis Leman, I. c., extr., p. 10 ?; R. rubiginosa var. micrantha Lindley, I. c., p. 87; Seringe, in DC., prod., II, p. 617; R. rubiginosa var. nemoralis Thory, 1. c., p. 110; Seringe, l. c., p. $616 ; R$. rubiginosa var. nemorosa Du Mort., fl. belg., p. 95; Carion, cat. de Saone-et-Loire, p. 42; R. rubiginosa b. Mutel, 1. c., I, p. 5449; R. rubiginosa var. grandiflora Grodet, 1. c., p. 214; R. micrantha var. A. Grenier, fi. juras., p. 251 ; R. micrantha var. nemorosa Du Mort., monog. Ros. belg., p.55; R. nemorosa Libert, in Lejeune, f1. Spa (1815), II, p. 5111; Boreau, l. c., éd. 2, no 686, éd. $2, n^{\circ} 872$ et catal. M.-et-Loire, p. 80 ; Arrondeau, 1. c., p. 126; Déséglise, 1. c., p. 15้/, extr., p. 114; de Martr.-Don., I. e., p. 255; Cariot, 1. c., II, p. 189; Fourreau, I. c., p. 76 ; R. Libertiana Tratt., I. c., II, p. 80 ; Bl. et Fing., I. c., p. 640 . 
Icon. Engl. bot., XXXV, pl. 2490, et third edit., pl. 469; Roessig, die rosen, tab. 10, mala; Redouté, les roses (1824), livrais. $51, \mathrm{C}$.

Exs. Billot (suites), nº 5598 .

Hab. Juin. Broussailles, haies. - Angleterre. Yorkshire : Masham (Baker); - Kent : Erik (Woods, in herb. Déséglise); - Sommerset : Alcombe (Miss Giffard); - Cornwall : Saint Johns (Briggs); - Devonshire : Saltash, Jamerton, Blaxton (Briggs), - Belgique, Prov. de Namur: Rochefort (Crépin); - prov, de Luxembourg : Grune, Awenne (Crépin). - France. Vosges : Saulxures (Pierrat); - Maine-et-Loire : Chenchutte (Boreau); - Sarthe : Saint-Parin-des-Champs (Boreau); - Saone-etLoire : Autun, Broye (Carion); - Haute-Garonne : Toulouse, Cagire (Timbal-Lagrave); - Hautes-Pyrénées : Gèdre (Bordère); - Gard : le Vigan (Tuezkiewiez); - Savoie : Salins près de Moutiers (Pugel); Haute-Savoie : le Salève! - Suisse. Valais : Bovernier. - Autriche. Tyrol : mont Baldo, Madona del monte Roveredo (Kerner). - Prusse. Guberthal près de Rastenburg (Caspary). - Espagme. Guadarama (Lange, in herb. Boissier). - Italie. Vénétie : S. Vigilio (Kerner).

OBs. R. Pommaretii Puget, in Crépin, primit. mon. Ros., fasc. I, p.65.

J'ai reç de M. Puget un R. Pommaretii, sur lequel je ne puis trop me prononcer, n'ayant vu que des échantillons incomplets; je donne en entier la description établie par M. Puget.

Arbrisseau élevé; aiguillons du vieux bois robustes et crochus; rameaux florifères inermes; pétioles aiguillonnés en dessous, légèrement glanduleux et parsemés de poils qui disparaissent avec l'âge; 5j-7 folioles ovales ou arrondies, glabres, d'un vert sombre en dessus, plus pâles et par'semées de glandes en dessous, spécialement sur les nervures, la médiane un peu velue dans le jeune âge, doublement dentées, à dents secondaires glanduleuses; stipules assez larges, glabres, les inférieures portent quelques glandes en dessous, bordées de glandes, à oreillettes étroites ou peu divergentes; pédoncules peu glanduleux, quelquefois lisses, longs; tube du calice allongé-ellipsoïde, lisse; divisions calicinales 2 entières velues, כ appendieulées, égalant la corolle, non persistantes; styles courts, glabres; fleur rose; fruit en fuseau très-allongé, d'un rouge presque noirìtre à la maturité(Puget).

Lot-et-Garonne : Chemin de IJanau à Sainte-Colombe (Puget). 
545. R. Aoribunda Steven, n Besser, cat. hort. Crem., ann. 1816, sup. 4, p.19; M.-Bieb., I. c., IV (1819), p. $\overline{3} 4 \overline{5}$; Tratt., I. c., II, p. 78; Seringe, in DC., prod., II, p. 621; R. rubiginosa M.-Bieb., I. c., I, p. 598 exel. syn.

Hab. Mai, juin. - In Tauriae sylvaticis (M.-Bieb.).

5/4. IR. dimiunta Boreau in litt.!; R. micrantha Boreau, l. c., éd. 2, II, p. 182, obs., éd. 5, no 876 (non Smith); Déséglise, I. c., p. 155 , extr., p. 115\%; Cariot, I. c., p. 189; Fourreau, I. c., p.76; R. micrantha var. b. Grenier, f1. juras., p. 231?; R. micrantha a. vulgaris Du Mort., І. с., p. 丂้น?

Haв. Juin, juillet. Broussailles. - France. Haute-Vienne : Saint-Hilaire, Bonneval (Lamy); - Cher : Carrières de la Chapelle-Saint-Ursin, Brécy, Berry à Fontiley, forêt d'Allogny; - Puy-de-Dòme : Saint-Pardoux (Lamotte), Saint-Nectaire (Lamy); - Aude : le Mas-Cabardès, Carcassone (Ozanon); - Rhône : Lyon à Iseron (Boreau); - Jura : Lons-le-Saulnier, route de Bourg (Puget). - Suisse. Cant. de Bâle : Jura de Bâle (Christ).

Oss. Je ne connais pas le R. parvula Grenier, in Crépin, I. c., fasc. 1, p. 65 (non Sauzé et Maillard). Ce rosier aurait, d'après l'analyse de M. Crépin, les divisions calicinales plus ou moins glanduleuses sur le dos et aux bords, le pédoncule abondamment hispide-glanduleux, le tube du calice ovoïde, arrondi, les folioles très-petites, glabres en dessus, à côte seulement un peu velue en dessous, les pétioles presque glabres, la corolle très-petite, ne dépassant guère 9 centimètres de diamètre.

IIAB. - Gard : Aulas (Diomède ex Crépin)

245. R. Inctiflora Déséglise, in Fourreau, 1. c., p. 76, sine deseript.; R. Vaillantiana Cariot, I. c., 187 (non Boreau).

Exs. Billot (suites), n 5595 .

Arbrisseau de 1 mètre à $1^{\mathrm{m}} \breve{0} 0$, ayant le port du 
R. sepium; aiguillons dilatés à la base, comprimés, presque droits ou inclinés; pétioles glabres, parsemés de poils courts, brillants ef de glandes fines, aiguillonnés en dessous ; 5-3-7 folioles petites, ovales-arrondies ou ovales, elliptiques, glabres ou parsemées de quelques poils rares en dessus, un peu velues en dessous sur la nervure médiane qui a de longs poils mous blanchatres disparaissant en partic avec l'àge, les folioles sont parsemées en dessous de glandes fines peu abondantes, doublement dentées, à dents secondaires glanduleuses; stipules glabres en dessus, glabres et parsemées de quelques glandes en dessous, bordées de glandes; oreillettes aiguës, droites ou peu divergentes; pédoncules glanduleux, portant à leur base des bractées ovales-cuspidées, glabres, bordées de glandes ordinairement plus longues que les pédoneules; tube du calice petit, grêle, ovoüde, un peu hispide à la base; divisions calicinales tomenteuses aux bords, parsemées de glandes fines, 2 entières, 5 pinnatifides à appendices étroits, saillantes sur le bouton, plus courtes que la corolle, réfléchies à l'anthèse, puis eadurues; styles glabres, disque plan; fleur blanc de lait; fruit petit, ovoüde, arrondi à la base; un peu rétréci au sommet, rouge-orangé.

II (Ozanon), Roncière (Chabert), Craponne près de Lyon (Boullu).

246. R. Lemanii Boreau, I. c., éd. 5 (183̌7), n 87 引 et catal. M.-et-Loire, p. 80; Déséglise, I. c., p. 142, ex1r., p. 102; Grenier, fl. juras., p. 250; Cariol, 1. c., p. 187 ; Pérard, 1. c., p. 82; Verlot, I. c., p. 117; R. hystrix Leman, l. c., p. 10, n 19 (non Lindley); Desportes, I. c., no 1951; Boreau, 1. c., éd. 2 (1849), II, p. 182; R. micrantha var. hystrix Baker, I. c., p. 222; R. micrantha var. Lemanii Du Mort., p. 53. 


\section{( 295$)$}

Exs. Déséglise, herb. ros., $\mathrm{n}^{\circ} 71$.

HaB. Juin. Bois, haies. - Angleterre. Indiqué par M. Baker dans les comtés de Surrey et de Glocester. - Belgique. Indiqué par M. Du Mortier dans sa monographie des rosiers de la Flore belge. - France. Vosges: Rambervillers (Boulay); - Loiret : hois de la Caille près de Tigy; Mainc-et-Loire : indiqué comme C. dans ce département par M. Boreau daus son catalogue; - Cher : le Corpouay, Marmagne, Fublaine près de Sainte-Thorette, vignes de Couët près de Mehun, Valio, Fontiley; Allier: Montlucon, collıne de l'Abbaye (I'érard, catal.); - Haute-Garome : bois de Grammont, Saint-Paul-de-Fenouilhade, Mancioux (Timbal-Lagrave); - Rhône: Roncieux, Vaugneray, Balmes-Viennoises, bois de l'Étoile (Chabert), Lyon au pont d'Alay (Ozanon); - Isère : Paris et près de Grenoble, la bastille de Grenoble (Verlot), la Salette (Ozanon); - Savoie : Salins près de Moutiers (Puget), entre les Charmettes et Montagnole près de Chambéry (Songeon).

Ons. $R$. densa Timb.-Lagrave, une excurs. bot. à Bagnères-de-Luchon, in bull. Soc. bot. de Fr., IX (1864), extr., p. 17.

Je suis loin d'être fixé sur ce rosier, dont j'ai vu un exemplaire très-incomplet, que M. Timbal-Lagrave a bien voulu me communiquer.

547. R. notuadifolia Rau, enum. ros., p. 156, sub R. rubiginosa var. rotundifolia; Tratt., I. c., II, p. 75; Rchb., I. c., p. 617; Mutel, I. c., I, p. 549; Boreau, I. c., éd. 5, no 877; Déséglise, l. c., p. 156, extr., p. 116; Cariot, I. c., P. 189; Cottet, I. c., p. 45; R. rubiginosa var. rolundifolia Lindley, I. c., p. 88; Thory, I. c., p. 112; Seringe, in DC, prod., II, p. 616; BI. et Fing., I. c., p. 659; Desportes, 1. c., n 1950; Du Mort., l. c., p. 52.

II ar. Juir, juillet. Broussailles. - Angleterre. Yorkshire : haies près de Newton (Baker); - Amongst Whins in Ogle thorpe pastures (Hailstone, 185̄5, in herb. Déségl.); - Devonshire : Egg Buckland (Briggs). - Belgique. Prov. de Namur : Rochefort (Crépin). - France. Loir-et-Cher : Beallmont (Franchet); - Loiret: Pare de la Caille près de Tigy! - HauteGaronne : Toulouse (Timbal-Lagrave); - Rhône : Francheville, pont d'Alay (Chabert); - Savoie: Méry (Puget). - Alsace. Ruines de Falkenstein près de Niederhronn! - Suisse. Valais : Bovemicr (Cottet). - Prusse. Lyck 
près de Thalussen, Schloswald (Caspary). - Espagne. Sierra de Villa Verda (Bourgeau).

Var. B. pedunculis laevibus. - Exs. Déséglise, herb. ros., nº 75 ; Reliquiae Mailleanae, $n^{0} 1086$.

Haв. - Iautes-Alpes : La Grave (Mathonnet, Ozanon): - Isère : les étages à Saint-Christophe-en-Oisans (Boullu).

OBs. Le R. Puymaurea Grenier, inéd., n'est pas une Sepicene, mais appartient au groupe du $R$. rotundifolia, d'après l'échantillon que j’ai recu de M. Grenier. Peut-être appartient-il à la variété pedunculis luevibus? Je ne puis rien affirmer sur un échantillon incomplet.

IIAB. - Hautes-Alpes: Puy Maure, près de Gap (Grenier).

\section{Sect. XV. - Tomentosae.}

Déséglise, obs. on the differ. meth. proposed for the classif. of the spec. of the genus Rosa, in the Naturalist, $n^{\circ} 20$ (1863), p. 515, ,extr., p. 16, et révision de la sect. Tomentosa, in mém. Soc. acad. de M.-ct-Loire, XX (1866); Cariot, étud. des fleurs (1863), II, p. 190; Du Mort., monog. des roses de la flore Belge (185̋6), p. 48; Crépin, primit. monog. ros. (I869), in bull. soc. roy. bot. de Belgique, VIII, p. 246, extr., p. 25, part.; Cottet, énum. ros. du Valais, in bull. soc. Murith., fase. III (1874), p. 44; Villosae DC., in Seringe, mus. helv. (1818), I, p. 5 et p. 4, part.; Lindley, monog. ros. (1820), p. 72, part.; Besser, enum. Pod. et Volh. (1822), p. 61; de Pronville, monog. genre rosier (1824), p. 75, part.; Rehb., fl. excurs. (1852), II, p. 615, excl. R. glandulosa Bell.; Crépin, 1. c., p. 247, extr., p. 26; Boissier, fl. Orient. (1872), p. 681; Cottet, 1. c., p. 44; Caninae Seringe, in DC., prod. II (1825), p. 611, part.; Diaslylae trib. Orthocanthae Godet, fl. Jura (1855), p. 204. 
Aiguillons assez grèles, peu comprimés à la base, droits ou un peu arqués, dégénérant rarement en aiguillons sétacés au sommet des rameaux; folioles grisâtres, pubescentes ou mollement velues sur les deux faces, rarement glabrescentes, glanduleuses ou non glanduleuses sur la face inférieure, simplement ou doublement dentées; divisions calicinales persistantes ou caduques; pédoncules hispides-glandulcux, rarement glabres ou velus; fleur blanchâtre ou d'un rose pâle ou d'un rose vif.

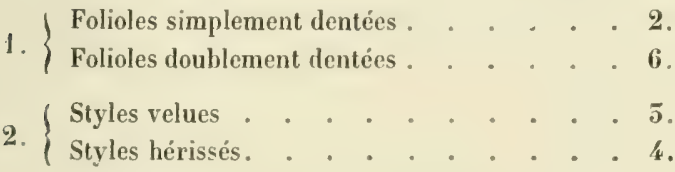

Pédoncules et tube du calice couverts de soies glanduleuses, fleur presque blanche, fruit

5. \{voïde . . . . . . . . dumosa.

Pédoncules glabres ou pubescents, tube du calice glabre, fleur d'un rose pâle . . . farimulenta.

Rameaux floraux pubeseents, fleur d'un rose

4. vif, fruit d'un beau rouge, ovoïde ou subglobuleux . . . . . . . . micans.

Rameaux floraux non pubescents au sommet . 5 .

Aiguillons des rameaux floraux dégénérant en

5. $\left\{\begin{array}{l}\text { soies glanduleuses, fleur grande, d'un beau } \\ \text { rose, fruit assez petit, subglobuleux . . . }\end{array}\right.$ Aiguillons ne dégénérant pas en soies glanduleuses, fleur rose, fruit subglobuleux . - cinerascens.

6. $\left\{\begin{array}{l}\text { Folioles parsemées de glandes en dessous . . } 7 . \\ \text { Folioles églanduleuses en dessous . . . } 16 .\end{array}\right.$

7. $\left\{\begin{array}{l}\text { Aiguillons dégénérant en soies glanduleuses } \\ \text { ou rameaux floraux velus au sommet } \\ \text { Aiguillons ne dégénérant pas en soies glandu- } \\ \text { leuses, rameaux floraux lisses au sommet }\end{array}\right.$ 
Rameaux floraux velus au sommet, feuilles à villosité brillante, styles obscurément hé-

8. rissés. . . . . . . . . . .

fameaux floraux lisses, aiguillons dégénérant

floccida. en soies glanduleuses, styles hérissés, folioles grandes, ovales, fleur d'un beau rose.

Genevensis

(Tiges et rameaux floraux inermes, pédoncule

9. et tube du calice couverts de soies glandu-

leuses, styles velus, fleur rose.

Tiges et rameaux floraux aiguillonnés . . 10 .

Styles velus ou hérissés . . . . . . 11.

10. Styles glabres, folioles ovales aiguës, à odeur de térébenthine, fleur rose clair . . . foctida.

11 Styles velus . . . . . . . . 12.

. Styles hérissés. . . . . . . . 14.

12. Pédoncule glabre supérieurement, velu à la

rose . . . . . . . . farinosa

Pédoncule hispide-glañduleux . . . . 15.

Pétioles tomenteux, glanduleux, inermes, fleur d'une rose pâle, sépales persistant sur le fruit, fruit ovoïde, attenué au sommet, folioles couvertes d'une pubeseence veloutée, vestila.

15.

Pétioles pubescents, parsemés de glandes, aiguillonnés, folioles pubescentes, à nervures plus ou moins chargées de glandes visqueuses odorantes, fleur grande, rose, sépales non persistants sur le fruit . . . .

terebinthinacea.

Tube du calice ellipsoïde, glabre, sépales persistants, folioles ovales-elliptiques, fleur

14. blanche nuancée de rose, fruit gros, ellipsoide.

scabrinescula.

Tube du calice ovoïde, hispide-glanduleux . .

15.

Folioles ovales-aiguës, glabres en dessus, velues sur les nervures, la côte glanduleuse, fleur d'un rose clair . . . . . . . .

13.

Folioles ovales-lancéolées, pubescentes sur les abietina. deux faces, glanduleuses en dessous, fleur rose

cuspidatoides. 


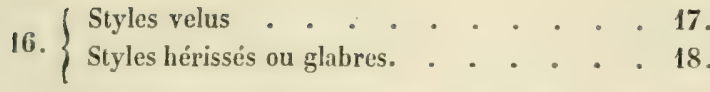

, Tube du calice subglobuleux, fleur d'un rose pâle, pétioles souvent inermes, fruit ovoïde, couronné par les sépales redressés persis-

17.

Tube du calice ovoìde, fleur assez grande, rose, pétioles aiguillonnés, fruit gros, obovoïde, couronné par les sépales redressés, caducs avant la maturité du fruit. . . .

intromissa.

Tunoniensis.

Styles glabres, folioles ovales-aiguës ou oblon-

18. $\{$ gues, fleur grande, d'un rose clair, fruit gros, ellipsoïde allongé. . . . . . confusa.

Styles hérissés. . . . . . . . . . 19

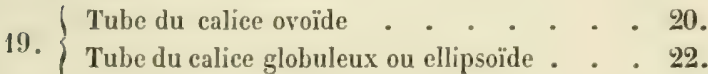

Tube du calice globuleux ou ellipsoïde . . . 22.

20. $\left\{\begin{array}{l}\text { Fleur rose ou d'un rose clair. . . . . } 21 . \\ \text { Fleur blanchâtre, folioles ovales-elliptiques, } \\ \text { fruit globuleux. . . . . . dimor }\end{array}\right.$

Folioles ovales-elliptiques, fleur d'un rose

21. $\begin{aligned} & \text { clair, fruit ovoïde allongé, sépales caducs tomentosa. } \\ & \text { Folioles elliptiques-subarrondies, fleur rose, }\end{aligned}$ fruit globuleux, sépales persistants . . . Andrzeiouskii.

Tube du calice globu!eux, folioles ovales-aiguës, fleur d'un rose pâle, sépales non persistants,

22. fruit subglobuleux . . . . . . subglobosa.

Tube du calice ellipsoïde, folioles ovales-ellipti. ques, fleur rose pâte, fruit gros, ellipsoìde, d'un rouge sanguin, sépales persistants . . Annesiensis.

\section{A). Verae-tomentosae.}

* Folioles toutes simplement dentées.

548. R. Wdoensis Boissier, 1. с., p. 683 .

HAB. - In Jaghestania superiori ad Dido (Ruprecht, in herb. Boissier). 


\section{( 298 )}

- M. Boissier place ce rosier dans les Caninae; je pense, d'après le peu d'échantillons que j'ai pu voir', qu'il appartient plutôt aux Tomentosae.

Var. B. biserrata Boissier, 1. c.

Le seul échantillon que j'ai vu evistant dans l'herbier de M. Boissier est étranger au type ci-dessus et à la section Caninae! Les folioles sont glabres sur les deux faces, les nervures sont parsemées de glandes, les aiguillons sont longs, droits. Il est diflicile de se prononeer sur un échantillon très-incomplet.

349. R. Ammena Boissier, 1. c., p. 674.

Exs. Bourgeau, année 1862, sans numéro.

Нав. - Arménie. Baibout (Bourgeau, in herb. Boissier).

5̋0. R. Vanheurckiana Crépin, in Boissier, I. c., p. 683 .

Exs. Kotschy, $\mathrm{n}^{\text {os }} 569 ! 789$, sup.!

Hab. - Musch, Arménie australe (Kotschy, sup. n 789), et dans la vallée alpine de Nerga Sauk à une altitude de 7000 pieds (Kotschy, iter cilicico-kurdic., $n^{\circ} 569$ ).

531. R. Boissieri Crépin, primit. monog. rosar., p. 119; Boissier, 1. c., p. 682.

Exs. Balansa, pl. d'Orient, $\mathrm{n}^{\text {os }} 510,514$.

Ilaв. - Lazistan, vallée de Djimil (Balansa, in herb. Boissier).

552. R. Balansaca Nob.; R. Boissieri b. spinulosa Boissier, l. c.

Exs. Balansa, pl. d'Urient, ann. 1866, $1^{\circ}$ 518. Mon étiquette n’a pas de numéro sans doute par omission, car la mème plante qui se trouve dans l'herbier de M. Boissier porte le $n^{\circ} 518$ !

Décrit sur deux magnificues fragments de tige florifère ayant six rameaux florifères, récoltés par M. Balansa.

Arbrisscau......; aiguillons des tiges florifères épars, 
peu nombreux, faibles, blanchattres, dilatés à la base en forme de disque, droits ou un peu arqués, ceux des rameaux florifères grèles, épars ou géminés; écorce vineuse ou verditre; pétioles tomenteux, les uns aiguillonnés, d'autres incrmes; folioles grandes, elliptiques (d'un vert cendré sur le see), parsemées de poils eourts, apprimés en dessus (qui doivent disparaître avec l'àge? ear sur le mème rameau je vois quelques folioles glabres en dessus), velues en dessous prineipalement sur les nervures, nervures saillantes et blanchatres surtout dans les folioles supéricures, simplement dentées; stipules trèsgrandes, larges, glabres, oreillettes aiguës, divergentes, la partie intrastipulaire velue; pédoncules solitaires, chargés de petites soies spiniformes blanchâtres terminées par une glande (noiratue sur le sec), cachés par des bractées larges, ovales, glabres; tube du ealice ovoüde, couvert de petites soies comme celles des pédoncules; divisions calicinales spatulées au sommet, couvertes sur le dos de petites soies spiniformes blanchàtres, glanduleuses, 2 entières à bords tomenteux, 5 pinnatifides à appendices larges relativement, les appendices laryes inférieurs ont en outre 1-2 petites dents, plus longues que la corolle, étalées à l'anthèse; styles hérissés; lleur grande; fruit?

IIsв. Juin, juillet. - Région sub-alpine du Lazistan près de Djimil, ver's 2000 mètres d'altitude (Balansa).

OBS. Peut-ètre ce rosier est-il étranger à cette section? Il a cependant les earactères des Tomentosae; il peut se faire aussi que ce rosicr ait déjà reçu un nom spécilique, qui m’est inconnu.

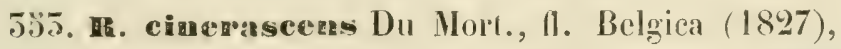
p. 95 et monog. ros., p. 50; Tinant, f1. Luxemb., p. 25:3; Déséglise, revue sect. Toment. in mém. soc. acad. de 
M.-et-Loire, XX (1866), cxtr., p. 51; Crépin, l. c., p. 7\%̈; Fourreau, 1. c., p. 76 ?; R. tomentosa var. cinerascens Crépin, notes sur qq. pl. rar. ou crit. de la Belgique (1862), fase. 2, p. 5ั3; R. pellita Ripart! in litt.

Exs. Wirtgen, pl. crit., $n^{\circ} 78$.

IIAB. Mai, juin. Haies, bois. - Belgique. Prov. de Namur : entre Éprave et Rochefort (Crépin); - prov. de Luxembourg : entre Neupont et Hamaide (Crépin). - France. Deux-Sèrres : rochers de l'Argenton (Boreau); - Haute-Vienne : Rochechouard (Lamy); - Cher : Gérissai, les Aixd'Angillon (Ripart), forêts de Vierzon, d'Allogny, de Fontmoreau et du Rhin-du-bois à la fosse du Dragon, Graire, Turly. — Sarthe : Le Mans (Boreau); - Saône-et-Loire : Autun (Carion); - Haute-Garonne : Toulouse (Baillet); - Rhône : Limonet (Chabert), Beaumont, Tassin (Boullu), Lyon au pont d'Alay (Ozanon); - Haute-Savoie : mont de Sion (Puget); Isère: Saint-Pierre-de-Chartreuse (Verlot). - Alsace. Forêt de Gros W ald près de Reichoffen! - Autriche. Autr.-inf. : Oberbergern près de Mautern vallée du Danube (Kerner); - Tyrol : Dölsach, mont Baldo (Kerner). - Prusse. Coblence (Wirtgen).

5\%.4. IR. micas Nob.; R. velutina Chabert! in Cariot, 1. c., p. 677 (non Clairville).

Arbrisseau à racine non traçante, élevé de 1-5̄ mètres, touffu, rameaux retombants flexueux, les rameaux floraux ont une pubescence blanchitre, aiguillons droits horizontaux, dilatés à la base en forme de disque; pétioles veloutés, canaliculés, munis de quelques rares glandes fines et de quelques petits aiguillons en dessous; $\lcm{5-7}$ folioles veloutées à villosité brillante, ovales-elliptiques ou ovales-aiguës, d'un vert cendré en dessus, simplement dentées, à dents ciliées; stipules étroites, à oreillettes aiguës, un peu divergentes, pubescentes en dessous, à bords eiliés-glanduleux; pédoneules solitaires ou en bouquet, poilus et hispides-glanduleux, villosité disparaissant avec l'àge, munis de bractées veloutées, celles des bouquets 


\section{(501)}

sont ovales-acuminées, quelques-unes sont terminées par un petit appendice foliacé, ordinairement plus longues que les pédoncules; tube du calice ovoïde contracté au sommet, ce qui lui donne plutòt une forme subglobuleuse, un peu hispide à la base; divisions calicinales spatulées au sommet, pubescentes, parsemées de glandes fines sur le dos, 2 entières, 5 pinnatifides, réfléchies à l'anthèse, plus courtes que la corolle, non persistantes; styles hérissés, disque plan; fleur d'un rose vif; fruit d'un beau rouge ovoïde ou subglobuleux.

IIab. - Juin. Ilaies. - France. Cher : vignes de Couët près de Mehun ! bois de Ma magne ! - Ain : Villette (Boullu); - Rhône : Charbonnière (Chabert), Tassin à l'Aiga (Boullu); - Haute-Savoie : Habère-Lullin (Puget). - Suisse. Cant. de Fribourg : Chatel-sur-Montsalvens (Cottet).

Ons. Dans ma révision de la section Tomentosa (1S66), j’ai admis en synonyme au $\boldsymbol{R}$. cincrascens, le $\boldsymbol{R}$. velutina Chabert non Clairville. En 1869, М. Crépin, primit. monog., p. 98, a appelé mon attention par ses judirieuses observations sur la tendance de plusieurs tomenteuses à avoir les rameaux finrifères veloutés de poils. Cette pubescence se trouve sur un certain nombre d'espèces orientales; M. le docteur Ilse a trouvé aussi dans la Thuringe un $R$ tomentosa, pour lequel II. Crépin propose le nom de $R$. Thuringinca, dont les rameaux florifères sont entièrement couverts d'un fin tomentum assez dense blanchâtre II faut croire que cette pubescence remarguable est un fait rare pour nos rosiers français, car depuis l'année 1860, sur les milliers de spécimens que j'ai pu voir, j’ai constaté le fait pour deux ou trois départements seulement. M. Cariot, l. c., dit du $R$. velutina Chab. : "Voisin des R. tomentosa et R. mollissima; " il ne fait aucune mention de la dentition des folioles, caractère qu'il semble pourtant important de connaitre ! M. Cariot cût beaucoup mieux fait de donner la description qu'il tenait de Chabert, au lieu de faire une comparaison qui en définitive n'explique rien.

5วั5. Re. Varcyama Boullu, in litt.!

Voici la description que M. Boullu ma fait parvenir avec son type. 
Arbrisseau de 1 mètre at $1 \mathrm{~m} 30$, à tiges droites, armées, ainsi que les branches principales, d'aiguillons trèsallongés, dilatés, presque droits; rameaux florifères courts, roides, un peu flexuctix, munis d'aiguillons fins, longs, droils, entremèlés d'aiguillons sétacés glanduleux; stipules glabres en dessus, tomenteuses et un peu glanduleuses en dessons, bordées de glandes, oreillettes courtes, aiguës, presque droites; pétioles tomenteux, parsemés de glandes, inermes ou munis de rares petits acieules; $5-3-7$ folioles, les inférieures ovales-arrondies, les supérieures ovaleselliptiques, vertes en dessus, cendrées en dessous, mollement pubescentes, à dents presque simples, mueronéeseiliées; pédoncules longs, 1-5-4, chargés de petites soies spiniformes terminées par une glande d'un rouџe noiràtre, hractées ovales-euspidées, glabres en dessus, tomenteuses en dessous, bordées de glandes plus courtes que les pédoncules, les pédoneules latéraux portent en outre au-dessus de leur base deux petites bractées lancéolées; tube du calice subglnbuleux, hispide-glanduleux; divisions ealicinales spatulées an sommet, glanduleuses sur le dos, 2 entières, 5 pinnatifides, égalant la corolle, réfléchies à l'anthèse, cadurques à la maturité du fruit; styles courts, hérissés; fleur grande, d'un beau rose; fruit subglobuleux, assez petit (Boullu). - M. Boullu a oublié un caractère qui me parait important, e'est celui des rameaux florifères velus au sommet.

Haв. Juin. IJaies, broussailles. - France. Rhône : Marey-l'Étoile, T'assin, Méginant (Boullu).

5̈6. R. Aumosa Prget, in Déséglise, rev. sect. toment., p. 40; Fourreau, l. c., p. 76; R. dimorpha Grenier, 1. c., p. 25̄, part. (non Besser).

Exs. Billot (suites), nos $58: 33,58: 3 ; 3$ bis?, 58333 ter? 
II Ar. Juin, juillet. Bois montagneux. - Belgique. Province de Namur: Rochefort (Crépin). - France. Rhône : Tassin (Boullu); - Savoie : Margeriaz près de Chambéry (Songeon); - Ilaute-Savoie : Bellevaux (Puget), le Salève au dessus de Veyrier.

25̈7. R. farianuenta Crépin, 1. c., p. 25 et p. 75 ; R. farinosa Déségliss., I. c., p. 17 (non Rau); Cottet, l.c., p. 44; R. villosa var. nuda Seringe, in DC., prod., II, p. 618, part.

Exs. Sendtner, ann. 1847, $\mathrm{n}^{\circ} 941$.

IIAB. Juin. Haics, broussailles. - France. Vosges : Moyemont (Boulay); - Iautes-Alpes : Briancon (DC. 1807 !); - Rhône : Saint-Genis-lesAllières. - Suisse. Valais : Salvan ! mont Clou au dessus de Bovernier ! Prusse. Koenigsberg (Caspary). - Turquie d'Europe. Bosnic (Sendtner, in herb. Boissier).

** Folioles doublement dentées.

† Folioles à glandes éparses en dessous ou à nervures secondaires glandulcuses.

5̋8. T. Portida Bastard, sup. fl. M.-et-Loire (1812), p. 29; DC., fl. fr., V (1813), p. 5̄̌k, excl. syn. Jacq.; Tratt., I. c., II, p.110?; Desportes, I. c., n 1926; Rchb., I. с., II, p. 624; Mutel, I. c., I, p. 5ร̌̈; Boreau, bull. soc. ind. d'Angers (1844), extr., p. 12, fl. cent. éd. 2, $n^{\circ} 688$, éd. 5, no 878 et catal. M. et-Loire, p. 80; Guépin, fl. de M.-ei-Loire, sup. (1842), p. 42; Gr. et Godr., I. c., I, p. 5̋ว9?; Déséglise, essai monog., p. 117; Lloyd, l. c., p. $179 ; R$. mubiginosa var. 4, foetida Desvaux, obs. (1818), p. 156?; R. collina var. foetida Thory, 1. c., p. $72 ; R$. tomentosa var. foetida Seringe, in DC., prod., II, p. 618; R. villosa var. foetida Lois., I. c., I, p. 561? Icon. Redouté, les Roses (182/), livrais. 1̋̈, D. mala. Exs. Baker, herb. ros. brit., n 11 . 
НАв. Mai, juin. Haies. - Angleterre. Yorkshire : Studley, Thirsk, (Baker); - Cheshire : Burton (Webb); - Devonshire : Newton, Brixlon, Revelstoke, Britjend, Killey (Briggs); - Cornwall : Elmgate, SaintStephens, Saltash (Briggs). - France. Mane-ct Loire: colline Ardenai (Bastard, 1810, in herb. DC.), Angers, Rablai (Boreau); - Vendée : Napoléon-Vendée (Lloyd); - Calvados : bois de Manerbe prés de Lisieux ! - Mayenne : Belgeard, Grenhart près de Wayenne (Boreau); - Isc̀re : Saint-Komain près de Crémieux (Boullu).

OnS. Je croyais que le $\boldsymbol{R}$. Jundzilliana Baker (non Besser), était un rosier nouveau, d'où le nom de $R$. Britunnica Déségl., inéd. Depuis, en étudiant ce rosier, je me suis convaicu que c'était le $R$. foctida Bast., opinion déjà émise par M. Boreau en 186\%3. M. Baker dit styles thinly hairy; dans les échantillons que j’ai reçus de. II. Baker et de plusieurs botanistes anglais, les styles sont very glabrous! Du no $/ 1$ de l'herb. rosar. de M. Baker, les \& échantillons que j’ai ct qui portent onze fleurs ont les styles glabres.

\section{5y9. R. abietima Grenier, fl. juras., p.154; R. foetida Grenier, l. c. (non Bastard).}

IIAB. Juin. Région montagneuse. - France. Doubs : La Fresse près de Pontarlier (Grenier).

Oss. R. Uriensis Lag. et Pug., in Crépin, I. c., p. 25, sine descript.; R. tomentella forma Uriensis Christ, 1. c., p. 15\%. - Ce rosier m'étant inconnu, je donne in extenso la description que M. P'uget m'a fait parvenir sans échantillon à l'appui.

Arbrisseau peu élevé; aiguillons nombreux, recourbés, mêlés au sommet des tiges d'aiguillons plus petits, droits ou inclinés; pétioles velusglanduleux, aiguillonnés en dessous; 5-7 folioles obovales ou ovalesarrondies, d'un vert sombre et parsemées de poils apprimés en dessus, plus pâles et velues, plus ou moins glanduleuses en dessous, à côte médiane glanduleuse, doublement dentées, à dents eiliées, les secondaires glanduleuses; stipules étroites, glabres, glanduleuses en dessous, à oreillettes courtes et divergentes; pédoncules ord. réunis par 2-4, hispidesglanduleux, munis à leur base de petites bractées ovales, glabres, les égalant; tube du calice ovoïde ou or oïde-arrondi, hispide-glanduleux; divisions calicinales 2 entières, 5 pinnatifides foliacées au sommet toutes très-glianduleus's, atteignant la corolle, redressées aprè̀s l'authèse 
et persistantes jusrqu'à la maturité du fruit; styles saillants velus; fleur rougeâtre; fruit ovoüde-arrondi, les latéraux presque sphériques, hispidesglanduleux (Puget).

IIab. Juillet. Bois. - Suisse. Cant. d'Uri : entre Wasen et Göschenen (Gisler, teste Puget). - M. Christ, 1. e., indique aussi ce rosier dans le cant. du Tessin à Airolo, et dans le cant. des Grisons.

560. He. Gislewi Puget, in Crépin, 1. e., p. 26 et p. 76, sine descript.

Arbrisseau de 1-2 mètres, chargé d'aiguillons assez nombreux, longes, droits ou un peu inclinés, en forme de disque à la base, plus robustes sur les vieilles tiges; pétioles pubescents-glanduleux, portant de petits aiguillons en dessous; כ̋-7 folioles un peu grandes, pétiolées, ovaleselliptiques allongées, d'un vert sombre et parsemées de poils en dessus qui disparaissent avec l'àge, pubescentes en dessous, à cote médiane portant qq. glandes, les folioles les plus inférieures de charjue rameau ont des glandes éparses en dessous, doublement dentées, à dents ciliées glanduleuses, la terminale ord. arrondic à la base et aiguë au sommet; stipules érroites, glabres en dessus, pubescentes glanduleuses en dessous, à oreillettes courtes, droites ou peu divergentes; pédoneules longs, solitaires ou réunis par 2-4, portant à leur base de petites bractées glabres, bordées de glandes, les pédoncules extérieurs portent en outre 2 petites bractées opposées, glabres, bordées de glandes, toutes beaucoup plus courtes que les pédoncules; tube du calice ovoïde on cllipsö̈de, hispide ylanduleux au moins à la base; divisions calicinales pinnatifides, toutes glanduleuses en dehors, égalant la corolle, réfléchies à l'anthèse, non rechessées ou persistunles; styles courts, glabres; fleur's grandes, d'un rose clair; fruit ovoide, d'un rouge sanguin à la maturité, plus ou moins hérissé de soies grlanduleuses (Pugret). 
IIn. IIaies, bois. - Suisse. Cant. d'Uri : Oberhalb Altorf (Puget). Je connais impariaitement ce rosier, par un échantillon incomplet recu de M. Puget.

561. R. Snfrerti Kirsehleger, fl. Als., I, p. 247; $\boldsymbol{R}$. glandulosa Kirschl., prod. (non Bellardi).

Ilab. Mai, juin. - France. Vosges : bois de Saint-Morand à Ribeauvillé (Kirschleger!).

562. R. spiunlifolia (1) Dematra! ess. monog. (1818),

(1) J'ai publié précédemment une étude critique sur cette espèce dans le Bulletin de la Société royale de Bolanique de Belgique, XIV, pp. 528-545, séance du 5̋ décembre 187\%. J'ai donné en même temps la description détaillée du type et des six variétés que j’y rattache.

Depuis la publication de ect article, II. Godet vient de m'écrire relativement au R. spinulifolia distribué par feu Thomas; voici un passage de sa lettre que je transeris in extenso :

..... Je crois, comme vous, que la plante distribuée par Thomas, dont " je possède un exemplaire de lui, provient d'un pied cultivé provenant " d'un buisson des environs de Genève, car Thomas n'a pas mis le pied, * que je sache, dans notre Jura neuchatelois, tandis qu'il allait assez " souvent à Genève. " (Godet, lettre du 10 mai 1876).

Je m'explique difficilement comment II. Godet n'a pas exprimé plus tôt son doute sur la localité assignée par Thomas à la plante qu'il a distribuée aux uns et aux autres et publiéc dans l'exsiccata de Reichenbach! Si le premier devoir d'un floriste est de soumettre à une juste critique les travaux de ses prédécesseurs, il est aussi de son devoir de signaler les supereheries de ces petits marehands de plantes, parmi lesquels malheurcusement il s'en trouve de peu délicats en matière de localité et de provenance. Il y a là un abus de confiance révoltant, qui peut induire en erreur les auteurs consciencieux, et les savants futurs feront justice de ces supercheries!

Il me semble que M. Godet aurait dû mettre, en 1832, dans sa flore du Jura, son doute sur la provenance de cette espèce pulliée par Thomas dans l'exsiccata de Reichenbach; car je présume qu’à cette époque. la première pensée de $\mathbf{~ I . ~ G o d e t ~ e ́ t a i t ~ l a ~ m e ̂ m e ~ q u e ~ c e l l e ~ q u ' i l ~ v i e n t ~ d e ~}$ m'exprimer dans sa lettre d'aujourd'hui. 
p. 8; Tratt., I. c., II, p. 108; Gaudin, f1. helv., III, p. 556 ; Koch, 1. c., p. 230; Déséglise, in bull. Soc. bot. Belg., XIV, p. 5.57; R. spinulifolia Dematratiana Thory, I. c., p. $115 ; R$. mbiginosa var. spinulifolia Seringe, in DC., prod,, II, p. 616.

Icon. Thory, I. c., fig. I; Redoute, les roses (1824), liv. 57, A. mauvaise.

IIs f. Juin, juillet. Rémion des montagnes, - Suisse. Cant. de Fribourg : Châtel-sur-Montsalvens (Dematra, in herb. Gaudin! in herb. Kew; Cottet, 1874!).

Obs. Le $R$. spinulifolia des auteurs présente un groupe de formes affines qui demandent à être étudiées avec soin. Je donne ici sous la rubrique de variétés ee que j’ai pu étudier d'après les échantillons de l'herbier de II. Boissier et ceux du mien, afin d'appeler l'attention des botanistes sur ces formes qui, micux étudices, pourraient un jour constituer des espèces, tout aussi légitimes, pour ne pas dire plus, que plusieurs de celles créées dans ces derniers temps.

B. grandifolla Déséglise in bull. soc. bot. Belg, XIV, p. 5559; R. spinulifolia Rehb., exs., $\mathrm{n}^{\circ}$ 1899! (non Dematra); Billot, exs., $\mathrm{n}^{\circ} 3077$ bis; $R$. tomentosa var. scabriuscula Seringe! in DC., prod., II, p. 618; R. Csailletir Désége, ms.

II $\downarrow$ в. Juillet. Région montagnarde.-France. Savoic: pentes occidentales du unont Margeriaz, près de Chambéry (Songeon). - Suisse. Cant. de Neuchâtel : environs de Neuchâtel (Chaillet, in herb. DC., 1807!) - canton de Fribourg : Châtel-sur-Montsalvens? (Leresche, in herb. Boissier!), près Fribourg? (Em. Thomas, Charin, in herb. Boissier).

C. mlabreseens Déséglise, 1. c., p. $540 ; R$. spinulifolia Christ, die Rosen der Schiveiz (1875), p. 87, pro parte; $\boldsymbol{R}$. glabrescens Déséglise, mss.

IIAB. Région des montagnes. - France. Isère : la Ferrière d'dllevard (Boullu). - Suisse. Cant. de Bâle : Jura de Bâle (Christ).

D. vlllosula Déséglise, 1. c., p. 541; R. spinulifolia Godet, fl. Jura, p. 209, pr. part. (non Dematra); Reuter, cat. Genève, p 6:3; Déséglise, ess. monog., p. 118; Cariot, l. c., p. 190; Grenier, 11. juras., p. 250, part; Fourreau, I. c., p. 76; R. multivaga Déséglise, mss. 
Exs. Billot, n 5077!

II a . Juin, juillet. Broussailles des montagnes. - France. Moubs : Pontarlier (Grenier); M. Grenier indique aussi le Grand-Combe-des-Bois; je n'ai pas vu la plante de cette localicé; - Ain : la Faucille, Gex (Reuter, in herb. Boissier); - Haute-Savoie : montagne de l'Offiège (Puget), le mont Salève! - Savoie : les Voirons (Reuter, in herb. Boissier). - Suisse. Cant. de Neuchâtel : Lignières (Chaillet, 1810, in herb. DC! sub. 110 420).

E. ambigua Déséglise, 1. c., p. 5ł2; R. Cumberiensis Déséglise, mss.

IIar. Juin. Région des montagnes. - France. Savoie : Margeriaz près de Chambéry, pentes au dessus de Thoiry (Songeon).

F. slabrata l)éséglise l. c., p. 545; R. spimulifoliu Verlot, 1. c., p. 118 (non Dematra); R. propinqua Déséglise, mss.

Hab. Région des montagnes. - France. Isère : sommet du mont SaintEynard près Grenoble (Verlot).

G. hispidella Déséglise, I. c., p. 54 ; R. spimulifolic Godet, I. c. ! part., non Dematra; $R$. Jurana Déséglise, mss.

Hab. - Suisse. Cant, de Neuchâtel : Chaumont (Godet, juin 186\%, in herb. Cottet!)

565. 及. vestita Godet, fl. Jura (1855), p. 210; Reuter, I. c., p. 65 ; Grenier, I. e., p. 252; Déséglise, révis. sect. Toment., extr., p. 6; Fourreau, l. c., p. 76; Verlot, l. c., p. 118; R. montana DC., 11. fr., V (1813̈), p. 2022 (non Vill.); R. spinulifolia var. b. restita Rapin, guide, p. 191. Exs. Billot, $n^{\circ} 5078$.

Has. Juin. Région des montagnes. - France. Isère : Saint-Romain près de Crémieu (Verlot, catal.); - Haute-Savoie : le mont Salère, les Voirons, Reyvroz (Puget); - Savoie : mont Nivolet (Songeon).

OBS. M. Lodet, I. c., dit : « il se trouve dans l'herbier DC., envoyé en 1807 par Chaillet, des environs de Lignières."

M. Godet doit faire certainement une confusion en citant la plante de Lignières d'après Chaillet, d'une part pour son $R$. spinulifolia, enfin pour son R. vestila? D'abord l'étiquette de Chaillet ne porte pas de loculité pour ce qui est le $\boldsymbol{R}$. vestita; l'étiquette est sans numéro et de l'année 1819 ! - Voici la copie de cette étiquette : s Arbuste ressemblant 
"à l'Alpina; fleurs et calices de la Pyrenaicr, mais les feuilles velues; "M. de Ilaller le regarde conme une variété de l'Alpina qu'il appelle " pubescens, et dit que si on veut la séparer de l'Alpina, il faut aussi a séparer la dumetorum de la canina; si vous persistez dans votre sépa" ration, ce sera une espèce nouvelle. Il me dit aussi qu'il l'a trouvée près " de Saint-Cergues; effectivement j'ai trouvé dans mon herbier un échan" tillon de Thomas sans indication qui se rapporte à cette espèce ou « variété. Juin.»

56/4. Terebenthimacea Besser, enum. Pod. et Volh. (1822),p.21 et p.61; Tratt., l.c., II, praef. p.XIV; Boreau, 1. c., éd. 2, n 689, éd. 3, n 879; Arrondeau, f1. Toulous. (1854), p. 127?

Exs. Unio itiner., ann. 1859?; sans numéro.

IIaв. Juin. Bois. - Russie. Tyrae prope Zaleszngki (Besser!), Volhynie? (Iohenacker); - France. Yonne : bois du Bouchard près d'Irancy (Boreau, fl. cent.).

365้. R. Andrennensis Crépin, notes sur qq. pl. rar. et crit. de la Belgique, in bull. Acad. roy. de Belg., 2e série, XIV (1862), nº 7 et extr. p. 50, Déséglise, 1. c., p. 7; R. villosa var. suberecta Woods, 1. c., p. $192 \mathrm{et}$ herb. no 50 ; R. spinulifolia b. Foxiana Thory, 1. c., p. 116?; R. pseudo-rubiginosa Lejeune, f1. Spa, I, p. 229; Seringe, in DC., prod., II, p. 615้; R. mollissima b. Lejeune et Court., comp., II, p.142; R. mollissima var. Arduennensis Du Mort., l. c., p.49; R. mollissima var.pseudo-rubiginosa Baker, l. c., p. 214.

HIAB. Mai, juin. Haies, buissons Angleterre : Yorkshire, Thirsk (Baker). - Belgique. Prov. de Luxembourg : Saint-Ilubert (Crépin).

566. AR. pulveacalenta M.-Bieb., fl. Taur.-Cauc., I, p. 599 et III, p. 544 (non Guss., nee Baker); Sprengel, syst., II, p. 352; Lindley, l. c., p. 95; Tratt., l. c., II, p. 79; Seringe, in DC., prod., II, p. 617; de Prouville, 
l. c., p. 94; Desportes, I. c., n 1995; R. pruinosa Don, hort. cant. (1812), p. 199, ex Desportes.

Icon. M.-Bieb., cent. pl. rar., II, tab. 62.

HAB. - Les collines du Caucase (Bieb.); un échantillon cultivé venant de Besser (1824), se trouve dans l'herbier DC. - Je ne vois pas à la plante de Besser les feuilles "uirinque glanduloso-villosis, " comme Ie dit Bieberstein. Les folioles sont d'un vert clair, légèrement velues, églanduleuses en dessus, pubescentes glanduleuses en dessous.

567. R. caryophyllacea Besser, cat. hort. Crem. (1811), sup. 4, p. 18, ann. 1816, p. 117 et enum. Pod. et Volh., p. 20; Tratt., l. c., p. 68; Rehb., l. c., p. 618; R. rubiginosa var. caryophyllacea Seringe, in DC., prod., II, p. 617.

HAB. - La Podolie (Besser! 1820, in herb. DC.).

568. R. capmoides Kerner! in litt.

Arbrisseau....., tige florale aiguillonnce, à aiguillons épars, grèles, assez nombreux, entassés par trois ou géminés sous les pétioles, dilatés à la base, droits ou un peu inclinés, ceux des jeunes pousses stériles plus robustes; pétioles tomenteux blanchàtres, églanduleux ou parsemés de quelques glandes fines, aiguillonnés ou inermes; folioles médiocres, ovales, parsemées de poils courts apprimés en dessus, pubescentes-glanduleuses en dessous, doublement dentées; stipules courtes, glabres en dessus, pubérulentes et portant quelques glandes en dessous, oreillettes aiguës, droites; pédoncules solitaires ou billores, hispides-glanduleux, cachés par des bractées ovales, glabres, bordées de glandes; tube du calice ovoïde, hispide; divisions calicinales courtes, spatulées au sommet, glanduleuses sur le dos, 2 entières, 5 pinnatifides à appendices étroits, saillantes sur le bouton, réfléchies à l'anthèse, caduques avant l'entière coloration du fruit; 
styles hérissés; fleur médiocre, d'un beau rose; fruit ovoïde.

Il ${ }_{A B}$ - - Autriche. Tyrol : Zirhenhof près de Mieders vallée de Stubai, Saint-Martin près de Hall, Lienz (Kerner).

Obs. M. Christ, die rosen schw., regarde ce rosier comme étant le R. abietina Grenier. Je ne comprends pas ce rapprochement, ni d'après le texte ni d'après le type authentique de $\mathbf{M}$. Grenier. Le $\boldsymbol{R}$. capnoides me semble plutôt appartenir au groupe du $\boldsymbol{R}$. cuspidata.

569. A. cuspidata M.-Bieb., I. c., I, p. 596, III, p. 559; Seringe, in DC., prod., II, p. 618; Crépin, I. c., fasc. 2, p. 88.

HAB. - Le Caucase.

570. ar. cuspidatoides Crépin, in Scheutz, stud. öfv. de Skand. arten. af släg. rosa (1872), p. 57 , et primit. monog. ros., fase. 2, p. 127; R. cuspidata auct. gall. (non Bieb.); R. tomentosa Woods, l. c., p. 19 et herb. $\mathrm{n}^{\text {os }} 58$, $403,46,47,48,31$ (non Smith); Grenier, fl. juras., p.254; R. tomentosa var. Seringeana Du Mort., l. c., p. š1; R. Seringeana Godr., fl. Lorr., èd. 2, I, p. 253.

Exs. Seringe, ros. desséch., nº ̋̈; Baker, herb. ros. brit., $\mathrm{n}^{\circ} 9$; l'échantillon en fruit et la tige stérile sont certainement étrangers et pris sur un autre buisson que celui en fleurs. Je considère ee que j'ai en fruit et la tige stérile comme étant le $\boldsymbol{R}$. mollissima Fries; l'échantillon en fleurs est le $\boldsymbol{R}$. cuspidatoides Crép.; Wirtgen, pl. crit., $n^{0} 544$; Van Heurck et Martinis, herb. des pl. crit. et rar. de la Belgique, $\mathrm{n}^{\circ} 214$.

IIı. Juin, juillet. Haies, bois. - Angleterre. Northumberland : Nolywell (Baker); - Yorkshire : Thirsk, Cleveland, Thomton, Iluker (Baker); - Deronshire: Stretchley (Brigrs); - Cornwall : Lundulph (Briggs). Belgique. Prov. de Hainaut : Masnuy (Martinis); - prov. de Namur: Rochefort, Vignée, Ilan-sur-Lesse (Crépiu). — France. Calvados : Lizicux 
(Boreau); - Cher : Bouy commune de Berry, Achères; - Doubs : mont Brégille près de Besançon (Paillot); - Saône-et-Loire : Parepas près d'Autun (Carion); - Rhône : Charbonnière (Boullu), Lyon (Ozanon); IIaute-Savoie: Saint-Martin au-dessus de Pringy, Argonnex (Puget). Suisse. Cant. de Berne : Belpberg (Seringe); - cant. de Bâle : Jura de Bûle (Christ); - cant. de Schaffhouse : Schaffhouse (Christ); - Valais : Bovernier (de la Soie). - Autriche. Tyrol : Vorderfsoder, Gebhardsberg (Kerner). - Allemagne. Silésie (Baker); Coblence (Wirtgen), Posen, Lyck (Caspary).

Var. B. - Tous les caractires du $R$. cuspidatoides, dont il diffère par ses rameaux floraux poilus au sommet, ses pétioles blanchâtres à tomentum feutré, les folioles à villosité plus abondante et brillante, elles sont aussi doublement dentées, parsemées de glandes en dessous, les tiges florales sont inermes ou peu aiguillonnées dans mes échantillons; styles obscurément hérissés. $R$. floccida Déséglise.

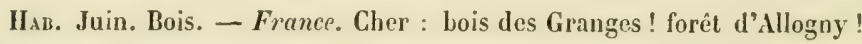
- Rhône : Francheville, Charbonnière (Boullu).

571. R. Genevensis Puget, mss.; R. tomentosogallica Rapin!; $\boldsymbol{R}$. fimbriata Grimbi ?

Arbrisseau peu élevé, à rameaux faibles, flexueux, aiguillons grêles, longs, inégaux, dilatés à la base en forme de disque, droits ou inclinès, dégénérant au sommet des rameaux en aiguillons fins, sétacés, aciculaires; pétioles tomentcux, glanduleux, aiguillonnés; 5-7 folioles larges, ovales, pubescentes sur les deux faces, vertes en dessus, plus pâles en dessous, parsemées de glandes sur les nervures, doublement dentées, les secondaires terminées par une glande; stipules glabres en dessus, pubescentes en dessous, les unes portent des glandes et d'autres sont églanduleuses; pédoneules longs, solitaires ou triflores, hispides-glinduleux, bractées ovales, pubescentes en dessous, beaucoup plus courtes qu'cux; tube du calice ovoïde, hispide-glanduleux; divisions calieinales spatulées au sommet, glanduleuses 
sur le dos, 2 entières, $\overline{5}$ pimmatifides, rélléchies à l'anthèse, puis redressées, caduques avant la maturité du fruit; styles courts, hérissés, disque plan; fleur assez grande, d'un beau rose; fruit ovoüde.

Description reçue de M. Puget.

IIAs. - Suisse. Cant. de Genève : Onex (Rapin), Pinchat! - cant. de Schaffhouse : Schaffthouse, près de la ville (Gremli !).

572. R. scabriuscula Smith, engl, bot., XXVII, no 1896; Winch, bot. guid., 2, préf. p. $\ddot{3}$ ? ex Smith; Woods, l. c., p. 195, herb. $\mathrm{n}^{\text {os }} 51-5 \overline{5}$; Tratt., I. c., I, p. 120̈; Déséglise, révis. seet. Toment., p. 52; R. tomentosa var. b. Smith, engl. f1., II, p. 585; R. tomentosa var. scabriuscula Baker, 1. c., p. 217.

ICon. Engl. bot. XXVII, tab. 1896.

HAB. - Angleterre. Yorkshire: Thirsk, Sowerby (Baker).

575. Pr. Fariuosa Bechst., Forstl)., p. 245 et p. 1046; Rau, I. c., p. 147; Lindley, 1. c., p. 141; Thory, l. c., p. 68; Tratt., I. c., I, p. 115; Bl. et Fing., I: c., I, p. 655; Desportes, 1. c., no 1790; Rchb., fl. excurs., II, p. 616 ; Déséglise, 1. c., p. 17, part.; R. tomentosa var. farinosa Seringe, in DC., prod, II, p. 618?; R. tristis Kerner, in litt.

Icox. Redouté, les roses (1824), livrais. 50, B. mala.

II в. - Écosse. Perthshire? (Hailstone). - Autriche. Tyrol : mont Calvarienberg près de Trins, vallée de Gschnitz sur un terrain schistcux it une altitude de 4000 pieds (Kerner). - Bavière. Mainbernlseim (Rau).

† Folioles églanduleuses en dessous.

574. TR. Morkhasesnig Tratt., 1. c., 1, p. 11/; R. hispida Borkh., Forshh., II, p. 15.52, 11" 486 (non 
Krocker); Poir., encycl., sup., VI, p. 286; Lindley, 1. c., p. 156.

IIAs. - La Germanie.

575. R. collivaga Cottet, in Crépin, 1. e., fasc. I (1869), p. 26 et p. 76 , sine descript.

Arbrisseau de deux mètres de hauteur, rameaux droits non flexueux ni retombants, écorce verte, aiguillons nombreux, inégaux, droits, dilatés à la base en forme de disque, blanchàtres, les autres plus petits et de mème forme, ceux des jeunes pousses rougeâtres; pétioles tomenteux, aiguillonnés; 7 folioles cendrées-grises, tomenteuses sur les deux faces, douces au toucher, nerveuses, ovaleselliptiques, la terminale quelquefois en pointe au sommet et cordiforme à la base, doublement dentées, à dents ouvertes, aiguës; stipules assez grandes, de mème couleur que les folioles, glabres en dessus, velues en dessous, oreillettes aiguës, divergentes; pédoncules réunis en bouquet par 5-4-7, hispides-glanduleux, la base du bouquet porte trois bractées grisàtres, ovales-cuspidées, dépassant ou égalant les pédoncules; les pédoncules extéricurs sont munis en outre de deux petites bractées opposées plus courtes qu'eux; tube du calice d'un vert cendré, obovoïde-allongé, hispide-glanduleux; divisions calicinales ovales, spatulées au sommet, glanduleuses en dessous, 2 entières, 5 portant de petits appendices, saillantes sur le bouton, plus courtes que la corolle; fleur assez grande, d'un beau rose à onglet blane; styles hérissés, disque plan; fruit obovoïde, gros, couronné par les divisions calicinales persistantes jusqu’à la maturité du fruit.

Hab. Juin, juillet. Haies. - Suisse. Cant. de Fribourg : Montbovon, où M. l'abbé Cottet me l'a fait récolter. 


\section{$(515)$}

576. R. velutisa Clairville, man. herb. en Suisse et en Valais (1819), p. 165 ; Lindley, I. c., p. 140; Seringe, in DC., prod., II, p. 622.

$\mathrm{HAB}_{\mathrm{A}}$ - Suisse. Bruel, Winterthour (Clairville).

Ons. Il m'a été impossible de voir un type de ce rosier dans les collections de Genève. - Clairville dit : " fruit rond, calices et pédoncules hispides-glanduleux, feuilles cotonneuses en dessous, bords glanduleux." R. myriacantha DC.? - Peut-ètre ce rosier est-il étranger à cette section? je suis porté à croire que e'est le $R$. pimpinellifolia var. pilosa Lind. ? En présence d'une aussi courte description et en l'absence d'un type authentique, je ne puis faire qu'une supposition.

577. R. tomesutosa Smith, fl. brit. (1800), II, p. 559; DC., fl. fr., IV, p. 440; Gmelin, I. c., IV, p. 569; Pers., l. c., II, p. 50; Lejeune, fl. Spa, I, p. 250 ; Tratt., l. c., I, p. 117; Balbis, l. c., I, p. 262; Rchb., l. c., II, p. 616; Host, 1. c., II, p. 21; Boreau, 1. c., èd. 2, II, p. 690, éd. $\overline{5}, n^{\circ} 881$ et eatal. M.-et-Loire, p. 80; Gonnet, I. c., p. 478; Godet, l. c., p. 212 ; Déséglise, ess. monog., p. 122 et révis. sect. Toment., p. 28; de Mart.-Don., I. c., p. 25ّ̋; Cariot, l. c., p. 190; Fourreau, l. c., p. 76 ; Pérard, I. c., p. 82; Verlot, I. c., p. 119; Coltet, I. c., p. 44; Reuter, I. c., p. 78; R. heterophylla Woods, I. c., p. 195ٌ? herb. $n^{\circ} 54, n^{\circ} 58 \%$ R. pulchella Woo's, I. c., p. 196 , herh. $n^{\circ} 56 ; \boldsymbol{R}$. tomentosa var. Smithiana Scringe, in DC., prod., II, p. 618, excl. syn. Besser et Rau; R. villosa b. Hudson, fl. Angl. (1798), p. 219; R. insidiosa Grenier, 11. juras., p. 25j; R. villosa Lapeyr., pyrén., p. 285, ex Clos, révis. herb. Lapeyr., p. 260; $\boldsymbol{R}$. eriosa Ripart! in litt.; R. umbellifera Sw. ?

Icos. Engl. bot., first edit., tab. 990, third edit., 1ab. 467, mala!

Exs. Cnio itiner., an. 1859?; Baker, herl). ros. Mrit., 
$n^{\circ} 8$; Billot, nos 1662 et 16621 is, (suites), 5726,5727 ; Wirtgen, pl. crit., ${ }^{\text {ns }} 252$, 27l; Fries, herb. norm., $n^{\circ} 46$;

IL $\mathbf{a}$. Juin. IIaies, bois. - Angleterre. Norihumberland : Seaton (Baker); - Cumberland : Nston (Miss Unthank); - Westmoreland (Watson); Cornwall : Saltash, Saint-Stephens, Latehbrook, Burraton (Briggs); près de Newcastle! (Winch, 1829, in herls. DC.). - Belgique. Prov. de Luxembourg : Grune (Crépin). - France. Vosges : ruines du château de Romont (Boulay), forêt de Rambervillers ! - Maine-et-Loire : Brissarthe (Boreau); - Aisne : Villers-Cotterêts (Mabile); - Loiret : Orléans! - Cher : AC. forèt d'Allogny, Mehun; - Doulss : Saint-Ferjeux près de Besançon (Grenier), mont Brégille, Saint-Martin (Paillot); Saône-et-Loire : la Chicolle (Carion); - Aveyron : Mondalazac (Revel); Hautes-Pyrenées : Gavarnie (Bordère); - Tarn : bois de Brassae (de Larembergue); - Ifaute-Garonne: Toulouse, Boussens (Timbal-Lagrave); - Khône : Tassin (Puget); - Isère : mont Rachet (Verlot); - HauteSavoie : Pringy, Arenthou, Thonon, Allonzier (Puget); - Savoie : SaintCassin près de Chambéry (Songeon). - Suisse. Cant. de Fribourg : Charmey (Louis Thomas); - Valais : Choex (Cottet). - Prusse Rhénene. Coblence (Wirtgen).

Var. b. Tuprechti Boissier, fl. Orient., II, p. 682.

Folia obscurius duplicato-serrata saepe simpliciter serrata, calycis laciniae integrae rarius 1-2 lacinulosae (Boissier).

Isa. - In Tuschetia Caucasi orientalis prope Diklo (Ruprecht, in herb. Boiss.).

L'échantillon que j'ai vu dans l'herbier de M. Boissier me semble plutòt appartenir au groupe du $R$. mollissima gu'à celui du R. tomentosa, mais il est difficile de se prononcer en présence d'un seul échantillon très-incomplet.

578. R. intuomissa Crépin, 1. c., fase. 1 (1869), p. 77; R. intricata Crépin, olim; R. praccox Boullu, in Cariot, non Lodd., nee Waitz; R. properata Boullu, in litteris!; R. cinerascens var. intricala Du Mort., l. c., p. §0.

IIA . Juin. Ilaies, broussailles. - Belgique. Prov. de Namur : Rochefort (Crépin). - France. Haute-Loire : Ceyssac près de Puy (Boullu); - 
lihône: Beatmont près de Lyon, Craponne aux aquedues (Boullu); Haute-Savoie: le Salève (Rapin).

579. GR. dianosplat Besser, eat. hort. Crem., an. 1811 , sup. 万, p. 19 et an. 1816, p. 117, et enum. Pod. et Folh. (1822), p. 10; M.-Bieh., I. c., III, J. 5/40; Tratt., I. c., I, P. I22; Rchb., l. c., II, J. 617; Boreau, I. e., él. 5, II, p. 2.j2, olss.; Déséglise, l. c.. p. 121 et rév. sect. Toment., extr., p. $15 \%$. tomentosa var. dimorpha Du Mort., 1. e., p. כ้1.

Exs. Reichenbach, n 1955; Billot, n 1481 .

Haв. Juin, juillet. - France. Vosges : Bamont (Boulay); - Doubs : Pontarlier (Grenier), mont d'Or ! mont Brégille! — Saône-et-Loire : Macon (Fontaines). - Belgique. Prov. de Namur : Waulsort (Crépin). Autriche. Croatie autrich. : Banat pris de Csiklowa (Wierzbicki, in herb. Boissier).

OBs. Le type de Besser qui se trouve depuis 1820 dans l'herb. DC., provient du jardin de Besser. Dans le $R$. dimorphe distribué par Besser, il doit y avoir certainement une confusion? L'échantillon du Musée de Cambridge et celui que j'ai vu dans l'herbier du comte Jaubert, sont certainement étrangers au type. La villosité des folioles est plus abondante et les faces supéricure et inférieure sont glanduleuses! $R$. cuspidata Bicb.?

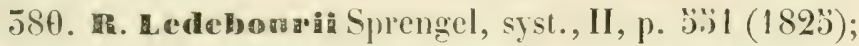
R. mollis Ledebour, mém. Acarl. Saint-Pétersb., I, p. :i:31; I.-Bieb., 1. ‘, III, p. 5/41; Tratl., I, c., I, p. 124; Seringe, in DC., prod., II, p. 618.

HAr. - Le Caucase, mont Kaischaur.

581. HR. stobglobosa Smith, engl. f1. (182/), II, p. 584; Borean, 1. c., éd. 2, n 691, éd. 5, nº 882 et eatal. M.-et-Loire, p. 80; Reuler, I. e., p. 67; Déséglise, ess. monog., p. 125; Cariot, I. c., p. 191; Verlot, 1. c., p.119; R. Sherardi Smith, l. e., IV, 1. 269 (non rectifié); Déséglise, révis. sect. Toment., extr., p. $\bar{\jmath} \overline{5}$; Fourreau, 
l. c., p. $76 ; R$. tomentosa var. E. Woods, l. c., p. 201 et herb., $n^{\circ} 4 \overline{5} ; R$. tomentosa var. subglobosa Carion, catal. Saòne-t-Loire (18399), p. 45; Du Mort., l. c., p. "3il; Baker, I. c., p. 217; R. villosa Bastard, I. c., p. 188 (non L.); Desvaux, fl. de l'Anjou (1827), p. 526; Guépin, fl. M.-ct-Loire (1858), p. $556 ; R$. villosa sylvestris Desvaux, journ. bot. (1815), II, p. 117; R. ciliato-petala Godet, l. c., p. 211 ex Reuter.

Exs. Wirtgen, pl. crit., $n^{\circ} 25 \overline{5}$; Billot, $n^{\circ} 1481$ bis ?; Déséglise, herb. r'os., $\mathrm{n}^{\circ} 57$.

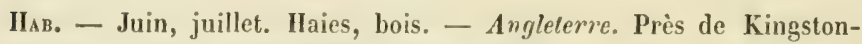
Upon-Thomes; Thunbridge Wells et Dove d'après Smith; - Devonshire : haies près de Plymptôn (Briłgrs). - Belgique. Namur (Crépin). - France. Vosges: Schirmeck (Billot), Presle, Basse-sur-le-Rupt (Pierrat); - Côtesdu-Nord : Dinan (Mabile); - Loire-inférieure: Arche-Gaubert près de Mauves (Lloyd); - Maine-et-Loire: C. Ia Haie-Longue (Boreau); - Sarthe : le Mans (Boreau); - Vendée : forêt de Mervent (Letourneux); - Loiret: Orléans (Jullien); - Loir-et-Cher : Souëme! - Cher : Saint-Florent!; Haute-Vienne : Thias (Lamy); - Saône-et-Loire : Autun (Carion); Rhône : pont d'Alay, Charbonnière (Chabert); - Isère : forêt de Porte, de Corenc au Sappey (Verlot); - Ilaute-Savoie : le Salève, Thonon. Suisse. Cant. de Bàle : Bâle (Christ). - Autriche. Tyrol : Pustaria (Kerner). - Prusse. Winningen, vallée de Conde (Wirtgen), Lyck (Sanio).

Var. B. macrocarpa. Fruit très-gros, globuleux. - Haute-Savoie : Habère-Poche (Puget), Salève (Rapin).

582. R. Tumoniensis Déséglise, révis. de la sect. toment., p. 10; Crépin, l. c., fasc. 1, p. 76.

Exs. Déséglise, herb. rosar., $n^{\circ} 56$; Billot (suites), $n^{\circ} 5599$.

Haß. NIai, juin. Buissons des montagnes. - France. Haute-Savoie : Thonon, grèves du lac derrière Ripaille, Reyvroz (Puget).

585. R. confusa Puget, 1. e., p. 76, sine deseript.; Verlot, 1. c., p. 118, obs. 
Arbrisseau peu élevé, chargé d’aiguillons longs, droits ou un peu inclinés, géminés sous les pétioles, rougeàtres sur les jeunes pousses; pétioles tomenteux, glanduleux, aiguillonnés; $\dddot{\partial-7}$ folioles toutes pétiolées, ovales-aiguës ou oblongues, souvent sensiblement rétrécies à la base, pubescentes en dessus, tomenteuses-blanchatres en dessous, la nervure médiane parsemée de quelques rares glandes, doublement dentées, à dents ciliées glanduleuses; stipules glabres en dessous, à oreillettes aiguës, droites; pédoncules solitaires ou réunis par 2- $\ddot{3}$, longs, hispides-glanduleux, portant a leur base des bractées lancéolées, glabres ou glabrescentes en dessous, plus courtes qu'eux; tube du calice ovö̈le, hispide-glandulcux; divisions calicinales 2 entières, 5 pimnatifides it appendices courts bordés de glandes, grlanduleuses sur le dos, plus courtes que la corolle, persistant jusqu’a la coloration du fruit; styles courts, glabres; disque saillant; fleur grande, d'un rose clair; fruit ellipsoïde-allongé ou ovoïle, contracté en col au sommet, hispide-glanduleux.

$\mathrm{H}_{\mathrm{AB}}$. Juin, juillet. Région des montagnes. - France. IIaute-Savoie : Reyvroz, Ilabère-Lullin (Puget); indiqué dans le départ. de l'Isère à Saint-Romain, par Fourreau dans son catalogue.

584. R. Anesiensis Déséglise, révis. de la sect. toment. (1866), p. 14; Crépin, 1. e., fasc. 1, p. 76.

Exs. Déséglise, herb. rosar., $n^{\circ} 74$.

IIs. Juin, juillet. Buissons de la région montagneuse. - France. Ilaute-Savoie : Pringy (Puget); - Savoie : mont Joigny près de Chambéry (Paris).

583. R. Andrzeiowscii Steven, in Besser, cat. hort. Crem., an. 1811, sup. 5, p. 19, an. 1816, p. $117 \mathrm{et}$ enum. Pod. et Vollı., pp. 19, 66; Bicberst., I. c., II, 
p. 559, sul, R. villosa; Tratı., I. c., I, p. 120; Borcau, l. c., éd. 5, n 885; Déséglise, essai monog., p. 12/ et révis. sect. toment., p. 53; Cariot, 1. c., p. 191; Fourreau, I. c., p. 76 ; Verlot, l. e., p. 119; Coltet, I. c., p. 4k; R. villose var. syleestris Seringe, in DC., prod., II, p. 618 , part.; R. permutata Ripart!, in litt.

Exs. Wirtgen, pl. crit., no 179 ?; Baker, herb. ros. brit., $n^{\circ} 10$.

IIAB. Juin. Bois. - Angleterre. Yorkshire : Sowerby, Thirsk (Baker), Forcett (Hailstone); - Devonshire : vallée de la Plym (Briggs); Cornwall : haies près de Downderry (Briggs.) - France. Cher : forêt du Rhin-du-bois, Contremoret près de Bourges, pie de Montaigu, SaintFlorent, forêt de Vierzon; - Saône-et-Loire : Châlons-sur-Saône(Ozanon); - Rhône : Saint-Laurent-de-Vaulx (Boullu); - Ilaute-Savoie : Pringy (Puget). - Suisse. Valais : au dessus de Lourtier vallée de Bagnes (Cottet). - Russie d'Europe. Podolie (Besser, 1820, in herb. DC.).

\section{B) Pomiferae.}

Villosae DC., I. c. (sub sect.), part.; Crépin, 1. c., p. 26, part.; Cottet, 1. c., p. 4/4.

Aiguillons grèles et droits; folioles tomenteuses, glanduleuses ou églanduleuses sur le parenchyme ou les nervures secondaires; corolle d'un rose vif; divisions calicinales persistantes; fruit ordinairement gros, pomiforme, rarement petit.

Le nom de $\boldsymbol{R}$. villosa est aluandonné dans la science moderne comme olseur et ambigu; heauroup d'espèces des autres sections précédentes sont aussi velues; le nom de Pomiferae, emprunté à l'espèce principale de la subdivision, caractérise parfaitement ce petit groupe. 
1. Folioles églanduleuses en dessous . . . . 2

Folioles plus ou moins glanduleuses en dessous . ";.

2. $\left\{\begin{array}{l}\text { Folioles grandes ou moyennes . . . . . . . } \\ \text { Folioles très-petites, pétales très-petits, d'un rouge }\end{array}\right.$ foncé, fruit petit, sọhérique . . . . . . minulu

(Folioles grandes, elliptiques, fleur rose, pétales

5. ciliés-glanduleux à la base, fruit très-gुros,

Violacé, hérissé

Folioles moyennes

Fleur rose, pétales ciliés à la base, fruit globuleux,

4. d'un rouge brun

f Fleur rose, pétales jaunes à l'onglet, non ciliés, fruit ovoïde, rouge. . . . . . . . .

pomifera.

4.

mollis.

Grenierii.

:i. $\left\{\begin{array}{c}\text { Folioles grandes, elliptiques, fleur grande, d'un } \\ \text { beau rose, fruit très-gros, obovoïde, hérissé. . . . . . . . . . . . . . . . . . . }\end{array}\right.$

(Fleur d'un beau rose, fruit obovoïde, affectant

6. $\{$ une forme pyriforme, glabre . . . omisse.

Fleur d'un beau rose, pétales à onglet jaune, fruit arrondi, rouge, hérissé . . . . resinosoides.

586. R. omissa Déséglise, réris. sect. Toment. (1866), extr., p. 12; Fourreau, 1. c., p. 76; Verlot, 1. c., p. 118.

Exs. Déséglise, herb. ros., $1^{0}$ 57; Billot (suites), $n^{\circ} 5600$.

IIAB. Juin. Région des montagnes. - France. Isère : forèt de Porte près de Grenoble (Verlot), Villard-de-Ians (Boullu). - Haute-Savoie : Tessy, Pringy, bois du Barioz, Saint-Martin, Épagny, mont de Sion, Argonnex (Puget).

587. Baprechti Boissier, f1. Orient., II, p. 682.

IIAs. - In Tusehetia Caucasi orientalis (Ruprecht, in herb. Boissier).

588. R. Whedureichii Boissier et lieut, diagn., sér. 2, fase. 2 , p. 49; Boissier, 1. c., p. 681.

Exs. de Ifeldreich, no 2245. 
I

589. R. mollis Smith, engl. bot. XXXV (1812), $n^{\circ}$ 2459; Winch, geogr. distrib., p.42; R. mollissima Fries, novit., ed. 2 (1828), p. 151 et sum. veget. Scand., p. 17/; Godet, I. c., p. 212; Boreau, I. e., éd. J̄, n 884; Carion, l. c., p. 45; Reuter, 1. e., p. 66; Déséglise, ess. monog., extr., p. $12 \%$, et révis. sect. Toment., extr., p. 56; Cariot, l. e., p. 191; Grenier, I. c., p. 251; Fourreau, 1. c., p. 76; Verlot, I. c., p. 119; Boissier, 1. c., p. 681?; Baker, I. c., p. 215, part.; Cottet, 1. c., p. 44; R. villosa L. sp. 70/, part.; Woods, l. c., p. 188 et herlo. $n^{\circ s} 23$ it 50; Hooker, brit. fl., p. 255; Reuter, cat. de Genève (1852), part.; R. ciliato-petala Koch, syn. p. 255 (non Besser); R. Andrzeiouskii Boreau, l. c., éd. 2, n 692 (non Besser); $\boldsymbol{R}$ villosa var. mollissima Rau, enum. ros. p. 154; R. dubia Wibel, 11. Werth., p. 265, ex Rau; R. tomentosa var. mollissima Du Mort., fl. Belg., p. 95.

Icon. Engl. bot., tab. 2459, third edit. (186/4), tab.466.

Exs. Fries, herb. norm., no 4k; Baker, herb. ros. brit., $\mathrm{n}^{\text {os }} \check{3}, 6$ ?

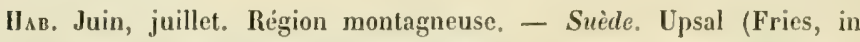
herb. Boissier), Wexiö (Scheutz), Stockholm (Nyman). - Angleterre. Northumberland (Baker); - Yorkshire : Thirsk (Baker), Sowerby (Addison); - Cheshire : Kirby (W ebb); - Derbyshire : Middleton (Purchass). - Ecosse. Glasgow (W ebb); - Perthshire : banks of loch Earn (Hailstone). - France. Finistère : Brest à Guipavas (Boreau); Puy-de-Dôme : Murol près du lac Chambon, mont Dore (Lamy); Nièvre : entre Planchez et Houx (Boreau, in herb Jaubert); - HauteLoire: Ceyssac près le Puy (Boullu); - Lozère (Prost, 1815, in herb.DC.); - Ilaute-Savoic : Saint-Nicolas-de-la-Chapelle (Puget), le mont Saleve! Savoie : Chambéry (Songeon). - Suisse. Valais : Obergestler (Lagger), Zermatt (Cottet); - cant. de Bâle : Bâle (Christ). - Autriche. Bohème : Babina-Menthan près de Leitmeritz (Kerner). - Allemagne. Bavière 
Stein près de Würzbourg (Christ); - Posen : Lyck (Sanio). - Italie. Montenero, Viaggio al Valtuve (Tenore).

Var. b. coerulea Baker, exs., no 7; Déséglise, réris. sect. tom., p.58.; R. villosa var. coerulea Woods, I. c., p. 189 et herb., $n^{\mathrm{es}} 26$ et 28.

Il diffère du type par les pédoncules et le tube du calice lisses, ses feuilles parsemées de glandes. Le no 7 de la collection de II. Baker a les fruits lisses et seulement quelques rares soies au sommet.

IIAB. - Angleterie. North-Yorkshire; Cumberland; Northumberland (Baker).

590. Rehentzii Christ, flora, 1874, extr., p. 24.

HAB. - Danemark. Ile de Sceland : Gurze (Scheutz, Christ).

Pétioles tomenteux, inermes; folioles à villosité courte apprimée en dessus, pubescentes-glanduleuses en dessous, douhlement dentées; stipules assez larges, vertes, glabres en dessous, pubérulentes-glanduleuses en dessous, oreillettes divergentes; pédoncules courts, solitaires ou biflo.es, hérissés de petites soies blanches spiniformes; tube du calice ovoïle, hérissé de soies blanches spiniformes terminées par une glande noirâtre; bractées ovales-cuspidées, glabres en dessus, pubérulentesglanduleuses sur le dos, cachant les pédoncules; divisions calicinales, spatulées et denticulées au sommet, hispides-glanduleuses sur le dos, 2 cntreres, 5 pimnatifides à appendiees filiformes bordés de glandes; styles courts, velus, occupant tonte la surface du disqur; fleur blanche.

Notes prises sur l'échantillon que je tiens de M. Christ.

591. R. vemasta Scheutz, stud. öfver de Skand. art. af slïgtet Rosa (1872), p. 56; Crépin, primit. mon. ros., fase. 2 (1872), in bull. soc. roy. bot. de Belg., XI, p. 245, extr. p. 127; Christ, l. e., p. 24.

$\mathrm{II}_{\mathrm{AB}}$ - Suède. Blekinge (Scheutz, Christ).

592. IR. Cremsensis Kerner, in litt.

Arbrisseau.....; aiguillons des rameaux floraux grèles, fins, droits, dilatés à la base en forme de disque; pétioles pubérulents, glanduleux, aiguillonnés en dessous; folioles elliptiques, vertes, parsemées de poils apprimés 
en dessus, blanchitres, pubescentes, glanduleuses en dessous, doublement dentées; stipules inférieures glabres en dessus, glanduleuses en dessous, les supéricures glandulcuses au sommet, oreillettes aiguës, divergentes, à bord glanduleux; pédoncules en houruet ou 1-2-ð̄-4, hispidesglanduleux, bractées lancéolées, acuminées, glahres sur les deux faces, la côte un peu velue et glanduleuses au sommet en dessous, égalant ou dépassant les pédoncules; tube du calice violacé, subglobuleux, hispideglanduleux; divisions calicinales longues, glanduleuses sur le dos, les intérieures tomenteuses aux bords, 2 entières, pimnatifides à appendices lancéolés, hordés de glandes; styles courts, très-velus ; lieur rose; fruit....

Il AB. Région montagneuse. - Aulriche. Mont Kegel entre Krems et Stein; mont Baldo (Kerner).

595. anstaris Kerner, in Crépin, l. e., fase. 1, p. 25 , sine descript.

D’après mes échantillons, rameaux florifères incrmes, rameaux stériles incrmes; pétioles tomenteux, glauduleux, inermes ou faiblement aig̣uillonnés; $; \mathbf{j} 7$ folioles elliptiques, pubesecntes sur les deux faces, glanduleuses en dessous, nervures banchitres, saillantes, doublement dentées; stipules larges, vertes, glabres en dessus, glanduleuses en dessous, oreillettes presque droites; pédoncules 1-2-5, hispides-glanduleux, bractées larges, glabres en dessus, glanduleuses en dessous, dépassant ou atteignant les pédoncules; tube du calice ovoüde, glahre; divisions calicinales spatulées au sommet, hispides-glanduleuses sur le dos; styles velus; fleur rose; fruit non parrenu à sa maturité, globuleux.

Ces notes sont prises sur les échantillons recus de 
M. Kerner; M. Kerner aura ì nous dire le port de l'arbrisseau, la forme des aiguillons des tiges et si les divisions calicinales sont persistantes ou caduques.

Ce rosier est une Tomentoste et non une Rubiginosae.

IIAb. - Aulriche. Tyrol austr. : Ritten près de Botzen (Kerner).

594. R. resiznosa Sternb., in Rechb., fl. excurs., II, p. $616, \mathrm{n}^{\circ} 5997$.

Exs. Reichenbach, no 1271 ?

Ons. Rehb. dit du $R$. resinosa: " laciniis calycis integris, foliolis ovatoa ellipticis duplicato-glanduloso-serratis viridibus pubescentibus subtus " sparsim glandulosis. " J'ai admis ce rosier sous ee nom avec un point de doule dans ma révision de la section tomentosa; depuis cette époque, j'ai pu voir, dans l'herbier de H. Brissier, le $R$. resinosa publié par Rehb., dans son exsiccata no 1271, venant de Lofer près de Salzbourg. - Ce numcero est représenté par deux brins pris sur des sommités florales, l'un en houton non épanoui, l'autre en fruit trop avancé. Ces deux brins ont-ils été pris sur le mème buisson? C'est une question qu'il est permis de faire en présenee des échantillons qui ont été distribués ! Il faut dire d'abord que ces spécimens ne correspondent pas avec la description donnée par Reichenbach.

10 Le spécimen portant un bouton non épanoui a les folioles glabres et parsemées de glandes en dessus, pubescentes en dessous sur les nervures principalement, le parenchyme est plus ou moins parsemé de glandes sessiles, la côte médiane églanduleuse; pétioles tomenteux-blanchâtres, églanduleux, aiguillonnés; stipules larges, vertes, glabres en dessus, pubcrulentes-glanduleuses en dessous; pédoncule très-court, entièrement cachén, ainsi que le tube du calice, par une large bractée, glabre en dessus, puliérulente-glanduleuse en dessous; tube du calice petit, arrondi, chargé de pelites soies spiniformes terminées par une glande; divisions calicinales hérisscies de petites soies et glanduleuses sur le dos, les intéricures à lords tomenteux, 2 entières, 5 pinnalifids à aprendices filiformes bordés de glandes.

20 L'échantillon en fruit, e'est-ì-dire le brin qui porte deux pétioles et un fruit, a les folioles finement velues, à villosité courte et églanduleuses ch dessus, grisilres, tomenteuse's, églanduleuses en dessous, la còte médianc 
est dépourvue de glandes; les pétioles tomenteux-blanchâtres, parsemés de quelques glandes fines, faiblement aiguillonnés en dessous; les stipules étroites, tomenteuses en dessous; le ramuscule qui porte le fruit est parsemé de poils au sommet; le fruit est ti ès-gros, dans le genre de celui du $\boldsymbol{R}$. pomifera, mais les soies spiniformes sont beaucoup plus petites que dans le $\boldsymbol{R}$. pomifera; les divisions calicinales couvertes de glandes et de petites soies, les exlérieures pinnatifides.

595. T. resinosoides Crépin, in Cottet, 1. c., p. 4/4; R. resinosa Boreau, l. c., éd. 5, no 883 (non Sternb.); Déséglise, essai monog., extr., p. 126, et révis. sect. Toment., extr., p. 58; Cariot, 1. c., p. 192; Verlot, l. c., p. $594 ; \boldsymbol{R}$. pomifera Lec. et Lamotte, eat. du plat. centr., p. 150 (non Herm.).

Exs. Déséglise, herb. ros., $n^{0} 75$; Billot (suites), $n^{\circ} 5601$.

Hab. Juin, juillet. Broussailles des montagnes. - Suède. Blekinge, Elleholm (Scheutz). - Angleterre. Yorkshire : entre Thirsk et Woodend, Westerdale (Baker). - France. Vosges : ballon de Saint-Maurice (Pierrat); - Puy-de-Dôme : Puy de Pariou ! pelit Puy-de-Dòme! - Rhône : Craponne (Boullu); - Loire : Planfoy (Chabert); - ItauteSavoie : Ilabère-l'oche, montagne de l'Offìge (I'uget). - Suisse. Cant. de Fribourg: la Tine près de Montbovon! - Valais : Bovernier (de la Soie), Dixain de Conches (Lagger).

596. Fr. minuta Boreau in Déségl., ess. mon., $\mathrm{n}^{\circ} 105$ et rév. sert. Toment., p. 42; Verlot, I. c., p. 119; R. villosa var. minuta Rau, enum. ros., p. 156; Desportes, ros. gall., $\mathrm{n}^{\circ} 1764$; Bl. et Fing., comp., I, p. 625; Seringe in DC., p. II, pr. 619.

Exs. Déséglise, herb. ros., $n^{\circ} 76$ ?

$\mathrm{II}_{\mathrm{aB}}$. Juillet. Région des montagnes. - France. Hautes-Alpes : La Grave (Ozanon).

597. F. ciliato-petala Besser, enum. pl. Pod. et 
Volh. (1822), p. 66; Tratt., 1. c., II, praef., p. 7; Seringe, in DC., prod., II, p. 619.

IIAB. - Russie. La Lithuanie (Besser, 1821, in herb. DC.). - Prusse. Posen, Lyck (Sanio, Caspary).

598. ER. Grenierii Déséglise, essai monog., extr., p. 128, rév.sect. Toment., extr., p. 45; Verlot, l. c., p. 119; Cottet, l. c., p. 4k; R. Perusiana Timbal-Lagrave, in litt.!

Exs. Déséglise, herb. ros., $\mathbf{n}^{\text {os }} \overline{\mathrm{j}} 8$ et 58 bis; Billot (suites), $\mathrm{n}^{\text {os }} 5602$ et 5602 bis.

II.ı. Juillet. Région des montagnes. - France. Hautes-Alpes : La Grave (Ozanon), mont Bayard près de Gap (Gariod), Villard-d'Arène (Verlot); Isère : Saint-Christophe-en-Visans (Boullu); - Lozère : rochers à Loubet (Timbal-Lagrave); - Pyrénées-Orient. : Montlouis près le village d'Eynes (Timbal-Lagrave); - Haute-Savoie : Reyvroz, Habère-Lullin (Puget). Suisse. Valais : Dixain-de-Conches (Lagger), mont Catogne! Bourg-SaintPierre!

599. pomifen. Herm., diss. (1762), p. 16; Roessig, die rosen (1800), $n^{\circ} 50$; Gmelin, 1. c. (1806), II, p. 410; Tratt., I. c. (1825), I, p. 108?; IIost, l. c. (18.51), 1I, p. 23; Koch, syn. (1845), p. 255; Godron, f1. Lorr. (1845), I, p. 222; Gr. et Godr., I. c. (1848), I, p. 560 , part.; Boreau, l. c., éd. 2 (1849), n 695, éd. 5 (1857), no 886; Kirschleger, 1. c. (1852), I, p. 250; Godet, 1. c. (1855), p. 210; Reuter, 1. c. (1861), p. 67, part.; Déséglise, 1. c. (1861), extr., p. 129 et révis. sect. Toment. (1866), extr., p. 4/; Di Mort., I. c. (1867), p. 48; R. villosa L., sp., p. 704, part.; Villars, fl. Dauph. (1789), III, p. 531; Mocneh, meth. (1794), p. 688; Willd., spec. plant. (1797), II, p. 1069 et enum. plant. (1809), p. 5/4; Krocher, 1. c. (1798), II, p. 14/; Gilibert, pl. d'Europe (1800), I, p. 585; Smith, fl. Brit. (180/), II, p. 558; DC., f1. fr. (1805), IV, p. 440, exel. 
var. b.; Pers., I. c. (1807), II, p. 48; Lindley, I. e. (1820), p. 74; de Prouville, I. c. (182/), p. 77; Du Mort., fl. Belgica (1827), p. 95, exel. var. b.; Rehb., l. c. (1850), II, p. 615, part.; Lorey et Duret, I. e. (1851), I, p. 508; Mutel, I. c. (1854), I, p. 547, excl. syn. Besser; Gomnet, I. c. (1847), p. 478; R. villosa var. pomifera Desvaux, journ. bot. (1815), II, p. 117; Thory, 1. c. (1820), p. 63; Seringe in DC., prod., II (1823), p. 618; Desportes, 1. c. (1827), $1^{\circ} 1761 ; \boldsymbol{R}$. villosa var. A. vulgaris Rau, I. c. (1816), p. I3̆l; Bl. et Fing. (1823), I, p. 624; Duby, I. c. (1828), I, p. 179.

Icon. Flora Danica, IX, tab. 145̌8; Swenk bot., V, tab. 515̄; Roessig, I. c., tab. 50; engl. bot., IX, pl. כૅ45.

Exs. Billot, $n^{\circ}$ 14.82!; Wirtgen, pl. erit., $n^{\circ}$ 24!; Thielens et Devos, Kickxia belgica, $n^{\circ} 307$; Fries, herb. norm., $n^{\circ} 47$ ?

II ab. Juin, juillet. Région des montagnes. - Belgique. Prov. de Namur : forteresse de Namur (Devos). - France. Vosges : le Bohneck (Pierrat); Loir-et-Cher : spont.? les Montils (Franchet); - Seine-et-Marne : spont.? rnvirons de Fontainclbleau (Naudin); - Pyrénées-Orient. : Saint-Martin en montant au Canigou (Iuet du Pavillon, in herb. Boissier); - Savoie : Granier (Puget). - Alsace. Hagueneau (Billot), - Suisse. Valais : Wiestin à Zermatt, Dixain de Conches (Lagger). - Autriche. Indiqué en Bohème et en Moravie par Host. - Allemagne. Coblence (Wirtgen). - Ilalie. Piémont : val Pesio (Bornet).

400. Rr. Fribargensis Lagger et Puget, in Crépin, primit. monog. ros., fase. I, p. 27, sine descript.

Sous-arbrisseau ì racine traęante, peu élevé, d'un mètre à peine de haut, ne formant pas buisson; rameaux courts chargés d’aiguillons petits, droits, inégaux, entassés, dilatés à la base en forme de disque; pétioles glanduleux, 
parsemés de poils en dessus, aiguillonnés en dessous ; 引-7 folioles ovales-elliptiques, portant en dessus quelques poils qui disparaissent avee l'àge, fermes, nerveuses, doublement dentées, glabres et glanduleuses en dessous ; stipules glabres en dessus, glanduleuses en dessous; oreillettes courtes, droites; pédoncules très-courts, hispides-glanduleux, droits à la floraison, penchés à la maturité du fruit; tube du calice ovoïde ou subarrondi, couvert de longues pointes sétacées terminées par une glande; divisions calirinales 2 entières, 5 un peu appendiculées, couvertes de glandes et de petits acicules, plus courtes que la eorolle, redressées, conniventes, charnues ì la base, persistant sur le fruit; styles courts, velus; fleur grande, d'un beau rose; fruit très-gros, obovoüde-pyriforme, d'un beau rouge, couvert de soies spinuliformes.

IIAB. - Suisse. Cant. de Fribourg : pâturages de la Gotalaz où M. l'abbé Cottet me l'a fait récolter.

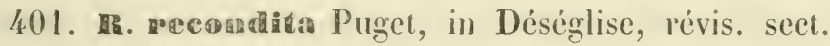
Toment. (1866), extr., p. 46; Verlot, l. c., p. 119; Cottet, I. c., p. \&k; R. Clusiana Bouvier! bull. soc. bot. de Fr., sess. d'Annecy, XIII, p. 14 (non Waitz).

\section{Exs. de Heldreich, $n^{\circ} 512$.}

$\mathrm{II}_{\mathrm{AB}}$ - - Juillet. Région des montagnes. - Angleterre. Yorkshire : haies entre Thirsk et Woodend (Baker); - Glocestershire : près de Painswick (IV ebb). - Écosse. Lanarkshire : Borriton, cascade sur la Clyde (Ilailstone, juill. 1829). - France. Isère : Saint-Christophe-en-Oisans (Boullu); Alpes-maritimes : la Mairis près de Luceram, Colmiane (Bornet); Pyrén.-Orient. : Eynes (Ripart); - Haute-Savoie : les Gets, Reyvroz, vallée de Lullin (Puget), Argentière ! - Saroie : Saint-Nicolas-la-Chapelle, Ilauteluce (Puget). - Suisse. Valais: Münstiger (Lagges), mont Arpile! la Forclaz! le Levron, vallée de Bagnes! - Autriche. Tyrol : val di Ledro, Hinterssoder (Kerner). - Prusse. Posen : Lyek (Sanio): - 
Italie. Saint-Oyen, vallée d'Aoste près de Saint-Rémy! - Perse. Laristan, vallée Djimil (de Heldreich, in herb. Boissier).

402. R. Wromith Puget, in bull. soc. Murith., fasc. 5 (1874), p. 5\%; Cottet, I. c., p. 44.

Hab. Région des montagnes. - Suisse. Valais : Münstiger, Halden, Dixain de Conches (Lagger); - cant. de Fribourg : les Combes (Puget).

405. IR. Gaudini Puget, in Déséglise, révis. sect. Toment. (1866), p. 47, obs.; Cottet, l. c., p. ๖̌

II Obergestelen, Dixain de Conches (Lagger).

404. R. Gombensis Puget, in bull. soc. Murith., fase. 5 (1874), p. 5̌4; Cottet, 1. c., p. 44.

$H_{A B}$. Région des montagnes. - Suisse. Valais : Dixain de Conches (Lagger).

405. R. proxima Cottet, in Crépin, 1. c., p. 27, sine descript.; $\boldsymbol{R}$. glutinosa Dematra, I. c., p. 6, ex loco natali (non Sibth.).

Arbrisseau peu élevé (40 à 60 centim.), touffu; souche pourvue de rejets souterrains plus ou moins longuement traçants; tiges principales roides, droites, ordinairement à écorce d'un brun noirâtre; aiguillons caulinaires épars, peu nombreux, inégaux, subulés, blanchâtres, horizontaux ou un peu inclinés, dilatés à la base en forme de disque; rameaux florifères flexueux, d'un glauque violacé, munis de petits aiguillons grêles, droits; pétioles tomenteux, glanduleux, plus ou moins aiguillonnés en dessous ; $\breve{-}-7$, rar. 9 folioles assez grandes, ovales-elliptiques ou elliptiques-oblongues, les inférieures obtuses parsemées en dessus de petites glandes, les supérieures et les florales plus ou moins atténuées au sommet, pubescentes en dessus, grisàtres-velues et couvertes en dessous de glandes résineuses 
odorantes; dents composées, larges, plus ou moins ouvertes, portant 2-5 dents secondaires terminées par une glande fine; stipules larges, glabres en dessus, velues et chargées de glandes en dessous, oreillettes aiguës, droites ou peu divergentes, cilices-glanduleuses aux bords, les stipules supérieures sont en outre parsemées de glandes en dessus; pédoncules solitaires ou réunis par 2-4, glauques, souvent violacés, légèrement hispides-glanduleux ; bractées ovales, souvent terminées par unc pointe foliacée, glabres en dessus, velues-glanduleuses en dessous, égalant les pédoncules ou plus courtes; tuje du calice arrondi, glauqueviolacé, glabre, entièrement lisse, rarement muni vers le haut de quelques petites glandes stipitées; divisions calicinales spatulées, ciliées au sommet, couvertes sur le dos de glandes, 2 entic̀res, 5 pinnatifides à appendices étroits, linéaires, saillantes sur le bouton, égalant la corolle ou un peu plus longues, étalées à l'anthèse puis redressẻes, comniventes, persistantes; styles courts, velus; fleur d'un beau rose, pétales non ciliés-glanduleux ; fruit d'un rouge violacé pruineux à la maturité, de grosseur médiocre, obovoïde (Cottet).

II An. - Suisse. Cant. de Fribourg : les Mérils-verts-Champs (Cottet). 



\section{INDEX.}

Les noms spécifiques admis sont en italique.

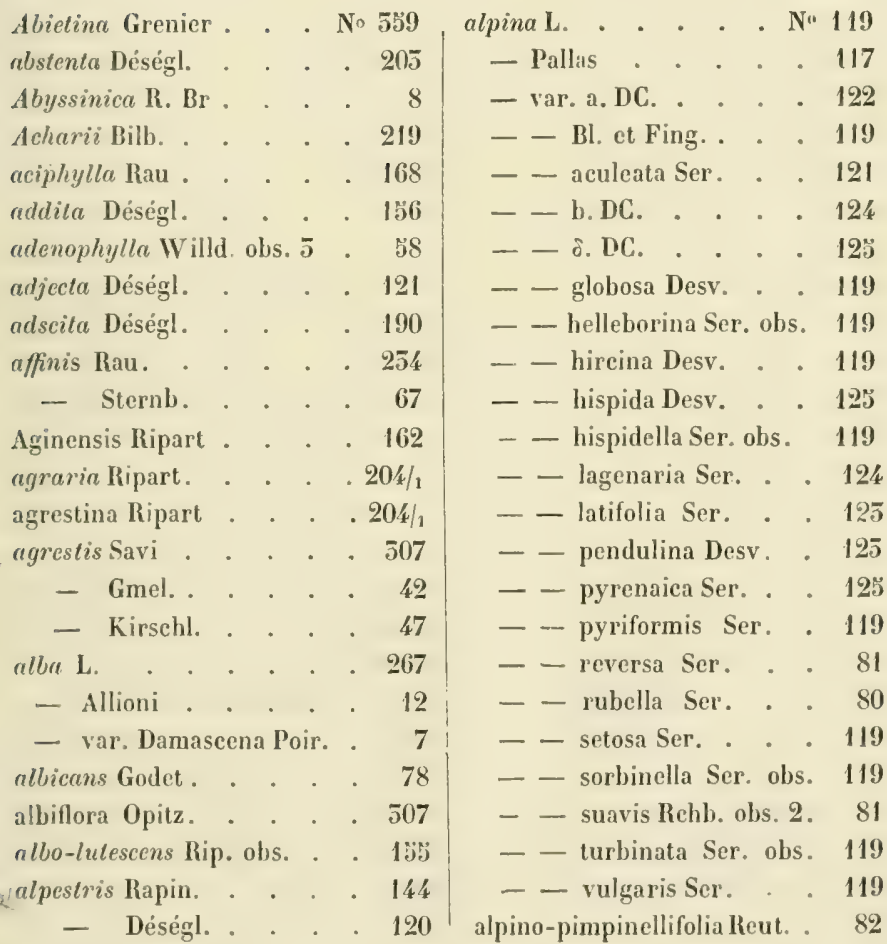


alpiphila Arv.-Touv. . . . 157

Altaica Willd. . . . . 75

Amansii Rip. et Déségl. . 162

amoena Kerner . . . . 296

ambigens Gremli . . . . $4 \overline{5}$

ambigua Lejeune . . . . 211

amblyphylla Rip. obs. . . $2 / 7$

Amoyensis Hance. . . . 59

Andegarensis Bast. . . . 204

Andrewsii Tratt. . . . 125

Andrzeiowscii Besser. . . 583

Andrzeiouskii Boreau. . . 589

Annesiensis Déségl. . . . 584

anserinaefolia Boiss. . . . 103

apricorum Ripart. . . . 524

approximata Déségl. . . 255

Arabica Crépin . . . . 519

arborea Pers. . . . . . ร

arcana Déségl. obs. . . . 2:50/1

Arduennensis Crépin . . . 563

arenaria Bieberst. . . . 94

arenivaga Déségl. . . . 43

arguta M.-Pur. . . . . 253

- Steven . . . . 501

aristata Lapeyr. obs. . . 95

armata Steven. . . . . 158

armatissima Déségl. et Rip. 188

Armena Boiss. . . . . 549

Armidae W ebb. . . . . 196

arvatica Puget. . . . . 509

arvensis Huds. . . . . 19

- pl. auct. . . . . 20

- Roth. . . . . . 258

— var. Ayreshirea Ser. . 14

- - bibracteata Ser.

- Dracteata G. et G.

13

18

- Candolleana Thory

14

- - hybrida Lindl

12 arvensis var. multiflora Boreau 15

- var. obtusata Ser. . . 17

- - ovata Desv. . . . 21

_ - parvifolia de II.-Don. 19

- - prostrata Thory. . 14

- - umbellata Godet . 15

arvensis-gallica Gremli . . 45

arvina Krocker . . . . 44

- Lloyd. . . . . . 49

aspernata Déségl. . . . 214

aspratilis Crépin . . . . 214

assimilis Déségl. . . . . 60

atropurpurea Brot. . . . 52

atrovirens Viv. . . . . 12

Aucheri Crépin . . . . 553

Aunieri Cariot. . . . . 225

australis Kerner . . . . 595

Axmanni Gmel. . . . . 42

Ayreshirea hort. brit. . . 1/4

Bukeri Déségl. . . . . 276

Balansaen Déségl. . . . 252

Baldensis Kerner, obs. - 20

Balearica Desfont. . . 118

balloniana Herm. . . . 74

balsamica Besser . . . . 520

Baltica Roth. . . . . . 90

Banksiae R. Br. . . . . 57

Bellavallis Puget . . . . 252

Beggeriana Schr. . . 18

Belgica Brotero . . . 58

Bengalensis Pers. . . . 52

- var chinensis Pers. . 52

Besseri Tratt. . . . . 69

Libracteal Batés. . . 15 e33

liricolor lacquin . . . 268

Biebersteiniana Tratt. . 290

Billetii P'uget. . . . . $5 / 4$ 
biserrata Mérat . . . No 195

blanda Jacquin . . . . 97

- Brotero. . . . 58

Blondaeana Ripart. . . 281

Boissieri Crépin . . . 551

- b. hispidula Boiss. . 552

Boraeana Béraud . . . . 49

borealis Tratt. . . . . 67

Boreykiana Besser . . . 267

Borkhausenii Tratt. . . . 574

Borkhausii Gaud. . . 25 j

Borreri Woods . . . . 275

Boullui Gand. . . . 515

Boverneriana Lag. et de la Soie 260

Urachyata Déségl. . . . 253

brachypoda Déségl. et Rip. . 193

bracteata IV endl. . . . 53

- var. scabricaulis Lindl. 55

bractescens Woods . . . 245

brevistyla var. a. DC. . . 29

- - b. DC. . . 25

- var. Ianceolata Tratt. . 25

Broteri Tratt. obs. . . 15

Brownii Tratt. . . . . 9

Brunonii Lindl. . . . . 9

- var. arborea Ser. . . 5

- - nudiuscula Lindl. . 9

Bungeana Bois. et Buhse. . 102

Cabulica Boiss. . . . 106

- var. latispina Boiss. . 106

eaesia Smith . . . . . 237

- var. incana llook. . 247

Caledoniae Borrer. . . . 145

calycina Bieberst. . . . 157

Camberiensis Déségl. obs. e. 562 campestris Du Mort. . . $\mathbf{2 8 9}$

- Fries. . . . 238 campestris var. myriacantha

Wallr... No 85

- yar. pimpinellifolia

Wallr. . . . . 67

Canariensis Déségl. obs. . 524

Candolleana var.elegans Thory $\mathbf{8 0}$

canescens Baker . . . . 247

canina L. . . . . . 147

- Mandon. . . . . 172

- Auct. . . . . 177

- var. aciphylla Lindl. . 168

- - ambigua Ser. . . 171

- Andegavensis Desp. 204

_ - Du Mort. . . 211

- - arvatica Baker . 509

- b. Bieberst. . 175

- Bakeri Baker . . 276

- - biserrata Cheval. . 195

- - Borreri Baker . . 273

— collina Boissier . . 203

- - Gr. et Godr. . 258

- - Reuter . . . 25 5ั

- coriacea Boissier . 156

- coriifolia Baker. . 248

- - dumalis Baker . 177

- - dumetorum Desv. . $23 \%$

- - fastigiata Desv. . 25

- - glandulifera Woods. 204

- glandulosa Rau . . 177

- - - Grenier . . . 204

- - glauca Desv. . . 157

- - glaucescens Desv. . 148

- - grandidentata Desv. 204

- - hirtella Gr. et Godr. 204

- - hispida Desv. . . 204

- _ b. Boreau . . 258

- - c. Boreau . . 267

— - incana Baker . . 247 
canina var. insignis Gren. No 182 - - intermedia Desv. . 212 - Lejeunii Du Mort. 211 - - leucantha Delast.obs. 252 — - - Guépin . . 252 - macrocarpa Cheval. 192 - Malmundariensis Cheval. . . 171 — - Neratiana Ser. . 195 - - nitens Desv . . . 149 - - obtusifolia Desv. . 252 - - pilosiuscula Ser. 262,501 - - ramosissima Bl. et Fing . . . 161 - - rotundifolia Desv. 212 - - sarmentacea Godet. 177 - - senticosa Rehb. . 135 - - squarrosa Ser. . . 175

— - stipularis Cheval. . 177

- - suberistata Bak. . 140

- - surculosa Smith. . 202

- - sylvestris Roth. . 19

- - tomentella Bak. . 272

- - urbica Baker . . 256

_ - vulgaris b. Mutel . 155 canina-gallica Gremli. . . 45 - - Timb.-Lagr. . 44 capnoides Kerner . . . . 568 capreolata Niel obs. . . . 14 Carelica Fries. . . . . 9ב̆ Carioti Chabert . . . . 169 Carolina var. laevis Ser. . 118 caryophyllacea Besser. . . 567 Cancasica Bieberst. . . . 245 celsicola Arv-Touv. . . . 141 centifolic L. . . . . 66 cerasifera Timb.-Lagr. . . 261 cerea Roessig.
Chaberti Déségl. .

No 220

Chaboissaci Grenier . . 185

Chailletii Déségl. obs. B. . 562

chamaerhodon Villars. . . 67

Chapusii Godet . . . . 258

Chavini Rapin. . . . . 199

Cheriensis Déségl. . . . 515

Cherokensis Don . . . 40

Chinensis Jacquin. . . . 52

chlorophylla Ehrh. . . . 268

ciliato-petale Besser . . . 597

- Godet . . 581

— Koch. . . $\mathbf{5 8 9}$

rinerascens Du Mort. . . 555

Carjol. . . 246

- var. intricata Du Mort. 578

cinerea Rapin . . . . . 259

- Swartz ..... 95

cinerosa Déségl. . . . . 246

cinnamomea L. . . . . 95

- Gmel, obs. 1 . . 95

- Herrm. . . . . 119

- Karel. et Kir. . . 116

- Mérat . . . 97

- var. b. Lindl. . . . 95

_ collincola Ser. . . 95

- - foecundissima Koch. 95

— - glauca Desv. . . 128

- - globosa Desv. . . 97

— - latifolia Ser. . . 95

- - majalis Rau. . . 95

- - oblonga Desr. . . 128

cladoleia Ripart . . . . 180

clivorm Scheutz . . . . 256

Clotildea Timb.-Lagr. . . 24

Clusiana Bouv. . . . 401

clynophylla Thory . . . 54

coerulescens Kerner . • . 12 
collina Jacquin - . . No 258

- Boreau . . . . 258/1

- DC. . . . . . $25 ว$

- Fries. . . . . 234

- Lloyd. . . . 4 . $258 / 2$

- Schrank . . . . 67

- var a. Thory: . . . 258

-. - 3. el \% Woods . . 256

- constricta Guép.obs. 247

- Deseglisei Du Mort. ّコ̆0

- - dumelorum Thory . 253

- - fastigiata Thory. . 25

- - flexuosa Du Mort. 297

- - foetida Thory . . 558

- - leucantha Thory . 252

- obtusifolia Du Mort. 252

— urbica Du Mort. . 256

collincola Ehrh. . . . . 95

collivaga Cottet . . . . 573

commutata Scheutz . . . 292

comosa Ripart. . . . . $52 \%$

complicata Grenier - . . 158

concinna Lag. et Pug. obs. 2. 272

condensata Pug. obs. . . 204/3 confusa Puget. . . . . 585

conica Chabert. . , . . 䛃

consanguinca Gren. . . . 285

consimilis Déségl. . . . 71

conspicua Boreau . . . 16

controversa Ripart obs. . . 281

- Leman. . . . 21

cordifolia Host. . . . 58

- Chabert . . . 60

coriacea Crépin . . . 156

coriifolic Fries . . . 245

coronata Crépin . . . . 91

- var. subnuda Crépin. 91

coruscans Waitz . . . 111 corymbifera Borkh. - . No 248

corymbosa Bast. . . . 16

Cotteti Puget . . . . 287

crassifolia Wallm. . . 245

Cremsensis Kerner . . . 592

Crepiniana Déségl. . . . 142

Cretica Tratt. . . . . . 557

cucumerina Tratt. . . 41

curticole Puget . . . . 185

curvula Timb.-Lagr, obs. 4. 252

cuspidata Bieberst. . . . 569

cuspidata Auct. Gall. . 570

cuspidatoides Crépin . . . 570

— var. b. . . . 570

cymosa Tratt. . . . . 58

Czackiana Besser. . . .

Daënensis Boissier . . 105

Dahurica Pallas . . . 100

Damascena Miller. . . . 66

- var. subalba Red. . 7

decipiens Boreau . . . 56

decora Kerner. . . . . 296

Delasoiei Lag. et Pug. . 140

densa Timb.-Lagr. obs. . . 546

depressa Gremli . . . . 282

Deseglisei Boreau. . . . 230

dichroa Lerch. . . . . 83

Didaensis Boissier. . . . $\mathbf{5 4 8}$

diminuta Boreau . . . 544

dimorpha Besser . . . 579

- Grenier. . . . 5ă6

dimorphacantha Nartinis. . 552

disereta Ripart. . . . 140

dissimilis Déségl. . . 116

diversifolia Vent. . . . 52

Djimilensis Boiss. . . . 229

Doniane W Woods . . . . 87 


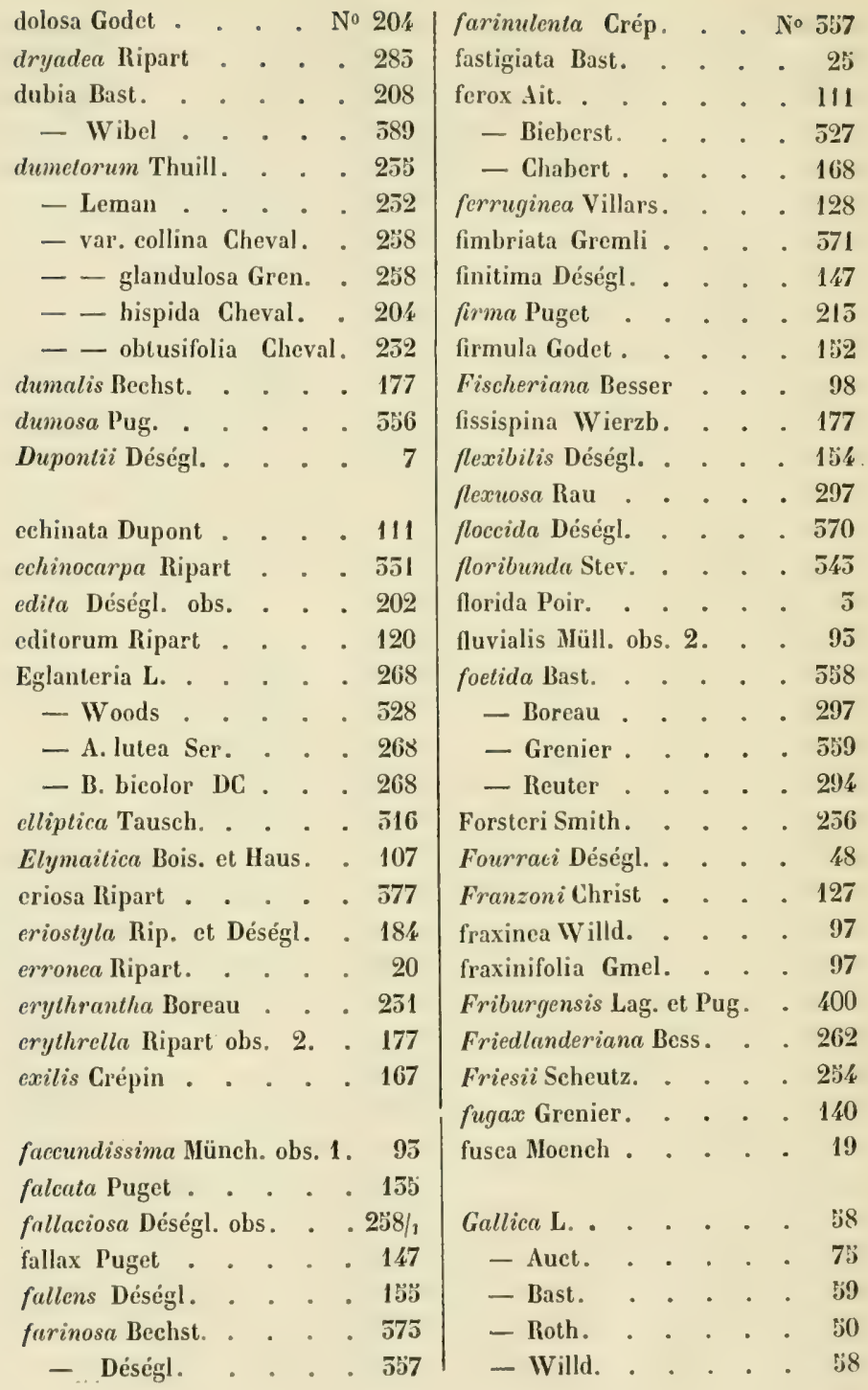


Gallica var. A. DC. . . No ๖̊ | glutinosa Sibth. . . . No557

- - arvina Ser. . 4 44 - Dematra. . . . 405

- - hispida Ser. . . 50 - Schultz . . . . 505

- - hybrida Ser. . . 42 - var. B. Rehb. . . . 538

- - inermis Ser. . . 52 - tomentella Bois. . 108

- marmorea Ser. obs. 58 Gmelini Bunge. . . . . 117

- - officinalis Ser. . . 58 Godeti Grenier. . . . . 286

- - pygmaea Boiss. . . 60 Gombensis Puget . . . . 404

— - velutinaeflora Cariot b5 Gorenkensis Besser. . . . 155

- - versicolor Tratt. . 58 gracilis Woods. . . . . 88

Gallico-canina Reuter. . . 224

Gallico-serpenti Kirschl. .42,44

Gallico-stylosa Timb.-Lag. 44

Gallico-umbellata Rapin. . 285

gallicoides Déségl. . . . 22

Gaudini Puget. . . . . 405

geminata Rau. . . . . 47

Genevensis Puget . . . 571

gentilis Sternb. . . . . 82

Gisleri Puget . . . . . 560

glaberrima Du Mort. . . . 178

glandulifera Roxb. . . . 5

glandulosa Bellardi . . . 126

- Besser . . . . . 500

- Boreau . . . . . 214

— Déségl. . . . . . 129

- Kirschl. . . . . . 561

glauca Villars . . . . 157

- Desfonl. . . . . 128

- Desy. . . . . . 128

- Dierb. . . . . . 19

- Schot. . . . . 218

glaucescens Desv. . . . 148

- Wulf. . . , 128

glaucophylla Ehrh. . . . 271

— Winch. . . . . 177

globatu Déségl. . . . . 257

globularis Franch.

165

granatensis Willk. . . 85

grandiflora Wallr. . . . 504

- Lindl. . . . . . 75

graveolens G. et G. - . 197, 114

- var. Corsica G. et G. 512

- - eriophora Gren. . 514

- - nuda Gren. . . 515

Grenierii Déségl. . . . 598

Guepini Desv. obs. 2. . . 247

Gustensteinensis Cat. hort. . 128

IIaberiana Puget . . . . 221

haematodes Boiss. . . . 227

Ialleri Krocker. . . . . 19

IIampeana Griseb. . . . 128

Hardii Paxton. . . . . 54

Ileckeliana Tratt. . . . . 559

Heldreichii Bois. et Reut. 588

hemisphaerica Herm. . . 271

hemitricha Ripart obs. . . 256/3

herporhodon Ehrh. . . . 19

helerophylla Timb.-Lag. obs. 518

- Woods. . . . . 577

IIbernica Smith. . . . 145

- var. glabra Bak. . . 143

Ilispanica Bois. et Reut. . 198

- var. Nevadensis B. et R. 278

hispida Krock . . . . 123

- Borkh. . . . . 574 
hispida Münch. . . . No รั0 hispidocarpa Chabert. . . 294 hispidula Ripart olss. . . 250/2 hololeia Ripart. . . . . 152 horrida Fisch. horridula Déségl. obs. . . 168 humifusa Tratt. humilis 'Tausch. IIungarica Kern. hybrida Schl. hystrix Lindl.

- Leman

Iberica Stev.

Ilseana Crép. imitala Déségl. immitis Déségl. implexa Grenier imponens Ripart incurnata Miller incerta Déségl. obs. inclinata Kern. incomparabilis Chab. . inconsiderala Déségl. inconspicue Déségl. Indica $\mathbf{L}$.

- var. acuminata Ser.

- - humilis Ser.

- Lawranceana Ser.

_ - longifolia Lindl.

- - semperflorens Ser. inermis Bieberst. . - Miller. inodora Fries.

- Ilook.

- Rehb.

- Timb.-Lag. obs. insidiosa Ripart insidiosa Gren. . . . . 577 insignis Déségl. et Rip. . . 182 intercalaris Déségl. . . . 120 intermedia Carr. . . . . 2 - Chab. . . . . 45 interveniens Déségl. . . . 209 intricata Gren. . . . . 159

- Crép. . . . . . 578

— Déségl. . . . . . 121

intromissa C’ép. . . . . 378 involucrata Roxb. . . . 54 involuta Smith. . . . . 90

- Winch. . . . . 87

- var. Doniana Bak. . . 87

- - gracilescens Bak. . 87

- - lacvigata Bak. . . 90

- - Moorii Bak. . . 90

- - occidentalis Bak. . 90

- Robertsoni Bak. . 87

- - Sabini Bak. . . . 86

- - Smithii Bak. . . 90

- - Wilsoni Bak. . . $\$ 9$

Isaurae Tratt. . . . . . 554

Iwara Sieb. . . . . . 112

jactata Déségl. . . . . 241

Jordani Déségl. . . . . 5̄lร

Jundzilliana Bess. . . . 500

Jundzilli Bess. . . . . . 500

Jurana Déseggl. obs. g. . $\overline{5} 62$

Kamschatica Vent. . . 110

- Thory. . . . . 111

- var.ferox Ser.... 111

Klukii Bess. . . . . . 520

- Borcau. . . . . 521

Kotschyana Boissier . . . 109 


\section{( 541$)$}

lacerans Bois. et Buhse. . No 104 lactiflora Déségl. . . . . 543 ladanifera Timb.-Lag. . . 518 laevigata Michx. . . . . 40 laevis Boullu. . . . . 5 lagenaria Villars . . . . 124 Laggeri Puget. . . . . 226 latebrosa Déségl. . . . . 210 latispina Boiss. . . . 106 Laurentiae Andr. . . . . 5 ! - var. subinermis Tratt. 51 Lawranceana Sweet ... . 51 laxu Retz... . . . . 99 Ledebourii Spreng. . . . 580 Lehmanniana Bunge . . . 103 Lemaitrei Ripart . . . . 204/2 Lemanii Boreau . . . . $\mathbf{5 4 6}$ Leschenaultiana Red. . . 1 leucantha Bieberst. . . . 290 - Bast. 252

- Loisel, obs. I. . . . 252 leucochroa Desv. . . . 29

- var. angustana Desv. . 23

_ B. Loisel. . . . 28

Libanotica Boiss. . . . . 557

Libertiana Tratt. . . . . 542

Lindleyana Tratt. . . . 5/4 livescens Besser . . . . 502 — var. major Bess. . . 502 livida Host. . . . . . 128 longepedunculata de la Soie. 129 longicuspis Bertol. . . . 11 longifolia Willd. . . . . 55 Luciae Fr. et Roch. . . . 4 lucida Koch. . . . . . 96 Lugdunensis Déségl. . . . 514 - var. macrocarpa. . . 514 lurida Andr.
Lusseri Lag. et Pug. . No 528

lutea Dalechamps . . . . 268

- Brot. . . . . . 271

lutescens fl. albis Link. . . $\quad 75$

lutetiana Leman. . . 147

Lyellii Lindl. . . . . 56

Macartnea Dum.-Cours . . 5:) macrantha Desp. . . . . 年6:3 macroacantha Rip. . . . 151 macrocarpa Mérat. . . . 192 - Boiss. . . . . 191 macrophylla Lindl. . . . 114 macropoda Ripart obs. . . 84k majalis Herm. . . . . 93 Malmundariensis Lej. . . 171 Mandoni Déségl. . . . 172 Maracandica Bunge . . . 269 Mareyana Boullu. . . . $53 \%$ marginate Wallr. . . . 2₫8 Martini Gren. . . . . 201 Mathonneli Crép. . . . 70 medioxima Déségl. . . . 170 megalocarpa Déségl. . . . 191 melanocarpa Link. . . . $\quad 69$ mentita Déségl. . . . . 508 micans Déségl. . . . 5̋̈k micrantha Smith. . . . 5/22

- Boreau . . . . . 544

- DC. . . . . . 197

— var. A. Grenier. . . 542

- - B. Grenier . . 544

- liystrix Bak. . . 5 \&6

- Lemanii Bak. . . 546

- nemorosa Du Mort. j/42

- - permixta Gren. . 528

- - septicola Gren. . 529

- Valesiaca Christ. . 277 
mierantha var, vulgaris

Du Mort.

microcarpa Lindl.

- Besser

microphylla Roxb.

- Desfont.

- Willd.

minula Borcau. . . . . 596

mirabilis Déségl. . . . . ร̌4

mitissima Gmel. . . . 74

mixta Tratt. obs. . . . 119

- Chabert . . . . 48

modesta Ripart . . . . 27

mollis Smith . . . . . 589

- Ledeb. . . . . 580

- var. b. coerulea Bak. . 589

mollissima Fries . . . . 589

- var.Arduennensis Dum. 563

- - B. Lej. et Court. . 563

- - pseudo-rubigin. Bak. 563

Monspeliaca Gou. . . . 122

montana Vill. . . . . . 129

- DC. . . . . . 565

monticola Déségl. . . . 164

- A. Reuteri Rap. . . 157

- B. alpestris Rap. . . 144 montivaga Déségl. . . . 164 moschata Miller. . . . .

- DC. . . . . 10

- Lapeyr. . . . . . 12

- Mutel. . . . . . 15

- var. rosea Ser. . . 7

mucronulata Déségl. . . . 152

multiflora Thunb. . . . 5

- var. A. Ser. . . . 5

multivaga Déségl. obs, d. . 562

Murithii Puget . . . . 102

muscose Ait. obs. . . . 66 mutica Müll. . . . . No 95

myriacantha DC. . . . 85

- var. pumila Desv. . 85

myrtifolia Hall. . . . . 507

Nebrodensis Guss. . . 278

neglecta Ripart. . . . . 510

nitens Desv. . . . . . 149

nivalis Don. . . . . . 800

nivea Dupont . . . . . 7

- DC. . . . . . 40

nemorivaga Déségl. . . 295

nemoralis Leman. . . 542

nemorosa Libert . . . . $\mathbf{5 4 2}$

nemorum Ripart . . . . 56

nitidula Besser. . . . . 501

nuda Woods . . . . . 142

oblonga Déségl. et Rip. . 179

obscura Puget, obs. . . . 256/5

obtusa Ripart, obs. . . 204/5

obtusifolia Desv. . . . . 252

occulta Crépin. . . 209,210

OEderiana Tratt. . . . . 68

omissa Déségl. . . . 586

Onensis Kerner . . . 217

opaca Grenier. . . . 258

opacifolia Chabert. . . . 57

operta Puget . . . . . 550

oplisthes Boissier. . . . 154

opsostemma Ehrh. . . . 5

oreinosa Ripart . . . . 119

Orientalis Dupont. . . . 108

- hort. Genev. . . 255

- var. Olympica Clem. . 108

Orphanidis Bois. et Reut. . 541

orthacantha Kern. . . . 155

ovata Lejeune . . . . . 21 
oxyacantha Bieberst. - No 76 oxyphylla Ripart . . . . 152 oxyodon Boissier . . . . 79 Ozanonii Déségl. . . . 73 parviflora Ehrh. obs. . . 66 parvifolia Pall. . . . 85 parvula Sauz. et Maill. . . 27

- Grenier obs. . . . 544 pellita Ripart . . . . . 555 pellucina Arv.-Touv. . . 70 pendulina Ait. . . . 125 - Rchb. . . . . 124 pennina de la Soie . . 158 permixta Déségl. . . . . 528 permutata Ripart. . . . 58 Perrieri Songeon. . . 151 persicifolia hortul. . . . 55 Perusiana Timb.-Lag. . . 598 petrogenes Ozan. obs. . . 75 Phoenicea Boissier. . . . 6 Phrygia Boissier . . . 270 pilosa Dupont . . . . . 255 pimpinellifolia L. . . . 67

- Auct. . . . . . . 68

- Hook, et Th. . . . 77

- Pall. . . . . . 75

— var. A. Duby. . . . 62

- affinis Koch. . . 67

- Altaica Ser. . . . 78

- B. Kirschl. . . . 68

- - C. Lloyd. . . . 84

- - grandiflora Ledeb. . 75

- - inermis DC. . . 74

- - involuta Ser. . . 90

- Mariaeburgensis Ser. 68

- - microcarpa Ser. . 69

_- mitissima Koch. . 74 pimpinellifolia var. montana Kirschl. . . . No 74 - - myriacantha Ser. . 85 _ oxyacantha Pall. . 76 - - pilosa Ser. . . . 90 - rosea Ser. . . . 67

- tomentella Boiss. . 68 platyphylla Rau . . . 258 platyphylloides Déségl. et Rip. 259 Polliniana"Spreng. . . 45 Pollinaria de Pronv. . . 40 pomifera Herrm . . . . 599 — Lec. et Lam. . 595 Pommaretii Pug. obs. . . $\mathbf{3 4 2}$ pomponia DC. obs. . . 66 poteriifolia Besser. . . 68 Pouzini Tratt. . . . 197 praecox Boullu. . . . 578 praeterita Rip. obs . . . . 281 procera Salisb. . . . 267 properata Boullu . . . $\mathbf{3 7 8}$ propinqua Déségl. obs. F. . 562 prostrata DC. . . . . 44 - var. obtusiuscula. . . 14 protea Ripart . . . . 284 provincialis Ait. . . . . 59 - Bieb. . . . . 527

- Willd. obs. . . . . 59 — var. parvifolia Bieb. . 85 proxima Cottet. . . . 403 pruinosa Don. . . . . 566 pseudo-flexuosa Ozan. . . 298 pseudo-lucida Besser. . . 96 pseudo-rubiginosa Lejeune . 565 pseudo-sepium Call. . . . 503 psilophylla Rau. . . . . 224

- Boreau. . . . . 225

— Déségl. . . . . 282 


\section{( 544$)$}

Pugeti Boreau.

pulchella Willd obs.

- Boreau.

- Woods. . . . . 577

pulverulenta Bieberst.

- Guss.

pumila Lin. fil

- Jacquin. .

- var. B. Poll.

- - hispida Rau.

punicea Miller.

purpurascens Rip. obs.

pusilla Maurit. Cat.

pustulosa Bertol. . . . . ว๊̄ 8

Puymaurea Gren. obs. . . 547

pygmaer Bieberst. . . . 61

- var. Olympica Clem. . 557

Pyrenaica Gou.

pyriformis Déségl.

ramealis Pug. obs.

ramosissima Rau.

rampant Reyn.

ramulosa Godr.

Rapini Boissier.

Ratomsciana Bess.

Raui Tratt.

Ravaudi Boullu obs. 4 .

recondila Puget.

recurva Roxb. olss .

Regeliana Lind. et And.

repens Scop.

resinosa Sternb.

- Boreau.

- Lejeune obs. 2

resinosoides Crépin.

reticulata Kerner.

Reuteri Godet.
No 294

66

560 558

63

50

45

50

268

$204 / 4$

51

8

61

$12 j$

$2 \% 1$

$256 /$ 161

$$
177
$$

271

$26 k$

204

129

401

41

111

19

594

593

5ㄴ)

593

500

157
Reuteri var. adenophora Gr. No 140 - B. Reut. . . . $\mathbf{1 5 8}$

- - intermedia Gren. 158

- - transiens Gren. . . 159

reversa W. et Kit. . . . 81

Reynieri Hall. . . . 129

Rhactica Kerner. . . . . 159

rhyncocarpa Rip. obs. 5 . . 177

lhodani Chabert. . . . 42

rigida Willd. . . . . . 527

rigidula Lag. et Pug. . . 238

Ripartii Déségl. . . . . 84

rotundifolia Rau. . . . . 547

- ped. laevib. . . . . 547

Rousselii Ripart. . . . 208

Roxburgii Tratt. . . . 115

mbeila Smith. . . . . \$0

- Godet. . . . . 82

rubelliflora Ripart. . . 174

rubescens Ripart. . . . . 175

rubicunda Hall. . . . . 128

rubiginosa Ait. . . . . 528

- Auct. . . . 524,529

- Bicberst . . . . 545

- Coss.. . . . . 198

- Guimp. . . . . 516

- Lin. . . . . 524,528

- Wahl. . . . . $\overline{5} 13$

- var. Arabica Boiss. . 519

- A. vulgaris Rau. . 528

- - B. Mutel. . . . 542

- C. Mutel. . . . 512

- C. Rapin. . . . 272

- caryophyllacea Ser. 567

- - comosa Dum. . . $52 \%$

- Cretica Thory. . . 557

- D. Bechst. . . . 513

- _ echinocarpa Gren. . 551 


\section{$(545)$}

rubiginosa var. flexuosa

Lindl.

- foetida Desy.

- flore albo Poll. . . $\mathbf{5 0 7}$

- - grandiflora God. . 312

- - grand. Ser. . . 304

- glabra Rau. . . . 515

- - Iberica Boiss. . . 554

- - inodora Lindl. . . 273

- - micrantha Lindl. . 542

- - minor Ledeb. . . 527

- nemoralis Thory. . 342

- nemorosa Dum. . 542

- parviflora Saint-Am. 551

- - parvifolia Ser. . 278

- - permixta Bak. . . 528

- - resinosa Wall. obs. 2. 529

- - rotundifolia Lindl. . 547

- - sepium Saint-Am. . 503

- - spinulifolia Ser. . $\mathbf{5 6 2}$

- - sylvicola Bak. . 553

- - triflora Willd. . . 526

- - umbeliata Bourg, obs. 524

— - Lindl. . . . 526

rubra Lam. . . . . . 58

rubrifolia Vill. . . . 128

— var. glandulosa Ser. . 129

- - hispidula Ser. . $\quad 128$

- - laevis Ser. . . . 128

- montana Gaud. . 129

- - pinnatifida Ser. . 137

— - Reuteri Godet. . . 157

migosa Thunb. . . . 111

- var. glabriuscula Crép. 111

rupestris Crantz. . . . . 119

Ruprechti Boissier. . . . 587 ruralis Déségl. . . . . 62

Ruscinonensis Déség, et Gren. rustica Leman. . . . No 23

rusticana Déségl. . . . . 17

Sabauda Rapin. . . . 92

Sabini Woods. . . . . 86

- var. B. Smith. . . . 87

- Doniana Lindl. . . 87

- - gracilis Rchb. . 88

Salaevensis Rapin. . . . 150

- Verlot. . . . . 129

- var. longepedunculata. 129

- sanguisorbella Chr. . 129

Salvanensis de la Soie. . 528

Sambrancheriana de la Soie. 140

sancta Richard obs. . . . 66

sanguisorbifolia de la Soie. $\quad 129$

sarmentacea Woods. . . . 177

saxatilis Steven. . . . . 263

- Boreau.. . . . 214

- hort. Genev. . . . 98

scabriuscula Smith. . . . 372

scandens Miller. . . . . 13

- Brot. obs. . . . 15

- Mœnch. . . . . . 19

Schergiana Boissier. . . 244

Scheutzii Christ. . . . 590

Schimperiana H. et Steud. . 8

Scholtiana Ser. . . . 218

Schultzii Ripart. . . . 146

Scopoliana Tratt. . . . . 93

Scotica Miller. . . . . 67

semi-glabra Rip. obs. . . 256/,

semi-glandulos $\alpha$ Rip. obs. . 281

semperforens Willd. . . 32

- Desv. . . . . . 58

- var. longifolia de Pronv. 35

- - minima Sims. . . 51

10 sempervirens $\mathrm{L}$. . . . 12 


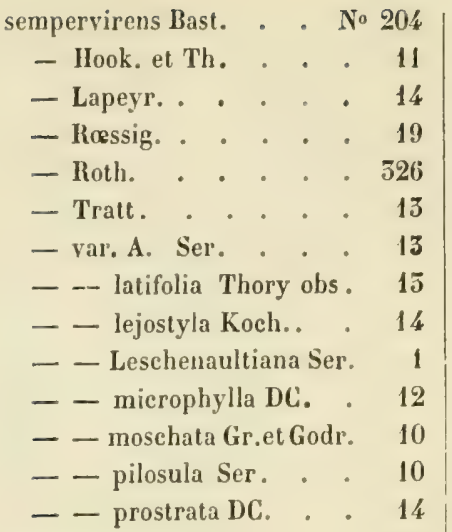
senticosa Achar. . . . 150 seperina Sauz. et Maill. . . . 21 sepium Thuill. . . . 503

- Rau. . . . . 248

$-\mathrm{Sw}$. . . . . . 245

— var. agrestis Carion. . 507

— - alba Desv。 . . . 507

- - intermedia Desv. . 204

- - myrtyfolia Hall. . 507

- - nitens Desv. . . 195

_ - parviflora Bast. . 507

- - pubescens Rap. . 503

— - rosea Desv. . . 50 ว

- - sphaerocarpa Cariot. 510

- - stipularis Desv. . 177 septicolt Déségl. . . . . 329 Seraphini Viv. . . . . 512 sericea Lindl. . . . . . 115 Seringeana Godr. . . 570 serrulata Chabert obs. . . 175 serpens Wibel. • . . . 19 serpenti-canina Kirschl. .25,25 setulifera Timb.-Lagr. . . 293 Sherardi Smith. . . . 581
Siberica Tratt. . . . No 73

Sicula Tratt. . . . . . 540

Silverhielmii Schr. . . . 101

similata Pug. . . . . . 273

simplex Scop. . . . . . 95

Sinica Murr. . . . . . 40

- Tratt. . . . . . 32

solstitialis Besser. . . . 233

- Gren. . . . 245

— var denudata Giren. . 240

- - glandulosa Gren. . 259

Soongorica Bunge. . . . 250

speciosı Déségl. . . . . 299

sphaerica Gren. . . . . 166

- var. aciphylla Gren. . 166

sphaerocarpa Pug. . . . 242

sphaeroidea Ripart. . . . 194

spinosissima L. . . . $\quad 68$

- Gou. . . . . . . $8 \overline{5}$

- Pesneau . . . . . 96

- Tratt. . . . . . 84

- Wall. . . . 95

- var. flore roseo Ilerrm. 67

_- Pallasii Tratt. . . 75

- - pilosa Lindl. . . 90

- - pusilla Woods. . 68

spimulifolia Demat. . . . 562

— var. Dematratiana Thory. 262

- - denudata Gren. . 288

- - Foxiana Thory. . 563

_ - vestita Rapin. . . 565

squarrosa Rau. . . . . 175

stenocarpa Déségl. . . . 186

stephanocarpa Déségl. et Rip. 189

stipulacantha Bast. . . . 212

stipularis Mérat . . . . 177

stylosa Desv. . . . . . 25

- Gand. . . . . . 23 


\section{$(547)$}

stylosa Mérat - . . No 21

- var. bracteata Saint-Am. 15

- - corymbosa Desv.obs. 252

- Desvauxiana Ser.

- gallicoides Bak. . 22

- - glandulosa Ser. . 50z

- L lanceolata Ser.

- - leucochroa Ser. . 25,29

- - trivialis Gren.

suavifolia Light. . . . . 528

suavis IVilld. obs. 2. . . 8 !

subcristata Bak. . . . . 145

subdola Déségl. . . . . 521

Suberli Ripart. . . . . 207

subglobosa Smith. . . . 581

- var. macrocarpa Déségl. 581

subinermis Chabert . . . 46

subintrans Gren. . . . 578

submitis Gren. . . . . 250

subolida Déségl. . . . . 295

Sufferti Kirschl. . . . . 561

sulphurea Ait. . . . . 271

surculosa Woods. . . 202

S̄wartziana Fries. . . 147

sylvatica Tausch. . . . ร็

- Gater. . . . . 58

sylvestris Herrm. . . . 19

- Seguier . . . . 507

- Tabern..... . 248

sylvicola Déségl. et Rip. . 535

sylvularum Ripart . . . 181

syntrichostyla Ripart. . . 150 systyla Bast. . . . . . 2ö

- Woods... . . 15

- var. lanceolata Lindl. 25

tenuiglandulosa Mérat. $\mathbf{3 2 6}$

terebenthinacea Besser. . . 564 tcrebenthinacea Déségl. . No 295

- Gren. . . . . . 245

ternata Poir . . . . 40

Thomasii Pug. obs. . . . 293

Thunbergii Tratt. . . . 5

thyrsifora Leroy . . . . 2

Timeroyi Chabert. . . . 219

tomentella Leman . . . . 272

- Bak. . . . . . 276

- var. Uriensis Chr. . 559

tomentosa Smith . . . . 577

- Woods . . . . . . 570

- var. B. Smith . . 572

- - cinerascens Crép. . 533

- - dimorpha Dum. . 579

- - dumetorum Gaud. . 272

- - E. Woods.. . 581

- - farinosa Ser. . . $57 . \overline{5}$

— - foetida Ser. . . 508

- - incana Woods. . 247

- - marginata Rapin . 288

- - mollissima Dum. . 589

- - Ruprechti Boiss. . 577

_ - scabriuscula Bak. . 572

- - Seringeana Dum. . 570

— - Smithiana Ser. . 577

- - subglobosa Carion . 581

tomentoso-gallica Rapin . . 571

tortuosa IVierzb. . . . 204

Touranginiana Déségl. et Rip. 160

trachyphylla Rau . . . . 289

- Boreau . . . . 281

- Gren. . . . . . 288

- var. arvatica Dum. . 501

- Blondaeana Dum. . 281

- - campestris Dum. . 28!

- nuda Gren. . 281

transiens Kerner . . . . 159 
( 548 )

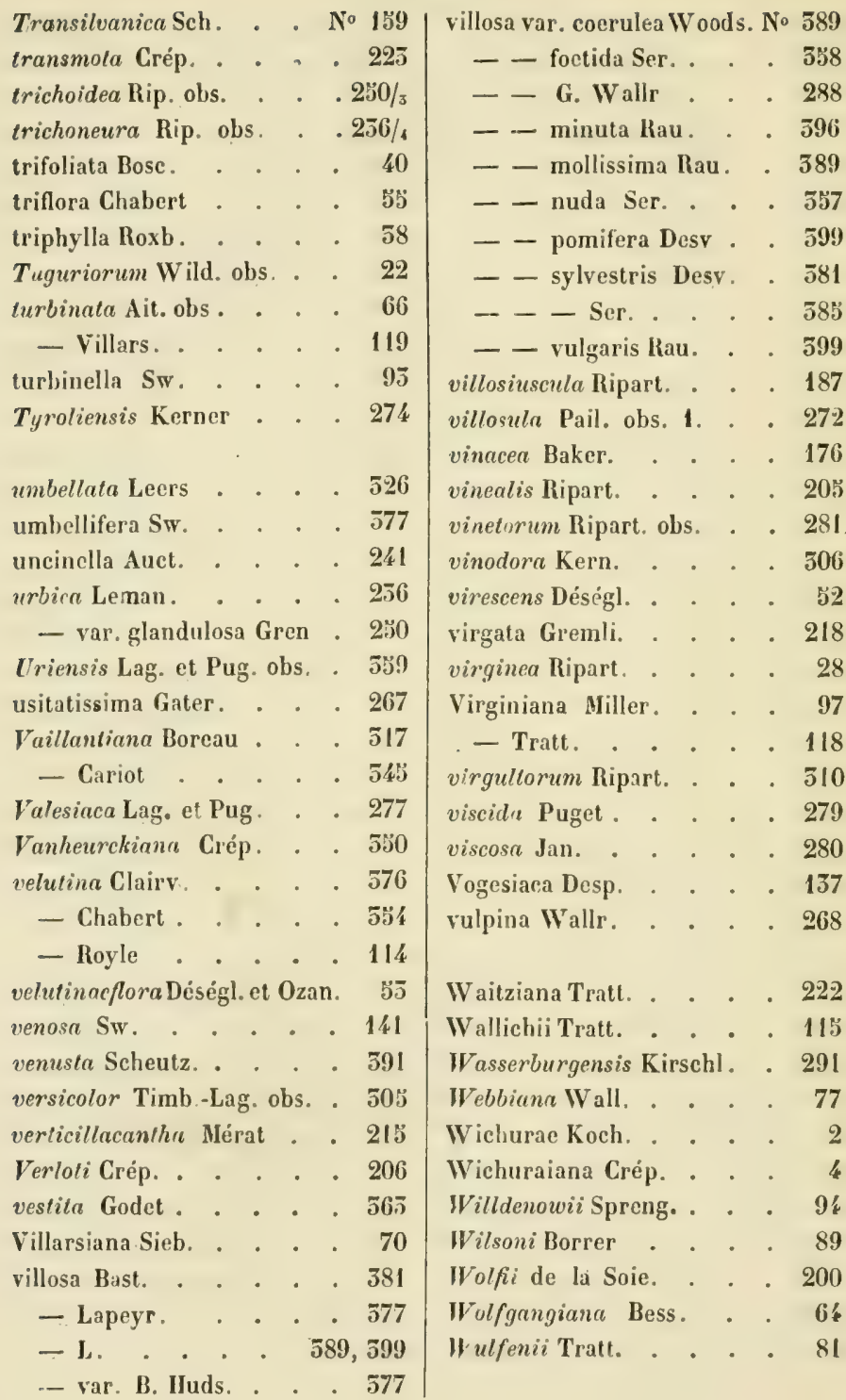




QL 145.R6 D4

Deseglise, Pierre A/Catalogue raisonne 0

35185000739951 


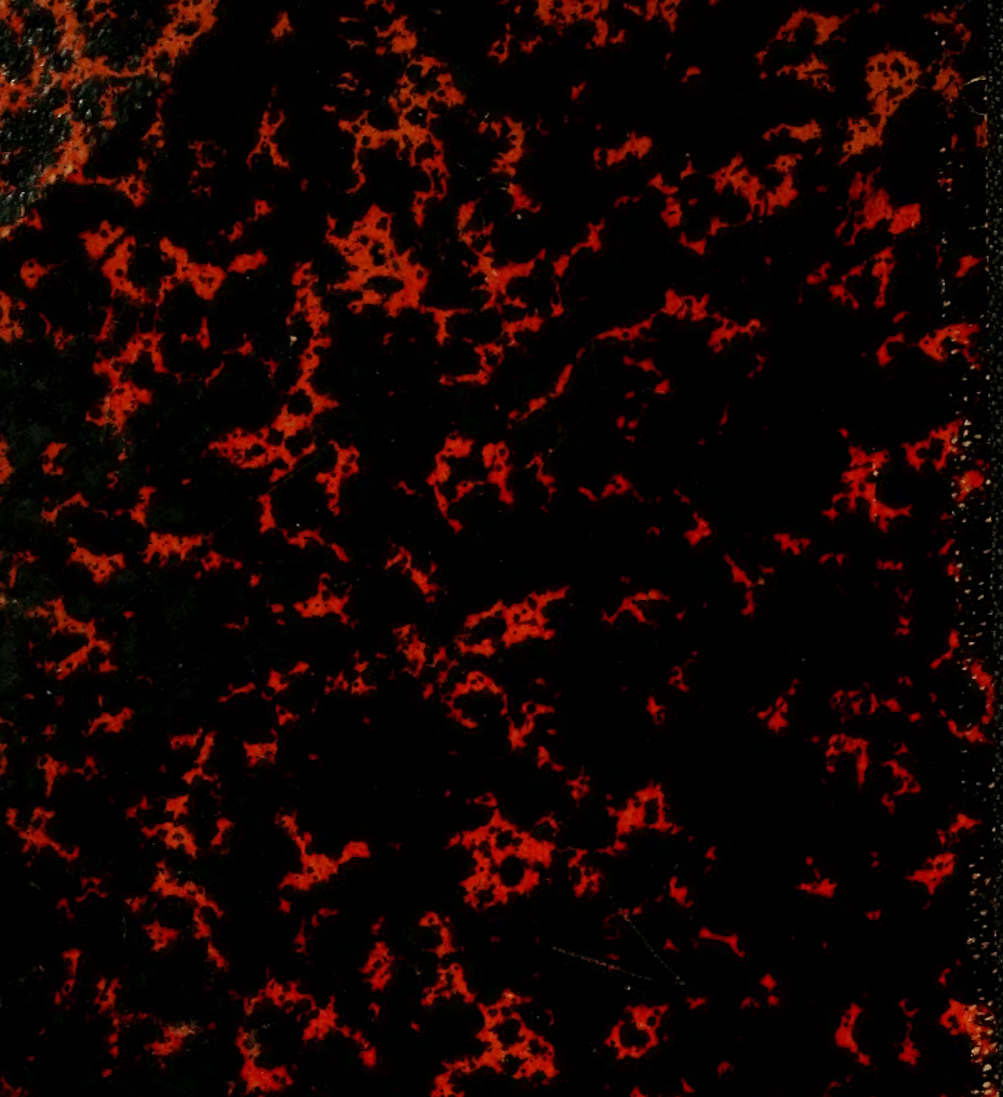

